s. 



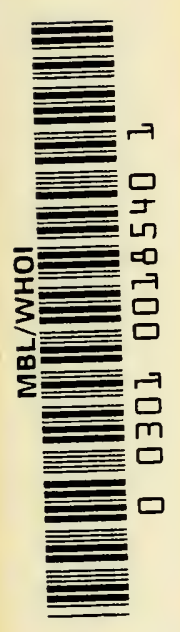





\section{PHYSIOLOGICAL MAMMALOGY}

\section{VOLUME I}

Mammalian Populations 


\section{Physiological Mammalogy}

VOLUME I

Mammalian Populations

CONTRIBUTIONS BY

JOHN B. CALHOUN J. J. CHRISTIAN

VOLUME ॥

Mammalian Reactions to Stressful Environments 


\title{
PHYSIOLOGICAL MAMMALOGY
}

\author{
EDITED BY \\ WILLIAM V. MAYER \\ Department of Biology, Wayne State University, Detroit, Michigan \\ and \\ RICHARD G. VAN GELDER
}

Department of Mammalogy, The American Museum of Natural Histary

New York, New York

\section{VOLUME I}

Mammalian Populations
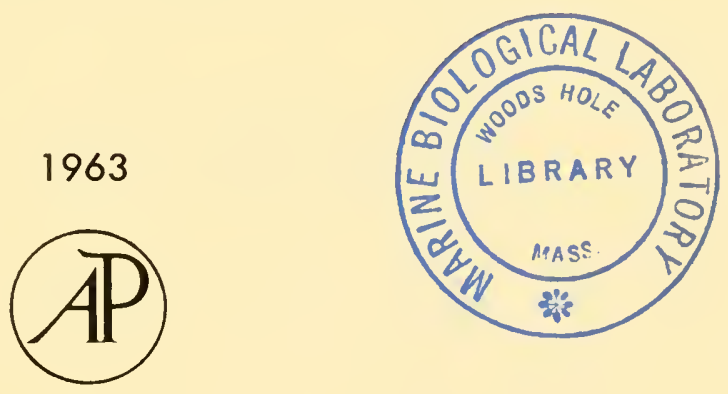

ACADEMIC PRESS-New York and London 
Copyright (C) 1963, By Academic Press Inc.

ALL RIGHTS RESERVED

NO PART OF THIS BOOK MAY BE REPRODUCED IN ANY FORM, BY PHOTOSTAT, MICROFILM, OR ANY OTHER MEANS, WITHOUT WRITTEN PERMISSION FROM THE PUBLISHERS.

ACADEMIC PRESS INC.

111 Fifth Avenue, New York 3, New York

United Kingdom Edition published by ACADEMIC PRESS INC. (LONDON) LTI).

Berkeley Square House, London W.1

IJBRARY of Congress CARD NUmble: 63-23:22

PRINTED IN THE UNITED STATES OF AMERICA 


\title{
Contributors to Volume I
}

\author{
JOHN B. CALHOUN \\ Laboratory of Psychology \\ National Institute of Mental Health \\ Bethesda, Maryland \\ J. J. CHRISTIAN \\ Division of Endocrinology and Reproduction \\ Research Laboratories, Albert Einstein Medical Center \\ Philadelphia, Pennsylvania
}





\section{PREFACE}

The field of mammalogy has, until very recently, been largely concerned with morphology and systematics; investigators have done little to bring together experimental work on the widely divergent mammalian types of which we have knowledge. The treatise "Physiological Mammalogy" was, therefore, conceived as a device to bring together the existing knowledge of an experimental nature on those animals usually regarded as "wild." Approximately fifteen themes, on which a considerable body of evidence has been accumulated over the years, provide the central organizing core around which various authors have been asked to make their contributions. The amount of material available on the non-classic laboratory animals has been difficult to synthesize; but the experts who were asked to contribute to the planned original single volume have proven so knowledgeable about the areas of their specialities and have made such a truly impressive survey of the experimental literature on their topics that it has become necessary to expand the work from the original single volume of relatively short essays to a minimum of three and perhaps even more additional volumes.

The plan of this work is such that anyone working with mammals will find it an indispensable reference. It is particularly valuable to experimentalists working with mammals in the areas of physiology, mammalogy, and ecology. Within these volumes will be found comprehensive essays on specific topics in physiological mammalogy, as well as a cogent analysis of the experimental field developing both what is known and what needs yet to be done. The research worker will find, in perusing these pages, challenging observations to which he might well address future researches. The student will find reference material and previous observations which will make these volumes useful as a baseline from which additional studies can be begun. Most of the authors have, in addition to providing a comprehensive review article, intercalated their own observations and commentary to the point where the articles themselves constitute original contributions to the field. This treatment provides a comprehensive analysis of the over-all specified topic.

These volumes will provide the investigator with information that will enable him to choose experimental animals previously little used in the laboratory because their specific physiological properties and behavior were formerly not well known. As a compendium on physiology of normally non-laboratory mammals, this treatise will be of value to anyone who works with manmals and to any experimental animal biologist. 
The first volume consists of two comprehensive articles dealing with the physiology of populations. Calhoun's "The Social Use of Space" presents many interesting new ideas on the behavior of animals in populations and the effects of grouping of individuals upon the physiology of the organism. Where many workers have thought of the experimental animal only as an individual apparently divorced from his environment and other members of the species, Calhoun points out the fallacy of this view in providing experimental data that demonstrate the effects of numbers of individuals on the behavior of each individual. Christian's article on population growth treats the problem largely from an ecological viewpoint in dealing with limiting factors of natural populations and population interrelationships.

Volume II will consist of three contributions dealing with natural populations and their adaptations to stressful environments. Dr. Charles Kayser treats the mammalian phenomenon of hibernation as a mechanism for avoiding periods of unfavorable environment, and Dr. Robert Chew deals with water balance in desert rodents. Inasmuch as reproduction is considerably affected by the environment, it too can be considered a process modified by environmental stresses, and is discussed in the second volume. Subsequent volumes will include articles on such topies as temperature and metabolism, physiological geneties, photoperiod, and orientation by echolocation. The Editors will conclude the series with a summary article on the phylogeny of physiology.

The Editors feel particularly fortunate in that they have received fine cooperation from outstanding authorities in the specific subject matter topics covered. It is the caliber of the individual author on which these volumes base their contribution to science; and while the Editors assume any responsibility for defects of organization or inadvertent errors, the credit for the success of the volumes, as a whole, rests on the individual contributors.

October, 1963

William V. Mayeli

Richard G. Van Geldek 


\section{INTRODUCTION}

The development of the biological sciences has been largely through the observational method. In the nineteenth century descriptive biologists came to occupy a preeminent and leading position in the field of biology. With the advent of the twentieth century, however, biology began to emphasize the experimental; and today, biology is an experimental science in practically all of its branches. However, experimentation in animal biology has very largely concentrated on a relatively few species which have come to be classic. These animals were selected because of their tractability and availability, and because of the fund of existing knowledge about them. Therefore, animal experimental biology has been largely oriented around the dog, cat, rabbit, mouse, guinea pig, and white rat. Despite the fact that again and again it has become obvious that there are wide varieties of metabolic patterns and inter-specific differences in physiology as well as morphology, the bulk of experimentation continues to be done with relatively few classic types. Despite this concentration of effort, over the years numerous researchers have investigated the use of different animals for experimental purposes, including mammals ranging from aardvarks to zebras.

The writings of these investigators, however, are scattered widely both in literature and in time, making it difficult for an experimental biologist to draw on the fund of already available knowledge about animals other than those most frequently used. The basic thesis guiding the preparation of "Physiological Mammalogy" has been to make available to the experimental biologist the wealth of data in the work of widely geographically scattered research scientists of diverse experimental interests.

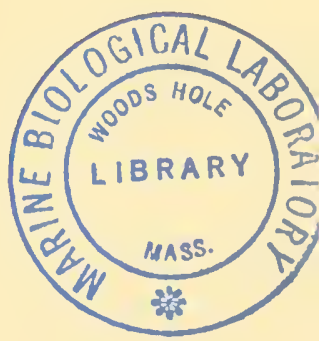





\section{TABLE OF CONTENTS}

LIST OF CONTRIBUTORS_.............................. V

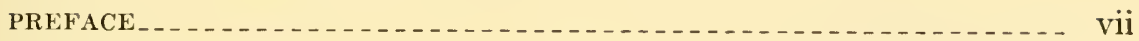

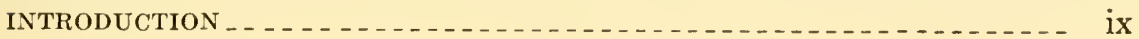

\section{CHAPTER 1}

\section{The Social Use of Space}

\section{John B. Calhoun}

I. Introduction

II. The Bivariate Normal Type of Home Range

III. Behavioral Origins of the Bivariate Normal Type of Home Range_..... 8

IV. Use of a Two-Dimensional Field _ _ _ _ 19

V. Summary of the Concept of Home Range

VI. Continuous Removal Trapping of Small Mammals _ _ _ _ _ _ _ _ _ _ 26

VII. Toward a General Theory of Interspecific and Intraspecific Use

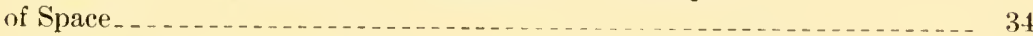

VIII. Interpretations of Observed Data Derived from Removal Trapping of Small Mammals _._._.

IX. A Theoretical Conceptualization of the Evolution of a Social Hierarchy among Species in the Utilization of Space_...

X. Psychological Dominance as the Primary Component of the Niche _._. _ 77

XI. An Induced Invasion . . . _ _ _

XII. Derivation of Compact Colonies from Constellations__._.

XIII. A Formulation of Group Dynamies _.. . . . . _ _ _ _ 101

XIV. Consequences and Examples of Social Interaction Systems _. _ _ _ _ _ 148

XV. Conclusion _......... 184

References............. 185 


\section{CHAPTER 2}

\section{Endocrine Adaptive Mechanisms and the Physiologic Regulation of Population Growth}

J. J. Christian

General Introduction 189

PART 1. THE ENDOCIRIN ADAPTIVE MECHANISMS

1. Introduction $\ldots \ldots \ldots$

II. The Endocrine Glands of Adaptation $\ldots \ldots \ldots 2$

PART 2. PHYSIOLOGIC ADAPTION AND MAMMALIAN POPULATIONS

I. Introduction _. .

II. Endocrine Responses to Social Pressures and to Population Density _._._. 263

III. Conclusion ......

References. . . . . . . . . . 328

AUTHOR INDEX _.

SUBJECT INDEX _... 


\title{
$-1$ \\ The Social Use of Space
}

\author{
JOHN B. CALHOUN
}

Laboratory of Psychology, National Institute of Mental Health, Bethesda, Maryland

\section{TABLE OF CONTENTS}

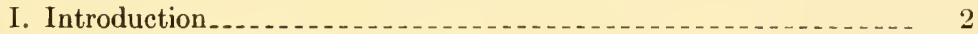

II. The Bivariate Normal Type of Home Range............ 4

III. Behavioral Origins of the Bivariate Normal Type of Home Range.. 8

A. Activity in a One-Dimensional Habitat ............... S

IV. Use of a Two-Dimensional Field . . _ _ _ _ 19

A. Theoretical Origin

B. Travel-Path Home Range............... 24

V. Summary of the Concept of Home Range _._. 25

VI. Continuous Removal Trapping of Small Mammals _ _. _ _._. _ 26

A. Rich Lake Island, New York, 1952, Sixty-Day Removal StudyData Contributed by William L. Webb_............... 27

B. Chadwick Woods, Montgomery County, Maryland, Removal Study, 1958-1959-Data Contributed by Kyle R. Berbehenn 29

C. Comparative Catches, Huntington Wildlife Forest, 1952-1953Data Contributed by Earl F. Patric and William L. Webb...

D. Comparative Catches, Huntington Wildlife Forest, 1951-Data

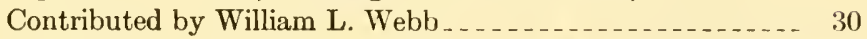

E. Comparative Catches in Maine (1950) and Maryland (1953) _._ 31

F. Comparative Catches of Peromyscus and Clethrionomys _..... 32

VII. Toward a General Theory of Interspecific and Intraspecific Use of Space._._._.

A. A Two-Species System _........ 36

B. The Nature of the Inhibitory Influence . . . . . . . . . . 35

C. The Learning of Signals__._._. 39

D. The Distance between Neighbors of the Same Species _._. _... 42

E. Methods of Calculating Data Relative to the Distance between

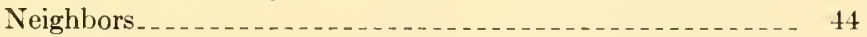

F. Further Comment on the Impact of All Individuals on the

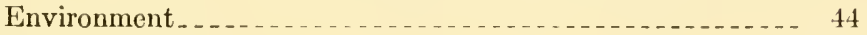

G. Contacting Neighbors _........ 45

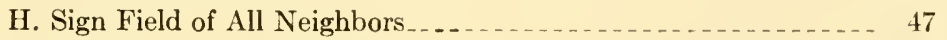

I. Signal Field of Neighbors _ . . . .

J. Hum Field . . . 50

K. General Conclusion Concerning the Distance between Neighbors 50

L. The Number of Neighbors Perceived . . . . . . 
VIII. Interpretations of Observed Data Derived from Removal Trapping of Small Mammals_................................. 52

A. The Relationship between Two Dominant Species_.......... 52

B. Removal Captures of Socially Dominant Species_.......... 55

C. Constellation Formation-An Intraspecific Phenomenon _... _ 57

D. Expected Variability in the Number of Individuals Forming Constellations

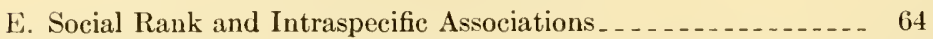

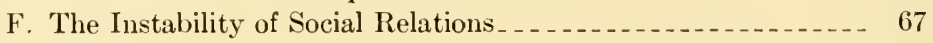

IX. A Theoretical Conceptualization of the Evolution of a Social Hierarchy among Species in the Utilization of Space_........ 70

1. Psychological Dominance as the Primary Component of the Niche _ 77

XI. An Induced Invasion _................................. 80

XII. Derivation of Compact Colonies from Constellations__.___.__ 86

A. Compaet Coinny Formation in the Norway Rat_........ 87

B. Howler Monkey's, a Compact Colony Living Species_._._._. 90

C. Behavioral Sink Lovelopment by the Norway Rat ........ 92

D. Yarding by Deer in Northern Wisconsin _._._.

E. Concerning Basic Numbers, $N_{b}$, for Man _._.

XIII. A Formulation of Group Dyı amics_._.

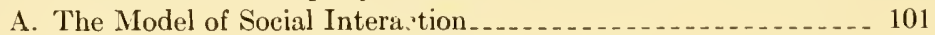

B. Basic Processes Involved in Social Interaction . . . . . . . . 116

XIV. Consequences and Examples of Social Interaction Systems_... . _ 148

A. Velocity Reduction in a Hierarchy of Mice... . . . . . . . . 148

B. The Choosing of a Partner $\ldots$

C. The Response-Evoking Capacity Circumplex _._._._._. 155

D. Conformity, Withdrawal, and Creativity_._.

E. Velocity and Home Range._._._.

F. Velocity in High-Density Rat Societies _.

G. Exploratory Behavior. . .

XV. Conclusion _... 184

References . . _

\section{Introduction}

During recent years many investigators have considered the physiological consequences to the individual of altering the size of the group of which it is a member. Inherent in such studies is the assumption that for a particular species there is some optimum group size, above or below which the altered frequency or type of interactions are either stressful or fail to elicit optimum physiological states. My purpose will be to develop formulations concerning the social use of space to determine whether there might be certain optimum group sizes.

I shall consider only indirectly the physiology of individuals. Instead, emphasis will be upon relationships which determine the "physiology" of the community. The basic particle is the individual mammal. In any total 
assembly of such particles inhabiting a particular environment, taxonomic categories, such as species and genera, represent general classes of particles. Although several classes may share certain characteristics, each possesses characteristics peculiar to itself, which on the average differentiate it from all other classes. Furthermore, the properties of any particular particle may change through time as a function of maturation and experience. Such changes are reflected in the individual's internal milieu. Only at this level are we concerned with physiology in its classical sense.

A social system consists of particles moving through space and time. In the course of evolution and maturation, such particles may develop enhanced capacities for affecting others and, in turn, for being more affected by them. This inquiry seeks that essential nature of these particles which influences the course of social evolution. It concludes that each category of particles which we designate as a species must develop a basic group size, designated as its basic number, $N_{b}$. Then, assuming the validity of the concept of an $N_{b}$, the inquiry is extended to encompass the following questions:

1. How do changes in $N$ from $N_{b}$ alter the social system?

2. Given a species, whose groups are of the optimum $N_{b}$ type, how do changes in the environment or in the nature of the particles affect the social system?

3. What are the basic organizational and interactional properties of the group?

I found very few prior studies in the literature suitable for guiding me in developing formulations adequate to satisfy these objectives. Therefore, this discourse will be neither a review of the literature nor even an adequate presentation of empirical data. Instead, the major theme concerns development of a logically sound, theoretical framework of processes underlying social phenomena.

In the course of this development some empirical data will be presented. These data are not meant to prove the correctness of the formulations. They are presented simply as background for the origin of ideas or to show that there at least exist some data which are harmonious with the developed concepts. Much of such data is presented here for the first time. I am indebted to many persons for their permission to cite such original data.

I have attempted to place many of the concepts in sufficiently precise mathematical form to permit a better understanding of the dynamics involved. My inadequacy in the field of mathematics has been buffered by the advice of several competent mathematicians: James U. Casby, Murray Eden, Samuel W. Greenhouse, Seymour Geisser, Clifford Patlak, and 
John Gilbert. However, I assume full responsibility for any errors, inadequate presentation, or overextension from their initial guidance.

I have found this effort a rewarding one for the development of insight into complex social systems, and I can only hope that in some small measure it may serve as a bridge for others in their design of experiments or in their further theorizing.

\section{The Bivariate Normal Type of Home Range}

Home range denotes the area covered by an individual in its day-today activities. Field studies of many species of mammals have revealed that each individual customarily stays within a restricted area for long periods. The individual utilizes the center of such an area most intensively. With increasing radial distance from this home range center (HRC) the relative frequency of visitation per unit of area decreases. Calhoun and Casby (1958) found that the bivariate normal distribution function adequately describes home range. The following is a summary of their analyses.

In home range studies, "density function" is a mathematical expression representing the probability of an animal being present in some arbitrarily small area. Three assumptions are made:

The home range is fixed. In other words, the statistics of the home range are stationary or time independent.

There is a true center of activity although the apparent center, the mean coordinate point of capture, of activity may deviate from it.

The probability of an animal being in a unit of area decreases with increasing distances from the true center of activity. This and the second assumption suggest a bivariate normal distribution of the density function:

$$
f(x, y) d x d y=\frac{1}{2 \sigma^{2} \pi} \exp \left[-\left(x^{2}+y^{2}\right) / 2 \sigma^{2}\right] d x d y
$$

where $\sigma$ is the standard deviation of the distances in the $x$ and $y$ direction and is assumed to be equal for both, and $x$ and $y$ are measured from their respective means. This density function may be used to represent the percentage of time spent in the area $d x d y$ located at the Cartesian coordinates $x, y$, or in polar coordinates:

$$
f(r, \theta) r d \theta d r=\frac{1}{2 \sigma^{2} \pi} \exp \left(-r^{2} / 2 \sigma^{2}\right) r d \theta d r
$$

Here, the area $r d \theta d r$ is determined by $r$.

The density function in terms of the Cartesian coordinates is more 
meaningful from an ecological standpoint because it states in comparative terms the amount of time spent by an animal in a small standard area at any position in the home range. However, for the initial mathematical manipulation, it was found more convenient to express the density function in terms of polar coordinates. Then the probability of finding the animal between the radii $r$ and $r+d r$ about the true center of the home range is:

$$
f(r) d r=\frac{2}{2 \sigma^{2}} \exp \left(-r^{2} / 2 \sigma^{2}\right) r d r
$$

If Eq. (3) is integrated over the range 0 to $\sigma$ we have

$$
\int_{0}^{\sigma} \frac{2 r}{2 \sigma^{2}} \exp \left(-r^{2} / 2 \sigma^{2}\right) d r=1-e^{-1 / 2}=0.3940
$$

In the above equations $\sigma$, the standard deviation of the normal distribution function, is the value of a radius within which the probability of the animal being present is $39.4 \%$, if its movements can be described by a bivariate normal density function.

Similarly, integrating Eq. (3) over the range 0 to $2 \sigma$ gives

$$
1-e^{-4 / 2}=0.8645
$$

Similarly, integrating Eq. (3) over the range 0 to $3 \sigma$ gives

$$
1-e^{-9 / 2}=0.9888
$$

The above sigma thus delineates a single distance term by which home range may be described. The term "home range sigma" will be so utilized in following sections.

Although this sigma may be calculated from a series of coordinate points of capture by equations presented in the original paper, use of recapture radii provide a more direct means, adequate for most purposes. Calculate the mean coordinate point of capture, the approximate home range center. Then on a large scale grid map of the study area measure recapture radii, $r$, from this mean coordinate point of capture. Unbiased estimates of sigma, $s$ and $s_{i}$ may be calculated by the following equations:

$$
\begin{gathered}
s=\left[\frac{\sum_{i=1}^{n} \sum_{j=1}^{K_{i}} r_{i j}^{2}}{2(N-n)}\right]^{\frac{1}{2}} \\
s_{i}=\left[\frac{\sum_{j=1}^{K_{i}} r_{j}^{2}}{2\left(K_{i}-1\right)}\right]^{\frac{1}{2}}
\end{gathered}
$$


where:

$s=$ unbiased estimate of the home range sigma for all the animals in a sample

$s_{i}=$ unbiased estimate of the home range sigma for any particular animal

$K_{i}=$ number of captures of $i$ th animal

$n=$ number of animals

$N=$ total captures $=\sum_{i=1}^{n} K_{i}$

$i j=j$ th observation of the $i$ th animal

A detailed analysis of the home range for 25 male harvest mice (Reithrodontomys) on which there were 10 to 24 captures each indicated that there was a significant variation of sigma among animals. In other words, some animals had significantly larger home ranges than others. Therefore, in order to compare the observed recapture radii with the theoretical (Table II in Calhoun and Casby, 1958), each recapture radius was normalized into a standard measure denoted by $Z$ in which the home range sigma for each animal was assigned a value of 1.0 and all recapture radii expressed as a proportion of this.

As may be seen from Fig. 1, the theoretical closely approximated the observed. Although this detailed analysis has been applied only to this one species, it shall be assumed for the purpose of developing further formulation that the bivariate normal distribution function adequately describes fixed home ranges of other species.

Comparison of observed and theoretical distribution of home range radii required viewing home range as a probability of capture which changes with radial distance from the home range center. Bands of equal width increase in area with radial distance from the home range center, while probability of capture per unit area decreases with increase in radial distance. Interaction of these two factors results in more captures at about one sigma from the home range center than at any other distance (Fig. 1).

However, the ecologically important aspect of the bivariate normal distribution as an expression of home range is the relative probability that an animal will be in a unit of area with respect to the radial distance of that unit area from the home range center (Fig. 2). For any given sigma characterizing a particular species, its density function in terms of area curve may be obtained by multiplying the relative sigma value on the abscissa by the observed sigma and dividing the density function values on the ordinate by the square of the observed sigma. 


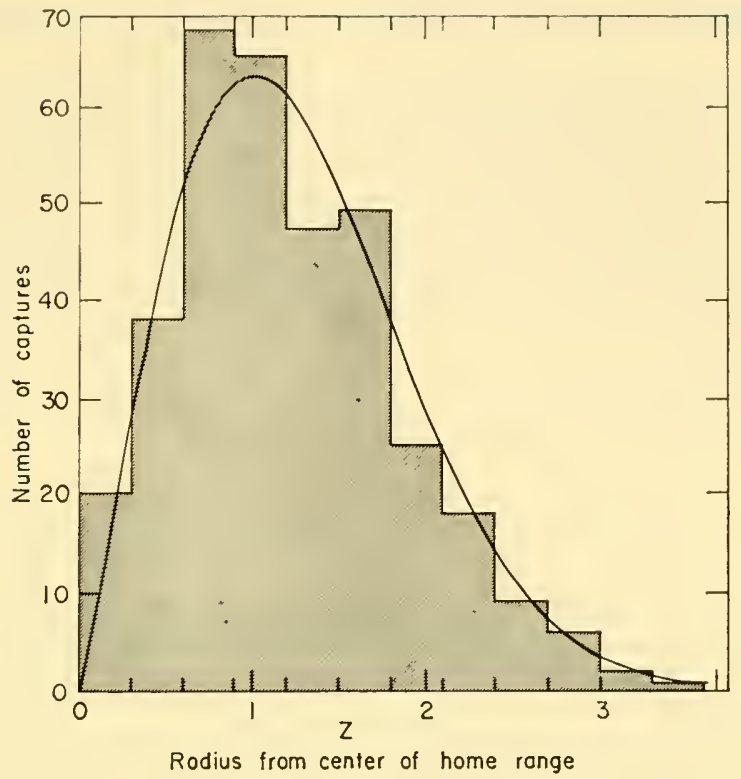

Fig. 1. Observed (histogram) and theoretical distribution of 348 recapture radii $(Z)$ of 25 male harvest mice from the center of their home range. $Z$ here represents a normalized measure of the bivariate normal home range sigma.

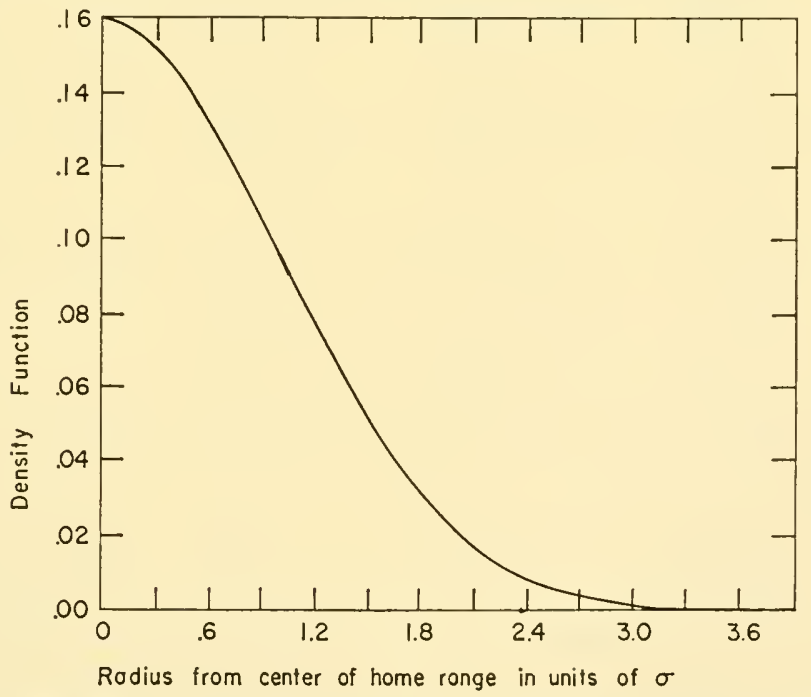

FIg. 2. Cross section of the density function of home range in terms of area. Rotation of this curve about its axis reveals the mountain-shaped topography of home range. 


\section{Behavioral Origins of the Bivariate Normal Type of Home Range}

The fact that a particular equation happens to describe home range enables derivation of several principles regarding the use of space by an entire community. Discussion of these principles follows in Section VII. However, as a background to this discussion it will be advantageous to seek an understanding of the biological basis for the bivariate normal type of home range.

\section{A. Activity in a One-Dimensional Habitat}

Admittedly, animals rarely live in essentially one-dimensional environments. However, I suspected that if animals were placed in such environments certain regularities of behavior might be revealed which would provide insight into their use of two-dimensional environments. To this end, four 14-foot long alleys were constructed. Each had a channel 8 inches wide. Each $8 \times 12$-inch segment of the floor was so suspended that when a domesticated Norway rat, used as a subject, stood on such a segment a microswitch closed. This closure initiated a signal such that the exact position at every point in time was recorded on a recording oscillograph. A partition between the first and second treadles formed a home compartment. A $3 \times 3$-inch opening through this partition provided access to the rest of the alley. Food and water placed in this compartment further enhanced the role of this compartment as a "home." A ground glass plate, through which shone the light from a 100 -watt lamp, formed the opposite end of the alley. This light served to concentrate the activities of the rats emanating from the home compartment. It was as if every foot of the alley were several feet long. Details of the effect of varying light intensity at the end of the alley on explorations will be presented elsewhere. This apparatus is referred to as the Ferguson Activity Alley.

Suffice it to consider the results from 73 rats, each run for 72 hours in the alley. Each rat made from 10 to 30 excursions out into the alley each night. Despite the presence of the bright light at the end of the alley, onefourth of the trips terminated at the end of the alley. In other words, the end of the alley formed a barrier. Most of the trips thus terminated at the end of the alley presumably would have represented trips of greater length had the alley only been longer. Most of the time a rat would go out to some intermediate distance, stop momentarily, and then turn around and go directly back home. Occasionally, a rat would wander back and forth from the point of initial termination. All trips with such vacillations and those ending at the barrier were excluded from the initial analysis. 


\section{Trip-Terminations in an Unstructured One-Dimensional}

\section{HaBITAT}

The initial investigation focused upon examination of the distances from the home compartment at which nonvacillating trips terminated in the unstructured alley. "Unstructured" denotes the absence along the alley of any stimuli likely to elicit responses and so induce a rat to stop. Rats were placed in the alley during the middle of the afternoon, a time of minimal activity within their normal 24-hour rhythm of activity. And yet when placed in the alley every rat exhibited a 2- to 3-hour period of hyperactivity. Further details of the decay curve of this hyperactivity are discussed in Section III, A, 3.

For 73 rats complete records (Table Ia) were available for all trip-

TABLE Ia

Number of Trips Terminated with Reference to Distance from Home

\begin{tabular}{|c|c|c|c|c|}
\hline \multirow{2}{*}{$\begin{array}{l}\text { Distance in } \\
\text { feet of } \\
\text { termination }\end{array}$} & \multicolumn{2}{|c|}{ Unstructured alley } & \multicolumn{2}{|c|}{ Structured alley } \\
\hline & $\begin{array}{l}\text { During first } \\
2.5 \text { hours }\end{array}$ & $\begin{array}{c}\text { During next } 3 \\
6 \text { A.M. }-6 \text { P.M. } \\
\text { periods }\end{array}$ & $\begin{array}{l}\text { Strips of } \\
\text { paper }\end{array}$ & $\begin{array}{l}\text { Pellets } \\
\text { of food }\end{array}$ \\
\hline 1 & 274 & 558 & 1053 & 955 \\
\hline 2 & 204 & 407 & 839 & 643 \\
\hline 3 & 150 & 31 กั & 723 & 509 \\
\hline 4 & 104 & 277 & 302 & 375 \\
\hline 5 & 99 & 294 & 194 & 288 \\
\hline 6 & 81 & 219 & 230 & 258 \\
\hline 7 & 56 & 139 & 1.51 & 163 \\
\hline s & 58 & 115 & 149 & 137 \\
\hline 9 & 47 & 98 & 112 & 119 \\
\hline 10 & 39 & 113 & 57 & 104 \\
\hline$\Sigma$ & $1112^{a}$ & $253.5^{a}$ & 3810 & 3551 \\
\hline $\begin{array}{l}\text { Barrier and vacillat- } \\
\text { ing trips }\end{array}$ & 502 & 1458 & $59^{b}$ & $127^{b}$ \\
\hline Total trips & 1614 & $402: 3$ & 3869 & 3678 \\
\hline Trips/rat/hour & 8.844 & 1.531 & & \\
\hline
\end{tabular}

a Only nonvacillating trips included.

${ }^{b}$ Vacillating trips and trips at whose end an object was transported home are not included since the termination of trips determined was solely by the number of transported objects. 
terminations during both the initial 150 minutes of hyperactivity and the following three, 6 P.M. to 6 A.M., 12-hour periods of normal heightened nocturnal activity. The frequency, $y$, of terminating trips as a function of distance, $x$, from home is described by the equation:

$$
y=\exp (a+b x)
$$

where $b$ is the slope. The slope for trip-terminations during the hyperactive period, $b_{1}$, is -0.2099 ; while $b_{2}$, the slope for trip-terminations during the 36 hours of normal nocturnal activity, is -0.1924 . The $t$ test

$$
t_{32}=\frac{b_{1}-b_{2}}{\sqrt{\text { Var. }\left(b_{1}-b_{2}\right)}}=-0.682
$$

has a $p$ value of 0.051 which indicates that slopes $b_{1}$ and $b_{2}$ do not differ significantly.

Therefore, it is concluded that the neural mechanism producing termination of trips is unaltered by the nearly sixfold increase in the incidence of initiating trips accompanying initial exposure to a strange environment. For this reason, the mean slope of -0.20115 , i.e., $\left(b_{1}+b_{2}\right) / 2$, was fitted to both these sets of data shown in Fig. 3 as trips per rat per hour for comparative purposes to emphasize the hyperactivity of initial exposure to a strange environment.

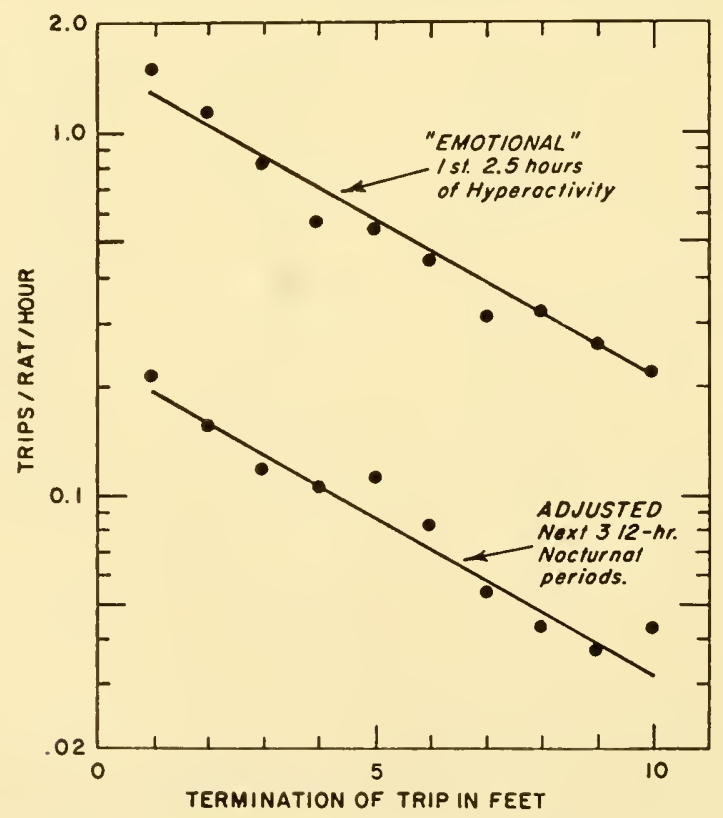

Fig. 3. Frequency of terminating trips at successive distances from the home compartment in the unstructured Ferguson Alley. See Table Ia. 
2. The Role of a Structured Environment on the Termination OF TRIPS

Natural habitats possess structures which elicit responses. Items of food and nesting material represent structures normally causing animals such as rats to terminate trips. When such items are transported home the trip resembles the nonvacillating ones in the one-dimensional alley in the sense that there is a direct outward phase, terminated by the object being picked up, followed by a direct homeward trip transporting the item. In order to explore the effect of such structuring in the one-dimensional habitat upon termination of trips, one of two procedures was followed: At each onefoot interval from home along the alley, there was placed a pad of paper strips or an open hopper of food pellets. During any particular rat's stay of 3-12 days in the alley, only nesting material or only food pellets were available. Periodic replenishment of each source ensured a continuous supply at each distance. Nevertheless, the rats removed items from each distance (Table Ia) even though this necessitated passing by opportunities to respond while on the outward journey. Each item removed at a particular distance from home is considered to indicate a trip-termination at that distance. Examination of the oscillograph record confirmed this interpretation.

The frequency of termination of such trips as a function of distance is also described by the equation, $y=\exp (a+b x)$. The slope for trips terminated by picking up paper strips, $b_{3}$, is -0.3027 ; while $b_{4}$, the slope relating to securing food pellets, is -0.2481 . The $t$ test,

$$
t_{32}=\frac{b_{3}-b_{4}}{\sqrt{\operatorname{Var} \cdot\left(b_{3}-b_{4}\right)}}=-2.128
$$

has a $p$ value between 0.05 and 0.01 which indicates a statistically significant difference between these two slopes. However, examination of Fig. 4 reveals a marked dispersion about the best-fit line of the observed points relating to nesting material. For this reason, the interpretation that the $b_{3}$ and $b_{4}$ slopes differ statistically is open to question that this difference in slope implies biological significance. I therefore believe it wisest to assume that $b_{3}$ and $b_{4}$ are really identical, or nearly so.

If this is so, we may compare the slopes of the mean of $b_{1}+b_{2}$ with that of $b_{3}+b_{4}$. Here the $t$ test

$$
\frac{\frac{1}{2}\left(b_{1}+b_{2}\right)-\frac{1}{2}\left(b_{3}+b_{4}\right)}{\sqrt{\operatorname{Var} . \frac{1}{2}\left(b_{1}+b_{2}\right)-\frac{1}{2}\left(b_{3}+b_{4}\right)}}=4.0925
$$

with a $p$ value less than 0.001 . 


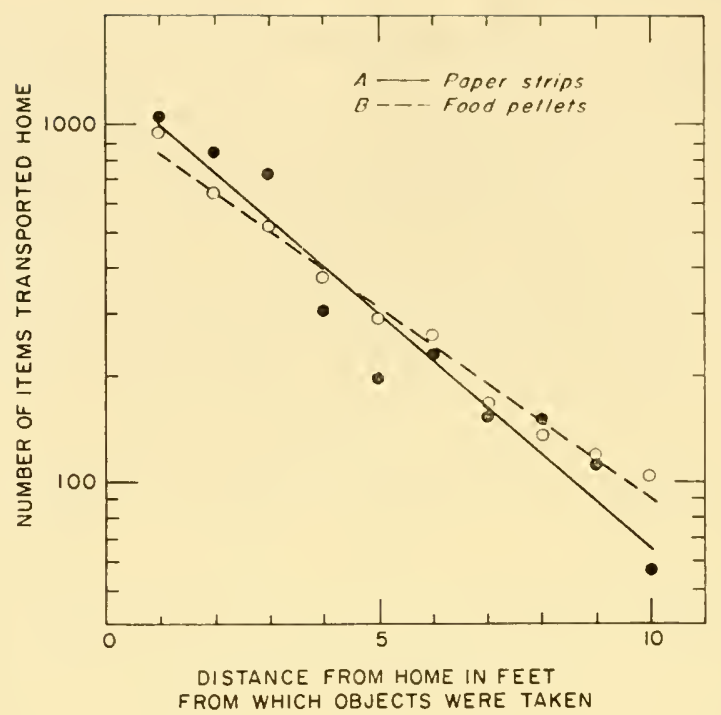

FIG. 4. Frequency of transporting nesting material and food into the home compartment from points at successively greater distance from it. $A, 3510$ strips of paper; $B, 3551$ pellets of food. See Table Ia.

It is therefore concluded that structuring the environment with items inducing responses leads to a reduction of the distance from home at which trips are terminated.

\section{The Probability of 'lerminating Trips}

The two prior sections merely demonstrate an effect produced by structuring the environment. They do not further our understanding of the underlying biological process.

The behavior of rats in the structured environment provides the clue. During any period of intensive transportation, one trip almost immediately followed the preceding one. And yet the distance at which a particular trip terminated bore no relationship to the distance at which the previous or following one terminated. It was as if the rat was blind to its surroundings on the outward trip until some neural switching mechanism became activated in a random fashion with reference to the time of the trip's initiation. This switching on (or off?) placed the rat in a perceptive phase at which time it responded by picking up the nearest relevant object and transporting it into the home compartment. Therefore, it will be helpful to deter- 
mine the probability of this switching, which is synonymous with the probability of terminating a trip.

Let: $t_{j}=$ the number of trips reaching any $j$ th distance from home.

$N_{j}=$ the number of trips that stop at the $j$ th distance.

$p_{j}=$ probability of stopping at the $j$ th distance.

Then:

$$
\begin{aligned}
t_{j-1}-t_{j} & =p_{j-1} t_{j-1} \\
N_{j} & =p_{j} t_{j} \\
N_{j}-N_{j-1} & =p_{j} t_{j}-p_{j-1} t_{j-1}
\end{aligned}
$$

If $p_{j}=p$ (a constant independent of $j$ ), then:

$$
\begin{aligned}
N_{j}-N_{j-1} & =p\left(t_{j}-t_{j-1}\right) \\
& =-p\left(t_{j-1}-t_{\cdot j}\right) \\
N_{j}-N_{j-1} & =-p\left(p t_{j-1}\right)
\end{aligned}
$$

And by analogy to Eq. (11):

$$
N_{j-1}=p t_{j-1}
$$

Substituting Eq. (15) into Eq. (14):

Therefore

$$
N_{j}-N_{j-1}=-p N_{j-1}
$$

$$
p=\left(N_{j-1}-N_{j}\right) / N_{j-1}
$$

This $p$ represents a constant probability of terminating trips which arrive at a point regardless of the distance from home. Rigorous proof that this $p$ actually is a constant is difficult from present data because of the barrier produced by the relatively short alley. However, the validity of a constant $p$, independent of distance, may be arrived at intuitively since an equation of the form $y=\exp (a+b x)$ best represents the observed data. In other words, $\log y$ plotted against $x$ forms a straight line. Whenever this is so, Eq. (17) must be true.

Utilizing Eq. (9) stated in the form:

$$
\log _{e} y=a-b x
$$

the expected number of trips terminating at $N_{j-1}$ and $N_{j}$, where $j=2$, were found to be as shown in Table Ib, along with the $p$ values calculated from Eq. (17). Thus, the probability of 0.182 of terminating trips arriving at any distance in the unstructured alley is increased to 0.24 by structuring. 


\section{TABLE Ib}

Probability of Terminating Trips with Respect to Environmental Structure AND Level of ACTIVITY

\begin{tabular}{|c|c|c|c|c|c|}
\hline \multirow{2}{*}{ Alley } & \multirow{2}{*}{ Secondary condition } & \multicolumn{2}{|c|}{$\begin{array}{l}\text { Expected number of } \\
\text { trips terminating at: }\end{array}$} & \multirow{2}{*}{$p$} & \multirow{2}{*}{$\begin{array}{l}\text { Mean } \\
\quad \bar{p}\end{array}$} \\
\hline & & $N_{j-1}$ & $N_{j}$ & & \\
\hline \multirow{2}{*}{ Unstructured } & $\begin{array}{l}\text { During first } 2.5 \mathrm{hr} \text {. of hyper- } \\
\text { activity }\end{array}$ & 237.5 & 194.3 & $0.182^{a}$ & \multirow{2}{*}{0.182} \\
\hline & $\begin{array}{l}\text { During normal nocturnal ac- } \\
\text { tivity }\end{array}$ & 510 & 417 & $0.182^{a}$ & \\
\hline \multirow{2}{*}{ Structured } & Paper transportation & 999 & 783.3 & 0.261 & \multirow{2}{*}{0.240} \\
\hline & Food transportation & 837 & 653.5 & 0.220 & \\
\hline
\end{tabular}

${ }^{a}$ The mean $b$ value of -0.20115 used in the calculation of $p$.

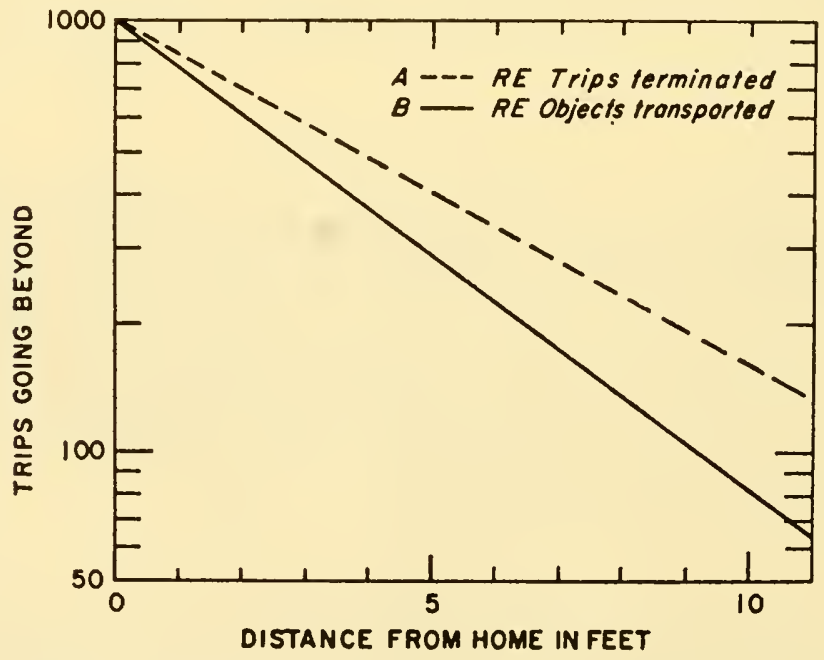

FIG. 5. Theoretical curves depicting relative likelihood of trips going beyond successive distances from home based upon an initial 1000 departures. $A$, in an unstructured alley (see Fig. 3); $B$, in an alley structured with objects available for transport back home. 
The amount of structuring used here increased the probability of stopping by $32 \%$ [i.e., $(0.240-0.182) / 0.182$ ]. The extent to which this difference modifies the use of a field may be visualized by considering the following: Of every 1000 trips arriving 1 foot from home in the unstructured alley, 30 would stop at the tenth foot and 134 would continue farther, whereas in the structured environment only 20 would stop at the tenth foot and only 64 would continue farther (see Fig. 5).

In elosing this section it should be emphasized that the probability of stopping is basically a function of time. In the particular situation, the $p$ 's refer to the time required for the rats to travel a distance of 1 foot.

A further question may be asked: "How does structuring increase the probability of stopping if the animals are perceptually 'blind' to specifie stimuli to the extent of being unable to exhibit directed responses to them?" One may visualize the situation in general terms: There is some assembly of neurons which provides a signal terminating an ongoing behavior, such as outward locomotion from home. The magnitude of this signal necessary for behavioral termination requires simultaneous firing of some $x$ number of neurons. This assembly of neurons may be called a "governor." It must be located anatomically in some subeortical portion of the brain precluding conscious awareness of its functioning. During any ongoing behavior all perceivable stimuli unrelated to the ongoing behavior initiate impulses which arrive at the behavior terminating governor. Each unit of impulse causes an increase in the rate of firing of the neuronal net forming the governor. The greater the intensity of such stimuli, or the greater the number of stimuli of a given intensity, the more rapidly will the neurons of the governor fire and thus the shorter will be the interval between emission of effective signals by the governor. This signal both terminates the ongoing behavior and produces awareness of stimuli appropriate to eliciting those responses appropriate to initiating some other behavior.

\section{Initial Hyperactivity in a Strange Environment}

For the rats discussed in Section III, A, 1 the level of activity in terms of trips per rat per hour (Fig. 3 and Table Ia) was approximately six times as high during the first 150 minutes of exposure to a strange environment in eomparison with the later periods following adjustment. This observation requires postulation of a second governor, one which determines the probability of initiating trips. Initiating trips is here considered to be synonymous with initiation of periods of diffuse undirected motor activity. Examination of the expression of hyperactivity during the first $2-2 \frac{1}{2}$ hours of exposure to a strange environment provides insight into the functioning of this governor of trip-initiations. 
For 71 of the 73 rats previously discussed with reference to the distance of trip-terminations, a count was made of the total feet traveled each halfhour during the first $2 \frac{1}{2}$ hours of exposure to the alley. A similar analysis was prepared for the 26 rats, considered further in Section III, A, 5, which emerged into the alley more than momentarily and for which the recording system functioned properly.

For each set of data, results are quite similar (Fig. 6). Activity contimuously declines during initial exposure to a strange environment. But the important point is that this decline begins from an extremely elevated state of hyperactivity. From Table Ia it may be calculated that the average

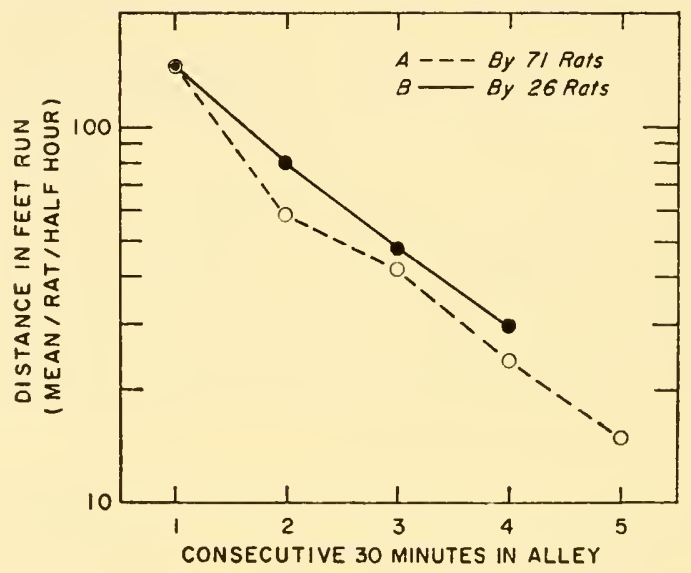

FIG. 6. Hyperactivity in a strange environment: Curve $A$ represents the mean activity level of 71 rats immediately after first exposure to the Ferguson Alley. Curve $B$ similarly gives the mean response of 26 rats to the NIH Emotional Activity Alley.

round trip approximates 10.44 feet. During the normal nocturnal period of activity, trips occur at the rate of 1.53 per hour. This means that in this one-dimensional alley rats travel 8 feet per half-hour on the average when adjusted to their environment. As may be seen from Fig. 3 the activity is increased nearly twenty times normal during the initial half-hou in this strange environment. Projection of the curves, shown in Fig. 6, indicates that a normal level of activity will be reached by $3-4$ hours of adjustment.

Such decay curves of hyperactivity suggest that the rats secrete some humoral agent upon their initial exposure to strange stimuli such as represented by the activity alley. This humoral substance increases the rate of firing of the neuronal net comprising the governor which determines onset of diffuse motor activity, such as trips out into the alley. As this substance is degraded, the frequency with which this governor emits signals initiating trips decreases until normal behavior is achieved. 


\section{Avoidance of a Strange Field}

During the pursuit of these studies on behavior in a one-dimensional habitat, a few rats failed to emerge into the alley until after the lapse of several hours. Most frequently such rats were members of subgroups having had less opportunity to adjust to novel stimuli. This suggested that the less opportunity an animal had to make adjustments to strange stimuli, or the more novel was the strange environment, the more likely it would be avoided.

New alleys were constructed to permit exploration of this hypothesis. These alleys consisted of an $8 \times 8$-inch chamel 1; feet long. At one end a home nest box could be attached from which the rat gained access to the alley by way of a $3 \times 3$-inch door. At the opposite end of the alley, light from two 60 -watt lamps shone through a ground glass plate. Unless otherwise modified, the floor was stationary. A photoelectric cell at each 3-foot interval along each alley initiated a signal to an Esterline-Angus event recorder each time a rat passed. This apparatus is called the "NIH Emotional Activity Alley."

Each of the 76 subjects, Osborne-Mendel male rats, was housed alone for 3 months from weaning in a small cage precluding the visual perception of any object outside the cage. Cages were not opened. Food and water were delivered into the cage through channels making it unnecessary to open them. These procedures minimized opportunity for adjusting to new configurations of stimuli. In the terminology of Section XIV, G, 1, the isolation cage represents an $E_{1}$ configuration of stimuli. Under this terminology $E_{1} \cdots E_{n}$ represent a series of discrete configurations of stimuli which an individual may encounter for the first time in that order.

Twenty rats were transferred directly from the isolation cages into the activity alley for a '2-hour exposure. Of the remaining 56 rats, 24 were exposed to an $E_{2}$ configuration for 2 hours for 10 days prior to being placed in the alley. Similarly, 16 others were exposed to an $E_{3}$ configuration, while the remaining 16 were exposed to $E_{2}$ for 2 hours, then immediately exposed to $E_{3}$ for 2 hours for 10 days. Three days after these exposures, which provided opportunity to adjust to the new stimuli of $E_{2}, E_{3}$, or $E_{2}$ and $E_{3}$, each rat was given a 2 -hour trial in the activity alley, which represented an $E_{4}$ configuration. Half of each of these four groups were placed in the alley with a stationary floor, an $E_{4 \mathrm{~A}}$ configuration; while for the other half the alley contained sections of tilting floor, an $E_{4 \mathrm{~B}}$ configuration, which clanged when the rats passed over them.

Greater detail of these studies are presented in Section XIV, G, 4 and in Table IIb. However, the results presented in Table IIa suffice for the present purposes. Both aspects of the hypothesis were confirmed. The 
Table IIa

Effect of Training and Degree of Strangeness of a Field upon Its Being Entered during 2 Hours of Exposure by Each of 76 Rats $^{a}$

A

"Training" in adjusting to

Response

to

alley strange stimuli

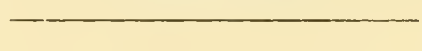

Yes

No
B

Degree of strangeness of alley

High

Low

(tilting floor) (stationary floor)

Entered

Avoided

37

19

4

16

13

30

25

8

${ }^{a}$ A data: $\chi^{2}=10.8045 ; p$ less than $0.001 ; \mathrm{B}$ data: $\chi^{2}=13.7110 ; p$ less than 0.005 .

stranger a field or the less opportunity there has been to adjust to novel stimuli, the greater will be the tendency for an animal to avoid that field.

\section{Probability of Vacillating at the Termination of Trips}

In the unstructured Ferguson Activity Alley, rats usually went directly out to the point where the trip terminated, then turned around and went directly back to the home compartment. Yet occasionally when a rat made an excursion out into the alley, it would wander back and forth

TABLE IIb

Effect of Kind and Sequence of "Training" in Adjustment to Strange Stimeli upoN Avoldaxice of ax ACtivity Alley

\begin{tabular}{|c|c|c|c|c|c|c|}
\hline \multirow{2}{*}{ Group $^{a}$} & \multicolumn{3}{|c|}{$E_{4 A}$ Alley } & \multicolumn{3}{|c|}{$E_{4 B}$ Alley } \\
\hline & $\begin{array}{l}\text { Total } \\
\text { trials }\end{array}$ & $\begin{array}{c}\text { Trials } \\
\text { entered }\end{array}$ & $\begin{array}{c}\text { Proportion } \\
\text { entered }\end{array}$ & $\begin{array}{l}\text { Total } \\
\text { trials }\end{array}$ & $\begin{array}{c}\text { Trials } \\
\text { entered }\end{array}$ & $\begin{array}{l}\text { Proportion } \\
\text { entered }\end{array}$ \\
\hline A & 40 & 11 & 0.275 & 40 & 2 & 0.050 \\
\hline B & 48 & 33 & 0.688 & 48 & 24 & 0.500 \\
\hline $\mathrm{C}$ & 32 & 31 & 0.969 & 32 & 22 & 0.688 \\
\hline $\mathrm{D}$ & 32 & 24 & 0.750 & 32 & 6 & $0.18 \mathrm{~s}$ \\
\hline Total & 152 & 99 & 0.651 & 152 & 54 & 0.355 \\
\hline
\end{tabular}

a See Seetion XIV, G, 4 for details of treatment of the four groups. 
about the place where the trip terminated. Two hundred and fifty-six of the total 2357 trips studied with reference to the initial period of hyperactivity, terminated in such vacillations. As graphically shown in Fig. 7 the probability of vacillating is proportional to the length of the trip.

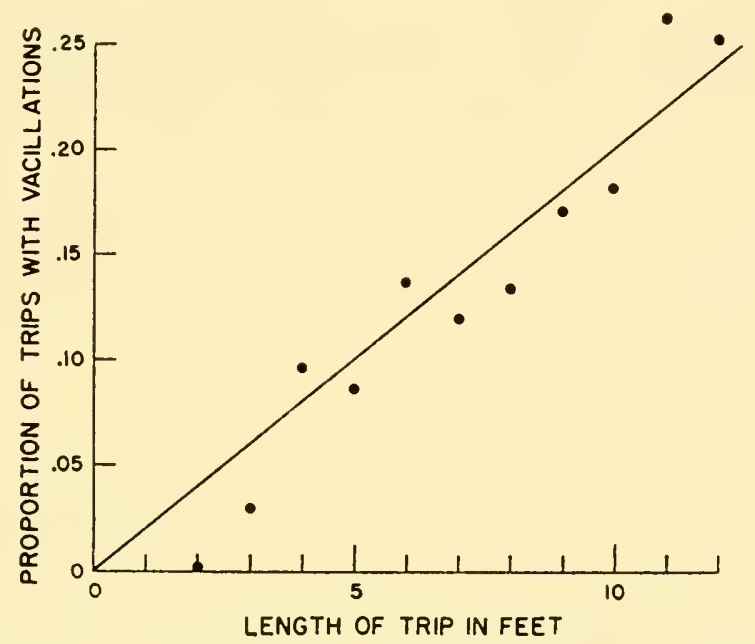

FIG. 7. Probability of trips including a period of vacillation, wandering back and forth about the point of termination. Curve based on 2357 trips by 136 rats during their first 150 minutes in the Ferguson Activity Alley.

'These results are given here because of their bearing on the question of the proportion of time an animal is capable of responding to static components of its environment. As I have stated above, I am proceeding with this formulation, utilizing the hypothesis that directed responses can be made only at the ends of periods of diffuse activity, which are represented here by the outward termination of trips. If this responsiveness at ends of trips persists throughout the wandering about at the end of trips, then it follows that the farther a familiar type of object is from home the more likely it will be responded to in proportion to trips terminating there. This conclusion is subject to the qualification that the object is so sparsely distributed in space as to require more searching than mere detection of the surroundings at the point of terminating a trip.

\section{Use of a Two-Dimensional Field}

\section{A. Theoretical Origin}

We are here considering animals whose movements emanate from a fixed home base. For them a two-dimensional field differs from a one-dimensional 
one in that in a two-dimensional field the area available at successive radial distances from home increases with radius. Considering this fact, will the hehavior exhibited by rats in a one-dimensional field lead to an equation for home range closely resembling the bivariate normal distribution [Eq. (3)]?

When Casby and I originally found that the bivariate normal distribution did conform with the observed home range resulting from captures, we were merely culminating a search for a means of deseribing the distribution of captures about the mean coordinate point of capture. This conformity revealed nothing about the biological mechanisms involved. We shall now inquire whether the phenomena of (a) decreasing frequency of arriving at successively greater distances from home, and (b) the probability of wandering increasing with distance from home suffice to explain the origin of the bivariate normal type home range.

A critical issue concerns the origin of the observation or "capture." Two types of observation are possible. First, the observer may record the physical presence of the animal at successive points independent of the activities of the individual. Second, the observation may arise as a consequence of the animal responding to an object placed by the investigator. Captures in traps represent this type of observation.

An assumption is made regarding where responses, such as entering baited traps, will be made. This is that such responses to continuously present and unrarying stimuli occur only during the period of wandering at the end of trips. This assumption implies that the animal remains in a perceptually blind state during the outward and return phases of a trip. The circumstantial evidence suggesting this assumption will not be considered here.

Let: $P_{1}=$ probability of terminating a trip at radius $r$.

$t=$ time spent wandering at $r$ if it stops there.

$C=$ probability of capture at $r$, which equals $t P_{1}$ times geometry factor of two-dimensional space.

Then:

$$
\begin{aligned}
P_{1}(r) & =A e^{-r / \alpha} \\
t(r) & =B r
\end{aligned}
$$

These two equations, in which $A, B$, and $\alpha$ are constants, represent the two basic assumptions regarding use of one-dimensional space. Then considering the geometry factor:

$$
C=K r e^{-r / \alpha} \cdot r d r d \theta
$$


And normalizing, it is found that:

$$
C=\frac{1}{2 \alpha^{3}} r^{2} e^{-r / \alpha} d r
$$

Where $r / \alpha=R$, the cumulative probability of capture, Cum, as a function of the radius $R$ from home becomes:

$$
\mathrm{Cum}_{\text {II }}=1-\left(\frac{R^{2}}{2}+R+1\right) e^{-R}
$$

Equation (23) above will be called Curve II as shown in Table III and Fig. 8. It may be compared to a similar cumulative probability curve for the bivariate normal distribution, which will be called Curve I, and which has the form:

$$
\mathrm{Cum}_{\mathrm{I}}=1-\exp \left(-r^{2} / 2\right)
$$

Curve II, Eq. (23), may be compared to Curve I, Eq. (24), by converting $R$ into units of $r$, where $r$, the radius from the center of the home range, is measured in the $\sigma$ units of the bivariate normal distribution, provided the constant $\alpha$ of $R=r / \alpha$ is known. This conversion was arbitrarily accomplished as follows: It can be shown that the cumulative probability of "capture" of Eq. (23), when expressed in terms of $\alpha$ and $r$, has the form:

$$
\mathrm{Cum}=1-\frac{1}{2 \alpha^{2}}\left(r^{2}+2 \alpha r+2 \alpha^{2}\right) e^{-r / \alpha}
$$

By Eq. (24), Curve I, when $r=1.2$, Cum $=0.513$. Therefore, by successive approximations, utilizing Eq. (25), it was found that when $\alpha=0.44$, Cum $_{\text {II }}=0.511$ at $r=1.2$. Therefore, 0.44 is the $\alpha$ conversion factor applied to $R=r / \alpha$, so that Curves I and II may be compared in terms of the bivariate normal home range $\sigma$ distance.

The values for these two curves, as shown in Table III, are shown in Fig. 8. Note that up to about $2 \sigma$ radius these two curves are so nearly identical that they are either likely to approximate actual field data equally well. There is considerably more "tail" to Curve II, but since so few observations occur in the greater than $2 \sigma$ range, it will still be difficult to decide which of these two curves most nearly approximates actual field data for the longer recapture radii.

However, the objective was to determine how well phenomena observed in the use of one-dimensional space could lead to a curve approximating the bivariate normal distribution. One of the assumptions was that the wandering responsive phase was proportional to radius from home. In the analysis of wanderings, vacillations at ends of trips in the one-dimen- 


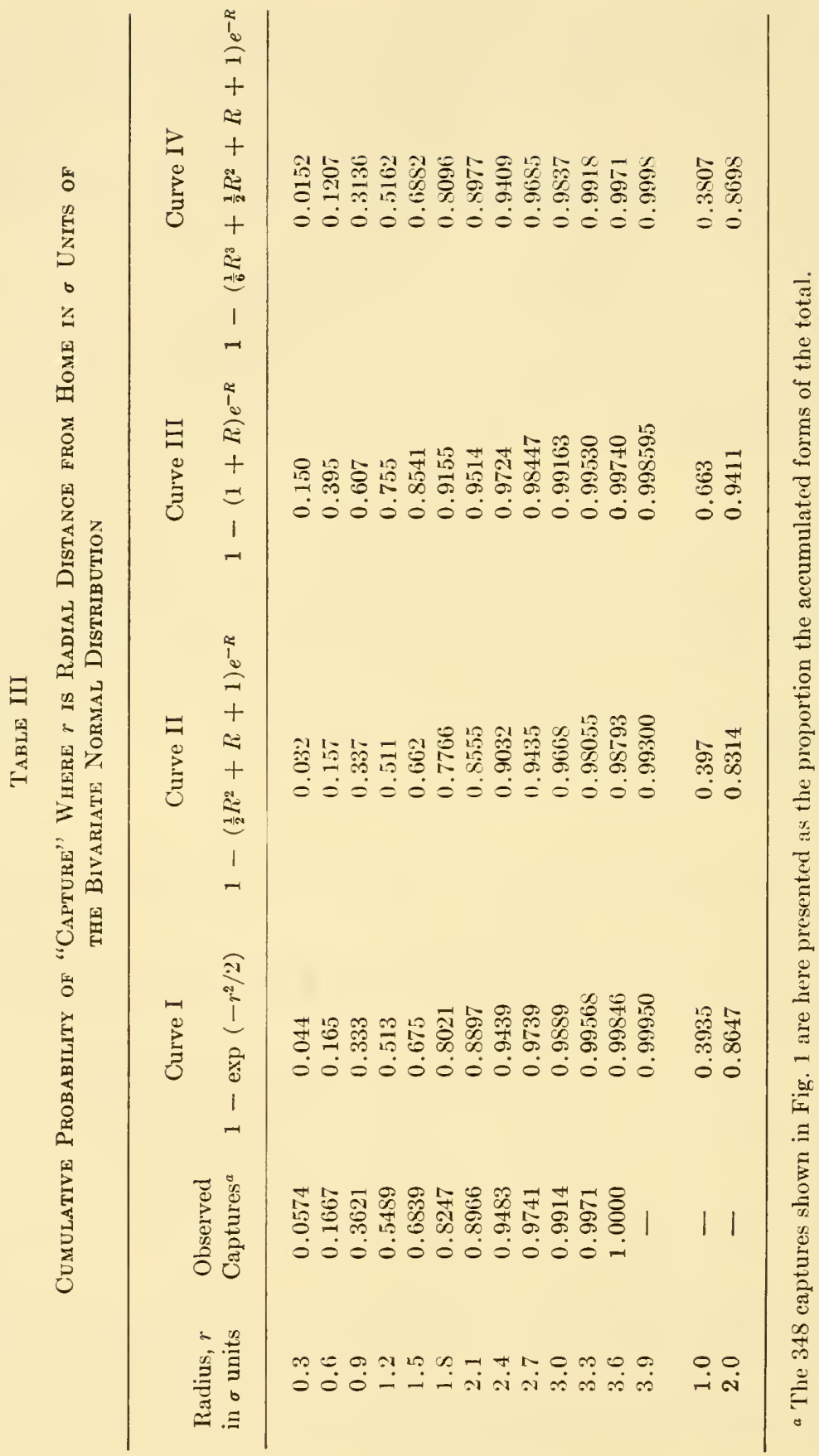




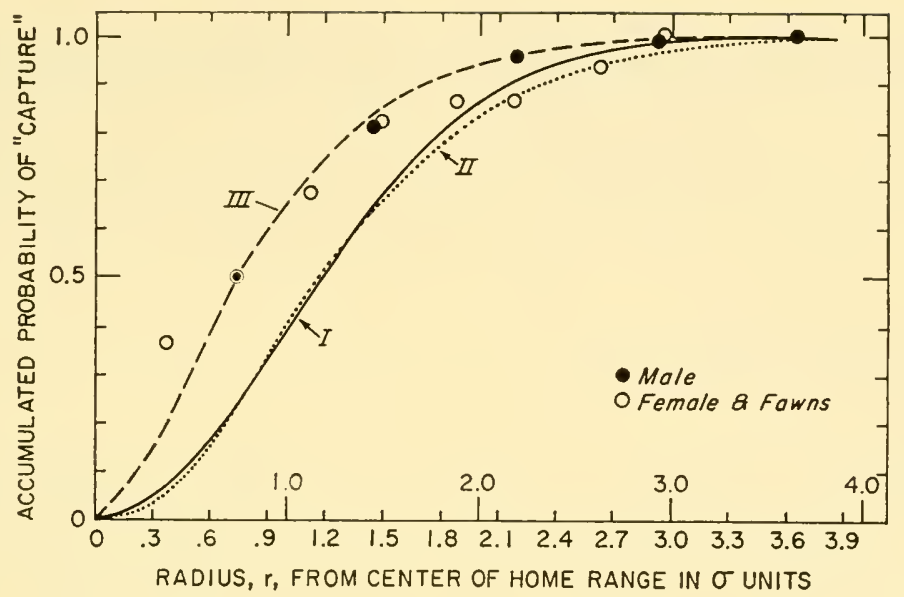

Fig. 8. Accumulated probability of "capture." Curve $I$ is the expected from the bivariate normal distribution function. Curve $I I$ is the expected based upon (a) termination of trips according to the equation, $y=e^{a+b x}$ in which the slope $b$ is negative, (b) probability of wandering at end of trips is proportional to radius, and (c) captures only occur during the wandering phase at end of trips. Curve $I I I$ is the accumulated travel path with no wandering at end of trips and "captures" represented by observation of the animal in motion.

sional alley shown in Fig. 7, it was only recorded whether or not a wandering occurred at the end of trips. No measure was made of the length of the wandering. However, it is my impression that the longer a trip, the greater will be the amount of wandering at the end of a trip if wandering is initiated. This would mean that the amount of responsive wandering at the end of trips is proportional to $r^{2}$ rather than just to $r$. If this is really the case, then we can derive a fourth cumulative probability curve, Curve IV, which has the form:

$$
\mathrm{Cum}_{\mathrm{Iv}}=1-\left[\frac{R^{3}}{6}+\frac{R^{2}}{2}+R+1\right] e^{-R}
$$

By successive approximations utilizing Eq. (25), it was found that when $\alpha=0.32, \mathrm{Cum}_{\mathrm{IV}}=0.516$ at $r=1.2$. Therefore, 0.32 is the $\alpha$ conversion factor applied to $R=r / \alpha$, so that Curves I and IV may be compared in terms of the bivariate normal home range $\sigma$ distance. Note that thus there is forced conformity of both Cum II and Cum $\mathrm{Cu}_{\mathrm{IV}}$ to Cum $\mathrm{I}$ at the 1.2 value of $r$.

Near the home range center, Curve IV predicts fewer captures than is implied by Curve II or found by observed captures, Table III. However, at the longer radial distances from the home range center, Curve IV more 
closely approximates Curve I and the observed data than does Curve II. At present parsimony demands assuming only that the farther an animal moves from his home range center, the more likely it will terminate an outward trip and respond to stimuli near the place of stopping. Extremely careful observation is required to determine if animals tend to wander still farther about the points of termination of trips as these points occur farther from home.

\section{B. Travel-Path Home Range}

C'aptures or responses represent only one method of assessing home range. I have indicated that one assumption regarding behavior is that on the outward trip from home and on the return trip to home, the animal is in a preceptually blind state during which static stimuli fail to elicit responses. And yet it is possible to observe and record the presence of such nonresponsive individuals on this outward and return trip. Utilizing Eq. (19) and considering the effect of the geometry factor in the sense that the observer in a two-dimensional field can record an animal only if it passes directly by the observer, and assuming that the amount of wandering at end of trips is minimal, the equation for the cumulative probability of observing an animal during its travels, Curve III, becomes:

$$
\text { Cum }_{\text {III }}=1-(1+R) e^{-R}
$$

Data for Curve III with the $\alpha$ constant $=0.44$ are shown in Table III and on Fig. 8. Obviously, if "captures" represent such observations, the animal will appear to spend more time closer to home. Leopold et al. (1951, Fig. 50) provide data on home range based upon risual observation of marked mule deer. They presented their data in terms of number of observations within successive 100-yard bands from the site of capture. The actual home range center will be on the average somewhere to the right or left of the line of length $d$ connecting the point of eapture and the point of later observation. the actual radial distance from the true home range center would be $K \cdot d / 2$ when $K$ is between 1.0 and 1.414 . Without going into the origin of $K$, it is still apparent that the distances given by Leopold et al. (1951) can be utilized as approximating proportionality to radial distances of observation from the home range center. The cumulative probabilities of observation for 102 observations of males and 103 observations of does and fawns are with distance, respectively: 100 yards $(0.363,0.495), 200$ yards $(0.500$, $0.815), 300$ yards $(0.678,0.952), 400$ yards $(0.726,0.980), 500$ yards $(0.862,1.0), 600$ yards $(0.862,-), 700$ yards $(0.932,-), 800$ yards $(1.0,-)$. Noting that 0.50 of the males were observed within 200 yards 
of the point of eapture, and that 0.495 of the females and fawns were observed within 100 yards of the point of capture, and utilizing Eq. (27) and the fact that $R=r / \alpha, \sigma$, in the sense of $d$, for males is 270 yards and for females and fawns is 137 yards. On this basis the observed cumulative probabilities of observation were converted into sigma units of distance from the point of capture and posted on Fig. 8.

There is forced conformity with Curve III, Eq. (27), at 0.74 $\sigma$. However, the further proximity of the observed points, up through 1.5 $\sigma$, to Curve III provides credence to the general formulation of Eq. (27). Observations may occur when the animal is on either the outward or return portions of a trip or during the wandering at the end of a trip. The more an animal wanders at ends of trips relative to the cumulative travel path, excluding wandering at the end of trips, the farther the observed points may be expected to diverge from Curve III and approach Curves I and II. In fact, were it possible to obtain adequate assessments of home range in terms of observations made of the individual during its travels as well as of data derived from responses such as entering traps, it would be possible to calculate wandering distance at $r$.

\section{Summary of the Concept of Home Range}

Developing an attachment to a restricted region to which an animal returns after wandering outward from it requires that the individual belong to a species which has evolved the capacity to retain the memory of prior experience. If an animal remains at the site of its birth there is no question that the immediate surroundings of this site will be known better than more distant ones. However, many individuals are forced from the site of their birth. The term "forced" is here used as a postulate derived from the observed avoidance of fields containing strange stimuli. Parsimony demands the assumption that an animal will remain at home unless forced away, until it can be shown that an animal will change its home in the absence of any factors which might tend to expel it.

Even if an animal is expelled from the site of its prior residence and begins to wander at random, it will be chance cover some areas more frequently than others. As it thus becomes familiar with a restricted area, it will increase its avoidance of less frequented areas. This process will lead to a repeated frequenting of a particular restricted location which can be defined as "home." Home may acquire other attributes, such as the construction of a den, but such additional attributes are not necessary for a site to become a home.

At irregular, probably random, intervals the individual engages in ex- 
cursions from home. During these outward excursions the animal remains in a refractory state during which it is unable to respond to stimuli encountered. Only at the termination of trips, including the period of wandering at end of trips, will the animal be able to respond to objects or situations such as it had already passed on the outward trip. However, those objects which were bypassed on the outward trip do alter the probability of terminating trips. The greater the number of such objects, or the greater the intensity of stimuli emanating from them, the greater will be the probability of a trip stopping after reaching every distance from home. Should novel stimuli be encountered during a trip, the shorter will be the interval after reaching home before initiating another trip. This holds in so long as the novel stimuli are not excessive. In this case, the animal will remain at home for unusually long periods before again venturing forth. The probability of wandering increases with distance from home. These several aspects of locomotion and responsiveness to stimuli lead to a probability of responding within the region about an animal's home, which so closely resembles that of the bivariate normal distribution function that the latter may be used to represent home range.

\section{Continuous Removal Trapping of Small Mammals}

When a large number of snap-traps are set within a habitat where mice and shrews live, it is usually observed that more individuals are captured on the first than on the second or third day, and that fewer animals enter traps on the third than the second day. It has generally been assumed that this decline in catch through time would continue were the traps left set for a longer period. Based upon this assumption, various equations (e.g., Zippin, 1956; Calhoun and Casby, 1958) have been developed for estimating the number of individuals which would eventually enter the traps.

Furthermore, it has also generally been assumed that the catch for a 3or 4-day period would reflect the relative species composition of the community being sampled. This assumption served as the basis for establishing the North American Census of Small Mammals (Calhoun, 1949-1957). During the nine years, cooperators provided results (see NACSM Release No. 9) for 1615 standard trap-lines consisting of 20 stations, usually 50 feet between stations, 3 traps per station, run for three consecutive nights. Despite the laudable objectives of this cooperative endeavor, this tremendous effort has, for reasons we shall shortly see, provided inadequate data for insight into either relative density or species composition.

Questioning the usefulness of short-term removal trapping developed 
slowly. It began in 1950 when I asked A. Dexter Hinekley, then a summer student at the Jackson Memorial Laboratory, to continue eight NACSMI lines for 15 days (see Section VI, E and Fig. 13A). Despite the expectation of a continually declining catch after the first 3 days, it turned out that the number taken from day 4 onward actually increased each successive day until on day 1.) three times as many individuals entered traps as did on day 1.

These results initiated an intensive effort to explore the results obtained from prolonging the period of removal trapping. I am particularly indebted to Drs. William L. Webb and Earl F. Patric of the Huntington Wildlife Forest, and to my colleague, Dr. Kyle R. Barbehenn. Without their permission to utilize here some of the data from their extensive studies of removal trapping, it would have been impossible to develop the concepts elaborated in succeeding sections of this paper. I have also profited from a number of 30-day census contributed by other NACSMI cooperators.

Our concern here will be with the contribution of this program, and allied research developing from it, to furthering our understanding of home range and the organization of small mammal communities. The following few sections merely present results. Note that within each study presented, the several species present markedly different patterns of capture by traps over time. Interpretations based upon the data in Section VI are given in Sections VIII, IX, and $\mathrm{X}$.

\section{A. Rich Lake Island, New York, 1952, Sixty-Day Removal Study-Data Contributed by William L. Webb:}

Four NACSM traplines were rum on this island in the Huntington Forest for 60 consecutive days, beginning August 16. The red-backed mouse, Clethrionomys, and the shrew Sorex formed the majority of the catch. Figure 9 gives the results for the first 30 days. Note that Clethrionomys, which entered the traps in large numbers during the first few days, was by the end of 30 days represented by only half the total ratch as that for Sorex, whose peak in catch per day did not come until the ninth day. Both species exhibited a secondary minor period of increase in captures.

During the same summer, 34 NACSM lines were run for the standard 3-day period in similar forests on the mainland (Table IV). As on the island, comparatively few Sorex entered traps on mainland areas during the first 3 days of trapping. And yet, continuous removal trapping for an 80-day period (Section XI) in one tract of the mainland forest produced $(117$ Clethrionomys and 1225 Sorex. 


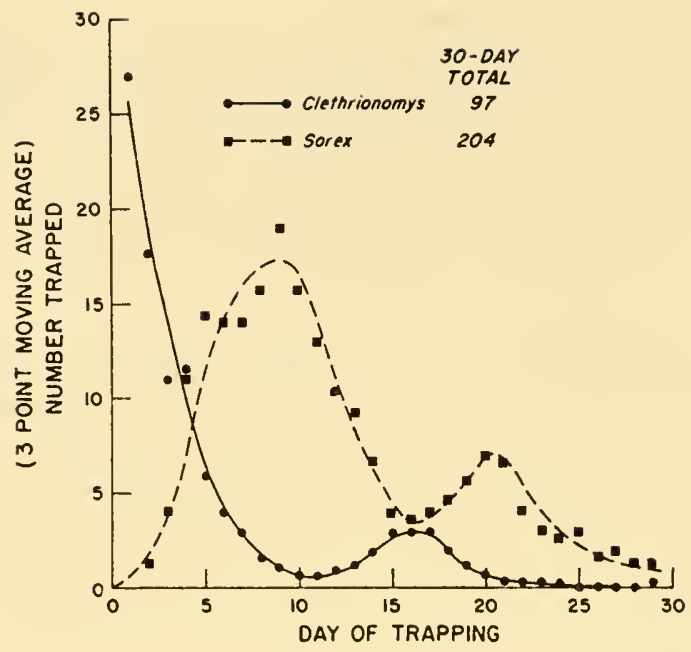

FIG. 9. Daily removal captures of the two most abundant species inhabiting a 30acre island; recorded by Dr. William L. Webb.

\section{TABLE IV}

Comparison of Results between Short-Term and Long-Term Rhmoval, Trapping on the Huntington Forest during 1952

\begin{tabular}{|c|c|c|c|}
\hline \multirow{2}{*}{ Location } & \multicolumn{3}{|c|}{ Genus } \\
\hline & Clethrionomys & Sorex & Peromyseus \\
\hline $\begin{array}{l}\text { 80-Day total for continuous removal } \\
\text { trapping on a } 30 \text {-acre mainland area } \\
\text { (see Section XI) }\end{array}$ & 617 & 1225 & 77 \\
\hline $\begin{array}{l}34 \text { NACSM lines each for } 3 \text { days on main- } \\
\text { land }\end{array}$ & $753(22.1)^{a}$ & $17(0.5)$ & $38(1.1)$ \\
\hline $\begin{array}{l}4 \text { NACSM lines on Rich Lake Island } \\
60 \text {-day total } \\
\text { Total 1st } 3 \text { days }\end{array}$ & $\begin{array}{r}133 \\
53(13.3)\end{array}$ & $\begin{array}{r}243 \\
4(1.0)\end{array}$ & $\begin{array}{c}27 \\
0\end{array}$ \\
\hline
\end{tabular}

a Mean 3-day totals shown in parentheses. 
B. Chadwick Woods, Montgomery County, Maryland, Removal Study, 1958-1959-Data Contributed by Kyle R. Barbehenn

Five circular traplines, each with a radius of approximately 300 feet, were used. The number of traps per station and the interval between stations varied among the lines. However, for the present purposes these differences are unimportant. As with the Rich Lake Island study, the genus, here Peromyscus, with the initial most rapid input was represented by only half as many (57) captures as another genus, Blarina, which entered the traps more slowly (116 taken) (see Fig. 10). A third genus, Sorex, began its period of maximum captures only after the majority of the other two genera had been removed, but only 48 were captured. Note that Peromyscus exhibited a fairly rapid rate of capture until approximately $65 \%$ of the 30 -day total had been taken. Following an intervening period with very few captures, there ensued a secondary period of increased (aptures.

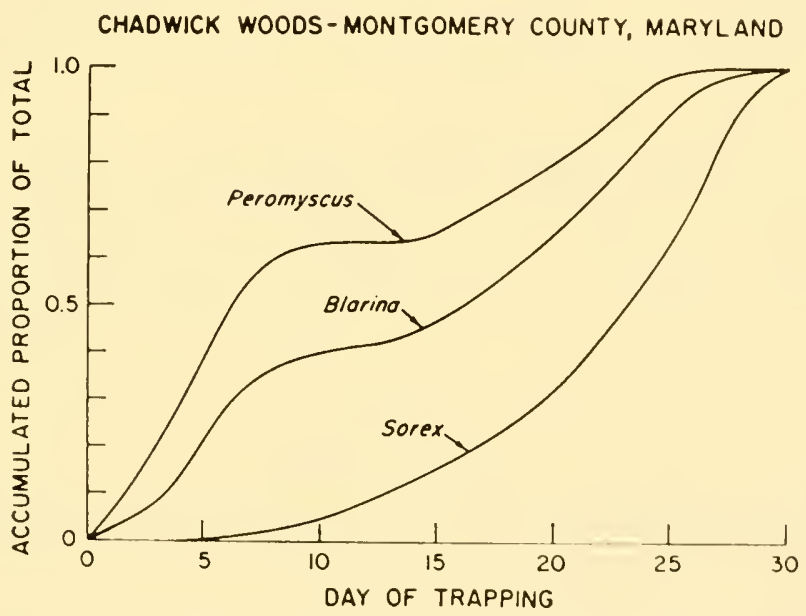

FIG. 10. Differential "schedules" of entering traps expressed by the three species recorded by Dr. Kyle Barbehenn in his study of continuous removal trapping in an upland hardwood forest.

C. Comparative Catches, IIuntington Wildlife Forest, 1952-1953-Data Contributed by Earl F. Patric and William L. Webb

During these two years Peromyscus and Blarina were universally scarce on the Huntington Forest. In order to determine the general pattern of their input, data from five separate plots must be pooled. 
Four plots consisted of concentric circular traplines, 75 feet between circles, with one trap each 10 feet along the lines. Two plots consisted of three lines, and two of four lines. The fifth plot is represented by the first 15 days' results of the Rich Lake Island study presented in more detail in Section VI, B. All areas were sampled for at least 15 days (Fig. 11). Each day all animals captured were removed.

Note that whereas the catch of Clethrionomys initially declines through

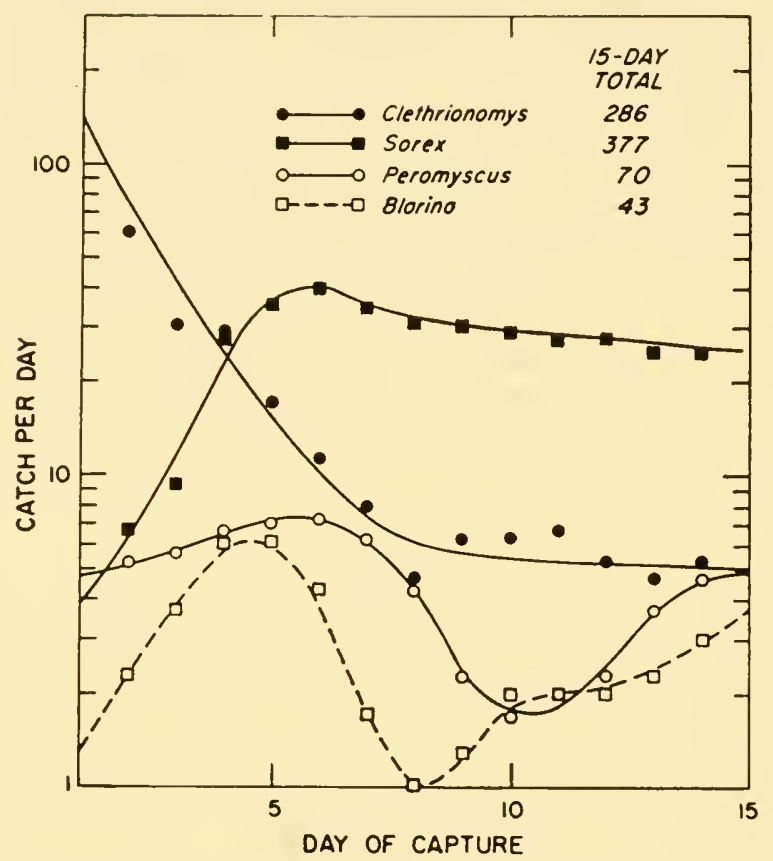

FIg. 11. Summated results from five continuous removal studies conducted by Dr. Earl Patric and Dr. William Webb during 1952 and 1953 on the Huntington Forest, New York.

time, all other species exhibit an increase in catch per day for the first 4 or 5 days.

D. Comparative Catches, Huntington Wildlife Forest, 1951-Data Contributed by William L. Webb

Nine NACSM lines were each run for at least 24 days during September of 1951 (Fig. 12). In contrast to the following two years, Peromyscus nearly equaled Clethrionomys in abundance. Likewise, Blarina was abun- 


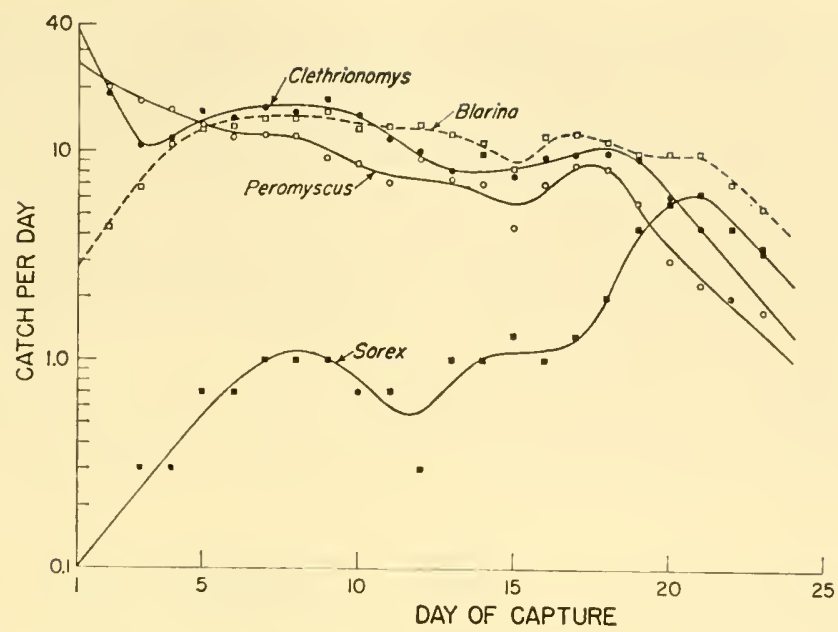

FIG. 12. A continuous removal study conducted by Dr. William L. Webb on the Huntington Forest in 1951. This year represented a time when the dominance relationship of the three most abundant species was not yet clarified, the small mammal community was in a state of social flux. See Table V. Points shown are 3-point moving averages.

dant. Only Sorex was rare. The relative likelihood of capture during the initial and terminal days of trapping forms a most interesting series (Table V). Initial and terminal likelihood of capture are inversely related despite the fact that usually over $50 \%$ of each genus were taken during the middle period of trapping.

TABLE V

Huntington Forest (1951) 24-Day Continuous Removal Trapping

Genus 24-Day total

Proportion of 24-day total during

First 5 days $\quad$ Last 5 days

$\begin{array}{lrrr}\text { Peromyscus } & 217 & 0.418 & 0.042 \\ \text { Clethrionomys } & 267 & 0.311 & 0.079 \\ \text { Blarina } & 229 & 0.188 & 0.166 \\ \text { Sorex } & 41 & 0.024 & 0.585\end{array}$

E. Comparative Catches in Maine (19.50) and Maryland (1953)

Eight NACSM lines within an 80-acre tract of a much more extensive continuous forest opposite the Hamilton Station of the Jackson Memorial 
Laboratory on Mt. Desert Island formed the trapping procedure in the Maine study. Four concentric circular traplines, each of a 75 -feet greater radius than the next innermost one, having one trap each 10 feet along the lines, formed the trapping procedure in the Maryland study. During the years these studies were conducted, I was still working under the assumption (see Calhoun and Webb, 1953) that the large numbers of animals taken shortly after the first 3 days of trapping resulted primarily from invasion from beyond the periphery of the trapping area. These relatively short-term removal studies are presented here (Fig. 13) since they provide further insight into the differential rate of input for the several species making up the small mammal community. Total 15-day catch by genus
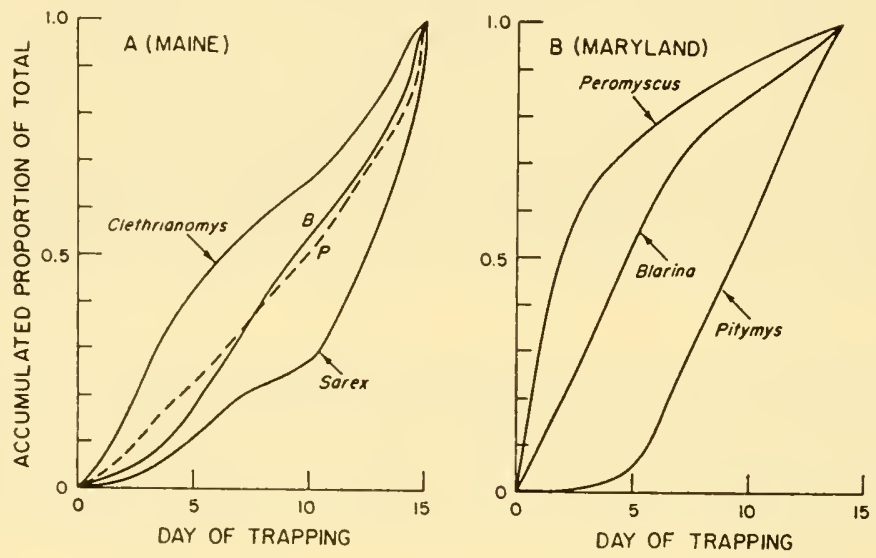

Fig. 13. Two short-term removal studies. The more dominant a genus happens to be the sooner will $50 \%$ of its members be trapped. In Fig. 13A: $B=$ Blarina; $P=$ Peromyscus.

for the Maine study (Fig. 13A): Clethrionomys 80, Sorex 53, Peromyscus 75, and Blarina 132. Total 14-day (atch by genus for the Maryland study (Fig. 13B): Peromyscus 45, Blarina 76, and Pitymys 62. The Maine study was conducted by A. Dexter Hinckley under the author's direction, and the Maryland study was condueted by the author.

\section{F. Comparative Catches of Peromyscus and Clethrionomys}

Relationships between these two genera, revealed by NACSM census data, have been particularly helpful in developing insight concerning community organization. Census from three localities, where both genera occur, are represented by a large number of traplines. Those run in each 
locality encompass a period of several years, and so should provide a representative picture of relationships in the respective habitats. Results in Table VI derive from 37,080 "trap-days" of effort.

Drs. Earl IF. Patric and William L. Webb provided the New York data from the Huntington Wildlife Forest at Newcomb, New York. The majority of the animals shown were taken in the years of high density, 19.5.3-1954, although the few taken during the low density years of 1955 and 1956 are included. Dr. J. E. Moore of the University of Alberta, Edmonton, pro-

TABLE VI

Comparative Catch of Two Genera in Three localities

\begin{tabular}{|c|c|c|c|c|c|c|c|}
\hline \multirow{2}{*}{ Genus } & \multirow{2}{*}{ Location } & \multirow{2}{*}{$\begin{array}{l}\text { Number } \\
\text { of lines }\end{array}$} & \multicolumn{3}{|c|}{$\operatorname{Day}^{a}$} & \multirow{2}{*}{$\begin{array}{c}\text { Total, } \\
\text { days } 1-3\end{array}$} & \multirow{2}{*}{$\begin{array}{c}\text { Mean } \\
\text { per line }\end{array}$} \\
\hline & & & 1 & 2 & 3 & & \\
\hline \multirow{3}{*}{ Clethrionomys } & New York & 90 & $\begin{array}{c}586 \\
(0.408)\end{array}$ & $\begin{array}{c}470 \\
(0.327)\end{array}$ & $\begin{array}{c}3 S 0 \\
(0.265)\end{array}$ & 1436 & 16.0 \\
\hline & Alberta & 36 & $\begin{array}{c}117 \\
(0.390)\end{array}$ & $\begin{array}{c}103 \\
(0.33 S)\end{array}$ & $\begin{array}{c}83 \\
(0.272)\end{array}$ & 303 & 8.5 \\
\hline & Maine & 80 & $\begin{array}{c}90 \\
(0.489)\end{array}$ & $\begin{array}{c}5 \mathrm{~S} \\
(0.315)\end{array}$ & $\begin{array}{c}36 \\
(0.196)\end{array}$ & 184 & 2.3 \\
\hline \multirow{3}{*}{ Peromyscus } & New York & 90 & $\begin{array}{c}93 \\
(0.274)\end{array}$ & $\begin{array}{c}117 \\
(0.344)\end{array}$ & $\begin{array}{c}130 \\
(0.382)\end{array}$ & 340 & 3.8 \\
\hline & Alberta & 36 & $\begin{array}{c}241 \\
(0.577)\end{array}$ & $\begin{array}{c}122 \\
(0.292)\end{array}$ & $\begin{array}{c}55 \\
(0.132)\end{array}$ & 418 & 11.6 \\
\hline & Maine & so & $\begin{array}{c}132 \\
(0.434)\end{array}$ & $\begin{array}{c}103 \\
(0.399)\end{array}$ & $\begin{array}{c}69 \\
(0.227)\end{array}$ & 304 & 3.8 \\
\hline
\end{tabular}

${ }^{a}$ Proportion of 3-day $\Sigma$ shown in parentheses.

vided the Alberta census including the year's 1948-1956. Dr. John A. King and two U. S. National Park Rangers, Clifford Senne and L. S. Winsor, and the author conducted the Maine census on Mit. Desert Island between 1949 and 1952 .

Figure 14 shows the decline in catch from day 1 through day 3 for these two genera for Maine and New York.

In Maine where both genera have low densities, their respective patterns of decline in catch through time are very similar. However, the relatively greater catch of Clethrionomys on day 1 should be noted with reference to 
the trends of the 15-day trap-out as shown in the left-hand graph of Fig. 13 for eight NACSM lines also run on Mt. Desert Island.

In New York, where Peromyscus had the same density as in Maine but Clethrionomys was four times as numerous as Peromyscus, a striking difference in the trends resulted. Clethrionomys exhibited a typical, though somewhat slow, decline through the three consecutive days. In contrast, despite removal trapping and thus fewer deer mice available for entering traps, a greater number of Peromyscus entered traps each successive day. I wish to emphasize that competition for entering traps contributed negligibly to these trends. Three times as many traps were set each day as there were total animals caught for the entire 3-day period.

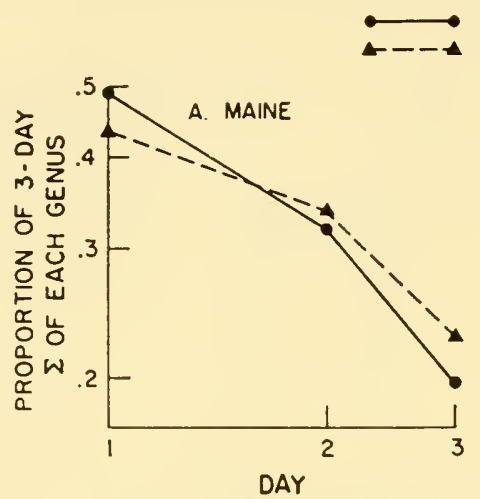

Clethrionomys
Peromyscus

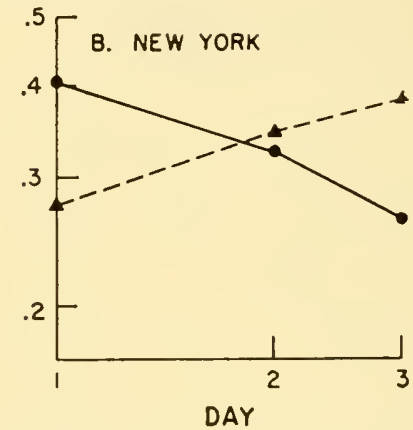

FIG. 14. Capture rates for Clethrionomys and Peromyscus in Maine and New York. Nearly codominance, or lack of dominance, is reflected by the Maine data, whereas in New York Peromyscus is clearly subordinate to Clethrionomys. See Table VI.

In Alberta, where both species are on the average relatively abundant (Table VII), there exists a marked seasonal difference (Fig. 15) in the trend of input over time between these species. During the spring, when low densities characterize both species, each exhibits a rapid rate of decline. In contrast, by fall when high densities have developed for both species, Clethrionomys shows a relatively constant input. Note the reversal of the trends of input for Clethrionomys and Peromyscus when Fig. 11 and Fig. 15B are compared.

\section{Toward a General Theory of Interspecific and Intraspecific Use of Space}

The data presented in Section VI reveal that a continuous decline in catch from one day to the next during removal trapping is the exception 
TABle VII

Mean Catch per Trapline ox Dr. Moore's Alberta Study Area

\begin{tabular}{lccc}
\hline \multirow{2}{*}{ Cienus } & \multicolumn{2}{c}{ Season } & \multirow{2}{*}{ Fall/Spring } \\
\cline { 2 - 3 } & Spring & Fall & \\
\hline Clethrionomys & 5.00 & 11.90 & 2.18 \\
Peromyscus & 8.06 & 15.56 & 1.93 \\
\hline
\end{tabular}

rather than the rule. Even where the numbers taken per day do initially decline, a secondary increase usually ensues by the fifteenth day of trapping. Furthermore, many species actually exhibit an increase in catch per day as their associates are removed. Any trend of increase in catch after previous removal of associates can only mean that the survivors have in some way altered their behavior so as to increase their exposure to traps. Furthermore, we must conclude that those caught earlier in time must, while still alive, have suppressed this change of behavior. We shall now consider how these results make possible a conceptualization of spatial, temporal, and social organizations of the small mammal community.
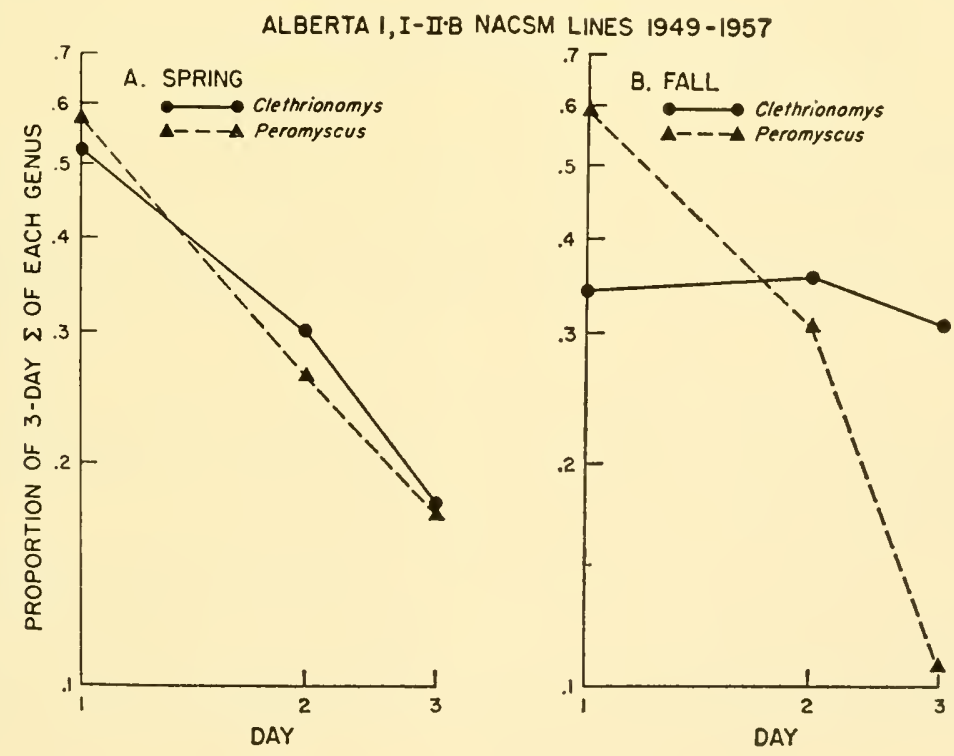

Fig. 15. Seasonal capture rates for two species. With the increase in density from spring to fall (see Table VII) Peromyscus apparently becomes dominant to Clethrionomys. 


\section{A. A Two-Species System}

The relationship between Clethrionomys and Sorex on the Rich Lake Island (Section VI, A, Fig. 9) and between Peromyscus and Blarina in the Chadwick Woods study (Section VI, B, Fig. 10) form the basic data leading to this formulation. The shrews - Sorex and Blarina, respectively,in these two studies exhibited a delay in entering traps until many of the mice, Clethrionomys or Peromyscus, had been removed. Yet despite this slowness of entering traps, twice as many shrews as mice were taken during the 30 days of trapping in each study.

These data pose two questions:

1. Why were there twice as many shrews as mice?

2. In what way (and why) did the behavior of the shrews change so that after several days of trapping they were more exposed to traps than initially?

The first assumption will be that the larger the home range the greater the likelihood an animal will encounter a trap and be caught. Since during the first few days of trapping many mice but few shrews were taken, despite the greater abundance of shrews, the mice must have had considerably larger home ranges than the shrews. Furthermore, since the number of shrews taken per day increased during the first 10 days of trapping, it follows that their home range expanded as the mice were killed off. This leads to the conclusion that the mice in some way inhibited the extent of home range of these shrews. We may now designate the mice as being dominant or alpha species and the shrews as subordinate or beta species.

Formulation of a theory depicting the social and spatial aspects of such a two-species system requires the assumption of a uniform distribution of centers of home range for the alpha species. This represents the simplest assumption leading to a 1:2 ratio of number of alpha and beta species. In the preparation of Fig. 16, a field of uniformly spaced dots (not shown in Fig. 16) was plotted. These dots represented home range centers for members of the alpha species. A circle of radius half the distance betrieen centers was drawn about each center. Each of the larger circles in Fig. 16 encompasses some portion of an alpha individual's home range.

Now we can ask: Where is it most logical to find the home range centers of beta species? They should be located at points minimizing encounter by members of the beta species with members of the alpha species. The interstices formed by juncture of each set of three neighboring home ranges of alpha species represent such locations.

Here a beta individual is equidistant from three alpha individuals. Displacement of the home range of a beta species member from such a point 
will increase its probability of encountering at least one member of the alpha species. About each such home range center a smaller circle was drawn (Fig. 16). This smaller eircle represents the same proportion of the beta species home range as does the larger eircle for the alpha species.

Examination of Fig. 16 shows that according to this formulation there will be in an ideal steady state exactly twice as many individuals of the subordinate beta speeies as there are of the dominant alpha species. This is because there are twice as many interstices between uniformly distributed alpha home ranges as there are alpha home ranges.

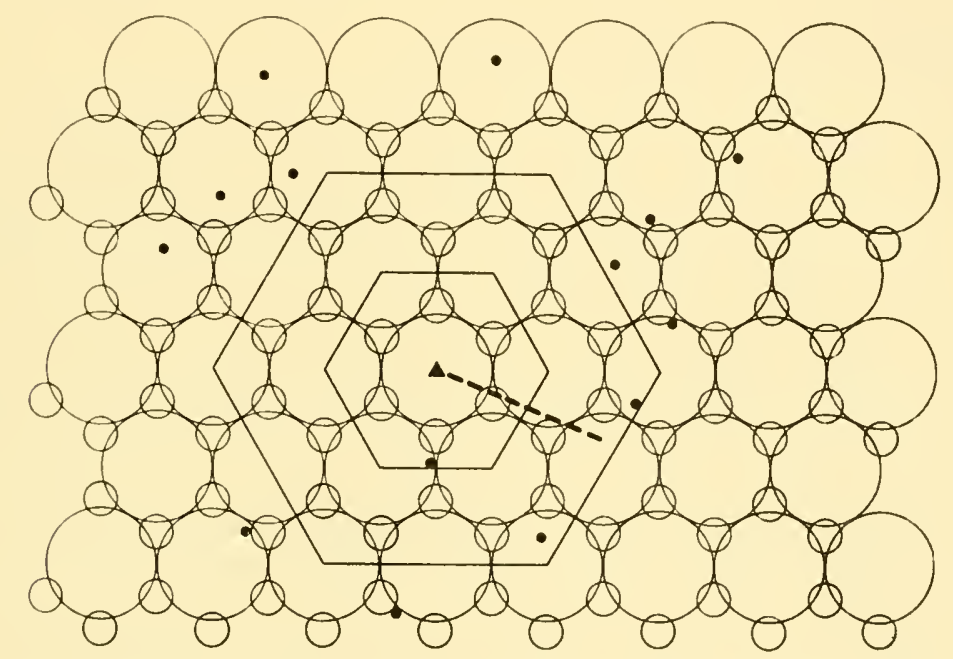

FIG. 16. Spatial distribution of a dominant and a subordinate species. Large circles represent a uniform distribution of one sigma radius portions of the home ranges of a dominant, alpha, species, while the smaller circles represent a similar proportion of the home range for members of a subordinate, beta, species. See text for other details, Section VII, A.

Now, suppose that a few traps are placed at random within a habitat characterized by inhabitation by such an alpha and a beta species. The fifteen dots in Fig. 16 represent such random points. It is readily seen that there is an alpha individual exposed to nearly every trap, but few individuals of the beta species are exposed to traps.

Now suppose we do set traps in such a system and remove the individuals caught. It is the general experience in continuous removal trapping that $25-50 \%$ of the members of the alpha species which are taken during 30 days are actually taken during the first 3 days.

This can only mean that by the end of 3 days there must be many members of the beta species whose neighbors of the alpha species are then no 
longer present. In the absenee of the inhibiting influences emanating from the former alpha neighbors, the beta individuals then make a complementary expansion of their home range. As they do so, some beta individuals come into contact with traps and are also removed. Inspection of a number of continuous removal census in which there is an alpha speeies and one or more subordinate species indieates that maximum expansion of home ranges of the subordinate speeies is generally reached by the fifteenth day of trapping. After this time, the cateh by day for subordinate speeies also deelines over time sinee fewer and fewer remain to be eaught.

\section{B. The Nature of the Inhibitory Influence}

Both Sorex and Blarina in Fig. 10 and Sorex in Fig. 9 exhibit an inerease in cateh even beginning on the second day of removal trapping. Similar results apply to the subordinate speeies included in Figs. 11-14, although not so apparent in those graphs where the ordinate represents accumulated eateh. These results indicate that even remoral of a small proportion of the alpha species is sufficient to induce home range expansion by subordinate species. Thus, the means of communication through which inhibition operates must be sufficiently effective and repetitive that a change in the general field intensity (or frequeney) of stimuli emanating from an alpha species is detected within a few hours at least by subordinate species.

Bodily contact by random movement is unlikely to be effective. By the same token that it takes several days before all alpha species are taken in traps, it follows that in many instances subordinate species would be unaware of the absence of their alpha neighbors if this detection were a consequence of a change in frequency of contact. Production and detection of scent, at least where scent signposts are concerned, would likely operate to inhibit home range expansion because of the persistence of scent beyond the death of alpha individuals. Sight is unlikely to be an effectual means of detection of alpha by beta species, both because of concentration of activity during the night by many species and because many of these beta speeies aetually spend considerable time under the leaf mold.

There remains vocalization and audition as the means of eommunication. Although there is as yet no proof that such is the means of eommunication whereby individuals ean detect the presence of unseen neighbors, it stands out as the most likely possibility. Most small mammals do vocalize.

Fewer barriers exist that might prevent or distort the passage of sound through the environment than is true with regard to light stimuli or odors. In the following discussions vocalization and audition will be assumed to be the means of communication within and between species. However, 
identification of the means is unessential to the general argument; only recognition of the existence of some effective means of communication is necessary.

\section{The Learning of Signals}

There exists the possibility that the response of one individual to a signal emitted by another has become through evolutionary processes one which does not require a learned association between the signal and some act on the part of the emitter for its development. In the prior history of such species there must have been the opportunity for associating the signal with its emitter and there must have been survival value in the receptor developing an innate response to detection of the signal. However, until such responses to signals are demonstrated to be innate, it shall be assumed that they are learned.

We may now ask, "How may the members of a species learn a signal when the individuals are characterized by fixed home ranges which may be described by the bivariate normal distribution function?" In order to gain insight into this question, we shall consider two neighbors, $A$ and $B$. $A$ 's home range center is fixed whereas $B$, who lives some distance away, gradually shifts its home range center toward that of $A$. When the home range centers are six home range sigma or more apart, it is apparent that the probability of their meeting by ehance will be extremely remote. This relative probability of meeting is proportional to the product of their density functions at any particular point (see Table 2 in Calhoun and Casby, 1958).

However, as the home range center of $B$ approaches that of $A$, these two individuals will meet by chance on very rare occasions. Three examples of the relative probability of $A$ and $B$ contacting are given in Fig. 17 . When the home range centers (HRC's) are 3.9 sigma apart, one peak is 1.5 sigma from $A$ 's HRC and the other is 1.5 sigma from $B$ 's HRC. In examining Fig. 17 it is well to keep in mind that we are considering the probability of contact at points along the line connecting the two home range centers. At all distances intervening between home range centers, from slightly over 3 sigma up to 6 sigma, there are always two peaks in this curve of probability of contact between two neighbors. As the home range center of $B$ approaches 3 sigma to that of $A$, these two peaks approach each other until at 3 sigma they coincide for the first time. This single peak of highest probability of eontact of two neighbors, which lies exactly halfway between the two home range centers, characterizes all distances less than 3 sigma intervening between the home range centers. 
Let us assume that when $A$ and $B$ meet there is some interaction between $A$ and $B$. That is, $A$ responds to $B$ and $B$ responds to $A$. At the same time, each emits a signal. If such chance contact occurs frequently enough there exists the opportunity of each individual associating the other with the signal emitted at the time of interaction. Furthermore, we may assume that learning is enhanced by other factors of the environment being constant

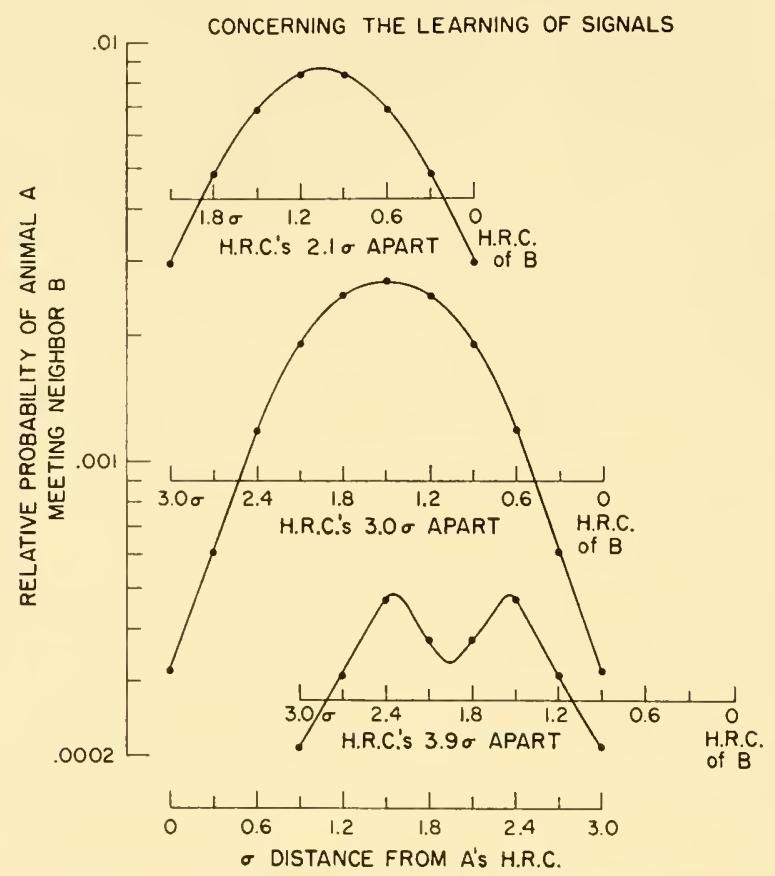

Fig. 17. Concerning the learning of signals. Not unless home range centers are 3 sigma or less apart will there be a single point of most probable contact of two animals meeting by chance. This point lies halfway between the line connecting the two home range centers. The closer home range centers approach, the higher will be the probability of chance contact and thus the more likely the association of any simultaneous signal with the consequences of meeting.

at the time of interaction. When home range centers are more than 3 sigma apart, the two points of greatest probability of contact are separated from each other and therefore are unlikely to have identical surroundings. This nonidentity of surroundings, that is the absence of identical secondary reinforcers, may be expected to retard learning.

However, at a 3 -sigma interval between home range centers, there is only a single point of greatest probability of contact; thus, at this intervening distance between home range centers there is not only an increased 
probability of contact because the home range centers are closer together, but there exists a greater constancy of secondary reinforcers at the single point of greatest probability of contact. It is for this reason that I suspect that learning of signals is not likely to be effective unless home range centers are 3 sigma or less apart.

Once an animal has learned to associate a signal with the animal which emitted it, there then exists the opportunity that the detecting individual (an perceive (hear) the signal at some distance from the emitter and make the appropriate response of approach or withdrawal. How far the signal may be detected depends upon both the intensity of the signal emitted and the ability of the detector to hear it. Presumably, sensory capacities for detection and motor capacities for emission have evolved simultaneously and in harmony. We may then wonder as to the distance over which such evolution of capacities permits the detection of an emitted signal. In the absence of any experimental data, introspection suggests that one might anticipate evolution of capacities to the point that an individual can just detect a signal emitted at the maximum distance between home range centers which still permits the learning of such signals. As we have seen, this distance is equivalent to 3 home range sigma. In other words, when animal $A$ is at its home range center, a signal emitted by animal $B$ at the border of $A$ 's home range (as represented by a 3-sigma distance) reaches $A$ in just the sufficient intensity to elicit a response by $A$. As $B$ moves farther than 3 sigma away from $A$, the signal exhibits further decrease in intensity. Such reduced intensity may well be pereeived by $A$ until $B$ gets at least 6 sigma away. Thus, between 3 and 6 sigma, it is suspected that the signal itself is perceived but is below the threshold necessary for eliciting a response by the receptor. Signals arising between 3 and 6 sigma from the receptor are here designated as contributing to what I shall call "hum."

These characteristies of the signal are represented schematically in Fig. 18. A signal emitted by one animal when nearly in contact with another may be given a rating of 1.0. For the purpose of later calculations, it is assumed that there is an inverse decrease of intensity of the signal at successive distances from the emitter until at a distance of 3 sigma it has reached one-tenth of the intensity that might be recorded at the emitter.

Investigations of this formulation requires that the sound signals emitted by typical individuals of a species be recorded and other individuals of the same species trained to exhibit a response upon perception of a recorded vocalization. Then, in the native habitat of the species, the trained subject must be moved continually farther away from the sound source until it no longer exhibits the characteristic response which it had been trained to perform following presentation of the signal. This distance may then be 
compared with the animal's home range sigma, as determined by live trapping and related observational procedures.

\section{The Distance between Neighbors of the Same Species}

From the observed tendency of a 1:2 ratio between alpha and beta species when coexisting in the same habitat, it was concluded that the home range centers for the alpha species should approximate a uniform distribution. However, this analysis provided no insight into whether there

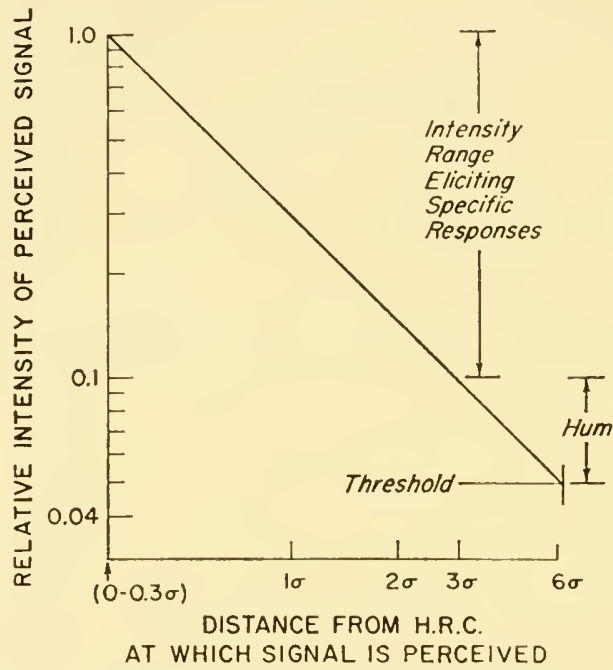

FIG. 18. Theoretical conceptualization of signal characteristics with reference to emission at a constant intensity at the home range center and detection and response by others at distances from it.

might be some ideal distance which should intervene between home range centers. In order to seek this insight we shall consider a single species community in which all individuals have exactly the same-sized home range, center's of home range are uniformly distributed, and the bivariate normal distribution function describes the home range.

Answers to two questions will be sought with reference to the relative distance between home range centers:

1. How does distance between home range centers affect the impact of the community on the environment?

2. How does the interval between home range centers affect the probability of one individual meeting or detecting its neighbors?

The impact of an individual upon its environment should be proportional 
to the amount of time per unit area it spends at successive distance from its home. This relative impact is described by the bivariate normal distribution function (Fig. 2). For any particular animal this means that at 3 sigma distance from its home its impact per unit area will be only 0.011 of what it was adjacent to its home. We may visualize the impact of any one animal upon its environment as having a mountain-shaped topography. Where home range centers are at least 6 sigma apart, there lies between them a "valley" where neither animal has a significant effect on the en-

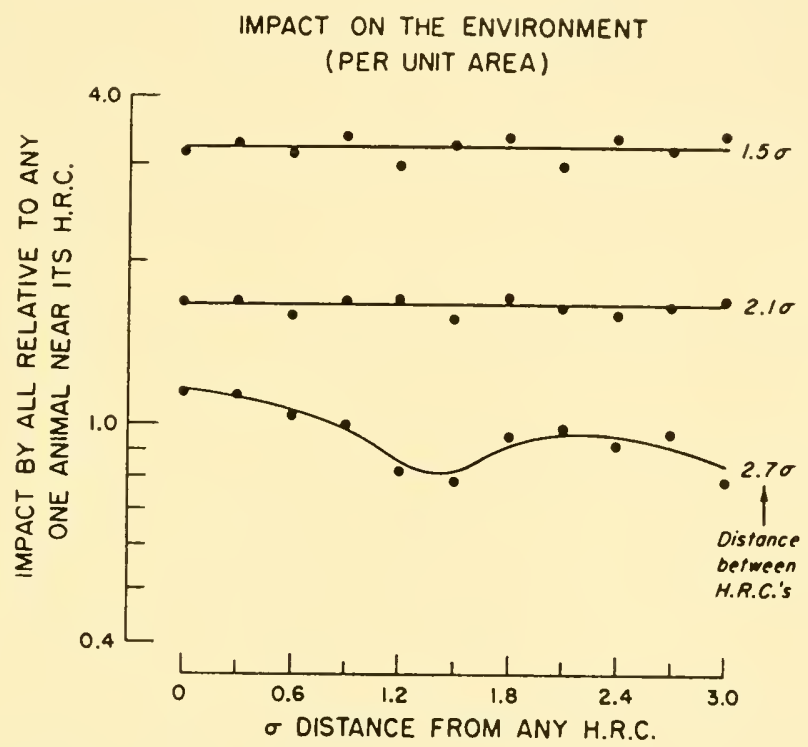

FIG. 19. Impact on the environment (per unit area). The value 1.0 represents the effect one individual will have near its home range center. Since home ranges increasingly overlap as their centers approach each other, i.e., density increases, the summated impact of all animals on any one point not only increases, but the relative impact on all points becomes more nearly equivalent.

vironment. As soon as home range centers get closer than 6 sigma to each other, the home ranges overlap and neighbors can both affect those portions of the environment falling within both neighbor's home ranges. As soon as home range centers become less than 3 sigma apart, some portion of the environment can be affected by more than two individuals.

At any point in the environment, the impact of all animals which can arrive at that point during this normal ranging about their home is proportional to the sum of their separate density functions at that point. Utilizing the normative data of density function as a function of the sigma radius from home, given in Table 2 of Calhoun and Casby (1958), several curves of summated density function were calculated (Fig. 19). 


\section{E. Methods of Calculating Data Relative to the Distance between Neighbors}

In Fig. 16, which illustrates the uniform distribution of an alpha species and of a beta species lying in the interstices between the home ranges of the alpha individuals, we can select any single alpha individual and note certain characteristics of the geometric distribution of its alpha neighbors. One such individual, whose home range eenter is indieated by a triangle, is shown in Fig. 16. A line drawn between the home range centers of its adjoining nearest neighbors forms a hexagon about this individual. Just as there are six nearest neighbors, there are twelve next-nearest neighbors. Lines connecting the home range eenters of these next-nearest neighbors also form a hexagon. Successively more distant neighbors form concentric hexagons, each containing six more individuals than the next innermost hexagon. For the purpose of investigating the effeet of neighbors on each other or upon the environment, a system of four "concentric" hexagonal sets of neighbors was prepared on a large sheet of graph paper. This procedure was repeated three times, forming spatial sets in which home range centers between nearest neighbors were respectively 1.5, 2.1, and 2.7 sigma apart. Ruler scales representing density functions (Table 2, Calhoun and Casby, 1958) at suceessive sigma distances from the home range eenter, as well as ruler seales representing intensity of signal (Fig. 18) were prepared. Using these ruler seales, several types of erents were calculated with regard to their changes in intensity or frequency along a 3sigma route such as is shown by the heavy dashed line in Fig. 16 .

At each of eleven points along this typical route of travel, a sum of the density functions of all neighbors whose home ranges overlapped one or more of these eleren points was ealculated (see Fig. 19).

\section{F. Further Comment on the Impact of All Individuals on the Environment}

Each of these sums of density functions were divided by 0.159 , the relative density function of an animal near its own home range center. By so doing we ean obtain a fairly good idea of the impact of all individuals who may arrive at any particular point with reference to the effect that one individual would have near its home range center. It may be seen that when home range eenters are 2.7 sigma apart, considerable inequality between points exists. In other words, points near home range centers are relatively intensively used in comparison to distances about halfway between home range centers. This inequality of usage of the environment is even more pronounced when home range centers are more than 2.7 
sigma apart. However, by the time home range center's are uniformly distributed at 2.1 sigma apart, all portions of the environment are approximately equally utilized although every point is more intensively utilized than when home range centers were farther apart. Every further increase in density, as represented by home range centers coming closer together, merely increases the intensity of usage of every part of the environment and all parts continue to be equally utilized.

These curves (Fig. 19) are particularly instructive in gaining an insight into an "ideal" interval between home range centers. It is logical to assume that portions of the environment which are less utilized than others serve as a trap to catch wandering individuals who have not yet established a home range. As long as the process of equalizing distance between adjoining home range centers continues, no remaining pockets of less utilized habitat will occur by the time adjoining home range centers are nearly 2.1 sigma apart.

Any increase in density, that is any shortening of the interval between home range centers below 2.1 sigma, will merely increase the probability that available objects will be overutilized.

At the maximum interval between HRC's at which uniform utilization of the environment arises, aggressive actions exhibited by individuals with resident home ranges may be expected to prevent excess members of the populations from settling down within such an established area. Such wandering individuals may be expected to wander through and out of such established areas and into marginal habitats.

Thus, if minimizing the opportunity for aggressive encounters and the development of a uniform utilization of resources represent forces affecting evolution, we may anticipate development of capacities for communication which will most readily assure that the members of a population of a single species will be able to distribute themselves uniformly through space with an approximate 2.0 home range sigma distance intervening between any two adjoining home range centers.

\section{G. Contacting Neighbors}

The product of the density functions of any two individuals at a particular point determines the relative probability that these individuals will meet by chance. Similarly, the product of the density function of any one individual at a point with the sum of the density functions of all other individuals determines the relative probability that this one individual will contact neighbors at that point. Such latter calculations were made for uniformly distributed home range centers at $2.7,2.1$, and 1.5 sigma 
(see Fig. 20). As might be expected, as home range centers get closer together the probability of any individual contacting neighbors at every distance from its home range center increases. There may be some frequency of contacting neighbors which becomes so unbearable to the individual that his resultant aggressive actions prevent further contraction of the interval between home range centers. However, we have no basis for gaining insight as to what this frequency might be.

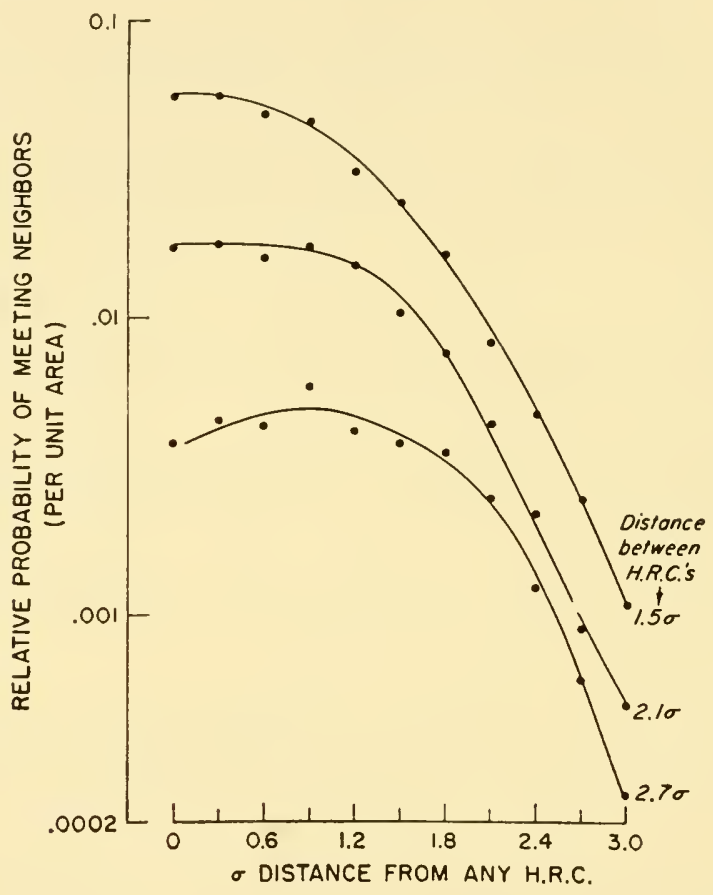

FIG. 20. Contacting neighbors. The relative probability of any one individual meeting others with reference to its distance from its own home range center, and to the distance between home range centers of all individuals.

One characteristic of these curves of relative probability of contacting neighbors does lend itself to suggesting a condition leading to an optimum interval between home range centers. When HRC's are 2.1 sigma apart the probability of contacting neighbors up to half the distance between home range centers is for all practical purposes constant. Applying this insight to all members of the population, it is apparent that with IIRC's this distance apart, the probability of contact between neighbors becomes relatively constant everywhere. If we accept the principle elaborated by 
Fredericson (1951) that animals attempt to make their euvironment predictable, and if we accept constancy of consequences as assuring greater predictability, then it follows that where the members of a community have their home range centers approximately 2.1 sigma apart, greatest predictability with regard to contacting neighbors characterizes this interval.

\section{H. Sign Field of AlliNeighbors}

Urination, defecation, and activities relating to the removal of materials used for food or nests represent signs by which one individual might recognize the presence of neighbors. Signs left by neighbors may be expected to be proportional to the sum of the density functions of neighbors at points considered. Unless home range centers are extremcly close together, there will arise a noticeable increase in signs of neighbors as the individual moves away from its own home range center. Obviously, the closer home range centers are to each other the relatively greater will be the sign of neighbors at any particular radius from the individuals's own home range center. We may then wonder what standard a particular individual may utilize in judging the intensity of signs left by its neighbors. Any individual's own sign is maximal near its own home range center. Therefore, an individual may resort to comparing the relative amount of sign of neighbors at any point to that which it would leave in a similar area near its own home range center.

Dividing the sum of the density functions of all neighbors at a particular point by the density function of a particular individual near its home range center provides such an index of the relative intensity of sign of neighbors (Fig. 21). The optimum interval between home range centers with regard to the sign field should be that interval at which throughout the home range of a particular individual the total sign left by neighbors nearest approximates that individual's own standard and in which there is greatest predictability with regard to sign, that is in which there is the least variation in intensity of neighbors' signs from point to point. Judging from the three curves presented in Fig. 21 an inter-home range center interval of somewhere near 2.1 sigma would lead to the development of an optimum sign field.

\section{Signal Field of Neighbors}

Following the formulation presented in Section VII, C, it is assumed that the signal emitted by one individual can be perceived by another in- 
dividual with sufficient intensity to produce a response by the latter in so long as the individuals are separated by a distance no greater than 3 home range sigma. In all probability, signals in the sense of vocalizations are emitted by each individual periodically as they wander through their home range. In order to simplify calculation of the signal field of neighbors, the particular condition was taken where all signals are emitted only from the home range centers. Thus, along a typical route of travel, as shown by the heavy dashed line in lig. 16 , the sum of the intensity of signals from all neighbors was calculated.

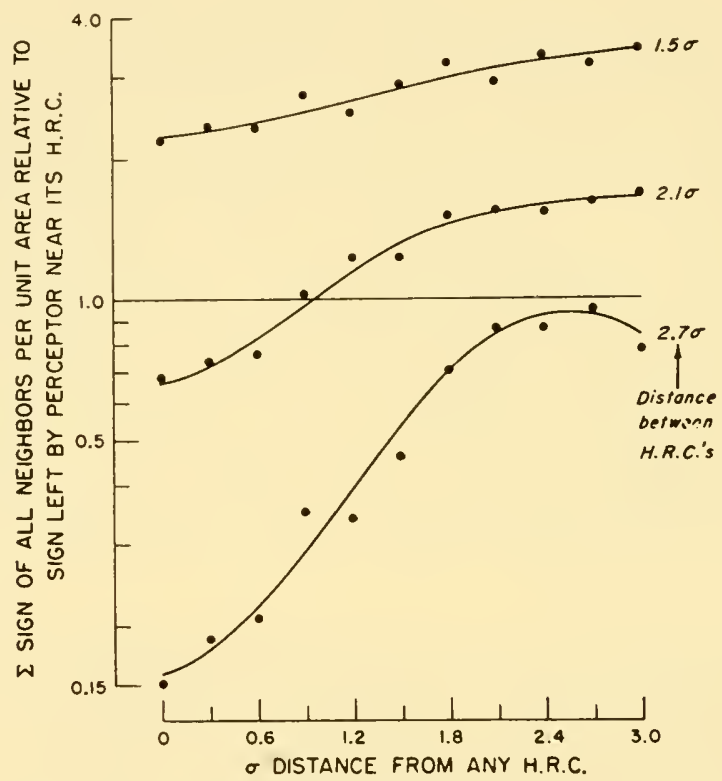

FIG. 21. Sign field of neighbors. Signs are considered as any persisting indication of an animal having made a response, i.e., defection, gnawings, or removal of food items. Thus Fig. 21 essentially represents the subtraction of the density function of one individual from the sum of the density functions of all individuals as shown in Fig. 19.

Again, we might wonder what standard the individual might utilize in judging the total intensity of signals received. Since the learning of the signal presupposes emission by one individual and detection by the other when they are in contact, this level of intensity with an assigned value of 1.0 may be taken as the standard. Since the intensity of signals probably drops off inversely proportional to distance, the sum of signals at any point in place and time may be less than 1.0. A further complication to the problem is that all neighbors may not emit signals simultaneously. Simultaneity other than by chance will arise regularly only if the detection of the 
signal by one individual elicits a similar response by the perceiver. However, if this is so, and if each individual after emitting a burst of sequential signals enters a refractory period (see Section XIII, A) of some given mean length before it can emit signals again, then we have a situation in which there occur recurrent periods during which most nonsleeping individuals in the community emit signals nearly simultaneously. Although no proof of the validity of this assumption can be offered at present, my formulation will accept the existence of such a process. Such an assumption is inherent in the utilization of Fig. 22 in arriving at some insight as to the

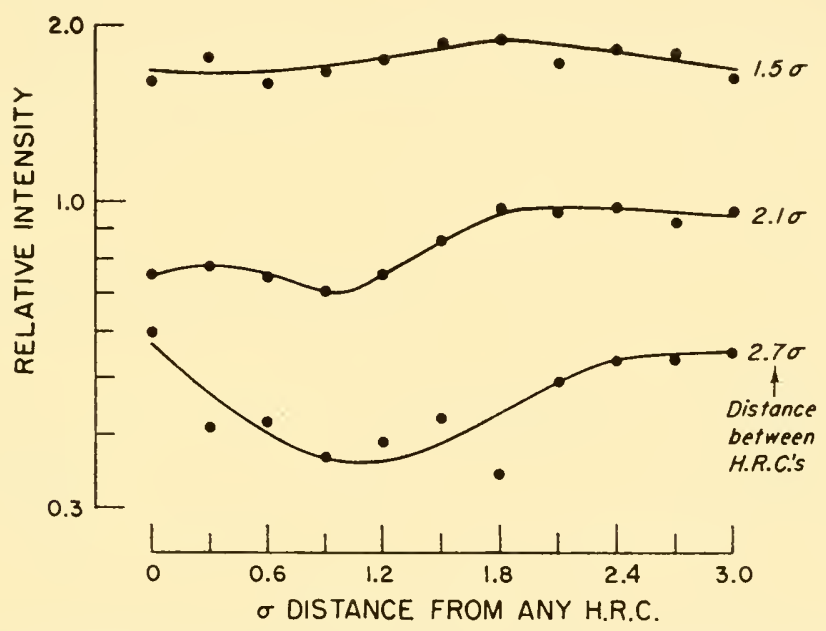

F1G. 22. signal field produced by neighbors. If all neighbors emit signals simultaneously at their home range centers, and these signals have the properties shown in Fig. 18, then their summated intensity will form a "topography" as here shown with reference to any particular animal moving through its home range. The value 1.0 represents the intensity of a signal at the point of emission.

influence of the signal field on determination of an optimum interval between home range centers.

As with the sign field of neighbors, the signal field of neighbors is presumed to influence interval between home range centers through the members of the community seeking that interval between home range centers which will ensure most closely attainment of both constancy of the signal field, leading to predictability and to approximation of the standard signal intensity. Judging by the three curves in Fig. 22, operation of these two criteria indicates an optimum interval between home range centers slightly less than 2.1 sigma. 


\section{J. Hum Field}

In Section VII, C it was suggested that when the emitter is between 3 and 6 sigma from the receptor the signal given by the emitter can be perceived by the receptor but is insufficient in strength to elicit the appropriate responses. The sum of all such signals below threshold for inducing a response is here termed "hum." When this value exceeds the standard intensity of 1.0 (see Section VII, I), the receptor will become restless even though perhaps not exhibiting a specific response to the signals. What effect this general state of restlessness may have upon the receptor is unknown, but it is logical to assume that the members of the community

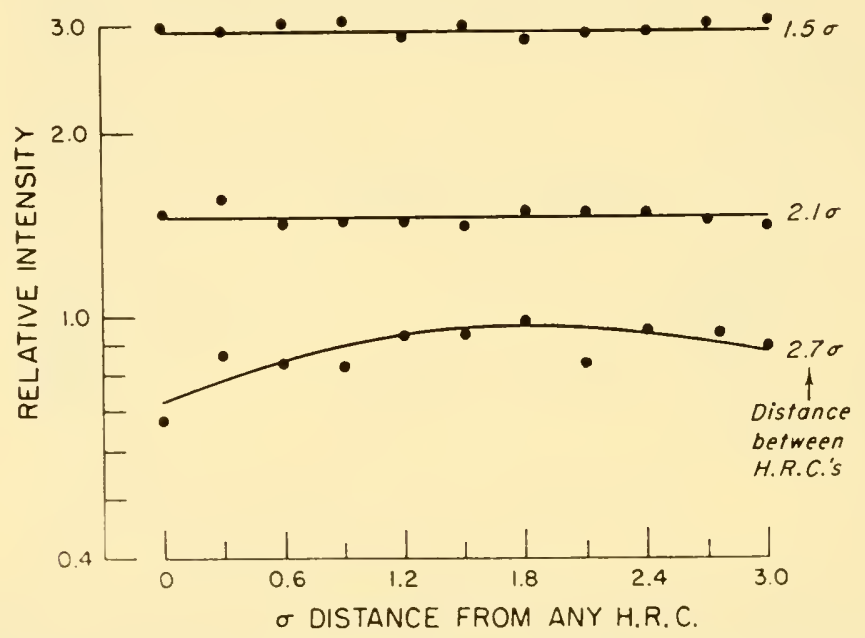

FIG. 23. Hum field refers to the sum of the intensity of all signals, any one of which is below that minimum intensity required to elicit a specifir response. The value, 1.0, denotes the intensity of a signal at the point of emission.

will attempt to adjust the interval between the home range centers in such a way as to reduce the likelihood of the "hum" exceeding 1.0. To do so implies that home range centers must be of the order of 2.4 sigma apart (Fig. 23).

\section{K. General Conclusion Concerning the Distance between Neighbors}

Effective learning of signals probably does not begin until home range centers come at least 3.0 sigma from each other, and even further shortening of this interval must increase the effectiveness of learning. Several 
factors examined all suggest that the optimum interval between home range centers should be of the order of a 2 home range sigma distance. From an evolutionary standpoint, the most important of these probably is the fact that utilization of the environment becomes uniform at slightly more than 2 sigma distance between home range centers. This factor should have been the major one in the evolution of those characteristies of individuals pertaining to the frequency of trips, the velocity of the individuals, and the aggressive acts elicited when two individuals meet. It is probably strictly coineidental that the properties of the sign and signal field are also likely to be such as to make an interval of about 2 sigma between home range centers optimum. It is at this distance between home range centers that both signs and signals become most constant and thus more predictable. Also, the intensity of the signs and signals most nearly approximate any receptor's own behavior near its home, which can serve for it as a standard in evaluating the intensity of the actions of its neighbors impinging on it.

\section{The Number of Neighbors Pereeived}

When home range centers are 2.7 sigma apart, an individual at its home range center can just perceive all its six nearest neighbors. However, as it begins to move away from home it begins to lose contact with those nearest neighbors lying on the opposite side of its home range center. By the time it reaches half the distance to its nearest neighbors in the direction in which it is traveling, it ean detect only three of its nearest neighbors. Toward the periphery of its home range, it can detect only two of its nearest neighbors. In addition, it ean detect one, and only one, of its next-nearest neighbors, which are members of the group of twelve forming the second hexagonal tier of neighbors about its home range center. Thus, with this fairly large interval between home range centers, any one individual has poor contact with its associates. When home range centers are 2.1 sigma apart, any one individual ean maintain contact with all six of its nearest neighbors out to about 0.75 sigma. Even when an individual has journeyed halfway in a direction of a nearest neighbor it is still in contact with four of its six nearest neighbors, in addition to one of the twelve neighbors lying in the next tier. As he proceeds still farther, losses of nearest neighbors are compensated for by next-nearest neighbors. Thus, at all times when home range centers are 2.1 sigma apart, an individual is in contact or potential contact with five or six of its neighbors.

By the time home range centers are of 1.5 sigma apart, an individual is in potential contact with ten other individuals though not always the same 
ten, no matter where it is in its home range. Since the dynamics of the use of space relating to uniformity of utilization of resources and the characteristics of the sign and signal field all point to an optimum interval between home range centers of somewhere near' 2 sigma, it follows that there should have been evolutionary adjustment of tolerance to simultaneous or near simultaneous communication with five to ten others.

\section{Interpretations of Observed Data Derived from Removal Trapping of Small Mammals}

At this stage in the development of a concept of community organization, one must resort to a certain amount of quasi circular reasoning. Regularities in observed results lead to theoretical formulations. Then these formulations can be used to reexamine the data for further insight. This is my present intent. In time, many aspects of the concept may be subjected to more rigorous study. However, for the present we must content ourselves with a search for a best approximation to a very complex set of phenomena.

Section VI, "Continuous Removal Trapping of Small Mammals," presented results from scveral extensive studies. Specifie interpretations follow.

\section{A. The Relationship between Two Dominant Species}

The dominant species in the community reveals itself during removal trapping through its members having such large home ranges that every individual living near a trap has a high probability of encountering it. Thus, for them, fewer days lapse from initiation of trapping until $50 \%$ of the population is caught. As can be seen from the two Maryland studies presented (Fig. 10 and Fig. 13B), Peromyscus fulfills this criterion. For species associated with Peromyscus, whether they be Blarina and Sorex, or Blarina and Pitymys, the dates of $50 \%$ removal arrive much later. The later the date of $50 \%$ remoral, the more subordinate a species, and the more slowly its members expand their home ranges as the dominant species is removed.

In more northern forest habitats, Peromyscus rarely is found in the absence of Clethrionomys. In fact, it is as if the red-backed mouse is just superimposed upon the simpler Peromyscus-Blarina-Sorex community of more southern forests. Typieal dominance of Clethrionomys over Peromyscus may be seen in Figs. 9, 11, and 13A. Although I am convinced that Clethrionomys usually can develop the ability to inhibit the home range of 
Peromyscus, there exist conditions limiting the extent to which this ability may develop.

The typically low relative density of both species (Table VI) on MIt. Desert Island, Maine, represents such a condition. As revealed in Fig. 14, both species decline at about the same rate from day 1 through day 3 . This can happen only when no alteration in home range size transpires over time or when the survivors of each species make equivalent but slight increases in extent of home range. Appreciation of why inhibition of home range fails to develop at low densities demands knowledge of variables we lack.

It demands that we know actual densities. The NACSM census provide only relative densities. However, we can make approximations. Run long enough (30 days), the 950-foot-long B-type NACSM census procedure should take all residents within 3 home range sigma on either side of the trapline and for a radius of this distance about the end of the line. If we take 50 feet as approximating the average home range sigma of small manmals, uninhibited by dominants, then approximately 8 acres are exposed to such a trapline. Furthermore, examination of 30-day censuses reveals that for species with uninhibited home ranges, $2.5-50 \%$ of the residents are taken during the first 3 days of trapping. On this basis, there was on the average less than one Clethrionomys and less than two Peromyscus per acre in this Maine study. Since juveniles, with as yet probably little influence on the spatial distribution of associates, comprised a portion of the catch, it is quite likely that the average distance between home range centers for each species exceeded 3 sigma. As shown in the prior theoretical sections, learning of signals would most likely be fairly ineffective here because contact between neighbors would be infrequent.

Under these circumstances the signals emitted by each species should have acquired little in the way of negatively stimulating characteristics for its own members. It follows that inhibition of home range size will have been negligible and thus the two species, which probably have nearly the same size home range, should encounter traps with nearly equal frequency, and thus the rate of decline in catch from days 1 through 3 should be nearly equal. However, in the one Mt. Desert Island, Maine, study where eight NACSM lines were run for 15 days (Fig. 13A), it is apparent that home ranges of Peromyscus were slightly contracted. Fifty per cent of the 15-day total for Clethrionomys was attained by day 6 , but not until day 10 for Peromyscus. Thus, where both species regularly occur at low densities, Peromyscus is only moderately subordinate to Clethrionomys.

In the Adirondacks, where Peromyscus most frequently has a low density and Clethrionomys a much higher one (Table VI), Peromyscus is markedly subordinate. Its home ranges not only are markedly contracted, but also 
they expand immediately as Clethrionomys are removed. The validity of this interpretation is revealed in Fig. $14 \mathrm{~B}$, which shows that during the first 3 days of trapping as the Clethrionomys population is reduced, as indicated by fewer numbers taken on successive days, the numbers of Peromyscus taken increases. This increasing catch can only result from sufficient expansion of home range by survivors to bring about an increased probability of encountering traps.

One might argue that these latter data for Peromyscus merely indicate that the snap-trap is initially a sufficiently strange object to elicit avoidance. As time elapses these mice become accustomed to the presence of the trap and thus later in time more individuals will enter traps. Two lines of evidence of reasoning suggest the fallacy of this interpretation. If it were correct, we must conclude that Peromyscus in Maine lack this strange object response but those in New York have it highly developed (see Fig. 14). There exists no logical basis for beliering that such a difference characterizes the populations of these two areas. Furthermore, in the trapping of both Peromyscus and Clethrionomys it is not an uncommon experience to find either of these mice dead in a trap with a bloody stump of one hind leg, while another trap, 2-5 feet away, is covered with fresh blood and fur. The conclusion as to what happened is clear. The mouse happened to get caught in one trap by one leg, it chewed or pulled itself loose, then went fairly directly to another trap, bit at the bait on the treadle and was thus killed. If these mice have a strange-object reaction it must be of a sufficiently low order of magnitude that even the recent loss of a leg in one trap fails to increase it to the point of avoiding the next trap encountered.

In the third area, Alberta, from which adequate data are available for these two genera, both are relatively abundant but Peromyscus exceeds Clethrionomys (Table VII). During the fall season, nearly twice as many of each genus are trapped as during the spring. During the period of low spring densities, these genera exhibit nearly identical rates of decline (Fig. 15A) accompanying removal trapping. As with the Maine data, such trends may be interpreted as indicating that at such densities neither species is capable of markedly inhibiting the home range of the other. However, by fall many Clethrionomys have contracted their home ranges as a response to their exposure not only to more of their own kind but also to more Peromyscus. Home ranges of Peromyscus remained unaltered, as indicated by the similarity of rate of decline during both spring and fall (Fig. 15). However, the daily catch for Clethrionomys remained nearly identical through three successive days of removal trapping. Sufficient enlargement of home ranges by surviving Clethrionomys must have taken place each day to lead to an equivalent frequency of traps being encountered on the following day by red-backed mice, despite their fewer number's 
than on the preceding day. At this Alberta site, the inhibition of Clethrionomys home ranges by Peromyscus must have been less than the inhibition of home ranges of Peromyscus by Clethrionomys in the Adirondacks.

Despite the paucity of areas from which extensive comparable data are available, it looks as though Clethrionomys has a slight advantage over Peromyscus in gaining psychological ascendency. At this point one may suspect that the characteristics of some environments will markedly favor the reproduction and survival of one of these genera. Whichever genus this happens to be will then become psychologically dominant to the other, as evinced by the contraction of home range of the less numerous genus. Consideration of other aspects of the relationship between these two genera requires familiarity with the concept of the constellation dealt with in the following sections.

\section{B. Removal Captures of Socially Dominant Species}

In several studies already presented (Figs. 9-13) we have seen that one species tends to be caught in large numbers during the first few days, and that the time of maximum input for the remaining species comes during a successively later period. Very frequently a secondary increase in daily catch starts near the 15th day of trapping for the species with initially the greatest rate of capture. Such species will henceforth be designated as the socially dominant or alpha species of the small mammal community. For example, see Clethrionomys in Fig. 9 and Peromyscus in Fig. 10. Where there are several species taken, usually only one is characterized by this secondary input. Four censuses examined included one such species and a fifth included two with definite secondary inputs. ${ }^{1}$

Although several species are involved in this phenomenon, the assumption is here made that they all so behave because of similar properties leading to their alpha rank in the community. If this is so, we are justified in pooling the data. A table of the total catch per day of trapping was

1 The five censuses utilized in preparing Fig. 24:

1. By Dr. J. E. Moore, Sept. 1959, Edmonton, Alberta: 128 Peromyscus maniculatus borealis, $65 \%$ of 30 -day total by day $14-15$.

2. By Dr. A. I. Roest, Oct.-Nov. 1959, San Luis Obispo, California: 75 Dipodomys heermanni, $65 \%$ of 30 -day total by day $15-16$.

3. By Dr. William L. Webb, Fall 1952, Rich Lake Island, Newcomb, New York: 97 Clethrionomys g. gapperi, $65 \%$ of 30 -day total by day 7 .

4. By Dr. Earl F. Patric, Fall 1953, Arbutus Area, Newcomb, New York: 86 Clethrionomys g. gapperi, $65 \%$ of 30 -day total by day $12-13$.

5. By Dr. Kyle R. Barbehenn, Nov.-Dec. 1959, Chadwick Woods, Montgomery County, Maryland: 57 Peromyscus leucopus, $65 \%$ of 30 -day total by day 14; 116 Blarina brevicauda, $65 \%$ of 30 -day total by day $20-21$. 
prepared. These data are shown as a three-point moving average in Fig. 24.

There results a continuous decline in catch until about the 12th day. After this, the daily rate of capture increases, reaching a second maximum five to seven days later. Following this, the number of animals taken continuously declines, but it is not until about the 27 th day of removal trapping that the numbers taken per day reaches the low level characterizing the 12 th day.

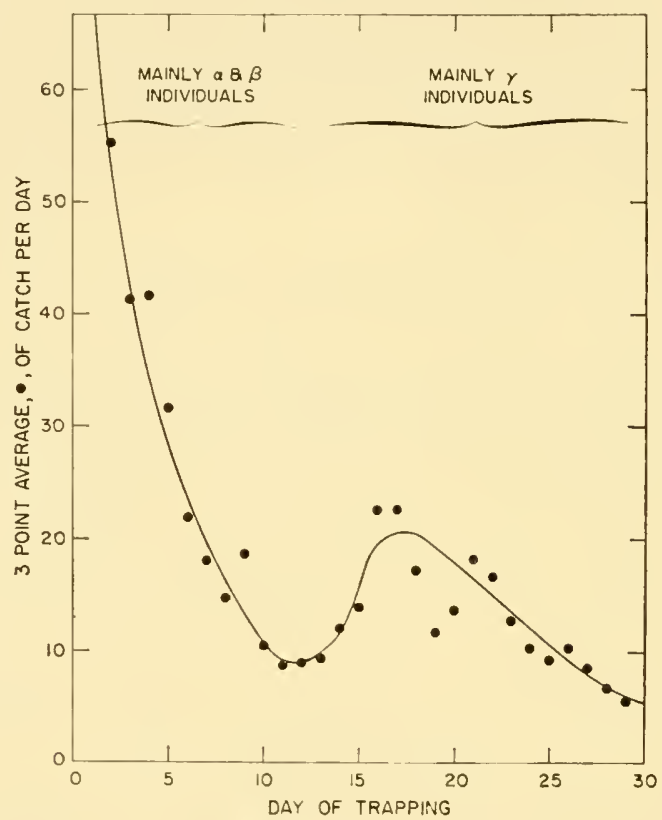

FIg. 24. Removal captures of 559 small mammals who are representatives of species, socially dominant in their community. Alpha and beta represent the intraspecific dominant individuals with large home ranges. The gamma individuals represent the intraspecific subordinates which enlarge their home ranges following removal of the alphas and betas.

If the assumption that practically all the resident population exposed to the traps is removed during 30 days of trapping is correct, then the accumulated catch plotted as the proportion of the 30-day total over time will reveal both the proportion of the total comprising the initial input and the time at which the secondary input begins (Fig. 25). This shows that the intersection of the two rates of input occurs on day 1.5 after removal of $64^{\circ}$, of the resident population. 


\section{Constellation Formation-An Intraspecific Phenomenon}

After the removal of $64 \%$ of the resident population of socially dominant species, whose home ranges are sufficiently large to give them a higher probability of encountering traps, there arises a secondary input. These latter individuals must have enlarged their home ranges as a response to the absence of their former associates. It follows that certain individuals

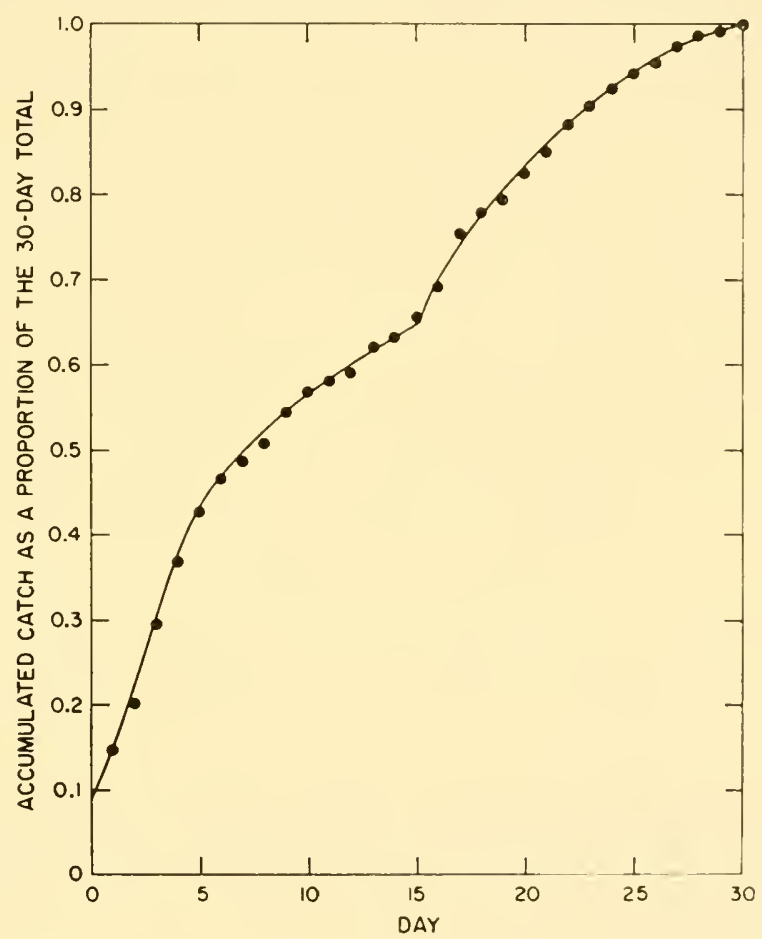

Fig. 25. Removal captures of socially dominant species. Same data as in Fig. 24, but here shown as an accumulated total.

have the ability to restrict the home ranges of other members of their own species. At this point in my analysis I began to wonder whether there were circumstances relating to the spatial distribution of the population which might lead to a $64: 36$ ratio of larger:smaller home ranges.

In the previous discussion we have seen that one possible spatial equilibrium is that in which home range centers become uniformly distributed with an inter-home range center interval of about 2.0 sigma. It was further pointed out that the mechanics of moving through space are such as to favor the evolution of a signal emitted by one individual which has the 
potentiality of eliciting a response by another up to a maximal distance of about 3 sigma between individuals. We may then ask, "How many other" individuals in such a uniformly distributed population can any one individual know?" Figure 26 shows that a line connecting the home range center of any one individual's six nearest neighbor's forms a hexagon. With the hypothesized communication system operating, this one individual can remain in constant communication contact with all its six nearest neighbor's except under the condition when it and one of them happen to

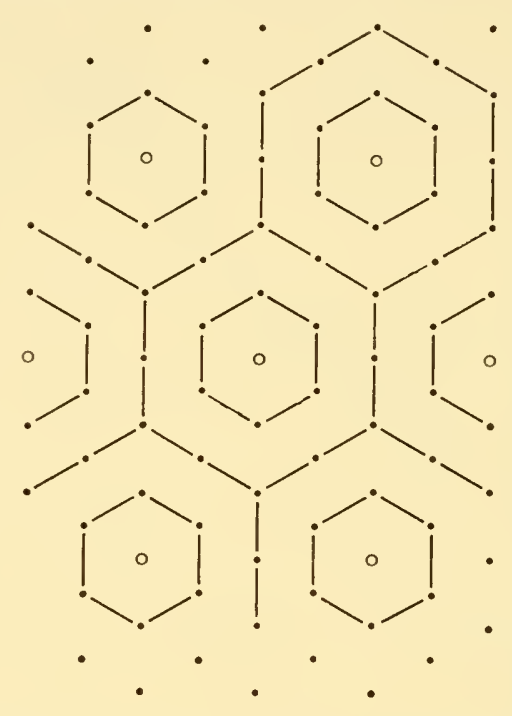

FIG. 26. Spatial distribution of home range centers during the preconstellation phase. Small open circles represent centers for individuals destined to become alpha members. Dots represent home range centers for all other individuals. Home range centers are uniformly distributed.

move in opposite directions away from the line connecting their respective home range centers. Such actions can increase the distance between them to greater than 3 sigma.

Lines connecting the home range centers of this individual's next-nearest neighbors also form a hexagon. Home range centers for all these twelve next-nearest neighbor's lie nearly 4.0 sigma from the selected individual. Therefore, each of these next-nearest neighbors can be in contact with the selected individual when they approach each other, such that the distance intervening between them becomes less than 3.0 sigma. Any more-distant neighbors, those living 6.0 or more sigma from the selected one, will have 
such a low frequency of communication with the selected individual that mutual effects will be of little significance.

Examination of the spatial distribution of home range centers in a uniformly distributed population reveals that one-twelfth of the members each have six nearest neighbors whom they can influence withont competitive influence being exerted by any other individuals in this one-twelfth population. Such individuals will hereafter be designated as alpha individuals. Home range centers for these alpha individuals are designated by small open circles in Fig. 26. It will further be noted that two such alpha individuals, who live closest to each other, share next-nearest neighbors.

Antagonistic relations, uniform utilization of the environment, and attainment of uniform sign and signal fields approximating in intensity the sign and signal intensity expressed by an individual at its own home range center, and equalization of contact rate with all neighbors throughout any one individual range, all serve as forces leading to a uniform distribution of home range centers at near 2.0 sigma between centers (Section VII, K). Yet, we may anticipate an opposing force of attraction between members in close communication with each other. This will lead to all nearest neighbors shifting the centers of their home ranges slightly toward their single alpha associate. Such nearest neighbors will hereafter be designated as beta individuals.

Now we can turn our attention to the alpha's next-nearest neighbors. It may be seen from Fig. 26 that in each group of twelve next-nearest neighbor's, six are equidistant to two neighboring alphas. Therefore, since the probability of each of these six nearest neighbors is 0.5 of being attracted to either of two alpha individuals to which they are equidistant, each alpha on the average will have attracted to it three of these nextnearest neighbors. The remaining six next-nearest neighbors to any alpha are equidistant to three alphas. It similarly follows that on the average any one alpha will have attracted to it two of six such next-nearest neighbors. Next-nearest neighbors which so move toward an alpha assoeiate will hereafter be designated as gamma individuals. Thus, each alpha will have attracted toward it six beta individuals and on the average five gamma individuals. This process leads to a clumping of the population into groups ranging in size from 7 to 19 , with a mean of 12 (see Section VIII. D).

The central alpha individual because of its more favored position in the communication network may be assumed to be dominant to both its beta and gamma associates. Gamma individuals, because of their peripheral location in the developing clump, here designated a constellation, will be subordinate to both the beta and alpha associates. Now let us consider the 
situation where home ranges contract as individuals become more subordinate.

How much beta and gamma members may be expected to contract their home ranges requires consideration of phenomena treated in Section XIT, E, titled "Velocity and Home Range." There, it is shown that in terms of $\sigma_{\alpha}$ units of distance, the home range sigmas for alpha, beta, and gamma individuals become, respectively, $1.0,0.9575$, and 0.6457 . Since an optimum uniform utilization of the environment requires a $2 \sigma$ interval between home range centers, the distance between alpha and beta home range centers will become $1.9575 \sigma_{\alpha}$, and $1.6032 \sigma_{\alpha}$ between beta and gamma home range centers.

The periphery of each constellation (Fig. 27) may be arbitrarily designated as that radius from the center of the alpha member's home range extending to one $\sigma_{\alpha}$ beyond the center of each gamma individual's home range. This radius is $3.1478 \sigma_{\alpha}$. And since the home range centers of the alpha members of adjoining constellations are $6.92 \sigma_{\alpha}$ apart, it is obvious that between constellations there lies what may be called an interconstellation matrix, receiving very little usage from the dominant species forming constellations. Furthermore, this interconstellation matrix must markedly reduce communication between members of adjoining constellations. How extensive this reduction becomes must be viewed against the requirement of a $2 \sigma$ inter-HRC interval for an optimum state. Yet, the HRC of any beta or gamma member of one constellation will lie on the average $4 \sigma$ units of distance away from the nearest beta or gamma members of an adjoining constellation with reference to their own reduced home ranges.

On the average, such constellations will consist of twelve individuals, 1 alpha, 6 betas, and 5 gammas. Of these, the gammas with smallest home ranges form 0.417 of the population. These are the individuals who, after removal of their dominant alpha and beta associates, will enlarge their home ranges. Actually, some gammas will be trapped before the 1.5th day and some alphas and betas will be taken after it. Furthermore, Fig. 2.5 clearly shows that a few more gammas would have been taken after the 30th day of trapping. Therefore, the observed proportion of the population consisting of gamma individuals, estimated at about $36 \%$, reasonably well approximates the theoretical expected of $41.7 \%$.

To date, this approximation stands as the only direct supporting proof (see Section XI) that populations of dominant species of small mammal communities do, in fact, tend to form such constellations. If they really do, we may anticipate that there has been evolution relative to physiology such that its optimum state is most compatible with interactions among individuals transpiring in groups with a mean size of twelve adults. In 
later sections, I shall present data which support the hypothesis that a group size of twelve has heen phylogenetically retained in more highly evolved forms.

For the present, let us examine further implications of constellation formation upon the structure of the small mammal community. Con-

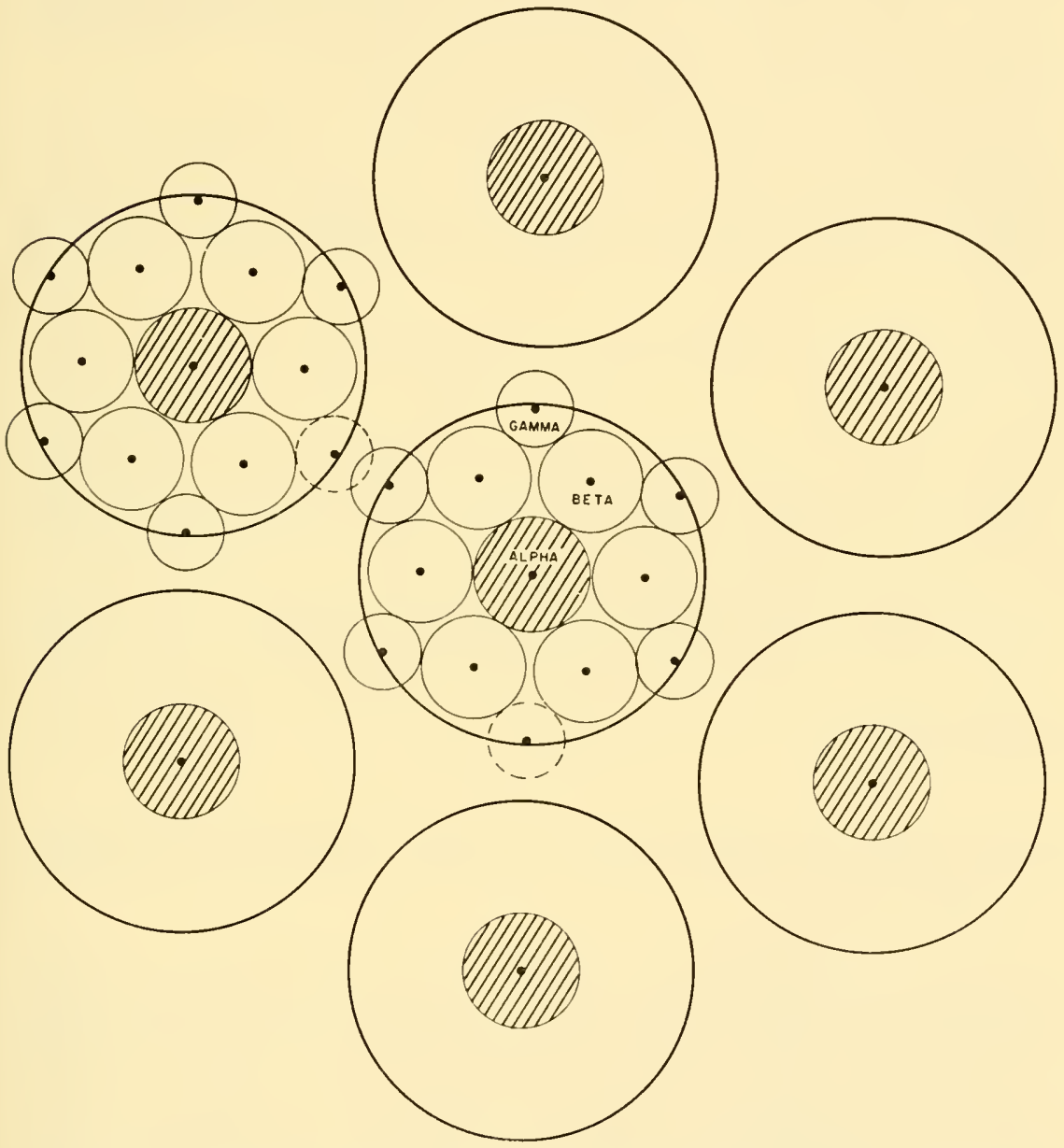

FIG. 27. The theoretical constellation phase of intraspecific community organization of dominant species. A 3 -signia radius home range for alpha individuals is represented by the large circle. Small circles represent 1-sigma radius portions of all members of a constellation. Contraction of home range by beta and alpha members permits the more intensively used portion of the home ranges of all individuals to fall mostly within the 3 -sigma radius home range of the dominant alpha members. Crosshatched circles represent the 1-sigma portion of the home range of alpha individuals. 
stellation formation presupposes passing through a stage of uniform distribution of home ranges. In the interstices between the home ranges of alpha species there is the opportunity for very subordinate species with highly contracted home ranges to establish themselves. As the constellation forms, certain members of the subordinate species will be retained within the interstices of the constellation while others will lie at its periphery. However, as constellations form, there develops an interconstellation matrix rarely frequented by members of the alpha species. IVe may then wonder what members of the community will live here.

At this point, the Huntington Forest censuses of 1952 and 1953 (Fig. 11) are particularly instructive. The red-backed mouse, Clethrionomys, is obviously the alpha species. Just as obviously, the shrew Sorex is the most subordinate species, which has highly contracted home ranges lying in the interstices between those of Clethrionomys. Had all these census been run for longer than 30 days, it looks as though the $2: 1$ ratio of the alpha species to one of its most subordinate associates would have been realized. Censuses shown from other areas indicate that both Peromyscus and Blarina are dominant to Sorex in the community. And yet, in these Huntington Forest censuses, relatively few of either were caught. What I suspect has happened is that as the constellations developed, both species were relegated to the interconstellation matrix, where they existed in somewhat contracted home ranges, as indicated by the eatch per day increasing from day 1 to days 4-6. In fact, for both these speeies it looks as though a certain portion of their members had even more markedly contracted home ranges. This is indicated by the secondary increase in eatch per day starting between days 8 and 10 .

\section{Expected Variability in the Number of Individuals Forming Constellations}

In terms of the formulation of Section VIII, C the six nearest neighbors to any alpha individual will always be attracted toward it as the members of a population with a uniform distribution of home range eenters begin to form diffuse elumps, termed constellations. The twelve next-nearest neighbors to any alpha individuals may be divided into two types, $a$ and $b$, aceording to their probability of being attracted toward any given alpha neighbor.

Let: $a$ represent the six individuals who have a probability, $p_{a}=\frac{1}{2}$, of being attracted to any given alpha.

$b$ represent the six individuals who have a probability, $p_{b}=\frac{1}{3}$, of being attracted to any given alpha. 
The probability, $p_{a}(i)$, that $i$ number of the six $a$ individuals will be attracted to the given alpha will be:

$$
p_{a}(i)=\left(\begin{array}{l}
6 \\
i
\end{array}\right)\left(\frac{1}{2}\right)^{6}
$$

Similarly the probability, $p_{b}(i)$, that $i$ number of the $\operatorname{six} b$ individuals will be attracted to the given alpha will be:

$$
p_{b}(i)=\left(\begin{array}{l}
0 \\
i
\end{array}\right)\left(\frac{1}{3}\right)^{i}\left(\frac{2}{3}\right)^{6-i}
$$

Therefore, the probability, $p(m)$, where $m=0$ to 12 , of $m$ members of the 12 next-nearest neighbors joining any given alpha and its six nearest neighbors to form a constellation will be:

$$
p(m)=\sum_{i=0}^{m} p_{a}(i) \cdot p_{b}(m-i)
$$

For example, if $m=4$ this becomes:

$$
\begin{aligned}
p(4)=p_{a}(0) \cdot p_{b}(4)+p_{a}(1) \cdot p_{b}(3)+ & p_{a}(2) \cdot p_{b}(2)+p_{a}(3) \\
& \cdot p_{b}(1)+p_{a}(4) \cdot p_{b}(0)
\end{aligned}
$$

$p(0) \cdots p(12)$ were calculated and are shown in Table VIII as $p(7+0)$

\section{TABLE VIII}

Expected Variability in the Number of Individuals Formixg a Constellation

Probability of group developing

0.01234568

0.05041152

0.12345679

0.20190329

$0.2322530 \mathrm{~s}$

0.19266546

0.11612654

0.05047582

0.01543210

0.00315072

0.00038580

0.00002143 
$\cdots p(7+12)$ since the constellation becomes composed of the addition of these next-nearest neighbors, or gamma individuals to the basic core of the one alpha and its six beta nearest neighbors.

\section{E. Social Rank and Intraspecific Associations}

In the 14-day removal study shown in Fig. 13B, trapping results indicated large home ranges for Peromyscus, moderate-sized ones for Blarina, and highly contracted home ranges for Pitymys. Estimated home range sigmas, $\sigma$, of 50,25 , and 12.5 feet, respectively, probably closely enough approximate the real values to permit their use in a study of these data. If we knew the actual home range centers of all animals trapped, a 1-sigma radius circle plotted about each on a map of the study area should provide more insight into spatial relationships.

Since such centers were not known, they were approximated by making the following assumptions:

1. Peromyscus maximize inter-home-range-center distance from other Peromyscus.

2. Blarina similarly maximize distance, not only from others of their own kind, but also from Peromyscus.

3. Pitymys maximize distance from both Peromyscus and Blarina, as well as from others of their own kind.

4. The later an animal was trapped the farther its home range centers were located from the trapline.

For Peromyscus some of the HRC's were shifted to one side of the line of capture, and the remaining to the other side, until every adjacent 3 HRC's approximated equilateral triangles. A similar procedure was applied to Blarina captures except that where possible their HRC's also were placed in the center of triangles formed by the HRC's for Peromyscus. Then the HRC's for Pitymys were, insofar as possible, placed in the centers of triangles connecting the HRC's of the other two species.

Utilizing these assumptions, centers of home ranges for Peromyscus were plotted first, Blarina second, and Pitymys last. Originally these were all plotted on a single figure, but for the sake of greater clarity in examining intraspecific relations the home ranges, in terms of 1-sigma circles, are shown separately in Figs. 28-30.

Moisture conditions in the habitat varied markedly. Steep xerophytic slopes of oak and pine covered the three sectors encompassed between the $\mathrm{E}$ and SW radii. The sector between the $\mathrm{N}$ and $\mathrm{NW}$ radii was quite steep and dry. A damp drainage area lay roughly along the W and NE radii. 


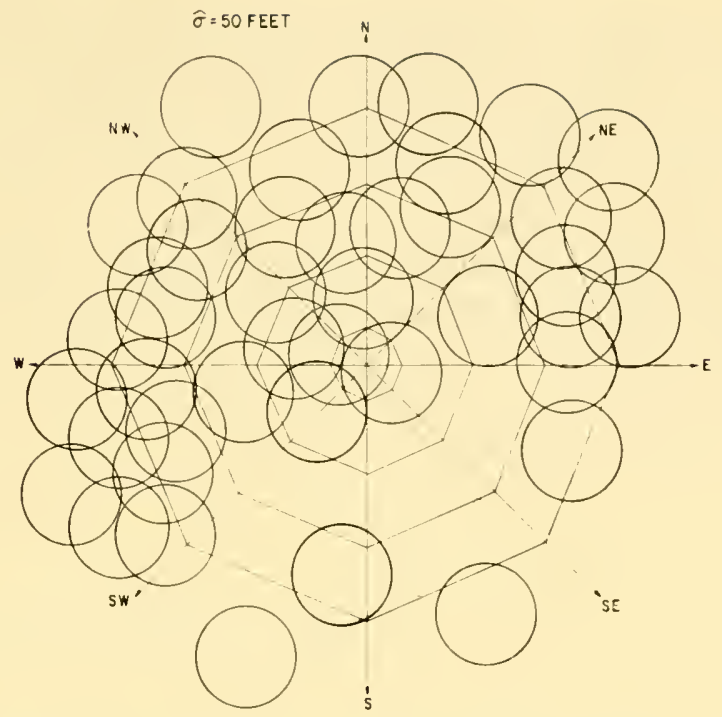

Fig. 28. Schematic home ranges for Peromyscus. The octagons represent traplines along which removal trapping was conducted.

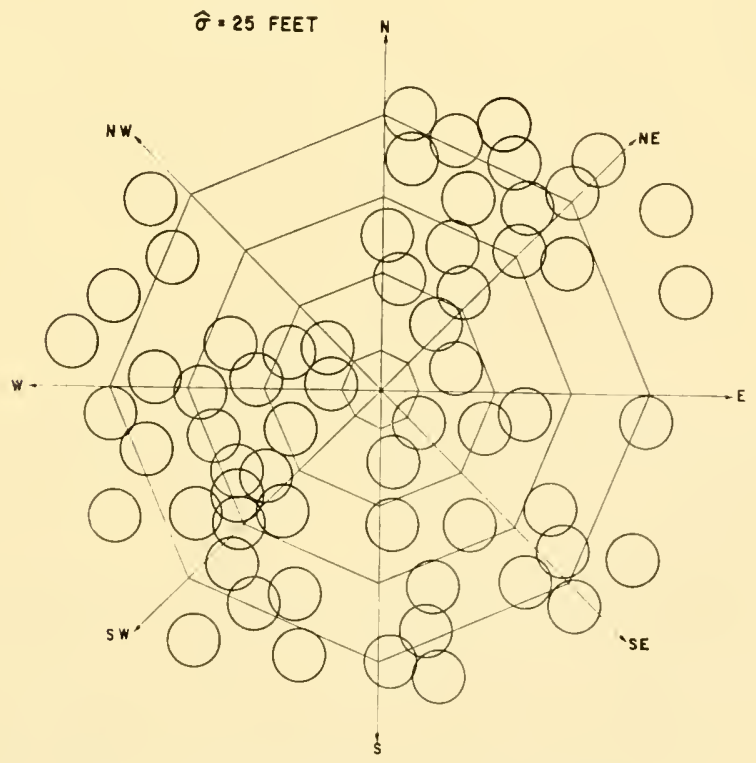

FIG. 29. Schematic home ranges for Blarina. 
Away from the narrow drainage area the other four sectors were moderately mesophytic. Deep leaf mold covered the entire forest floor. No attempt was made to obtain a detailed cover map. Total timbering a year later for a housing development revealed that many of the trees in the forest exceeded 150 years in age.

It may be noted that both Peromyscus and Blarina tended to avoid the more xerophytic areas, whereas Pitymys was more abundant in these drier areas. However, it is interesting to note that no Blarina occurred in the $\mathrm{N}$ to NW sector and yet quite a number were taken in the even more

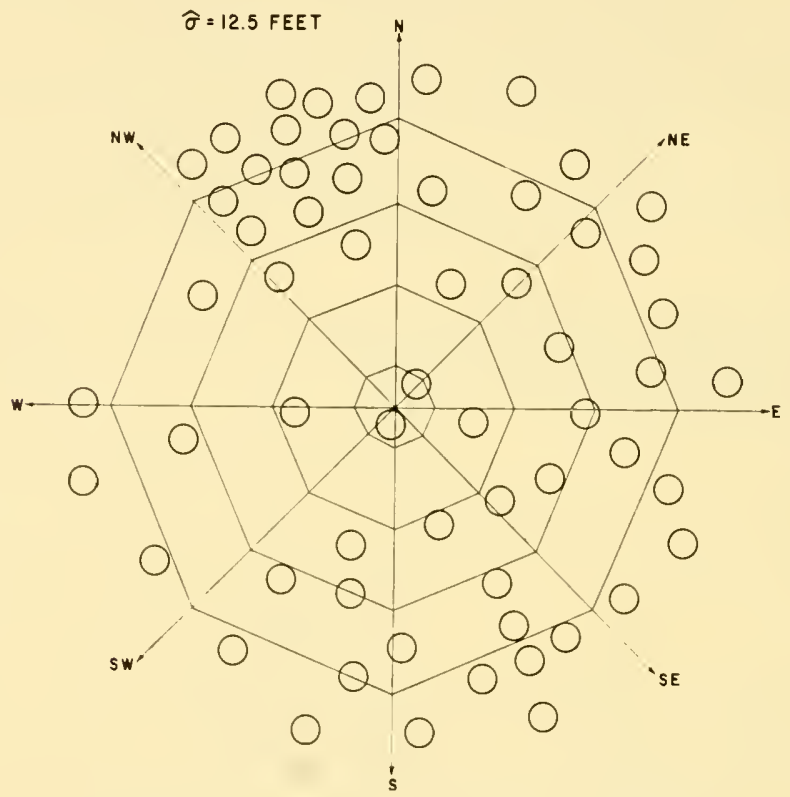

FIG. 30. Schematic home ranges for Pitymys.

xerophytic E to SIV sectors. The marked clumping of Pitymys in the $\mathrm{N}$ to NW sector suggests that a tendency toward colony formation in this very subordinate species may actually serve to exclude from that region the more dominant Blarina.

However, despite such a local tendency for Pitymys to cluster, no overlapping of the 1-sigma radius home ranges occurred anywhere in the study plot. In fact, the mean interval between home range centers of nearest neighbors is of the order of 4 sigma. This must mean that the probability of one Pitymys associating with a neighbor is very low. With their larger home ranges, there is more opportunity for Blarina to associate with others 
of their own kind. Yet even with them their movements are sufficiently inhibited as to reduce contacts far below that otherwise possible. However, with Peromyscus so much overlapping of home ranges existed that most individuals must have had frequent associations with others of their own kind. Reduction of communication with others of their own kind accompanies interspecific social subordination. The lower the rank of a species, in terms of the degree other species in the community cause it to restrict its home range, the more its intraspecific systems of communication will be reduced. Even, as the analyses shown in Figs. 28-30 suggest, if there develops some compensatory clumping of home ranges by members of subordinate species, there must still be a greater degree of isolation between such elumps than between any comparable number of groups of the dominant species.

\section{F. The Instability of Social Relations}

Results from the extensive censusing of small mammals conducted by Drs. Patric and Webb and their associates at the Huntington Forest form a major key in unraveling the process involved in community structure. Fortunately, their records (Patric, 1958) include some of the years before 1952 (see Table VI for 1952-1956).

An effort of 9650 trap-nights during 1940 and 1941, in which traps were set for five consecutive nights, caught 173 Clethrionomys and 1280 Peromyscus, or 7.4 Peromyscus for each Clethrionomys; in contrast, during the years of 1952-1956, 4.1 Clethrionomys were captured for each Peromyscus taken (Table VI). However, during these two years the trends of capture over time were so nearly identical that only the greater numbers of Peromyscus can argue for its having been more dominant. Actually, both showed increases in catch per day as associates were removed. The day 1 to day 5 captures were: for Peromyscus, 117, 225, 313, 317, 308; for Clethrionomys, $17,19,61,46,30$. Apparently many members of both species were characterized by reduced home ranges, which they expanded as associates were removed. There being so few Clethrionomys present, they could not represent the species producing the inhibition of home ranges. Thus at this time we must suspect that actions by members of the genus Peromyscus not only eaused many of its own kind to contract their home ranges, but also caused similar contraction by Clethrionomys. In the light of the apparent reversibility of social roles of these two genera, previously discussed when comparing different localities, these comparisons between different eras within the same habitat suggest that Peromyscus and Clethrionomys in this habitat are really codominants, but that one will nearly exclude the 
other. At such times whichever one became more numerous than the other would cause members of the less numerous species to contract their home ranges and live within the interconstellation matrix of the more abundant species.

A small amount of trapping during 1946-1948 revealed that Peromyscus still held a 3:1 relative abundance over Clethrionomys. The intensive trapping was resumed during 1951, an apparently critical year in the social balance of the population. Forty-one NACSMI traplines, each run for three consecutive days, for a total of 7380 trap-nights of effort (NACSM Release No. 5) provided total catches for the three consecutive days as follows: Clethrionomys: 143, 114, 77; Peromyscus: 167, 132, 108; Blarina: $52,58,50$; Sorex: 2, 0, 2. Although Peromyscus was slightly more numerous than Clethrionomys, 9.93 versus 8.15 per trapline per three days, its slower rate of decline in captures from days 1 through 3 suggests that it was slightly subordinate and that some of its members were enlarging home ranges as their associates were being trapped off. However, the nearly constant total catch per day for Blarina clearly indicated its subordinate status to the other two genera.

The 24-day continuous remoral study of 1951 (Table V, Fig. 12) provided further insight into this realignment of social relations. Despite nearly equal numbers taken for the three most abundant species, Blarina is clearly subordinate to both Peromyscus and Clethrionomys. Its more marked contraction of home ranges is revealed by the continuously increasing daily catch orer the first few days of trapping (Fig. 12). Comparison of the proportion taken the first 5 days with that during the last 5 days provides an index of expansion of home range. The relatively more that are taken during the first 5 days, the less has been the expansion of home range, and thus the more dominant the species. On this basis, the four genera are listed in order of decreasing rank in Table V.

One of the most remarkable aspects of this set of data is the nearly constant daily eatch for each of the three more abundant genera between the 4 th and 17 th day of trapping. This means that within each genus, survivors increase their home range each day sufficiently to result in as many enrountering traps as on the previous day despite their fewer numbers. Furthermore, an individual member of each of these genera must be receptive to the inhibitory signals from the other two genera as well as of others of its own kind. Otherwise, the capture curves would have resembled that for clearly alpha species (Fig. 9).

This set of data also suggests that in the presence of several more dominant species actively contending for rank status, the very subordinate Sorex not only is markedly reduced in numbers, but also is much slower in expanding its home ranges. Peak captures, and thus maximum expansion 
of home range, did not take place until day 21, which was just after the final maximum expansion of home range for the other three species.

By the following year (Table Ib, NACSMI Release No. 6) this uncertain social state had completely clarified. Three-day totals for 34 NACSM lines were Clethrionomys (753), Peromyscus (38), Sorex (17), and Blarina (1). Other details of the resultant social organization have already been treated in Section VIII, A.

The studies (e.g., Figs. 10 and 13B) conducted during the past few year's in Montgomery County, Maryland, by Dr. Barbehenn and me, further substantiate the indeterminancy of the small-mammal community as a dynamic system. We have mostly sampled woodlands of 50 to 1000 acres. Peromyscus, Blarina, Sorex, and Pitymys are the more abundant species. In every case Peromyscus is the dominant species. For it, the largest catches per day occur during the first few days, and usually from day 1 there is a continuously declining catch per day with the exception of the slight secondary increase resulting from expansion of home ranges by gamma individuals (Fig. 10). Blarina is also nearly universally present. The date by which $50 \%$ of the total is trapped consistently arrives several days later for Blarina than for Peromyscus. In actuality, peak numbers taken per day usually occur several days after initiation of trapping. Thus, in these communities the home ranges of most Blarina are socially contracted. When either Sorex or Pitymys is present, they are definitely subordinate to both Peromyscus and Blarina in terms of the degree to which their home ranges are contracted. Their peak captures per day never occur until after most of the two dominants have been removed. Relative numbers are quite another matter. It seems to be purely a matter of chance whether either Pitymys or Sorex is present in any particular woodlot. Either, both, or neither may be present. Their absence appears not to be due to absence of requirements for food and shelter, but merely due to failure to reproduce under circumstances of spatial isolation, when the processes of social adjustment within a particular woodlot happens to markedly reduce the numbers of some one species. These woodlots in suburban to semirural Montgomery County, adjacent to the district of Columbia, exist as ecological islands which must be characterized by a rather low probability of receiving colonizers of these subordinate species. Furthermore, any one of the three subordinate species can become the most abundant species in the community. In each case the most abundant species has small home ranges whose centers lie within the interstices of the larger home ranges of the dominant Peromyscus (as shown in Fig. 16). Blarina was such a species in the study shown in Fig. 10. Had trapping been continued longer in the study shown in Fig. 13B, Pitymys would undoubtedly have had a nearly 2:1 ratio of abundance to the dominant Peromyscus. 
In other unpublished studies by Dr. Barbehenn, Sorex eatches nearly doubled those of Peromyseus.

\section{A Theoretical Conceptualization of the Evolution of a Social Hierarchy among Species in the Utilization of Space}

The eoneept of social inhibition of home range represents an inference derived from the observed differential probability of capture by snap traps. Reduction of extent of home range must result from an inerease in the probability of terminating trips away from home. We have seen that an increase in the strueturing of the environment, with stimuli which elicit responses, does lead to an increase in the probability of terminating trips. This structuring of the environment represents an increase in the amount of stimuli impinging on the organism. For this reason I believe that one is justified in making the following tentative generalization: Any increase in the frequency or intensity of stimuli to which the animal has responded in the past will lead to an increase in the probability of terminating trips.

It follows that genera such as Sorex or Pitymys frequently are characterized by such small home ranges that we may conclude that they experience a high frequency or intensity of relevant stimuli. At the same time their associates, Peromyscus or Clethrionomys, have large home ranges. This being so, we may conclude that the latter are not unduly exposed to an excess of relevant stimuli. And yet we may suspect that any stimulus which Sorex or Pitymys can detect can also be detected by Peromyscus or' Clethyrionomys. Thus, their differential response to stimuli must involve some internal mechanism through which stimuli produce an effect indieating relevancy or irrelevancy.

So far it has appeared that auditory stimuli of the class represented by vocalizations of members of the small mammal community might represent the elass of stimuli producing alterations of home range. If this inference proves correct, it means that some species respond to a broad spectrum of different auditory stimuli whereas others "ignore" all except those emitted by their own species.

Broadbent (1958) elaborates a theory of perception and communication which may serve in conceptualizing how vocal communication functions in leading to an organization of the small mammal community. Briefly this theory is as follows:

The eentral nervous system may be conceptualized as a signal flow system possessing the following connections and characteristies. Stimuli impinging upon the sense organ generate signals which pass into a tempo- 
rary store. Storage here is in terms of seconds only. From this short-term store, signals must pass through a limited capacity channel before they can serve to initiate immediate responses or reach a long-term store where the signal can be preserved to affect later action. Signals passing through the limited eapacity channel from the temporary store may be passed back through another circuit and reenter the temporary store. Likewise, responses made to a stimulus or a sequence of stimuli, in turn, serve as stimuli which generate signals entering the temporary store. Furthermore, several signals may arrive simultaneously at the temporary store through separate sensory channels. Only a portion of these signals in the temporary store can get through the limited capacity channel.

There has evolved a neural mechanism which Broadbent calls a "filter," intervening between the temporary store and the limited capacity channel. This filter "selects" which signals may get through the limited capacity channel and thus be available for (a) recirculation into the temporary store, (b) inducing immediate response, or (c) entering the long-term store.

The following conditions affect the probability of signals passing through the filter:

1. The signal is of the same class as that of the prior signal. That is, the related stimulus has similar characteristics in terms of frequency, intensity, pattern, or location of origin. In other words, the filter tends to pass in secunence several signals from stimuli with related characteristics.

2. However, the longer a given category of signals has been passing the filter, the more likely the filter will switch to signals arriving from a different sensory channel.

3. Signals generated by intense or infrequent (novel) stimuli exhibit a high probability of passing through the filter.

4. Given any three signals in the temporary store and one is passed through the filter, the one of the remaining two most likely to follow it is the one which followed it most frequently on prior occasions.

A special case will particularly concern us. An animal may exhibit both bodily response and vocalization to a given external stimulus. Each of these responses also beromes a stimulus with a high probability of association, each with the other, and each with the external stimulus. As the external stimulus becomes weaker, only the bodily response is preserved. Presumably the reason for this is that the bodily response represents a more intense stimulus and for this reason develops a higher conditional probability of association with the external stimulus. That is, the signal from the bodily response stimulus is more likely to pass through the filter immediately after the signal from the external stimulus. 
Although I have treated Broadbent's theory only sketchily here, its importance in the context of the present discussion is his documentation of the necessity for postulating the existence of some neural mechanism having the properties he attributes to the "filter." His thesis evolved primarily from studies with human subjects, and to a much lesser extent from studies of rats and dogs. He accepts the existence of a filter, and even that there are intraindividual differences in the effectiveness of its maturation. His concern with the evolution of the filter extends only to his belief that animals with a smaller cortex probably also have a less well developed filter. He does not discuss how a less well developed filter would differ from a more highly evolved one. We might anticipate the four attributes of filters listed above to be less well developed. That is, signals sequentially passing the filter represent a class of stimuli having a wider range of variability; shifts from one sensory channel to another occur with greater frequency; a stimulus need be only slightly more intense or novel to generate signals capable of having priority over other signals in passing through the filter, and two stimuli must be associated in time much more frequently for their signals reaching the temporary store to have a higher conditional probability of passing in sequence through the filter.

Let us turn to a consideration of how the social use of space may have encouraged the evolution of animals with more efficient neural filters. At the dawn of mammalian evolution, we can visualize a type having elose equivalence both morphologically and physiologically to contemporary shrews of the genus Sorex, with the exception that they had developed essentially no neural filter mechanism for screening signals passing from the temporary store to the limited capacity channel. All stimuli arriving separately would get through the limited capacity channel and it was purely a matter of chance which of two simultaneously arriving stimuli might find passage. Under these cireumstances, no diserimination may be made between aversive stimuli emitted by neighbors at a distance. This would lead to a uniform distribution of home range centers at approximately 2.0 sigma distance between centers.

Paleontological evidence suggests that these early diminutive mammals preyed upon insects and other small invertebrates. Furthermore, since among present day shrews Blarina is dominant to Sorex in the hierarchy of use of space, it follows that evolution permitting such differential control of space must have proceeded prior to further marked alteration with reference to feeding habits. Therefore, it appears that early in the history of shrew-like mammals there was sufficient evolution to permit one species to inhibit the extent of home range of another. For simplicity's sake let us designate the earlier form as $A$, and the later derived one as $B$. Two characteristics gradually become fixed in $B$. It evolved an altered vocaliza- 
tion. This vocalization preserved much of the characteristics of $A$, but entailed an addition of components. Following Broadbent's analysis of stimulus charaeteristies we may suspect that an attribute of some portion of the added vocal eomponents included an inerease in intensity. Likewise, $B$ 's filter developed alterations which enabled $B$ to filter out selectively those voealizations not ineluding the new attributes developed by $B$. Thus, members of the new species $B$ eould develop conditioned associations with the vocal signals emitted by its own kind while ignoring those emitted by species $A$. At maximum stability of such a two-species community there would exist a $2: 1$ ratio of $A: B$ in a similar fashion exemplified by the Blarina: Peromyscus eommunity previously described for Dr. Barbehenn's Chadwiek Woods study (Section VI, B) and Dr. Webb's Rich Lake Island study (Section VI, A) for Sorex: Clethrionomys.

The interesting aspect of this $2: 1$ ratio of species $A: B$ is that it enabled three animals to live where only one lived before. In other words, the evrolution of dominant speeies $B$ not only enabled as many of species $B$ to live in the habitat as was formerly the case with reference to the time when speeies $A$ only existed there, but it also enabled twice as many of the more primitive species $A$ to live in the habitat as had been the case when $B$ was absent. For such a pattern of evolution to have transpired, it means that intraspeeific dispersal of home range eenters in a one-speeies community as a consequenee of the repulsive eharaeter of vocal stimuli must have been sufficient to ensure an average utilization of resourees far below maximum earrying eapaeity. To clarify further what is intimated above: Members of $A$, as a result of antagonistic interactions with others of its own kind, develop eonditioned avoidanee responses to intraspecific voealizations. Furthermore, the greater the frequency of these vocalizations, as represented by inereases in density of the species, the greater is the probability of outward excursions from home being terminated, thus the smaller home range. Laeking a suffieiently effective neural filter, $A$ responds to $B$ 's vocalizations as if they were their own. If $B$ emits signals with the same frequency as does $A$, it follows that in a stable two-species system $A$ will be responding to three times the signal load as $B$. For this reason, $A$ 's home range becomes markedly reduced in eontrast to its area when $B$ was absent.

The next step in the evolution of the soeial hierarehy of space utilization, resulting in speeies $C$, entailed similar alterations to vocalizations and to enhancement of the filter in restrieting the spectrum of stimuli which would likely be assoeiated with intraspecifie interactions. Judging by the fact that the omnivorous mouse Peromyscus is dominant to both Sorex and Blarina, one may conclude that evolution of altered food preference facilitated further evolution of interspecific social domination of space. 
In fact, it was this difference in food preference which led me to conclude that the interspecific aspects of dominance in the utilization of space most likely fails to involve direct physical interaction between members of different species. Objects desired by species holding opposite ranks in the hierarehy gradually became more and more different. At this level of evolution species $A$ responds not only to vocalizations of its own kind but also responds to vocalizations of both $B$ and $C$ as if they were by members of species $A$. Species $B$ can ignore signals from $A$ but treats both its own signals and those from $C$ as $B$ signals, while $C$ "filters out" $A$ 's and $B$ 's signals and responds only to those of its own species. In other words, $C$ functions as if it alone were in the environment.

The fourth step involving evolution of speeies of type $D$, which is similarly dominant to species types $A, B$, and $C$, again is accompanied by further specialization toward a nearly total use of plant material as food. Judging by the results of field studies presented earlier in this paper, the redbacked mouse, Clethrionomys, represents a species at the D level. In most situations where both it and Peromyscus are present, Clethrionomys dominates. Following the previous line of reasoning we may anticipate that such a $D$-type species emits vocalizations having not only the basic chalacteristies of species $A, B$, and $C$, but, in addition, possesses vocalization characteristics peculiar to itself.

At each level a species responds not only to its own vocalizations, but also to those of all species ranked above it, as if they were emitted by its own kind. The lower the rank of a species, the greater are the number of vocal stimuli to which it responds and thus the smaller its home range. Reasoning back from present day small-mammal communities to the probable course of evolution, it appears that there is a correlation between (a) social rank in the domination of the use of space, and (b) the shift from camivorous to herbivorous diet. I do not believe that an herbivorous diet per se contributes in any way to social dominance. Rather, it has relevance only because of the later development of flowering plants and grasses. Evolution of more advanced types of plant permitted erolution of small mammals specialized to utilize these new resources. To a certain degree snch feeding specialization would enable an incipient species to avoid direct competition with its progenitor. Once removed from overt competition with its progenitor, psychologieal dominance by the incipient species could then proceed through the process of increased complexity of vocalization and development of a more effective filter.

A major aspect of my thesis is that psychological dominance, resulting from a greater complexity of vocalization and an increased effectiveness of the neural filter, far outweighs all niche specializations in determining the relative abundance of species comprising the small nammal community. 
Such psychological dominance also develops among members of a single species, at least at the higher levels of the interspecific hierarchy. We have already presented the data and logic which gave rise to the concept of constellation formation. On the average, each constellation has one central alpha member with a very large home range, six beta members with home ranges slightly smaller than for alpha individuals, and five gamma members with markedly restricted home ranges. Such intraspecific differences in home range size suggests that, among alpha $(C$ or $D$ types in above discussion) species at least, developmental alterations in vocalization arise. Gamma members of alpha species exhibit a minimum complexity of vocalization. Successively beta and alpha members increase the complexity of their vocalizations. All members of the species presumably have the same level of filter development. Even so, the differences in complexity of vocalization should result in alpha members mainly ignoring vocalizations of beta and gamma individuals insofar as these signals have a negative valance. Beta members respond not only to other beta individuals but to their alpha associates. Gamma members not only treat the vocalizations of other gamma members as inhibitory stimuli, but are likewise similarly influenced by those from their beta and alpha associates.

It must be borne in mind that this whole discussion of vocalizations and filters in the context of the small mammal community is strictly theoretical. However, it not only provides a conceptual framework offering one interpretation of empirical data, but also enables formulations capable of experimental analysis.

A study of the complexity and intensity of vocalizations is suggested as having priority in testing the theory. Sorex, Blarina, Peromyscus, and Clethrionomys, respectively representing theoretical types $A, B, C$, and $D$ discussed above, should serve as particularly useful subjects, especially since they all may occur in the same small-mammal community. The vocalization of each higher member of the series should include the basic characteristies of all lower ones and in addition possess characteristics not held by lower members in the series. Furthermore, if each of these species is experimentally exposed to a conditioned avoidance situation where vocalizations of their own species serve as a conditioned stimulus, one may anticipate that rocalizations of the other member's of the series will equally well induce avoidance upon their replacement of the intraspecific stimulus only if the vocalization represents a higher member of the series. Unfortunately, the extreme paucity of our knowledge of vocalizations of small mammals necessitates these suggestions in lieu of any firm experimental evidence.

Development of cryptic behavior forms an ancillary aspect of this concept. The lower-ranked shrews typically spend much of their time in under- 
ground runways. Presumably such behavior enables these species to reduce the frequency or intensity that they experience by the rocalizations of their dominant associates. When Dr. Barbehenn first joined me in these studies of small-mammal communities he insisted that the only effective way of trapping Blarina was to set traps at points where excavations in humus revealed underground runways. However, it had been my experience with continuous removal trapping, where traps were set at fixed intervals from stations without any regard to underground runways, that once the dominant Peromyscus has been removed Blarina were caught on surface sets with equal ease as earlier for Peromyscus. Therefore, when he initiated his Chadwick Woods study (Fig. 10) he set his traps on the surface without regard to runways. During the initial days while many Peromyscus still survived, most Blarina were taken only by traps accidentally set by underground runways. As the number of Peromyscus became fewer and fewer by the removal trapping, not only were more Blarina caught, but also an increasing percentage of these had so entered traps as to indicate clearly that they had been wandering about the surface and were not emerging through the leafmold below the trap. Thus, a reduction in crypticism accompanied enlargement of home range. This same change in behavior characterizes the typically subterraneous mouse Pitymys following removal of its dominant associates. The prior discussion of the special case of both bodily movement and vocalizations of another individual become important here. We might thus expect that vocalization of shrews would become reduced as they become more cryptic in the presence of dominant mice. Also as mice are removed from the habitat, shrews should not only spend more time out on the surface, but they should vocalize more.

This section cannot be closed without brief reference to the meadow mouse Microtus. Data from a recent unpublished study by Dr. Barbehenn in an abandoned orchard in Maryland suggest that this genus represents one terminal phase in this evolution of types, which on the psychological level enables successively evolved types to aequire a more dominant position with reference to the use of space. At points isolated from each other by at least 600 feet he placed covered feeding stations which contained rolled oats mixed with dyes which stained the fur of animals eating there. Daily removal of food increased for nearly a month, after which the daily removal fluctuated about an asymptote for another month. At the end of this time removal trapping was conducted along a circular trapline having a radius of 150 feet from the feeding station. Practically all the Peromyscus taken were marked with the red dye, but no Microtus were so marked. This indicated that Microtus living at this distance from the feeding stations were not only unaffected by it, but their home ranges were unaffected 
by Peromyscus, even those living much farther out in the habitat, altering their movement and passing with increasing frequency and in an altered pattern through the home ranges of Microtus. This trapping was followed by additional removal trapping along several concentric circular traplines between the original one and the bait station. Many more Microtus were trapped, but practically all Peromyscus had been removed in the initial trapping. Furthermore, the drop-off in catch of Microtus in this later trapping, as the concentric lines came nearer the initial one, indicated that only Microtus living within 37 feet of the initial circular line had been taken by it. It furthermore indicated that Microtus were not wandering about, as we had supposed they might be, but regardless of distance from the feeding stations had maintained fixed home ranges despite many strange Peromyscus focusing their movements toward this one central spot. Parenthetically, it might be added that this altered behavior by Peromyscus did disrupt Sorex home ranges. They were taken in relatively large number beginning with the very first day of trapping along the initial circular trapline.

Now the question arises, "What do these data indicate?" Since Peromyscus altered its behavior by readjusting its movements toward the feeding station as if Microtus was not there, I conclude that one of Microtus's adaptations has been the loss of vocalization. On the other hand, since Microtus appears to be unaffected by the altered movements of Peromyscus and the periodic concentration of many Peromyscus in one spot, which must increase the vocalizations at that spot, I conclude that Microtus has evolved a filter system so effective that they can ignore signals from other species. This leads me to suspect that Microtus represents what might be called a secondary herd-type species. See later discussion on the evolution of colonialism and herding.

\section{Psychological Dominance as the Primary Component of the Niche}

Hutchinson (1957) includes the relationships one species has with others as comprising aspects of its niche requirements equally to be considered along with food, shelter, and climatic factors. Implicit in his inclusion of interspecific social factors in niche characterization is the relative capacity for any two given species to compete for some given environmental commodity or condition. The more nearly the identity of their nonsocial niche requirements, the more important become social relationships both as a component of the niche and in leading to the elimination of one species by the other via the principle of "competitive exclusion" (Hardin, 1960).

In terms of this viewpoint and the marked difference in diet, morphology, 
and behavior of such distantly related genera as Clethrionomys, Peromyscus, Blarina, and Sorex, we would suspect minimum niche similarities and thus little influence of one genus upon the other.

If their niche requirements are really quite dissimilar, if within any individual's home range each requirement is represented at many points, and if in most home range-sized plots most of the niche requirements for each genus abundantly occur, then, depending upon the intraspecific factors leading to fluctuations in density, we might in fact anticipate that one or several of these genera might occur simultaneously in high densities. In fact, observed relative densities do behave in such a fashion. Were we content to rest our case solely on relative densities, we would remain content with the satisfactoriness of such logic in revealing true relationships.

However, the time sequence analysis of removal captures leads to a formulation of community dynamies suggesting the nearly complete invalidity of the concept of lack of influence of one species upon another if their niche requirements markedly differ. In fact, the concept of competition has little relevance. Instead, "home range inhibition" becomes the most useful concept. Home range inhibition is the consequence of processes through which the individual reacts to a signal as if it were identical to a different signal with which it shares certain physical characteristics. In other words, an animal may react to a signal emitted by a member of another species as if it were the signal emitted by another member of its own species with whom its interactions have led to its characterizing the signal as noxious. Such a species becomes a subordinate member of the community.

Signals of the dominant species must contain not only the basic characteristics of the subordinate but some characteristies peculiar to itself. Thus, when both a dominant and a subordinate species occur simultaneously in a habitat, members of the dominant species will come to associate noxious qualities only to that portion of the signal which is species-specific since it is the only portion of the signal which always accompanies a negative intraspecific interaction. If we consider a community composed of four species $A, B, C$, and $D$ (following the nomenclature of Section IX) in which $D$ is most dominant and $A$ most subordinate, and signal components $a, b, c$, and $d$ are observed, then $D$ 's signal should include components $a, b, c$, and $d$; $C$ 's should include $a, b$, and $c$; $B$ 's only $a$, and $b$; and $A$ 's only $a$.

Even though $A$ learns to define signal $a$ as noxious only by reacting with other $A$ associates, he will respond similarly to detection of the a components of his $B, C$, and $D$ associates. I have cited data indicating that the greater the frequency of relerant stimuli in the environment the greater will be the probability of the origin of a neural signal leading to termination of the behavior of outward movement from home. In this situation, every 
time $A$ emerges and starts on an outward excursion he experiences a bombardment by so many a signals that his trip is shortly terminated and he returns home. In a similar fashion, $B$ 's home range will become contracted but not so much as $A$ 's because $A$ does not emit $b$ signals, whose sum at any one time is contributed to ouly by species $B, C$, and $D$. $C$ will have only a slightly inhibited home range since it is responsive only to signals contributed by itself and $D$. At the apex of the system members of species $D$ are influenced only by $d$.

At the psychological apex of the community, further differentiation occurs among the members of the dominant, $D$ species. According to the formulation developed in Section VIII, C, members of this species have the capacity to differentiate into alpha, beta, and gamma members. Their basic $d$-type signal becomes differentiated into $d_{\alpha}, d_{\beta}$, and $d_{\gamma}$ components. Alpha individuals possess all three; beta members only $d_{\beta}$ and $d_{\gamma}$; while gamma members emit only $d_{\gamma}$.

This purely theoretical formulation predicts that home range size and complexity of signals emitted are positively correlated. The observed data on removal captures lead to inferences of home range expansion following removal of associates. Intra- and interspecific inhibitions are so apparent as to demand the minimum assumptions made abore for maintaining such a complex spatial organization of the small-mammal community. Compare these formulations with those of "velocity" developed in Sections XIII, A; XIV, A; and XIV, E and F. Such comparison leads to the conclusion that the greater an individual's velocity, the more complex will be the pattern of signals he emits.

Psychological dominance is then the ability to inhibit the home range of others resulting from the fact that the dominant shares certain signal characteristies with the subordinate, but in addition possesses signals which the subordinate lacks. The sharing of a signal by a species with another species which it usually dominates may lead to mutual inhibition of home range or actual reversal of roles.

Consider the Clethrionomys and Peromyscus relationship. The greater frequency with which Clethrionomys appears to be dominant to Peromyscus suggests that it has the $c$-d-type signal while Peromyscus has only $c$. We may ignore other shared characteristics of their signals. In fact, we may focus only on the shared $c$ component. Chance vagaries of the system may from time to time, after a erash in the populations of both species, result in a marked preponderance of Peromyscus over Clethrionomys. Similarly, at the southern periphery of its range, we can expect Peromyscus frequently to be more dense than Clethrionomys. Clethrionomys will then meet too infrequently to make any associations with their own species' specific signal. However, encounters will occur more frequently between Clethri- 
onomys and the more numerous Peromyscus. Regardless of which species dominates in the actual encounter, the interspecific common characteristic of the signal may be expected to assume only a negative quality by Clethrionomys. Some interactions among Peromyscus must be of a positive nature. Thus, even though both species may detect the interspecies common component of their signals with equal frequency, Peromyscus might be expected to exhibit less contraction of its home range because this signal is less aversive than it is for Clethrionomys.

This line of reasoning applies also at times to relationships among three species. In the unusual situation (Fig. 12) in which Blarina, Peromyscus, and Clethrionomys were all quite abundant, the sequential trapping data clearly indicate that inhibition of home range nearly equally characterizes all three species.

In such a system, the extent and center of an animal's home range depends not so much on characteristics of the habitat as on the current density and origins of signals, and the temporal and spatial history of interaction among members of the community during the most recent generations. The absence of an animal in a locality cannot be construed to mean unsuitability of the habitat. It is conceivably possible to delineate microhabitat characteristics contributing to the animal's niche even under such circumstances. However, it requires that we know an individual's home range center and that we mark out stations along a circumference of a circle having a radius which will result in a high probability of the animal crossing that circumference. A 1-sigma radius should prove effective. Examination of stations from such a series, which have a greater than chance frequency of visitation, might lead us to isolate those conditions which do contribute to an animal's niche.

\section{An Induced Invasion}

The major portion of this section presents the author's interpretation of a study conducted by Webb and Rosasco (1953). It describes the response of the red-backed mice, Clethrionomys, surrounding a 30 -acre tract within which continuous removal trapping was conducted for 80 consecutive days. In this account it will become apparent that the concepts elaborated remain inconclusive. Even so, their implications, when taken in conjunction with the other sections of this paper, warrant their presentation.

A brief history of events leading up to Dr. Webb's study provides a background for appreciating the objective. During the summer of 1950 , while I was in residence at the Roscoe B. Jackson Memorial Laboratory as a National Institute of Mental Health Special Fellow, Dr. A. Dexter 
Hinckley, then a sophomore at Yale University, was assigned to me for supervision on a research project. I assigned him the task of placing eight NACSM traplines in an 80-acre central portion of a much more extensive tract. He ran the 480 traps for 15 consecutive days. At that time it was generally accepted that if one plotted a regression line through points representing catch per day as a function of total prior catch, this line would intersect the abscissa at a point denoting the total population (see Calhoun and Casby, 1958, pp. 15-16 for a summary of this procedure). Previously, Hayne (1949) had made such estimations on the basis of 3 days of consecutive trapping as employed by the North American Census of Small Mammals. Thus, it was my anticipation that Hinckley's longer-term trapping would merely result in the anticipated continuous decline from day to day, and that by the 15th day essentially no more animals would be entering the traps. Furthermore, by the end of the 15th day the total should approximate that predicted by the intersection of the abscissa by the regression line as noted above. During the first 3 days the catch per day did decline. However, on the fourth day Hinckley reported a larger catch than on the first day. Well, I thought this was just due to the vagaries of chance or some umrecognized climatic factor. On every successive day his report was the same-more animals than yesterday. On the 15 th day, three times as many animals were taken as on the first day. All of this was very perturbing.

After several months of reflection I came up with this formulation: During the first 3 days the number of residents in the 80 -acre tract had been drastically reduced. Animals at the periphery of this tract would then find themselves with the normal number of neighbors centrifugal to the trapped area but with very few remaining centripetally. Now, suppose that in the normal state maintenance of a uniform distribution through the environment is facilitated by vocalizations and audition. Each individual's customary state would be that of detecting an equal intensity of signals in all directions from the center of its home range. In these terms mice and shrews at the periphery of the trapped area would detect few signals toward it but comparatively many away from it. Their normal response being to move so as to equalize signals coming from all directions, their response to the neighboring depleted area would be to move in toward it. They then became exposed to traps still set in the central area and many of these invaders were killed. This left the next peripheral group of animals in the same situation so that they also began moving inward toward the trapped depleted area. By this process, a chain reaction was set in motion in which the entire population for a great distance from the trapped area began moving toward it. If we visualize the trapped area as a circle, rather than its actual rectangular shape, it becomes apparent that if animals are 
moving in at a constant speed of travel per day, those invading the trapped area each day will represent residents from successive bands of equal width. Each successive day the majority of the captures will be from a more distant band than on the prior day. In a system of concentric bands of equal width, each band more distant from the center contains a larger area than the next innermost one. Thus, with density proportional to area and invasion into the central area transpiring from a constant rate of morement torrard it, catch per day should increase with time.

At the time when this formulation was just crystallizing in the fall of 1950 , Dr. Webb wrote me concerning their developing plans for longrange studies of small-mammal populations of the Huntington Forest near Newcomb, New lork. I mentioned the interesting results obtained by Hinckley, and the hypothesis generated by them. His response was to replicate Hinckley's study.

Details of these two studies have been included here (Figs. 12 and 13. A). Howerer, at the time of these two studies the failure of catch per day to decline over time blinded us to the story which the differences of input for different genera could tell us. That is, it was not realized at that time that expansion of home range by subordinate members of the dominant species and by all members of the remaining subordinate species could lead to results by removal trapping in which catch per day did not decline even though there were no invasions. Not recognizing this possibility the results of these two studies were described (Calhoun and Webb, 1953) as supporting the hypothesis that continuous removal trapping did in fact lead to inrasion of the trapped-out area by residents from surrounding areas.

By that time I had become associated with the Neuropsychiatry Division of the Walter Reed Army Institute for Medical Research. If this hypothesis were correct it might have relevance both to lemming migration (Elton, 1942) and to certain panic phenomena of troops (Ranson, 194?; Caldwell, et al., 1951). In order to explore this phenomenon in more detail. it was possible for Dr. Webb to negotiate Contract Number DA-49-00MD-325 between the Medical Research and Development Board (Office of the Surgeon General, Department of the Army) and the College of Forestry, State University of New Tork. This enabled him and his associate to execute a large series of studies on the response of small mammals to remoral trapping. The major details of these studies will be published elsewhere by Dr. Webb. I merely wish here to present a brief outline of one of these studies because of its importance to the general theme being dereloped in this paper.

In the center of an extensive forested tract they established a circular trapline with a 562 -foot radius. Along this trapline 781 snap-traps were 
placed, one at approximately every 5 feet. Along two diameters at right angles to each other, 156 additional traps were placed, 3 to a station and with a 50-foot interval between stations. This central cross of traps was intended to facilitate removal of residents and afterward capture any invaders "filtering" through the peripheral circular trapline. All 937 traps were run for 80 consecutive days. No Clethrionomys entered the traps on the 21 st day. Prior to that, 101 were caught and, in addition, six died in a preceding period of live trapping. These 107 individuals presumably lepresent the majority of residents. Between the 21st and 80th day of trapping, 501 additional redbacks entered traps. Furthermore, these 501 represented four fairly definite waves, roughly 15 days elapsing between the beginning of each wave and the start of the next one. Each later wave exceeded in numbers that of the preceding one (Fig. 31).

Let us now return to a consideration of the hypothesis, originally conceived from reflecting upon the results of Hinckley's 1950 Maine study. Although it is now recognized that the hypothesis does not apply to the study from which it originated, we shall now ask the question: "Does it

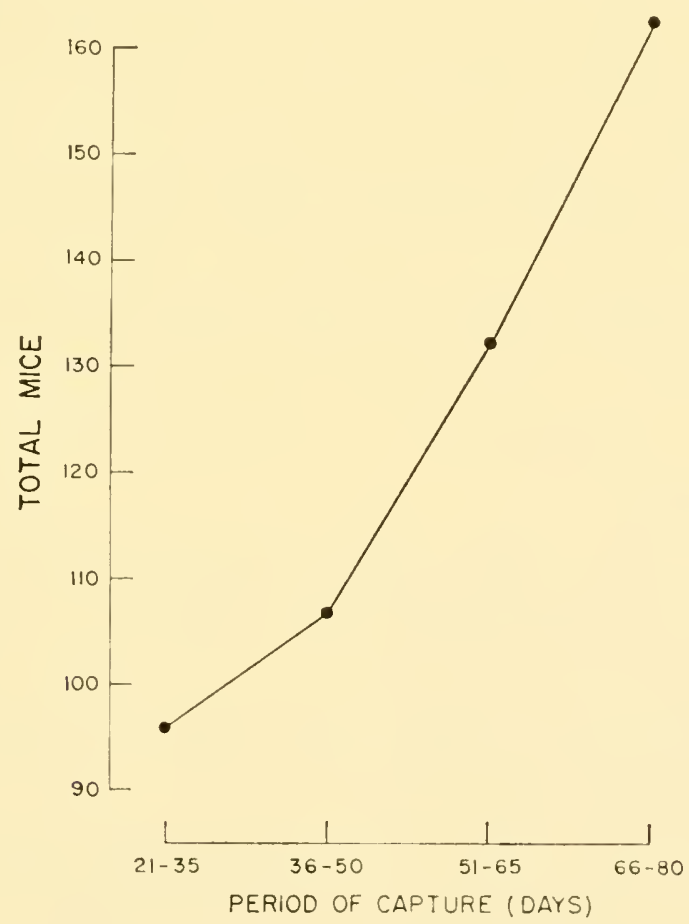

Fig. 31. Invasion of redbacked mice into an area from which most of the residents had presumably been removed. 
apply to the present data with regard to captures following presumed removal of all residents during the first 20 days?" If it does, we shall also wish to know whether the apparent waves might reflect some basic property of intraspecific social organization.

The first problem concerns determination of the area inhabited by those animals taken during the first 20 days. It must include all the $\pi r^{2}$ area delimited by the trapline. Furthermore, animals from some distance outward from the trapline must also have been eaught. On first thought, it might be logical to anticipate that all mice whose home range centers lay within 3.0 home range sigma of the circular trapline, and away from it, would be the only ones to exposed. However, as we shall see, the distance outward from the trapline to which animals are affected by it more likely approximates the maximum distance at which they ean perceive signals from other mice.

But, this is getting ahead of the analysis. For the present let us assume that each wave of mice entering the traps represents the inhabitants of a band of width $w$. Furthermore, assume that during the first 20 days mice from a band of width, $w$, immediately outward from the trapline, get (aught by it in addition to those internal to the circular trapline.

The radius from the center of the area being trapped to the trapline was 562 feet. Thus, the area sampled during the first 20 days equals $\pi(562+w)^{2}$. Since each wave of invaders is presumed to represent a band of equal width, $w$, then the entire area sampled during the entire 80 days equals $\pi(562+5 w)^{2}$. One hundred and seven mice were taken from the central area, and 608 from the total area. Thus, to the extent that number of mice is proportional to the area they inhabit, 5.626 as many mice inhabited the total area as the central area. It follows that:

$$
5.626\left[\pi(562+w)^{2}\right]=\pi(562+5 w)^{2}
$$

Thus $w=302$ feet.

Radii to the limits of the central area and the four successive bands become $864,1166,1468,1770$, and 2072 feet (Table IX). From these the areas within the central area and the four bands may be calculated. These are areas as proportions of the total within a circle having a radius of 2072 feet as given in Table IX. These proportions ean then be utilized to calculate the expected number of mice residing within the central area or invading it during later suecessive periods.

The assumptions force identity between observed and expected for the central area, but not for the four bands. However, the observed eatch for the four bands will approximate that of expected only to the extent that the formulation is in harmony with reality. As may be seen from Table IX, observed and expected numbers approximate each other so elosely as to 
TABLE IX

Invasion of Redbacked Mice into an Area Subjected to Continuous Removal Trapping

$\begin{array}{ccc}\text { Location } & \begin{array}{c}\text { Radius to } \\ \text { outer edge } \\ \text { of area }\end{array} & \begin{array}{c}\text { Proportion of total } \\ \text { area from which } \\ \text { trapped mice originated }\end{array}\end{array}$

Central area,

$$
r=w+562 \mathrm{ft} \text {. }
$$

Band No. 2

Band No. 3

Band No. 4

Band No. 5

$\begin{array}{rrrr}864 & 0.174 & 107 & 107 \\ 1166 & 0.145 & 8 S & 96 \\ 1468 & 0.183 & 111 & 107 \\ 1770 & 0.227 & 138 & 135 \\ 2072 & 0.271 & 165 & 163 \\ & \overline{1.000} & -609 & 608\end{array}$

make a test of significance superfluous. This, of course, only proves that the formulation is not wrong; it does not prove its validity. In the absence of any evidence to the contrary, the formulation will be accepted as approximating reality, and I will proceed with exploring further questions.

First, what about $w=302$ feet? Webb and Rosasco (1953) and Patric (1958) offer considerable evidence indicating that the home range sigma for Clethrionomys must be close to 50 feet. Thus, $w=6.0 \sigma$. According to the independently arrived at formulation, rocal signals can be detected up to a distance $6.0 \sigma$ from the emitter (Fig. 18 and Section VII, C). Also, the diameter of the constellation approximates $6.0 \sigma$ (Fig. 27, Section VIII, C). Reeall that the constellation represents the inferred basic unit of social organization among such animals. Thus all members of constellations which overlap the eircular line, or even just touch it although the home range centers of their members all live peripheral to the trapline, can detect a signal void centripetal to the trapline.

Before going on, I would like to emphasize that the diameter of 1121 for the circular trapline was dictated by the desire to ensure that mice arriving at the trapline would not be able to hear others across the roid on the other side. In fact, if 302 feet does represent the threshold distance of perception, then the angle of signal void facing a mouse arriving at the trapline would be that angle subtended by chords of 302 feet from that point, in this case 159 degrees.

The existence of constellations assumes prior development of bonds of attachment among its members. Once the central cross of traps and the circular trapline had removed all mice exposed to it, survivors of constella- 
tions in contact with the trapline would then tend to move simultaneously toward it. There would then arise the situation in which out to a $w$ distance from the trapline few mice remained. At this time the mice in the second band would begin moving more or less together after a lag in time resulting from their attachment to home and to each other. No mouse could leave home until several of its associates were ready to move together. Similarly, residents of bands 3,4 , and 5 would start inward as soon as most of their neighbors of the adjoining inner band had moved $w$ distance toward the central trapping area. In this way a "chain-reaction" was set in motion in which several bands simultaneously were moving toward the central area.

For the present this interpretation seems the most likely one. Whether the phenonemon has any analogies to lemming migrations or troop panic need not concern us here. Of importance are the indications that the influence of a signal void extends for approximately ti.0 home range sigma. The wavelike nature of invasion suggests that most of the mice from a band of 6.0 sigma move together. That they should do so is in harmony with the concept of constellation formation into groups whose mean size is 12 individuals and whose spatial diameter is about 6.0 home range sigma.

In the context of the present paper this additional support of the concept of a constellation as a real phenomenon represents the prime importance of this study on an induced invasion. For it is the evolution of the constellation and its later condensation into the compact colony way of life that $I$ believe represents the reason why a basic group size of 12 adults is the most important one in the mammalian series.

\section{Derivation of Compact Colonies from Constellations}

Accentuation of the social bonds among members of a constellation must have increased reproductive effectiveness and permitted survival of individuals exhibiting a decreased antagonism toward those neighbors who become familiar through repeated contacts. As the aggressiveness became reduced, beta and gamma members gradually shifted their home range center toward that of their dominant alpha associate until at last theirs coineided with his. A compact colony will then be formed in which all individuals share the same home range. Furthermore, the aggressive actions of the single dominant alpha individual of a compact colony serves to protect the other members of his colony from intrusion by extracolony members of the same species. Whereas constellation formation may be observed to take place within one or a few generations from a prior uniform distribution of home range centers, the development of compact colonies from constellations must have entailed considerable genetic change over 
sufficient generations to permit the evolution of a new species or genus. Stages intermediate between that of constellations and compact colonies may be recognized by the mean interval between adjoining home range centers decreasing from 2 sigma toward zero signa.

\section{A. Compact Colony Formation in the Norway Rat}

Over a 27 -month period I observed the development of social organization in a population of Norway rats confined in a one-(quarter acre enclosure. Preliminary details have already been presented (Calhoun, 1949, 1952),

\section{TABLE $\mathrm{X}$}

Chatracteristics of Norway Rat Colonies

\begin{tabular}{lcccccc} 
Colony & $\begin{array}{c}\text { Number } \\
\text { of } \\
\text { rats }\end{array}$ & $\begin{array}{c}\text { Proportion } \\
\text { female }\end{array}$ & $\begin{array}{c}\text { Mean } \\
\text { weight, } \\
\text { males } \\
\text { (gm.) }\end{array}$ & $\begin{array}{c}\text { Mean weight, } \\
\text { nonpregnant } \\
\text { females } \\
\text { (gm.) }\end{array}$ & $\begin{array}{c}\text { Proportion } \\
\text { of females } \\
\text { reproducing }\end{array}$ & $\begin{array}{c}\bar{x} \text { young } \\
\text { per } \\
\text { female }\end{array}$ \\
\hline a & 14 & 0.928 & 548 & 457 & 0.769 & 4.3 \\
b & 6 & 0.666 & 511 & 449 & 0.750 & 3.8 \\
c $+\mathrm{d}$ & 11 & 0.600 & 500 & 488 & 0.428 & 3.4 \\
$\mathrm{e}$ & 14 & 0.642 & 512 & 435 & 0.555 & 2.3 \\
$\mathrm{f}$ & 15 & 0.534 & 456 & 427 & 0.250 & 2.0 \\
$\mathrm{~g}$ & 16 & 0.812 & 432 & 413 & 0.153 & 0 \\
$\mathrm{~h}$ & $\mathrm{~S}$ & 0.500 & 477 & 357 & 0 & 0 \\
$\mathrm{i}$ & 13 & 0.000 & 442 & - & - & - \\
$\mathrm{j}$ & 10 & 0.100 & 448 & - & 1.0 & 0 \\
$\mathrm{k}$ & 13 & 0.000 & 429 & - & - & - \\
\hline
\end{tabular}

and further details will shortly be published (Calhoun, 1963). By the end of the study the population had increased essentially from a single reproducing female to 120 adults. These formed 11 clear-cut local colonies (Table $\mathrm{X}$ ). Earh colony inhabited a single burrow or group of neighboring boxes, placed kelow the surface, to which the rats had access by a drain tile from the surface. Each such burrow or cluster of inhabited boxes was separated from adjacent ones by an average distance of about 35 feet. These are rank-ordered from $a$ to $k$ in a descending order of social rank. For the present purposes we may equate social rank with sex ratio and reproductive success. High-ranking colonies had few males and many females, most of whom successfully reared litters or were pregnant at the time of terminating the study. As social rank of the colony decreased there 
gradually ensued a change toward more males per female, and these females were less successful in reproduction. At the lowest level a colony consisted of only males, or if females occurred they were essentially asexual with regard to reproductive effectiveness. The lower the colony's rank, the lower the mean weight.

Nembers of each colony represented more than one place of birth in the pen. Members of the highest-ranked colony, $a$, mostly still lived at the place of their birth or had come there from adjacent colonies. As social rank of the colony decreased, its members represented ever more different places of birth. Despite this disparity in places of birth characterizing each colony, the total number of adults forming each colony varied little from the mean number of 12 characterizing the loosely knit constellation of such simple social types as Peromyscus and Clethrionomys.

At this point we may consider possible events which fostered the evolution of the compact colony from the loosely knit constellation. The major impact of the constellation way of living is that the most frequent group size would be 12 individuals. It might, therefore, be anticipated that evolutionary processes would adjust the physiology and behavior of such species to be most effective and appropriate to interactions transpiring in such a sized group. In accordance with the conservatism of evolution we might anticipate restriction of group size about this optimum of 12 as other factors caused the constellation to contract into a compact colony. Restriction of location of food stands is the most likely candidate for an appropriate environmental change. Provided the location of food became restricted but abundant and relatively permanent at these locations, we could expect types like the Norway rat to develop. They build burrows not too far away from such spatially restricted sources of food and all members of the colony participate in transporting this food into the burrow where large eaches are formed.

Compact colony evolution produced a situation which necessitated further erolution of the nervous system. If groups of 12 adults assembled, either as a consequence of being born at the same place or from random mixing, a more nearly equal sex ratio would characterize most colonies than was the case in my study within the experimental enclosure. This presents the opportunity for development of marked aggression among males and consequent stress experienced by associated females. Actually this was the initial situation always characterizing an incipient colony of Norway rats. In the process one or more males were driven off and sufficient females remained, although some always left to keep the colony at near 12 individuals. The rejected males either joined another developing colony lacking a male as dominant as the one at the colony they left or they joined to form an all-male colony. Where there was a single very 
dominant male (as in colony $a$, Table $\mathrm{X}$ ) his actions kept away most other males at times when his harem females were in estrus. This reduced the stress experienced by females in estrus, such as followed the thousands of mountings or attempted mountings experienced by females in colonies lacking such dominant males. In this sex-ratio restructuring of the population, a few colonies contribute most of the young.

Although this readjustment ensures the survival of the species, it is not the consecuence most important with regard to further social evolution. Each time a rat is excluded from one colony it attempts to join another aggregate in order that it will again find itself in a group of a size most compatible with its physiology. As soon as a group exceeds the optimum size, some of its members are excluded from it. This results in a marked reshuffling of the population from the time of puberty of young born in one season up to the beginning of the next breeding season. At this time the population is relatively stable with regard to membership of each colony.

In the process of attaining colony stability, the social enviromment is in a constant state of turmoil. Each individual is forced to make many adjustments to such changes. It continually has to learn new social relationships. These are learned so well that a group can shift its place of residence over a sufficient distance that passage by or through other colonies is necessitated. Even so, they can maintain their group integrity. I am convinced that the necessity of making such changes of membership from one colony to another required for reproductive survival of the species has resulted in the evolution of the Norway rat into a species which is not only highly perceptive of changes within the environment but has the capacity to learn required adjustments of behavior.

Although such capacities for perception and learned adjustment must have arisen in the context of a changing social environment, these same capacities then become available for perceiving and adjusting to nonsocial changes in the environment. Among ecologists this extreme awareness to changes in their environment by Norway rats has been termed the "strange object reaction" (e.g., Chitty and Southern, 195t). Among psychologists it is reflected in the studies falling under the broad rubric of "open-field emotional behavior" (e.g., Hall, 1934, Schneirla and Tobach, 1962).

If I were to make my evolving thesis concerning the social use of space complete, I should substantiate the role of vocal communication at the compact colony level of social evolution. Unfortunately, I cannot cite any adequate proof regarding the nature of its function. Norway rats do have a wide scope of vocalization ranging from the loud signal accompanying the termination of a fight to the low chirping and whining one can detect if one lies on the surface of a burrow with his ear at an entrance hole. Many 
other vocalizations also oceur when rats are on the surface near their burrows and also while at the source of food. I can only suspect that among these there is specific communication among alpha members, the dominant males, neighboring colonies, and that rats at the food source emit a signal which might be termed a "here is food" signal. Unless there are such signals, it is difficult for me to understand many of the observed behaviors of rats which clearly indicated that they were aware when other rats were or were not at the food source, even though they could not see it.

\section{B. Howler Monkeys, a Compact Colony Living Species}

Carpenter (1962) summarized the results of field research on this species (Alouatta palliata) during the past thirty years. Tabular data on 136 distinct groups show the number of adult males and females and the number of immature individuals in each group. I derived Fig. 32 from these data. Two large groups containing 27 and 31 adults, respectively, were omitted from the analysis.

Regardless of group size all groups contained more females than males. Excluded males live in a state of near isolation and have very little association either with each other or the organized groups. As with the Norway rat, reproduction within compact colonies apparently requires a reduction in the number of males in groups for effective reproduction to take place.

One of the central hypotheses in my formulation of the social use of space is that constellation formation must have served as a mold which so guided evolution that behavior and physiology would become fixed so that they would have optimum expression in a group of 12 adults. For howler monkeys, groups of 9 to 11 are encountered more frequently than smaller and larger ones (Curve A, Fig. 32). And although the decline in frequency of groups containing more than 11 adults is not so rapid as expected (Table VIII), the observed data do reveal a marked decrease in frequency of larger groups. Furthermore, only $3 \%$ of the 136 groups exceeded the maximum of 19 anticipated by the theory. The theory indicates no expectation of groups containing less than 7 adults, yet $26.5 \%$ of the 136 groups of howler monkeys did contain less than 7 adults. However, it must be pointed out that such theory presupposes completion of all social processes culminating in a group having considerable stability of membership. Incipient groups formed from the fragmentation of larger ones and larger ones approaching the point where fragmentation is imminent should logically both be excluded in comparing observed and theoretical frequencies of group sizes. However, present knowledge prevents such a comparison. 
Production of young is fairly ineffective in small groups in comparison with those containing 10 or 11 adults (Curve $C$, Fig. 32). In general, the trend of young per female also decreases as groups get larger than 12 individuals. The two very large groups not included in this figure and which had 27 and 31 adults were characterized by only 0.18 and 0.29 young per female, respectively. We must conclude that group size does affect those behaviors and physiology culminating in the production and survival of young.

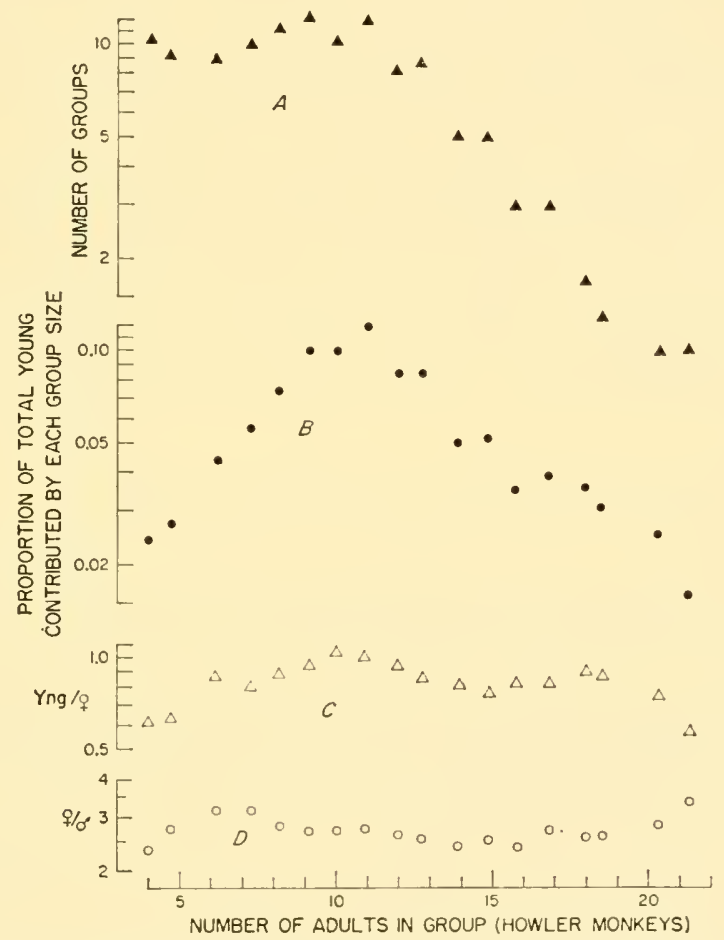

FiG. 32. Group dynamics of howler monkeys based upon Carpenter (1962).

Another way of looking at this problem is to ask: "What proportion of the total young are contributed by each group size?" For any group size the proportion will depend upon (a) the proportion of females in the group (Curve D, Fig. 32), (b) the number of young per female (Curve $C$, Fig. 32), and (c) the number of groups of each size (Curve A, Fig. 32); where such data for each group are weighed against similar data for all other group sizes. Resultant data are given in Curve B, Fig. 32. This curve clearly shows that more individuals gain their initial social experience 
in groups of about the size anticipated by theory as most nearly optimum than they do in any smaller or larger sized groups. In fact, the mean number of adults with which the 838 young in these 136 groups had their early social experience was 12.22 !, even though Curve $C$, Fig. 32 , is flatter than predicted by the theory of Seetion VIII, D and Table VIII.

My selection of the Norway rat and the howler monkey as examples of compact colony types is open to the eriticism that I selected those species which would support my theory that group size in higher evolved types is dependent upon their evolution from species which had been characterized by the loose constellation form of social use of space. At present, this criticism eannot be aroided. I wish merely to say that my intensive study of a few groups of Norway rats, and the extensive study by Carpenter and his colleagues of a large number of howler monkey groups, are the only ones known to me that appear adequate for the present purpose.

All we ean really say at present is that available data show that optimum group size in some compact colony living species appears to approximate 12 adults and that this number is in harmony with that number anticipated by the physies of communication characterizing more primitive and more dispersed types.

\section{Behavioral Sink Development by the Norway Rat}

Much of the prior sections have been devoted to documenting theory and evidence supporting the hypothesis that groups of 12 individuals represent a major category of optimum density. Yet many species customarily live as aggregates much larger than this. Why such large groupings should have evolved remained a puzzle until insight developed from an unexpected phenomenon arising in the situational content of some experimental populations of rats I was studying (Calhoun, 1962a).

The upper portion of Fig. 33 presents in diagrammatic fashion the salient aspects of the environment affecting the rats. Four 35 square-foot pens, separated by 2 -foot high partitions, formed a linear communication network through the opportunity of access between adjoining pens via the V-shaped ramps, $R$, surmounting barriers between pens. $F$ and $W$ represent a superabundant supply of food and water in each pen. "Apartment" houses, $H$, connected to the floor by ramps provided ample place of retreat and rearing young by most residents. The $H$ 's of the left-hand pens I and II were 3 feet from the floor, while in pens III and IV a 6 -foot distance separated the $H$ 's from the floor. Height formed an intentional environmental factor designed to produce a $2: 2: 1: 1$ ratio of density across pens I:II:III:IV as a consequence of the inverse-proportionality-to-effort usage principle. 
In addition the endedness of the environment biased movement. After some period of time every rat tended to leave the pen it was then in and go into an adjoining pen. Rats in an end pen could only go to the adjoining center pen, while a rat in one of the two center pens could go to the other center pen or to the adjoining end pen. In other words, when a rat is in an end pen it has a probability of 1.0 of going into the adjacent center pen, but if it is in a center pen it has a probability of 0.5 of going into the adjacent end pen or 0.5 of going into the other center pen. Repetition of shifting by all members by the operation of this principle alone soon leads to a

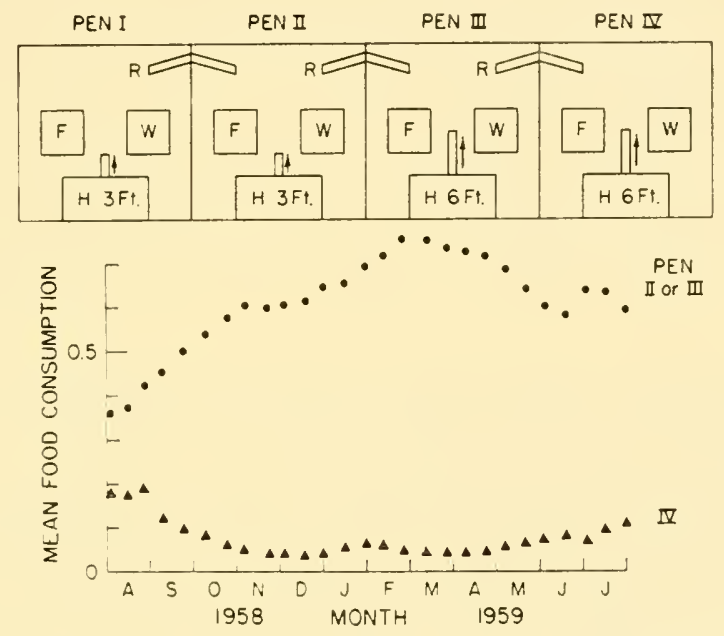

FIG. 33. The upper portion of the figure represents in schematic fashion the environment in which large social groups of albino rats were maintained. See text for details. The lower figure contrasts the amount of food consumed in the most used and the least used pen from the inception of the behavioral sink during the sixth month (August, 1958) of the study.

steady state in which a $1: 2: 2: 1$ ratio will characterize the density of rats across pens I:II:III:IV. See pages 298-299 of Calhoun (1962a) for details of the mathematies involved in the origin of this ratio.

Populations in four such 4-pen environments were studied from February 1958 to July 1959. By the eighth month, September 1958, each population consisted of three generations, each artificially fixed at near 30 individuals. The first and second generations were sexually adult, and the third was recently weaned. Distribution of adults at this time proved to be of considerable importance in producing a phenomenon $\mathrm{I}$ have termed a "behavioral sink." Five surveys of place of residence gave a total count by pen of 343 for pen I, 467 for pen II, 331 for pen III, and 245 for pen IV. 
If the two movement biasing principles described above operated independently and equally, the expected ratio of density would be $3: 4: 3: 2$ across pens I:II:III:IV, thus giving an expected distribution for the September 1958 observation of $347: 462: 347: 232$. The observed and expected values are so nearly the same as to support strongly the belief that these two movement biasing principles were in fact the only effective ones operating at this time. These same factors also affected the third generation then maturing.

Taking mortality into consideration, each population consisted of about 80 rats with on the average 20 living in pen I, 27 in pen II, 20 in pen III, and 13 in pen IV. Only in pen IV did the density approximate the ideal of 12. Elsewhere, partieularly in pen II, density far exceeded this. From this time on a remarkable change in the differential use of space, particularly as reflected by food consumption, set in. In one pen (in three instances it was in pen II and in the fourth in pen III) food consumption increased at the expense of that in the other three pens. See the lower half of Fig. 33. By the time seven months had elapsed, most rats were eating all their food in this "favored" location and all rats were doing most of their eating there.

The explanation for this change appears rather simple. Gnawing food through the wire mesh of the food hoppers required considerable time. In the one pen where more rats fed than in the other pens, the probability of two rats eating side by side increased. Gradually rats redefined the eating situation as requiring presence of other rats. Thus, all rats shifted most of their eating to that pen where this condition was most likely to be met. It must be kept in mind that such a system is stochastic and not deterministic, so it was not unexpected that pen III beeame the favored place of eating by one of the four groups. However, the likelihood of pen IV ever beeoming the favored pen is extremely remote.

The learned need for social proximity while engaging in an act which might have been expressed alone assumed priority over the simple original hunger drive. Food was not food without the presence of a comrade. This whole process of developing excessive aggregations in order to satisfy a secondarily acquired social drive is what I mean by a "behavioral sink." Gradually more rats also shifted residence to this favored place of eating. Such behavioral sinks result in every member encountering more associates than the ideal, and even more than necessitated by the operation of those principles of spatially structuring the environment which biases movement.

In this situation marked alterations in mortality and behavior resulted. Males became pansexual in the sense that they mounted other rats irrespective of sex or age. Nest building and maternal behavior became so disrupted in most females as to prechude the possibility of most young 
surviving. In each experimental setting the rats experienced less disturbance in one pen, usually pen $I V$, than elsewhere, since they were somewhat less trapped in the behavioral sink. And yet even here only half the young born survived to weaning and their growth was markedly retarded. In contrast, in the pen where most rats assembled only half as many young were born and only $1 \%$ of these survived to weaning.

In addition, the abnormal frequency of social interaction resulted in marked disturbance to female reproductive physiology. Near-term fetuses died. Some females with such dead fetuses shortly succumbed from apparent toxemia. Others died from massive hemorrhaging in many organs, an accentuation of the event likely to have been associated with fetal death. Many females who survived such events later died as a consequence of a site of resorption of a near-term fetus becoming the focal point for the development of a large abscess. Normally death occurred by the time the abscess reached a diameter of $50 \mathrm{~mm}$. As an example of this scourge, $56 \%$ of second-generation females died by a year of age, by which time only $10 \%$ of males had died.

Were a species to survive for many generations in an environment fostering development of a beharioral sink, it is obvious that selection must proceed to produce individuals whose behavior and physiology were in harmony with such a heightened frequency of social interaction. Wherever an environmental resource which was formerly so widely distributed as to be readily available within each individual's or group's home range becomes restricted, then conditions are ripe for production of a behavioral sink. It is my belief that just such happenings have been the usual alterations which have forced the evolution of horde or herd type species from one previously characterized by an optimum group size of 12 adults.

\section{Yarding by Deer in Northern Wisconsin}

Characteristically since 1935 white-tailed deer (Dahlberg and Guettinger, 1956) in Northern Wisconsin assemble during the winter in a restricted area known as yards. These cover only $5-10 \%$ of their range. Conifers, which comprise the major cover in the yard, provide protection from deep snows. However, such cover provides only a secondary quality food. One or more feeding stations were established in most yards. During the 1930's food supplements represented a small amount of total food requirements. By 1953, when artificial feeding was largely terminated, most of the food requirements were supplied at these feeding stations in many yards. Nevertheless, many deer died of "starvation" even in yards where the most food was provided. 
The restricted locations where food was provided and the striking aggregations of deer in their vicinity reaching 350 per square mile strikingly resemble my experimental populations of rats from which developed the concept of the behavioral sink. Even though many deer died in the yards, the question stands: "Is this really an instance of a behavioral sink?" Gaining insight into this question has proved to be a difficult detective job. Shiras (1921), Sanders (1939), Swift (1948), Kabat et al. (1953), Schorger (1953), and Dahlberg and Guettinger (1956) proved to be particularly helpful.

Before the days of lumbering, deer were so scarce in the primeval forests of Northern Wisconsin as to contribute very little to the diet of Indians. Between 1860 and 1880 a marked increase in deer followed lumbering operations with the consequent development of openings and second growth which provided abundant food. Although the deer did not reach the densities of 1935 to 1953 , they supported a major industry as a commercial source of meat. There is some mention during this era of herds up to 200 being seen, of aggregations about salt licks, and about concentrations in white cedar swamps during heavy snows. However, one gets the impression from Schorger's eitations that it was more customary for deer to be seattered. After heavy snows the commercial hunters trailed the deer until they found them exhausted and trapped by the deep snow. Schorger (1953, p. 210) writes, "It is stated by Harvey Braein that about Christmas, 1857 , a crust about one-half inch in thickness formed on the deep snow in Buffalo County, and that nearly every deer perished. Following the spring, their bodies were found in nearly every coulee." Unfortunately, this is the sort of data one has to rely on. Even so, it suggests a typical pattern of scattering.

As early as 1920 when the Northern Wisconsin deer herd was well on its way to recovery after its prior decimation by forest fire and unrestricted hunting, private hunting clubs and the operators of tourist camps had begun the practice of feeding deer during the winter months. After 1935 Civilian Conscrvation Corps camps and the Wisconsin Conservation Department greatly increased this artificial fceding. However, the intensive artificial feeding characterizes only the 1943-1953 period. Swift (1948) states that yarding had not commenced rery extensively until after 1941 even though astonishingly high populations existed in many locations. It is difficult to escape the conclusion that the accentuation of yarding was a direct outgrowth of the artificial feeding.

Daily movements rarely exceeded one-quarter mile from the feeding stations. Thus, available food outside the yards remained unutilized. Even with the advent of warm weather deer exhibited considerable reluctance in leaving the yard despite increasing new growth outside it. Fren cessa- 
tion of artificial feeding has not disrupted the marked yarding tendencies of Wisconsin deer. Through many generations they have developed a culture demanding an excessive frequency of contact with others during the winter season when they would otherwise be more scattered.

I will freely grant that this interpretation of the origin of yarding may be oversimplified. Nevertheless, available observations warrant consideration of the concept of the behavioral sink as helping us understand the historical development of yarding to an excessive degree.

\section{E. Concerning Basic Numbers, $N_{b}$, for Man}

During the past half million years, density of Homo sapiens has exhibited a continued increase over the inhabited portions of the earth (Deevy, 1960; von Foerster et al., 1960). Most who have concerned themselves with studying such change restrict their emphasis to changes in density accompanying advance in extractive efficiency of natural resources. Less attention, even by anthropologists, has been devoted to determining sizes of social groupings forming partially closed systems. Such partially closed systems range from a male-female pair to that of a nation such as the United States among which interactions among all members may be conceived of in terms of population potential (Stewart, 1948; Calhoun, 19.57). It will not be my purpose here to treat the entire range. Rather, I shall merely present a few highly selected examples of some of the smaller groupings which presumably reflect evolutionary limitations to group structure. On the assumption that these group sizes represent the consequences of underlying basic forces, they will be used in later sections (XIII, A and B) as data for developing a general formulation of group size and social interaction.

For about $98 \%$ of his history during the past half-million years, simple food gathering limited man's economy and social life. One of the earliest known settlements at the Star Carr site in east-central England of nearly 10,000 years ago consisted of five families, ten adults (Braidwood and Reed, 1957). Thus, it appears that an adult group size not diverging greatly from my hypothesized ideal of 12 may have characterized the human species up until at least 10,000 years ago.

The Australian aborigines (Birdsell, 1953, 1957) provide further insight into the basic numbers of human groups. In terms of the typical number of adults, five levels are recognizable: (a) the family with 2 adults; (b) the "horde" or extended family with 16 adults; (c) the supra-horde of 50 adults; (d) the tribe with 200 adults; and (e) the supra-tribe with 2200 adults. The horde forms the most basic social group, ranging generally 
between 10 and 20 adults. When the group exceeds 20 adults a budding process occurs, 10 adults forming a minimal-sized horde. 'These approximations of Birdsell's again suggest a basic group size not diverging greatly from the 12 presumably fixed by much earlier evolution. Occasionally, the horde may fragment temporarily into single family groups when scarcity of food demands such dispersal.

Also, oceasionally an average of five hordes, 50 adults, may temporarily assemble into a supra-horde. However, this grouping appears to be a less basic one than the other four. The third grouping, the tribe, lacks any form of authority, and only on rare oceasions do the 12 or so hordes forming the typical tribe assemble. However, common bonds of culture, their customs and value systems, elearly delineate the tribe as a social entity. Furthermore, marriages are primarily restricted to those between indiriduals of different hordes within the same tribe. Only in one local region of Australia has a higher-order grouping evolved. Three supra-tribes, averaging 2200 adults, represent an assembly of tribes bound together by a more advanced type of political organization characterized by matrilineal deseent.

These data suggest that eultural evolution has proceeded by saltatorial steps, each characterized by some accretion to the culture. It will be my hypothesis, to be developed in more detail in Sections XIII, B, 2 and B, 3, that the sole function of culture is to provide a mold which enables interactions to transpire in a larger group such that their physiological consequences to the average individual closely approximate those that would result were the individuals still living in a closed social group of about 12 individuals.

Hallowell (1960, pp. 345-346) states that “. . a a normative orientation becomes an inherent aspect of the functioning of all socio-cultural systems, since traditionally recognized standards and values are characteristic of them. Techniques are appraised as good or bad; . . . . Knowledge and beliefs are judged true or false. Art forms and linguistic expression are evaluated in relation to ethical values. All cultures are infused with appraisals that involve cognitive, appreciative, and moral values," and "if the total ramifications of the normative orientation of human societies are taken into account, we have a major clue to the kind of psychologieal transformation that must have occurred in hominoid evolution which made this level of adaptation possible and some measure of its depth and significance for an understanding of the dynamics of human social systems of social action." Culture so conceived as normative orientation in which individuals play sanctioned roles provides the structure which allows individuals to reap the maximum rewards (the theta, $\theta$, of Section XIII, A) from participation in the social system. 
If culture really does permit individuals to function in the context of a larger social group as if they were still only in the basic $N_{b}=12$ group compatible with their physiology, then any disruption in the culture should reduce the group size since its unstable state would then no longer buffer the individuals from the excessive contacts with their associates. I am assuming that, depending upon the extent of the cultural disturbance, physiological disturbances comparable to those of my rats caught in the behavioral sink (Section XII, C) would arise.

In fact, Birdsell (1953) demonstrates that such a phenomenon has characterized Australian aborigines in recent times. Tribes which have recently adopted the rites of circumcision or subincision generally have a size less than one-third that of tribes which have either not been exposed to these practices or adopted them long ago. Furthermore, the historical records indicate that tribes once reduced in numbers after they first adopted these rites now after several generations have recovered their typical numbers.

These data on the Australian aborigines further suggest that an individual can shift his participation from one level of social organization to another, provided there are cultural means for channeling such participation. Duff and Kew (1957) provide an account of the recently extinct Kunghit Haida Indians of British Columbia, which enables similar insights into basic group sizes in a food-gathering people.

Their winter village consisted of 16 to 20 large houses (1600 sq. ft. of floor space each). From various of the accounts it appears that the tribe totaled about 500 individuals, of which slightly over 200 were adults. This means about 10-12 adults on the average per house. Each house was inhabited by a kinship group or lineage. During the warmer months of the year each lineage group left the winter village for its own hunting territory. Like the Australian aborigines, these British Columbia Indians also appear to have a basic group size not diverging far from 12 and an assembly of these into a tribe of around 200 adults.

Incipient agriculture, in which plow and draft animals are absent, represents an even more advanced efficiency of food extraction, characterized by a permanent village. The Jarmo site in Iraq, inhabited some 6700 years ago, presumably represents a typical village at this level (Braidwood and Reed, 1957). Braidwood and Reed estimate that 150 persons (50 adults) inhabited the 25 houses located there. This type of village structure extends into the present. The mean size of 185 villages in this part of Iraq is 140 , which presumably represents $46-56$ adults.

From the scanty examination of lower-order basic group sizes in man we shall skip to the urban society of a modern nation, the United States. The social organization represented by Australian aborigines and the 
Kunghit Haida suggests that each larger semiclosed social system includes within it all the culturally limited basic group numbers. But even if some are skipped or unrecognizable, the one group structure which must be preserved is that of 12 adults. Recent studies by Zimmerman and Broderick (1954) and Zimmerman and Cervantes (1960) confirm this suspicion. Their approach has been to focus on any given family, designated the ego family, and then to determine with how many other families its members have frequent and close associations. These latter are designated as friend families. Absence of divorce or desertion, juvenile arrest, or children not completing high school comprised criteria for judging a family as "good" or "successful." Presence of these traits were used to delimit the "bad" or "unsuccessful" families. Values held by a family were judged on the basis of their religion, region of origin, income level, and kinship bonds. The good ego families typically have five friend families with whom they have a high coincidence of values, and furthermore, if the ego family is characterized as good most of the friend families are likely also to be so characterized. On the other hand, bad ego families generally have fewer friend families and they are likely to differ from them with respeet to the value traits. The fewer the values shared by the several families forming such a cluster, the smaller the eluster will be and the greater the probability that each family will be characterized by one or more of the traits denoting it as an unsuccessful family.

The ideal state then appears to be six families, 12 adults, composed of an ego family and five friend families. Shared values bind such a eluster despite the dispersal of the member families through the local community. Furthermore, each friend family in a particular cluster is, as an ego family, the center of another cluster. In this way an extension of the cluster develops to include 26 total families. Although similar bonds between families may include a larger network, insofar as any particular family is concerned the 25 friend families and extended friend families form the limit of dependence and social support relationships. This approximation of 50 adults of the family-friend cluster further argues for the reality of $N_{b}=50$ as a basic grouping revealed also in Birdsell's supra-horde of Australian aborigines and of the incipient agrieultural village of the Jarmo type. Reduction of the size of the family cluster below the optimum of six when values held by member families diverge from each other represents another example of the principle of group fragmentation, enunciated by Birdsell, which follows a clash in values. Zimmerman and Cervantes refer to this conflict as a "confusion of values."

All the information in this section, when viewed as a whole and in the context of the earlier sections concerning the evolution of a basic group size, suggests the following tentative generalization: Modern man derived 
from his primate and preprimate ancestors a physiology transpiring in groups within the range of 10-20 adults. This physiology was fixed some half a million years ago and has not significantly diverged from it since. Development of a larger social group is made possible by a culture in which a normative orientation prescribes values, and sanctions roles of behavior such that the total effect of participation in a larger group so buffers the individual that at any particular time the individual functions socially as if he were a member of a group of 12 individuals. Furthermore, genetic changes of the central nervous system making learned value systems of cultures possible must have arisen under circumstances which prevented division of the basic group size when it reached twice this level. Either an ecological-psychological trap like the behavioral sink (Section XII, C) or any isolated but very abundant source of a needed resource would be adequate to demand either a genetic change of physiology making life in large groups tolerable, or a genetic change endowing the central nervous system with the capacity to learn and culturally transmit values. Each increase in group size is associated with a reorientation of the value system. Such increases in group size are saltatory. The theoretical basis of why such changes must be saltatory and not transitional is discussed in Section XIII, B, 3.

\section{A Formulation of Group Dynamics}

Twelve individuals represent the approximate optimum group size for certain species (Sections VIII, C and XII, E). Furthermore, such a sized group might be expected to have evolved from home range dynamics. Evidence could readily be assembled that other basic $N$ 's, $N_{b}$ 's, characterize other mammalian species. Some typically live as pair's while others assemble in herds excecding 100 or 1000 individuals. However, circumstances may force $N$ to diverge markedly from $N_{b}$. Elaboration here of the model of social interaction presented in pages 349-354 of Calhoun (1957) provides insight into the consequences of such divergence of $N$ from $N_{b}$.

\section{A. The Model of Social Interaction}

On a presumptive basis there are three variables which should determine the mechanics of contact and interaction. These are (a) the number of animals moving about and having opportunity of contacting each other, (b) the length of the refractory period following the response of one animal upon contacting another until it is again capable of exhibiting a similar 
response, and (c) the amount of space in which the movement of $N$ individuals takes place. Random distribution of positions of individuals at any moment in time is assumed. We choose to ignore a small correction factor arising from the fact that all individuals move. Velocities of all individuals are initially assumed to be a constant. Furthermore, we assume that all individuals are identical. Thus our concern is not which individuals meet, but rather which state, responsive or refractory, the contacting individuals happen to be in.

$N=$ Number of animals forming the group.

$d=$ The diameter of interaction for each animal, that is, that distance between the centers of two individuals at which a physical or psychological collision or contact occurs. In the simplest case animals may be considered equivalent to billiard balls. Then $d$ represents the diameter of the ball, the individual. See Section XIII A, 1 for further elaborations.

Assume an animal moving in some direction on the plane in a population, $N-1$, of other animals.

Each of these other individuals presents a target of dimension $d$, normal to the $x$ direction. The expectation that the incoming animal will make a collision while moving a distance $\Delta x$ (in time $t$ ) is the ratio,

$$
\frac{d(N-1) \Delta x}{A}
$$

of surface covered by the targets to the total surface, where $A$ is the area available to the animals.

It should be emphasized that the unit of time must be sufficiently large so that the number of collisions in that time interval is large enough to justify using the statistical law of large numbers in the derivation. For similar reasons, it must be assumed that the mean free path of the individuals must be large in comparison with the target diameter.

Since the velocity $r$ may be considered equal to $\Delta x / t$, the arerage number of contacts $n_{c}$, per individual in time $t$ is

$$
n_{c}=\frac{d(N-1) v t}{A}
$$

For present purposes we are concerned only with the average $n_{c}$ in $t$ and not in the variability in contacts in $t$. The frequency of contacts by a given individual will be:

$$
f_{c}=\frac{n_{c}}{t}=\frac{d(N-1) v}{A}
$$


Since $d, v$, and $A$ will be considered constants for this presentation, we set

$$
(d v / A)=\mu \quad \text { so that } \quad f_{c}=\mu(N-1)
$$

$\mu$ reflects the ease of communication in the sense of contacts per unit of time. Basically, $d, v$ and $A$ may be specified in terms of linear unit, $L$. Therefore,

$$
\mu=\frac{d v}{A} \approx \frac{L \cdot L t^{-1}}{L^{2}}=\frac{1}{t}
$$

The symbol $\approx$ is here used in the sense of "dimensionally equivalent to." So by selecting appropriate units of time, $\mu$ can be made equal to 1.0. In following discussions $\mu$ will be considered equal to 1.0 in this sense whenever the basic $N, N_{b}$, of a species is in an evolutionarily steady state.

We will assume that the population of individuals can be divided into two classes: $\left[\mathrm{N}_{a}\right]$, those individuals who are in a responsive state, and $\left[\mathrm{N}_{p}\right]$ those individuals who are in a refractory state. We further assume that the individuals in $\left[N_{a}\right]$ will be rendered refractory either after a contact with a member of the same class or with a member of $\left[\mathrm{N}_{p}\right]$. After any such contact, an individual will remain in the refractory state for a length of time, $\alpha$, the refractory period, and after this time has elapsed return to membership in $\left[\mathrm{N}_{a}\right]$. It is also assumed that any contact that an individual undergoes while it is in the refractory state has no influence on the duration of its refractory period.

The duration of such refractory periods must be a function of the behavior of each nember of the contacting pair toward the other. It is assumed that the critical aspect of this behavior is its intensity. At the steady state of an $N_{b}$ we shall first consider every individual to be identical with reference to the intensity of its behavior toward others.

Let:

$i_{a}$ be the intensity of action of any nember of $\left[N_{a}\right]$ toward every associate it encounter's.

$i_{p}$ be the intensity of action of any member of $\left[\mathrm{N}_{p}\right]$ toward every associate it encounters.

It is further assumed that the most likely way that the duration of the refractory period, $\alpha$, becomes a function of the behavior of two individuals toward each other is that it results from the product of the intensities of their behaviors. Furthermore, there must be some factor, which will be called $B$, which governs whether a refractory period will result from the interaction. 
Let :

$B_{a}=1.0$ be the value of this factor in all members of $\left[\mathrm{N}_{a}\right]$.

$B_{p}=0$ be the value of this factor in all members of $\left[N_{p}\right]$.

$\alpha_{a a}$ represent the refractory period resulting in each of two members of $\left[N_{a}\right]$ who meet.

$\alpha_{a p}$ represent the refractory period resulting in each member of $\left[N_{a}\right]$ which encounters a member of $\left[N_{p}\right]$.

$\alpha_{p a}$ represent the refractory period resulting in each member of $\left[N_{p}\right]$ which encounters a member of $\left[N_{a}\right]$.

$\alpha_{p p}$ represent the refractory period resulting in each of two members of $\left[N_{p}\right]$ which meet.

It follows that:

$$
i_{a} i_{a}=\alpha_{a a} B_{a}=i_{a} i_{p}=\alpha_{a p} B_{a}, \quad \text { and all are real values }
$$

and that:

$$
i_{p} i_{a}=\alpha_{p a} B_{p}=i_{p} i_{p}=\alpha_{p p} B_{p}=0
$$

In all following discussion $B_{a}$ and $B_{p}$ will be omitted in discussing $\alpha$, but every mention of $\alpha_{a a}$ and $\alpha_{a p}$ will assume the action of $B_{a}$, and likewise any mention of $\alpha_{p a}$ and $\alpha_{p p}$ will assume the action of $B_{p}$.

It may be objected that no distinction is being made between the two kinds of contacts, responsive-responsive, and responsive-refractory. It is perfectly feasible to introduce two refractory periods, $\alpha_{a c}$ and $\alpha_{a p}$, of different duration to answer this objection. At the present juncture the experimental data are so scanty that it does not appear to be fruitful to introduce additional complexity, and we have chosen to consider $\alpha_{a a}=\alpha_{a p}$ for the working model insofar as duration is concerned.

An alternative model would have been to choose $\alpha_{a p}=0$, that is, the only contact inducing a refractory period being a contact between two individuals both of whom are responsive. Under such an assumption the number of contacts between responsive individuals in a unit interval of time would increase asymptotically to the value $1 / \alpha$, whereas, as we shall show, the model adopted provides that the number of contacts between responsive individuals passes through a maximum as $N$ increases.

Given sufficient proximity of an individual in the responsive state to some other individuals requisite to the usual elicitation of an interaction or response to denote a contact, evidence from certain mammals suggests a mechanism capable of blocking a social response. Such a mechanism 
which defines the probability of a contact being socially "perceived" shall be called $\mu^{\prime}$.

$$
\begin{aligned}
n_{a}= & \text { number of contacts made while the given animal is in the "re- } \\
& \text { sponsive" state. } \\
f_{a}= & \left(n_{a} / t\right) \text { is the frequency of responsive contacts when the animal in } \\
& \text { question is in the "responsive" state over all time. }
\end{aligned}
$$

Since each contact between two individuals, at least one of whom is in the responsive state, is followed by a refractory period $\alpha$, characteristic of each of the responsive individuals, and since there are $n_{a}$ such contacts in time $t$, then the individual is in the refractory state for a total time $\alpha n_{a}$. Clearly, the total time $t_{a}$ in which the individual is in a responsive state is $t-\alpha \mu^{\prime} n_{a}$.

Since $n_{a}=t f_{a}$ then

$$
t_{a}=t-\alpha \mu^{\prime} n_{a}=t-\alpha \mu^{\prime} t f_{a}=t\left(1-\alpha \mu^{\prime} f_{a}\right)
$$

In this sum of refractory intervals, $t_{a}$, contacts will be made at frequency $f_{c}$, but all such contacts are made while the animal is responsive so that

$$
n_{a}=f_{c t_{a}}=f_{c} t\left(1-\alpha \mu^{\prime} f_{a}\right)=t f_{a}
$$

or, since $f_{a}=n_{a} / t$

$$
f_{a}=f_{c}\left(1-\alpha \mu^{\prime} f_{a}\right)
$$

Thus

$$
f_{c}=\frac{f_{a}}{1-\alpha \mu^{\prime} f_{a}}
$$

It will also be helpful to rearrange Eq. (39) to obtain $f_{a}$ as a function of $f_{c}$ :

$$
f_{c}=f_{a}+\alpha \mu^{\prime} f_{a} f_{c}=f_{a}\left(1+\alpha \mu^{\prime} f_{c}\right)
$$

So

$$
f_{a}=\frac{f_{c}}{1+\alpha \mu^{\prime} f_{c}}
$$

We may also define the frequency of refractory contacts by each individual over all time

$$
f_{p}=f_{c}-f_{a}
$$

Contacts between individuals will be of three kinds: (a) both individuals responsive; (b) both refractory; and (c) one individual responsive and the other refractory. A given individual meets $f_{c}$ other individuals in unit 
time. Of these $f_{c}$ contacts, $f_{a}$ are with individuals in a responsive state. Hence, the probability $p_{a}$ that any given encounter will be with a responsive individual will be

$$
p_{a}=\left(f_{a} / f_{c}\right)
$$

Therefore, of all the encounters $f_{a}$ in unit time which the given individual makes while it is responsive, the number

$$
f_{a a}=p_{a} f_{a}
$$

will be with other responsive animals. Thus, $f_{a a}$ may be considered the (absolute) frequency of responsive-responsive encounters. Substituting (43) into (44) we obtain

$$
f_{a a}=\frac{f_{a}^{2}}{f_{c}}
$$

Using Eq. (41)

$$
f_{a a}=\frac{f_{c}^{2}}{f_{c}\left(1+\alpha \mu^{\prime} f_{c}\right)^{2}}=\frac{f_{c}}{\left(1+\alpha \mu^{\prime} f_{c}\right)^{2}}
$$

In like manner we can define $f_{p p}$ as the frequency of contacts of individuals both of whom are refractory and of $f_{a p}$ for the frequency of contacts in which one individual is responsive and the other refractory.

$\mathrm{By}$ an argument analogous to that given above we arrive at the formulation

$$
f_{p p}=\frac{f_{p}^{2}}{f_{c}}=\frac{\left(f_{c}-f_{a}\right)^{2}}{f_{c}}
$$

Using Eq. (41)

$$
\begin{aligned}
f_{p p} & =\left[f_{c}-\left(\frac{f_{c}}{1+\alpha \mu^{\prime} f_{c}}\right)\right]^{2} / f_{c}=f_{c}\left(\frac{1+\alpha \mu^{\prime} f_{c}-1}{1+\alpha \mu^{\prime} f_{c}}\right)^{2} \\
& =\frac{\alpha^{2} f_{c}{ }^{3}\left(\mu^{\prime}\right)^{2}}{\left(1+\alpha \mu^{\prime} f_{c}\right)^{2}}
\end{aligned}
$$

Again:

$$
f_{a p}=\frac{2 f_{a} f_{p}}{f_{c}}=\frac{2 f_{a}\left(f_{c}-f_{a}\right)}{f_{c}}
$$


And using Eq. (t1)

$$
\begin{aligned}
f_{a p} & =\frac{2 f_{c}}{1+\alpha \mu^{\prime} f_{c}}\left[f_{c}-\left(\frac{f_{C}}{1+\alpha \mu^{\prime} f_{c}}\right)\right] \cdot \frac{1}{f_{c}} \\
& =\frac{2}{1+\alpha \mu^{\prime} f_{c}}\left[\frac{f_{c}\left(1+\alpha \mu^{\prime} f_{c}-1\right)}{1+\alpha \mu^{\prime} f_{c}}\right] \\
& =\frac{2 \alpha f_{c}^{2} \mu^{\prime}}{\left(1+\alpha \mu^{\prime} f_{c}\right)^{2}}
\end{aligned}
$$

We can obtain an explicit relation between $f_{a p}$ and $N$ and $f_{a a}$ and $N$ by substituting Eq. (34) respectively into Eqs. (50) and (46) :

$$
f_{a p}=\frac{2 \alpha \mu^{\prime} \mu^{2}(N-1)^{2}}{\left[1+\alpha \mu \mu^{\prime}(N-1)\right]^{2}}
$$

And

$$
f_{a a}=\frac{\mu(N-1)}{\left[1+\alpha \mu \mu^{\prime}(N-1)\right]^{2}}
$$

The function $f_{a a}=0$ when $N=1$ and also $f_{a a}$ approaches 0 as $N$ tends to infinity. Since $f_{a a}$ is continuous and differentiable for all positive values of $N$, it has a maximum $N_{m}$ for some value of $f_{a a}$ at which the derivative of $f_{a a}$ with respect to $N$ is zero

$$
\frac{\mathrm{d} f_{a a}}{\mathrm{~d} N_{m}}=0=\frac{\mu\left[1+\alpha \mu \mu^{\prime}(N-1)\right]\left[1-\alpha \mu \mu^{\prime}(N-1)\right]}{\left[1+\alpha \mu \mu^{\prime}(N-1)\right]^{4}}
$$

and

$$
1-\alpha \mu \mu^{\prime}\left(N_{m}-1\right)=0
$$

Hence

$$
N_{m}-1=\frac{1}{\alpha \mu \mu^{\prime}} \quad \text { or } \quad \alpha \mu \mu^{\prime}=\frac{1}{N_{m}-1}
$$

Thus, the larger $\alpha$, the smaller $N_{m}$. In other words, the position of the maximum shifts to the left as $\alpha$ increases. In order to find the maximal value of $f_{a a}, f_{a a}^{(m)}$, we may substitute Eq. (55) into (52) obtaining

$$
f_{a a}^{(m)}=1 / 4 \alpha \mu^{\prime}
$$

In other words, the number of responsive-responsive contacts in unit time decreases as the refractory time increases. Since the refractory time, 
$\alpha$, is assumed to increase when the intensity of interaction, $i^{2}$, increases, $f_{a a}$ decreases as intensity of interaction increases.

When the $N$ of a species has attained an evolutionary steady state, designated as $N_{b}, \mu$ and $\mu^{\prime}$ will each have values of 1.0. When $\mu=1.0$ it will be designated $\mu_{b}$. Existence of an $N_{b}$ steady state does not mean that the temporal $N$ cannot fluctuate within the lifetime of a species or the history of a population. Rather, it means there is a particular $N$ compatible with $\mu_{b}$. At this $N_{b}$, with its $\mu_{b}$, all contacts are perceived. That is, $\mu^{\prime}=1.0$, and whenever $\mu^{\prime}=1.0$ it will be designated $\mu_{b}^{\prime}$. Not ouly will all contacts by responsive individuals be perceived, but each member of $N_{b}$ will interact with the same average intensity and, thus, $\alpha$ becomes $\alpha_{b}$. Obviously $N_{b}$ is the $N_{m}$ toward which a species "strives." In this "striving," which may be either maturational or evolutionary in terms of units of time, $N$ may vary as a function of $\alpha$, or $\alpha$ may vary as a function of $N$. At that $N$ it follows from Eqs. (55) and (56) that:

$$
\alpha_{b} f_{a a}^{(m)}=0.25
$$

This holds for all $N_{b}$.

$\alpha_{b} f_{a a}^{(m)}$ defines the maximum satisfaction from social interaction and will hereafter be referred to as $\theta_{b}$.

The usual intensity, $i$, of interaction, which determines $\alpha$, since $i^{2}=\alpha$, may be considered as basically under genetic control. Similarly, $\mu$ and $\mu^{\prime}$ may be considered to be normal expressions of genetic factors in so long as $N_{b}$ is approximately realized and the members of $N$ experience conditions in harmony with their genetic constitution, that is to say that the environmental conditions approximate those usually experienced by the species for many prior generations. However, abnormal environmental circumstances may so alter physiology and condition behavior that $i, \mu$, and $\mu^{\prime}$ diverge from the $i_{b}, \mu_{b}$, and $\mu_{b}^{\prime}$ appropriate to $N_{b}$. In these circumstances $\mu$ and $\mu^{\prime}$ no longer each equal 1.0, nor is $i_{b}$ in harmony with $N_{b}$ in the sense that $\alpha f_{a a}$ will lead to maximum satisfaction from social interaction. Yet, regardless of how $i, \mu$, and $\mu^{\prime}$ have diverged during maturation, this maximum may be attained if the species adjusts by attaining that $N$, different from $N_{b}$, such that:

$$
\alpha f_{a a}^{(m)}=0.25=\theta_{a}^{(o)}
$$

Theta, the maximal and also optimal satisfaction from social interaction, is here designated as $\theta_{a}^{(o)}$ or just $\theta_{a}$ to indicate its possible attainment at some other $N$ than $N_{b}$.

Interactions whose frequency has been designated by $f_{a p}$ require special consideration. 
For clarification: $f_{a p}=f_{a p}^{\prime}+f_{p a}^{\prime}$

And $f_{a p}^{\prime}=$ frequency with which a given responsive individual interacts with refractory individuals, while it is itself in the responsive state.

And $f_{p a}^{\prime}=$ frequency with which a given refractory individual interacts with responsive individuals, while it is itself in the refractory state.

It can be demonstrated that $f_{a p}^{\prime}=f_{p a}^{\prime}$.

Therefore

$$
f_{a p}^{\prime}=0.5 f_{a p}
$$

If we let $\alpha_{m}$ represent that $\alpha$ appropriate to $N_{m}$ and $f_{a a}^{(m)}$, then from Eq. (55)

$$
\alpha_{m}=\frac{1}{\mu_{m} \mu_{m}^{\prime}\left(N_{m}-1\right)}
$$

Similarly, for $N_{b}$ with $\mu$ and $\mu^{\prime}=1.0$

$$
\alpha_{b}=\frac{1}{N_{b}-1}
$$

If we assign $f_{a p}^{(m)}$ as the $f_{a p}$ characterizing $N_{m}$ when $f_{a a}^{(m)}$ represents the maximal value of $f_{a a}$ (e.g., see Eq. (56)), then by utilizing Eqs. (51), (52), (55), and (60) and considering the fact that $\mu^{\prime}=1.0$, it follows that

$$
f_{a p}^{(m)}=2 f_{a a}^{(m)}
$$

And from Eq. (59) it follows that, when $\mu$ and $\mu^{\prime}$ each equals 1.0,

$$
f_{a p}^{\prime(m)}=f_{a a}^{(m)}
$$

For clarification, it is to be noted that Eqs. (62) and (63) refer to the condition when $\mu$ and $\mu^{\prime}$ remain unchanged at the $\mu_{b}$ and $\mu_{b}^{\prime}$ values appropriate to $N_{b}$, but $\alpha$ adjusts to the existing $N$ according to Eq. (60) so that the existing $N$ becomes an $N^{(m)}$ differing from $N_{b}$ in most instances.

As already demonstrated

$$
\theta_{o}=\alpha f_{a a}^{(m)}
$$

where $\theta_{o}$ represents the maximal, and for this special case also the optimal, amount of time an individual can remain in that refractory state denoting satisfaction from social interaction. At $N_{m}$, where $\theta_{0}$ is realized $\alpha f_{a p}^{\prime(m)}$ 
amount of time is spent in frustrating refractory periods.

Here

$$
\theta_{f}^{(m)}=\alpha f_{a p}^{\prime(m)}
$$

And from Eqs. (63), (64), and (65) it is obvious that

$$
\theta_{f}^{(m)}=\theta_{o}^{(m)}
$$

Since $N_{b}$ is a special, and the most important, ease of $N_{m}$, Eq. (66) represents a significant consequence of evolution, as well as adjustment to current group size different from $N_{b}$. It means that when members of a group attempt to maximize satisfaction from social interaction, they will of necessity spend an equivalent amount of time experiencing frustration from social interaction. Evolution having transpired in such a system of social physics, physiology must be in harmony with this normal degree of frustration. Likewise, any marked decrease or increase of $f_{a p}^{\prime}$ from $f_{a p}^{\prime(m)}$ should prove stressful.

From Eqs. (51), (59), and (61), when $\mu \mu^{\prime}$ and $\alpha$ remain appropriate to $N_{b}$ but $N$ fluctuates, it follows that

$$
f_{a p}^{\prime}=\frac{\alpha_{b}(N-1)^{2}}{\left[1+\alpha_{b}(N-1)\right]^{2}}
$$

As $N$ approaches zero, $f_{a p}^{\prime}$ approaches zero. As $N$ approaches infinity, $f_{a p}^{\prime}$ approaches the $f_{c}$ characteristic of $N_{b}$, that is when $f_{c}=N_{b}-1=1 / \alpha_{b}$. Yet at the same time (see discussion following Eq. (52)) $f_{a a}$ approaches zero as $N$ approaches infinity with reference to its divergence from $N_{b}$. At $N$ 's much larger than $N_{b}$ the frequency of contacts resulting in refractory periods (i.e., $f_{a a}$ and $f_{a p}^{\prime}$ ) comes to approximate the total contacts transpiring in $N_{b}$; however, practically all of such contacts are of the type frustrating to individuals having returned to the responsive state.

\section{Terms and Equations}

The following assembly of definitions will facilitate understanding later discussions. Insofar as possible the $N$ animals in the group will serve as the basis of the definitions. Some terms utilized in later sections will also be included here.

$N=$ Total number of individuals in the group. In the strictest sense, a group is defined by habitation of an exclusive area in which each resident member has a good chance of contacting all others.

$d=$ Target diameter of an individual. In the simplest sense, $d$ specifies the actual physical diameter with the "animal" having no more $d$ properties than a billiard ball. Included under $d$ are 
any characteristics such as bright color, vocalizations, odor, or upright posture which enhance the likelihood of an individual being perceived by its associate. Through evolution and maturation certain species, particularly man, acquire the capacities to utilize nonphysical characteristics to alter target diameter. These nonphysical characteristics include attitudes and values whose possession influences the likelihood of the holder being perceived and responded to by his associates.

$v=$ "Velocity" with which an individual "moves" through its environment. It includes all properties which enhance the likelihood of one individual approaching its associates. Thus, in addition to including actual velocity, it includes all sensory mechanisms which extend the individual's perception of others in any direction along its travel path. Thus, where $r$ is the radius of perception beyond the physical bounds of the individual, $v$ becomes $r L t^{-1}$, see Eq. (35). Furthermore, $v=r L t^{-1}$ must actually become more complex than this. Animals further vary in the number of trips per unit time. See previous discussion in Sections III, A, 1 and $\mathrm{A}, 4$ which deal with how emotionality alters the frequency of trips. Therefore, if we let:

$v_{1}=r=$ radius of perception

$v_{2}=$ number of trips per unit time; or any time or place pattern of movement which alters probability of contacting others

$v_{3} \approx L t^{-1}=$ actual velocity

Then biological velocity, $v$, becomes:

$$
\begin{aligned}
v & =v_{1} v_{2} v_{3} \\
& =v_{1} v_{2} L t^{-1}
\end{aligned}
$$

Note: Here the product is used in the sense of a function of.

When $v$ is considered in later discussions it will have all these connotations.

$r=$ radius of perception as discussed above.

$A=$ area inhabited by the $N$ individuals, each of whom has a good opportunity of contacting any other member of $N$.

$\mu=(d v / A)$ is a communication-enhancing or contactproducing factor. By considering $v$ in its simplest sense, $\mu$ becomes $(d r v / A)$ as soon as the perception swath 
determined by the individual's rapacity to perceive beyond its own physical bounds comes into play.

$\mu^{\prime}=$ A communication-inhibiting or contact-blinding factor. It reflects a psychological property permitting the individual to ignore a contact resulting from $\mu . \mu^{\prime}$ must derive from $\left(d^{\prime} r^{\prime} v^{\prime} / A^{\prime}\right)$ factors. See Section XIII, B, 4 for further treatment of $\mu^{\prime}$.

$i=$ Intensity of action of one individual toward another upon contact.

$\alpha=i^{2}$, the duration of the refractory period following the contact of a responsive individual with some other individual. Contacts made by an individual while it is in a refractory state have no influence upon its $\alpha$. In some way the refractory period is a consequence of the intensity of interaction. It is thus the result of the interplay between the action of each individual toward the other. I have, therefore, assumed that the product of these intensities of action represents a first approximation of a proportionality to the duration of the refractory period.

$f_{a a}=$ The frequency with which one individual, while in the responsive state, meets other individuals, who are also in the responsive state.

$f_{a p}^{\prime}=$ The frequency with which an individual, while in the responsive state, meets nonresponsive ones (i.e., those in the $\alpha$ refractory state).

$\alpha_{a a}=$ Refractory period produced in each individual after each of the $f_{a a}$ interactions in which it is involved. $\alpha_{a a}$ produces satisfaction.

$\alpha_{a p}=$ Refractory period produced in the responsive individual after each $f_{a p}^{\prime}$ interaction. $\alpha_{a p}$ produces frustration at least in the sense of being a nonspecific stressor of physiology.

$\alpha_{a a}=\alpha_{a p}$ with regard to duration.

$\theta_{a}$ or

$\theta^{(a)}=\alpha f_{a a}$, the amount of time per unit time spent in satisfying refractory periods. $\theta_{\alpha}$ represents the consequences of positively affective interaction.

$\theta_{f}$ or

$\theta^{(f)}=\alpha f_{a p}^{\prime}$, the amount of time spent in the frustrating and physiologically stressful state.

$\theta_{f}$ approaches zero as $N$ approaches 1.0 
$\theta_{f}$ approaches 0.25 at $N_{b}$

$\theta_{f}$ approaches 1.0 as $N$ approaches infinity with reference to $N_{b}$.

$\theta_{f}$ represents the consequences of negatively affective interactions.

$\theta_{a}^{(m)}=\alpha_{m} f_{a a}^{(m)}=$ maximal $\theta_{a}=0.25$; see Eq. (57).

$N^{(m)}=$ That $N$ at which $\theta_{a}^{(m)}$ results. In other words, at $N^{m}$ satisfaction from social interaction is maximized, but at $N^{(m)}, \theta_{f}^{(m)}=\theta_{a}^{(m)}$, that is, there is as much frustration as satisfaction from social interaction.

$N_{b}=$ The basic group size of a species living under those conditions to which it is most adapted. $N_{b}$ is a special case of $N^{(m)} \cdot \theta_{a}^{(b)}, \theta_{f}^{(b)}, \alpha_{b}, i_{b}, \mu_{b}$, and $\mu_{b}^{\prime}$ represent values appropriate to $N_{b}$. Here $\theta_{a}^{(b)}$ and $\theta_{f}^{(b)}$ always $=0.25$, as may be seen from Eqs. (57) and (63). At $N_{b}$ both $\mu_{b}$ and $\mu_{b}^{\prime}$ must equal 1.0. $\theta_{a}^{(m)}, \theta_{f}^{(m)}$ represent values appropriate to $N^{(m)}$ in which $\theta_{a}^{(m)}=\theta_{a}^{(b)}$ and $\theta_{f}^{(m)}=$ $\theta_{f}^{(b)}$.

However, $\alpha^{(m)}, i^{(m)}, \mu^{(m)}$ and $\mu^{\prime(m)}$ at $N^{(m)}$ may all differ from comparable values appropriate to $N_{b}$.

$\theta_{a}^{(o)}=\theta_{a}^{(m)}$ for all $N$ other than $N_{b}$, although quantitatively $\theta_{a}^{(o)}, \theta_{a}^{(m)}$, and $\theta_{a}{ }^{(b)}$ all $=0.25$.

$N_{o}=$ Any $N$ when $\theta_{a}=\theta_{a}^{(o)} . N_{o}$ may equal $N_{b}$, but when it differs from $N_{b}$, some alteration in $\mu, \mu^{\prime}$, or $\alpha$ permits attainment of the optimum $\theta_{a}$, that is $\theta_{a}^{(o)}$.

\section{Interaction Functions Stated in Terms of $N$}

The number of individuals inhabiting an area is more readily measured than any other function relating to this model of social interaction. Therefore, it will be helpful to state all other functions in terms of $N$ :

$$
f_{c}=\mu(N-1)
$$

If intensity of interaction is labile to the point that $\theta_{a}^{(m)}$ ('an always be attained, then

$$
\alpha_{m}=\frac{1}{\mu_{m} \mu_{m}^{\prime}\left(N_{m}-1\right)}
$$

which means that:

$$
i_{m}=\left(\frac{1}{\mu_{m} \mu_{m}^{\prime}\left(N_{m}-1\right)}\right)^{1 / 2}
$$


A core aspect of this thesis is that whenever $\mu$ increases above $\mu_{b}$ there will be compensatory shifts in $\mu^{\prime}$ such that $\mu \mu^{\prime}$ will again equal 1.0. Therefore, $\alpha$ and $i$ will gradually become a function of $N_{m}$, or we might rather say that the members of the group attempt to adjust their intensities of interaction to make any existing $N$, regardless of how much it has diverged from $N_{b}$, become $N_{m}$. Thus, Eqs. (60) and (68) become:

$$
\begin{aligned}
\alpha_{m} & =\frac{1}{N-1} \\
i_{m} & =\left(\frac{1}{N-1}\right)^{1 / 2}
\end{aligned}
$$

At $N_{b}$ it follows from Eqs. (56) and (69) that the maximum frequeney of interaction of one responsive individual with other responsive ones becomes:

$$
f_{a a}^{(m)}=\frac{N_{b}-1}{4}
$$

Accepting the logic above that in time all individuals will attempt to adjust their intensity of interaction compatible with any existing $N$, it follows that:

$$
f_{a a}^{(m)}=\frac{N-1}{4}
$$

It further follows from Eqs. (52) and (69), where $\mu \mu^{\prime}$ tend to adjust to 1.0 and $i$ adjusts to maximize $\theta_{a}$ regardless of change in $N$, that:

$$
f_{a a}^{(m)}=\frac{\mu(N-1)}{[1+\alpha(N-1)]^{2}}=\frac{\mu(N-1)}{4}
$$

And similarly at $f_{a a}^{(m)}$, it follows from Eqs. (51), (59), and (69) that:

$$
f_{a p}^{\prime(m)}=\frac{\alpha \mu^{2}(N-1)^{2}}{[1+\alpha(N-1)]^{2}}=\frac{\mu^{2}(N-1)}{4}
$$

Equations (73) and (74) must be kept in mind while reading Section XIII, B. It has already been pointed out in the discussion following Eq. (66) that in the evolutionary steady state $\theta_{f}^{(m)}=\theta_{o}^{(m)}$. Here [see Eq. (63) $], f_{a a}^{(m)}=f_{a p}^{\prime(m)}$. This is a major premise of this paper, that animals "strive" to experience equal amounts of satisfaction and frustration from social interaction. But note what happens according to Eqs. (73) and (74) when $\mu$ varies. If $\mu$ increases and the members attempt to optimize 
satisfaction, to attain $\theta_{o}^{(m)}$, then

$$
\frac{\theta_{f}}{\theta_{o}^{(m)}}=\mu, \quad \text { or } \quad \theta_{f}=\mu \theta_{o}^{(m)} .
$$

Thus, excess frustration will increase proportional to the increase in $\mu$. For this reason, animals will always be conservative in that they will attempt to reject any changes leading to an increase in $\mu$.

Where $\mu=\mu_{b}=1.0$ and $N$ becomes $N_{m}$ [also refer to Eq. (2:5)]

$$
f_{a a}^{(m)}=f_{a p}^{\prime(m)}=\frac{N-1}{4}
$$

In some circumstances $\mu$ may be more labile than $i$ as an adjustive mechanism to changes in $N$ from $N_{b}$. Where intensity of interaction remains constant at that level appropriate to $N_{b}, \alpha$ remains $\alpha_{b}$. And yet E(1. (5.)) reveals that the $N$ differing from $N_{b}$ can become $N_{o}$ provided:

$$
N_{o}=1+\frac{1}{\alpha_{b} \mu_{o} \mu^{\prime}}
$$

Also by analogy to Eq. (69):

$$
\alpha_{b}=\frac{1}{N_{b}-1}
$$

Then substituting Eq. (77) into (76):

$$
N_{o}=1+\frac{N_{b}-1}{\mu_{o} \mu^{\prime}}
$$

Therefore:

$$
\mu_{o} \mu^{\prime}=\frac{N_{b}-1}{N_{o}-1}
$$

In the original change of $N_{o}$ from $N_{b}, \mu$ and $\mu^{\prime}$ were $\mu_{b}$ and $\mu_{b}^{\prime}$ and each was therefore equal to 1.0. However, we are here concerned with the case when $\mu$ is labile, that is, it can become different from $\mu_{b}$. Furthermore, $\mu_{b}^{\prime}$ can be ignored since any change in $\mu_{b}^{\prime}$ must await some stability in the change of $\mu$. Thus, when $\alpha$ remains at $\alpha_{b}$

$$
\mu_{o}=\frac{N_{b}-1}{N_{o}-1}
$$

Where intensity of interaction remains constant, and $N_{i}$ represents the 
value of $N$ at the inflection point of $f_{a a}^{\prime \prime}$, that is at the point where the second derivative of $f_{a a}$ as a function of $N$ is zero, it may be shown that

$$
N_{i}=1+\frac{2}{\alpha_{b} \mu_{o}}
$$

Then inserting Eq. (77) into Eq. (81)

$$
N_{i}=1+\frac{2\left(N_{b}-1\right)}{\mu_{o}}
$$

'Then inserting Eq. (80) into Eq. (82)

$$
N_{i}=1+2\left(N_{o}-1\right)
$$

Where $N$ is $N_{i}$, Eq. (83) becomes

$$
\begin{aligned}
N_{i} & =1+2\left(N_{b}-1\right) \\
& =2 N_{b}-1
\end{aligned}
$$

\section{B. Basic Processes Involved in Social Interaction}

\section{Satisfaction and Frustration as a Function of Group Size}

Satisfaction and frustration from social interaction are by definition measured, respectively, by $\theta_{a}$ and $\theta_{f}$. Full satiation attains at $\theta_{a}^{(m)}$ and optimum frustration at $\theta_{f}^{(m)}$. These equivalent quantities are equally necessary for the individual to persist in an optimum state. We shall here be concerned with the effects upon $\theta_{a}$ and $\theta_{f}$ resulting from varying $N$ when $\alpha_{b}, \mu_{b}$, and $\mu_{b}^{\prime}$ remain constant and appropriate to $N_{b}$. It must be recalled from the statement preceding Eq. (65) that $\theta_{f}^{(m)}$ is not used in the sense of the maximum $\alpha f_{a p}^{\prime}$ but rather as the amount of time spent in frustrating refractory periods at $N_{m}$, that $N$ where the maximum amount of time, $\theta_{a}^{(m)}$, is spent in satisfying refractory periods. As implied in Table XI, B and Fig. 35, $\alpha f_{a p}^{\prime}$ attains a maximal value when $N=$ infinity.

As $N_{b}$ increases $i$ and $\alpha$ must decrease in order to maintain $\theta_{a}^{(m)}$ [see Eqs. (56), (61), and (69-71)]. Likewise, for any arbitrary series of $\alpha$, such as $1.0,0.75,0.5,0.25,0.1,0.05,0.025$, and 0.01 , there must be respective $N_{b}$ at which $\alpha f_{a a}^{(m)}=\theta_{a}^{(b)}$. Each such $\alpha$ with its corresponding $N_{b}$ might be considered as representing a distinct species. For each species circumstances may cause $N$ to diverge from its $N_{b}$. In any such divergence $\theta_{a}$ diminishes, and for the species where $\alpha$ remains constant, $f_{a a}$ will exhibit changes proportional to $\theta_{a}$. Thus, $f_{a a}$ may be taken as an index of the degree to which changes in $N$ from $N_{b}$ diminish satisfaction from social interac- 
tion. Such reductions of $f_{a a}$ are shown in Fig. $34 ; f_{a a}^{(m)}, \mathrm{Eq} .(75)$, is that $f_{a a}$ denoted by the point where the dashed line intersects each solid line curve. Dropping vertically to the abscissa from each such intersection defines the $N_{b}$ for which that $\alpha$ is appropriate in the sense of optimizing satisfaction, $\theta_{a}^{(m)}$. In each case, regardless of the size of $N_{b}, \alpha f_{a a}^{(m)}=0.25$.

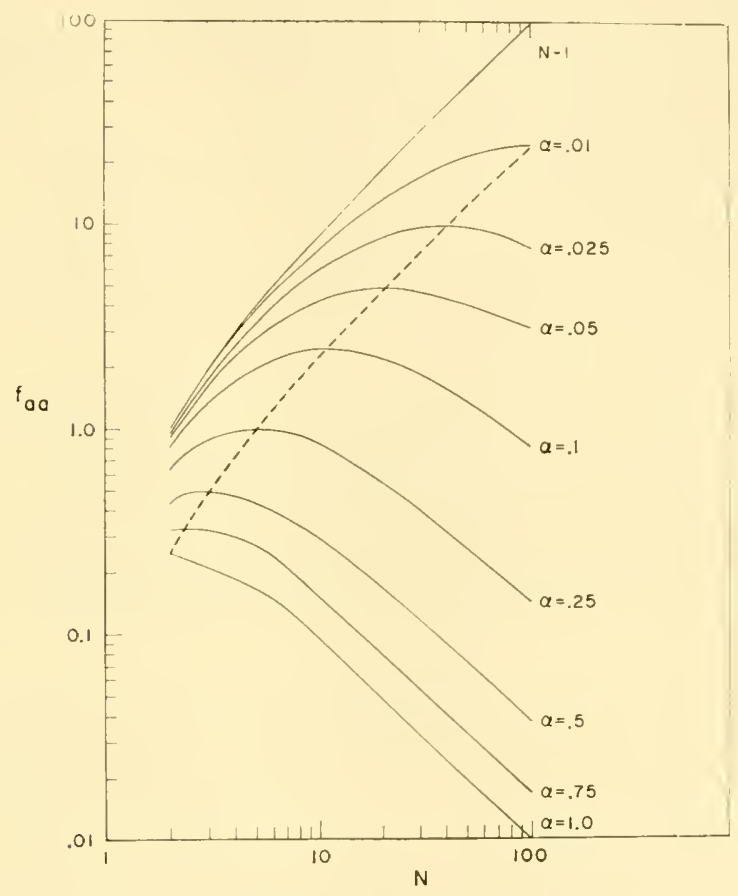

FIg. 34. Frequency of satisfactory social interactions $\left(f_{a a}\right)$ as a function of density $(N)$ and refractory period $(\alpha)$. The intersection of the dashed line and any solid line curve defines the basic group size, $N_{b}$, appropriate to that $\alpha$. All $\alpha f_{a a}$ defined by these intersections represent a constant, $\theta_{a}^{(b)}$, which defines the optimum amount of satiation which can be experienced by any member of an $N_{b}$. The values on the ordinate indicated by $N$ intersecting the dashed line represent $f_{a a}^{(m)}=(N-1) / 4$ when $\mu$ and $\mu^{\prime}$ each equals 1.0 or $\mu \mu^{\prime}=1.0$. For any given $f_{a a}^{(m)}, \alpha$ can be determined by finding the $N$ at which $f_{a a}$ intersects the dashed line. Here $\alpha=1 /(N-1)$.

Each $N_{b}$, so defined, represents a distinct species in the sense that there have arisen genetic alterations in $i$, such that there exists the highest probability of a steady state in which $\alpha f_{a a}$ optimizes satisfaction from social interaction.

Some of the insights revealed in Fig. 34 may be more explicitly comprehended by examining Table XI. In the left-hand part A of this table, suc- 
cessive doublings of $N_{b}$ are presented. For each $N_{b}$ those values for $f_{c}, i_{b}$, $\alpha_{b}$, and $f_{a a}^{(m)}$ are given which are requisite for each member to attain on the average an optimum satisfaction, $\theta_{o}=\alpha f_{a a}^{(m)}$, from social interaction. It is apparent that as $N_{b}$ increases, $\alpha_{b}$ and $i_{b}$ decrease, while $f_{c}$ and $f_{a a}^{(m)}$ increase. As the $N_{b}$ group size increases, each individual will have more $f_{c}$ contacts with associates, of which one-fourth will be satisfying (i.e., $f_{a a}^{(m)} / f_{c}=\frac{1}{4}$ ). For clarification, I might add that for each individual on the average at every $N_{b}$, another one-fourth of the contacts are of the frustrating $f_{a p}^{\prime}$ type, while the remaining one-half of the contacts transpire while in the refractory state which involve $f_{p a}^{\prime}$ or $f_{p p}$ contarts. Values for $N_{b}=12$ are shown in italics for reference because of the apparent importance of groups of this size. It may be seen that $i$ for $N_{b}=12$ is only one-third that for $N_{b}=2 . N_{b}$ must increase from 12 to 121 for a similar decrease in intensity of interaction to be necessary.

In the now voluminous literature on "stress," many papers deal with the physiologieal repereussions aceompanying change in group size. Yet these reveal little concerning how much physiological disturbance might be expected to result from a given change in group size. The prior model of social interaction will now be examined to determine what insight the model provides, under the assumption that it approximates reality.

TABLE XI

Normative Relative Values of Interaction Factors ${ }^{a}$

\begin{tabular}{|c|c|c|c|c|c|c|c|c|c|}
\hline \multirow[b]{2}{*}{$N$} & \multicolumn{4}{|c|}{ A. When $N=N_{b}$} & \multicolumn{5}{|c|}{ B. When $\alpha=0.091^{b}$} \\
\hline & $f_{c}$ & $\alpha$ & $i$ & $f_{a a}^{(m)}$ & $f_{a a}$ & $0.5 f_{a p}^{c}$ & $\theta_{a}$ & $" \theta_{d} "$ & $\theta_{f}$ \\
\hline 2 & 1 & 1.000 & 1.000 & 0.25 & 0.84 & $0.0 \mathrm{~s}$ & 0.07644 & 0.1736 & 0.007 \\
\hline 4 & 3 & 0.333 & 0.577 & 0.75 & 1.85 & 0.51 & 0.16835 & 0.0816 & 0.046 \\
\hline 8 & 7 & 0.143 & $0.37 \mathrm{~S}$ & 1.75 & 2.62 & 1.67 & $0.23 S 4$ & 0.0116 & 0.142 \\
\hline 12 & 11 & 0.091 & 0.302 & 2.75 & 2.75 & 2.75 & 0.250 & 0.00 & 0.25 \\
\hline 16 & 15 & 0.067 & 0.258 & 3.75 & 2.68 & 3.66 & 0.2439 & 0.0061 & 0.333 \\
\hline 32 & 31 & 0.032 & 0.180 & 7.75 & 2.12 & 5.99 & 0.1929 & 0.0571 & 0.545 \\
\hline 64 & 63 & 0.016 & 0.126 & 15.75 & 1.39 & 7.97 & 0.1265 & 0.1235 & 0.725 \\
\hline 128 & 127 & $0.00 \mathrm{~s}$ & 0.089 & 31.75 & 0.80 & 8.71 & 0.0728 & 0.1772 & 0.793 \\
\hline
\end{tabular}

a All values are relative to the intensity of interaction, 1.0, appropriate to a group of 2 individuals, when $\mu=1.0$.

${ }^{b}$ The $\alpha$ appropriate for $N_{b}=12$.

${ }^{c} 0.5 f_{a p}$ when $\alpha$ remains constant approaches $1 / \alpha$ as $N$ approaches infinity. In this (ase with $\alpha=0.091,1 / \alpha=11.0$. In other words $0.5 f_{a p}$ approaches $N_{b}-1.0 .5 f_{a p}$ is used in the sense of $f_{a p}^{\prime}$. 
Two deviations from the consequences of social interaction appropriate to $\mathrm{N}_{b}$ stand out as the logical candidates as physiological stressors. First there is the situation in which an existing $\theta_{a}$ is less than the optimum $\theta_{a}^{(m)}$ or $\theta_{0}$. This difference is designated as the satiation deficit, $\theta_{d}$, where

$$
\theta_{d}=\alpha_{b} f_{a a}^{(m)}-\alpha_{b} f_{a a}=\theta_{o}-\theta_{a}
$$

The second stressor is $\theta_{f}$. It presents a philosophical problem with regard to its assessment as a stressor. At $N_{b}, \theta_{f}=\theta_{0}$. The mechanies of interaction according to the model are such that optimizing (i.e., maximizing) satisfaction from social interaction leads to an equivalent amount of frustration. During evolution, physiology must have been altered such that it became compatible with this amount of frustration. $\theta_{f}$ may be thought of as a nonspecific stressor whose presence in $\theta_{f}^{(m)}$ amount, that is the amount which will arise when $N_{m}$ is also $N_{b}$, is necessary for stimulating physiology to an optimum level. When $\theta_{f}$ is below optimum, $\theta_{f}<\theta_{f}^{(m)}$, there will be a deficit in the nonspecific stressors required to maintain physiology at normal levels. Above optimum levels, when $\theta_{f}>\theta_{f}^{(m)}, \theta_{f}$ may be considered truly as a stressor to the extent that it exceeds $\theta_{f}^{(m)}$. As $N$ becomes greater than $N_{b}, \theta_{f}$ becomes greater than the optimum value of 0.25 , and as $N$ approaches infinity $\theta_{f}$ approaches 1.0. This means that nonspecific stressors in the sense of frustration from social interaction can never exceed four times the optimum level. For these reasons I choose to examine merely how $\theta_{f}$ varies as a function of $N$ rather than making any effort to evaluate any possible differential effect resulting from $\theta_{f}$ being greater or less than the optimum.

To see directly how these two stressors, $\theta_{d}$ and $\theta_{f}$, vary as $N$ changes, a specific case for $N_{b}=12$ is given in Part B of Table XI. Here again, as in Fig. 34, it may be seen that the frequency of satisfactory interactions, $f_{a a}$, declines following either decreases or increases in $N$ from $N_{b}$. However, frustrating interactions, $f_{a p}^{\prime}$ decline as $N$ declines below $N_{b}$, and likewise increase as $N$ increases above $N_{b}$. There results an approximately $70^{\%}$, deficit in satiation when $N$ declines to 2 or increases to about 122 from the $N_{b}=12$. Somewhat more marked changes from the optimum frustrations follow changes in $N$ from $N_{b}$.

In order that the change in $\theta_{f}$ as a function of the deviation of $N$ from $N_{b}$ may be visualized, they were calculated (Fig. 35) for $N_{b}=2$ and $N_{b}=12$, for which appropriate intensities of interaction are, respectively, 1.0 and 0.302 . Only increases in $\theta_{f}$ at $N$ 's above $N_{b}$ are shown. As $N_{b}$ increases from 2 to 12 , the respective intervening curves for $\theta_{f}$ shift to the right, that is, it takes slightly greater relative increases from $N_{b}$ to produce an equivalent increase in $\theta_{f}$. For all practical purposes the $\theta_{f}$ curves for all $N_{b}$ above 12 are identical with that of $N_{b}=12$. Initial increases in $N$ 
above $N_{b}$ produce the greatest increase in $\theta_{f}$. Later equivalent increases in $N$ produce less and less increments to $\theta_{f}$, frustration.

Satiation deficit, $\theta_{d}$, increases (Fig. 35 ) in a somewhat similar fashion as $\theta_{f}$, but it takes somewhat larger increments in $N$ to produce comparable increments in $\theta_{d}$. Whereas $\theta_{f}$ most likely represents a quantity of social nonspecific stressors, $\theta_{d}$ most likely reflects emotion of a kind which on the human level we call sadness, foreboding, apprehension, or home sickness. It represents the physiological consequences resulting from needed and perhaps known social interaction.

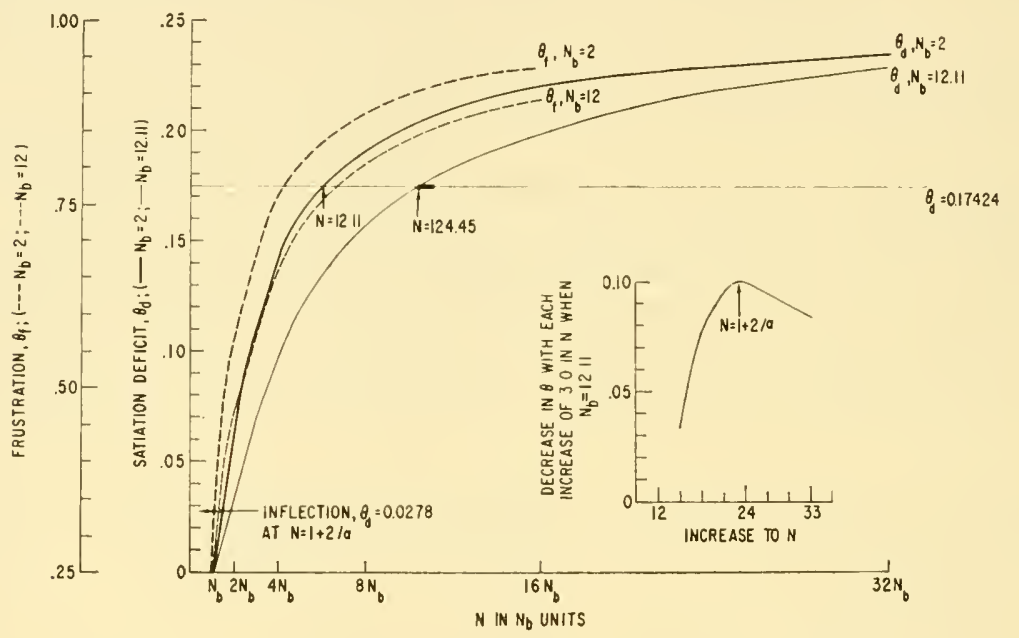

FIG. 35. Satiation deficit and frustration at greater than optimum group size. The inset figure defines the $N$ of maximum decrease in the satiation $\theta$, per unit increase of $N$.

A word of explanation is in order to reveal why I selected the nonfinite $N_{b}=12.11$ instead of 12.0 for examining $\theta_{d}$ in Fig. 35. In brief, I reasoned that there must be some $\theta_{d}$ not compatible with maintaining social life at that corresponding $N$. When this $N$ is reached there must be some genetic change transpiring which so reduces intensity of interaction that $\theta_{o}$ may be restored. On a rather arbitrary basis, I selected $\theta_{d}=0.174$, which is equivalent to a $70 \%$ decrease in $\theta_{a}$ from $\theta_{o}$. Reacquisition of $\theta_{o}$ at this $N$ requires $i$ to decrease to 0.3 of its former level. By such criteria, successively larger $N_{b}$, starting with $N_{b}=2$, will form a series of $2,12.11,124.45$, etc. Any such saltatorial series of $N_{b}$ 's may be designated $N_{b}^{(1)}, N_{b}^{(2)}, N_{b}^{(3)}, \ldots$., $N_{b}^{(n)}$. The $\theta_{d}$ curves for all $N_{b}$ of 12.11 and above will cross the 0.174 horizontal $\theta_{d}$ line within the small black rectangular area superimposed on 
this line in Fig. 35. Turther elaboration of such saltatorial series of $N_{b}$ is given in Section XIII, B, 3.

It must be kept in mind that a basic assumption underlying this general formulation of social interaction is that the mean free path of an individual must be large in comparison with the target diameter represented by any other individual. That is, animals must not be so crowded that one individual becomes so physically hemmed in or surrounded by a few others that opportunity to contact many of its associates becomes markedly reduced. When an experimental study violates this condition, what has been said in the above statements will not apply.

\section{The Budding Off of Social Groups}

As the group size increases beyond $N_{b}$ both the deficit in satiation, $\theta_{d}$, and the amount of frustration, $\theta_{f}$, increase in so long as intensity of interaction remains constant. Nembers of the group will find participation in it both less and less satisfactory and more and more stressful. The question arises, "At what point will members find conditions so unbearable that they will leave or at which the group will split?" Changes in $\theta_{a}$, or $\theta_{d}$, as a consequence of changes in $N$, are proportional to $f_{a \alpha}$. Therefore, we would like to know if there is some $N$ from which any given change in $N$ brings about a greater change in $f_{a a}$ than a similar change from any other $N$. The second derivative, $f_{a a}^{\prime \prime}=0$, occurs when: $N=1+2 /\left(\alpha_{b} \mu_{o}\right)$ [see Eq. (81) ]. This $N$ shall be referred to as $N_{i}$.

For $N_{b}=12.11$ this arithmetic inflection point comes at $N=23.22$. As may be seen from the inset graph in Fig. 35 this $N$ marks the point of maximal change in $\theta_{a}$ with a given change in $N$. At this point an increase in $N$ produces a greater decrease in $\theta_{a}$ than a similar change at any other $N$ greater than $N_{b}$. Furthermore, at $N_{i}, \theta_{d}$, as calculated by Eq. (86), for any $N$ will always be 0.0278 , which represents an $11 \%$ deficit in $\theta_{0}$.

For howler monkeys and man, where the basic $N$ appears to be about 12 adults, the social group size rarely exceeds $2 N_{b}$ unless, as in the case with man, the next well-defined larger group is much larger. It is for this reason that I suspect that $N$ will split or bud off another group when $N$ approaches $\left(2 N_{b}-1\right)$, the point of maximal rate of change in satiation deficit as given by Eq. (8.5). In essence, this line of reasoning says that by the time a group nearly doubles in size from its basic $N$, its members will begin to feel uncomfortable in the sense that they do not find participation in the group sufficiently satisfactory. This will lead to enough members leaving the former group so that within each of the two new groups interaction will produce near optimal results. 


\section{Saltatorial Changes in the Basic Group Sizf;}

Within most orders, and many lesser taxonomic categories, related species may be found between which there exist marked differences in the typical group size. Caribou and elk characteristically maintain large herds in contrast to the small groups or even isolated pattern of living by mule deer or moose. During the active breeding season, bats of the species Myotis lucifugus and $M$. yumanensis roost singly or in small clusters, whereas $M$. grisescens and $M$. velifer maintain large assemblies even during the breeding season. Woodchucks, Marmota monax, tend to live in isolation, whereas black-tailed prairie dogs, Cynomys ludoricianus, live in large colonies.

Obriously these represent a select group of comparisons. Although I shall not attempt to substantiate here the typical group sizes found within any fairly closely related series of species, examination of many series suggests that there are within each series several discrete basic group sizes with an extensive range between any two nearest sizes not represented by any species. For the present purpose, this conclusion will be accepted as approximating reality. Then the question follows: "What characteristics of physiology and group interactions might lead to saltatorial steps in group size which become fixed by natural selection or cultural evolution?"

In the first place, there must be some condition which induces animals to assemble in far greater group sizes than their $N_{b}$, and this condition must remain sufficiently strong to prevent splitting of the group as it approaches $2 N_{b}-1$ [see Eq. (8.5)]. Any spatially restricted but locally abundant resource might well so act, particularly if response at the source favored the establishment of a behavioral sink as described in Section XII, C. Increase in group size beyond $2 N_{b}-1$ would accentuate social discomfort and stress in the sense of increasing $\theta_{d}$ and $\theta_{f}$ (Fig. 3.j). At some point these factors must become so intense as to produce sufficient decrements in reproduction and survival to threaten the survival of the species. There is no a priori basis for judging what this threshold might be. Beyond $2 N_{b}-1$ each increment in $N$ produces a smaller increment of $\theta_{d}$ and $\theta_{f}$. Examination of the curves in Fig. 35 reveals that when $\theta_{d}$ is about 0.70 of its maximum and $\theta_{f}$ is slightly over trice its optimum level, any further increments to $N$ produce little further change in $\theta_{d}$ and $\theta_{f}$. The horizontal line through all curves defines this point on each curve and shall be considered empirically as a limit beyond which further increases in $N$ cannot be tolerated.

If $N_{b}^{(1)}=12$, the limit is $N_{b}^{(2)}=82$ for $\theta_{f}$ and $N_{b}^{(2)}=123$ for $\theta_{d}$. It will be recalled from Section XII, A that $N_{b}$ for the Norway rat appeared to be about 12. Although local colonies approximated this number, all members of all colonies were forced to interact at the single source of food and 
water. Furthermore, the entire population in the quarter-acre pen surrounded by a rat-proof fence made a closed system out of the entire population. At 123 adults (Table $\mathrm{X}$ ), marked disturbance was in evidence, with only a minority of the females reproducing successfully. In the closed systems contained within a smaller area, described in Section XII, C, severe reproductive disturbance eharacterized an $N$ of slightly less than 80 adults. Therefore, for the Norway rat at least, when the actual $N$ approaches the increase above $N_{b}$ presumed to represent the tolerance limit, a degree of physiological disturbance of sufficient magnitude arise as to indicate a necessity for some evolutionary adaptation to the increase in group size for continued survival. Parenthetically, I might add that this tolerance limit for $\theta_{d}$ and $\theta_{f}$ was arbitrarily assigned simply with reference to the slope of the eurve, and without prior knowledge that this level would lead to tolerance limit $N$ 's so closely approximating those observed in my experimental studies.

One type of evolutionary ehange which will reinstate $\theta$ to its optimum level, that will eliminate $\theta_{d}$, involves reducing intensity, $i$, of interaction. It will be recalled that $i^{2}=\alpha$, the refractory period following interaction. Now if $N_{b}=12$, and the tolerance limit $N$ is 12:3, a reduction of $\alpha$ from 0.091 , the $\alpha$ appropriate to $N_{b}=12$, to 0.0082 , the $\alpha$ appropriate to $N_{b}=$ 123 , will retum $\theta$ to its optimum value of 0.25 . The change in $i$ is 0.3 of its level at the former $N_{b}$, that is, from $i=0.3$ to $i=0.09$.

With a hereditary change in behavior amounting to a reduction of $i$ to 0.3 its former level, $N_{b}$ changes from 12 to $\left.12:\right)$. These would then represent two species, the stem one having an $N_{b}^{(1)}=12$ and residing under those environmental conditions not necessitating an evolutionary change in physiology and behavior, and the derived one having an $N_{b}^{(2)}=123$ and residing in the presence of those environmental conditions forcing the maintenance of group size far above that of the stem species. If this process is repeated each time $\theta_{d}$ reaches a tolerance limit of approximately 0.607 of the minimum $\theta_{d}$ possible, there arises what might be termed a "satiation deficit saltatorial series of basie $N$ 's" which are as follows, starting with $N_{b}^{(1)}=12$.

$\begin{array}{lr}N_{b}^{(1)} & 12 \\ N_{b}^{(2)} & 1223 \\ N_{b}^{(3)} & 1,359 \\ N_{b}^{(4)} & 15,088 \\ N_{b}^{(5)} & 167,645 \\ N_{b}^{(6)} & 1,862,544 \\ N_{b}^{(7)} & 20,695,365 \\ N_{b}^{(8)} & 229,937,917\end{array}$


Each successive $N_{b}$ will have an intensity of interaction approximating 0.3 that of the preceding. At the 8 th and last listed $N_{b}$, intensity of interaction would be only 0.0002 that when $N_{b}=12$. It seems rather patent that no meaningful behavior could transpire with such a reduced intensity (duration) of interaction. Two-hundred thirty million adults in a semiclosed social system can only apply to the world as a whole for the human species. Reduction of intensity of activity as a means of recovering satiation from social interaction could, in evolutionary terms, likely suffice in mammals to the third stage of 15,000 adults which entails a reduction of $i$ to 0.09 of that appropriate to $N_{b}=12$.

A similar series of $N_{b}$ can be calculated with reference to $\theta_{f}=0.775$ or the tolerance limit involved in shifting $N_{b}$ from 12 to 82 . Optimum $\theta_{f}=$ 0.25 can be regained if at this limit $i$ is reduced to about 0.36 of its intensity at the former $N_{b}$. Such a "frustration saltatorial $N_{b}$ series" becomes:

$\begin{array}{lr}N_{b}^{(1)} & 12 \\ N_{b}^{(2)} & 82 \\ N_{b}^{(3)} & 597 \\ N_{b}^{(4)} & 4,491 \\ N_{b}^{(5)} & 32,343 \\ N_{b}^{(6)} & 238,153 \\ N_{b}^{(7)} & 1,753,772 \\ N_{b}^{(8)} & 12,914,892\end{array}$

Again this series becomes rather absurd at the upper limit because of the great demand for reducing intensity of interaction. Since semiclosed systems, at least on the human level, and occasionally with other mammals do approach some of these $N_{b}$, we must ask what other avenues of evolution exist.

For this we must assume that intensity of interaction remains constant at some level approximating that for $N_{b}=12$, but that a tolerance limit for $\theta_{d}$ and $\theta_{f}$ exists. At the $N$ of these limits a change in behavior may take place which insulates the individual by producing subaggregates in which, for all practical purposes, the individual at any particular time is a menber of a subgroup in which $N_{b}=12$, even though many other subgroups exist in the environs. The individual may be a member of several such groups but participates in only one at a time. Such changes in behavior can be considered to be of either genetic or cultural origin. In either case, so long as any tolerance limit for $\theta_{f}$ and/or $\theta_{d}$ exists, there must be saltatorial steps between successive $N_{b}$, and only a few such steps are possible even if the tolerance limit arises at a somewhat lower level than hypothesized above.

If later research supports this hypothesis it will have eonsiderable 
hearing on our understanding of the course of evolution involving change in group size. It will mean that gradual changes in heredity or culture will rarely have transpired. Rather, from the pool of gene variability accumulated in the species, there will be rapid shifts in gene frequencies of many genes, thus resulting in a new phenotype. In so long as environmental conditions facilitate maintenance of its $N_{b}$ by a species, its gene pool may become quite diverse through the accumulation of mutant genes. Then, once environmental circumstances force the species to maintain an elevated $N$ near its tolerance level for $\theta_{d}$ or $\theta_{f}$, an extreme selection pressure will arise for reducing the frequency of all genes except those which adapt the species to its new $N$. A genetically variable $N_{b}^{(1)}$ species will thus rapidly be transformed into a genetically rigid $N_{b}^{(2)}$ species.

On the cultural level such a process of saltatorial change in basic group size demands that the value system which dictates acceptable roles of action and communication be preserved even after the usual group size has far exceeded the $N_{b}$ appropriate for that value system. At the same time, under the pressure of increases in $\theta_{d}$ and $\theta_{f}$, small segments of the closed system will develop values divergent from the main group. At the tolerance limit of $\theta_{d}$ and $\theta_{f}$, when $N$ has so diverged from $N_{b}^{(1)}$, there will arise a marked and rapid shift to the prevalence of those newer values appropriate to $\theta_{o}^{(m)}$ and $\theta_{f}^{(m)}$ at $N_{b}^{(2)}$. Value frequencies and gene frequencies become isomorphic in these two avenues through which there can be a saltatorial evolution from one basic group size to another.

Basic group size for adults only in the primary steps of human cultural evolution seem to include the 10-16 range, 50, 200, and 2,000. This series resembles neither of the hypothetical saltatorial group size series except in its saltatorial character. The hypothetical series merely demonstrated the kind of changes following from stated assumptions. The exact series followed by any line of change depends upon the threshold tolerance limit for $\theta_{d}$ and $\theta_{f}$ as well as three factors ignored in our discussion up to the present. Discussion up to this point assumes $\mu=(d v / A)=1.0$, where $d$ represented the target diameter of other individuals, $A$ the area inhabited by the $N$ individuals, and $v$ the velocity of movement of individuals. In essence, $\mu$ represented the likelihood in time $t$ of one individual encountering another.

It can readily be shown from Eqs. (52) and (60) that $\theta_{a}$, the satiation from social interaction, i.e., $\alpha f_{a a}$, can remain constant regardless of changes in $\mu$. At least this is so if the physiology and behavior of the species is completely adjustive. From the general form of Eq. (60) where $\mu^{\prime}=1.0$, $\alpha=1 /[\mu(N-1)]$, it follows that each doubling of $\mu$, that is doubling the likelihood of one individual meeting another, necessitates a halving of $\alpha$, and thus reduces intensity of interaction from $(\alpha)^{1 / 2}$ to $(\alpha)^{1 / 2} / 2$. If we 
follow the prior assumption that each species has an optimum intensity of interaction, then each increase in $\mu$ will have an analogous effect to increasing group size. In other words, increasing $\mu$ above 1.0 will increase $\theta_{d}$ and $\theta_{f}$. When we are concerned with the effects of changes in $\mu$ but assume $\alpha$ remains static at the value appropriate to $\mu=1.0$, then $\alpha$ must be calculated from Eq. (60) with $\mu^{\prime}=1.0$ and $f_{a a}$ calculated with this $\alpha$ by using Eq. (52) above and some value of $\mu$ different from $\mu_{b}=1.0$.

For example, consider $N_{b}=12$. Then $\alpha_{b}=0.091$, and $\theta_{b}=0.25$ (see Table XI). If $N$ doubles and $\mu$ remains $1.0, \theta_{a}$ becomes 0.219 , but if $N$ remains constant at $N_{b}$ but $\mu$ doubles to $2.0, \theta$ becomes 0.19 . . Thus, a comparable increase in $\mu$ produces a greater deficit in satiation, $\theta_{d}$, from social interaction, than does a double of $N$.

Thus, saltatorial evolution of $N_{b}^{(1)}$ to $N_{b}^{(2)}$ may be neressitated either by an increase in $N$ or an increase in $\mu$. The rate of change in $N$ and $\mu$ may well offset the tolerance limit of $\theta_{d}$ or $\theta_{f}$ and thus affect the magnitude of the shift from $N_{b}^{(1)}$ to $N_{b}^{(2)}$. $d v$ essentially measures the rate of communication and $A$ the space within which this eommunication takes place. Thus, $\mu$ will increase if $A$ remains constant and $d v$ increases, or if $d v$ remains constant and $A$ decreases. If both the rate, that is means, of communication increases and the distance over which communication must take place decreases, $\mu$ will increase very rapidly. Detailed consideration of rommunication is given in the following section.

\section{The $\mu$ Communication Fuxction}

We have already seen that $\mu=(d v / A)$, as defined by the previous Eqs. (35) and (80), is a communication-enhancing or contact-producing factor. (See prior discussion under Terms and Equations, Section XIII, A, 1.) Other than for pointing out in the latter part of Section XIII, B, 3 that altering $\mu$ has much the same consequences as altering $N$, we have been content to consider consequences of variability in other functions when $\mu$ remains constant at that value $\mu_{b}=1.0$ appropriate to $N_{b}$.

I was led to examine the question of the consequences of varying $\mu$ as a result of the observation by Birdsell and by Zimmerman and Cervantes, cited in Section XII, E. They observed that where a conflict of values arises in a group there results a reduction in group size. Here, we are concerned with the speeial case where attitudes or values comprise a major aspect of the target diameter $d$. Each member of the group holds some $n$ number of values by which others recognize it as an appropriate object for interacting. When some particular value is shared by all members, it may be said to possess a unitary value in contributing to target diameter. In other words, under this circumstance all individuals possess the same 
target diameter, $d=1.0$. With reference to Eq. (35), a unity value for target diameter merely means that there has been genetic or cultural adaptation to the actual magnitude of $d$, such that $\mu=d v / A=1.0$. However, if an individual expresses a value shared by only a few of his associates, he will by this fact be much more likely to be perceived by his associates, and thus more likely to be reacted to by them. His target diameter will be increased. Furthermore, it is logical to assume that the larger an individual's target diameter, the greater will be the response evoked from associates. In so long as all other $d$ value characteristics remain identical among the members of the group, the one which does vary among members will assume the sole role of influencing target diameter. As a first approximation this response-evoking capacity, which I will call $S$, of a particular $d$ value can be taken as being inversely proportional to the probability $p$ of its being encountered among the members of the group of $N$ individuals.

Therefore:

$$
S=1 / p
$$

Where only one component of $d$ varies, and since those shared components of $d$ may be ignored, $d \approx S$. For the special case where all members have the same $d$ :

$$
\mu=(d v) / A=(S v) / A=1.0
$$

Furthermore, where area, $A$, remains unchanged at the value appropriate to $N_{b}$ it has the relative value of 1.0. Therefore:

$$
\begin{aligned}
S v & =1.0 \\
S & =1 / v
\end{aligned}
$$

And considering Eqs. (87) and (89)

$$
v \approx p
$$

Lastly:

$$
v=1 / s^{\prime}
$$

Variability of the target diameter $d$ among individuals means that $d$ comprises an assembly of traits, physical size, color, vocalizations, behavior, and attitudes or values. $d$ is the total complex. Components shared by all members will be referred to as $(d)$. Those remaining traits, through which an individual differs from its associates, represent a genetically and culturally determined phenotype to which the response evoked from associates is a function. By response I here refer solely to actions reflerting the choosing or rejecting of an associate. Such a response may be a function 
of the trait itself or may be a function of a recognizable degree of difference between one individual and its most similar associate. I believe that such degrees of difference form the primary basis for the maturation of social behavior and social organization within a group. Further treatment of this topic follows in Sections XIII, B, 5, a and b; XIV, A and B.

Such traits or degrees of difference comprise the units influencing social behavior. These units will here be called $d$-genes. As stated above they may be of either hereditary, or cultural origin. Any $d$-gene, $g^{(1)}$, may develop an allelic series of differing or "mutant" forms $g_{1}^{(1)} \cdots g_{n}^{(1)}$. When degrees of difference, and not the absolute amount or kind of difference, forms a $d$-gene there can only be two forms of a particular $d$-gene, $g_{A}^{(1)}$ and $g_{a}^{(1)}$, where $g_{(a)}^{(1)}$ represents a degree of difference from the ideal type, the ideal $d$, and $g_{A}^{(1)}$ represents the retention of the ideal traits for which $g_{a}^{(1)}$ represents the divergence. $d$-genes of the type $g_{A}^{(1)}$ will be called dominant $d$-genes, while those of the type $g_{a}^{(1)}$ will be called recessive $d$-genes. $d$ genes of the latter type are treated in detail in Sections XIII, B, 5, a and b. Without specifying the allelic nature of any $d$-gene it is obvious that the target diameter $d$ is a function of $(d), g^{(1)}, g^{(2)}, \cdots, g^{(n)}$.

Let

$S_{i}{ }^{A}$ represent the response-eroking capacity of any $i$ th individual with reference to the probability of its being chosen by associates as an object of affection. $S^{(A)}$ is related to Schaeffer's love-acceptance referred to in Section XIV, C.

$S_{i}{ }^{(a)}$ represent the response-eroking capacity of any $i$ th individual affecting the probability of his being rejected by associates. $S^{(a)}$ is related to Schaeffer's hostility-rejection referred to in Section XIV C.

$S^{(A)}$ is a function of both $(d)$ and the assembly of dominant $d$-genes, while $S^{(a)}$ is solely a function of recessive $d$-genes. The probability of encountering the common $(d)$ assembly of traits will be 1.0. Therefore, from Eq. (87) the positively affective stimulus-eroking capacity of this commonly held assembly of traits will contribute to the $S^{(A)}$ of an individual inversely proportional to the probability of its being encountered within the $N$ individuals forming the group. Thus where $p_{g}$ represents the probability of encountering a particular $d$-gene and $A, B, C, \cdots$, represents the dominant "allele" of $d$-genes (1), (2), (3), $\cdots$, and there are $N-1$ $d$-genes of the degrees of difference type, then:

$$
S_{i}^{(A)}=\frac{1}{p_{(d)}}+\frac{1}{p_{g}^{()}}+\frac{1}{p_{g \mathrm{~B}}^{(1)}}+\cdots+\frac{1}{p_{g(N-1)}^{(N-1)}}
$$

Similarly where a, b, c, $\cdots, N-1$ represent recessive $d$-genes of the 
degree of difference type:

$$
S_{i}^{(a)}=\frac{1}{p_{\theta_{\mathrm{a}}}^{(1)}}+\frac{1}{p_{g_{\mathrm{b}}}^{(2)}}+\cdots+\frac{1}{p_{g(N-1)}^{(N-1)}}
$$

Equation (92) applies strictly only for that single individual which has preserved all the dominant or ideal traits. For every other individual one or more terms in Eq. (92) will be missing, depending upon replacement of the dominant $d$-genes by recessive ones. Similarly Eq. (93) applies strictly only to that individual in which all traits, other than those commonly held $(d)$, have diverged from the ideal, that is in that individual in which all non- $(d) d$-genes are recessive. For all other individuals one or more terms in Eq. (93) will be missing.

Consider the case where all $d$-genes in a group had been identical up until a particular point in time, at which a particular $d$-gene, $g^{(1)}$, "mutated" to $g_{2}^{(1)}$ in half the members. In this mutation $g_{2}^{(1)}$ diverged sufficiently from $g_{1}^{(1)}$ to make quite distinct the derived from the original. Then the probability of each in the group will be only 0.5 and thus the responseevoking capacity of each will rise to a relative value of 2.0 . For the average individual $S$ will have increased from 1.0 to 2.0 .

The total $N_{b}$ members of a basic sized group will consist of $N_{1}$ type 1 individuals possessing $g_{1}^{(1)}$ and $N_{2}$ type 2 individuals possessing $g_{2}^{(1)}$. Thus $N_{b}=N_{1}+N_{2}$. Any individual will be considered as being able to encounter itself in the sense of being aware of its own characteristics.

Considering this premise it follows that the probability $p_{1}$ of any type 1 individual being met by associates becomes:

$$
p_{1}=N_{1} / N_{b}
$$

Similarly, the probability, $p_{2}$, of type 2 individuals being met by associates becomes:

$$
p_{2}=N_{2} / N_{b}
$$

From Eq. (87) it follows that response-evoking capacity, $S_{1}$, of any type one individual, and $S_{2}$ of any type two individual will be respectively:

$$
\begin{aligned}
& S_{1}=N_{b} / N_{1} \\
& S_{2}=N_{b} / N_{2}
\end{aligned}
$$

Therefore, the mean response-evoking rapacity, $\bar{S}$, of the $N_{b}$ individuals becomes:

$$
\bar{S}=\frac{N_{1} S_{1}+N_{2} S_{2}}{N_{1}+N_{2}}
$$


Substituting Eqs. (96) and (97) with (98)

$$
\bar{S}=2.0
$$

The $S$ of Eqs. (96) to (99) is essentially that of Eq. (93) in which $g_{1}^{(1)}$ and $g_{2}^{(1)}$ become, respectively, recessive $d$-genes, $g_{\mathrm{a}}^{(1)}$ and $g_{\mathrm{b}}^{(2)}$.

As may be seen from Eq. (98), whenever $N_{1}$ or $N_{2}$ is zero, that is, all members of $N_{b}$ have the same target diameter, the response-evoking capacity of each member of the group has a relative value of 1.0. However, Eq. (99) shows that as soon as $N_{b}$ becomes divided into subgroups $N_{1}$ and $N_{2}$, even though the divergent $N_{2}$ has only one member, the average response-evoking eapacity doubles. The probable consequence of this doubling depends upon the relative numbers of $N_{1}$ and $N_{2}$. Consider

\section{TABLE XII}

The Influence of Relative Size of Subgroups of $N_{b}=12$ on Response-Evoking Capacity

\begin{tabular}{rrrrr}
\hline$N_{1}$ & $N_{2}$ & $\bar{S}_{1}$ & $\bar{S}_{2}$ & $\bar{S}$ \\
\hline 12 & 0 & 1 & 0 & 1.0 \\
11 & 1 & 1.0909 & 12 & 2.0 \\
10 & 2 & 1.2 & 6 & 2.0 \\
9 & 3 & 1.3333 & 4 & 2.0 \\
$S$ & 4 & 1.5 & 3 & 2.0 \\
7 & 5 & 1.7143 & 2.4 & 2.0 \\
6 & 6 & 2.0 & 2.0 & 2.0 \\
\hline
\end{tabular}

$N_{b}=12$, then when $N_{1}$ and $N_{2}$ have the sizes given below, members of each will have $S_{1}$ and $S_{2}$ as shown in Table XII.

The $S$ of the members of larger subgroups can never exceed twice the optimum level, but the $S$ of the members of smaller subgroups has a maximum of $N$ times that where all members of $N_{b}$ have the same target diameter. To understand the consequences to an individual resulting from possession of a large $S$, we must inquire further as to its implication. In the first place, it may evoke more frequent responses from associates. If the group is essentially an $N_{b}$ one, such an individual will experience more contacts than otherwise would be anticipated. This will have the same deleterious consequences to him of being in too large a group. $S$ in this case may be thought of as increased target diameter, $d$. On the other hand, a heightened $S$ may evoke more intense reaction, $i$, from associates at time of contact. This will have the consequence of increasing the refractory periods, $\alpha$, and thus with frequency of contacts maintained harmonious 
with $N_{b}$, the thetas, both those of satiation and frustration, will be increased. In this situation the eritical point becomes the attitude of associates toward such individuals. If the attitude toward this individual possessing rarely encountered characteristics is one in which the desired characteristie is venerated or desired by the majority not possessing it, then the resulting intense interaction will have the consequence of more frequently resulting in an $\alpha_{a a}$ satiation type refractory period. However, it is much more likely that the strange, rare characteristic will elicit an aggressive or rejection type response leading to an $\alpha_{a p}$ frustrating type refractory period for the individual with the heightened $S$.

When the $N_{1}$ subgroup represents a majority, they can achieve a return of their $\mu$ and $\theta$ 's to more nearly normal levels by ejecting the aberrant $N_{2}$ individuals. However, the minority $N_{2}$ members of the $N_{1}+N_{2}$ group suffer most from heightened $\mu$ and $\theta$ 's. Therefore, their seeking escape from the group becomes a motivating force greater than that of ejection by their associates.

If neither $N_{1}$ or $N_{2}$ form a clear majority, the most likely result will be a splitting of the group in half, but with retention of both $N_{1}$ and $N_{2}$ type individuals in each smaller group. This consequence derives from the following:

When $S$ doubles, $\mu_{b}$ doubles, the new $\mu=2.0$.

The "effort" of the group will be to make the easiest adjustment which will make $\mu=2.0=\mu_{o}$. This route lies in reducing the $N$ which was an $N_{b}$ to an $N_{o}$. From Eq. (78) it follows that:

$$
N_{o}=1+\frac{N_{1}}{\underline{2}}+\frac{N_{2}}{2}-\frac{1}{2}
$$

The best approximation any group can make is to divide in half. Each half must contain nearly equal numbers of $N_{1}$ and $N_{2}$. If all $N_{1}$ formed a group spatially distinct from the $N_{2}$ members of the former $N_{b}$, the $\mu$ of every member in each group would automatically return to 1.0 since within each new and smaller group all members would have the same target diameter. Thus, with the $N$ of each new group being only $0.5 N_{b}$, every member would experience a marked reduction in satisfaction and frustration thetas below optimum since the frequency of contarts $f_{c}\left[E_{(1 .}\right.$ (34)] would automatically be reduced.

In discussing this concept of a few divergent individuals or even a single one doubling the $\mu$ of the entire group, one of my colleagues remarked that such an increase appears unreasonable. Three examples will suffice to demonstrate the reasonableness of this assumption. Barnett (195.5) main- 
tained established groups of Norway rats in large cages. Despite or because of the existing hierarchy characterizing the group, all rats exhibited relative amicable relations, one to another. All presumably had developed nearly identical target diameters. Introduction of a single alien rat immediately produced a state of turmoil within the group, particularly intense actions being directed toward the alien by all members of the established group. In such experiments the alien rat, the one with the markedly differing target diameter, frequently died within a day or two. This death came, not as a result of wounds received, but as a result of inability to accommodate physiologically to the intense action directed toward him. His $\theta_{f}$ exceeded a threshold compatible with survival.

Or we may take any one of the several incidents publicized by the press and television during 1960 and 1961 in which "Freedom Riders" engaged in action which challenged the established value systems of certain segments of the socially dominant whites in the Deep South of the United States. Such actions frequently evoke drastic reaction, including physical violence, from members of the established group. Furthermore, many cross currents of elevated intensity of interaction became generated within members of the established group as they considered appropriate adjustments to the threat to their held values and to their prior target diameter.

The point I have been making in both the theoretical formulation and in these examples is that a qualitative change in the target diameter of a portion of the group will produce both an increase in $\mu$ and an increase in intensity of interaction. Another pertinent example is one I have previously given (Calhoun, 1956, pp. 87-88). That example concerns the establishment of a new group, designated as "C57 Colony 1B," from two smaller groups of mice not previously having contact with each other. After provision of an access door through the wall previously separating the two groups, the males from the socially more integrated group invaded the living space of the other males. Not only did the former attack the latter, but the males from the more socially integrated group began a period of intense fighting among themselves such as had nerer previously been observed.

Admittedly, all three of these examples include introduction of aliens into an established group rather than the hypothesized divergence in target diameter of a portion of a single group. However, it is difficult to imagine a portion of a group altering their target diameter without temporary partial isolation from the remainder. So, in effect, the consequences of divergence of target diameters among members of a group will be the same regardless of how the group is assembled.

In actuality

$$
\mu=(S v / 1)
$$


However, in most instances, I shall continue to consider $\mu=(d v / A)$ as previously. But when so doing, it must be understood that $d$ is used in the sense of its $S$ response-evoking capacity.

When $S$ changes from 1.0 to 2.0 for the reasons relating to Eq. (99), $\mu$ will no longer be $\mu_{b}=(d v / A)=1.0$, but $\mu$ will then become $(2 d v / A)=$ 2.0. Inserting $\mu=2.0$ into Eq. (78), in which $\mu^{\prime}$ remains 1.0 , for the special case where $N_{b}=12$, then $N_{o}$ becomes 6.5 . This means that in the presence of conflicting values group size must be reduced for each individual to maintain its $\theta_{o}$, its optimum level of satiation from social interaction. Increase in $\mu$ follows increases in $d$ or $v$, or decrease in $A$. Regardless of the origin of the increase in $\mu$, reduction in group size should follow.

Such reduction in group size should not be instantaneous. Consider $N_{b}=12, \alpha_{b}=0.091$ and $\mu=2.0$ and the group has not yet fragmented. From Eq. (82) it is obvious that $N_{b}=N_{i}$, when $\mu_{o}=2.0$ and $\alpha=\alpha_{b}$, and that $\theta_{d}$, the deficit in satiation from social interaction, will be as great as if $\mu$ had remained unchanged at 1.0 and $N_{b}$ increased to $\left[1+2\left(N_{b}-1\right)\right]$. [Refer to Section XIII, B, 2.] This is a very interesting consequence for it means that when $\mu$ increases to $2.0, N_{b}=N_{i}$. Recall that $N_{i}$ is that $N$ at which an increment in $N$ brings about the greatest change in $\theta_{d}$. Since groups do resist division and since any increase in $\mu$ is likely to be gradual, the most likely time for fragmentation of the group is when $\mu$ becomes 2.0 and $N_{b}=N_{i}$. Then $N_{b}$ will divide into two groups approximating $N_{0}$ determined by Eq. (78). Roughly, this says that when the ease of communication doubles as a result of a doubling of the response-evoking capacity $S$, the group will approximately divide in half if it is to optimize satiation from social interaction.

This process of halving the basic group size each time the ease of communication becomes twice as efficient cannot continue long if $N_{b}=12$, because by the fourth doubling of $\mu$, sexual reproduction could no longer be tolerated. That is, $N_{o}$ would be less than two individuals. The practice of divorce by the human animal reflects this process. We now have another question raised: "What avenue of adaptation or adjustment is open if $N_{b}$ remains 12 and $i$ remains unchanged at $i_{b}$ ?"

Although $\mu$ theoretically may be defined in terms of attributes of $d, v$, and $A$ external to the organism, any solution to this question demands that $\mu$ must in effect be reduced back to 1.0 by some compensating mechanism.

This mechanism which alters the probability of a contact being socially perceived has been called $\mu^{\prime}$. In Eqs. (38) to (55) it was shown that $\mu \mu^{\prime}$ represents the appropriate interaction between these two factors. So far $\mu^{\prime}$ has been elaborated no further. $\mu \mu^{\prime}$ then becomes the communication constant, more explicitly stated as $(d v / A) \mu^{\prime}$. Since $\mu$ can vary as a result of any one of its contained factors, $d, v$, or $A$, fluctuating alone, one cannot 
eseape the conclusion that during evolution a separate compensating mechanism for each must have arisen. This means that there is a $d^{\prime}$, a $v^{\prime}$, and an $A^{\prime}$, and that $\mu^{\prime}=\left(d^{\prime} v^{\prime} / A^{\prime}\right)$. Furthermore, when $\mu \mu^{\prime}=1.0, \mu^{\prime}=$ $1 / \mu$. Having arrived at these insights, one is logieally lead to ask: "What do $d^{\prime}, v^{\prime}$, and $A^{\prime}$ most likely represent biologieally?" Although answering this question is not necessary for the general theoretieal formulations, an attempt to speeify their more likely nature may be helpful in searehing for their identification.

$d^{\prime}$ represents the degree to which the stimuli emanating from any configuration pass unselectively from the sense organs into the memory store of the central nervous system (CNS). Thus, an increase in $d^{\prime}$ means facilitation of passage of stimuli into the CNS, while a decrease in $d^{\prime}$ indicates impeding or preventing stimuli from getting to the CNS. When the target diameter of associates inereases through evolution by acquiring more $d$-genes, a compensating evolution of a $d^{\prime}$-mechanism will permit a discrimination among the $d$-genes such that in that brief span of time required for psychological contact only a portion of the $d$-genes of the other individual will be responded to. It must be kept clearly in mind that an inerease in the efficiency of the meehanism which serves to alter $d^{\prime}$, deereases $d^{\prime}$. Such a decrease in $d^{\prime}$ implies the evolution of a filtering deviee which reduces the amount of information about others per unit time arriving at the sense organs, which is permitted to pass from them into the integrative centers of the nervous system. Without speeifying what CNS structure serves the $d^{\prime}$ function, it meets the requirements hypothesized by Broadbent for his CNS "filter." See Section IX.

$v^{\prime}$ also represents a process internal to the individual. It cannot have any influence upon the motor eomponent of $v$. Therefore it must affect the consequences of those sensory capaeities which enable the individual to achieve a psychologieal eontact prior to an actual physical one. This is the $r$ component of velocity mentioned in Section XIII, A, 1. Just as an increase in $r$ increases $v$ by deereasing the time required for a contact, so must a decrease in $v^{\prime}$ function to increase the time from the moment of input of a signal from a $d$-gene at the sensory organ until this transformed signal reaches and evokes a response at an effector. This $v^{\prime}$ could represent either a struetural or biochemical alteration in the time required for an impulse to pass over a synapse, or it could be represented by an alteration in the number of neurons in the circuit which will also alter transmission time. But we must not eonfuse the magnitude of $v^{\prime}$ with the efficiency of the meehanism involved. An inerease in $v^{\prime}$ means a decrease in the effieiency of the mechanism, that is inereased synaptic transmission, while a decrease in $v^{\prime}$ follows from an increase in the efficiency of the meehanism in impeding the passage of the signal along the eireuit between the sense organ to the effectors. 
A word is required to differentiate clearly $d^{\prime}$ from $v^{\prime}$. $d^{\prime}$ governs the prohability of a signal relating to $d$-genes getting through the Broadbent type hypothesized "filter," while $v^{\prime}$ applies to the speed of transmission from the filter to effectors.

In a similar fashion, conceptualization of $\mathrm{L}^{\prime}$ must be in terms of counteracting $A$. Where an $N_{b}$ group is living under optimum conditions, $A$ may be considered to be equal to 1.0. Optimum conditions will continue even though $A$ changes in so long as $A A^{\prime}=1.0$. For the sake of simplicity we are considering the case where the only change in the system pertains to $A$. Recall that $A$ represents the area which the group shares. In essence then $A$ alters the time between eontacts. Therefore $A^{\prime}$ must operate in a similar but opposite direction to $A$. Suppose that $A$ increases. In effect this is equivalent to a decrease in density. Under such circumstances there ean be no internal mechanism enhancing the probability of an actual contact. Therefore, when $A$ increases, an $A^{\prime}$ compensating mechanism involves an imagined contact. To the extent that such an imagined contact leads to an equal $\alpha$ refractory period, an $A^{\prime}$ mechanism will be effective. Since an increase in $A$ implies a decrease in $A^{\prime}$, a deerease in $A^{\prime}$ means an increase in the capacity to store memories of associates, which can eompensate for their absence. Such an increased storage of memories implies an increase in cortical mass.

On the other hand, suppose that $A$ deereases. This will reduce the time elapsing between contacts, and since this will have the same consequence as increasing $N$ when we are concemed with an $N_{b}$ group, its members will experience an increased $\theta_{d}$ and an increase in $\theta_{f}$ above the optimum level. In this situation $A^{\prime}$ must function to increase the relative time between contacts. The only way for this to happen is for the intensity, $i$, of interaction to decrease. Reeall that intensity of interaction has been measured in terms of its duration, and that $i=(\alpha)^{\frac{1}{2}}$ when $\alpha$ represented the duration of the refractory period following interaction. Furthermore, Eq. (60) becomes:

$$
\alpha=\frac{1}{\mu \mu^{\prime}(N-1)}
$$

when we consider the several factors in the general sense where a variation in one may influence any other.

Then

$$
\alpha \mu^{\prime}=\frac{1}{\mu(N-1)}
$$

The reason for stating the equation in this fashion is that $\mu^{\prime}$ eannot alter $\mu$ as such but ean only alter its effects through ehanging something else. 
What Eq. (103) implies is that where $A^{\prime}$ is the factor which produces the variability in $\mu^{\prime}$, it can be effective in maintaining $\theta_{0}$ but not necessarily an optimum $\theta_{f}$ (see discussion following Eq. (7t)), provided it acts as a governor on the intensity of interaction independent from the influence upon $i$ exerted by $d$. A decrease in the $A$ component of $\mu$ indicates a decrease in the home range $\sigma$ (see Sections II-V). The significance of this line of reasoning is that this $A$ governor, which controls $i$, is likely to be identical to the one previously postulated for determining the duration of an outward trip from home. In the general sense, this governor controls the duration of behaviors. Social interaction merely represents one specific category of behaviors. Related to the above discussion, it may be noted that Eqs. (69) and (103) are equivalent since $\mu^{\prime}=1 / \mu$, so long as $\mu=1.0$ or $\mu>1.0$. As originally formulated in the discussion before Eq. (38), $\mu^{\prime}$ simply operated as a probability of an actual contact being perceived. However, the above and following discussion indicate that $\mu^{\prime}$ can exceed 1.0. By imagining contacts, which actually do not occur, through a decrease of the $A^{\prime}$ component of $\mu^{\prime}, \mu^{\prime}$ can exceed 1.0.

In terms of the model proposed in Section III, A, whenerer A decreases, the rate of firing of the neuronal net of the governor will increase. Thus, an increase in $A^{\prime}$ represents an increased rate of firing of the neuronal net of the governor.

Previously I pointed out that a decrease in $A^{\prime}$ to compensate for an increase in $A$ might be visualized as an hallucinatory process. There is no reason why $A^{\prime}$ cannot equally involve the governor of intensity of activity when $A$ increases above $A_{b}$ normally appropriate to $N_{b}$; a decrease in $A^{\prime}$ would represent a slowing of the firing of the neuronal net of the governor, which change would then permit a longer duration of interaction. This longer duration of interaction would compensate for the fewer $f_{a a}$ interactions possible in a larger $A$.

The concept of social perception of contacts subsumed under $\mu^{\prime}$ thus includes a wide variety of processes. It includes (1) selective acceptance of stimuli to be integrated in the central nervous system; (2) impedance or facilitation of passage of signals across neuronal synapses; (3) alteration in the length of a neuronal circuit; (4) hallucinating a contact; and (5) governing the intensity of interaction. These $\mu^{\prime}$ capacities for adjustment are most likely to be found farthest advanced in those species in which $d$, $v$, and $A$ fluctuate most markedly with reference to all members of a group within the life span of every individual. However, over long spans of time encompassing many generations, a gradual increase in $d$, $v$, and $A$ should increase the complexity of social life possible. At least this conclusion holds to the extent that enhanced synaptic transmission, increased duration and intensity of behavior, and increased discriminatory capacity foster more effective social behavior. 
The following summary of presumed relationships may serve as a basis for evaluating the consequences of change in the components of $\mu$ and $\mu^{\prime}$.

External Internal
change change

Increase in Decrease in

\begin{tabular}{|c|c|c|}
\hline$d$ & $d^{\prime}$ & $=$ Increase in discriminatory capacity \\
\hline$v$ & $v^{\prime}$ & $=$ Impeded synaptic transmission \\
\hline$A$ & $A^{\prime}$ & $\begin{array}{l}=\text { Increased duration and intensity of be- } \\
\text { haviors, or } \\
\text { Increased memory storage of social stimuli } \\
\text { permitting hallucinated interactions which } \\
\text { indicate increase in cortical mass }\end{array}$ \\
\hline
\end{tabular}

Decrease in Increase in

$d$

$d^{\prime} \quad=$ Decreased discriminatory capacity (i.e., less selection of stimuli passing the "filter")

$v \quad v^{\prime} \quad=$ Enhanced synaptic transmission

$A \quad A^{\prime} \quad=$ Decreased duration and intensity of behavior, or decreased memory storage of stimuli which are available for producing hallucinated social interactions which indicates decrease in cortical mass

These relationships represent intuitive logical deductions. $d^{\prime}$ and $A^{\prime}$ serve as fairly satisfactory first approximations. With progressive social evolution, $d$ and $A$ generally increase. Along with this trend, discriminatory capacity increases, cortical mass increases, and ability to maintain a behavior for longer periods of time increases, all of which tend to harmonize with the model. However, I am less satisfied with my formulation of $v^{\prime}$ (Section XIII, A, 1). The reason for this opinion is that with advancing social evolution there presumably arises an increase in $v_{1}$, that sensory extension of the self to produce psychological contact before bodily contact. This should produce the opposing phenomenon of impeded synaptic transmission. For the present, I can see no rational basis for reconciling impeded synaptic transmission with social advances.

$v^{\prime}$ merely connotes alteration of sensory perceptions of others involving some distance between the two individuals concerned. My supposition that alteration of synaptic transmission subserves $v^{\prime}$ may well be wrong. The important point for the present is simply recognition of the likelihood of some such mechanism whose evolution is influenced by social conditions. 
I have pointed out that since $\mu$ can change as a result of independent change in $d, v$, and $A$, consequently there must be diserete phenomena in the individual representing what I call $d^{\prime}, v^{\prime}$, and $A^{\prime}$, which can change independent of each other. Nevertheless, it is quite likely that the components of $\mu$ and $\mu^{\prime}$ do have interactions. The concept of intensity (duration), $i$, of social action includes control of $i$ by both internal and external factors. The internal factor is $A$ ', the "governor" previously discussed. The external factor is the $d$ of the other. The greater $d$, the greater $i$. Thus, $d$ can influence the governor. This means that an increase in the $d$ of associates can decrease the $A^{\prime}$ of self.

In studies with rats recently completed (Calhoun, 1962b) some rats develop a high $v$ while others develop a low $v$. Those with a very high $v$ exhibit high $i$ in terms of both intensity and duration. This suggests that in some way an increase in $v$ leads to a decrease in $A^{\prime}$. Furthermore, male rats with very low $v$ commonly respond as though they did not make adequate discrimination of the cues emanating from associates. They sexually mount associates without regard to their age, sex, or sexual receptivity. These observations suggest that lowering of the motor components of self's $v$ increases self's $d^{\prime}$, which suggests that somehow when an animal decreases its velocity its ability to discriminate among available social stimuli also becomes reduced. All I have attempted to do in the preceding paragraphs is to lay the groundwork for understanding the meaning of the contactmodifying factor $\mu^{\prime}$.

Decreases of $\mu$ re increase in $N$ : We are here concerned with the special case where the area, $A$, remains constant as numbers of individuals, $N$, increase. This means that density increases. We have already scen that an increase in $N$ with $\mu$ held constant leads to a deficit, $\theta_{d}$, in satisfaction from social interaction as well as an increase above optimum of the frustration, $\theta_{f}$, from such interaction. As density increases one should anticipate $\mu$ changing before $\mu^{\prime}$. Therefore we shall consider $\mu^{\prime}$ as remaining constant at the 1.0 value appropriate to $N_{b}$ but let $N$ increase. In each instance we wish $N$ to become $N_{o}$, which means that $\theta_{o}$ and $\theta_{f}^{(m)}$ will be optimum. Consider the case where $N_{b}=12$ and intensity of interaction remains at $i_{b}$, it may be seen from Eq. ( 80 ) that successive doublings of $N_{o}$ demands successively slightly more than halving of $\mu_{0}$ :

$\begin{array}{rl}N_{o} & \mu_{o} \\ 12 & 1.0 \\ 24 & 0.478 \\ 48 & 0.234 \\ 96 & 0.116 \\ 192 & 0.057 \\ 384 & 0.028\end{array}$


Where $\mu=\mu_{o}$ and $N=N_{o}$ it may be seen by substituting Eq. (80) into Eq. (34) that the frequency of contacts will remain constant at $\left(N_{b}-1\right)$ regardless of the increase in density. Since we are considering the special case where area, $A$, remains constant, the constancy in the absolute number of contacts despite inereases in density can only derive from decreases in the $d$ or the $v$ component of $\mu=d v / A$.

Where decreases in $\mu$ transpire within the lifetime of an individual as an adjustive ehange to increase in density, and where this decrease in $\mu$ results solely from a decrease in the target diameter, $d$, this change must be reflected through reductions in the frequency of usage of signaling mechanisms or of the intensity of such signals. Vocalizations should oecur less frequently, be less complex, and of reduced intensity. Bodily display characteristies under voluntary control should similarly be reduced, as should also the use of ehemical signals. Similarly, when a species, $A$, now exhibits an $N_{b}$ of $80-120$ or $800-1400$ (see Section XIII, B, 3) but there is reason to suspeet that at some earlier era it had had an $N_{b}$ of 2 or 12 , such as is still expressed by a related species (or genus), $B$, then a comparative examination of species of types $A$ and $B$ should reveal that members of species $B$ more frequently resort to the utilization of vocal, display, and chemical signals and that these are of greater complexity and intensity than in species $A$. In making any such comparisons it is well to keep in mind the earlier hypothesis that the change of an $N_{b}=2$ to an $N_{b}=12$ may lead to an increase in complexity of the signal. For this reason, it is more appropriate to compare a species whose $N_{b}$ has a typical range of 7-19 with one which rarely falls below 80 . Such comparisons will provide critical tests of the general formulation.

In like fashion, velocity, $v$, may be the factor which becomes reduced as density increases. This reduction may be expressed in any of the three aspects of $v$ listed in Section XIII, A, 1. Sense organs may become less effective with reference to the animals' perception. This reduces $v_{1}$. Such a tactic must be effective only through genetie changes and so can serve only as a long-term adaptive mechanism. The animal may also reduce its $v_{2}$ by way of reducing the number of trips it makes, or by altering the time and place of its activities. This strategy includes initiating activity while other's are resting, or by becoming cryptic in the sense of becoming arboreal or subterranean. Finally, the animal may reduce its $v_{3}$, its actual rate of movement.

Although such reductions in $d$ and $v$ will lead to a reduction in $\mu$ which will compensate for inerease in density, it is obvious that $\mu$ must decline so markedly as to present biological limits of effectiveness. Further accommodation to increases in density must require $\mu^{\prime}$ to decrease also. Reduction in this communication-inhibiting or contact-blinding factor means (a) decreased duration or intensity of behaviors or decreased memory 
storage (i.e., increase in $A^{\prime}$ ); (b) increase in discriminatory power in the sense of screening out portions of those stimuli of the $d$ of others requisite for eliciting responses (i.e., decreasing $d^{\prime}$ ) ; or (c) impeded synaptic transmission (i.e., decreasing $v^{\prime}$ ).

\section{Behavioral Origin of Response-Evoking Capacity, $S$}

a. The target diameter genotype as determined by varibility of behavioral traits. I now wish to present the logic of why variability of behavioral traits becomes inevitable. In fact, as aminals become more social, variability in physical traits must become of less importance in determining the kind and intensities of interaction. So let us start with the case where all indiriduals possess identical heredity and therefore identical physical characteristics. Even for so simple an organism as the house mouse, marked differences in capacities for social involvement develop despite the fact that the members of the group come from a stock made genetically homozygous by nearly a hundred generations of brother-to-sister inbreeding (Calhoun, 1956).

The initial formulation of social interaction dealt with a deterministic model in which all individuals were identical. It showed that half the time an individual was in the responsive state it would meet another responsive individual and half the time it would meet another in the refractory state. Thus, even under ideal conditions, an individual would be frustrated as frequently as it would be satisfied from social interaction. But satisfaction will not likely precisely alternate with frustration. Furthermore, if we consider some arbitrary relatively short span of time when the group first forms, determined by the average individual having, for example, a total pool of interactions equivalent to $2-5$ times the number of individuals in the group, then something like the following will have transpired:

Each individual's behavior toward another may be characterized by its form or pattern and by its timing with regard to whether the other individual involved in the interaction is also in the responsive state (the $a$ state) or whether it is in the opposite or nonresponsive state (the $p$ state). Initially the form of the behavior of all individuals in the responsive state will be identical. Identical form denotes possession of the entire assembly of traits, $d$, by every individual. With each individual contacting its associates in a random sequence over time, it is inevitable that some, who are in the responsive $a$ state, will purely by chance more frequently encounter others who happen to be in the nonresponsive $p$ state. Each such encounter will throw the responsive individual into an $\alpha_{a p}^{\prime}$ frustrating type refractory period. Thus, the appropriate behavior of this individual will not only not be rewarded, it will actually be punished. After this individual 
passes through its $\alpha_{a p}^{\prime}$ refractory period and again enters the responsive state, some random ehange may typify its behavior. The more frequently it is frustrated, the more likely will its behavior become deviant simply because there has been so infrequent reinforcement of its original appropriate form. Conversely, those individuals which, when they are in the responsive state, have met another also in the responsive state, will have the original appropriate behavior rewarded or reinforced. The behavior of such individuals will remain much in its original form.

In this way the members of a group may be rank ordered according to the degree to which their behavior has deviated from the original. Each recognizable unit of deviation represents a recessive $d$-gene. Each unit of retention of the original behavior pattern from which theirs has been a deviation represents a dominant $d$-gene. The more dominant $d$-genes an individual possesses, the more intensely will associates respond to him in a positive affective manner, and the more likely will he be chosen as a partner or leader. Conversely, the more recessive $d$-genes an individual possesses, the more intensely will associates impose restraints or sanetions on him, and the more likely will they reject him.

It is useful to borrow terminology from geneties which deals with heredity. Let upper case letters represent dominant $d$-genes, and lower case letters represent recessive $d$-genes. Such a system for an $N=11$ is shown in Table XIII. Here $(d)$ represents the common traits shared by all members of $N .(d)$ plus the remaining dominant and recessive genes specify an individual's $d$-genotype. That individual which has been exposed to the least number of circumstances producing changes in its $d$-genotype may be said to possess the "ideal" $d$-genotype. All other individuals will diverge more or less from this ideal. They may be rank ordered from the alpha individual with the ideal $d$-genotype to the omega $n$th ranked individual, which differs most from the alpha one. Each ean then be assigned a similarity rank, $R$. The alpha individual is represented by $R_{1}$; the one who differs least from the alpha has $R_{2}$; while the individual who differs most from the alpha has $R_{n}$. In Eqs. (92) and (93) $i=R$ (Tables XIII to XV).

Consider $R_{4}$. By utilizing Eq. (93) its $d$-genotype, by which we simply mean its $d$, becomes:

$$
\begin{aligned}
d=(d)+g_{a}^{(1)}+g_{b}^{(2)}+g_{c}^{(4)}+g_{D}^{(5)} & +g_{F}^{(6)} \\
& +g_{\mathrm{G}}^{(7)}+g_{\mathrm{H}}^{(8)}+g_{\mathrm{I}}^{(9)}+g_{\mathrm{J}}^{(10)}
\end{aligned}
$$

Obviously the $d$ for each similarity rank, $R$, will be different for every other one. Therefore $S$ for each individual will be unique. This responseevoking capacity, $S$, represents the $d$-phenotype. 
TABLE XIII

Trait, $d$-Gene, Differentiation in a Similarity Rank Hierarchy

\begin{tabular}{|c|c|c|c|c|}
\hline $\begin{array}{l}\text { Similarity } \\
\operatorname{rank}, R\end{array}$ & $N_{\mathrm{j}}$ & $N_{\mathrm{J}}$ & $\begin{array}{l}\text { Common } \\
\text { traits } \\
\text { (d) }\end{array}$ & Differentiating traits, $d$-genes \\
\hline
\end{tabular}

Dominant $d$-genes

\begin{tabular}{|c|c|c|c|c|c|c|c|c|c|c|c|c|c|}
\hline 1 & 11 & 1 & $(d)$ & A & B & $\mathrm{C}$ & D & $\mathrm{E}$ & $\mathrm{F}$ & G & $\mathrm{H}$ & I & $\mathrm{J}$ \\
\hline 2 & 10 & 2 & $(d)$ & $a$ & B & $\mathrm{C}$ & D & $\mathrm{E}$ & $\mathrm{F}$ & G & $\mathrm{H}$ & I & $\mathrm{J}$ \\
\hline 3 & 9 & 3 & $(d)$ & $\mathrm{a}$ & b & $\mathrm{C}$ & D) & $\mathrm{E}$ & $\mathrm{F}$ & G & $\mathrm{H}$ & I & $\mathrm{J}$ \\
\hline 4 & S & 4 & $(d)$ & a & b & c & D & $\mathrm{E}$ & $\mathrm{F}$ & G & $\mathrm{H}$ & I & $\mathrm{J}$ \\
\hline 5 & 7 & 5 & $(d)$ & a & b & c & $\mathrm{d}$ & $\mathrm{E}$ & $\mathrm{F}$ & G & H & I & $\mathrm{J}$ \\
\hline 6 & 6 & 6 & $(d)$ & a & b & c & d & e & $\mathrm{F}$ & G & $\mathrm{H}$ & I & $\mathrm{J}$ \\
\hline 7 & 5 & 7 & $(d)$ & a & b & e & d & $\mathrm{e}$ & f & $\mathrm{G}$ & $\mathrm{H}$ & I & $J$ \\
\hline 8 & 4 & s & $(d)$ & a & b & c & d & $\mathrm{e}$ & $f$ & $\mathrm{~g}$ & $\mathrm{H}$ & I & $J$ \\
\hline 9 & 3 & 9 & $(d)$ & a & b & c & d & e & $\mathrm{f}$ & $\mathrm{g}$ & $\mathrm{h}$ & I & $\mathrm{J}$ \\
\hline 10 & 2 & 10 & $(d)$ & a & b & c & d & e & f & $\mathrm{g}$ & $\mathrm{h}$ & $\mathrm{i}$ & $\mathrm{J}$ \\
\hline 11 & 1 & 11 & $(d)$ & $a$ & b & e & d & $\mathrm{e}$ & f & $\mathrm{g}$ & $\mathrm{h}$ & $\mathrm{i}$ & $\mathrm{j}$ \\
\hline
\end{tabular}


b. Response-eroking capacities. Prior formulations regarding the typing of hehavior and personality, exemplified by Schaefer $(1959,1961)$, suggest that the nature of one's own $S$ and the nature of the response eroked from others is influenced by the mood or attitude of the other individual involved. This mood or attitude determines how one riews the target diameters of others. It determines whether one focuses on the dominant $d$-genes or the recessive d-genes of others. That is, when an individual responds to another does he look at the other's desirable or undesirable characteristics. It will be a prime premise of my formulation that one can be affected only by the good or by the bad side of another at a particular moment in time, but not by both simultaneously. Furthermore, I shall show that there are two ways of assessing the good qualities, the dominant $d$-genes, of another. Likewise, there are two ways of assessing the undesirable qualities, the recessive d-genes, of another. Any individual's d-genotype in a social setting produces four types of $d$-phenotypes, that is four kinds of $S$.

Let

$S^{(A)}$ represent the response-evoking ('apacity of an individual affecting the probability of his being chosen by associates as an object of affection. $S_{A}$ is related to Schaefer's love-acceptance. $S_{A}$ depends upon both the common target diameter, $(d)$, and on dominant d-genes.

$S^{(a)}$ represent the response-evoking capacity of an individual affecting the probability of his being rejected by associates. $S^{(a)}$ is related to Schaefer's hostility-rejection. $S^{(a)}$ depends solely on recessive $d$-genes. It is dependent in no way upon the common traits, $(d)$.

$S^{(V)}$ represent the response-evoking capacity of an individual affecting how intensely he will be loved or approved of by associates. Love here implies intensity of positive response and thus connotes increase in probability of an individual realizing the objective of his behavior. $S^{(V)}$ determines the extent to which an individual's desires will be facilitated by associates. In this sense, $S^{(V)}$ is related to Schaefer's autonomy. $S^{(V)}$ depends upon both the common target channels, $(d)$, and on dominant $d$-genes.

$S^{(v)}$ represent the response-evoking capacity of an individual affecting the degree to which its velocity is altered. $S^{(v)}$ is related to Schaefer's control. $S^{(v)}$ is dependent solely upon recessive $d$ genes. It influences the intensity of negative sanctions imposed by associates.

Equations for $S_{i}^{(A)}$ and $S_{i}^{(a)}$ have already been given; i.e., Eqs. (92) and (93) subject to the restrictions there stated. 
Let:

$p_{i}^{(A)}=$ the probability of choosing any $i$ th individual.

$p_{i}^{(a)}=$ the probability of rejecting any $i$ th individual.

Then

$$
\begin{aligned}
& p_{i}^{(A)}=\frac{S_{i}^{(A)}}{\sum_{i=1}^{N} S_{i}^{(A)}} \\
& p_{i}^{(a)}=\frac{S_{i}^{(a)}}{\sum_{i=1}^{N} S_{i}^{(a)}}
\end{aligned}
$$

$i$ here, and in Eqs. (92) and (93) and (105) to (113), refers to specification of individuals by similarity rank, $R$. See discussion before Eq. (104).

Equations (105) and (106) in essence state that the probability of any other individual choosing or rejecting the $i$ th individual depends upon what proportion of the total dominant $d$-gene pool, or recessive $d$-gene pool, of the entire $N$ individuals is encompassed by this $i$ th individual. Note that these equations include evaluation of one's entire experience with members of the group, including awareness of one's own traits. This topic of self-awareness will be discussed later.

Conceptually, it is somewhat more difficult to understand $S^{(V)}$ and $S^{(v)}$, although the equations for their calculation are rather simple. Let us consider $S^{(v)}$ first, since earlier reference simply to $S$ was usually in the restricted sense of $S^{(v)}$.

Consider the individual in Table XIII with similarity rank $6, R_{6}$. When individuals $R_{1}$ to $R_{5}$ are in that state where they tend to impose restraints or sanctions on others, they will view $R_{6}$ as being more different from the ideal type than they themselves are. In this sense, $R_{1}$ to $R_{5}$ are type 1 individuals, in the sense of Eq. (9t). Similarly, $R_{7}$ to $R_{11}$ will perceive $R_{7}$ as being like themselves in that they all share the recessive $d$-gene, $g_{e}^{(5)}$. Thus, they along with $R_{6}$ may be considered as type 2 individuals, in the sense of Eq. (95), with reference to calculating the $S^{(v)}$ of $R_{6}$ by Eq. (97). $N_{j}$ for $R_{6}$ is 6 . By a similar logic the $S^{(v)}$ of each individual may be calculated. See Table XV for $S^{(v)}$ calculated for every member of an $N=11$ as depicted in Table XIII.

Each individual will belong to a different-sized $N$ of type 2 individuals. This $N$ will hereafter be referred to as $N_{j}$ to differentiate it from the $N_{2}$ given in Eq. (97). By analogy to Eq. (97):

$$
S_{i}^{(v)}=N / N_{j}
$$


And from Eq. (91) it follows that

$$
v_{i}=N_{\mathrm{j}} / N
$$

Equation (108) has proved a most useful one in the study of social groups of experimental animals because it leads to predieting the degrees of social withdrawal expected among any group of known size. In the discussion following Eq. (99) I pointed out that where $\mu$ inereases as a result of $\bar{S}^{(v)}$ becoming greater than 1.0, accommodation might be through ejection of those members with the largest $S^{(v)}$ or by a splitting of the group. Each of these possibilities presumes unused area $A$ into which the appropriate individuals may immigrate. However, when surrounding groups maintain territories, or other eireumstances preclude emigration, then the $A$ component of $\mu=(S v / A)$ remains constant. Thus, reduction in velocity, $v$, becomes the only avenue for reducing $\mu$ back to the 1.0 value appropriate to $N_{o}$.

By a similar line of reasoning to that leading to $\mathrm{Eq}$. (107), $N_{\mathrm{J}}$ represents the number of individuals with which the individual in question possesses a given uniqueness of dominant $d$-genes.

Reference to Table XIII will clarify the meaning of $N_{\mathrm{J}}$. For example, $R_{5}$ belongs to an $N_{\mathrm{J}}=5$ since it may be recognized by sharing the dominant $d$-gene, $E$, with four other individuals. $N_{\mathrm{J}}$ and the similarity rank, $R$, will always have identical numerical values.

By analogy to Eq. (107)

$$
S_{i}^{(V)}=N / N_{\mathrm{J}}
$$

And although I do not for the present see how one identifies $I$ in biological or social terms, although it may represent the seeking for positive affection, it is obvious that

$$
\mathrm{I}_{i}=N_{\mathrm{J}} / N
$$

In this sense behavioral d-genes do not represent retention or deviation from speeific behaviors. Characterization by two individuals of possessing at least three degrees of deviation does not mean that these degrees of deviation are identical.

Now consider a group consisting of four individuals, the pertinent data and calculations for which are given in Table XIV. The probabilities of the dominant and recessive $d$-genes are:

$$
\begin{aligned}
p_{A} & =1 / 4 & & p_{a}=3 / 4 \\
p_{B} & =2 / 4 & & p_{b}=2 / 4 \\
p_{C} & =3 / 4 & & p_{c}=1 / 4 \\
p_{(d)} & =4 / 4 & &
\end{aligned}
$$




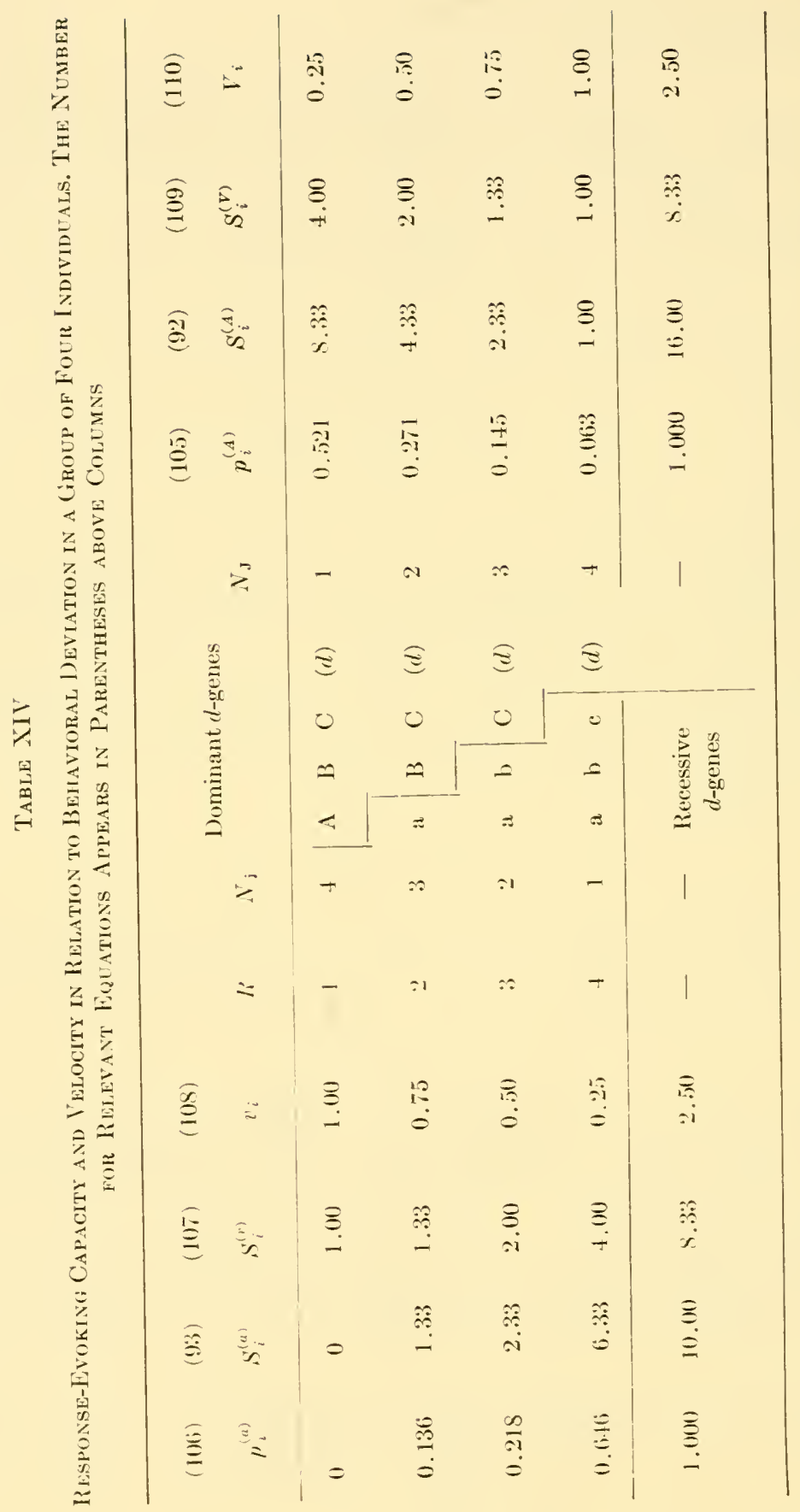


The pool of shared behaviors, $(d)$, acts as a single dominant $d$-gene. Where $R$ represents the rank of the animal in terms of the degree of retention of dominant $d$-genes, that is how closely it expresses the ideal prototype manner of behaving, $S^{(1)}$ and $S^{(a)}$ may be calculated by Eqs. (92) and (93) utilizing the above probabilities of dominant and recessive d-genes.

Then

$$
\begin{aligned}
& S_{R=1}^{(A)}=(4 / 1+4 / 2+4 / 3+4 / 4)=8.3 .3 \\
& S_{R=2}^{(A)}=(4 / 2+4 / 3+4 / 4)=4.33 \\
& S_{R=3}^{(A)}=(t / 3+4 / t) \quad=2.33 \\
& S_{R=4}^{(A)}=(t / 4) \quad=1.00 \\
& \sum_{R=1}^{N} S_{i}^{(A)} \\
& 16.00=4 N
\end{aligned}
$$

Restatement of the concept of behavioral $d$-genes in the context of a hypothetical example of their relationship to the origin of the various stimulus-evoking capacities and of velocity, $v$, will assist in clarifying the relationships. As described in Section XIII, B, .5, a the members of an established group may be ranked in terms of the degree to which deviation has developed from the ideal prototype. This ideal prototype will always be that individual characterized by the least absolute deviation. There will be $N-1$ degrees of deviation in a group of $N$ individuals.

Let:

$S_{1}, S_{2}, \cdots, S_{N-1}$ represent the characterization of an individual by 1 , $2, \cdots, N-1$ degrees of deviation

$\Delta_{1}, \Delta_{2}, \cdots, \Delta_{N-1}$ represent the absence of deviations $\delta_{1}, \delta_{2}, \cdots, \delta_{N-1}$ Then:

$$
\begin{aligned}
& \delta_{1}=\text { recessive } d \text {-gene a } \\
& \delta_{2}-\delta_{1}=\text { recessive } d \text {-gene } b \\
& \delta_{3}-\delta_{2}=\text { recessive } d \text {-gene } c \\
& \text { etc. }
\end{aligned}
$$

And

$$
\begin{aligned}
& \Delta_{1}=\text { dominant } d \text {-gene A } \\
& \Delta_{2}-\Delta_{1}=\text { dominant } d \text {-gene } \mathrm{B} \\
& \Delta_{3}-\Delta_{2}=\text { dominant } d \text {-gene C } \\
& \text { ete. }
\end{aligned}
$$

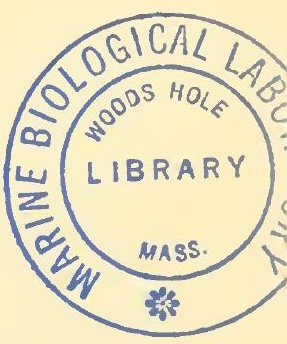

$$
\begin{array}{lll}
S_{R=1}^{(a)} & = & 0 \\
S_{R=2}^{(a)}=(4 / 3) & = & 1.33 \\
S_{R=4}^{(a)}=(4 / 3+4 / 2) & = & 2.33 \\
S_{R=4}^{(a)}=(4 / 3+4 / 2+4 / 1) & = & 6.33
\end{array}
$$

Then $\sum_{R=1}^{N} S_{i}^{(a)}$

$$
10.00=3 N-(N / 2)
$$


$N_{\mathrm{J}}$ and $N_{\mathrm{j}}$ in the sense of type one and type two individuals discussed in relation to Eqs. (94) and (95) and (107) are given in Table XIV. When $N_{\mathrm{J}}=2$ it means that the second ranked, $R=2$, individual is a member of a subgroup of two individuals which share the dominant $d$-gene B. Likewise when $N_{\mathrm{j}}=2$ it means that the third ranked, $R=3$, individual belongs to a subgroup of two individuals each of whom possesses recessive $d$-gene b. Every individual belongs to a unique $N_{\mathrm{J}}$ and $N_{\mathrm{j}}$. Given these uniquenesses of $N_{\mathrm{J}}$ and $N_{\mathrm{j}}$ as shown in Table XIV, the respective $S_{i}^{(V)}$, $S_{i}^{(v)}$ and $v_{i}$ were caleulated respectively by Eqs. (109), (107), and (108) and presented in Table XIV.

Up to the present I have intentionally maintained the discussion of l'esponse-evoking capacity, $S$, on a nearly strictly theoretical plane for the purpose of clarifying concepts. Even though such theoretical formulations may be justified in their own right, regardless of how well they approximate reality, still it is desirable to ascertain whether they are in harmony with observed data. To this end I selected two sets of empirically obtained data. One involves measurements for mice from which velocity, $v$, may be derived. The second involves the choosing of table partners among groups of delinquent girls. These latter data permit determination of how well the observed choosing can be predicted by Eq. (105).

\section{Consequences and Examples of Social Interaction Systems}

\section{A. Velocity Reduction in a Mierarchy of Mice}

I have previously described (Calhoun, 1956) the patterns of social relationships which develop among members of small groups of inbred domesticated mice. Whenever two mice passed within a few inches of each other they were recorded as having a contact, regardless of whether or not a detectable social interaction could be detected. During a "contact" two mice merely came within that range of each other for which contentions for status did at times develop. We shall consider the example provided by a group of eleven C57 black, inbred male mice which had developed a stable hierarchy prior to recording the frequency and kind of associations of each individual with his associates. Some pairs of mice contacted each other much more frequently than anticipated on a chance basis, while members of other pairs very infrequently met. These mice are rank ordered in Table XV according to the number of contacts each had with associates. For all practical purposes this order also represcnts the observed ability to dominate an encounter which precipitated in a fight or flight. That is, 
the most active mouse was the most dominant one and the least active one the most subordinate.

It can readily be shown that with $N$ 's as large as eleven, the number of contacts, $n_{c}$, is so nearly proportional to velocity, $v$, as to justify utilizing number of contacts as a measure of velocity in the present example. From Eq. (91) it is seen that $S_{i}^{(v)}$ varies inversely with $v_{i}$, and therefore on a theoretical basis mice with few contacts should be those with large responseevoking capacities.

TABLE XV

Velocity in a Hierarchy of Mice

\begin{tabular}{|c|c|c|c|c|c|c|}
\hline 1 & 2 & 3 & 4 & 5 & 6 & 7 \\
\hline \multirow[b]{2}{*}{$\begin{array}{l}\text { Mouse's } \\
\text { number }\end{array}$} & \multirow[b]{2}{*}{$\begin{array}{l}\text { Rank } \\
\quad R\end{array}$} & & \multicolumn{4}{|c|}{ Number of contacts, $n_{c}$, expected } \\
\hline & & $N_{i}$ & Obs. & $\begin{array}{c}\text { Exp. } \\
\text { Eq. (113) }\end{array}$ & ${ }^{v}{ }^{2} \cdot(108)$ & $\begin{array}{c}S^{(v)} \\
\mathrm{Eq} \cdot(107)\end{array}$ \\
\hline 315 & 1 & 11 & 123 & $12 S .17$ & 1.000 & 1.00 \\
\hline 311 & 2 & 10 & 116 & 116.63 & 0.910 & 1.10 \\
\hline 282 & 3 & 9 & 109 & 104.84 & 0.818 & 1.22 \\
\hline 286 & 4 & 8 & 93 & 93.18 & 0.727 & 1.38 \\
\hline 319 & 5 & 7 & 85 & 81.52 & 0.636 & 1.57 \\
\hline 303 & 6 & 6 & 65 & 69.85 & 0.545 & 1.83 \\
\hline 321 & 7 & 5 & 51 & 58.19 & 0.454 & 2.20 \\
\hline 317 & 8 & 4 & 46 & 46.65 & 0.364 & 2.75 \\
\hline 290 & 9 & 3 & 35 & 34.99 & 0.273 & 3.67 \\
\hline 288 & 10 & 2 & 26 & 23.33 & 0.182 & 5.50 \\
\hline 301 & 11 & 1 & 20 & 11.66 & 0.091 & 11.00 \\
\hline$\Sigma$ & & & 769 & & $\begin{aligned} \Sigma & =6.001 \\
& =(N+1) / 2\end{aligned}$ & \\
\hline
\end{tabular}

Where $v$ represents relative velocity as calculated by Eq. (10S) for any $N$, empirical calculation will show that:

$$
\sum_{i=1}^{(N)} v_{i}=\frac{(N+1)}{2}
$$

Then where $n_{c i}^{\text {(obs) }}$ and $n_{c i}^{(\exp )}$ represent respectively, observed and expected number of contacts in any arbitrary period of time, the number of 
contacts, $n_{e i}$, for any $i$ th animal becomes:

$$
\begin{aligned}
n_{c i}^{(\exp )} & =\left[\frac{v_{i}}{(N+1) / 2}\right] \sum_{i=1}^{N} n_{c i}^{(\mathrm{obs})} \\
& =v_{i}\left[\frac{2 \sum_{i=1}^{N} n_{c i}^{(\mathrm{obs})}}{N+1}\right]
\end{aligned}
$$

And by substituting Eq. (108) into Eq. (112) we obtain an equation more convenient for calculation:

$$
n_{c i}^{(\exp )}=\left[\frac{N_{j i}}{N}\right]\left[\frac{2 \sum_{i=1}^{N} n_{c i}^{(\text {(ubs })}}{N+1}\right]
$$

$n_{c i}^{(\exp )}$, so calculated, are given in Table $\mathrm{XV}$. Where

$$
\chi^{2}=\sum_{R=1}^{N}\left[\frac{\left(n_{c i}^{(\mathrm{obs})}-n_{c i}^{(\mathrm{exp})}\right)^{2}}{n_{c i}^{(\mathrm{exp})}}\right]
$$

$\chi^{2}=8.001$, which with 10 degrees of freedom has a $p$ of 0.629 . On this basis the observed certainly does not deviate significantly from the expected.

\section{Awareness of Self}

Three-fourths of the contribution to the above $\chi^{2}$ come from the single omega, $n$th ranked individual. Considering only the highest ten ranked individuals, $\chi^{2}=2.006$ which with 9 degrees of freedom has a $p$ of 0.99 ! I have already shown (Calhoun, 1956) that the paired contacts in this group diverged markedly from randomness, and so the divergence must reflect some fixed social system such as elaborated here with regard to reduction in velocity. Therefore, the marked divergence of this single omega individual is likely to reflect a basic process, not just a random variation; so I asked, "How would self-awareness affect the present formulation?" By self-awareness I mean that an individual recognizes and imposes self-sanctions which are of sufficient intensity to reduce his velocity just as much as do the sanctions imposed upon him by his associates. For this to happen it means that an individual can "meet" himself.

Equations (96), (97), (107), and (109) imply that an animal can meet itself. That an individual meets himself means that he must recognize himself. This raises the question of how an individual recognizes himself. One way is by comparison. Considering degrees of difference depicted by Table XIII, an individual can say, "I am at least as different as those 
which possess certain recessive $d$-genes that I possess." By this method of comparison with individuals who differ more than he does, an individual can know the entire extent of his difference. This is true for all except the $n$th ranked omega individual. As may plainly be seen from Table XIII, $R_{11}$ has no basis for comparing his $d$-gene $g_{j}^{(10)}$ since no other individual shares it. He can only be aware of his divergence including $g_{\mathrm{a}}^{(1)} \cdots g_{i}^{(9)}$ shared in entirety by $R_{\mathbf{1 0}}$. Therefore, by Eq. (107) his

$$
S^{(v)}=11 / 2=5.50
$$

and by Eq. (108) his

$$
v=2 / 11=0.182
$$

Therefore, by Eq. (113) his

$$
n_{c}^{(\exp )}=23.33
$$

Using this value in Table $\mathrm{XV}$ the $\chi^{2}$ for the entire group of 11 mice becomes 2.522, which with 10 degrees of freedom has $p=0.99$. I realize that this is only one case and I may justifiably be accused of making a "conceptual mammoth out of a mouse." Yet if animals do recognize their individuality, the only confirmation of such recognition can come through observing that the omega individual in an $N_{b}$ group exhibits twice the velocity or twice the number of contacts he would otherwise be expected to have.

It is realized that if the $N_{\mathbf{j}}$ of the $N$ th ranked animal, the omega individual, is equivalent to that of the $(N-1)$ th ranked animal, the $n_{c}^{(\exp )}$ for the omega animal will actually be slightly less than 23.33. Likewise the $n_{c}^{(\exp )}$ for all higher ranked animals would be slightly less than stated in Table $X V$, since

$$
\sum_{R=1}^{N} n_{c i}^{(\exp )}=\sum_{R=1}^{N} n_{c i}^{(\mathrm{obs})}
$$

\section{Sanctions and Facilitations}

When the target diameters of all members of $N$ are identical, then the $S$, in the sense of $S^{(v)}$, is identical for all individuals and is equal to 1.0. As target diameters vary in accordance with the system illustrated in Table XIII, $S$ increases except for the alpha first-ranked individual. Such increases in $S$ mean that the intensity (duration) of the response evoked from others will be greater. Therefore, intensity of response, the $i$ of the prior formulation, will increase by a factor $=S^{(v)}$.

Let $i_{e}^{(r)}$ represent the intensity of response eroked from erery member, 
$e$, of $N$ compatible with one's own $S^{(v)}$. Then it follows from Eq. (70) that for the $i$ th individual

$$
i_{e}^{(v)}=S_{i}^{(v)}\left[\frac{1}{N-1}\right]^{1 / 2}
$$

$i^{(v)}$ represents the imposed restraint or sanction. Sanctions in this sense lead to velocity reduction as exemplified by the mice. $i_{e}^{(v)}=i_{i}^{(v)}$ with reference to self action.

Where the target diameters of all individuals are identical, Eq. (70) with an $N$ of 11 gives an $i=0.316$. However, where target diameters differ as given in Table XIII, it may be seen from column 7 in Table XV that $S^{(v)}$ increases from 1.0 to 5.5 . (Here, I am also assuming that the $N$ th ranked individual has the same $S$ as the $(N-1)$ th ranked individual for reasons discussed in Section XIV, A, 1.) The mean $S^{(v)}$ will be 2.52. There fore, the mean $i^{(v)}$ becomes $2.52 \times 0.316=0.816$.

We are considering intensities of interaction involved during imposition of sanctions in the sense of restraints. Therefore, when we consider any individual in the a responsive state, the other individuals from which it evokes an $i^{(v)}$ sanction-type interaction must be considered to be in the $p$ nonresponsive state since the resulting interaction will not contribute to this individual's satiation from social interaction. Every member, $e$, of $N$, which is in the responsive state, $a$, will exhibit an $i_{e i}^{(v)}$ intensity of response toward any $i$ th individual, also in the $a$ responsive state, where

$$
i_{e i}^{(V)}=S_{i}^{(V)}\left[\frac{1}{N-1}\right]^{1 / 2}
$$

$i^{(V)}$ represents the intensity of interaction of the individual searching for satisfaction. Similarly the mean $i^{(V)}$ will also be 0.816 instead of the 0.316 which exists when all individuals in an $N$ of 11 have the same target diameter.

$$
\begin{aligned}
i_{i}^{(\boldsymbol{V})} i_{e i}^{(v)} & =\alpha_{a p}^{\prime} \\
& =0.666
\end{aligned}
$$

This $\alpha_{a p}^{\prime}$ represents the mean frustration-type refractory period resulting from the imposition of sanctions in an $N=11$. Since when all individuals have the same target diameter, $i^{2}=(0.316)^{2}=0.1$, hierarchy formation increases the average amount of stress more than sixfold. No wonder that the $i_{e i}^{(v)}$ elicited from others became so effective in reducing velocity so that each individual's $\mu$ again equals 1.0. That is, $v S^{v}=1.0$. Although 
intermittent application of sanction, $i^{(v)}$, may be required to reinforce the $v$ behavior, sanctions still must be primarily a phenomenon of hierarchy formation or role assumption.

When two individuals, $x$ and $y$, are both in the $a$ responsive state, their resulting interaction, $i_{x}^{(V)} i_{y}^{(V)}$ will lead to a satisfying $\alpha_{a a}$ refractory period. Being in the responsive state implies a need for satisfaction from social interaction. Furthermore, during such interactions each must be focusing its attention on the $S^{(V)}$ aspects of the other's target diameter. In like fashion, when individual $x$ in the responsive state approaches $y$ in the nonresponsive state, $x$ exhibits behavior appropriate to $y$ 's $S^{(V)}$, while $y$ retaliates with a response appropriate to $x$ 's $S^{(v)}$.

One individual which is in the responsive phase will have its behavior toward another judged by the latter as appropriate or inappropriate, depending upon whether or not the individual approached is also in the responsive state. Appropriate social behavior becomes synonymous with what I have termed "dominant $d$-genes." These behaviors must be appropriate both in form and in timing, such that the approached individual will be in a similar need state for obtaining satisfaction from social interaction. Even though a social behavior may seem appropriate with regard to form, if the timing of its expression is not harmonious with the refractory nonresponse state of the individual approached, then the approached individual will judge this behavior as being inappropriate. Inappropriateness in this sense becomes what I term a "recessive $d$-gene."

In a perfectly random system, with all individuals exhibiting identical behavior with regard to its form, some individuals will by chance more frequently encounter others who are in a refractory nonresponsive state. Thus, such individuals will be responded to by their associates in identical fashion as they would have been if the form of their behavior were actually inappropriate. To the degree that such structurally appropriate behaviors fail to be reinforced by similar behavior from associates, they may be expected to vary in some random fashion until their form becomes relatively distinct from the original. To the extent that these alterations in behavior become established, they represent clearly recognizable recessive $d$-genes. Due to this modification of behavior $S^{(v)}$ will increase and $S^{\left(V^{v}\right)}$ decrease, so even when the approached individual is also in the responsive state the approaching individual will experience less satisfaction from the interaction than it would have had these random processes not transpired.

Once $\mu$ becomes restabilized to 1.0 following the reductions in velocity accompanying hierarchy formation, it will then be possible for two individuals, each in the socially receptive $a$ state, to encounter each other and mutually contribute to each other's need state by an $i^{(V)} i^{(V)}=\alpha_{a a}$ interaction. In this case, each individual's own $i^{(V)}$ represents its searching 
for social satiation, while the other's $i^{(V)}$ represents a social facilitation, the opposite of a social sanction.

I shall leare the concept of social facilitation at this theoretical level without seeking empirical confirmation. However, excellent data have been presented by Moreno (1953; Moreno and Jennings, 1960) which permit an exploration of the probable validity of $S_{i}^{(A)}, \mathrm{E}_{(1}$. (92).

\section{B. The Choosing of a Partuer}

We hare already seen that $S_{i}^{(v)}, \mathrm{E}\left(1 .(107)\right.$, and $S_{i}^{(V)}$, Eq. (109), relate to those response-evoking capacities of an individual's target diameter which determine the intensity with which associates will respectively impose sanctions or facilitations. $S_{i}^{(-A)}$, Eq. (92), and $S_{i}^{(a)}$, Eq. (93), likewise represent aspects of one's target diameter influencing the response of others. One's own $S^{(-1)}$ determines the probability of being chosen by others as an appropriate object for social response, while one's own $S^{(a)}$ similarly determines the probability of being rejected. No doubt there are excellent empirical data for testing the validity of $S^{(a)}$. However, I shall confine myself to $S^{(A)}$. If I can show the likelihood of $S^{(A)}$ being an approximation of reality, it follows that $S^{(a)}$ can be similarly justified as a concept.

Moreno $(1953,1960)$ presents a set of data for which there has been no adequate formulation of their origin. In seven cottages each containing exactly 26 delinquent girls, he asked each girl to choose three others in their own cottage whom they would most like to sit close to at the dining table. This instruction presents marked complications in determining whether Eq. (105) will account for the observed results. However, Eq. (10.5) includes the possibility that one will choose oneself as a partner; that is, one will choose to eat alone. Moreno by his instructions excluded this possibility. Further, Moreno's instructions precluded the possibility of choosing the same person two or three times, which Eq. (105) permits on successive independent choices. Dr. Clifford Patlak worked out for me the full set of equations required to determine how many times each individual would be chosen, considering Moreno's restrictions, after the probability, $p$, of being chosen was calculated by Eq. (105) for each $i$ th individual in an $N=26$. Moreno's restrictions so complicated the calculations that it was concluded that a simple lottery would adequately test the applicability of the present theory, and at the same time avoid the time-consuming job of developing a computer program to the same end. This was done as follows:

1. $S_{i}^{(A)}$ was calculated by Eq. (92) for each of the 26 members of $N$, from $S_{1}^{(A)}$ for the alpha-ranked individual to $S_{26}^{(A)}$ for the omegaranked individual. 
2. Then the $p$ of being chosen was calculated for each individual by Eq. (105). For example:

$$
\begin{array}{ll}
p_{1}^{(A)}=0.152, & p_{2}^{(A)}=0.121 \\
p_{3}^{(A)}=0.092, & p_{10}^{(A)}=0.039 \\
p_{20}^{(A)}=0.012 &
\end{array}
$$

3. We then prepared $1000 \cdot p_{i}$ tokens for each individual.

4. All these 1014 tokens were placed in a large glass jar. For any "individual" to make three choices, three squares at random were picked out. If an "individual" picked himself, the token was returned to the jar" and another "choice" made at random. Similarly, if another individual was chosen twice, one of the two tokens was returned to the jar and another seleetion made until each "individual" had chosen three different "others." Between drawings all tokens were returned to the jar and the contents mixed. Each such test consisted of 78 "choices." At the end of each test it was possible to tally how many of the 26 "individuals" had never been chosen, chosen once, chosen twice, etc.

5. Fourteen such independent tests were performed and the mean number of individuals in each choice category was calculated. This mean represents an estimate of the expected.

These data in Table XVI are plotted in Fig. 36. A smoothed curve approximating the means of the observed and the lottery presumably approximates that conforming to Eq. (105), considering the restrietions imposed by Moreno. This smoothed curve is called "theoretical" in Fig. 36. Each of the two sets of points varies so closely about this curve as to provide confirmation that my formulation is adequate to account for the observed sociometric phenomenon of choice.

Moreno and his associates make much of the chains, triangles, ete. of reciprocal or nonreciprocal choices that became apparent in such a sociometric system. While such patterns may become fixed realities, there is no reason to attribute any condition other than chance to their origin.

\section{The Response-Evohing Capacity Circumplex}

Schaefer $(1959,1961)$ presents a conceptual model capable of describing the attitudes, personality or behavior of an individual in a two-dimensional space. This latter is determined by two orthogonal axes of polar opposites. As may be seen in Fig. 37, one axis consists of the polar opposites love and hostility, while the other is represented by control and autonomy. About 
TABLE XVI

Empirical and Theoretical Data Regarding the Choosing of a Partner

\begin{tabular}{cccc}
\hline & \multicolumn{2}{c}{ Mean number of individuals } & $\begin{array}{c}\text { "Theoretical" } \\
\text { Times chosen of }\end{array}$ \\
\cline { 2 - 3 } & $\begin{array}{c}\text { A } \\
\text { Moreno }(1953,\end{array}$ & $\begin{array}{c}\text { B } \\
\text { Present Lottery } \\
\text { based on } S^{(A)}\end{array}$ & \\
\hline 0 & $5.00)$ & 5.36 & 5.15 \\
1 & 4.15 & 5.36 & 4.76 \\
2 & 4.29 & 3.82 & 4.06 \\
3 & 3.72 & 2.29 & 3.01 \\
4 & 2.29 & 2.50 & 2.40 \\
5 & 2.00 & 1.79 & 1.90 \\
6 & 1.14 & 1.50 & 1.32 \\
7 & 1.43 & 0.86 & 1.15 \\
5 & 0.86 & 0.72 & 0.79 \\
9 & 0.57 & 0.57 & 0.57 \\
10 & 0.14 & 0.50 & 0.32 \\
11 & 0.43 & 0.36 & 0.40 \\
12 & 0.00 & 0.29 & 0.15 \\
13 & 0.00 & 0.07 & 0.04 \\
\hline
\end{tabular}

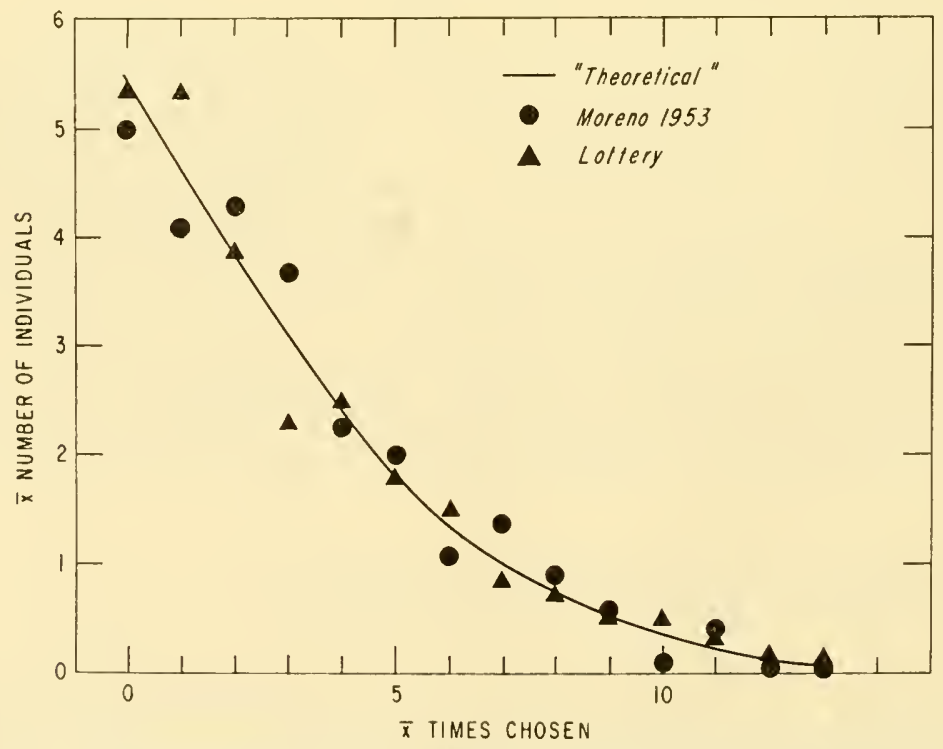

Fig. 36. The choosing of a partner. Moreno's empirical data are compared with a lottery conforming both to Moreno's procedure and the probabilities of being chosen as predicted by Eq. (105) in the present paper. 
or within the eircle determined by the extremes of these polar opposites, the typical characteristic of an individual may be oriented.

There exist remarkable similarities between Schaefer's model and that implied so far by my concept of response-evoking capacity, $S$, and the resultant or concomitant change in the behavior of the individual, such as reflected by change in relocity.

$S^{(v)}, S^{(V)}, S^{(A)}$, and $S^{(a)}$ represent factors not specifically treated by

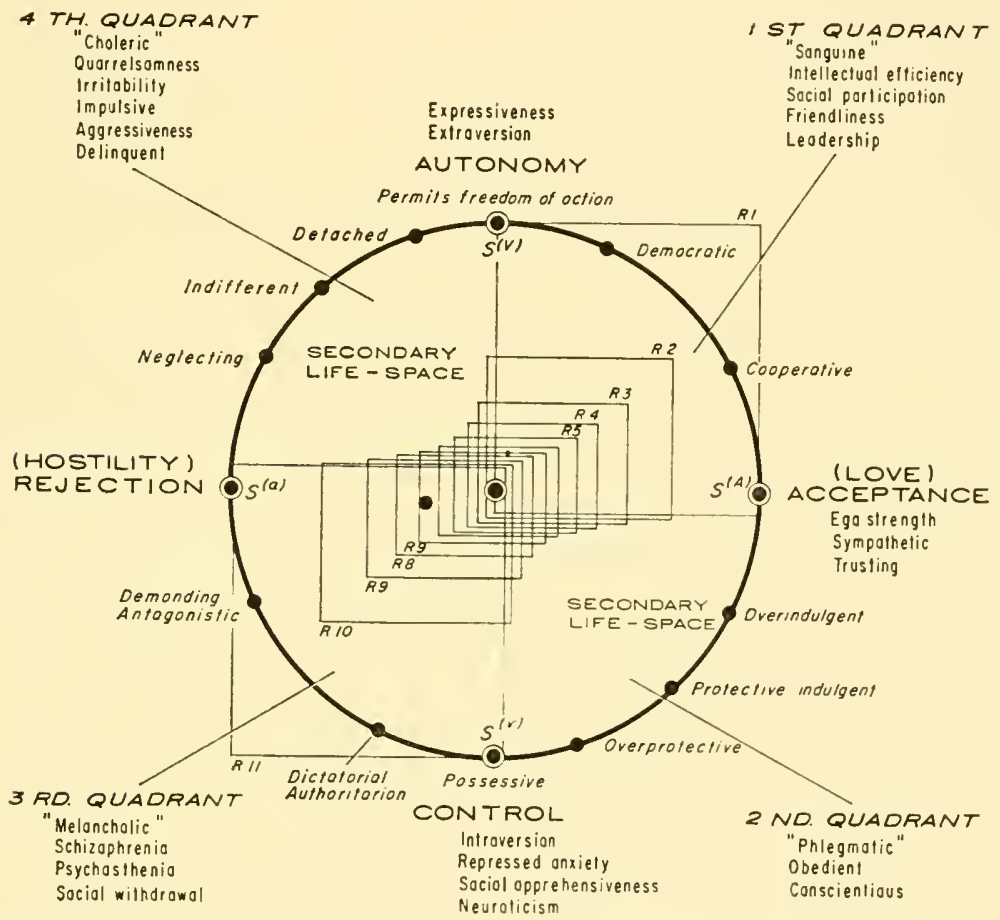

FIG. 37. The circumplex depiction of behavior and personality superimposed upon the coordinates of response-evoking capacity, $S$.

Schaefer. They relate to his formulation as follows: They represent those characteristies of the individual that determine the probability of aceeptance or rejection as a social object and that determine the kind and intensity of response elicited. Sehaefer eonsiders the second logieal step, the evoked response, such as the mother's response toward her children. He also considers the third-order phenomenon, that of the personality developed as a result of being the target of such responses.

I shall now attempt to place these three orders of phenomena in perspective. For an $N=11, S^{(v)}, S^{(v)}, S^{(A)}$, and $S^{(a)}$ were calculated by the 
above equations. 'These values were then recalculated as proportion of the maximum value. For examples, see tabulation.

\begin{tabular}{ccccc}
\hline Similarity rank & $S^{(v)}$ & $S^{(V)}$ & $S^{(A)}$ & $S^{(a)}$ \\
\hline 1 & 0.091 & 1.000 & 1.000 & 0.000 \\
6 & 0.166 & 0.166 & 0.244 & 0.220 \\
11 & 1.000 & 0.000 & 0.030 & 1.000 \\
\hline
\end{tabular}

$S^{(v)}$ and $S^{(V)}$ with values of 1.0 form polar opposites, as likerrise do $S^{(A)}$ and $S^{(a)}$. These polar opposites are shown as a two-dimensional coordinate system in Fig. 37. The four "response-eroking capacity" coordinate points for each individual are connected by lines. Thus, a square may be delineated for each ranked individual which represents its "life-space" with regard to eliciting responses from associates.

Schaefer's two-dimensional circumplex description of behavior and personality replaces the relative intensity values of my axis of correlation coefficients. In most comprehensive sets of measures of behavior or personality, he regularly found that two measures which can be equated with the terms love (acceptance) and hostility (rejection) are highly negatively correlated and so form polar opposites which may thus be plotted as polar coordinates at 180 degrees from each other. Similarly, concepts identified by the terms "control" and "autonomy" form polar coordinates opposite each other. Control and autonomy have zero correlation with acreptance and rejection and so the control-antonomy axis lies at right angles to the acceptance-rejection axis. From the center zero point each of the four axes extend outward to represent a maximum correlation coefficient of 1.0 at its extremity. Every other concept in the set is then correlated with each of the four "key" concepts. It is regularly observed that every other concept in the set has positive correlations with two of the neighboring polar concepts, and so each may be plotted in this two-dimensional behaviorpersonality field.

Such points approximately fall on the circumference of a circle intersecting the polar coordinates. Schafer calls such a set of points a circumplex. His schematic representation of types of maternal behavior is shown by dots in Fig. 37. Terms connected by arrows to these points denote various kinds of maternal behavior. These maternal behaviors are the types I would anticipate as being directed toward, and derived from, the responseevoking-capacity circumplex determined by $S^{(V)}, S^{(v)}, S^{(A)}$, and $S^{(a)}$.

Schaefer also reviews many studies by others which harmonize with this conceptualization of behavior and personality. I have included selected 
terms in boxes which carry the "flavor" of what each pole or each quadrant represents.

It will be noted that the response-eroking-capacity life-spaces of the members of any group, as I have described their origin, mainly fall in the first and third quadrant of this two-dimensional space. I shall, therefore, call these two quadrants the "primary life-space." We may inquire how individuals become identified with the other two quadrants, the second and the fourth, which may be called the secondary life-space.

My colleague, Dr. Kyle Barbehenn provided the solution. In examining Table XIII he noted its bilateral symmetry with reference to dominant and recessive $d$-genes. In any group recessive $d$-genes merely represent degrees of divergence from some ideal prototype. The alpha-ranked individual possesses only dominant prototype characteristics, whereas the omega-ranked individual, except for commonly held $(d)$ traits, possesses only divergent and therefore recessive $d$-genes. But suppose in the history of the group some circumstance led the omega's associates to consider his characteristics as being more desirable than the alpha's. Such a change in attitude would reverse the roles of all individuals, except for the medianranked individual. The individual who formerly was socially withdrawn and had a low velocity would immediately become a high velocity individual, participating in many social interactions. Likewise, the former alpha individual would become the omega one, losing his "leadership" role and becoming socially withdrawn.

This transformation of recessive $d$-genes into dominant ones, and vice versa, will still place most of the individuals again within the first and third quadrants of the circumplex life-space. The critical question involves the transition period. In this transition Eqs. (92) and (93) change roles with reference to their application to dominant and recessive $d$-genes, as likewise do Eqs. (107) and (109). There arises the likelihood that previous to this transition choosing and rejecting will be a more predominant activity than controlling or granting autonomy. If so, $S^{(A)}$ will switch to pertain to recessive $d$-genes and $S^{(a)}$ to dominant $d$-genes while $S^{(V)}$ and $S^{(v)}$ will retain their orientation toward dominant and recessive $d$-genes, respectively. Or the reverse could happen if the group were primarily oriented toward the control-autonomy axis. The consequence of these changes are summarized in Table XVII.

1. Shifts into the Second and Fourth Life-Space Quadrants BY RATS

No intent is here implied of proving the validity of the general formulations. All I have hoped for is to evolve a logical and reasonable formulation that may later prove to be a fair first approximation of processes that 
do in fact exist. This elaboration of Schaefer's circumplex life-space immediately brought recall of a striking transitory change in behavior which regularly occurs in structured, dense, socially closed systems of domesticated Norway rat populations.

When these populations have been permitted to attain a density of 60 to 100 adults in a space ideal for 40 or fewer adults, an extremely rigid social structure develops. All rats "know" their place and aggressive actions terminating in some individuals receiving even moderate-sized wounds become markedly reduced. Threat and avoidance becomes the predominant pattem where aggression and imposition of sanctions di-

\section{TABLE XVII}

Change of "Attitude" toward $d$-Genes Affecting Shifts into the Secondari LIFE-SPaCe OF THE 2ND AND 4TH QUadRaNTS

\begin{tabular}{|c|c|c|c|}
\hline Initial change & $\begin{array}{l}d \text {-Gene } \\
\text { involved }\end{array}$ & $\begin{array}{l}\text { Interaction of } \\
\text { neighboring poles }\end{array}$ & Quadrant \\
\hline $\begin{array}{l}S^{(A)} \text { oriented to recessive } \\
\quad d \text {-genes }\end{array}$ & Recessive & Control-acceptance & $2 \mathrm{nd}$ \\
\hline $\begin{array}{l}S^{(a)} \text { oriented to dominant } \\
\quad d \text {-genes }\end{array}$ & Dominant & Autonomy-rejection & 4 th \\
\hline $\begin{array}{l}S^{(V)} \text { oriented to recessive } \\
\text { d-genes }\end{array}$ & Recessive & Autonomy-rejection & 4th \\
\hline $\begin{array}{l}S^{(v)} \text { oriented to dominant } \\
\quad d \text { genes }\end{array}$ & Dominant & Control-acceptance & 2nd \\
\hline
\end{tabular}

rected toward maintaining status roles is involved. And yet in six of seven such populations studied, one or several males went temporarily berserk. Each such male abruptly began attacking all other members of the population except those that behaved as they did. They inflicted deep slashing gashes on the bodies and tails of associates of all ages and both sexes until fresh blood could persistently be observed splattered about the habitat.

For any particular rat such episodes persisted from one to several days and since usually several males were involved the total period of such disturbance might last up to 6 weeks. In every instance these bursts of males going berserk followed a period of at least three months during which the investigator had removed all young prior to weaning. Then a new generation of young were permitted to survive. The period of males going berserk 
coincided with the initial post-weaning integration of these young rats into the society when they ranged between 45 and 90 days of age.

All the males going berserk belonged to the high velocity, generally dominant, segment of the society, and so belonged in the "sanguine" most desirable first quadrant of the life-space circumplex. This meant that dominant $d$-genes predominated in their target diameter. The juvenile rats emerging into the society, being less differentiated, were therefore now prototypic and therefore resembled dominant adult males in their possession of mostly dominant $d$-genes. And yet these juveniles would still possess traits lost during maturation by most adults.

Due to the typical response of rejecting strange objects, the young would be rejected while still being permitted autonomy of action due to dominant $d$-genes shared with high-ranking adults. There being more juveniles in the population than adults belonging clearly in the first quadrant of the life-space eircumplex, the general response of rats to these adults would be to react to them as they did to juveniles by rejection. This rejection triggered the release of the muted aggressive eapacities of dominant males to the extent that it was expressed with great intensity even toward others, such as juveniles and adult females, who normally were not bitten.

Such an origin of an aberrant behavior in a rat society is patently an interpretation lacking the complete documentation to carry the conviction of its reality. Yet my intensive studies of rat societies permit identification of so many behavior-personality types as to suggest that rats are equally as complex as humans in this regard. The eight societies previously studied have been commented upon in general terms elsewhere (Calhoun, 1962a, b).

In the early history of a rat society, while its numbers and density are low, most individuals seem rather elearly to fall into quadrants one and three of the circumplex. This is a typical expectation when a straight-line hierarehy develops, as it always does in initial stages of social organization. Later on, histories and situations become more complex. Other types develop which may clearly be assigned to the second and fourth quadrant.

For example, there is the type I call a "prober," which appears to represent a rat having shifted from the third to the fourth circumplex quadrant. Earlier in their history they elearly belong within the lower echelons of the social hierarchy. Later on they are generally ignored by dominants with whom they live most closely. In consequence, they develop a marked persistent state of hyperactivity indieating autonomy of action. They seem to generalize this autonomy of action as permitting them freedom of action anywhere. Consequently, they persist in invading the domains of territorial males whenever members of their harems are in estrus. During such invasions they rarely contest the status of the territorial male, but in the process of being rejected by him receive wounds. These are received 
so frequently that their entire posterior becomes a mass of scar tissue devoid of hair. Like other males who still receive sufficient sanctions from associates to maintain their velocity at a low level, they become pansexual in the sense of including adult males and juveniles of both sexes as objects for sexual advances. They also share with the berserk males of quadrant four the property of heightened intensity of interaction. Theirs, howerer, reveals itself in sexual behavior toward adult females. Mounts, instead of lasting the usual 1 to 3 seconds, may continue for several minutes. This persistence of the mounting, without intromission, resembles that of frogs.

Low velocity male rats, those which belong in circumplex quadrants two and three, fall mostly into two distinct categories, those which have received many wounds and those which have received very few. The former, which belong in quadrant three, present no conceptual problem as to their origin. Straightforward operation of the four $S$ factors will always place the lower-ranked members of the hierarchy in the third quadrant. However, the latter, "phlegmatic" types must have arisen from a secondary 180 degree shift in response evoked by the $d$-genes. Their lack of wounds during their entire history indicates $S^{(A)}$ involves dominant $d$-genes. Their low velocity indicates that now as adults $S^{(v)}$ also involves dominant $d$ genes. They are rats for whom we may infer that their associates have always been "overindulgent" and "overprotective." Such rats are quite fat and have relatively small adrenals, rentricles, and kidneys. Though they exhibit some displaced sexual behavior, their "personality type" is one most characteristically involving failure either to elicit or initiate social interaction. They are types lacking social involvement.

This brief discourse on rat types suggests that my elaboration of Schaefer's $(1959,1961)$ circumplex behavior-personality complex may prove to be a fruitful framework for pursuing studies in comparative social psychology.

\section{Conformity, Withdrawal, and Creativity}

When $N$ increases above $N_{b}$ or $\mu$ increases above $\mu_{b}$ it has been shown that fragmentation of $N$ to appropriately sized discrete subgroups may lead to reacquisition of $\theta_{a}^{(o)}$ and $\theta_{f}^{(o)}$. Even when $N$ remains at $N_{b}$, the unavoidable variability in the four types of $S$ leads to $\mu$ differing among members of the group. Reduction of $v$ as $S^{(v)}$ increases enables the individuals to prevent excessive increments of $\theta_{f}$ above $\theta_{f}^{(o)}$. Even so, many individuals with reduced $v$ must experience either reduced $\theta_{a}$ or excessive $\theta_{f}$. We may inquire as to possible avenues for escaping these deviations from $\theta_{a}^{(o)}$ and $\theta_{f}^{(o)}$ without leaving the group. 
For those individuals who in their behavior do not differ markedly from the alpha-ranked, $R_{1}$, member, there exists the possibility of adopting the outward behavior of $R_{1}$. Such acquired conformity should permit elevation of velocity to basic levels and should produce a discontinuity in the range of velocities observed among members of the group. Whyte (1956) in his "The Organization Man" has emphasized the role of conformity as an adjustive mechanism accompanying increases in $N$.

At the opposite extreme of original behavioral divergence there exist individuals with such reduced velocity that their contacts with higherranked individuals proves insufficient to permit their developing conformity through emulating the behavior of their superiors. Recently I have had the opportunity of studying the behavior of all members of three populations of domesticated Norway rats for every member of which assessments of velocity had been made. Low-velocity rats develop the capacity to move about without engaging in interactions with their associates. They rarely initiate interactions nor do they elicit actions from associates. This social withdrawal becomes so complete that, despite being in the presence of many associates, they are characterized by small adrenals and small ventricles, just as are rats which have lived all their lives as members of $N$ 's of 3 . David Riesman et al. (1953) has movingly described such isolation and oblivion of surroundings among humans in his "The Lonely Crowd."

In between the velocity levels producing conformity and withdrawal, there lies a narrow but important range of velocity permitting a process which can terminate in creativity. Why this is so requires recall that according to my model or social interaction, interaction with self must be included along with interaction of self with others. One can choose oneself, one can reject oneself, one can facilitate one's own behavior, or one can restrain or impose sanctions on one's own behavior. All are possible.

Consider sanctions. These are mostly the $i_{e i}^{(v)}$, Eq. (11t), of others directed against oneself. Let $i_{i}^{(v)}$ represent self-sanctions. The self-interaction becomes:

$$
\begin{aligned}
{\left[i_{i}^{\left(v^{\prime}\right)}\right]^{2} } & =\left(S_{i}^{\left(v^{\prime}\right)}\left[\frac{1}{N-1}\right]^{1 / 2}\right)^{2} \\
& =\frac{\left[S_{i}^{\left(v^{\prime}\right)}\right]^{2}}{N-1}
\end{aligned}
$$

The $\left[i_{i}^{\left(v^{\prime}\right)}\right]^{2}$, which initially solely represents self-control but may evolve into creativity, is proportional to $\left[i_{i}^{\left(v^{\prime}\right)}\right]^{2}$. As may be seen from column 7 in Table XV, the intensity of this self-control mounts ever more rapidly as lower ranks in the hierarchy are approached. In so long as self-interac- 
tion only represents self-control, there can be no creativity. However, in this system what were recessive $d$-genes can become considered as dominant $d$-genes. When this happens for the whole group, a complete reversal of the social rank ordering develops. But such reversal can also take place within a single individual. In essence, this means that $\left[i_{i}^{\left(v^{\prime}\right)}\right]^{2}$, which is equivalent to an $\alpha_{a p}^{\prime}$ frustrating experience, became transformed into an $\left[i_{i}^{\left(V^{\prime}\right)}\right]^{2}$, which is equivalent to an $\alpha_{a a}$ satisfying experience, without losing any intensity in the process.

There need not be any outward manifestation of this transformed selfcontrol. $\left[i_{i}^{\left(V^{\prime}\right)}\right]^{2}$ only implies cortical associations among stored traces of external events lacking any aura of negativism. Other than this, there are no limits to the kinds of traces which may by chance attain a high conditional probability of association. External manifestation of $\left[i_{i}^{\left(V^{\prime}\right)}\right]^{2}$, that is creativity, implies sufficient contact or awareness of external events to permit symbolic or beharioristic alteration of the enivornment in harmony with these heightened conditional probabilities of association. $\left[i_{i}^{\left(V^{\prime}\right)}\right]^{2}$ must not be confused with learning. I shall not attempt to go into the question of learning here other than to say that it should be most effective in high velocity individuals.

It is well recognized that a feeling of ecstasy, of extreme well being, accompanies any instance of creativity. A unit of $\theta_{a}^{(o)}$ arising from a single interaction represents such ecstasy. Recall that $\theta_{a}^{(o)}=0.25$. Examination of Table XV reveals that not until $R_{8}$ is reached in the descent through such a hierarchy of $N=11$ individuals will $\left[i_{i}^{\left(v^{\prime}\right)}\right]^{2}=\left[S_{i}^{\left(v^{\prime}\right)}\right]^{2} /(N-1)$ exceed 0.025 . It will exceed 0.25 for $R_{8} \cdots R_{11}$ in an $N_{b}=11$. And yet I have already indicated that some of these, probably at least the last two, will be so withdrawn from reality as to preclude any opportunity of creativity. At most, we can therefore anticipate only $2 / 11$ or $18 \%$ of the group to possess potentialities of creativity. As $N$ increases, proportionately more indiriduals will accommodate by social withdrawal and relatively fewer will possess potentialities of creativity.

Realization of these potentialities requires another set of conditions, opportunity for having made many satisfactory accommodations to new configurations of stimuli. As discussed in the later Sections XIV, G, 1 to 3 , this means that there must have transpired an increase in one's psychological area, $A^{\prime \prime}$, as a compensation for the reduced $A$ associated with a lowered $v$.

\section{E. Velocity and Home Range}

In my search for adequate formulations of the social use of space and time I have been guided by several competent mathematicians. With 
regard to home range, Mr. James U. Casby (see Calhoun and Casby, 1958, pp. 16-17) derived a function, $K / 2 \pi \sigma^{2}$. He called this term "visitation frequency." It deseribes the relative frequency of visiting a particular place in the environment. Later, though published earlier (Calhoun, 1957), Dr. Murray Eden derived the function, $\mu=d v / A$ to represent a communieation function defining the relative probability of one individual meeting another. $K / 2 \pi \sigma^{2}$ concerns arrival at a stationary point, while $d v / A$ concerns arrival at a moving point. It was only after I began this elaboration of concepts concerning social use of space that I realized the isomorphism of these two functions. A and $2 \pi \sigma^{2}$ become equivalent expressions as likewise do $K$ and $d v . d$ here is used in the sense of $S^{(v)}$, Eq. (107).

I have already pointed out in the discussion pertaining to Eqs. (35), $(80)$, and (88) to (91) that when a group is in a steady state in harmony with its heredity and environment, $\mu=1.0$, provided appropriate units of time are considered. It followed that $v, S^{(v)}$, and $A$ also have relative values of 1.0 at $N_{b}$, the harmonious steady state $N$. Therefore

$$
v S^{(v)} / 2 \pi \sigma^{2}=1.0
$$

Given this relationship, can it assist us in determining relative home range $\sigma$ ? We have already seen in Sections VI-VIII that contractions and expansions result from both intraspecific and interspecific interactions. Let us consider home range of members of a constellation as discussed in Section VIII, C.

On the average the constellation consists of one alpha, 6 beta, and 5 gamma individuals, ranked in this order:

$$
N_{b}=N_{\alpha}+N_{\beta}+N_{\gamma}
$$

where $N_{\alpha}=1, N_{\beta}=6, N_{\gamma}=5$. The $\alpha$ individual represents the ideal prototype. Each beta diverges an equivalent amount from the alpha. Likewise, each gamma also diverges from the alpha, but more so than the beta. By analogy to the discussion pertaining to Table XIII, the $d$-genotypes of the 12 individuals in a constellation will be represented as shown in Table XVIII. $\sigma_{S}$ in the table denotes alteration in the home range $\sigma$ resulting from the individual's $S^{(v)}$.

The $N_{\mathrm{j}}$ for the alphas, betas, and gammas, aceording to the formulation of Section XIII, B, 5 become respectively 12, 11, and 5. From Eq. (107) it follows that their $S^{(v)}$ are, respectively, 1.0, 1.091, and 2.40 . This increase in response-evoking eapacity, $S^{(v)}$, among beta and gamma individuals will lead to their reduetion of veloeity, $v$, respectively to 0.9167 and 0.4167 , as given by Eq. (108), when compared with $v=1.0$ for the alpha member. 
In terms of home range the $S^{(v)}$ function in Eq. (117) may be ignored, which means that:

$$
v / 2 \pi \sigma^{2}=1.0
$$

For this relationship to maintain in the face of reduced $v$ for beta and gamma individuals, $\sigma$ must correspondingly decrease, such that

$$
\sigma=(v / 2 \pi)^{1 / 2}
$$

Since $2 \pi$ is a constant we may ignore it for the purpose of determining

TABLE XVIII

\begin{tabular}{|c|c|c|c|c|c|}
\hline $\begin{array}{c}\text { Constellation } \\
\text { member }\end{array}$ & $d-\mathrm{Ge}$ & notype & $N_{\mathrm{j}}$ & $v$ (Eq. (10s)) & $\sigma_{S}(\mathrm{Eq} .(120))$ \\
\hline$\alpha$ & $(d)$ & $\mathrm{A} B$ & 12 & 1.0 & 1.0 \\
\hline$\beta_{1}$ & $(d)$ & a $\mathrm{B}$ & 11 & 0.9167 & 0.9575 \\
\hline$\beta_{2}$ & $(d)$ & a B & 11 & 0.9167 & 0.9575 \\
\hline$\beta_{3}$ & $(d)$ & a B & 11 & 0.9167 & 0.9575 \\
\hline$\beta_{4}$ & $(d)$ & a $B$ & 11 & 0.9167 & 0.9575 \\
\hline$\beta_{5}$ & $(d)$ & a $B$ & 11 & 0.9167 & 0.9575 \\
\hline$\beta_{6}$ & $(d)$ & a $\mathrm{B}$ & 11 & 0.9167 & 0.9575 \\
\hline$\gamma_{1}$ & $(d)$ & $a b$ & 5 & 0.4167 & 0.6457 \\
\hline$\gamma_{2}$ & $(d)$ & $a \quad b$ & 5 & 0.4167 & 0.6457 \\
\hline$\gamma_{3}$ & $(d)$ & a b & 5 & 0.4167 & $0.645 \pi$ \\
\hline$\gamma_{4}$ & $(d)$ & $a b$ & 5 & 0.4167 & 0.6457 \\
\hline$\gamma_{5}$ & $(d)$ & a b & 5 & 0.4167 & 0.6457 \\
\hline
\end{tabular}

Relative Home Ranges of Constellation Members

home range $\sigma$ relative to that of the alpha individual. Then

$$
\sigma_{S}=(v)^{1 / 2}
$$

In essence, this line of reasoning predicts that as velocity is reduced the area eneompassed by the home range will develop similar reductions. Relative home range sigmas, derived by Eqs. (107) and (120), as given in Table XVIII formed the basis for the relative sizes of home ranges within a eonstellation (Fig. 27).

In eonsidering the home range of the individual in the context of membership within a constellation of $N_{b}$ individuals, it is obvious that the term "area" possesses dual meaning. $\left(2 \pi 3 \sigma_{\alpha}{ }^{2}\right)$ approximately measures the $A$ 
of the constellation (Section VIII, C), while (2 $\left.2 \pi \sigma_{S}^{2}\right)$ represents that portion of each individual's home range within which it spends 0.394 of its time. Wach individual simultaneously "inhabits" two life spaces, his own and that of the group of which it is a member.

For all members but the alpha member, qualitative differences in $S^{(v)}$ lead to reductions in $v$, which in turn bring about reductions in $A$ such that the reduced $A$ is proportional to $v$. This accommodation will result in $\mu$ again exceeding 1.0 and will thus expose the individual to stress from an increased frequeney of contacts. Further aceommodation, again reducing $\mu$ to the relative value of 1.0 , can come only through quantitative reduction of the target diameter, $d$, since the $S^{(v)}$ qualitative aspect has become a stable factor. It will be recalled that the eoncept of the constellation presupposes a eapacity for emitting and receiving signals sufficient for any member to gain contact with any other member despite their spatial separation. Such signals amount to an increase in $d$ at the moment of this emission. The easiest strategy for regaining a $\mu=1.0$ will be for each individual to reduce the frequeney of signaling as much as it had reduced its velocity. This line of reasoning culminates in the conclusion that $v, \sigma^{2}$, and $d$ (in the sense of frequency of signaling) will all be reduced to values inversely proportional to $S^{(v)}$. With respect to the unchanged relative values of 1.0 for the alpha individual, these values for beta members become respeetively $0.9167,(0.9167)^{2}$ and 0.9167 , while for gamma members they become respectively $0.4167 \quad(0.4167)^{2}$, and 0.4167 .

Consistent with my objective of developing formulations adequate for aequiring data in concrete experimental studies, the above theoretieal conclusions may be tested for their applieation to groups more compact than represented by constellations. Given an experimental $N=12$ restricted to an area $A$, one can estimate relative velocity be determining the proportions of a series of time samples in which each individual is active and exposed to situations where social interactions do oceur. This velocity we ean designate by $\hat{v}$. Velocity so estimated will reflect true velocity more accurately than the eruder measure of number of contacts, $n_{c}$, used in the specific case in the diseussion pertaining to Eq. (113). This is beeause an animal may be active when all others are at rest.

The more velocity is reduced, as predicted by Eq. (108), the more the individual should restrict his travels to a smaller portion of the area utilized by the group. Of an originally common home range shared by members of a "compact" group, low veloeity members will eome to utilize only a portion. Thus, the ideal design of an area within which social organization is being studied must provide for many subareas to which visitations may be recorded. Otherwise, reduction in home range cannot be detected.

Likewise, the more an individual reduees his velocity, the more he should 
reduce the emission of signals eliciting social awareness of him by associates. Such signaling may involve other than vocal modes and may even involve reduction of $S^{(v)}$ itself. In the end, such reductions of $d$ and $S^{(v)}$ will produce an individual which, when encountered, is judged by associates as being dull, lifeless, and with flat affect, lacking the attributes of an appropriate object for social interaction.

\section{F. Velocity in High-Density Rat Societies}

While these formulations of social dynamics were being developed I was simultaneously pursuing empirical studies of social dynamics revealed by large groups of domesticated Norway rats. We shall examine those data which indicate (a) that densities greater than appropriate for $N_{b}$ suppress velocity, and (b) that an increase in vitamin $A$ above normal levels buffers the social system against the velocity-suppressing force of increased density.

During a 16-month period of 1960-1961 further studies in the habitat (Fig. 33), discussed in Section XII, C, were conducted. In this second series of studies the only habitat change involved altering the method of providing food and water. This change precluded the development of the behavioral sink discussed in Section XII, C. [See Calhoun (1962b) for a general account of these two series of studies.] However, our concern here will be with results not previously presented.

During the 13th, 15th, and 16th months of this study estimates of velocity, $\hat{v}$ were made for each of the 32 adult males in each of the two societies considered here. All males were fully mature, ranging in age from 10 to 15 months of age.

Procedure for velocity estimation: As illustrated diagrammatically in Fig. 33, each of the four pens in the room defining area $A$ contained two areas where social interaction occurred most frequently. One was on top of the elevated artificial burrows; the other was on the floor in the immediate vicinity of the sources of food and water. During each half hour of observation, each rat was given one velocity score for each of the eight locations visited. On 2 days, not more than 3 days apart, Dr. Kyle R. Barbehenn and I each recorded such velocity scores for 16 half-hour periods during each of the three months mentioned above. For a particular month, the estimated velocity thus consisted of the sum of the velocity scores for 32 half-hour periods of observation. The estimated velocity, $\hat{v}$, is here taken as the mean for three 32 half-hour sums.

The two societies were designated as $1 \mathrm{~A}$ and $2 \mathrm{~A}$. Thirty-two males in $1 \mathrm{~A}$ and 32 males in $2 \mathrm{~A}$ survived through the 15th month. A few males died 
just before the third set of velocity estimations due to injections of a monamine oxidase inhibitor (Catron), which substance had no influence on the velocity of survivors. Thus, for a few individuals, $\hat{v}$ is based on the mean of only two estimates of velocity for the 13th and 15th months.

- These estimates of velocity are shown as data in Fig. 38. Approximate regression lines were fitted by eye through these points for both $1 \mathrm{~A}$ and $2 \mathrm{~A}$. Velocities of $1 \mathrm{~A}$ males are markedly lower than those for $2 \mathrm{~A}$ males. According to the general formulation of velocity, one would anticipate that males in $1 \mathrm{~A}$ had been exposed to many more social restraints or sanctions in the form of aggressive actions from associates. This, in fact, was

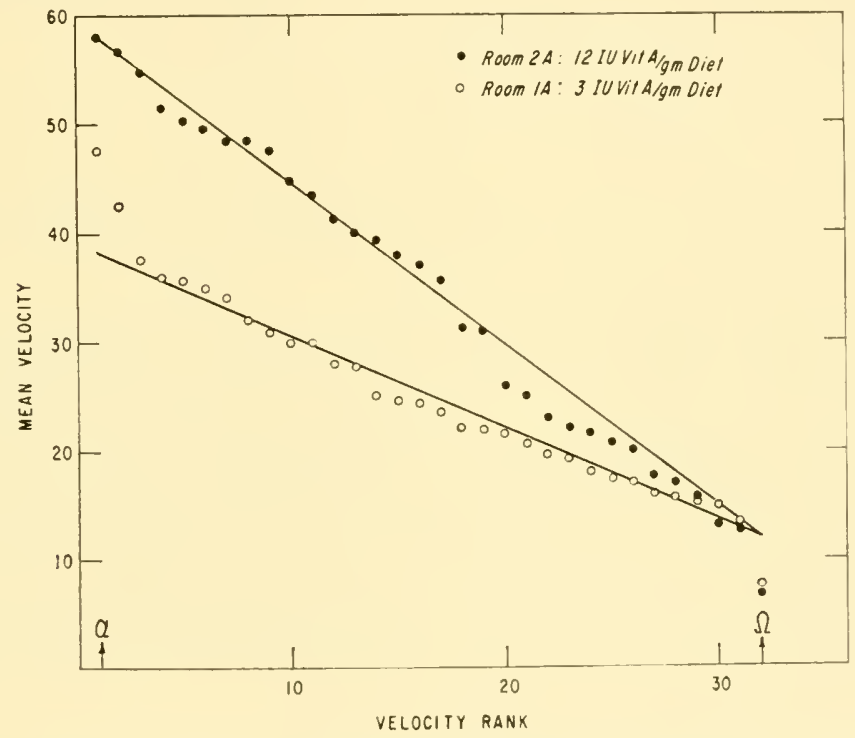

FIG. 3§. Velocity, velocity-rank relationships among male rats in a closed society.

the case as is reflected by the amount of scar tissue developed over the lumbar-sacral area from wounds received in fighting. On the basis of a 5point rating scale $(0=$ none, $5=$ most $)$, the mean scar tissue index for $1 \mathrm{~A}$ and $2 \mathrm{~A}$ males was, respectively, 3.24 and 2.49 .

The lowered intensity of fighting by $2 \mathrm{~A}$ males was associated with a higher vitamin $\mathrm{A}$ content of their diet. Both $1 \mathrm{~A}$ and $2 \mathrm{~A}$ rats were given an dentical synthetic diet except for vitamin A. $1 \mathrm{~A}$ rats received 3 international units per gram of diet, a high normal level in comparison to natural foods. However, the $2 \mathrm{~A}$ rats were given 12 international units per gram of diet, a level comparable to that given to humans in high potency vitamin pills. Although it is outside the objectives of the present discourse to detail 
the effects of increased levels of vitamin $A$ on behavior, it suffices to note that increases in vitamin A above normal levels acts as a kind of "tranquilizer" which reduces fighting but increases the prevalence of abnormal behavior (i.e., females become poorer mothers while males increase the frequency of exhibiting inappropriate sexual behavior).

Previously we have seen (Section XIV, A) that in an $N=11$ the observed velocities for mice, as calculated by Eq. (113), closely approximated the theoretical. We may ask the same equation of the present data. For $2 \mathrm{~A}$ the sum of velocity indices $\left[\sum_{i=1}^{Y} v_{i}^{\text {(obs) }}\right]$ for 16 hours of observation was 1089.2 , and for $1 \mathrm{~A}$ it was 804.7 . Let $v_{\alpha}^{(\exp )}$ and $v_{\Omega}^{(\exp )}$ represent the velocities of the alpha, 1st-ranked, individual and the omega, $N$ th ranked, individual, as calculated by Eq. (113). For $1 \mathrm{~A}, v_{\alpha}^{(\exp p}=48.77$ and $v_{\Omega}^{(\exp )}=$ 1.52 ; while for $2 \mathrm{~A}, v_{\alpha}^{(\exp )}=66.01$ and $v_{\Omega}^{(\exp )}=2.06$.

Had all the intermediate $v^{(\exp )}$ been calculated, as would have been represented by a straight line comnecting these extremes on Fig. 38 , it is quite obvious that the observed would differ significantly from the expected. One could drop the inquiry at this point and conclude that velocities for rats do not accord with theory as for the mice cited in Section XIV, A. However, there are two reasons for not dropping the inquiry at this stage. First, 32 males represent an $N$ almost three times the theoretical basic $N, N_{b}=12$ while the $N=11$ for the mice closely approximated this $N_{b}$. Second, the regression curve of relocity for the $1 \mathrm{~A}$ and $2 \mathrm{~A}$ males converge at the omega-ranked individual. Furthermore, this convergence is at a velocity 6 to 8 times that anticipated by Eq. (113). This fact suggests a minimum velocity, $v_{m}$, below which rats cannot reduce their velocity and long survive. Two such individuals with excessively reduced velocity are shown on Fig. 38. I can only say that, on the basis of the very few individuals which did develop such unusually low velocities, such individuals usually become bloated and usually shortly died.

Accepting the indication of the reality of $v_{m}$, is there a logical basis for recognizing its relative value? In the course of evolution, where the group becomes adjusted to an $N_{b}$, there will in any stabilized group be an $N_{b^{-}}$ ranked omega individual. In terms of Eq. (107) it is readily apparent that, where $R=$ rank in the sense used in Table XIII:

$$
N_{\mathrm{j}}=1+(N-R)
$$

Therefore, for any $i$ th individual where $i$ is equivalent to its rank $R$, Eq. (108) becomes

$$
v_{i}=\frac{1+N-R}{N}
$$


Since the rank, $R_{\Omega}$, of the omega individual is $N_{b}$ :

$$
\begin{aligned}
v_{\Omega} & =\frac{1+N_{b}-N_{b}}{N_{b}} \\
& =\frac{1}{N_{b}}
\end{aligned}
$$

Since $v_{\Omega}$ is the lowest velocity achicred in the normal $N_{b}$ selected by evolution, $v_{\Omega}$ most likely also represents $v_{m}$. Given a $v_{m}$ observed, $v_{m}^{\text {(obs }}$, we may calculate a $v_{\alpha}^{\text {(exp) }}$ appropriate to $N_{b}$. Recall that from Eq. (108) $v_{\alpha}^{\text {(rel) }}$ in relative terms $=1.0$ and the relative velocity, $v^{(\text {rel })}$, of any other ranked individual is by this equation represented as proportions of the alpha's velocity. $v_{\Omega}^{\text {(obs) }}$ or $v_{m}^{\text {(obs) }}$ from Fig. 38 is 12 . Therefore

$$
12=v_{\Omega}^{(\mathrm{rel})} \cdot v_{\alpha}^{(\exp )}
$$

And thus at $N_{b}$, and utilizing Eq. (123):

$$
v_{\alpha}^{\text {(expat } \left.N_{b}\right)}=v_{m}^{\text {(obs) }} / v_{\Omega}^{(\text {rel })}=v_{m}^{\text {(obs) }} \times N_{b}
$$

However, solving Eq. (124) requires that $N_{b}$ be known. The rats used were a domesticated albino strain, Osborne-Mendel. $N_{b}$ still might be 12, as we ean expect it to be for the wild type, but we have no way of knowing directly how domestieation has altered $N_{b}$. Furthermore, the artificial environment imposed possible changes on the area, $A$, factor in $\mu=d v / A$. So all that can be expected is that there is some optimum $N, N_{o}$, harmonious with the existing spatial structure of the environment and any changes arising through domestication. Now, using $N_{b}$ in the sense of $N_{o}$, values in relative (rel) terms become:

For $N=N_{b}, R=1: v_{\alpha}=1$

For $N=N_{b}$ or $N<$ or $>N_{b}, R=N_{b}$ or $N: v_{\Omega}=v_{m}=1 / N_{b}$

For $N>N_{b}, R=1: v_{\alpha}^{(\text {rel })}=1-\left[\frac{N-N_{b}}{N}\left(1-v_{m}\right)\right]$

When $N=\infty: v_{\alpha}=v_{m}=1 / N_{b}$

The general equation for $v$, where $v_{m}=1 / N_{b}$, and $R=$ velocity rank becomes:

$$
v^{\text {(rel) })}=R-N\left[\frac{v_{m}-\left\{1-\left[\left(N-N_{b}\right) / N\right]\left(1-v_{m}\right)\right\}}{N-1}\right]+v_{m}
$$


Assuming the validity of all assumptions inherent in Eqs. (124) to (126) we can now approximate the $N_{o}$ of the $1 \mathrm{~A}$ society of rats. The regression curve for $1 \mathrm{~A}$ shown in Fig. 38 gives $v_{\alpha}^{\text {(obs) }}=38$. Insertion of successive values of $N_{b}$ in Eqs. (124) and (125) shows that when $N_{o}=9$, $v_{\alpha}^{\left(\exp a t N_{b}\right)}=108$ and $v_{\alpha}^{(\mathrm{rel})}=0.36$. Since $0.36 \times 108=38.9$, it follows that, for the strain of rat under the existing environmental conditions, 9 individuals approximate $N_{o}$ for male rats.

Now we may return to the "tranquilizing" effect by which vitamin A "buffered" the 2A males from the velocity-inhibiting consequences of $N>N_{b}$. There needs to be a correction factor in Eq. (126) which, as a "tranquilizing" factor, $Z$, increases the slope of the velocity-velocity rank curve also increases, "piroting" about $v_{m}$. At present there is no
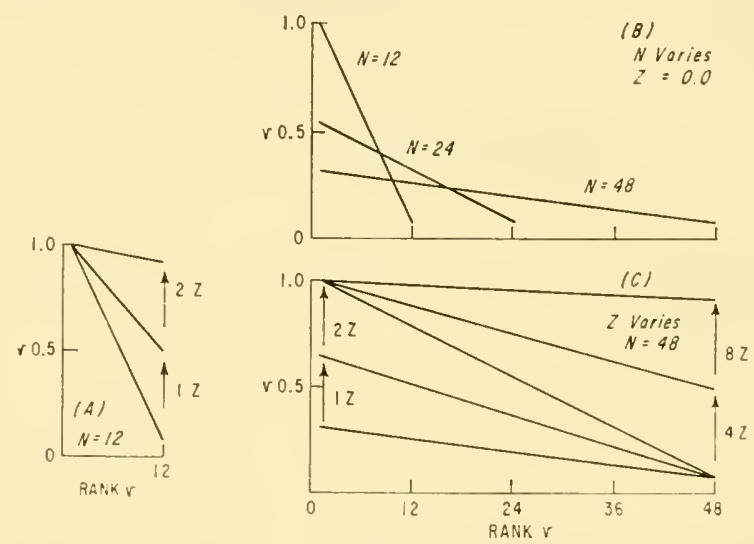

FIG. 39. Hypothetical effect of group size, $N$, and tranquilizer, $Z$, on velocity, $v$, when $N_{b}=12$.

a priori basis for determining this $Z$ factor, which can draw upon empirical evidence. However, one can visualize a likely formulation of $Z . Z$ implies a factor "blinding" awareness of the $d$ or $S^{(v)}$ of associates. Such perceptual blinding conforms with the $d^{\prime}$ factor of $\mu^{\prime}=d^{\prime} v^{\prime} / A^{\prime}$, previously alluded to. The critical issue concerns the influence of $Z$ upon $v_{\alpha}$. If the velocity of the first-ranked alpha individual never exceeds that appropriate at $N_{b}$, that is, if

$$
v_{\alpha}^{\left(N_{b}\right)}=v_{\max }=v_{M}=1.0,
$$

then we would have a partial basis for understanding how $Z$ alters $v$.

In the absence of adequate empirical data regarding the function of $Z$ on $v$, consideration of hypothetical relationships (Fig. 39) will facilitate our understanding to the point of enabling the design of critical experi- 
ments. First consider Eq. (125). Increasing $N$ reduces $v$ of all members. By the time $N$ is only a few times $N_{b}$, velocity of all members will be so reduced as to bias the probability of the social system surviving. Minimal velocity implies withdrawal from social interaction and restriction of activities to independent acquisition of food and water.

Now, assuming a $v_{M}$, even though it might exceed 1.0, Fig. 39 suggests that increasing $Z$ will eventually elevate $v_{\alpha}$ to $v_{M}$, while $v_{\Omega}$ remains at $v_{m}$ as shown in Fig. 38. Once $v_{\alpha}$ reaches $v_{M}$, further increase of $Z$ should reduce awareness of the $S^{(v)}$ of others to the point that $v_{\Omega}$ departs from $v_{m}$ and begins to approach $v_{M}$. At $Z=\infty, v_{\Omega}=v_{M}$. Far before $Z=\infty$, the velocity of all members of $N$ will be maximal for all practical purposes. A state of maximum conformity will then have been attained in which each individual views every other one as so like himself that no individual imposes restraints on the actions of any associate. All social organization must collapse, leaving a state of maximally moving independent particles, the random contact between any two of which will be equally satisfactory in consummating any interaction in which two individuals are necessary. This state demands equipotency of capacities. Complexity of behaviors will be limited to that degree possible by every individual having identical learning experience. Maximizing $Z$ becomes incompatible with a high state of learning and culture.

The human species appears to be embarked upon a journey of both maximizing $N$ and maximizing $Z$. If we are to avoid one of the other of the nirvana-like states of uniform $v_{m}$ or $v_{M}$, it behooves us to seek further insight from experimentation with animal groups.

Returning from theory to reality, we may consider some correlates of velocity. Most of the $1 \mathrm{~A}$ and $2 \mathrm{~A}$ rats discussed above survived to autopsy during the 17th month of the study. Each set of males was divided into five velocity class intervals, with as nearly as possible the same number of rats in each velocity range. Associated conditions are graphed in Fig. 40. As relocity increases, the amount of scar tissue derived from fighting increases. The somewhat S-shaped character of this curve conforms with historical events. In general, the more rats withdrew from social interaction by reducing their velocity, the fewer wounds they received. However, a few individuals, though having a very low velocity in late adulthood, were characterized by extensive scar tissue because they failed to withdraw as early in life as had their low velocity comrades. At the other extreme of velocity, territorial males or highly dominant individuals, who were territorial in the time dimension but not in space (i.e., the "changing-ofthe-guard" phenomenon through which several males share the dominant role in a particular area), by their status avoided attack and thus avoided being wounded even though they were extremely active and inflicted 
wounds on associates. Their lesser degree of scar tissue contributed to a reduction in the mean scar tissue index for high-velocity males.

Weights of adrenals, kidneys, and heart all tend to inerease as velocity increases. Despite lack of histological studies, what I suspect has happened is that as velocity drops as animals withdraw from social interaction, organ size decreases in accordance with decreased demands made upon them.

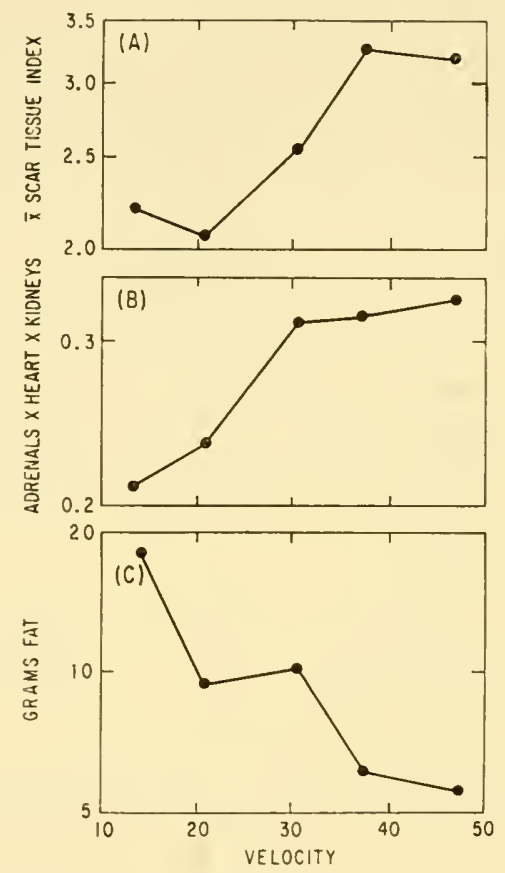

FIG. 40. Some major characteristics of rats affected by conditions that determine velocity.

Fig. 40 (B) simply shows the product of the weight of these three differeut organs.

Most sensitive to velocity and easy to measure is fat, Fig. 40 (C). Fat here represents those abdominal deposits most easily removed: the dorsal lumbar-sacral deposit, that in the genital mesentery, and those in the mesenteries of the gut. Though these deposits are rarely as large among males as for females, it is nevertheless quite clear that as rats slow down by social withdrawal they exhibit greater propensities for converting foodstuffs into fat. 


\section{G. Exploratory Behavior}

Ultimate exposure to some new configuration of stimuli represents the rommon factor in the three phenomena encompassed by the term, "exploratory behavior." These three phenomena are: (a) the rise and decline of a hyperactive state following exposure to a new eonfiguration of stimuli (see Section III, A, 4); (b) the rejection of new configurations (see Section III, A, 3); and (c) the seeking of new configurations. We shall now examine how the opportmnity for expressing such behaviors alters an individual's attitude toward its physical and social environment.

\section{The Hyperactivity Phexomexox}

In an animal's normal habitat this phenomenon may be anticipated to follow an encounter with a new configuration of stimuli at places in the normal home range where it has not occurred during customary travels. Field studies directed toward the elucidation of the consequences of such encounters are essentially nonexistent. Pearson (1960), by photographing marked mice as they move along their trailway systems, has found that, following the experience of being trapped and handled, mice not only become more active but also visit places within or near their normal home range which are normally infrequently visited. My study of the reaction of domesticated Norway rats following exposure to an activity alley (Section III, A, 4) represent this same type of situation with the exception that the induced state of hyperactivity must take place in the presence of the new configuration of stimuli represented by the alley.

Recall that the rat is placed in a compartment with an access door at one end of the alley. It does not have to enter, but many rats do so rather immediately. For example consider the 73 rats involved in the analysis of distance of termination of trips shown in Fig. 3. Analyses (Fig. 6) have been made of their hyperactivity during the initial 2.5 hours of their residence in the activity alley. From an initial high level, activity declines exponentially over an approximate $3-4$ hour period nearly to a base level maintained on the average through each of the 12 hours of normal heightened activity during the next 3 days. This pattern is shown diagrammatrically in sketch (1) of Fig. 41. Such heightened diffuse motor activity lacks any aura of goal direction and will be designated by the symbol, DMA. Such a configuration of new stimuli as is represented by the alley will be designated as $E$. If a rat is placed again in the alley for 2 hours on each of several consecutive days, no appreciable amount or duration of DMA oceurs on any day. This means that there has transpired an adjust- 
ment, $A$, to $E$ by the end of the initial 2 hours of exposure to $E$. After $A$, motor activity persists on the average at a base line intensity, $b$, unless some other new configuration $E$ is encountered. Thus, DMA represents increments of activity above $b$.

In the alley, $E$ can only include nonsocial physical stimuli. And yet in a social milieu of others of an animal's own species, the responses of an associate also represent an $E$. Provided such a social $E$ has not previously

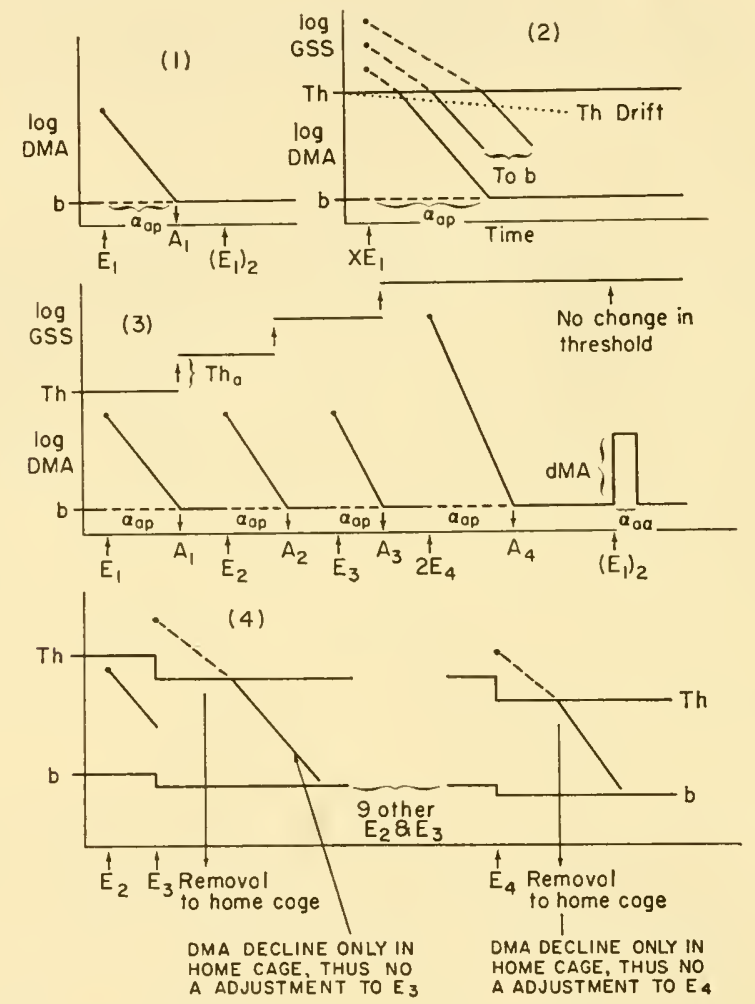

FIG. 41. Adjustment to configurations of stimuli. See text for comment.

been encountered or if the associate is in the nonresponsive, $[p]$, refractory state, then the latter's behavior, though perhaps experienced before, amounts to a previously unencountered $E$ in the sense that it does not permit directed motor activity leading to a satisfactory refractory period by the other individual involved in the encounter. For this reason, it will here be presumed that the period of decline of hyperactivity is equivalent to the $\alpha_{a p}$ frustrating type refractory period involved in normal social intercourse. 
If rats are maintained in isolation for several weeks or months and then placed in an emotional activity alley, a large proportion of them never go out into the alley from the "home" compartment. From this I infer that there is some upper threshold, Th, of DMA which "overloads" that neural cireuit permitting its expression. The three dots in sketch (2) denote such overloadings. In any sample of subjects, most of whom avoid entering the alley, there are a few with various lengthened latencies, causing delays of up to nearly the end of the 2-hour test period before initiation of the initial phase of intense hyperactivity. From this fact it is apparent that the normal DMA becomes replaced by some generalized stress state, GSS, which follows a similar (but lower?) rate of decline than the DMA. GSS lacks a striated muscle component. Once it has declined to Th, GSS becomes transformed to DMA. Such subjects, owing to their having been protected from impinging external stimuli for such a long time during their isolation, view the alley configuration as an increased intensity, $E$. An inference from this is that there is a slow drift downward of Th toward $b$ during the weeks of isolation when opportunities for adjustments $A_{1} \cdots A_{n}$ in response to $E_{1} \cdots$ to $E_{n}$ are absent. Thus, depending upon how extensive this drift has been, any particular $E$, such as represented by the activity alley, raises the GSS different amounts above Th. I suspect, though my data are not conclusive, that the more elevated GSS is above Th the lower will be its rate of decline. These differences are diagrammatically shown in sketch (2) of Fig. 41. Given sufficient time with no interference by other $E$ 's, DMA will eventually reach $b$ and an adjustment, $A$, transpires.

Now we may consider the more normal course of maturation, experience with a sequence of $E_{1} \cdots E_{n}$ [sketch (3) in Fig. 41]. One general observation first. 'The greater' has been the experience of rats in the sense of a larger number of different $E$ 's to which adjustments, $A$, have been made, the lower will be the probability of withdrawal in the form of failing to enter the alley from the starting "home" compartment. An explicit experiment concerning this point has already been eited with reference to Table Ib. Such results indicate an elevation of $\mathrm{Th}$ to $\mathrm{Th}_{a}$ following each $A$, and this elevation will be proportional to the magnitude of $E$ provided $E$ does not elicit a DMA exceeding Th. Thus, at some later time an intense $E$, designated as $2 E_{4}$ in sketch (3), will result in adjustment $A_{4}$, although had a $2 E$ configuration occurred earlier it would have resulted in the undesirable consequences accompanying elevation of DMA above 'Th. There is another conclusion, somewhat more tentative, though some of my results do support it. This is that with each successive $E$, the rate of decline of DMAA increases. In other words, the $\alpha_{a p}$ refractory period decreases. Thus, the more adjustments an individual makes, the better he will be able to eurtail $\alpha \int_{a p}=\theta_{f}$. I have already mentioned the failure of a second exposure to a 
particular $E$ to induce any material inerease in DMA. This means that if $E$ merely represents a configuration of strange stimuli with which the individual can interact in no meaningful way, the second exposure to $E$, that is $\left(E_{1}\right)_{2}$, will evoke no response. $\left(E_{1}\right)_{2}$ will merely be ignored. However, if some aspects of the $E_{1}$ configuration permit meaningful interaction, then $\left(E_{1}\right)_{2}$ will result in an interaction having an $\alpha_{a a}$ refractory period proportional to the evoked directed motor action, dMA. This $\alpha_{a a}$ will normally be of shorter duration than $\alpha_{a p}$ to $\left(E_{1}\right)_{1}$ since some components of the configuration are likely to be irrelevant to dMA. Furthermore, dMA may be expected to be maintained at near its initial intensity until the evoked behavior terminates. No further elevation of Th aceompanies $\left(E_{1}\right)_{1} \cdots\left(E_{1}\right)_{n}$. Persistent recurrence of any specific $E$ merely serves to prevent the drifting downward of Th.

Each adjustment to a new $E^{\prime}$ configuration resulting in an elevation in Th represents a contribution to the individual's psychological area, $A^{\prime \prime}$.

\section{The Seeking of New, Configurations}

Consider two groups of individuals, A and B, of which the members of each for a fairly long period merely reexperience particular sets of $E$ 's. Members of group A differ from those of B in that they are exposed to a larger assembly of different $E$ 's. Two such groups have been eonsidered in Seetion III, A, 5 and Table IIa. The fact that more of the A-type individuals entered the alley when exposed to this new $E$ configuration of stimuli indicates that reexperience of a larger assembly of different $E$ 's does maintain Th at higher levels despite some downward drift. The A-type individuals obviously have a larger psychological area, $A^{\prime \prime}$, than the B-type ones. $A^{\prime \prime}$ essentially connotes capacity to adjust. In any environment presenting frequent necessity for adjustment, a high $A^{\prime \prime}$ will prove advantageous. So we need to consider the question of maximizing $A^{\prime \prime}$.

Persistence in repeated interaction with certain E's is necessary or desirable because of acquired reward value accompanying the directed motor activity, dMA, appropriate to these E's. Such diIA can only preserve Th at a given level. Response to these $E$ 's involved in this dMA consumes time. If all waking time becomes relegated to dMA-eroking $E$ 's, the individual will develop a static $A^{\prime \prime}$. Furthermore, the more waking time becomes filled with repetitions of response to any given $E$, the more restricted will be $A^{\prime \prime}$. It is thus obvious that the best strategy for maximizing $A^{\prime \prime}$ will be to reserve a portion of one's waking hours simply for responding to new $E$ 's. Some as yet unknown but probably fairly long time, certainly of the order of several days even for rats, must elapse between one exposure to such a new $E$ and a reexposure, permitting sufficient extinetion of the $A$ 
adjustment so that at the re-exposure the $E$ elicits a DMA comparable to that of the initial exposure. Because of the limitations of time and space in which any individual's aetivities must transpire, maximizing $A^{\prime \prime}$ demands an aetire seeking of new $E$ 's. This seeking, I term $v^{\prime \prime}$. It represents a kind of velocity difficult to distinguish from the normal velocity, $v$, unless one is aware of the history of an individual with reference to its pattern of repeating specific dMA. In empirical experimental situations the relative magnitude of $v^{\prime \prime}$ may be determined by observing the probability of approaching a new $E$ introduced into an individual's home range so that normal movements will produce exposure. Ecologists concerned with controlling the density of species which damage human property or serve as hosts for diseases transmittable to man have been aware of $v^{\prime \prime}$ in a negative sense. They (e.g., Chitty and Southern, 19.5t) have noted the avoidance of new objects, such as poison baits or traps. This avoidance has been termed "the strange-object response."

\section{Active Rejection of New Configurations}

Distinct from the relative attraction to or avoidance of a new configuration is the phenomenon of physically rejecting or psychologically blo king awareness of new E's. Processes ineluded under this phenomenon of rejection may be designated as $d^{\prime \prime}$. A grasp of the types of phenomena subsumed under $d^{\prime \prime}$ may be obtained through considering a case observed in my laboratory.

Large "life-space" eages, LSC, were designed to provide an optimum situation for the breeding of the very sensitive wild Norway rats in the laboratory. A $16 \times 25$-ineh floor provided aceess to an aetivity wheel on one side and a lever on the other, which when pressed, provided a drop of water. From this floor two ramps led to a partitioned second floor of equal dimensions. From one side of the second floor rats had access to one $S \times$ $8 \times 6$-inch nest box, while two next boxes were accessible from the other side of the second floor. One male and two female adult rats lived in each of six cages. At the time in question three of these cages each also contained a recently weaned litter. Up to this time the water-providing lever apparatus had not been delivered by the manufacturer. In its place the adults were provided water through a drinking tute from a bottle, as had been the practice since they were eaptured in the wild as juveniles. When the lever apparatuses became available, one was inserted into each eage and the water bottle was removed. By the following morning when the cages were next examined, the situation in each cage was identical; all movable objects available to the rats, paper used as nesting material and orange peels, had been piled over the lever, completely hiding it. 
In those cages containing recently weaned young, the young soon scattered the pile of objects, exposing the lever, and in so doing accidentally pressed the lever and gradually learned its function. There then followed a repeated process of covering the lever by the adults and its removal by the young. Through this process the adults were forced to face $E$, represented by the lever and its attached water reservoir, sufficiently to permit an $A$ adjustment to $E$ through the DMA decline process. Several points may be deduced from these observations. Th for the adults had previously drifted downward toward $b$ so that the $E$ lever configuration caused DMA to exceed it. Furthermore, at weaning Th is sufficiently removed from $b$ that many $E$ 's will fail to evoke DMA elevation above Th. Had evolution not resulted in such a balance between neurology and physiology, animals just emerging out into the many $E$ 's of their environment would immediately be forced into a withdrawal state. Although I shall not go into this problem here, it is obvious that retardation of "weaning" increases the probability of withdrawal.

In the three cages lacking recently weaned young, the pile of material covering the levers remained undisturbed for several days until the rats were so weak from lack of water that it was apparent that their rejection of the lever was so complete that they would die before getting the opportunity to learn its function through chance depression of it. Replacing the former water bottle merely satisfied their thirst but failed to alter their rejection of $E$. "Teaching" the rats the lever was finally accomplished by taking all movable objects from the cage and gradually increasing the interval during which the water bottle was remored. It took 3 weeks to reach the same level of lever pressing by these rats that was obtained within 3 days by adults when young not only made rejection impossible but also set an example of adjusted interaction with the $E$ lever configuration.

Other examples of such $d^{\prime \prime}$ active rejection, but toward social $E$ 's, have already been given in Section XIII, B, 4 in connection with the three examples involving Barnett's Norway rats, the "Freedom Riders," and the formation of the $\mathrm{C} 57$ Colony $1 \mathrm{~B}$ of house mice. Thus, in terms of active rejection, $d^{\prime \prime}$ becomes essentially synonymous with intensity of action toward another, $i_{e i}^{(v)}$ as given by Eq. (114). But $d^{\prime \prime}$ must also encompass the more strictly psychological phenomena of psychological deafness or psychological blindness such as characterizes the "malingering type" of individual.

Note that this consideration of exploratory behavior has lead to formulations of $d^{\prime \prime}, v^{\prime \prime}$, and $A^{\prime \prime}$. As for prior comparable terms:

$$
\mu^{\prime \prime}=\left(d^{\prime \prime} v^{\prime \prime} / A^{\prime \prime}\right)
$$

Here $\mu^{\prime \prime}$ represents a third contact modifying function when relating to 
a social group. Just as with $\mu$ and $\mu^{\prime}$, so it is apparent here that the magnitude of $v^{\prime \prime}$ and $A^{\prime \prime}$ will normally change by comparable degrees in the same direction and that $d^{\prime \prime}$ will approximately vary inversely with $v^{\prime \prime}$.

\section{The Effect of Interval between E's on Velocity}

Further examination of the data in Table IIb, in the light of the formulations relating to exploratory beharior and configurations of stimuli, provides additional insight into the origin of reductions in velocity, both $v$ and $v^{\prime \prime}$. Refer to Section III, A, 3 for other comments. Presentation of the experiment in terms of the present formulations is as follows:

The subjects consisted of male albino Osborne-Mendel strain rats isolated at weaning. At this time each rat was placed in a $6 \times 6 \times 8$ inch eage from which it could not see out. Water and food were introduced through channels from the outside which prevented the rats from seeing the experimenters or being handled by them. All rats remained in such isolation for approximately three months before further treatment. These isolation cages may be termed an $E_{1}$ configuration of stimuli. Due to the long absence of opportunity for adjustment to new configurations of stimuli, the Th of all rats probably drifted toward $b$.

At the end of the three months of isolation the subjects were divided into four groups: A (20 rats), B (24 rats), C (16 rats), and D (16 rats). On each of 10 days during the next two weeks each member of Group $\mathrm{C}$ was exposed for two hours to a new configuration of stimuli, $E_{2}$, which was a Wahman activity wheel; all rats so exposed entered the wheel and ran during each of the 10 days. Similarly, members of Group $\mathrm{C}$ were exposed to an $E_{3}$ configuration of stimuli. This exposure consisted of placing the rats in a $2 \times 5$-foot pen where they had the opportunity to climb onto a central platform where a lever could be pressed to receive a drop of water. Each day of this 10-day training period half the members of Group D were exposed for two hours to $E_{2}$ and then, immediately following, for two hours to $E_{3}$. The other half of Group D were similarly treated but were exposed to $E_{3}$ just prior to $E_{2}$. Group A remained in their isolation cages during these two weeks.

During the third experimental week every rat in all four groups was given a two-hour exposure to the NIH Emotional Aetivity Alley on each of four successive days. This alley represented an $E_{4}$ configuration of stimuli. For half the rats in each group $E_{4}$ had a stationary floor, a condition we may designate as $E_{4 \mathrm{~A}}$. For the remaining rats $E_{4}$ had a tilting floor which clanged as the rats ran across it. This modifieation is designated as $E_{4 \mathrm{~B}}$, which represents a much more intense or strange configuration than $E_{4 \mathrm{~A}}$. Nany rats avoided entering the alley. 
Regardless of the amount of prior opportumity to adjust to novel configurations, the more intense $E_{4 \mathrm{~B}}$ elicited a more marked avoidance than did $E_{4 \lambda}$. (See Section III, A, 3.) Howerer, our present concern is with a different aspect of the results in Table IIb.

Upon exposure to $E_{4 \mathrm{~A}}$ it appears that prior experiences with $E_{3}$ was much more effective than with $E_{2}$ in reducing aroidance of the $E_{4 \mathrm{~A}}$ configuration. But members of both Groups $\mathrm{B}$ and $\mathrm{C}$ evinced much less avoidance of $E_{4}$ than did members of Croup A. This supports the formulation that prior opportunity to adjust to new configurations elevates $\mathrm{Th}$ so that at a following exposure to another new configuration of stimuli, DMA is less likely to exceed Th. Aroiding entering the alley is taken as evidence of DMA exceeding Th.

These results confirmed prior hypotheses. However, it was further assumed that rats of Group D would exhibit the most marked accommodation to $E_{4}$ since they would have had twice the opportunity for making adjustments to new $E$ 's. And yet even to $E_{4 \mathrm{~A}}$, the rats of Croup D showed little better capacity for adjustment than did members of Group B, and much less than did rats of Group C. Upon elevation of the intensity of $E_{4}$ to $E_{4 \mathrm{~B}}$, members of Group $\mathrm{D}$ exhibited an extremely more marked reduction in capacity to adjust than did the rats of Groups B and C, that presumably had less opportunity for "training" in making adjustments.

These results apparently contradict the theory. But consider the following. For rats exposed to new configurations of stimuli, such as $E_{4}$, but permitted to remain for several rather than for 2 hours, it has been noted that many individuals require up to 3 hours for DMLA to decline to $b$. In the 2000-odd tests where rats have been exposed to the $E_{4}$-type alley configuration to test for emotionality, the tacit assumption has been made that the remaining decline in DMA will take place after the return of the rats to their accustomed environment. However, this opportunity did not prevail when, after 2 hours in $E_{2}$ or $E_{3}$, rats were transferred to the opposite $E$.

Events presumably transpiring are diagrammed in the left-hand side of sketch (4) in Fig. 41. Cpon exposure to $E_{3}$ after only partial decline of DMA following exposure to $E_{2}$, the same increment in DMA is elicited, but its rise starts at the point of a still fairly high level of DMA. Thus, this second increment in DMIA forces it above the threshold, Th, where DMA is transformed from diffuse motor activity into the generalized stress state, GSS. Although GSS and DMA were not measured during $E_{3}$ (or during $E_{2}$, if it came second for Group D), both must have eventually completely disappeared after the usual return to the home cage. And yet the very failure of many members of Group $\mathrm{D}$ to enter the $E_{4}$ alley when given 
exposure to it suggests that for each degree of GSS induced by too close spacing of consecutive new $E$ 's, there had transpired a drop in Th and $B$ such that Th drops relatively more than $b$. Each of the ten opportunities for consecutive exposure to $E_{2}$ and $E_{3}$ must have narrowed the gap between $b$ and Th. Thus the later cxposure to $E_{4}$ must have caused "overloading" for most Group D rats to the extent that GSS had not declined to Th by the end of the two hours in $E_{4}$. This meant that decline in DNIA to base level took place in $E_{1}$. Return to $E_{4}$ for 2 hours on each of the following 3 days was characterized by persistence in avoiding entry into the $E_{4}$ alley configuration by most rats avoiding it on first exposure. Avoidance, $v^{\prime \prime}$, of any new $E$ will thus be proportional to the degree of CrSs "overloading" elicited by the new $E$ 's.

Although new $E$ 's have been considered above in the sense of physical nonsocial configurations, we may consider the consequences of too closely spaced new or undesirable $E$ 's in the social sense of $i^{(v)}$ 's of associates. Recall that such $i^{(v)}$ 's represent social restraints or sanctions imposed by associates. For such sanctions to become effective in reducing velocity, $v$, that is for causing a drop in baseline of activity, consecutive sanctions by the same or different associates must be sufficiently closely spaced to induce a GSS. No opportunity for $v^{\prime \prime}$ avoidance is possible. In fact, with the drop in velocity $v$ (synonymous with $b$ ), psychological area $A^{\prime \prime}$ must be restricted through failure to make adjustments $A$ to $E$ while still in the presence of $E$. And as we have seen, as $A^{\prime \prime}$ declines so will $v^{\prime \prime}$. This means that as velocity, $v$, declines, the individuals have even less capacity to avoid strange stimuli. However, recall that as $v^{\prime \prime}$ declines $d^{\prime \prime}$ increases.

I can cite no quantitative data to support this conclusion of $d^{\prime \prime}$ increasing as $v^{\prime \prime}$ decreases. However, the following observations support its reality. While making the observations on velocity of rats summarized in Fig. 38, I was consistently impressed by the manner in which most very low-velocity rats moved "through" their associates. During those rare times when active, they would pass by associates as if completely psychologically blind to their presence. Furthermore, their blasé, unaffective mode of posture equally failed to elicit response from associates. The completeness of this psychological rejection of reality is reflected in their smaller organ weight and larger amount of fat (Fig. 40), corresponding to states characterizing rats maintained in approximate isolation by restrictions to groups of 1 male with 2 females in small cages or pens.

A further corroborative observation comes from the study referred to in Section XII, A. Among the wild Norway rats in that study was a small group designated as possessing an array of aberrant symptoms and behavior which I called the "syndrome of the social outcast." From compari- 
son with rats described in Sections XII, C and XIV, F, I now know that these social outcasts must have been very low-velocity rats. Of all the rats involved in the study referred to in Section XII, A, they were the only ones ever caught in "Havahart" traps. These large, shiny wire-meshed traps with a door opened at either end and shiny metal treadles on the center floor were regularly placed on trials. All other rats invariably ran around these traps. Yet the social outcasts apparently ran into them without ever sensing their presence. This sensory unawareness is the low $v^{\prime \prime}$ factor characterizing low-velocity rats.

\section{Conclusion}

Man did emerge from the trials, successfully orercome, of a myriad of ever more simple forms. I have attempted to formulate some phenomena which have affected man's social evolution. Some of these phenomena appear no longer directly operative on the human animal. They nevertheless left their imprint on man's capacity to adjust physiologically and psychologically to the social system in which he lives. Foremost among these presumed legacies are the limitations imposed upon him from his origin out of an evolutionary line in which optimum adjustment demanded living in small groups, not exceeding twice twelve individuals.

Only yesterday, as one may measure evolutionary time in units of ten thousand years, did man begin his attempt to escape this evolutionary bond. But cultural evolution has not produced escape from this bond, merely accommodation to it. Human society has developed the form of a many-layered chain link armor. Each link is composed of not much less than, nor many more than, twelve individuals. The links have a fluid character. Through time, any one individual shifts his membership back and forth among several joining links. This poetic view embodies the essence of reality.

In contrast to such evolutionary legacies, there exist certain principles of social physics which must affect all social animals, man included. These principles derive from certain universals I have called velocity, target diameter, area, basic group sizc, the social refractory period, threshold for tolerance for change, and the like. I am fully cognizant that my formulations represent only crude approximations to reality, and may in fact contain several errors of logic. Yet we must develop adequate fornulations along the lines I have attempted or all efforts to gain insight into the individual's involvement in social action will prove sterile.

This sterility will crown the endeavors of both classical physiology and 
psychology. We can no longer afford to ignore the impact of the social setting on the individual's behavior and physiology. And without knowledge of evolutionary limitations and universal principles of social physics, consideration of the social setting will also prove of little avail. The search for conceptualizations, adequate for furthering this objective, serves as the justification for inclusion of my effort to introduce the more strictly physiological discussions by the other authors in these volumes.

\section{References}

Barnett, S. A. (1955). Competition among wild rats. Nature 175, 126.

Birdsell, J. B. (1953). Some enivronmental and cultural factors influencing the structuring of Australian Aboriginal populations. Am. Naturalist 87, 169-207.

Birdsell, J. B. (1957). Some population problems involving Pleistocene man. Symp on Quant. Biol. 22, 47-69.

Braidwood, R. J., and Reed, C. A. (1957). The achievement and early consequences of food-production. Symp. on Quant. Biol. 22, 19-31.

Broadbent, D. E. (1958). "Perception and Communication." Pergamon Press, New York.

Caldwell, J. M., Ranson, S. W., and Sacks, J. G. (1951). Group panic and other mass disruptive reactions. U.S. Armed Forces Med. J. 2, 541-567.

Calhoun, J. B. (1949). A method of self-control of population growth among mammals living in the wild. Science 109, 333-335.

Calhoun, J. B. (Ed.) (1949-1957). Anmual reports-North American Census of Small Mammals.

Calhoun, J. B. (1952). The social aspects of population dynamics. J. Mammal. 33, 139159.

Calhoun, J. B. (1956). A comparative study of the social behavior of two inbred strains of house mice. Ecol. Monographs 26, 81-103.

Calhoun, J. B. (1957). Social welfare as a variable in population dynamies. Cold Spring Harbor Symp. on Quant. Biol., 22:339-956.

Calhoun, J. B. (1962a). A behavioral sink. In "Roots of Behavior" (E. L. Bliss, ed.), Chapter 22. Harper Hoeber, New York.

Calhoun, J. B. (1962b). Population density and social pathology. Sci. Am. 206, 139-146.

Calhoun, J. B. (1963). The ecology and sociology of the Norway rat. U. S. Public Health Service Pub. No. 100s, Government Printing Office. 2 s pp.

Calhoun, J. B., and Casby, J. U. (1958). Calculation of home range and density of small mammals. U. S. Public Health Monograph No. 55.

Calhoun, J. B., and Webb, W. L. (1953). Induced emigrations of small mammals. Science 117, 35\$-360.

Carpenter, C. R. (1962). Field studies of a primate population. In "Roots of Behavior" (E. L. Bliss, ed.), Chapter 21, Harper (Hoeber), New York.

Chitty, D., and Southern, H. N. (1954). "Control of Rats and Mice." Oxford Univ. Press (Clarendon), London and New York.

Dahlberg, B. L., and Gucttinger, R. C. (1956). The white-tailed deer in Wisconsin. Wisconsin Conservation Dept. Tech. Wildlife Bull. No. 14.

Deevey, E. S., Jr. (1960). The human population. Sci. Am. 203:194-204. 
Duff, W., and Kew, M. (1957). Anthony Island, a Home of the Haidas. Report of the Provineial Museum of Natural History and Anthropology.

Elton, C. (1942). "Voles, Mice and Lemmings-Problems in Population Dynamics." Oxford Univ. Press, London and New York.

Frederieson, E. (1951). Time and aggression. Psychol. Rev. 58, 41-51.

Hall, C. S. (1934). Defecation and urination as measure of individual differences in emotionality in the rat. Psychol. Bull. 31, 604.

Hallowell, A. I. (1960). Self, society, and culture in phylogenetic perspective. In "The Evolution of Man." (S. Tax, ed.), pp. 309-372. Univ. of Chicago Press, Chicago, Illinois.

Hardin, G. (1960). The competitive exclusion principle. Science 131, 1292-1297.

Hayne, D. W. (1949). Two methods for estimating population from trapping records. J. Mammal. 30, 399-411.

Hutchinson, G. E. (1957). Concluding remarks (to Population Studies: Animal Ecology and Demography) Cold Spring Harbor Symposia on Quant. Biol. 22, 415-427.

Kabat, C., Collias, N. E., and Guettinger, R. C. (1953). Some winter habits of whitetailed deer and the development of eensus methods in the Flag yard of northern Wisconsin. Wiseonsin Conservation Dept. Tcch. Wildlife Bull. No. 7.

Leopold, A. S., Riney, T., MeCain, R., and Tevis, L., Jr. (1951). The jawbone deer herd. State of Calif. Dept. Nat. Resources, Game Bull. No 4.

Moreno, J. L. (1953). "Who Shall Survive?' Beacon House, Beacon, New York.

Moreno, J. L., and Jemnings, H. H. (1960). Statisties of social eonfiguration. pp. 19-51. In "The Sociometry Reader" (J. L. Moreno et al., eds.), The Free Press, Glencoe, Illinois.

Patric, E. F. (1958). Some Properties of the Small Mammal Populations of the Huntington Wildife Forest. Ph.D. Thesis, College of Forestry, New York State Univ., Syracuse, New York.

Pearson, O. P. (1960). Habits of Microtus californicus revealed by automatic photographic recorders. Ecol. Monographs 30, 232-249.

Ranson, S. W. (1949). The normal battle reaction: Its relation to the pathologie battle reaction. In "Combat Psychiatry," Bull. U.S. Army Med. Dept. 9, Suppl., 3-11.

Riesman, D., Glazer, N., and Denney, R. (1953). "The Lonely Crowd." Doubleday, Garden City, New York.

Sanders, R. D. (1939). Results of a study of the harvesting of white-tailed deer in the Chequamegon National Forest. Trans. North Am. Wildlife Conf. 4, 549-553.

Schaefer, E. S. (1959). A eircumplex model for maternal behavior. J. Abnormal Social Psychol. 59, 226-235.

Sehaefer, E. S. (1961). Converging conceptual models for maternal behavior and for child behavior. pp. 124-146. In "Parental Attitudes and Child Behavior" (J. C. Glidewell, ed.), C. C. Thomas, Springfield, Illinois.

Sehneirla, T. C., and Tobach, E. (1962). Eliminative responses in mice and rats and the problem of "emotionality." In "Roots of Behavior" (E. L. Bliss, ed.), Chapter 15. Harper (Hoeber), New York.

Schorger, A. W. (1953). The white-tailed deer in early Wisconsin. Trans. Wisconsin Acad. Sci., Arts and Letters 42, 197-247.

Shiras, G. III. (1921). The wildlife of Lake Superior, past and present. Natl. Geograph. Mag. 40(2), 113-204.

Stewart, J. Q. (1948). Coneerning soeial physies. Sci. American 178, 20-23.

Swift, E. (1948). Wiseonsin's deer damage to forest reproduetion survey-final report. Wisconsin Conservation Dept. Publ. $\mathbf{3 4 7}$. 
Swift, E. (1946). A history of Wisconsin deer. Wisconsin Conservation Dept. Publ. 323. von Foerster, H., Mora, P. M., and Amiot, L. W. (1960). Doomdsay: Friday, 13 November, A. D. 2026. Science 132, 1291-1295.

Webb, W. L., and Rosasco, E. M. (1953). An Investigation of the Properties of Induced Mass Movements of the Small Mammals of the Genera, Peromyscus, Clethrionomys, Blarina and Sorex. 100 pp. mimeographed, College of Forestry, State University of New York. Re Contract DA-49-007-MD-325, Medical Research and Development Board, Office of the Surgeon General, U. S. Army.

Whyte, W. H. (1956). "The Organization Man." Simon and Schuster, New York.

Zimmerman, C. C., and Broderick, C. B. (1954). Nature and role of informal family groups. Marriage and Family Living 16, 107-111.

Zimmerman, C. C., and Cervantes, L. F. (1960). "Successful American Families." Pageant Press, New York.

Zippin, C. (1956). An evaluation of the removal method of estimating animal populations. Biometrics 12, 163-189. 



\section{Endocrine Adaptive Mechanisms and the Physiologic Regulation of Population Growth*}

\section{J. J. CHRISTIAN}

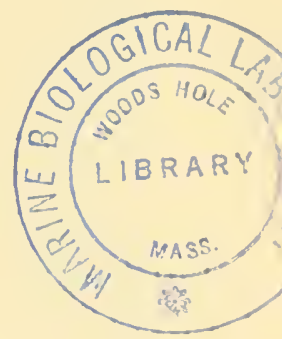

Division of Endocrinology and Reproduction, Research Laboratories, Albert Einstein Medical Center (North), Philadelphia, Pennsylvania

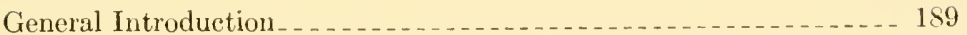

Part 1. The Endocrine Adaptive Mechanisms _. _ _ _ _. _. _ _ _ _ 191

I. Introduction _.

II. The Endocrine Glands of Adaptation ... _...

A. The Adrenal Glands_... . . . . . . . . . . . . . . . . . . . . . . 192

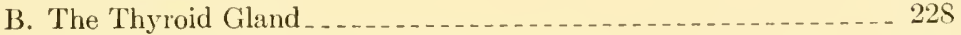

C. Other Endocrine Adaptive Factors _. . . . . . . . . . . . . . . 240

D. General Measurements of the Endocrine Adaptive Responses _ . _ 242

Part 2. Physiologic Adaptation and Mammalian Populations _. . . . . . . 261

I. Introduction $\ldots$

II. Endocrine Responses to Social Pressures and to Population Density _- 263

A. Experiments in the Laboratory with Populations of Fixcd Size - _ 263

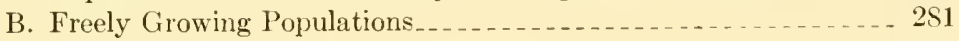

C. Natural Populations _... . . . . . . 300

III. Conclusion _. 325

References _...

\section{General Introduction}

Endocrine adaptive responses have become of particular interest to the mammalogist in recent years because of the likelihood that they play an important role in the regulation of the growth of mammalian populations. Sufficient evidence has aceumulated from the field and laboratory to warrant stating with fair certainty that these adaptive mechanisms are operative in and related to changes in the size of manmalian populations. However, there is still considerable uncertainty about their preeise role and relative importance in the regulation of population growth, especially with regard to generalizing to a large number of species from the few species for which data are presently available. There also exists a great deal of un-

* Note added in proof. For additional referenees pertaining to recent work on endocrines and population, the reader is referred to Christian (1961, 1963a \& b). 
certainty about the relationships of various environmental factors to the adaptive mechanisms. Then, besides the interest in adaptive mechanisms in relation to population growth, there is the frequently overlooked fact that these same physiologic reactions may effect profound morphologic changes in the members of a population and therefore directly affect the taxonomist who must use morphologic eriteria for distinguishing species and subspecies. It is entirely possible that many subspecific descriptions have been based on morphologic differences resulting from differences in the densities of the populations on which the descriptions are based. The mammalogist interested in reproduction in mammals must take adaptive mechanisms into consideration, as alterations in reproductive functions are an integral part of these same adaptive responses. Therefore there is adequate justification for this chapter on the endocrine adaptive responses, their effects, and their relationships to the densities of mammalian populations.

No matter how well an animal may be genetically adapted to its general environment, it still must have sufficient adaptive flexibility to meet the daily and seasonal environmental changes, as well as emergency situations, to which it will normally be subjected, and still maintain a constant internal environment. Nothing in the daily life and external environment of an animal remains constant; on the contrary there frequently are very sudden, often extreme, shifts in the environment which are stimuli that, if unopposed, would alter the internal environment of the animals. But the internal enviromment must remain constant if the animal is to survive. Therefore there must be a constantly active system of physiologic feedback mechanisms to compensate immediately for any tendeneies to shift the internal environment. Howerer, these adaptive responses do not take place without produeing measurable effects in the organs and glands primarily responsible for meeting the altered demands. Compensation for a life-maintaining change frequently occurs at the expense of some function less immediately important for survival, for example, reproduction. Consequently reproductive function declines measurably in the face of a need to maintain a constant internal physiologic state in the presence of adversity. The adaptive responses are changing constantly in degree to meet constantly changing daily circumstances, and it is generally thought that a certain amount of change is necessary to maintain the integrity of the system so that it will be capable of responding to more demanding circumstances. It is not surprising that at any given moment the physiologic status of a mammal reflects its total environment and that the whole system is in a constant stage of change, but for these same reasons it becomes difficult to study such a dynamic system in the complex environments of natural populations. Therefore a great deal of the existing evidence on the adaptive mechanisms of mammals, especially in relation to population density, has been gained by studies in the laboratory. 
Past research on the adaptive mechanisms has emphasized the adrenal cortices and to a lesser extent the thyroid gland and their hormones. Unquestionably the adrenal cortex is essential to life and plays a basic role in the adaptive responses, nevertheless there is a real tendeney to overlook the paramount importance of other systems and organs which also respond to adverse circumstances. In fact their actions are simultaneous with and inseparable from the actions of the adrenal cortex in many instances. A great many responses on the part of the organism act in concert to prevent any alteration in the basic physiology of the animal and to meet emergency needs. The central nervous system is a major and integral part of these adaptive mechanisms. Our understanding and interpretation of the physiologic changes taking place under a given set of circumstanees too frequently suffer from a tendency to think statically and in terms of isolated organs, systems, or hormones-a result of the kind of experimental approach necessary to understand the actions of various glands and their secretions. The isolated organ eoncept must give way to thinking in terms of dynamically interacting systems. However, to deseribe these mechanisms and their effects in dynamic inclusive terms is extremely difficult and has been made even more so by the recent elucidation of the key role played by the central nervous system in regulating the activities of the glands of internal seeretion, as well as by the realization that we are dealing with an enormously complex interacting system further complieated by the complex temporal relationships of the responses of these systems to the applied stimulus and to each other.

The balance of this chapter will be devoted to a more detailed discussion, first of the physiologic adaptive mechanisms themselves, and then with particular attention to the evidence implicating these responses in the regulation of the growth of mammalian populations. An attempt will be made to elarify some of these responses and to indicate areas where further research is needed, especially in relation to behavior and population density. Subjects which are adequately eovered in the usual textbooks of physiology will be omitted or only briefly summarized.

\section{Part 1. The Endocrine Adaptive Mechanisms}

\section{Introduction}

When a mammal is subjected to a stimulus which, if unopposed, would result at least in a change in its internal physiology, and more likely produce a circulatory collapse, a series of neural, neuroendocrine, endocrine, and vaseular responses follow which counteract the deleterious effects of the 
stimulus and also supply the increased needs of many tissues in order to meet the situation. Selye (1950) introduced the phrase "alarm stimulus" to describe such a stimulus which produces shock and evokes the usual physiologic responses to shock. In the present account an alarm stimulus is defined as any stimulus which, when applied to a mammal, tends to alter fluid and circulatory homeostasis, and therefore necessitates a physiologic adaptive response. This definition is somewhat circular insofar as it is in terms of a response, but it is not restrictive, and it does not imply that the adrenal cortex (at least that part responsible for the secretion of carbohydrate-active corticoids) is an essential participant, as is so often assumed. There may be qualitative similarities in the responses to different stimuli, but detailed studies suggest that there are all degrees of variation in the degree of participation of various systems and organs to a given stimulus. Probably the prime objection to the current concept of "nonspecific" response is the practical one that uncritical usage has tended to obscure important differences in the physiologic responses to different stimuli.

It should be pointed out that the degree of these responses appears to be relative, as the same responses qualitatively are essential for daily life, but must increase quintitatively in the face of adverse circumstances. A primary function of the endocrine adaptive responses is to insure an adequate circulation with an adequate supply of glucose and oxygen to tissues essential for emergency situations. Part of this function is the maintenance of an adequate circulatory volume and proper electrolyte and fluid balances. These adaptive responses will be discussed in greater detail in the following account.

\section{The Endocrine Glands of Adaptation}

\section{A. The Adrenal Glands}

\section{INTRODUCTION}

This discussion is primarily for the benefit of those who are interested in the physiological and comparative aspects of mammalogy. Therefore what is known of the endocrine adaptive mechanisms will be outlined without dwelling on details or becoming involved in the minor details or controversies of today's frontiers in endocrinology.

Rescarch on adaptive mechanisms to a large extent has centered around the adrenal glands, especially the cortex. One of the factors tending to synonomize "stress" with adrenocortical activity has been the measurement steroid secretion, weight, ascorbic acid depletion, and cholesterol 
content of the adrenals to determine whether and to what degree a stimulus produces "stress." Although the adrenals are of primary importance in physiologic adaptation to changing needs, it is important not to equate adaptation solely with adrenal function or to assume that the only function of the adrenals is to enable an organism to meet new and sudden demands. It is appropriate, especially for the mammalogist, to discuss adrenal glands in some detail because of their importance and beeause of the convenience of using them as indices of the degree of adaptive response to particular situations or stimuli. However, judgment must be used in interpreting the results of measurements of adrenal funetion, and one must realize that there are many other responses which are measured with extreme difficulty, and yet others may be completely masked by extraneous factors.

a. General morphology of the adrenal glands. The anatomy of the adrenal glands is diseussed in detail in many texts and papers on histology, gross anatomy, and comparative anatomy. Attention is called to the books by Bourne (1949), Hartman and Brownell (1949), Bachman (1954), and Jones (1957) for general treatments, especially from the comparative point of view.

The adrenal glands are yellowish paired organs lying at or near the anterior poles of the kidneys. Their position and form vary considerably from species to species. For example, in rabbits (Sylvilagus, Oryctolagus) they are oval discoid organs elosely applied to the vena cava; in woodchucks (Marmota) they are sausage-shaped and lie between the kidneys and the midline, usually closer to the latter; in mice and voles of almost all speeies they are round, oval, or pyramidal and lie approximated to the poles of the kidneys; and in the bats Myotis and Pipistrellus they lie beneath a layer of the renal capsule. These examples simply serve to illustrate the wide variations that oceur in their gross shape and position.

Two distinct portions of the adrenal are discernible when they are sectioned and examined grossly: a dark reddish brown or gray central core, the medulla; and a wide outer portion, the cortex, which is usually yellowish but may be gray or even translucent reddish brown, depending on the aetivity of the gland. The yellow color is imparted by lipids eontained in the cortical cells; thus color will vary with changes in the lipid content. Usually the cortex is quite wide, comprising from one-half to two-thirds of the radius of the gland. However, in some of the adult soricid shrews (Sorex fumeus, S. cinereus, S. palustris, S. dispar, and Microsorex hoyi) the gland consists almost entirely of medulla and has a very narrow cortex only a few cells wide. The extreme narrowness of the cortex is especially pronouneed in mature male shrews.

The adrenal gland is surrounded by a connective tissue eapsule from which a stromal framework of connective tissue descends into the cortex. 
The amount of eortical stroma may vary considerably; it is inconspicuous in most rodents whereas it is marked in most carnivores.

$b$. Zonation of the adrenal cortex. Three distinet major zones usually are identifiable histologieally in the cortex, although the zonation is diffieult to discern in a number of species (Bourne, 1949). An outer thin zona glomernlosa lies just beneath the capsule and consists of loops or balls of rather large cells with relatively clear eytoplasm. Central to the zona glomerulosa is a wide central zona faseiculata, which is composed of radially arranged straight cords of polyhedral cells that usually contain numerous cytoplasmie lipid racuoles. Lipid vacuoles oecur in the cortical cells of most species, but they may be absent in some, for example, the golden hamster (Mesocricetus auratus) (Alpert, 1950; Knigge, 1954a; Schindler and Knigge, $1959 \mathrm{a})$. Little or no lipid is present in the cortices of cattle, sheep, and pigs (Deane and Seligman, 1953). When present, the vacuoles may vary considerably in size and number, depending on variations in the activity of the eortex. The eells of the outer half of the zona faseiculata usually are larger and contain more lipid than those in the inner half of the zone. The fascicular cords are arranged as paired columns of eells lining vascular sinusoids in man and monkeys (Elias and Pauly, 1956), but is continuous in rats, the sinusoids penetrating the continumm (Pauly, 1957). The latter normally contain large amounts of blood circulating from the arteries in the capsule to the medullary venous sinusoids and adrenal vein. There are variations in the cireulatory arrangement with speeies, and it is more complex in detail than has been described here, but these matters are thoroughly covered elsewhere (Gersh and Grollman, 1941; IIartman and Brownell, 1949; Harrison, 1951, 1957; Elias and Pauly, 1956; Pauly, 1957). The cells of the fasciculata, when stained by routine procedures, bear a marked resemblance to the luteal cells of the ovary, interstitial cells of the testis, and, although less closely, the eells of the "brown fat" or "hiberuating gland" in its usual functional state. The zona retieularis forms a fairly wide cortical band between the medulla and the zona faseiculata in most speeies, but it is not always present (Hartman and Brownell, 1949). Its cords (or cortical continu'm) are more or less continuous peripherally with those of the zona fasciculata, but they rapidly break up into a reticular network as they proceed centrally toward the medulla. The eells are generally smaller than other cortical cells and usually contain no vacuoles. However, when vacuoles are present, they are usually very large.

There is need for a detailed, well illustrated, and thorough diseussion of the comparative morphology of the adrenal glands which would inelude a wide variety of species and a sufficient number of animals of each species to deseribe age and sex, as well as seasonal and environmental, relationships. It is not the purpose of the present discussion to dwell on the anatomy 
of the adrenal glands, but a brief summary of the partieularly useful and more recent publications on the subject will be given. The books which already have been listed discuss the adrenals of a large number of speeies, but the descriptions and illustrations are limited. However, the histology and histochemistry of the adrenals of a few species are discussed in considerable detail in a number of papers. The recent publications of Elias and Pauly (1956) and Pauly (1957) describe the microscopic anatomy of the adrenal glands of laboratory rats and humans. These papers are well illustrated, and the stereographic reconstructions of serial sections are helpful in understanding the adrenal morphology of these two species. One of the important facts brought out in these papers is that the adrenal cortex of the rat is not arranged in cords as it is in humans and monkeys. The parenchyma of the rat adrenal cortex is a continuum which is tunneled by vascular channels. A number of additional papers deal with the anatomy, circulation, or histochemistry of the adrenals of laboratory rats, especially with regard to function, zonation, and reactions to various stimuli (Howard, 1938; Flexner and Grollman, 1939; Greep and Deane, 1947, 1949a; Deane et al., 1948; Deane and Morse, 1948; Cain and Harrison, 1950; Feldman, 1950, 1951; Cater and Stack-Dunne, 1953, 1955; Josimovich et al., 1954; Jones and Spalding, 1954; Jones and Wright, 1954a, b; Christianson and Jones, 1957), and other more general papers on the histochemistry and function of the adrenals are based largely on material from laboratory rats (Dempsey, 1948; Greep and Deane, 1949a, b; Sayers and Sayers, 1949). The differenees in morphology between the adrenals of wild rats (Rattus norvegicus and Rattus alexandrinus) and those of Norway rats from the laboratory have been described by Rogers and Richter (1948), and the histology of wild and laboratory Norway rats has been deseribed and compared by Mosier (1957). A comparative study of the vascularization of the adrenals of rabbits, rats, and eats has been made by Harrison (1951) and followed by a description of the adrenal circulation and its regulation in the laboratory rabbit (Oryctolagus) (Harrison, 1957).

The histology of the adrenal glands of the prototherians Ornithorhynchus and Tachyglossus has been deseribed in considerable detail by Wright et al. (1957). The bulk of the chromaffin tissue was found in the lower pole in these species rather than in the more usual central position. The cortices of these species also differ considerably in their histologic appearance from those of eutherians. We have mentioned above that the adrenals of North American sorieids have strikingly little cortical tissue, although a critical study of this material has not been made (J. J. Christian, umpublished). Lanman (1957) has described the fetal zones of the adrenals of the following fetal or neonatal primates: macques (Macaca mulatta), potto (Perodicus potto), chimpanzee (Pan sp.), hybrids of Cercopithecus (Cerco- 
pithecus sp.), marmoset (Callothrix argentata), slow loris (Loris sp.), colobus monkey (Colobus polykomos), and humans. The anatomy of the adrenals of the macaque has been described by Harrison and Asling (1955). Variations in the histochemistry of the adrenals of cows, rats, and monkeys following "stress" or treatment with adrenocorticotropin, cortisone, or deoxycorticosterone were the subject of a paper by Glick and Ochs (1955). Additional descriptions of the adrenals of cows and other domesticated ungulates are subjects of papers by Elias (1948) and Weber ct al., (1950). Finally, Zalesky (1934) described in considerable detail the seasonal histologie ehanges in the adrenals of thirteen-lined ground squirrels (Citellus tridecemlineatus).

The morphology and histochemistry of the adrenals of laboratory and wild house mice have been thoroughly studied, largely beeause of the endocrine relationships of the transitory $\mathrm{X}$-zone which was first described by Howard (1927). Tamura (1926) wrote a detailed description of the changes during pregnaney in the adrenals of mice. This was followed by Howard's (1927) description of the X-zone and Waring's (1935) description of the development of the adrenal glands of the mouse. Following these there was a spate of papers deseribing the $\mathrm{X}$-zone and its reactions to various hormones and experimental treatments (Gersh and Grollman, 1939; Waring, 1942; MePhail and Read, 1942a, b; McPhail, 1944; Jones, 1948, 1949a, b, 1950, 1952 ; Miller, 1949; Benua and Howard, 19.50; Howard and Benua, 1950; Jones and Roby, 1954; Allen, 1954; Allen, 1957). The histology and histophysiology of the adrenals of hamsters (Mesocricetus auratus) have been deseribed by Alpert (1950) and Holmes (1955), and the effects of hypophysectomy and starvation on their adrenals by Knigge $(195 \mathrm{ta}, \mathrm{b})$.

The adrenals of a number of species of European small mammals have been studied and described by Delost, particular attention being paid to the presence or absence of an $\mathrm{X}$-zone and its relationships to the sexual eycle and sex accessories. The mammals in these studies included Microtus arvalis (Delost 1951; 1952a, b; 1954; 1956a, b) Microtus agrestis (Delost and Delost, 1955), Clethrionomys glareolus (Delost and Delost, 1954), Pitymys (Delost and Delost, 1955), Sorex arancus (Delost, 1957), and Crocidura (Delost, 1957).

Immature male and young nulliparous female house mice (Mus musculus) have an adrenocortical juxtamedullary zone, the X-zone, which unequivocally shows sex relationships (Howard, 1927; Deanesly, 1928; Jones, 1957). This zone is absent from mature male and parous or old females. Cortical X-zones have been described for a number of other species of small mammals including meadow voles (Microtus agrestis and Microtus arvalis), red-backed voles (Clethrionomys glareolus, pine voles (Pitymys 
subterraneus), and shrews (Sorex araneus and Crocidura russula) (Delost, 1951, 1952a, 1954, 1957; Delost and Delost, 1954, 1955), but there is some question whether the X-zones of these species are entirely analogous to the $\mathrm{X}$-zones of the house mouse. Delost $(1954,1956 \mathrm{~b})$ reports that cortisone involutes the so-called $\mathrm{X}$-zone of voles, which is a response not seen in house mice. The $\mathrm{X}$-zone of the shrew behaves like that of the house mouse with respect to its involution, but apparently has not been subjected to critical experiments in the laboratory (Delost, 1957). The $\mathrm{X}$-zone consists of cords of small, deeply acidophilic cells with intensely basophilic nuclei and which are about one-half the size of those of the zona fasciculata (Howard, 1927; Deanesly, 1928; Jones, 1949a, b, 1950, 1957; Benua and Howard, 1950). The cytoplasm of these cells, besides being more acidophilic than those of the fascicular cells, is unvacuolated ordinarily and lacks the sudanophilia of the other zones of the adrenal cortex (Jones, 1957). Criteria for critically distinguishing the $\mathrm{X}$-zone have been reviewed by Benua and Howard (1950) and Holmes (1955). The uniqueness of this zone rests on the fact that it is involuted by androgens and appears to depend on pituitary luteinizing hormone for its maintenance (Howard, 1927, 1959; MePhail and Read, 1942a, b; McPhail, 1944; Waring, 1942; Jones, $1949 \mathrm{a}, \mathrm{b}, 1950,1952,1957$ ). The function of this zone, if there is a specific function, is unknown. The so-called $\mathrm{X}$-zone of voles which has been described by Delost (1951, 1952a, 1954;) and Delost and Delost (1954, 1955) reappears after castration or after the hibernal periods of sexual inactivity in the males and persists through gestation and lactation in the females, and in these respects it differs markedly from the $\mathrm{X}$-zone of house mice. This zone may confound the use of adrenal weight as an index of increased cortical activity in the house mouse (Christian, 1956) and other species which possess it, but it provides a useful measurement for determining histologically the onset of androgen production, therefore puberty, in male house mice (Christian, 1956). A poorly defined X-zone has been described in mature nulliparous female hamsters, but not in males (Holmes, 1955), differing from the $\mathrm{X}$-zone of house mice in this respect. It is likely that an $\mathrm{X}$-zone will be described for other species when enough material from all age groups of both sexes has been critically examined, and that a variety of manifestations of this zone will be found.

The morphology and size of the adrenal cortex varies with its functional status (see also discussion under reticularis). The cortex undergoes rapid hyperplasia and hypertrophy in response to stimulation by adrenocorticotropin (ACTH) from the anterior pituitary. At first there is a rapid diminution in the size and number of lipid vacuoles, ascorbic acid, and cholesterol of the cortical cells (Sayers and Sayers, 1949). The vacuoles soon increase in number and size, providng the stimulation is not too 
severe (Dempsey, 1948; Sayers and Sayers, 1949; Greep and Deane, $1949 \mathrm{~b})$. The lipid vacuoles disappear first from the fasciculata next to the reticularis, so that it becomes indistinguishable from the latter. As stimulation continues, the disappearance continues centrifugally, and at the same time enzymes normally absent from the fasciculata, but present in the reticularis, make their appearance in the cells of the fasciculata, the innermost portion moving outward (Symington et al., 1958). Upon withdrawal of the stimulus of ACTH the lipid vacuoles increase considerably in size and may become very large. This stage presumably represents lipid storage. The cellular hyperplasia and hypertrophy mainly account for increases in the size and weight of the adrenal glands. Initially the cortex responds to stimulation with a marked decline in its cholesterol, neutral lipids, and ascorbic acid content (Greep and Deane, 1949b; Sayers and Sayers, 1949). These soon return at least partially to their original state, and in the inactive gland they may exceed their original levels. These matters are discussed in detail in the eited references in addition to discussions therein of the relationships of the cortex and its activity to various stimuli for varying lengths of time and with varying intensity.

c. The adrenal medulla. The adrenal medulla consists of rather irregular masses of polyhedral chromaffin cells derived, along with the ganglia of the sympathetic nervous system, from the primitive neurocetoderm. The medulla is homologous with the sympathetic ganglia and receives myelinated cholinergic preganglionic fibers from the greater splanchnic nerve. The medulla itself serves as the ganglion and the postganglionic tracts. There apparently are several types of cells in the medulla; these are discussed in more detail elsewhere (Hartman and Brownell, 1949; Eränkö and Raisanen, 1957). The cytoplasm of the medullary cells contains numerous minute deeply basophilic granules which stain blue with ferric chloride and brown with chromic acid (chromaffin) and which appear in some way to be related to secretory function.

The adrenal medulla generally is not thought to hypertrophy following stimulation in the same way that the adrenal cortex does. Rogers and Richter (1948) reported the absence of medullary hypertrophy with changes in adrenal size in rats. However, there is good evidenee that the medulla does hypertrophy, at least in some species and under some circumstances, even though it may not contribute significantly to an increase in the total weight of the gland, as a consideration of its geometry will show. Ifouse mice have been shown to exhibit a marked medullary hyperplasia and hypertrophy during pregnancy (Tamura, 1926) or chronic stimulation due to crowding (Bullough, 1952). Medullary hypertrophy also has been observed in a variety of species of captive wild ungulates subjected to ronditions in a zoological garden similar to the crowding of mice reported 
by Bullough (1952) (Ratcliffe, unpublished; .J. J. Christian, unpublished). These conditions which resulted in which medullary hypertrophy all constituted prolonged, ehronic stimuli. Medullary hypertrophy due to hyperplasia probably occurs simultaneously with cortical hypertrophy in many species but perhaps requires a more sustained stimulus and develops at a much slower rate. There also seems to be some suggestion that emotional stimuli may be important in this effect. Finally, it has been shown that treatment with pituitary growth hormone will produce a marked hypertrophy of the adrenal medulla (Moon et al., 1951; Lostroh and Li, 1958), and may eventually result in medullary tumors (Moon et al., 1950).

The role of sympathicomedullary function in physiologic adaptation requires more investigation, especially in regard to chronic stimulation, such as is produced by sociopsychologic pressures, and for a variety of species.

2. Hormones Secreted by the Adrexal Cortex: Their Actions ani) the Regulation of Their Secretion.

a. The zona glomerulosa. (1) The hormones. The adrenal cortex secretes two steroid hormones, aldosterone (18-aldocorticosterone) and deoxycorticosterone (11-deoxycorticosterone), which have their primary effects on salt-electrolyte and water metabolism. However, aldosterone is the only biologically important sodium-retaining corticoid secreted by the adrenal cortex and it is many times more powerful than deoxycorticosterone in its effects on electrolyte metabolism (Farrell et al., 195.5; Gaunt et al., 1955; Gross and Lichtlen, 1958). Also aldosterone is an important secretory product of the adrenal cortex, whereas deoxycorticosterone is secreted only in trace amounts (Farrell et al., 1955; Jones, 1957) and is probably a precursor in the formation of aldosterone (Giroud et al., 1958). The actions of these two hormones are very similar within the physiological range of dosages for each, but their actions with overdosage differ considerably: overdosage with aldosterone does not lead to the excessive sodium retention and the diabetes insipidus-like state which are seen after overdosage with deoxycorticosterone (Gross and Lichtlen, 1958).

It is appropriate at this point to comment on the general classification of the adrenal corticoids into the two broad categories which are used in the present account. The hormones of the adrenal cortex have been loosely grouped as "sodium-retaining" or "carbohydrate-active" according to whether their primary actions are on salt-clectrolyte metabolism or if they are among those steroids having marked effects on carbohydrate metabolism. The sodium-retaining steroids include aldosterone, deoxycorticosterone, and, to a much lesser extent, 17-hydroxy-11-deoxycorticosterone (Reichstein's compound S). The principal carbohydrate-actire steroids are 
hydrocortisone and cortisone (11-oxy-17-hydroxycorticoids). Corticosterone is included in this latter group, although it has moderate effects on both salt-electrolyte and carbohydrate metabolism. It is considerably weaker in all these actions than the principal corticoids in either of the categories (Jones, 1957). This classification into primarily carbohydrateactive and primarily sodium-retaining corticoids is useful, but by no means does it reflect the entire spectrum of activities of the hormones; in many instances there is a considerable overlap in these activities for a particular hormone, for example, corticosterone.

Recent morphologic and direct evidence shows that the secretion of aldosterone is a function of the zona glomerulosa, whereas the carbohydrate-active corticoids, except corticosterone, and probably the $\mathrm{C}_{19}$ steroids are secreted by the zona fasciculata and zona reticularis. Probably the most conclusive evidence for the relationship between specific secretory function and zonation of the adrenal cortex has been provided by the in vitro incubation and determination of the secretory products of selected segments of the adrenal cortex. Aldosterone was found to be secreted only by incubated portions of the zona glomerulosa of the adrenals of rats and beef cattle (Ayres et al., 1956; Giroud et al., 1956; Giroud et al., 1958); hydrocortisone was produced only by the zonac fasciculata-reticularis, and corticosterone was produced at approximately equal rates by all three zones of the adrenals of beef cattle (Ayres et al., 1956; Giroud et al., 1958 Stachenko and Giroud, $1959 \mathrm{a}, \mathrm{b})$. It was also shown in these experiments that ACTH or corticotropin peptides or other steroids were without effect on the production of aldosterone by the zona glomerulosa but that they markedly increased the production of total corticosteroids and of hydrocortisone by the fasciculata-reticularis (Stachenko and Cirond, 1959b). Additional evidence of functional zonation, less direct, has been obtained by relating changes in the composition of the secretory product with morphologic changes in the various zones of the adrenal cortex. A sodiumdeficient diet produces extreme hypertrophy of the zona glomerulosa and atrophy of the zona fasciculata of the adrenal cortices of rats (Hartroft and Eisenstein, 1957), and these changes are associated with a marked increase in the secretion of aldosterone and decreases in the secretion of corticosterone (Eisenstein and Hartroft, 1957). In somewhat comparable experiments it was shown that (1) sodium deprivation markedly increased the aliesterase activity of the zona glomerulosa but had no effect on its activity in the fasciculata of the adrenals of mice; $(2)$ deoxycorticosterone or sodium flooding depressed the aliesterase activity of the zona glomerulosa and increased it in the zona fasciculata; and (3) injected ACTH markedly increased the aliesterase activity of the zona fasciculata but did not affect it in the zona glomerulosa, whereas blocking $\mathrm{AC}^{\prime} \mathrm{TH}$ secretion with cortisone 
depressed the fascicular aliesterase activity (Allen, 1957). The results of the foregoing experiments provide convincing evidence in support of the hypothesis of Greep and his co-workers that the zona glomerulosa secretes the electrolyte-active, and the fasciculata the carbohydrate-active, corticoids (Greep and Deane, 1947, 1949b; Deane et al., 1948). This hypothesis was based on observations that (1) increased sodium intake or injections of deoxycorticosterone produced histochemical changes indicative of deereased activity in the zona glomerulosa of the adrenals of rats, and that (2) a reduction in sodium or increase in potassium produced cytological changes indicative of increased activity of the zona glomerulosa. There is little doubt that the glomerulosa is responsible primarily for the secretion of aldosterone and that the carbohydrate-active corticoids are secreted by the zona fasciculata and possibly by the zona reticularis. Convineing evidence of a functional separation between the zonae fasciculata and reticularis is not available, but the reticularis generally is not believed to be as active a secretory zone as the fasciculata.

The chief action of aldosterone is on sodium-potassium transport in the tubular cells of the renal nephron, and it is relatively more effective in promoting sodium retention than in promoting potassium excretion or water retention (Gaunt et al., 1955; Bartter, 1956; Jones, 1957; Gross and Lichtlen, 1958; Stanbury et al., 1958). It apparently stimulates the ionic exchange between potassium and sodium ions in the renal tubular cells (Bartter, 1956; Stanbury et al., 1958), although an overdosage of aldosterone will not produce excessive sodium retention and the animal therefore stays in sodium balance (Bartter, 1956; Gross and Lichtlen, 1958). In general the sodium-retaining corticoids act on the nephric tubular cells to promote an ionic exchange between sodium and potassium; so that sodium is retained and potassium is excreted (Bartter, 1956, 1957). Water is reabsorbed with the sodium or independently under the action of neurohypophyseal antidiuretic hormone (ADH) (Bartter, 1957). Proper fluid and electrolyte balance is maintained by these homeostatic endocrine activities acting in concert with water and salt intake and with hemodynamic and neural factors which affeet fluid volume, blood pressure, and renal glomerular filtration. The apparent anomaly of overdosages of aldosterone failing to produce excessive sodium retention depends on the fact that the blood pressure is raised and therefore the glomerular filtration rate is increased and sodium is lost accordingly (Stanbury et al., 1958). Proper fluid and electrolyte balance is vital to any animal, and adrenalectomized animals can be maintained with injected deoxycorticosterone or aldosterone, although they cannot adapt to added stress (Gaunt et al., 1955). The adrenalectomized laboratory rat or mouse also can be maintained alive by supplying $1 \%$ sodium chloride in its drinking water to replace the sodium loss 
accompanying adrenalectomy. However, adrenalectomized wild Norway rats (Rattus norvegicus) cannot be maintained in this fashion, even with the $\mathrm{NaCl}$ content of the drinking water as high as $4 \%$ (Richter et al., 1950). These facts emphasize the wide divergence between laboratory and wild strains of the same species. Evidently the requirements for adrenocortical hormones are much greater in mammals under feral conditions than for those raised or maintained in the laboratory or zoo. There is a marked disparity in the adrenal weights of mammals raised in the laboratory and in the same species under natural conditions, the differences due largely to differences in the amount of cortical tissue (Rogers and Richter, 1948; Nichols, 1950; Christian and Ratcliffe, 1952; Christian, 1955a); some of this difference, however, may be associated with the unconscious selection in breeding colonies for docility and good breeding performance.

(2) Regulation of aldosterone secretion. ${ }^{1}$ Since aldosterone acts primarily to maintain fluid and electrolyte homeostasis, it is not surprising that the secretion of this hormone is regulated largely by these factors. Changes in the volume of extracellular fluid (probably mainly the intravaseular volume), and the level of body potassium affect the rate of aldosterone secretion (Liddle et al., 1956; Bartter, 1957; Bartter et al., 1959), but to some extent the secretion of aldosterone in vivo can be stimulated by adrenocorticotropin (Farrell et al., 1955; 1958; Liddle et al., 1956), but apparently not in vitro (Stachenko and Giroud, 19.59b). This diserepancy may be explained by the increased production of the precursors of aldosterone by the fasciculata which then beeome accessible to the zona glomerulosa in the intact adrenal. Even though the secretion of aldosterone is moderately stimulated by $\mathrm{ACTH}$, the stimulation is not maintained in spite of continued treatment with $\mathrm{AC} T H$ (Liddle et al., 1956), and the response is considerably less than that seen following changes in the volume of extracellular fluid or in body potassium (Bartter et al., 1959). The glomerulosa will respond to inereased ACTH with increased secretion of aldosterone for only about 3 or 4 days, and then the rate of secretion declines in spite of continued $\mathrm{ACTH}$ and reaches base levels or even lower levels of secretion in about a week (Liddle et al., 1956). After this period, continued ACTH will not increase the secretion of aldosterone (Bartter et al., 1959). Finally, the secretion of aldosterone is only slightly depressed by suppressing ACTH secretion (Farrell et al., 1955; Liddle et al., 1956; Bartter, 19.57) or by hy-

1 Since completion of this chapter, there has been marked progresses in understanding the regulation of aldosterone secretion in response to hemodynamic changes. It is fairly certain that in response to decreased arterial pressure there is increased release of renin from the kidney. The end product of this release is angiotensin II which, in the presence of basal levels of ACTH, stimulates aldosterone secretion. [For a review see J. O. Davis (1963).] 
pophyscetomy (Farrell et al., 1955). These results, in addition to those discussed under the relationship between secretory function and zonation of the adrenal cortex, elearly indicate that the secretion of aldosterone is largely independent of adrenocorticotropin and the adenohypophysis and that its responses to ACTH may reflect the increased availability of aldosterone precursors from other parts of the cortex. Nevertheless, the secretion of aldostcrone in dogs appears to be dependent on an intact pituitary gland (Davis, et al., 1959a). However, as one might expect, a variety of stimuli which produce an increase in the secretion of ACTH may also stimulate an increase in the secretion of aldosterone. Farrell (1958) lists position, surgery, emotional factors, hypertension, insulin shock, and other stimuli among those resulting in an increased secretion of aldosterone, but probably none of these are without an effect on fluid and electrolyte balances which in turn would effect directly the mechanisms regulating the secretion of aldostcrone. On the other hand, there is a marked increase in the secretion of aldosterone in those diseases which are characterized by striking disturbances in fluid and electrolyte metabolism, such as congestive heart failure, hepatic cirrhosis, and nephrosis (Liddle et al., 1956). It seems likely that the increase in aldosterone secretion is slight in those circumstances which produce a marked increase in the secretion of ACTH and of the carbohydrate-active corticoids unless there is also involvement of fluid and electrolyte balances. It has been found that only one, the $\Delta-1$ fraction, of the several distinct fractions of ACTH has an appreciable effect on the secretion of aldosterone, and this fraction is a relatively small proportion of the total amount of ACTH which may be secreted (Farrell et al., 1958; Farrell, $1959 a)$.

The principal regulation of aldosterone secretion seems to be by a combination of neural and neurohumoral factors in response to changes in the volume of extracellular fluid or body potassium. However, there can be little doubt that a hormonal factor is involved in aldosterone secretion, as recently demonstrated with cross-circulation experiments by Yankopoulos et al. (1959). Recent experiments have indicated that the brain may secrete a hormone, glomerulotropin, not as yet isolated and characterized, from the region of the pineal body which stimulates the secretion of aldosterone from the adrenal zona glomerulosa (Farrell, 1959a).

Small changes in blood volume can effect striking changes in the rate of secretion of aldosterone (Bartter, 1957) possibly by affecting changes in pulse pressure (Bartter and Gann, 1960). Changes in blood volume elicit maximal reciprocal responses in the secretion of aldosterone and it appear's that this system is the most sensitive, as well as the most important, of those involved in the regulation of the secretion of aldosterone (Bartter, 1957; Bartter et al., 1959). A rise in blood volume reflexly depresses the 
secretion of aldosterone via stretch receptors in the region of the right strium or adjacent vena cava (Davis et al., 1956, 1957, 1958; Liddle et al., 1956; Bartter et al., 1958, 1959; Farrell, 1959a; Anderson et al., 1959), the vagus nerve (Mills et al., 1958), and central pathways possibly to depress the secretion of glomerulotropin from the pineal region of the brain althrough Davis et al. (1959b) indicated that the vagus is not involved in the afferent pathways of this control. Conversely, a decrease in blood volume stimulates the secretion of aldosterone (Bartter et al., 1959), although the exact pathways and mechanism by which this is achieved is unknown. Bartter and Gann (1960) have suggested that pulse pressure is a factor in changes in blood volume which affects aldosterone secretion. A drop in pulse pressure stimulates the release of aldosterone and a rise inhibits its release. These changes apparently come about through changes in the rate of tonie impulses over receptor nerves in the region of the thyrocarotid artery.

Another system that regulates the secretion of aldosterone involves the levels of potassium in the body. A deficieney of potassium, therefore a lowered concentration of body potassium, results in a lower rate of secretion of aldosterone if it was originally elevated, whereas an increase in body potassium results in an increase in the secretion of aldosterone (Bartter, 1956; Bartter et al., 1959). A rise in serum potassium, either absolute or relative to the concentration of sodium, is associated with an increase in the secretion of aldosterone, but it is not known whether a fall in potassium actively inhibits its secretion or permits it to return to base levels passively (Farrell, 1958). It has been shown that these changes in the rate of seeretion of aldosterone in response to changes in body potassium are independent of sodium concentration in the serum or the total amount of sodium in the body and are also independent of the sodium: potassium ratio in the serum (Bartter, 1956; 1957; Bartter et al., 1959). Similarly, there is no evidence to suggest that altered renal hemodynamies are responsible for the altered secretory rates of aldosterone (Bartter ct al., 1956; Cole, 1957). It is not known yet whether the regulation of the secretion of aldosterone by the body potassium is directly on the cells of the adrenal zona glomerulosa or is mediated through central channels (Bartter, 1956; Bartter et al., 1959). It eannot be said whether serum potassium, intracellular potassium, or a combination of both effects the control of the secretion of aldosterone, but there is evidence that the adrenal cortical cells themselves may respond directly to this type of stimulus (Bartter, 1956). On the other hand, Farrell (1958) suggests that the effect is through central channels. However, changes in potassium are not as important in the regulation of thesecretion of aldosterone as changes in the volume of the extracellular fluid (Bartter, 1957 ; Bartter ct al., 1959). 
In summary, three mechanisms are involved in regulating the secretion of aldosterone. The first and most important regulating factor is the volume of the extracellular, probably intravascular, fluid, changes in which act through atrial stretch receptors and other as yet unknown pathways to effect reciprocal changes in the rate of secretion of aldosterone. Decreased pulse pressure also stimulates increased aldosterone secretion and may be one way in which changes in blood volume act. Depression of the secretion of aldosterone by increases in blood volume requires an intact vagus nerve. The second mechanism responds to changes in body potassium; a rise in potassium resulting in elevation of the rate of secretion of aldosterone and a fall in potassium per'mit the secretion of aldosterone to fall back to normal. Finally, adrenocorticotropin, or at least a fraction thereof, is capable of stimulating the secretion of aldosterone in the intact animal, but only to a moderate degree and for a relatively short period of time, although the secretion of aldosterone or its regulation and the functional integrity of the adrenal zona glomerulosa apparently do not depend upon adrenocorticotropin. Glomerulotropin, a recently described hormone from the pineal complex region of the brain which stimulates the secretion of aldosterone, may be an important link in the regulating system depending on the volume of the extracellular fluid or body potassium or both, but this work requires confirmation.

The actions of aldosterone are essential in combatting incipient shock mammals, and this hormone apparently plays a vital role in the daily maintenance of fluid and electrolyte homeostasis. Aldosterone also may be more directly responsible for maintaining blood pressure and counteracting hemoconcentration through its activity in correcting alterations in blood volume.

b. The zona fasciculata. (1) The hormones. This zone of the adrenal cortex normally secretes hydrocortisone (Kendall's compound F), corticosterone (Kendall's compound B), small amounts of cortisone (Kendall's compound E), 11-deoxycorticosterone (Kendall's compound A), 11-deoxycorticosterone (DOC, DCA, or DOCA), 11-deoxy-17-hydrocorticosterone (Reichstein's compound S), and $\mathrm{C}_{19}$ ketosteroids, usually androgenic, the amounts and proportions depending on the species and the cireumstances. Although modification of this concept is required in the light of the work of Symington and his co-workers (19.58) ( $c f$. above). Their experiments indicate that the reticularis is the part of the cortex that normally produces corticoids and 17-ketosteroids at rest, and that the fasciculata becomes functional with increased stimulation. In other words, the reticularis is the active part of the cortex and the fasciculata is a resting portion. Actually this work indicates that the morphologic separation of the cortex into fasciculata and reticularis is unjustified. In addition to aldosterone, the 
normally important adrenocortical hormones are eorticosterone and hydrocortisone, and their respective ratios vary from species to species (Bush, 19533; Nelson, 195.5) and possibly with the degree of cortical stimulation (Bradlow and Callagher, 19.57). The ratio of hydrocortisone to corticosterone ( $\mathrm{F}: \mathrm{B}$ ratio) may vary from less than 0.05 in rats and rabbits to greater than 20 in monkeys (Bush, 1953; Reif and Longwell, 1958; Dorfman, 1959). Most species lie between these two extremes (Bush, 1953). However, there is little doubt that in most species studied, exclusive of rats and mice, these two steroids form from $80 \%$ to $95 \%$ of the total adrenal secretion of corticoids (Jones, 1957). Corticosterone is the principal carbohydrateactive corticoid secreted by mice, rats and, rabbits (Bush, 1953; Hofmann, 1956; 1957; Reif and Longwell, 1958; Wilson et al., Bloch and Cohen, 1960). whereas hydrocortisone is the prineipal corticoid in other species, including guinea pigs, hamsters, ferrets, eats, monkeys, sheep, and humans (Bush, 1953; Nelson, 1955; Jones, 1957; Peron and Dorfman, 1958, Schindler and Knigge, $1959 \mathrm{a}, \mathrm{b})$. The adrenals of house mice and rats apparently secrete large amounts of 11-hydroxy- $\star^{4}$-androstene-3, 17-dione (11-OH4AD) 11-hydroxyandrostene-3,17-dione, and other closely related steroids as major components of their natural adrenal seeretory product in addition to corticosterone and very small amounts of hydrocortisone and other corticoids (Sweat and Farrell, 1952; Bush, 1953; Iofmann, 1956; Bahn et al., 1957; Poore and Hollander 1957; Wilson et al., 1958 Bloch and Cohen 1960). Probably all species seerete 11-OH4AD and closely related $\mathrm{C}_{19}$ steroids, but usually in proportionately small amounts (Bradlow and Gallagher, 1957; Gallagher, 1958). However, it has been shown recently that the adrenal androgen, dehydroepiandrosterone, emprises about $50 \%$ of the total secretion of steroids by the human adrenal cortex (Vande Wiele and Lieberman, 1960). The general problem of the secretion of sex steroids by the adrenal cortex has not been studied until recently in the same detail as the carbohydrate-active corticoids, especially with regard to differenees among species, but there is no doubt that they are secreted by the cortex (Gallagher, 1958). These $\mathrm{C}_{19}$ steroids may be normal products, metabolites, or intermediate metabolites in the synthesis of other steroids (Dorfman and Shipley, 1956; Gallagher, 1958). Apparently there is considerable variation with species with respect to the secretion of androgens and androgen precursors (Bush, 1953; Jones, 1957; Gallagher, 1958; Wilson et al., 1958). In any event, the adrenal cortex is the starting point of $\mathrm{C}_{19}$ steroids which may act as weak androgens (Dorfman and Shipley, 1956; (Gallagher, 1958).

Normally cortisone, hydrocortisone, and eorticosterone appear in the urine as metabolites which ean be identified and related to the parent corticoid by the appropriate procedures (Gallagher, 1958; Dorfman, 1960). 
However, there are other metabolites in the urine in smaller quantities which cannot be related specifically to particular adrenocorticoids without radioactive labeling, but these ordinarily are not produced in appreciable (quantities (Gallagher, 1958). The types and quantities of the metabolites of a partieular hormone which appear in the urine usually provide a good index of adrenocortical activity for relatively longer periods of time than can be obtained by the measurement of the corticoids in the plasma, which ouly refleet the immediate situation (Nelson, 195.5). However, the urinary metabolites do not always refleet the actual adrenal secretory pattern, as has been shown for mice (Bradlow et al. 1954; Wilson ot al., 1958) although in many instances this may be surmised with confidence (Dorfman, 1960). In summary it may be said that hydrocortisone or corticosterone and $\mathrm{C}_{19}$ weak androgens are the major secretory components of the adrenal fasciculata, but that other carbohydrate-active corticoids, sodium-retaining corticoids, and adrenal androgens are also secreted, although usually not in appreciable quantities.

It is impossible to make hard and fast statements about the quantitative relationships of the adrenocortical hormones to one another because of a certain degree of inherent variability and beeause the techniques for their measurement are not sufficiently refined and certain for such detailed comparisons. A large number of steroids have been isolated from the adrenals of various species, frequently from perfused glands. Some of these may be biochemical artifacts, but many are probably intermediate products in the biosynthesis of the normal secretory products, or possibly steroids which are secreted only under unusual conditions (Jones, 1957; Bradlow and Gallagher, 1957; Gallagher, 1958). It is not known whether or to what extent, some of these steroids are secreted naturally. The picture is complicated further by the fact that the liver and other tissues metabolize the steroid hormones to new steroids which appear in the circulation and urine and which may have biological activity to varying degree (Gallagher, 1958). Therefore, the specific roles of the various adrenocortical steroids and their metabolites, especially those that appear in very low concentrations, in the economy of the whole mammal, and the variations in their secretory patterns from species to species and under normal and abnormal circumstances, needs clarification. Some of the discrepancies that appear in the literature regarding the relative amounts of various steroids secreted by the adrenals of a particular species seem to depend on whether the measurements were made in vivo or on perfusates of isolated glands (Bush, 1953; Jones, 1957). It seems evident that appreciably higher proportions of steroids which normally are seereted in low eoneentrations are found in perfusates than in vivo. However, it suffices for the present to know that the carbohydrateactive corticoids, hydrocortisone and corticosterone, are the major na- 
turally secreted corticoids of the zona fasciculata and to discuss the actions of these hormones as a class.

(2) Actions of the fascicular hormones. Hydrocortisone, cortisone, and corticosterone have important effects on carbohydrate metabolism and therefore are classed loosely as carbohydrate-active corticoids. They have in common either a hydroxyl group or ketonic oxygen at the carbon-11 position, and those with the most pronounced effects on carbohydrate metabolism, hydrocortisone and cortisone, have a hydroxyl group on the C-17 of the steroid nucleus. Corticosterone has a weaker action on carbohydrate metabolism than hydrocortisone or cortisone (Dorfman, 1949; Ingle, 1950; Parmer et al., 1951; Santisteban and Dougherty, 1954; Dougherty and Schnecbeli, 1955; Kass et al., 19.55; Noble, 195.5), but it has appreciably more effect on salt-electrolyte metabolism than either of the others (Noble, 1955 ; Farrell ct al., 1955; Jones, 1957). Because of these facts, the relatively small amounts of hydrocortisone which are normally secreted by the adrenals of mice and rats, along with corticosterone, have been held responsible for most of the carbohydrate-active corticoid activity, such as involution of the thymus, which has been observed in these animals (Wilson et al., 1958). The designation "carbohydrate-active corticoids" for this group of steroids by no means reflects all their activities. These corticoids have suppressive effects to varying degrees on inflammation and therefore are classified also as anti-inflammatory (antiphlogistic) hormones (Selye, 1950; Dougherty 1953). As a class they have profound effects on protein metabolism, fat metabolism, growth, oxygen consumption, and a number of other physiological functions (Noble, 1955; Jones, 1957). Hydrocortisone and cortisone are the most powerful of the fascicular carbohydrate-active corticoids and corticosterone the least powerful with respect to the enumerated activities (cf. above), although cortisone is not produced in biologically important quantities in any of the species so far investigated (Bush, 1953; Nelson, 1955). As a gencral rule, the degree of activity of a corticoid on carbohydrate metabolism is related inversely to its sodium-retaining ability. Finally, it should be noted that other steroids may affect the actions of the corticoids; for example, testosterone and estradiol potentiate the anti-inflammatory action of the carbohydrate-active corticoids (Taubenhaus, 1953), and testosterone enhances the thymolytic activity of cortisone (Selye, 1955; Dorfman and Shipley, 1956).

The carbohydrate-active corticoids, secreted by the zona fasciculata, will maintain life in adrenalectomized mammals (Ingle, 1950); nevertheless the exact functions of the adrenocortical hormones in the intact normal animal are difficult to delineate precisely, as these hormones are integral elements in a complex system of endocrine and neural responses which form a feed- 
back system to maintain homeostasis or to meet emergencies. Nevertheless, much has been learned about the specific activities of these hormones by the classic experimental approaches to such a problem: the substitution of pure hormones or extracts into intact and adrenalectomized animals and refinements of these procedures. The effects of injected carbohydrate-active corticoids are closely paralleled by those produced by injecting adrenocorticotropin (ACTH), the hormonal protein of the anterior pituitary responsible for stimulating the secretion of the earbohydrate-active and $\mathrm{C}_{19}$ steroids from the adrenal cortex (Poore and Hollander, 1957; Li et al., 1957; Lostroh and Li, 1957; Wilson, et al., 1958; Farrell et al., 1958).

The earbohydrate-active corticoids stimulate gluconeogenesis from proteins, and this activity is reflected by hyperglyeemia and glycosuria (Ingle, $1949 ; 1950 ;$ Jones, 1957). The inereased levels of glucose in the blood and urine are also partly due to the inhibition of glueose utilization (Jones, 1957). These hormones also inerease glyeogen deposition in the liver by accelerating its formation and depressing its release (Ingle, 19.50; Jones, 1957). Glyeogen deposition commonly is used to bioassay steroids for their glueoneogenic aetivity and other effeets of earbohydrate metabolism (Dorfman, 1949). The carbohydrate-active steroids not only increase protein catabolism, but they also depress protein anabolism (Engel, 1952). These two actions on protein metabolism are reflected by an increase in the nonprotein nitrogen of the blood as well as an increase in the excretion of urinary nitrogen (Selye, 1950). Lipogenesis is inhibited by the carbohydrateactive eorticoids, but their effects on lipid metabolism are poorly understood (Jones, 1957). There is considerable evidence to indicate that hydrocortisone and cortisone inerease the sensitivity of blood ressels to the aetions of epinephrine and norepinephrine, and that these steroids perform an essential function in maintaining normal tonus of the vasculature (Zweifach et al., 1953; Ramey and Goldstein, 1957). The carbohydrate-active hormones also decrease eapillary permeability and fragility and antagonize the spreading action of hyaluronidase, presumably by their effeets on the ground substance; these eortieoids appear to decrease permeability of the ground substance, and their ability to decrease capillary permeability may be dependent on this effect (Seifter et al., 1953; Zweifach et al., 1953). In these effects the carbohydrate-active corticoids are opposed by the actions of the sodium-retaining corticoids and growth hormone (Seifter et al., 1953: : Kass et al. 1953b; Dougherty and Schneebeli, 1955; Kramer et al., 1957). In addition to their eatabolie effect on protein, the earbohydrate-active corticoids have specific suppressive effects on osteogenesis, chondrogenesis, mitosis, growth in general, connective tissue growth, inflammation, phagocytosis, granulation, and antibody formation (Taubenluans and Amromin. 1950; Baker, 1950, Selye, 1951; Dorfman, 195.; Dougherty, 19.5; 'Tauben- 
haus, 1953; Bullough, 1955; Dougherty and Schneebeli, 1955; Kass et al., 1955; Irving, 1957). The effects on inflammation result from a failure of the usual inflammatory cells, lymphocytes and fibroblasts, to appear at the site of injury (Dougherty, 1953); Dougherty and Schneebeli, 1955). The lack of an adequate inflammatory response together with the failure of adequate granulation to take place markedly delays wound healing (Dougherty, 1953; Dougherty and Schncebeli, 1955). These effects, coupled with inhibition of phagocytosis and antibody formation, result in a marked decrease in resistance to infections, so that an animal may be rapidly overwhelmed by an infection (Thomas, 1953). There is ample experimental evidence to show that cortisone and hydrocortisone decrease host resistance to infection by a wide variety of pathogenic viruses, bacteria, protozoan and metazoan parasites (Thomas, 1953; Shwartzman and Aronson, 1953; LeMaistre et al., 1953; Kass et al. 1953b; Robinson and Smith, 1953; Whitney and Anigstein, 1953; Pollard and Wilson, 1955). Animals resistant to particular organisms may be made nonresistant by these steroids, and usually mild infections may become highly virulent.

High physiological doses of cortisone or hydrocortisone in the pregnant mammal may result in the development of malformations, especially cleft palate, in the fetus, the particular anomaly apparently depending on the stage of development of the fetus when it is subjected to the actions of the hormone (Glaubach, 1952; Fraser et al., 1953; Davis and Plotz, 1954; Kalter, 1954; Moss, 1955). Cortisone and hydrocortisone both produce eleft palates and other congenital defects in the fetus when injected into pregnant mice, the ineidence of these anomalies being greater when the injections were made on the tenth day than when later (Fraser et al., 19.33). The teratogenic effects of cortisone in mice have been shown to be decreased with inereased maternal body weight and to be affected by maternal genotype (Kalter, $1954 ; 1956$ ). Treatment of pregnant rats with high physiologie doses of cortisone results in a significant increase in intra-uterine mortality, occurring minly at mid-term and later (Seifter et al., 1951; Daris and Plotz, 1954). High doses of cortisone administered to nursing mice 9-12 days after parturition depress the growth of progeny, whereas ACTH and low doses of cortisone were without effect on the offspring, except to abolish the difference in growth rate normally seen between male and female mice (Glaubach, 1952). Cortisone, and to a lesser degree ACTH, depresses the growth of infant rats, stimulates the eruption of teeth, opening of the eyes, and development of the gingivae (Parmer et al., 1951). Cortisone in a total dose of $0.5 \mathrm{mg}$. given to newborn rats during the first week produced longterm damage, as indicated by the failure of the animals to attain normal body weight after three months (Parmer et al., 19.51). Corticosterone and pregneninolone were without effect in these experiments. Cortisone treat- 
ment of neonatal rats can also result in marked morphologic changes in the brain and skull (Moss, 1955). Cortisone or hydrocortisone may stimulate lactation (Selye, 1954), but the mechanism by which this is accomplished is unknown. These effects cannot be attributed to an inhibition of the secretion of gonadotropin by cortisone, as it has been shown that even relatively large doses of cortisone are without effect on the production of gonadotropins (Byrnes and Shipley, 1950), although it is well known that enormous doses of corticoids do exert some antigonadotrophic activity.

The carbohydrate-active corticoids also involute lymphoid tissues by producing degeneration and actual fragmentation of the lymphoid cells, inhibition of differentiation, and depression of lymphocytopoiesis (Selye, 1950; Dougherty, 1953; Santisteban and Dougherty, 19.54; Gordon, 19.5.; Weaver, 1955). These effects are also seen following injection of ACTH, with an increase in endogenous corticosteroid secretion (Baker et al., 1951). Lymphocytolysis evidently serves to release a readily available store of amino acids and may serve to provide a sudden flood of stored antibodies, which are normally produced in the lymphoid tissues (Keuning et al., 1950; Dougherty, 1953; Kass et al., 1953a; Sundberg, 1955). These actions result in involution of the thymus, lymph nodes, and malpighian corpuscles of the spleen. Therefore weights of these organs may provide useful indices of adrenocortical activity when they are used along with other indices of adrenal activity, such as adrenal weight, and appropriate controls. It should be remembered, however, that androgens, and to a somewhat lesser extent estrogens, are capable of involuting the thymus (Burrows, 1949; Weaver, 1955); therefore cognizance must be taken of this fact when using thymic involution as a means of appraising adrenocortical activity. However, the lymph nodes lose weight only after treatment of the animal with ACTH or carbohydrate-active corticoids (Weaver, 1955). Estrogen, testosterone, thyroid extract, adrenalectomy, thyroidectomy, and gonadectomy were without effect on the lymph nodes in these experiments (Weaver, 1955). The adrenal carbohydrate-active corticoids also depress the numbers of circulating eosinophils and lymphocytes, so that counts of these cells are frequently used to assess the functional integrity of the pituitary-adrenocortical system (Speirs and Meyer, 1949; 1951; Gordon, 1955; Speirs, $1955)$. In using counts of eosinophils or lymphocytes as indices of adrenocortical activity in wild mammals care must be taken (1) to standardize the procedures so that the results are completely comparable from count to count, and (2) not to elicit an adrenocortical response during the process of handling the animal.

The biological activity of $11 \beta$-hydroxy $\Delta^{4}$-androstene-3,17-dione (11OH$4 \mathrm{AD})$ and the closely related steroid $11 \beta$-hydroxytestosterone, as well as other related $\mathrm{C}_{19}$ steroids, deserve further comment, as one or the other of 
the first two, probably the first, is a major secretory product of the cortex, presumably of the zona fasciculata-reticularis of house mice, rats, and very possibly other rodents (Bush, 1953; Wilson et al., 19.58), and to these must be added dehydroepiandrosterone, which is now known to account for half of the steroid product of the adrenal cortex of human beings (VandeWicle and Lieberman, 1960). These steroids, as a group, are very weak androgens with insufficient activity to maintain the seminal vesicles and ventral prostate in hypophysectomized mice (Bahn ct al., 1957), although they evidently are sufficiently androgenic to produce histologically detectable changes in the epithelium of these organs, if not in changes in gross weight (Davidson and Moon, 1936; Lostroh and Li, 1957), and evidently, if they are secreted in large enough quantities, they can produce masculinization in humans (Dorfman and Shipley, 1956). In addition, Howard (1959) has shown that these weak androgens are more strongly androgenie if their activity is measured in terms of other assays, such as stimulation of the preputials and os penis. However, the $\mathrm{C}_{19}$ steroids with weakly androgenic activity, as measured by their ability to stimulate growth of the prostate or capon comb, can inhibit the secretion of gonadotropins in rats, especially in immature animals (author's italics), although it has been shown that the earbohydrate-active corticoids are incapable of producing this effect (Byrnes and Shipley, 1950; Byrnes and Meyer, 1951; Wilson et al., 1958). Therefore, it is possible that an increased secretion of these androgens steroids by the adrenal cortex in mice and rats can aceount, at least in part, for the suppression of reproduction commonly associated with cireumstances which increase the secretion of ACTH and carbohydrate-active corticoids, as described by Selye (1939).

These, in brief, are the actions of the important adrenal cortical hormones. Many questions remain manswered regarding the functions of the cortical hormones, especially with respect to their relationships to each other and to other endocrines, such as the thyroid and pancreatic islets. The carbohydrate-active corticoids in many respects are antagonistic to insulin and probably suppress thyroid activity, but these topies will not be dealt with here. The actions already listed are the major activities of the cortex which will enable an interpretation to be made of, as well as to anticipate, the results in other species. All the above effects have been duplicated by injecting adrenocorticotropin into intact mammals and thereby stimulating an increased secretion of endogenous adrenocortical steroids. They also have been produced by alarming stimuli, which increase the secretion of endogenous ACTH and in turn endogenous corticoids. Among these stimuli are cold, emotional trauma, physical trauma, toxie agents, and many others, although the general response to these stimuli is not necessarily qualititatively, or even quantitatively, similar in every case. 
There is much to learn about the differences in hormonal response to different stimuli. There are excellent reasons to believe that the various adrenocortical hormones are not always secreted in any fixed proportion to each other independently of the nature of the stimulus or its degree of severity (ef. above). Therefore it seems advantageous to speak of the responses evoked by the specific stimulus in each case rather than of a generalized nonspecific response to "stress."

(3) Regulation of secretion of the fascicular hormones. The regulation of the secretion of the hormones of the cortical zona fasciculata has been investigated intensively, and a great deal is known about the mechanisms involved, although many questions still remain unanswered. It is well established that adrenocorticotropin (ACTH), a protein hormone secreted by the anterior pituitary, stimulates corticoid production and release from the adrenal fasciculata and to a lesser extent stimulates the secretion of aldosterone from the zona glomerulosa (Wexler et al., 1955; Allen, 1957; Farrell et al., 1955; Jones, 1957; Li et al., 1957; Farrell et al., 1958, and other references cited earlier). ACTH also stimulates cellular hyperplasia and hypertrophy of the cortex, although pituitary growth hormone may play an important role in stimulating cortical hypertrophy (Cater and Stack-Dunne, 19533, 1955; Jones, 1957; Lostroh and Li, 1958), possibly acting synergistically with ACTH (Lostroh and Woodward, 1958). However, growth hormone has no effect on the release of corticosteroids (Rosenfeld and Bascom, 1956; Guillemin et al., 1958). ACTH also has a number of extra-adrenal actions; for example, it stimulates the preputial glands and other sex accessories, but not to the same extent as does testosterone (Davidson and Moon, 1936; Davidson, 1937; Jacot and Selye, 1951; Lostroh and Li, 1957). The regulation of the release of ACTH from the pituitary is not clearly understood, although there no longer can be any doubt that for the most part a neurohumor from the hypothalamus has a major role in regulating the production and release of ACTH (McCann, 1953; McCann and Brobeck, 1951; Harris, 1955a, b; Fortier, 1957; Woodbury, 1958). The regulation of the release of ACTH has been reviewed by Harris and Fortier (1954), Harris (1955b), and Fortier (1956), but advances in this field are rapid, and much additional information is available which is not included in these reviews. Some doubt has been east on the role of the hypothalamus in regulating ACTH secretion in response to surgical trauma (Story et al., 1959).

The adenohypophysis has a double arterial supply: a portal system draining from the hypothalamus and a direct systemic arterial supply (Green, 1951). Branches from the superior hypophyseal arteries form a tufted plexus of capillaries in the median eminence of the hypothalamus in 
intimate association with nerve fibers of the supraopticohypophyseal tract. These ressels drain into the adenohypophysis, where they break up to form a series of sinusoids. Here they are joined by eapillaries from the systemic arterial supply. The direction of the flow is from the median eminence to the adenohypophysis. Neural fiber tracts from hypothalamic nuclei deseend through the median eminenee and give off branches which terminate in intimate association with the arterial portal plexus in the median eminence (Scharrer and Scharrer, 1954). The remaining fibers continue on their way, to terminate in close approximation to capillaries in the neurohypophysis. Neurosecretory material is believed to traverse the sheaths of these nerves from hypothalamic secretory nuelei (Scharrer and Scharrer, 1954; Rennels and Drager, 195.5). There is evidence that a fraction of this neurosecretory material is responsible for stimulating the release of ACTH from the anterior pituitary (Drager, 1955; Rothballer, 1953; Saffran et $a l ., 19.5)$ ). Evidently the material is released by the appropriate stimulus from the terminations of the neurosecretory fibers into the pituitary portal vessels and is then borne, via the portal system, to the cells of the adenohypophysis proper (Scharrer and Scharrer, 19.54; Rennels and Drager, 1955). It is generally accepted that no nerve fibers terminate in the adenohypophysis and that there are no direct neural, only vascular, connections between the adenohypophysis and neurohypophysis (Green, 1951). The identity of the hypothalamic corticotropin-releasing factor (CRF) has not been determined, although it appears to be a small protein often associated with Pitressin (Saffran et al., 195.5; Porter and Rumsfeld. 1956). One group of investigators believes that the ACTH-releasing activity is associated with Pitressin, in particular with its pressor activity (ADH, rasopressin) (McCamn, 1957; McCann and Fruit, 1957). ADH exerts a powerful antidiuretic action on the kidney at many times the dilution that is necessary for it to exert a pressor effect or to stimulate the release of the ACTH (McCann and Fruit, 19.57). It has been suggested for this reason that the concentration of $\mathrm{ADH}$, when it is released from the posterior pituitary into the systemic cireulation, is sufficient to effect antidiuresis, but insufficient to stimulate the release of ACTH, but that when it is released directly into the pituitary portal circulation, it reaches the adenohypophysis in a sufficient concentration to effect the release of ACTH (McCann and Fruit, 1957). It should be noted that ADH evidently is released simultaneously into the pituitary portal system from the hypothalamus and from the neurohypophysis into the systemic circulation in response to stimulation.

Nevertheless, as attractive as this theory may seem, good evidence is aceumulating from a number of sourees which indicate's that the corticothopin-releasing factor is an entity separate from vasopressin, although 
the site of its origin in the hypothalamus and its exact identity remain unknown (Saffran et al., 1955; Porter and Jones, 1956; Porter and Rumsfeld, 1956; Clayton et al., 1957; Schally and Guillemin, 1959), and that the release of $\mathrm{ADH}$ may be completely unrelated to the release of ACTH (MCDonald et al., 1957; Schapiro et al., 1958). It appears that this substance is a small protein which is reeoverable from hypophyseal portal blood, from the neurohypophysis, or from extracts of appropriately selected portions of the hypothalamus, and that it is probably loosely bound to and travels with a much larger protein which is inactive with regard to the release of ACTH (Porter and Rumsfeld, 1956; Guillemin et al., 1957; Schally and Guillemin, 1959). The activity of the corticothopin-releasing factor may be angented by the simultaneous action of epinephrine (Saffran et al., 1955). As is so often the case, the solution to this problem may prove to be midway between, or to be a combination of, the two opposing views. It is possible that there are hypothalamic substances, such as vasopressin, other than a specific corticotropin-releasing factor which are eapable of effecting the release of specific ACTH fractions from the adenohypophysis. Recentevidence indicates that different stmulating factors are involved in activating different adrenocortical functions (Fortier, 19.56; Guillemin et al., 1958; Slusher, 1958; Nowell, 1959).

In spite of the existing uncertainty with regard to the nature of the speeific factor or factors from the hypothalamus which stimulate the release of ACTH, there is little doubt that hypothalamic substances earried to the adenohypophysis by the portal system can stimulate the release of $\mathrm{AC} T H$ in response to alarming stimuli, although Nowell (1959) has suggested that emotional and systemie stress involve different pathways for the release of ACTH. However, there are many other aspects of the overall regulation of the secretion of adrenocorticotropin which are not clear at the present time. One of these aspeets is the control of the normal daily lowlevel secretion of ACTH. There probably is a basal release rate of ACTH which is independent of the hypothalamic-hypophyseal portal system and which is sufficient for normal maintenance of adrenocortical function (Fortier, 1957). A number of workers maintain that the release of ACTH is subject to hypothalamic control only in response to acute stressful stimuli (Woodbury, 1958). The level of eirculating corticoids undoubtedly exerts a control over the rate of secretion of ACTH, as circulating adrenocorticoids are eapable of inhibiting the release of ACTH under most circumstances, although the more severe the stimulus, the greater the level of cireulating corticoids must be in order to block ACTH secretion (Sydnor and Sayers, 1954; Farrell and Laqueur, 1955; Sydnor et al., 1955; Richards and Pruitt, 1957). Furthermore, Fortier $(1959 \mathrm{a}, \mathrm{b})$ has shown that the corticotropinreleasing effect of stress is influenced markedly by the level of circulating 
corticoids. Withdrawal of the corticoids enhances both the release and synthesis of ACTH, its predominant effect being on the synthesis (Fortier, $1959 \mathrm{a}, \mathrm{b})$. The steroids appear to act on the pituitary itself, but they may act also on the hypothalamic center's, or on the releasing mechanism or mechanisms themselves, or even may act to some extent on the adrenal gland directly (Birmingham and Kurlents, 1958). However, the corticoids evidently are not capable of blocking the release of ACTH in response to all types of tissue damage, unless the steroids are present in very large quantities. It has been suggested in the case of tissue damage, such as burns, that substances are released from the site of the injury (Hume, 1953; Woodbury, 1958; Share and Stadler, 1958), which are able to stimulate directly the release of ACTH by direct action on the pituitary, although final proof of such a suggestion is lacking. It is unlikely that histamine, epinephrine, serotonin or a variety of other compounds which are released during tissue-breakdown are responsible for directly stimulating the release of ACTH (Sandberg et al., 1953; Guillemin, 1955; Guillemin et al., 1957; Woodbury, 1958) as has been suggested. Nervous impulses from the site of injury cannot be responsible for initiating the release of ACTH following injury, as complete denervation of the part or transection of the cord fails to block the release of ACTH (Hume, 1953). The mechanism by which adrenocorticotropin is released following tissue damage needs to be determined.

Finally, there is evidence that two or more substances from the hypothalamus may regulate different activities of the adrenal cortex. Slusher (1958) has shown that lesions in one region of the hypothalamus will bloek the usual fall in adrenal ascorbic acid which follows cortical stimulation without preventing the release of cortical steroids. On the other hand, lesions in a nearby region can prevent the release of corticoids without preventing the fall in ascorbic acid. Guillemin ct al., (1958) have shown that there may be a functional separation of corticoid secretion from ascorbic acid depletion. These investigators found that a marked secretion of corticoids could occur in the absence of detectable changes in adrenal ascorbic acid concentration when both variables were measured simultaneously following stimulation by ACTH in hypophysectomized rats. Finally, there is other evidence which suggests that the factors controlling cortical hypertrophy (adrenal weight factor: AWF) and ascorbic acid (ascorbic acid factor: $\mathrm{AAF}$ ) reduction are separate entities and may respond differentially to different stimuli (Nowell, 1959). Nowell (1959) has suggested that stimulation of the release of various AC'TH factors from the pituitary may involve different mechanisms for different stimuli; for example, emotional stimuli may require activation of the hypothalamic centers, whereas adrenal regeneration may not require hypothalamic activity. 
Plainly there are many problems still to be solved before a complete and detailed explanation of the regulation of the release of $\mathrm{ACTH}$ and the fascicular corticoids will be possible, but the evidence for the mechanisms which have been described is strong. There is no doubt that the hypothalamus has a major role in the regulation of ACTH release and of adenohypophyseal function in general, and there is no reason to question the existence of such a neurohumoral mechanism. The evidence for some of the details, however, is less conchusive. The cortical glomerulosa and the release of aldlosterone are largely independent of this system of regulation, as indicated earlier, except for a very moderate response to ACTH for a limited time.

c. The function of the zona reticularis. The zona reticularis has been thought to seerete sex steroids (Selye, 1947), especially androgens, but the evidence for such a function is poor and largely inferential (Dempsey, 1948; Greep and Deane, 1949b; Jones, 1957). This zone is made up of a continuation of the cords or continuum, as the case may be, of the zona fasciculata, the cells of which are in varying degrees of activity and respond accordingly to stimulation by ACTH (Jones, 1957). By and large they have the appearance of cells of the fasciculata which are declining in activity and becoming obsolete, but there is certainly some functional capacity in the reticular cells, as indicated by various histochemical procedures and the presence of mitosis, although it is apparently much less than that of the zona fasciculata (Dempsey, 1948; Greep and Deane, 1949b). Symington et al. (1958) have presented convineing morphologic and histochemical evidence, coupled with secretory studies, that the reticularis normally secretes the hormones usually attributed to the zona fasciculata and that the latter is in a resting state until further stimulated by ACTH. This suggestion is well worth considering especially as morphologic changes are related to steroid production by direct measurement. These studies neeessitate a revision of the classic concepts of the functional roles of the reticularis and fasciculata which have been based only on morphologic evidence.

d. The problem of the function of $\mathrm{Y}$-zone. The $\mathrm{X}$-zone of the adrenal cortex of the immature house mouse (Mus musculus) is maintained by gonadotropin rather than by ACTH or growth hormone (Jones, 1949b, 1950, 1952, 1957). Its function is unknown, and histochemically it does not present the appearance of being a secretory zone (MePhail, 1944; Howard, 1939; 1946; McPhail and Read, 1942a, b). The cells do not give the usual histochemical reactions for steroids or other lipids, although they do contain ascorbic acid (Jones, 1949a, 1950). There is no evidence that the X-zone of the house mouse secretes sex steroids (McPhail, 1944; Jones, 1957), although such a function has been postulated by several investigators (Jones, 1957). In fact the large amounts of $\mathrm{C}_{19}$ steroids secreted by the adrenals of mice in 
response to ACTH-secreting pituitary tumors were from the cortices of adrenals without $\mathrm{X}$-zones (Bahn et al., 1957). On the other hand, Delost (1951) suggests that the $\mathrm{X}$-zone may be that part of the cortex responsible for secreting the adrenal androgens which maintain the activity of the vas deferens and ventral prostate of meadow voles (1/icrotus arvalis) in winter and in adrenalectomized voles (Delost, 195t). The evidence for this suggestion is tenuous and requires further investigation and confirmation. The function of the $\mathrm{X}$-zone, if there is one, remains undiscovered at the present time.

\section{Adrenocortical-Gonadal Interrelationships}

This problem has been discussed by Jones (1957), and the reader is referred to this work for a more detailed discussion of this intriguing problem.

There is a very close relationship between the cells of the adrenal cortex and those of the gonadal cells with respect to their origin and function. Both groups of cells arise from the embryonic genital ridge of the coelomic mesothelium, both secrete steroid hormones in response to stimulation by pituitary hormones; and there is a definite overlap in their functional capacities. Even though the adrenal cortex is believed to be a source of sex steroids, gonadectomy in normal animals is followed by complete involution of the sex accessories to the immature condition (Jones, 1957). The involution occurs even in mammals such as mice and rats in which weakly androgenic $\mathrm{C}_{19}$ steroids normally are an appreciable portion of the cortical secretory product (Dorfman and Shipley, 1956; Wilson et al., 1958). Similarly, the sex accessories and secondary sex characteristics which are under holmonal control fail to develop in the prepubertal castrate mammals (Burrows, 1949; Jones, 1957). Finally, the transplantation of the adrenal to the uterus does not alter the morphology of that part of the uterus or of the ragina which is in direct contact with the graft (Sakiz, 1956). These facts cast considerable doubt on their ability of the normal adrenal cortex of any species to secrete steroids with strongly androgenic or estrogenic actions. There is much evidence, however, that under certain circumstances the adrenal cortex is capable of assuming sex steroidal activity, for example, in the androgenital syndrome and similar virilizing conditions in man (Dorfman and Shipley, 1956; Gallagher et al., 1958). A striking increase in the secretion of weakly androgenic adrenal steroids may account for these effects (Jones, 1957), but they may also result from the conversion of adrenal androgens into testosterone in these circumstances (Dorfman, 1960). These conditions are usually associated with functional tumors of the cortex or with a marked increase in the secretion of ACTH with an accompanying cortical hyperplasia, but without an appreciable increase in the secretion of 
the corticoids, althongh with a marked increase in the secretion of $\mathrm{C}_{19}$ steroids (Dorfman and Shipley, 1956; Bradlow and Gallagher, 1957, Dorfman, 1960). It is also possible that these effects are a result of aberrant biochemical pathways in the synthesis of adrenal steroids which produce greatly increased amounts of weakly androgenic steroids or possibly some compounds with more strongly androgenic activity (Dorfman and Shipley, 1956; Bradlow and Gallagher, 1957; Gallagher, 1958, Dorfman, 1960). Finally there may be abnormal or greatly increased androgenic metabolites of adrenal steroids (Gallagher et al., 1958). It is well known that gonadectomy is followed by a marked increase in the production of gonadotropins; therefore if the production of sex steroids by the adrenal cortex normally was controlled by gonadotropins, one would expect an inereased secretion of these sex steroids to follow gonadectomy and to be reflected by continued maintenance of the sex accessories. This is not the ease, as the sex accessories involute nearly completely following gonadectomy (Burrows, 1949; Jones, 1957). However, it has been shown that inereased secretion of $\mathrm{ACTH}$ is followed after a time by a marked increase in the secretion of $\mathrm{C}_{19}$ steroids (Bush, 1953; Dorfman and Shipley, 1956; Bradlow and Gallagher, 1957: Jones, 1957; Wilson et al., 1958, Vande Wiele and Lieberman, 1960). Apparently slight stimulation of the sex aceessories by adrenocortical androgens occurs following ACTH treatment of gonadectomized rats, but these effects can be detected only histologically (Davidson and Moon, 1936 ; Davidson, 1937; Lostroh and Li, 1957, Desclin, 1959). In marked contrast to the general tenor of these results, Delost (1951) reports that the adrenal cortex secretes hormones with androgenic actions on the sex accessories of male Microtus arvalis, as determined by morphologic criteria, but this work has not been confirmed. Delost (1956a) maintains that cortisone will stimulate some of the secondary sex organs in these mammals, but confirmation of these res ults also is needed before acceptance of his conclusions is warranted. It is best for the present to assume that the adrenal cortex of most species normally does not secrete appreciable amounts of steroids with pronounced androgenic or estrogenic activity and that, it usually represents abnormal function when appreciable quantities of hormones with these activities are secreted by the cortex, (Bradlow and Gallagher, 1957). The ability of the adrenal cortical and gonadal endocrine cells to secrete steroids with overlapping activity is an intriguing problem, and the reader is referred to Dorfman (1960) for a more detailed discussion of the steroidal biosynthetic pathways involved and the elucidation of some of these paradoxes. There is certainly variation between species in the types of adrenal steroids normally secreted. Under abnormal conditions there is no doubt that the adrenals produce increased amounts of steroids which may have sex steroidal activity and that the ovaries, for example, can be induced 
to secrete steroids with cortical or androgenic activity. Hill (1948) has shown that under the proper conditions, the ovaries of mice can assume adrenocortical function when transplanted to the ears. Under other circumstances the ovaries can be made to secrete androgens (Hill, 1937; Delost, 1955). It must be apparent that the whole field of adrenal-gonadal relationships is poorly understood, especially with regard to species differences.

There is one area of adrenal-gonadal relationships, however, which seems to be on relatively solid ground. Selye (1939) called attention to the fact that a variety of stimuli which elieited increased adrenoeortical activity also inhibited reproductive aetivity. Female rats subjected to a variety of alarming stimuli exhibited ovarian atrophy and more or less permanent anestrus. These observations have been confirmed and extended for a variety of species, including mice, voles, man, and others, and for a wide variety of stimuli (Christian, 1959b). The entire and exact mechanisms by which inhibition of reproductive function is brought about are not known with certainty. It may be due in part to a direct suppressive action of the carbohydrate-active corticoids on the reproductive organs; for example, it has been shown that cortisone and hydrocortisone depress the ovarian response to chorionic gonadotropins in intact rats (Smith, 1955), possibly by direet action on the vaseulature. However, it is more likely that the suppression results primarily from an inhibition of the secretion of gonadotropins from the anterior pituitary when there is an increased production of adrenocorticotropin. Selye (1939) suggested that there is a "shift" in pituitary function; the production of gonadotropins is sacrificed in order to increase the production of "life-maintaining" ACTH. But it is equally, if not more, likely that the seeretion of gonadotropin is inhibited by increased amounts of cortical androgens following stimulation by ACTH. The ability of androstenedione and other related weakly androgenic steroids to suppress pituitary gonadotropic aetivity has already been discussed and it has also been stated that these steroids appear to be major natural secretory products of house mice, rats, man and probably other species. It is partieularly interesting, especially to those studying population phenomena, that immature mice are significantly more suseeptible than mature mice to the suppressive effects of steroids on the secretion of gonadotropins (Byrnes and Shipley, 1950; Byrnes and Meyer, 1951). There is also the possibility that nervous stimuli, acting directly through the hypothalamus, can depress gonadotropin secretion.

Whether one or all of these mechanisms is involved, there is a decrease in the secretion of gonadotropin with the result that the secretion of sex steroids by the gonads is inhibited and the secondary sex organs may approach the appearance seen following gonadectomy or hypophysectomy (Selye, 1939; Christian, 1959b). The germinal cells of the gonads also 
reflect the withdrawal of stimulation by the gonadotropins and sex steroids. Inhibition of these endocrine functions can result in a depression of all reproductive activities, including the onset of puberty, lactation, fertility, normal maintenance of the embryos in utero, size of the secondary sex organs, and other functions assoeiated with full functional competence of the pituitary-gonadal system (Christian, 1956, 1959a, b). It is pertinent here to note that androgen injeeted into female mice or rats under 10 days of age will produce permanent sterility; this finding suggests a method for the production of sterility or markedly delayed maturation in natural populations (Barraclough, 1961; Barraelough and Gorski, 1961). Most of these effects presumably are brought about by a deereased seeretion of gonadotropins, but some of the failures in reproductive function in these situations undoubtedly result from the direct effeets of inereased levels of corticoids following increased secretion of ACTH. Certainly the ability of the carbohydrate-active corticoids to increase protein catabolism and suppress growth, especially of the connective tissue and its products, and to suppress mitoses, must have appreeiable effeets on the reproductive process, especially on the developing fetus, as we have indicated earlier. There is considerable evidence to indieate that alarming stimuli during pregnancy, presumably with a marked increase in the secretion of adrenocortical steroids, can result in congenital anomalies dependent on the stage of fetal development (Fraser et al., 1953; Aycock and Ingalis, 1946; Ingalls, 1956 ; Curley and Ingalls, 1957; Ingalls and Philbrook, 1958).

Finally it should be borne in mind that the degree of reproductive suppression will vary in some proportion to the severity of the inducing stimulus and that there will be some variation with species with respect to the particular part of the reproductive cycle which will be most severely curtailed. For example, intra-uterine mortality and resorption of the embryos is marked in house mice, but apparently is inconsequential in Norway rats, whereas the postparturitional loss of young is as great or greater in rats than it is in mice (Christian, 1959b). Voles evidently are quite susceptible to depression of fertility and development of maturity (Kalela, 1957), but house mice are affected similarly (Crowcroft and Rowe, 1957). These variations are of particular importance to the investigator involved in comparative studies, especially in studies of phenomena relating to population density. It should be kept in mind that the severity and duration of the stimulus and the age of the affected mammal also will have an important bearing on the particular stage of the reproductive cycle affeeted as well as the degree of its inhibition. There are numerous examples of modification of reproductive function in response to various environmental stimuli, probably representative of activity of physiologie adaptive mechanisms. Baker and Ransom (1932) found that winter temperatures $\left(5^{\circ} \mathrm{C}\right.$. $)$ signifi- 
cantly reduced the number of births on Microtus agrestis even though they were maintained on summer food. Chitty and Austin (1957) have ealled attention to the effects of environmental factors, especially social "stress," on changes is estrous pattern and behavior exhibited by Microtus agrestis. Additional examples of the effects of deleterions environmental factors on reproduction will be given later.

There is another aspect of the suppression of reproduction which should be mentioned at the present time, although it is not directly pertinent to adrenocortical-gonadal relationships. Inanition, starvation, protein deficiency, and probably other dietary inadequacies are capable of depressing or totally inhibiting reproduction, the degree depending on the severity of the inadequacy (Lutwak-Mann, 1958), without stimulating increased secretion of $\mathrm{ACTH}$ and therefore inereased adrenocortieal activity (Baker, 1952; Christian, 1959b, c; Eisenstein, 1959), and adrenocortical secretion is aetually depressed in rats deficient in pantothenic acid (Eisenstein, 1957). The experimental evidence on which this statement is based has been derived from laboratory mammals and therefore does not necessarily apply to other species or to natural populations, but the burden of proof lies with those who claim otherwise. Recent experiments with house mice have demonstrated rather conelusively that inanition does not stimulate the pituitary-adrenocortical system, although its effect on the reproductive system is striking (Lutwak-Mann, 1958; Christian, 1959b, c). Furthermore, a deficiency of vitamin $B_{6}$ or partial starvation does not increase the secretion of corticoids nor impair the ability of the adrenal cortex to secrete them, even though they may be a marked cortical hypertrophy (Eisenstein, 1959). These results are in agreement with those from a large number of earlier experiments (ef. Baker, 1952; Christian, 1959c). In addition Srebnik et al. (1958) have shown that a protein deficiency results in a failure in the secretion of gonadotropins, and Marrian and Parkes (1929) had shown earlier that anterior pituitary extracts could correct the ancstrus produeed by a dietary insuffieiency of vitamin B with the return of normal estrus and normal changes in the acessory organs of reproduction with the estrous cycle. One might hypothesize that an inadequate diet would impair the ability of the anterior pituitary to synthesize its protein hormones. However, the ability of the pituitary-adrenocortical system of mice to exhibit the usual responses to alarming stimuli, and therefore the ability to elaborate adrenocorticotropin, in spite of inanition indicates that this is probably not the case (Christian, 1959c). Therefore, the decreased production of gonadotropins by animals subjected to inanition, starvation, or other dietary inadequacies probably operates through an unknown mechanism, probably at the hypothalamic level, which does not reflect an inability of the anterior pituitary to synthesize protein hormones. 
4. Epinephrine and Nolrepinephrine; the Hormonles of till dolrexal Medulla and Sympathetic Nervous Systeu

The adrenal medulla and its hormones, epinephrine and norepinephrine, have been the subjects of numerous and voluminous reviews and are also discussed in considerable detail in most good texts of physiology and pharmacology. Therefore, except for a few aspects, a detailed account of these hormones and their physiologic and pharmacologic actions will not be given here. The reviews of the following investigator's may be referred to for a more detailed coverage of the subject: Hartman and Brownell, 1949; von Euler, 1951; Hagen and Welch, 1956; Gaddum and Holzbauer, 1957; Ramey and Goldstein, 1957; Elmadjian et al., 1958.

The adrenal medulla is an integral part of the sympathetic nervous system. The medulla is homologous with the sympathetic ganglia and is innervated by cholinergic preganglionic fibers of the splanchnic sympathetic nerves. Upon stimulation of the sympathetie nervous system, the adrenal medulla discharges norepinephrine and epinephrine into the systemic circulation, the proportions of these two compounds varying with the species and with the nature of the stimulus (von Euler, 1951; Hagen and Welch, 1956; Gaddum and Holzbauer, 1957; Gray and Beetham, 1957; Elmadjian et al., 1958; Goldfien et al., 1958). Norepinephrine is also secreted by the postganglionic sympathetic nerves and by the extra-adrenal chromaffin tissue of the sympathetic nervous system (von Euler, 1951; Hagen and Welch, 1956). Norepinephrine probably is the neurohumoral transmitter substance of the postganglionic sympathetic nerrous system, and apparently is released on nervous stimulation at the sympathetic nerve endings (von Euler, 1951; Hagen and Welch, 1956; Richardson and Woods, 1959). These two hormones have profound effects on the cireulatory system and glucose and fat metabolism, but by and large their effeets are short lived, owing to their rapid destruction in the body by the cytochrome oxidase system or by amine oxidases (Bell et al., 1950; Gaddum and Holzbauer, 1957). Norepinephrine and epinephrine have a variety of effects over the entire body which are brought about largely by their actions on smooth muscle and which in general parallel the effects of stimulating the sympathetic nervous system (Hartman and Brownell, 1949).

Epinephrine and norepinephrine both have profound pressor effects on the cardiovascular system and on the levels of blood sugar; but epinephrine in general has a greater effect on carbohydrate metabolism and produces a greater hyperglycemic response than norepinephrine, whereas norepinephrine has a greater pressor effect than epinephrine (Gaddum and Holzbancr, 1957). In many ways the actions of these two hormones are similar, but in others they have opposing actions. Norepinephrine in general produces a 
greater rise in blood pressure than epinephrine because it increases overall peripheral resistance, largely by constricting the vasculature of the muscles as well as of the skin (Bell et al., 1950). Epinephrine produces a greater constriction of the vasculature of the skin, but dilates the vessels of the skeletal muscles and increases the cardiac output by increasing the rate and strength of the heart beat. The effects of norepinephrine on cardiac output are variable. Both of these amines decrease the formation of urine, produce relaxation of the gut by inhibition of its smooth muscle, produce splenic contraction, dilate the bronchi, inhibit the bladder, and produce pupillary dilatation (Bell et al., 1950). Both produce a rise in blood sugar but, as we have mentioned, epinephrine produces a greater rise than norepinephrine. The rise in blood sugar and subsequent glucosuria result from the mobilization of glucose from the readily available stores in the liver, and secondarily from the muscles. The inmediate effect of epinephrine is to release glucose from the available stores of liver glycogen; therefore the magnitude of the resulting hyperglycemia depends on the amount of glycogen in the liver (Hartman and Brownell, (1949). The cventual effect of epinephrine, after an initial depletion of liver and muscle glycogen, is to shift carbohydrate from the muscles to the liver, as the uptake of glucose by muscle is depressed and it is well known that lactic acid derived from muscle glycogen is used by the liver to synthesize glycogen.

The actions of these hormones are the classic preparations for "fight or flight" in response to emergency situations (Cannon, 1915, 1932). The combined activity of epinephrine and norepinephrine ensure adequate blood and glucose to the muscles, increased oxygenation, and adequate blood flow. An increased supply of oxygen to the tissues is ensured by an increase in respiratory rate, bronchial dilatation, and contraction of the splenic capsule with release of stored red blood cells into the circulation. Other activities, unneeded in an emergency, are suppressed. The adrenal medulla and sympathetic nervous system respond to cold, fear, rage, trauma, pain, blood loss, anoxia, emotional tension, and a variety of additional alarming stimuli. A variety of chemical agents, such as potassium and serotonin, will release the catechol amines from the medulla (Gaddum and Holzbauer, 1957). The sympathico-adrenal system represents a major and immediate reaction system of the body to prepare for, or to counteract the effects of, an emergency situation. The acute response is relatively short lived and serves to maintain life and counteract shock until the cmergency passes or until longer-acting adaptive systems, such as the pituitary-adrenocortical system, take over and aid in physiologic adaptation to the situation.

Recent evidence has shown that norepinephrine is normally found in the walls of the arteries (Schmiterlöw, 1948), and that it plays a major, perhaps decisive role in the maintenance of normal vascular tonus and reac- 
tivity, achieving the latter by diminishing the sensitivity of the arterial musculature to epinephrine and norepinephrine by maintaining a constant low level of pressor amines in the arterial wall (Burn and Rand, 1958a, b). The source of the norepinephrine in the arterial walls is apparently the chromaffin tissue including the adrenal medulla, or sympathetic neural terminations which appear to release a low level of these cathechols amines constantly into the circulation (Bell et al., 1950; Gaddum and Holzbauer, 1957). Experiments with reserpine (Burn and Rand, 1958a, b; Eränkö and Hopsu, 1958), which depletes the epinephrine and norepinephrine from the adrenal medulla and sympathetic chromaffin tissue, depletes the content of catechol pressor amines from the arterial walls and thereby makes them excessively sensitive to circulating epinephrine and norepinephrine. However, the arteries are unresponsive to other noncatechol pressor amines which apparently exert their usual effects by releasing the norepinephrine in the arterial walls (Burn and Rand, 1958a, b). There is also evidence that the adrenal carbohydrate-active corticoids have a part in the maintenance of arterial tonus and reactivity by increasing the sensitivity of the vasculature to the action of epinephrine and norepinephrine (Ramey and Goldstein, 1957).

Reserpine is a pharmacologic agent which causes the disappearance of the catechol pressor amines from the chromaffin tissue and subsequently from the arteries (Burn and Rand, 1957, 1958b), but stimulation of the sympathetic nervous system also can exhaust the pressor amines from the sympathetic ganglia and adrenal medulla, although the stimulus must persist for 30 minutes or longer to achieve exhaustion of the adrenal medullas of dogs (Gaddum and Holzbauer, 1957). Therefore, it is conceivable that prolonged and intense emotional stimuli, such as one might expect as a result of social interactions between animals in populations of excessive density, might exhaust the stores of pressor amines, especially in the subordinate animals. If such an event occurs, one might anticipate that there would be a subsequent depletion of the arterial norepinephrine and loss of arterial tonus which might account for the occasional deaths due to the shock seen in mice shortly after they are first placed together (Christian, $1955 \mathrm{~b}$ ) or following more protracted periods of social strife (Frank, 1953). A loss of vascular tonus with a subsequent hypotension, and eventually shock with circulatory collapse, could explain the symptoms observed by Frank (1953) in dense populations of Microtus in the wild or in captivity or might be a part of the picture of "shock disease" (Green and Larson, 1938; Green et al., 1939; Christian and Ratcliffe, 1952). There is also the possibility of a simultaneous exhaustion of readily available glucose reserves by the action of epinephrine, especially in animals with a high metabolic rate, or, perhaps more likely, a loss of the ability to mobilize 
reserves due to exhaustion of the supplies of epinephrine. Such a mechanism, albeit conjectural, may provide a better explanation for the immediate and precipitate cause, the proximate eause, of "shock disease" than the previously postulated pituitary adrenocortical exhaustion (Christian, $1950 \mathrm{~b})$, although adrenocortical hyperactivity probably plays an additive or even synergistic role in the cause of the immediate mortality in "shock disease." These conjectures are not meant to relegate the pituitary-adrenocortical-gonadal system to a secondary role in the more prolonged and chronic effects of inereased population density or in the control of population growth, as we shall see later. However, the available experimental ovidence places the sympathico-adrenal medullary system in the forefront of the mechanisms which respond acutely and which need investigation in relation to "shock disease" and the sudden and mass mortality associated therewith, as well as in relation to those sudden deaths, resembling hypoglycemic shock, which oceur on first placing strange mammals together.

The development of techniques to measure the secretion of the catechol amines has led to a number of investigations on the secretion of epinephrine and norepinephrine in response to a variety of stimuli. One can almost predict which of these two amines will be secreted in response to a particular stimulus by knowing which has the greater effect on blood sugar or on blood pressure. Norepinephrine appear's to be released preferentially by the adrenal medulla during rest (Caddum and Holzbauer, 1957). The plasma concentration of norepinephrine rises sharply with acute muscular work, but the response of epinephrine varies from no ehange to a marked rise, depending on the individual (Gray and Beetham, 1957). Both return to normal levels within 15 to 30 minutes after cessation of work. Hypoglycemia is followed by a marked and sharp rise in the medullary secretion of epinephrine with a much less marked rise in norepinephrine (Gaddum and Holzbauer, 1957; Croldfien et al., 1958). Infusion of glucose promptly returns their secretion to normal levels. Repeated production of hypoglycemia with insulin eventually leads to a decline in the secretion of epinephrine, evidently due to medullary exhaustion (EImadjian et al., 1958). Hypotension produces a marked rise in the secretion of norepinephrine, but little or no rise in epinephrine (Elmadjian et al., 1958). Surgical shock or a change in position from reeumbent to standing leads to a sharp rise in the secretion of norepinephrine with or without a rise in epinephrine. (Elmadjian et al., 1958). Tense, anticipatory but passive emotional situations produce a marked rise in the secretion of epinephrine, norepinephrine being secreted in normal amounts, but active, aggressive emotional situations are related to a rise in norepinephrine (Elmadjian et al., 1958). If the emotional display is intense enough, both epinephrine and norepinephrine are elevated. It is of particular interest that in adrenalectomized patients the secretion levels of norepinephrine and their diurnal variations are completely normal, 
indicating that the normal daily secretion of norepinephrine is largely from extra-adrenal chromaffin tissues, whereas the secretion of epinephrine is largely from the adrenal medulla. These facts are particularly pertinent to investigations of the physiologic responses of mammals to sociopsychologic factors.

The secretion of epinephrine and norepinephrine evidently is controlled by separate hypothalamic centers (Elmadjian et al., 19.58). The secretion of norepinephrine apparently is related to an area in the posterior hypothalamus and that of epinephrine to a lateral area. However, the vasomotor center is in the region of the floor of the fourth ventricle in the medulla oblongata, and it is the most sensitive area relating to the secretion of epinephrine (Elmadjian et al., 1958).

In addition to the actions of epinephrine and norepinephrine listed above, these compounds of the sympathico-adrenal system have important activity relationships with other endocrine organs and their hormones. The medullary hormones, thyroid, and pituitary growth and thyrotropic hormones have a number of interrelated and interlependent actions. We have already mentioned that growth hormone stimulates adrenal medullary hypertrophy (Moon et al., 1950, 1951). Hypertrophy of the medulla with a pronounced increase in its epinephrine content also follows chronic poisoning of the thyroids of male and female rats with thiouracil (Marine and Bauman, 1945), whereas chronic nicotine poisoning causes a marked medullary hypertrophy mainly owing to an increase in the norepinephrinccontaining cells (Eränkö et al., 1959). The latter response cannot be elicited in mice or guinea pigs, although the adrenal medulla of the mouse has both epinephrine and norepinephrine-containing cells (Eränkö ct al., 1959). This difference clearly demonstrates the kind of difference one may anticipate between species, even as closely related as are the rat and mouse (Rattus norvegicus and Mus musculus). The ability of epinephrine to mobilize depot fat and produce a rise in unesterified fatty acids in intact animals is thoroughly established (Hartman andBrownell, 1949), but its ability to mobilize depot fat, as well as its hyperglycemic action, seems to depend on the integrity of the adrenal cortex and the carbohydrate-active corticoids (Levy and Ramey, 19.58; DeBodo and Altzuler, 1958). These results have led Levy and Ramey (1958) to suggest that the adrenal steroids and epinephrine may act in concert to regulate metabolism of fat cells. On the other hand, the ability of epinephrine to mobilize unesterified fatty acids depends on optimal thyroid function, but apparently is unrelated to adrenocortical function (Goodman and Knobil, 1959). The mobilization of fatty acids evidently provides a readily available souree of metabolites for the formation of glycogen (Hartman and Brownell, 1949) or for direct utilization by the muscles (Fredrickson and Gordon, 1958). 
It is well known that epinephrine produces a marked rise in calorigenesis and oxygen consumption in intact animals (Hartman and Brownell, 1949; Gaddum and Holzbauer, 1957). This ealorigenic activity of epinephrine and norepinephrine is important in adaptation to cold (Hsich ct al., 1957a, b) and is potentiated strikingly by thyroxine (Swanson, 1956, 1957) and further intensified by the action of growth hormone, apparently by the effect of the latter in increasing thyroid function (Evans ct al., 1958). Evidently both epinephrine and thyroxine (and an intact pituitary-thyroid system) are essential for survival in cold exposure and for adaptation to cold, as, in the absence of thyroxine, epinephrine does not exert its calorigenic action in rats (Swanson, 1956). It was found that oxygen consumption in thyroidectomized rats increased in proportion to the dose of epinephrine when thyroxine was supplied at a fixed standard dose (Swanson, 1957). On the other hand, oxygen consumption varied linearly with the log-dose of thyroxine when the animals were kept on a standard dose of epinephrine (Swanson, 1956). Epinephrine apparently is essential for increased calorigenesis, but requires thyroxine for its activity. Swanson (1957) has expressed the opinion that since reactivity to epinephrine is directly and intimately dependent on the level of thyroxine, the main role of the increased secretion of thyroxine in acclimitization to cold may be to potentiate the calorigenic activity of epinephrine. In any event, both epinephrine from the adrenal medulla and an intact properly functioning pituitary-thyroid system are essential for increased calorigenesis and adaptation to cold. The necessity of the adrenal medulla and an intact thyroid is shown by the fact that the calorigenic response to cold is abolished either by adrenal demedullation (Morin, 1946a, b) or by thyroidectomy (Nwanson, 1957).

It should be obvious from the foregoing discussion that the adrenal medulla and sympathetic nervous system and their hormones, epinephrine and norepinephrine, are vital components of a variety of adaptive mechanisms and, if anything, their importance has tended to be underestimated. It appears from the available evidence that the sympathico-adrenal hormones may play a key role in the physiologic responses to sociopsychologic factors associated with changes in population density, and therefore deserve more investigation.

\section{B. The Thyroid Gland}

\section{INTRODUCTION}

The thyroid gland and its hormones, primarily thyroxine and to a lesser extent triiodothyronine, are important components of the internal mechanisms which provide the organism with sufficient physiologic flexibility to 
be able to maintain a constant internal environment in the face of stimuli from and changes in the external environment. The thyroid is intimately associated with a number of adaptive mechanisms and has important interractions with the adrenal cortex, as well as with the adrenal medulla and its hormones. This is not the place for a detailed account of thyroid physiology, but a brief review of the functions and actions of the thyroid and its hormones will be given, largely derived from standard accounts and reviews. Emphasis will be placed on the role played in physiologic adaptation to environmental changes, especially in response to adverse stimuli.

It has long been known that thyroid hormone is essential for normal growth and development, as well as for the normal metabolism of most tissues. Furthermore it has a vital role in permitting a mammal to adapt to changes in the temperature of the external environment, especially in adaptation to cold, by acting synergistically with the calorigenic action of epinephrine, as we have seen in the preceding section, as well as by playing an important physiological role of its own (Swanson, 1957). The evidence that will be discussed subsequently shows that the thyroid also is involved intimately in the physiological responses to alarming stimuli. Therefore the thyroid, like the adrenal gland, assumes particular importance in a discussion of adaptive meehanisms.

\section{The Thyroid Hormones and Their Actrons}

The thyroid hormones, tetraiodothyronine (thyroxine) and $l-3,5,3^{\prime}$ triiodothyronine, have an overall action of increasing heat production by increasing the oxidative processes of many tissues and therefore their oxygen consumption (Sollman, 1957). This metabolie effect is in part brought about by the augmentation or facilitation of the calorigenic action of epinephrine, and it has been suggested that thyroxine and epinephrine influence consecutive rate-limiting reactions in the metabolic eycle, thyroxine acting at a later stage than epinephrine (Swanson, 1956). The metabolic effect of thyroxine, however, is not exerted equally on all tissues. The rate of oxidation by brain tissue, for example, is not influenced at all (Tata ct al., 1957), whereas the metabolism of the liver is increased more than of the body as a whole (Barker and Schwartz, 1953). However, the electroencephalogram excitability of the brain, electrolyte distribution, and circulation of the brain are profoundly affected by thyroid hormones (Tata et al., 1957). Increased thyroid hormone first affects carbohydrate, then fat, and finally protein metabolism (Sollman, 1957). Conversely, a deficiency in thyroid hormone reduces the oxidative activity of tissues in general. It has been suggested that all the actions of the thyroid hormones 
on metabolic processes may reflect a primary action at one biochemical site, possibly on cytochrome c (Rawson et al., 1955). Sollman (1957) has listed the following additional effects of thyroxine. Increased levels of thyroid hormones usually are accompanied by an increased pulse rate, increased nervous excitability, weight loss, and deereased liver glycogen. Thyroxine also sensitizes the tissues, especially the blood vessels, the actions of sympathomimetic compounds such as epinephrine (see above) as well as to the toxic effects of poisons. The increased sensitization apparently occurs at the receptor mechanisms. Thyroxine also effects the circulation, but mainly as a result of increased heat production. Thyroxine has a direct effect on the heart in increasing its oxygen consumption, but it also has an indirect effect on the heart and the rest of the circulatory system in the following way. The delayed, indirect, effect is due to an increased metabolic demand of the tissnes which results in an increase in carbon dioxide and decreases in oxygen at the artcriolar level. These effects result in a subsequent decrease in peripheral resistance, increased venous return, and, via cardiac reflexes such as the Bainbridge, an increased cardiac output and increased pulse pressure. The increased heat resulting from increased oxidation must be dissipated, and this is accomplished by dilatation of the vessels and opening of the arteriovenous anastomoses in the skin and other tissues with an accompanying increase in the amount of heat loss due to radiation. The normal calorigenic action of the thyroid hormones is essential for normal growth, maturation, and tissue differentiation.

Proper functioning of the thyroid and the production of thyroid hormones is completely dependent on an adequate dietary intake of iodine. Inorganic iodine is essential for the formation of the thyroid hormone by the thyroid gland, as the thyroid is incapable of trapping organic iodine (Salter, 1949; Halmi et al., 195.3; Rawson et al., 195.5). The way in which trapped inorganic iodide and tyrosine are converted into the thyroid hormone(s) has been critically reviewed by Rawson et al., (195i)). Inorganic iodide is trapped and presumably momentarily oxidized to active iodine in the thyroid epithelium. Pituitary thyrotropic hormone promotes the trapping of iodide by the thyroid gland, although the gland has some autonomy in this activity (Vander Laan and Greer, 1950; Halmi et al., 195.3; Vander Laan and Caplan, 1954; Vander Laan, 1955). The trapped and activated iodine is then used in converting tyrosine to diiodotyrosine, probably in the presence of peroxidases and cytochrome oxidases. Two molecules of diiodotyrosine are condensed to form a single molecule of thyroxine (tetraiodothyronine), which combines with thyroid globulin (thyroglobulin) and is stored as such in the colloid of the thyroid follicles. Other iodinated thyronines are found in the thyroid, but in much smaller amounts than thyroxine. These probably result from partial iodination and may represent other pathways 
in the formation of thyroxine than the one given above (Rawson et al., 1955). However, the metabolic pathway given is the usually accepted scheme for the synthesis of thyroxine. Thyroxine presumably is released from thyroglobulin by the action of proteolytic enzymes (Sollman, 1957). Thyrotropin promotes the iodination of tyrosine and the release of thyroxine from thyroglobuliu. A small amount of thyroxine loses one of its iodine atoms to become $(-3,5,3,3$-triiodothyronine in the thyroid, probably more in the peripheral tissues, and small amounts of this compound are found in the thyroid gland and in circulation (Gross, 19.5.5) $l-3,5,5,3^{\prime}-$ Triiodothyronine has a more pronounced action on oxidative processes than thyroxine, and its action is much more rapid, but less prolonged (Sollman, 1957). It has been postulated that the triiodothyronine provides the immediate thyroid response and is the substance which enters the cells and exerts the ultimate thyroid action, whereas thyroxine is the circulating form of the thyroid hormone (Gross, 1955). The differential distribution of these two hormones in the circulation and in the cells of rarious tissues supports this hypothesis (Gross, 195.5). Since inorganic iodide is essential for the normal functioning of the thyroid gland, the rate at which radioactive iodine is trapped and accumulated by the gland is a good index of thyroidal activity provided there is not an increased renal excretion of iodine. The rate of release of radioactive iodine from the thyroid is considered a more reliable and reproducible index of thyroid activity, as each animal can serve as its own control in experimental procedures (BrownGrant et al., 1954a).

\section{Factors That Regulate the Activity of the Thyroin}

The secretory aetivity and hormone synthesis by the thyroid is largely under the eontrol of the protein hormone thyrotropin (TSH) secreted by the basophils of the anterior pituitary (D'Angelo, 1955). However, the thy roid is capable of a low level of autonomous function without stimulation by the pituitary (Brown-Grant et al., 1954a). The rate of release of TSH from the anterior pituitary is apparently related to the level of eireulating thyroid hormone (D'Angelo, 1955), although the mediation of the hypothalamus appears to be required (Harris and Woods, 1958; D'Angelo and Traum, 19.58); therefore a low level of cireulating thyroid hormone stimulates an increased secretion of TSH and a high level inhibits its secretion. This is the classie eoncept of an endocrine feedback mechanism which operates to regulate the release of hormone from the target gland and to maintain a more or less constant level of circulating hormone under normal circumstances. However, there is recent evidence to suggest that the seeretion of TSH may not be a direct response to the level of eireulating thyros- 
ine, but rather to one or more of its peripheral metabolic actions (Goldberg et al., 1957). The secretion of thyrotropin from the pituitary is undoubtedly under the control of the hypothalamus (D'Angelo and Traum, 1958; Harris and Woods, 1958, Harris, 1959). Like the secretion of adrenocorticotropin, the secretion of thyrotropin appears to be controlled by a humoral factor from the hypothalamus (Brown-Grant et al., 1957). Section of the pituitary stalk leads to a loss of the inhibitory response of the thyroid to restraint or pain, and regeneration of the pituitary portal vessels is accompanied by a return of this response (Harris, 1955a, b; Reichlin, 1957a, b). The thyroid remains at least partially responsive to exogenous TSH when the pituitary stalk is sectioned (Reichlin, 1957a, b; Harris and Woods, 1958). There is also evidence that the hypothalamus is necessary for a decreased blood concentration of thyroid hormone to effect an increased secretion of TSH (Greer, 1951; 1952; Bogdanove and Halmi, 1953; Harris and Woods, 1958; D'Angelo and Traum, 1958). Increased activity of the thyroid accompanies electrical stimulation of the anterior portion of the median eminence, but not other parts of the hypothalamus (Harris and Woods, 1958). The hypothalamic factor responsible for stimulating the release of TSH does not appear to be contained in or associated with Pitressin (Reichlin, 1957a; D'Angelo and Traum, 1958), but little else is known of the nature of this factor. In addition to these regulating factors, the release of TSH is also affected by cortisone and ACTH (Brown-Grant et al., 1954c). Cortisone and ACTH inhibit the release of radioiodine from the thyroid of rabbits and rats, probably by inhibiting the release of TSH, although the mechanism by which this is accomplished is unknown (BrownGrant et al., 1954b, c; Brown-Grant, 1955). Cortisone and ACTH have also been reported to inhibit the uptake of radioiodine by the thyroid of rats (Money et al., 1950; Albert et al., 1952; Perry, 1951; Verzár and Vidovic, 1952) and of humans (Perry, 1951; Kuhl and Ziff, 1952; Albert et al., 1952; Berson and Yalow, 1952). However, reports of decreased uptake of radioiodine must be interpreted with caution, as it may reflect a lowered concentration of circulating iodide as a result of increased renal clearance of iodide following treatment with cortisone or ACTH (Brown-Grant et al., 1954c). Adrenalectomy inhibits the release of iodine and therefore of thyroid hormone from the thyroid glands of rats (Flückiger and Verzár, 1955). Bastenie and Ermans (1958) have shown that cortisone, in addition to its effect on the secretion of TSH, inhibits the stimulating effect of thyroxine on oxygen consumption and phosphorus turnover but fails to inhibit these actions of triiodothyronine. These authors concluded that cortisone inhibits the peripheral degradation of thyroxine to triiodothyronine. Nevertheless, there is little doubt that cortisone and ACTH do inhibit 
thyroid activity, probably by inhibiting the release of TSH from the pituitary.

Epinephrine, in addition to its synergism with thyroxine with respect to calorigenesis, appears to have more direct effects on thyroid aetivity, although the reports are eonflieting. Badrick and his co-workers $(1954,1955)$ reported that epinephrine inhibited the uptake of radioiodine by the thyroids of intact and hypophyseetomized rats, and similar results were reported for intaet rats by Money et al. (1950). Brown-Grant et al. (1954b) found that epinephrine decreased the release of radioiodine by the thyroids of intaet rabbits. On the other hand, epinephrine has been reported to inerease the release of radioiodine by the thyroid (Williams et al., 1949) and to inerease the content of radioiodine in the thyroid (Botkin and Tew, 1952), although the latter eould result from an inhibition of its release. More recently Ackerman and Arons (1958) have reported that epinephrine increases the release of radioiodine from the thyroids of intaet and hypophysectomized dogs. These divergent results are diffieult to reconcile, although the fact that epinephrine aeted in hypophysectomized as well as in intaet rats and $\operatorname{logs}$ (Badrick et al., 1955; Ackerman and Arons, 1958) suggests that the effects were directly on the thyroid gland. It is possible that these varying results may reflect the effeets of epinephrine on the thyroidal vaseulature in different animals and different circumstances, as was suggested by Badrick et al. (1955).

From the foregoing aecount it should be clear that the regulation of thyroid function is complex and involves a number of faetors. However, there seems to be little doubt that the hypothalamus has a relatively basic role in regulating the secretion of thyrotropin from the pituitary, and evidently is eapable of inhibiting its release (Brown-Grant et al., 1954b; Reichlin, 1957a; Harris and Woods, 1958; D'Angelo and Traum, 1958). D'Angelo and Traum (1958), in an extensive series of experiments on hypothalamichypophyseal-thyroidal relationships in rats, concluded that the hypothalamus funetions as a modulator, modifying the production and release of thyrotropin from the adenohypophysis not only under conditions of inereased demand, but also in meeting day-to-day needs. Normal blood levels of TSH cannot be maintained in the absenee of stimulation by the hypothalamus even with decreased levels of thyroid hormone. However, the adenohypophysis keeps its capacity to produce and release TSH, but regullated by the level of circulating thyroid hormone or by peripheral metabolic aetions thereof (ef. above). The action of thyroid hormone may be directly on the pituitary (D'Angelo and Traum,(1958). The injection of minute amounts of thyroxine into the hypothalamus is followed by a relatively long Iatent period before the release of TSH is inhibited, whereas 
injection into the anterior pituitary is followed by a more immediate response (Yamada and Greer, 1959; Yamada, 1959).

The thyroid was blocked in these studies (D'Angelo and Traum, 1958) by hypothalamic lesions or by treatment with propylthiouracil. Thyroid function was evaluated by the rate of turnover of radioiodine, bioassay of the pituitaries and plasma for their content of TSH and thyroid hormone, and was finally correlated with the results of detailed histologic studies of the glands of experimental and bioassay animals. The results appear to be definitive. These workers were further able to demonstrate the independence of ovarian, adrenocortical, and thyroid-regulating parts of the hypothalamus. How the adrenal corticoids and adrenocorticotropin fit into the regulation of thyroid function is unknown at present.

\section{Thyroid Responses to External Stimuli}

Probably the best-known action of the thyroid is its ability to increase the secretion of thyroid hormone in response to cold and to decrease its release in response to heat (Ring, 1942; Brown-Grant et al., 1954a; Stevens et al., 1955; Hellman and Collins, 1957). The gland also becomes hyperplastic with prolonged exposure to cold (Money, 1955). Upon exposure to low temperatures, there is an increased release of thyroid hormone from the thyroid gland (Brown-Grant et al., 1954a; Woods and Carlson, 1956), apparently in response to increased secretion of TSH by the pituitary (Stevens et al., 1955). However, Ring (1942) found that the thyroid per se can account for only a small part of the increase in heat production required to meet the demands of mice exposed to $2-4^{\circ} \mathrm{C}$, and that epinephrine and thyroid hormone are required for a maximum continued metabolic response to cold. He therefore concluded that the principle action of thyroid in response to cold exposure was to sensitize the animal to the calorigenic action of epincphrine. Swanson $(1956,1957)$ essentially confirmed and extended Ring's findings and conclusions, as we have seen above. Thyroidectomy abolishes the response to cold and deprives the animal of the ability to survive exposure to cold (Swanson, 19.57).

Exposure to cold increases pituitary-adrenocortical as well as thyroidal activity, contrary to the more usual reciprocal relationship between ACTH and TSH sccretion by the pituitary described below. Increased thyroidal activity apparently is the more vital of these two adaptive responses to cold. Thyroxine permits survival of hypothyroid rats subjected to cold, whereas adrenocorticotropin and adrenocortical extract do not (Freedman and Gordon, 1955). Similarly, minute amounts of thyroxine permitted $100 \%$ survival of rats treated with thiouracil lipon exposure to cold, whereas adrenocortical extracts failed to increase survival (Ershoff, 1948). 
Heat, on the other hand, climinishes thyroid activity (Hellman and Collins, 1957). Rats and mice have been shown to have reduced thyroid activity in the warm summer months (Hurst and Turner, 1947). Puntriano and Meites (1951) suggested that the seasonal changes in thyroid activity might be due to changes in day length rather than to seasonal ehanges in temperature, on the basis of their findings that prolonged exposure to light inhibits thyroid activity in mice and exposure to darkness increases thyroid activity. However, these results could not be confirmed in rabbits (Brown-Grant et al., 1954b). The rate of release of radioiodine by the thyroids of rabbits was unaffected by prolonged exposure either to darkness or to light. Therefore the validity of the hypothesis that day length affects thyroid activity is dubious.

In contrast to the thyroidal-adrenocortical relationship in response to exposure to cold, there is abundant evidence indicating that the activity of the thyroids is inhibited in response to alarming stimuli which evoke an increased pituitary-adrenocortical activity. In fact it has come to be generally accepted that there is a reciprocal relationship between ACTH and TSH secretion in response to "stress" (Harris, 1955a). Anoxia, nephrectomy, vitamin deficiencies, tourniquet shock, fasting, injected typhoid vaccine, swimming in cold water $\left(15^{\circ} \mathrm{C}\right.$.), injections of formalin, spinal cordotomy, electroshock, and gastrointestinal and peripheral trauma all inhibit the activity of the thyroid glands of rats (IVilliams et al., 1949; Paschkis et al., 1950; Bogoroch and Timiras, 1951; Hamolsky et al., 1951; Van Niddlesworth and Berry, 1951; Badrick et al., 1954, 195.j). Similar results were obtained with rabbits, using the release of radioiodine as the basis of measurement (Brown-Grant et al., 19; 4 b). Hemorrhage, anesthesia, laparotomy, intraperitoneal injections of turpentine, draining subeutaneous abscesses, or emotional stress in the form of restraint, subcutaneous faradic stimulation, or sudden changes from light to dark or vice versa were all found to inhibit thyroid activity in rabbits for 1-2 days. After this time the rabbits evidently became used to the procedures and were no longer alarmed by them, as thyroid function gradually returned to normal with continued exposure to these emotional stimuli. In some instances of these experiments the release of radioiodine from the thyroid was totally inhibited (Brown-Grant et al., 1954b). Emotional stimuli also were followed by a time lag of somewhat less than 3 hours between their first application and the first thyroid response. These experiments demonstrate that emotional factors, visual stimuli, and the central nervous system can affect thyroid function, presumably via the hypothalamus, just as they can increase the secretion of ACTH or adrenal medullary hormones (BrownGrant et al., 1954b). Kracht (1954) described a "true thyrotoxicosis" in wild rabbits stimulated by fear in response to being chased by ferrets and 
stated that this reaction was a "model of a thyrotrophic alarm reaction." His evidence was, for the most part, indirect and his conclusions that there was increased thyroid activity on the basis of increased renal excretion of injected radioiodine is untenable, especially for animals with a marked increase in adrenocortical activity. Finally, Brown-Grant et al. (195+b) found that the release of radioiodine by the thyroids of wild rabbits was inhibited by emotional stress, just as it was in the usual domestic rabbit. In view of these facts, the concept of a "fright thyrotoxicosis" is inacceptable. The inhibition of thyroid activity by emotional stress was not as consistent in adrenalectomized as it was in intact rabbits, otherwise their responses were similar. There is little doubt that the inhibition of thyroid activity in these experiments was due to a decrease in the secretion of thyrotropin (Brown-Grant $c t a l ., 1954 a, b)$. It is true that cortisone and ACTH inhibit the release of TSH from the pituitaries of rats (BrownGrant, 1955) and rabbits (Brown-Grant et al., 1954a, b; Harris and Woods, 1958), but the adrenal corticoids cannot be responsible for the major part, if any, of the inhibition of the release of thyrotropin in the above experiments, as adrenalectomy did not affect the inhibition of the thyroid, and cortisone was without effect on the release of radioiodine by the thyroids of rabbits injected with TSH. Finally, injected TSH abolishes the inhibition of the thyroid produced by ACTH and cortisone (Harris and Woods, 1958).

There has been some confusion in the literature about the effect of alarming stimuli on the thyroid. The experiments which have been discussed so far indicate that the thyroid is depressed under these circumstances, but it is also well known that emotional factors can precipitate thyroid crises in humans (Selye, 1950). These apparently contradictory experiences may depend on species differences, dose-time relationships, or both, as the recent work of Gerwing et al. (1958) has indicated. These investigators have shown that thyroid activity in mice, rats, and rabbits subjected to chronic toxic "stress" (injection of small doses of bacterial endotoxins repeatedly for 24 days) is inhibited at first, but returns to normal and eventually cxceeds the original normal level as the stimulation continues (Gerwing, 1958; Gerwing et al., 1958). On the other hand, guinea pigs and rhesus monkeys exhibit increased thyroid activity from the beginning. Injected thyrotropin stimulates the thyroids of mice, rats, and rabbits with inhibited thyroids following injection of the toxin, indicating that the depressed thyroid function was duc to a diminished secretion of TSH. It may be coincidental, but it is nevertheless interesting that these differences in thyroid function between species in response to bacterial toxin coincide with the differences in the secretory patterns of the major corticoids in these same species (cf. above): rats, mice, and rabbits secrete primarily corticosterone, and the other two species, hydrocortisone. These 
investigators (Gerwing, 1958; Gerwing et al., 1958) conclude that the hypothesis of a reciprocal relationship between the secretion of TSH and ACTH by the anterior pituitary is compatible with the evidence for rats, mice, and rabbits, but not for guinea pigs and monkeys. They suggest that in the latter two the inhibitory effect of increased corticosteroids does not occur. However, we have seen that the corticosteroids probably have no effect in the inhibition of TSH release and thyroid function in rats and rabbits in response to alarming stimuli; so that some other explanation must be sought. Man is probably similar to guinea pigs and rhesus monkeys in the above responses of the thyroid (Gerwing et al., 1958). These studies emphasize the importance of careful comparative work as well as the importance of dose-time relationships in physiologic functions in different species of mammal.

Starvation causes a profound depression of thyroid activity, probably by suppressing the secretion of TSH, as it has been shown that acute starvation markedly depresses the blood level of TSH in mice and rats (Money, 1955). However, if starved animals are subjected to cold, the degree of thyroid depression is inversely related to the degree of the reduction in environmental temperature (Reichlin, 1957a). The thyroidal iodine release rates at the lower temperatures, even though reduced, are appreciably higher than they are in starved animals at higher temperatures. The maintenance of body temperature appears to take precedence over the conservation of nutritive reserves and tissues in the regulation of thyroid activity activity (Reichlin, 1957a).

In contrast to some of the above relationships, increased thyroidal activity increases adrenocortical activity in white rats (Wallach and Reineke, 1949). Administration of thyroxine decreases adrenal ascorbic acid to minimal levels after 2-4 days and is followed, upon continued treatment with adequate doses of thyroxine, by a progressive increase in adrenal size and ascorbic acid content which reaches maximum in 4 weeks. The increase in adrenal weight in these circumstanees is roughly proportional to the dose of administered thyroxine. There is a narrow dose range of thyroxine in which there is no effect on the adrenals. Pcrhaps this dose range represents the normal physiologie daily secretion rate. It may be that the adrenal hypertrophy observed in rats exposed to cold is in part a result of the increased thyroid activity. On the other hand, adrenocortical function does not seem to depend on the presence of a normally functional thyroid, as thyroidectomy does not cause adrenal atrophy in rats although the zona fasciculata is reduced in width (Hess, 1953), nor does thyroidectomy decrease the ability of the adrenals to respond to stress (Hess and Finerty, 1952). The adrenals of rats treated with antithyroidal compounds (thiour:acil) also (were) responsive to injected adrenocorticotropin (Freedman and 
Gordon, 1955), although others report adrenal atrophy following blockade of the thyroid with antithyroid eompounds (Scifter et al., 1949). Finally, thyroxine augments the adrenal hypertrophy produced by growth hormone, although it does not augment the adrenal hypertrophy produced by ACTH (Bois and Selye, 1957).

There are many other factors which affeet thyroid function. A high dietary salt intake ean increase thyroid activity, even with a reduced iodine intake, in a rather circuitous way (Isler et al., 1958). The mechanism appears to be that with increased $\mathrm{NaCl}$ intake, and consequently an inerease in the excretion of $\mathrm{NaCl}$, there is apparently an increasc in the excretion of iodine with the sodium chloride. The loss of iodine produees a low serum concentration of iodine and seeondarily a low output of thyroxine. The low thyroxinc in turn stimulates increased secretion of TSH from the pituitary and a resulting stimulation of the thyroid gland.

\section{Thyroidal-Goxadal Ixterrelationships}

In addition to its more or less reciprocal relationship with the adrenal cortex, the thyroid, and therefore thyroidal adaptive responses, has important effects on reproduction and the reproductive organs. These effeets are of particular interest here, because they may occur in response to alarming stimuli and must be evaluated as possible factors producing the (hanges in reproductive funetion seen in mammals, especially in relation to the density of the population. A thyroidal-gonadal relationship is indicated by the tendeney for goiters to oceur at puberty, during pregnaney, or during estrus; also the serum protein-bound iodine is elevated during pregnancy, and stilbesterol increases the release of iodine by the thyroids of rats and mice (Sollman, 1957). There is eonsiderable evidence indicating that hypothyroidism is associated with abnormal ovarian funetion in humans (Rawson et al., 1955). Cold exposure causes a marked increasc in the length of the estrous cycle in rats which is prevented by administering thyroxine (Dempsey and Uotila, 1940; Denison and Zarrow, 195.5). Thyroxine also augments the recovery of function of the testes of immature rats from the atrophic effeets of starvation (Horn, 1955). The recovery of the testes in this ease is not related to the recovery in body weight, and the recovery of the sex accessories following treatment with thyroxine was only partial and required a greater period of time. Maqsood and Reineke (1950) found that high temperatures $\left(30^{\circ} \mathrm{C}\right.$.) decreased the weights of the testes and seminal vesicles of mice, but the administration of thyroprotein increased the weights of these organs at that temperature. Mild hyperthyroidism stimulated, and hypothyroidism inhibited, sexual development in growing male mice (Maqsood and Reineke, 1950). Higher doses of thyroid 
hormone decreased the weights of the reproductive organs. However, thyroidectomy was followed by an increase in the relative weights of the testes and seminal vesicles of rats (Hess, 1953). Hess (1953) concluded that thyroidectomy appears to sensitize the gonads and secondary sex organs to the actions of gonadtropins. Hyperthyroidism is associated with abnormal reproductive function in female rats (Denison and Zarrow, 195.5). Prolonged exposure to $2^{\circ} \mathrm{C}$. resulted in a marked increase in the length of the estrous cycle: proestrus and estrus were markedly prolonged, while metestrus and diestrus were shortened. Treatment with $50 \mu \mathrm{g}$. of thyroxine corrected these changes. On the other hand, brief (3 days) treatment of female rats with thyroid hormone just prior to mating apparently resulted in an increase in subsequent litter size, but the same amount of thyroid hormone given during hot weather or an increased amount of thyroid hormone adversely affected reproduction (Kraatz, 1939). These results seem to indicate that there is an optimal level of thyroid funetion for proper functioning of the reproductive system, and that any appreciable deviation from this level in either direction leads to diminished reproductive function.

The role of the thyroid in reproduction has been investigated by using radiothyroidectomized mice (Bruce and Sloviter, 1957). Radiothyroidectomy apparently had no effect on male fertility, female fertility, or litter frequency, but estrus and gestation were prolonged. Litter size possibly was reduced owing to increased resorption of embryos, but the matter requires further investigation. In contrast to these results, Chu (1944) found that surgical thyroidectomy in rabbits during early pregnancy resulted in total loss of fetuses due to resorption or abortion. The same operation in later pregnancy resulted in the young being stillborn. Induction of pregnancy in thyroidectomized rabbits was followed by resorption or abortion of the embryos or a prolongation of gestation, the young subsequently being born dead. Feeding desiecated thyroid tended to prevent the adverse effeets of thyroidectomy (Chu, 1944). Hypothyroidism induced by thiouracil in rats apparently was without effect on fertility in males and females but interferred with gestation in pregnant females, causing resorption in $100 \%$ of the animals (Jones et al., 1946). Thiouracil treatment of rats for shorter periods of time, less than 100 days, did not prevent some of the rats from delivering litters, but the young exhibited abnormal growth and development. It is quite erident that pregnancy is adversely affected by severe hypothyroidism with a marked increase in intrauterine mortality.

In general reproductive function is altered by marked inereases or decreases in thyroid activity, and it seems that the depression in thyroid activity in response to alarming stimuli must play a role, along with increased adrenocortical activity, in the commonly observed suppression of 
reproductive function. However, a great deal more investigation is necessary to elarify thyroidal-adrenal-gonadal relationships.

\section{Other Thyroid Relationships}

In addition to its relationship to the adrenals and gonads and to its general metabolic effects, the thyroid hormones play specific roles in the maintenance of other organs, and these may offer means of evaluating thyroid function in intact mammals in the field, providing they are used in conjunction with other information. For example, the harderian glands of rats and mice are maintained by thyroid hormones and as yet unknown pituitary factors (Boas and Bates, 1954; Hellman and Collins, 1957), although the thyroid may not be essential for the maintenance of these glands in guinea pigs (Smelser, 1943). Thyroid hormone, in conjunction with androgens, is required for the maintenance of the size and granulation of the cells of the serous tubules of the submaxillary glands of male rats (Grad and Leblond, 1949). The thyroid alone restores the number of these cells to normal, but does not affect the size or granulation.

Thyroid function in general declines moderately with age and increased size (Hurst and Turner, 1947), but there are no marked effects of this decline on growth or other functions.

It should be clear from the above aceount that the thyroid and its hormones participate actively in the adaptive responses and probably play a significant role in many aspects of these responses. Investigations on the reactions of the thyroid with changes in the size of mammalian populations and its relationship to adrenal and reproductive functions are needed.

\section{Other Endocrine Adaptive Factors}

\section{Pancreatic Islets}

A discussion of the physiology of the pancreas with respeet to glueose metabolism is not properly the function of this chapter, but it should be mentioned as one of the important endocrine organs regulating glucose utilization. The hormone best known of the islets of Langerhans is insulin, which is derived from the beta cells. More recently glucagon, presumably from the alpha cells, has been described. Insulin, in brief, inereases the utilization of glucose and in many of its actions is antagonized by cortisone. Glucagon, on the other hand, is a hyperglycemic factor. The function of these hormones with respect to the generalized adaptive responses has been little studied, but they certainly must play an important part in the general economy of the mammal under adverse circumstances. Anything affecting 
the mobilization and utilization of glucose is inevitably going to have an important bearing on the adaptive responses when a more or less final picture is available of the entire scheme of responses. The frequency of diabetes in, and its effect on, natural populations is unknown, although recent work indicates that under certain circumstances it is far from being a rare disease.

\section{Posterior Pituitary}

The posterior pituitary, or neurohypophysis, has been mentioned repeatedly in the discussions on the regulation of the secretion of the hormones of the anterior pituitary, but in addition the posterior pituitary has important functions of its own in physiologic adaptation. A lengthy discussion will not be indulged here, as these functions more properly come under the purview of discussions elsewhere or are insufficiently well known to warrant elaboration in detail.

a. Antidiuretic hormone. The posterior pituitary secretes an antidiuretic factor $(\mathrm{ADH})$ which is important in regulating the reabsorption of water from the renal tubules. Section of the pituitary stalk with a resultant denervation of the posterior pituitary is followed by diabetes insipidus as a result of the lack of antidiuretic hormone. ADH is apparently manufactured in the hypothalamus and traverses the fibers of the supraopticohypophyseal tract and is released into the systemic circulation in the posterior pituitary. A vasopressor activity is associated with the antidiuretic factor of the pituitary, but the neurohypophyseal hormone is much less effective in this activity than in its antidiuretic action. There is evidence that the secretion of $\mathrm{ADH}$ is increased in response to alarming stimuli as well as to dehydration, but there is also evidence that its secretion is independent of the release of ACTH from the anterior pituitary (cf. above). For a more detailed account of the pituitary antidiuretic hormone, the reader is referred to the recent review by Thorn (1958).

b. Lipid mobilizing factor. The recent work of Seifter and his colleagues (1959) has indicated the existence of a posterior pituitary lipid-mobilizing factor (LMF) which is released in response to adrenal corticoids. The hormone appears to be a peptide capable of mobilizing triglycerides from the mesenteric fat depots following injections of cortisone, exposure to cold, or subjection to other stimuli which induce an increased adrenocortical secretion. The lipid mobilization is blocked by adrenalectomy or hypophysectomy. The pathway for lipid mobilization by LMF in response to alarming stimuli appears to be anterior pituitary, adrenal cortex, and posterior pituitary (Seifter et al., 1959). 


\section{Other Gland Systems}

Many other glands, organs, and organ systems participate in physiologic adaptation, either directly or in response to increased adrenal or other hormones or diminution thereof. The essential integrative role of the central nervous system has been implicit throughout the foregoing discussion. The gastrointestinal tract and its appendant organs such as the pancreas, liver, and salivary glands also actively participate in adaptation (Ehrich and Seifter, 1948; Selye, 1950; Baker and Bridgman, 1954; Baker and Abrams, 195t). The responses of the stomach to the adrenocortical hormones and to "stress" have been reviewed recently by Gray and Ramey (1957) and will not be discussed further here. One example of the participation of the digestive organs in the adaptive responses is the response of the serous glands to increased adrenocortical secretion, first, with a loss of zymogen granules followed by a depletion of cytoplasm nucleic acids (Ehrich and Seifter, 1948). However, as most of these reactions are secondary to the increased activity of the pituitary and adrenal cortex, it does not seem appropriate here to dwell on them in detail sinee the entire organism responds to one degree or another to the physiologic alterations subsequent to increased activity of the primary adaptive mechanisms.

\section{General Measurements of the Endocrine Adaptive Responses}

\section{CinNerat}

The actions of and responses to specific hormones and adaptive mechanisms have been diseussed, but often there are responses in the intact animal that cannot be attributed to a particular hormone or system. The splenic hypertrophy and enlargement of the nucleus pulposus which is seen in mice and voles are examples of such responses (Clarke, 1953; Chitty et al., 1956; Christian, 1959c). The lymphoid tissue of the spleen is involuted by the adrenal glucocorticoids, but the resultant atrophy is more than offset by increased hematopoiesis in the spleens of mice and voles subjected to social stress or other alarming stimuli (Dawson, 1956). However, it is not known at present what specifieally is responsible for the inereased hematopoiesis. This seems to be the proper place to review the physiologie adaptive reactions and to do it in terms of these responses which are found in the intact animal in response to specifie stimuli. Wherever possible, the responses will be related to the specific mechanisms responsible for their occurrence.

It is appropriate first to discuss the stimuli which are known to elicit endocrine adaptive responses. These stimuli have typical dose-time-response relationships: the severer or the longer the stimulation, the greater 
the response (Sayers and Sayers, 1949). Nevertheless, it should not be assumed that all adaptive responses are qualitatively similar. Some stimuli may elieit quite similar responses in kind and clegree, whereas others will be manifested in qualitatively and quantitatively different manners. This statement should be apparent from the preceding accounts of the meehanisms involved. All have in eommon that if uneompensated they will produee widespread physiologic changes which come under the heading of shock (Selye, 1950). However, whether or not the symptoms commonly assoeiated with shoek are elieited depends on the severity of the stimulus (Selye, 1950). In short, almost any ehange in the external environment of the animal, physieal damage to the animal, or emotional upset is potentially eapable of producing profound deleterious effects on the animal, but within reasonable limits the adaptive mechanisms provide it with the flexibility to accommodate most changes or adverse stimuli. It is not until the latter reach rather serious proportions and evoke marked responses that we eustomarily consider the animal to be subject to "stress" or that the stimuli are labeled "alarming."

These stimuli act through or are received through a number of receptor pathways in the host. For example, physical damage, including burns, surgery, aente physieal trauma of all sorts, will evoke adaptive responses probably by direct neural pathways as well as via unknown chemical mediators, resulting from tissue damage which may stimulate the release of ACTH or other adaptive responses direetly. Hemorrhage, by deereasing blood volume, can effect the release of aldosterone directly. Many stimuli act directly through the central nervous system, such as light, noise, fear, and rage. Heat, cold, and similar environmental factors act through recetors in the individual and produce physiologic responses directly. Regardless of the receptor or pathway, all these primary environmental stimuli, if severe enough or chronic enough, have the ability to produce the symptoms of shoek through several common pathways. In so doing they produce physiologie ehanges, either directly or through the central nervous system, which in turn activate a chain of responses leading to defensive reaction against any adverse physiologie shift which may have been produced by the initial stimulus. The rariety of stimuli which have been found to elieit adaptive reactions is legion (Selye, 1950). In fact, one could eonclude that almost every experience eneountered in daily life by any mammal, if serere enough, is capable of producing shock and evoking marked adaptive responses, but for the purpose of the present discussion, the importance of emotional stimuli in evoking these reactions will be emphasized. These may be acute stimuli, and all of us are personally familiar with some of the reactions that they are eapable of produeing. However, it should be noted that emotional stimuli of a more ehronic nature ean produce profound 
long-term physiologic reactions which may have widespread effects on the host (Elmadjian ct al., 1958; Rateliffe and Cronin, 1958; Christian, 1959b; Mason, 1959). Close confinement, for example, can result in atrophy of the adaptive mechanisms with a resultant marked susceptibility to any subsequent alarming stimulus (Christian and Rateliffe, 1952). Social competition, social pressure, or chronic anxiety have been shown to produce marked physiologic responses (Christian, 1959a; Mason, 1959) Emotional factors, of necessity, have their beginning in the eentral nervous system as a result of sensory stimuli received from the external environment via the visual, aural, and olfactory sensory reeeptors. Therefore they are part of the complex system responsible for integrating the mammal with its immediate environment.

Whatever the particular adverse stimulus may be and regardless of the pathway of its reeption by the animal, whether sensory, traumatic, or otherwise, all stimuli have the ability to produce shock and evoke physiologic adaptive responses in the manmal. Shock is a vaguely defined entity, and there is no general agreement with regard to its exact nature or cause (see Selye, 1950; Scudder, 1952; Bing, 1952; Zweifach, 1952; Engel, 1952; Agate, 1952; Randall, 1952). Moreover, its primary effeets and the responses to it are often difficult to separate. Fundamentally the principal manifestations of shoek involve changes in the circulatory system leading to inadequate circulatory function and eventually circulatory collapse. If one keeps in mind that the fundamental changes seem to be blood sludging (Knisely ct al., 1947), ehanges in selective permeability of the peripheral vascular bed (Zweifach, 1952), capillary and arteriolar atony and hyporeaction of the vascular musculature to epinephrine and even direct stimulation with a resultant visceral vasodilatation (Zweifach, 1952), then the secondary effects of hemoeoneentration, hypotension, fall in blood volume, decreased cardiac output, and increased hematocrit are more easily understood. In general shock seems to involve a generalized failure of eirculatory integrity. With a superimposed relative cireulatory stasis and hypoxia, these alterations became more profound and the whole process becomes a vicious eircle (Zweifach, 1952). It is not known by what specific means these alterations in circulation are effected, but that they involve both neural and humoral components ean hardly be questioned. We have repeatedly emphasized that the basic role of the adaptive reactions is to maintain vascular integrity in opposition to forces tending to destroy it as well as to prepare the organism to meet emergency situations. Most alarming stimuli also produce an immediate discharge of the medullary hormones epinephrine and norepinephrine with mobilization of glueose reserves, increased heart rate and strength of beat increasing cardiac output, and constriction of viseeral and cutaneous blood vessels with a re- 
sulting shift of the blood to the museulature and central nervous system. If at the same time the output of ACTH is stimulated, adrenal glucocorticoids will also be increased, and among their effects is an increase in the reactivity of the blood vessels to the effects of epinephrine and norepinephrine. None of the reactions is completely isolated, but the balance between them may shift considerably depending on the nature of the stimulus. There are also temporal relationships. In many ways the immediate and short-lived activity of epinephrine is mimicked more chronically by the glucocorticoids. The response of the adrenal medulla is immediate, whereas the cortex responds somewhat more slowly and can be sustained for a great deal of time.

It is worth describing some of the measurable changes effected in a mammal by activation of the adaptive responses. Many of these have been described earlier under actions of the various hormones, but it is appropriate to discuss these as measurable changes in such a way that they might be useful in detecting and interpreting the effects of physiologic adaptation to potentially harmful stimuli. Many of the actions of the adrenocortical and other hormones serve specifically to restore the equilibrium of the internal environment after an alarming stimulus. These actions may be a decided disadvantage to the animal when they are prolonged. For example, increased secretion of the carbohydrate-active corticoids serves to maintain proper fluid and clectrolyte balances and to provide readily available glucose reserves in an emergency, but they also suppress inflammation, granulation, and antibody formation and thereby reduce host resistance to infection. The following list of adaptive responses is by no means complete, those effects having been selected which might prove useful or of basic conceptual importance to the investigator wishing to study the effects of physiologic adaptation.

\section{Measurbments Indicating Increased Adrexocortical Function}

a. Adrenal weight. This is a presumptive measurement of ACTH activity and response to an acute or chronic distress. Weights are the best available index of adrenocortical activity for many studies. For many long-term changes, weights are much more useful and much less subject to pitfalls than techniques such as changes in adrenal ascorbic acid, lipids, cholesterol, the production of plasma corticosteroids, or circulating cosinophils or lymphocytes. All these measurements are labile and reflect rapid changes in adrenal function: they indicate the status of the animal at the moment of making the measurement, thus they often may reflect nothing more than the process of trapping or handling an animal and may completely obscure longer-term changes, especially with excitable, highly reactive 
wild mammals. The problem of adrenal hypertrophy with weight changes and the relationship of these changes to the length and severity of the stimulus has been diseussed at length by Sayers and Sayers (1949).

Adrenal weight ehanges largely reflect changes in the zona fasciculata, which undergoes hyperplasia and hypertrophy following stimulation by ACTH and therefore is indicative of changes in the secretion of glucocorticoids. Under different circumstances, already discussed, the zona glomerulosa may hypertrophy, but changes in its size are relatively unimportant with respect to changes in the total weight of the gland because of its relatively minor contribution to the total mass of the adrenal. The same statcment is generally true for the adrenal medulla. Finally, changes in adrenal weight, when used as an index of cortical activity, should be evaluated in the light of histologic studies, as the amount of lipid in the cells of the faseiculata may vary sufficiently with various functional states to have a marked effect on adrenal weight. For example, stimulation of sufficient intensity to deplete the cortex of visible lipids may result in a decrease in weight whereas activity may actually be greater than in a heavier gland containing a large amount of stored lipid (Christian, 1959b).

The adrenal medulla in some speeics may hypertrophy and contribute to changes in adrenal weight. Although the contribution of the medulla to adrenal weight ehanges is usually negligible, it would be appreciable for the Soricidae, in which the adrenal is composed largely of medulla. Changes in the medulla and eortex of the long-tailed shrews (soricids) have not been studied, although these medullary-eortical relationships have been observed in mature individuals of Sorex cinereus, S. dispar, S. fumeus, S. palustris, and Microsorex hoyi. There may be a relationship between body size, adrenocortical mass, and metabolic rate in these minute animals.

Adrenal weights are useful for field studies, as they may be obtained on fresh or fixed material. From a practical standpoint it is almost essential to obtain adrenal weights from fixed material in mammals the size of mice or smaller, as it is very difficult to clean the glands properly and obtain reliable weights on the fresh glands. Rapid water loss from fresh glands of such small size further complieates the problem. In most cireumstances increased adrenal weight is an acceptable indicator of increased adrenocortical activity, but an effort should be made to obtain the adreuals from suddenly killed specimens, not from captive animals or from those held in a live trap for extended periods, if one wishes to assess adrenocortical function in the animal in its natural state. Animals which have been killed suddenly are not subjeet to changes in adrenal woight resulting from capture or handling

A word of caution is appropriate here with regard to sample size and adrenal weight-body weight relationships. Adrenal weight varies consider- 
ably from individual to individual in the same speeies, as well as with sex, size, and therefore presumably age, in most mammals. Consequently, it is necessary to obtain large enough samples of each sex in each age category (at least mature and immature) to have reliable eriteria for evaluating adrenal ehanges in a population and with time.

In most studies of natural populations the investigator does not have the privilege of selecting animals for size, weight, and sex each time a sample is colleeted, and it is therefore neessary to determine adrenal weight-body weight, adrenal weight-body length or some similar relationship for the speeies and population with which he is working in order to establish a common baseline for all samples. For example, it has been found that the logarithm of adrenal weight in milligrams on body length (exclusive of the tail) gives the best straight-line relationships for all sizes and for both sexes of Norway rats (Christian and Davis, 1955). In other species, for example Microtus pennsylvanicus, there are marked differences in body-adrenal relationships between the sexes. The oft-used relationhsip of milligrams of adrenal weight per 100 grams of body weight is quite arbitrary and usually overcorreets for lighter and undereorreets for heavier animals, therefore this relationship is useful for only a very narrow range of body weights. Too often adrenals are collected as an afterthought along with other data for which the investigation was originally designed. As a result the data on adrenal weights usually are inadequate. A study of changes in adrenal weight must be designed speeifieally to obtain this and the necessary related data. Samples should be consistent with respect to time, size, and sex and age composition. Many potentially useful field studies involving an immense amount of effort have been of little value beeause of poor sampling, usually a result of treating the collection of data on adrenal weights as a secondary consideration in the investigation. Sampling usually does not alter the populations, as the normal rate of reeruitment more than offsets losses due to sampling, in addition to whieh compensatory changes in mortality rate will usually eompensate for sampling losses, unless the sampling is very frequent and intense.

b. Routine histologic measurements. It is very useful in evaluating adrenal activity to measure the widths of the various zones and to eount the number of cells in cortical cords in an area where cords are in straight columns (Zwemer, 1936; Zwemer et al., 1938; Christian, 1956). A variation of this is the somewhat more preeise teehnique of projeeting a seetion through the center of the gland and outlining the various zones, eutting them out and weighing them or else measuring their areas with a planimeter. It is possible with serially seetioned glands to repeat such a procedure every so many seetions and in this way obtain preeise information on the contributions made by the individual zones to the total weight of the gland. These tech- 
niques necessitate selection of appropriate sections and therefore require serial sections, especially on animals with adrenals weighing less than $20 \mathrm{mg}$. For glands heavier than this it is possible to select a portion through the middle of the gland grossly. All these measurements can be made on sections stained routinely with hematoxylin and eosin.

c. Adrenocortical sudanophilia. Fat stains, such as Sudan IV, are useful in evaluating the secretory activity of the adrenal cortex. The normally active cortex ("resting cortex") usually exhibits marked sudanophilia of the fasciculata and glomerulosa with the fat in vacuoles of moderate size. The sudanophilia becomes markedly reduced in acutely stimulated glands, and upon continued stimulation the sudanophilia may return to some degree, but the size of the racuoles is reduced. Upon cessation, sudanophilia becomes intense and the vacuoles large. Sudanophilia may be completely absent from some species, such as the hamster, under all conditions. These details are discussed in greater detail elsewhere (Dempsey, 1948; Sayers and Sayers, 1949; Greep and Deane, 1949a, b; Symington ct al., 1958).

d. Adrenal ascorbic acid declines on stimulation with ACTH, and this has been used as a means of assaying ACTH in blood and other fluids (Saycrs and Sayers, 1949; Greep and Deane, 1949b). In general adrenal ascorbic acid is a useful histochemical means of assaying adrenal stimulation, although it may not reflect cortical secretory activity in the intact animal (Slusher, 1958).

e. Adrenal cholesterol also declines on stimulation and can be used to assess cortical activity (Dempsey, 1948; Sayers and Sayers, 1949; Greep and Deane, 1949b). Measurement of adrenal steroids in the adrenal venous affluent, plasma, or their urinary metabolites can be used to assess cortical activity directly. These are subject to daily variation and also respond rapidly to stimulation. In addition, they are involved techniques to carry out properly. There is also some question concerning the biologic significance of the particular steroids measured. These measurements are therefore of limited use for investigations outside the laboratory and are subject to numerous pitfalls in dealing with species which cannot be handled in the laboratory without stimulating adrenal activity. Measurements of urinary corticosteroids when practical and appropriate would probably be more useful than blood levels for studies relating to mammalian populations. Measurement of the products of corticosteroid metabolism in the urine is somewhat intermediate between weight and direct functional measurements, such as plasma steroids as a measure of chronic cortical stimulation. Urinary steroids may be collected daily ad infinitum under laboratory conditions and are unquestionably very valuable. However, steroid determinations are complex and difficult to interpret, and in the last analysis represent only those steroids which escape as such via the urine and consist 
largely of metabolites of steroids. Furthermore, a large proportion of the steroids are excreted in the feces in some species (Barry et al., 19.52; Bradlow et al., 1954). Finally, techniques for the measurement of urinary steroids have not been developed for use in the field. It should be apparent from the foregoing discussion that assessing adrenocortical activity by physiologic or biochemical means in living wild animals or animals which have been live-trapped is fraught with difficulty, and under most circumstances measurements as such of the blood steroids will probably reflect the immediate situation of the animal. In general, weight and histologic criteria from animals killed suddenly seem to offer the most dependable information at the present time for studying population phenomena, although the other procedure should be explored further. Even though there is always a question of the presumptive relationship between hypertrophy, morphologic change, and function, in most circumstances it is generally accepted that cortical hypertrophy reflects functional change. The probable departures from this general statement have been discussed above.

f. Thymicolymphatic system. In the foregoing account it has been stated that the earbohydrate-active corticoids produce involution of the thymicolymphatic system, mainly by effecting involution of the lymphoid elements proper. Therefore the weights of the thymus, lymph nodes, and spleen provide useful indicators of increased adrenocortical activity, especially if appropriate histologic checks are used.

g. Thymus weight. Cortisone, hydrocortisone, and endogenous adrenal corticoids cause thymic involution (Dougherty, 1952a, b; Weaver, 1955). Increased phagocytosis, edema of the comnective tissue stroma, and hyperplasia of the reticulum are associated with the destruction of the lymphoid tissue proper (Gordon, 1955). If the reaction is severe enough, only the stroma and a modified reticulum may remain, with no distinction between the cortex and medulla of thymus and lymph nodes in degree of involution (Weaver, 1955). The weight of the thymus therefore will be greatly dedecreased. Within a few hours following stimulation there is a marked edema, and during this period of edema there is a marked reduction in the number of lymphocytes; those remaining exhibit degenerative changes (Dougherty, 1952b). The effect is greatest on the small and medium-sized lymphocytes and thymocytes. These exhibit pyenosis, karyolysis, fragmentation, and other degencrative changes. The fragments are phagocytized by macrophages in reaction centers of the lymph nodes and are carried off in the lymphatics. Mitosis ceases in the lymphoid organs with treatment with cortisone, hydrocortisone, or with chronic stimulation of the adrenal cortices by endogenous or exogenous ACTH (Weaver, 1955; Gordon, 1955). These changes are common to the lymphoid follicles whereever they occur-splecn, nodes, gastrointestinal tract-but are most 
marked in the thymus. The greater sensitivity of thymocytes over the lymphocytes of the lymph nodes and spleen may be due to their greater rate of proliferation. Hydrocortisone is more effective in involuting lymphoid tissue than cortisone or corticosterone and tends to produce degencrative changes among immature lymphocytes of the lymph nodes to a much greater degree than cortisone (Dougherty, 1953; Santisteban and Dougherty, 1954). On the other hand, pituitary growth hormone and deoxycorticosterone appear to promote the growth of lymphoid tissue. The apparent increase in lymphoid tissue in gonadectomized or adrenalectomized animals (or in those animals with adrenocortical atrophy from inactivity or overfeeding) (Christian and Ratcliffe, 1952) apparently results from protection against involuting agents rather than a true hyperplasia (Dougherty, 1953 ; Santisteban and Dougherty, 1954).

The thymus is also involuted by androgens, estrogens, and to a variable degree by thyroidectomy (Weaver, 1955); androgens also potentiate the ability of the carbohydrate-active corticoids to effect thymic involution (Selye, 1955; Dorfman and Shipley, 1956). The cortex and medulla remain as distinct zones after this type of involution, although they are less clearly defined than in normal (Weaver, 1955). All elements of the thymus share equally in the involutional process. Thymocytes are reduced, but no acute destructive changes are noted. Therefore involution of the thymus by sex hormones is a nonspecific action (Weaver, 1955). The so-called involution due to aging of the thymus is prevented by gonadectomy in either sex (Selye, 1947); it is therefore logical to assume that the normal involution of this gland, seen especially at puberty (Christian, 1956), is due to the sex steroids.

It should be clear that thymic involution, and therefore thymic weight, can be a useful index of adrenocortical activity providing adequate consideration is taken of the action of the sex steroids.

h. Lymph node weight is a useful index of adrenocortical activity as their lymphoid tissue is involuted by adrenal corticoids as discussed above, but to a less marked degree than for the thymus (Weaver, 1955). The iliac lymph nodes are involuted by prolonged administration of ACTH, acute administration of cortisone (Weaver, 1955), or a variety of stimuli evoking increased adrenocortical activity, but the sex steroids or thyroidectomy are without effect on the lymph nodes (Wearer, 1955) or may even cause a hypertrophy of the nodes (Money et al., 1950). A weight loss of the lymph nodes should therefore more specifically reflect increased adrenocortical activity than the thymus. However, thymic weight, properly controlled, is more frequently used because of its much greater sensitivity to the carbohydrate-active corticoids. Selye (1950) has indicated that the lymph nodes 
even may hypertrophy in infectious disease or other cireumstances which demand increased phagocytosis of particulate matter.

$i$. Splenic weight. The lymph follicles of the spleen also are involuted by corticoids. A decrease in splenic weight is sometimes used to indicate increased adrenocortical secretion. However, contraction of the splenic capsule further decreases splenic weight. This may be a ralid procedure in the laboratory with injected hormones, particularly in hypophysectomized or adrenalectomized animals, but often may not be a ralid indication of increased adrenocortical activity, especially in intact small mammals. Voles (Microtus agrestis) and house mice, and possibly other species, exhibit a marked splenic hypertrophy when exposed to stimuli which cause increased pituitary-adrenocortical activity (Clarke, 19.53; Chitty, 1957; Christian, 1959c). Involution of the lymphoid follicles oceurs in these circumstances as might be expected, but the decrease in weight from this cause is overridden by the hypertrophy resulting from congestion and markedly increased hematopoietic activity. The increased hematopoiesis in these animals is accompanied by a reticulocytosis of the cireulating blood. The cause of increased hematopoiesis in these animals has not been demonstrated.

$j$. Lymphocyte counts. After an alarming stimulus there is an immediate rise in the number of circulating lymphocytes, which is then followed by a characteristic lymphopenia and eventually a return to normal levels. The initial rise in circulating lymphoeytes probably is at least in part due to release of lymphocytes from lymphoid tissues in response to an initial release of epinephrine, but this release by no means accounts for the entire rise (Crordon, 1955). The prolonged phase of lymphopenia which begins in the early stages of the increased pituitary-adrenocortical activity probably results from the destruction of medium and small-sized lymphocytes within the lymphatic organs by the corticoids (Gordon, 195.5). However, there is also some reason to believe that lymphocytes may migrate to depot situations. Over a prolonged period the main cause of the lymphopenia appears to be due to the reduced amount of lymphoid tissue and decreased lymphocytopoiesis with a decreased delivery of lymphocytes into the circulation. However, with small amounts of adrenal factors there may be an acutal hyperplasia of lymphoid elements, so that interpretations must be made with caution.

Lymphocyte counts as criteria of stress in wild mammals are subject to the same criticism as other measurements made on the living mammal: the response is rapid enough and the counts labile enough so that there is real danger that counts may reflect alarming stimuli induced by handling, thus masking any other effects which may be the main point of the study. 
$k$. Eosinophil counts. The numbers of circulating eosinophils are beyond doubt diminished by adrenal factors and eosinophil counts are commonly used to assess the response of the adrenal cortex to ACTH or indicate increased pituitary-adrenocortical activity (Speirs and Meyer, 1949; Speirs, 1955). The reduction in eosinophils appears to result mainly from their increased destruction under the influence of cortical hormones. A reduction in cosinophils can be effected by epinephrine as well as by cortical hormones, and there is evidence that the presence of cortical hormone is required for epinephrine to produce an eosinopenia (Gordon, 1955). It is mainly with respect to whether or not cortical hormones are necessary for the eosinopenic action of epinephrine that the specificity of eosinopenia as an indicator of increased adrenocortical activity has been questioned. Nevertheless, if epinephrine produces a marked eosinopenia in the intact animal, eosinophil counts in mammals, especially wild, appear to have limited value. Fear resulting from handling, trapping, etc., not only would produce eosinopenia, but could produce it without necessarily having an increase in adrenocortical activity. Nevertheless Louch (1958) used eosinophil counts to assess adrenocortical function in relation to changes in populations of voles and with adequate precautions the use of eosinophil counts for assessing adrenocortical activity in natural populations seems to have considerable value in these experiments. However it is not possible to state definitely whether the declines in cosinophils were due to cortical or medullary hormones, although it seems logical that the former were responsible and there was cortical hypertrophy. Southwick (1959) has also related declines in eosinophil counts in mice to increased adrenocortical functions, but again the role of epinephrine has not been evaluated. Eosinophils decline about an hour after acute stimulation of the pituitary-adrenocortical systems (Louch, 1958); therefore using counts as criteria of cortical function with respect to chronic stimuli has many inherent hazards. Acute responses to handling, fear, or line trapping could easily mask any effects produced by the chronic stimulus of changing population density. The relative roles of epinephrine and of the cortical hormones in producing the observed declines in eosinophils following emotional stimuli in mice must be assessed before one can state with finality that the cause was increased cortical activity.

l. Neutrophil counts. A rise in circulating neutrophils accompanies increased pituitary-adrenocortical activity, but similar changes can be induced by so many factors that, even though they may involve increased adrenocortical secretion, neutrophil counts are not very useful indices with which to measure pituitary-adrenal activity.

$m$. Liver glycogen. The accumulation of glycogen by the liver has been used as a means of assaying various steroids for their activity on carbohy- 
drate metabolism in adrenalectomized rats (Dorfman, 1949; Ingle, 1950). This response is a reflection of the ability of adrenal corticoids to stimulate gluconeogenesis. Glycogen in the liver can be measured chemically (Martorano, 1957) or can be visualized histochemically by the appropriate procedures (Dempsey, 1948; Lillie, 19.54). Liver glycogen presumably falls as a result of exhaustion of the animal's ability to adapt (Frank, 1953). Exactly what becomes exhausted under these circumstances is problematical, but it apparently is not the ability of the adrenal cortex to secrete steroids (Rosenfeld, 1958). Nevertheless, it is true that blood glucose and liver glycogen eventually fall to extremely low levels following intense and prolonged stimulation by alarming stimuli. This result may follow prolonged stimulation of the sympatho-adrenal system. Exhaustion of glycogen, consequently of glucose reserves, has been observed in natural populations of snowshoe hares (Green and Larson, 1938; Green et al., 1939) and voles (Frank, 1953) during episodes of mass mortality and may possibly be explained on the basis of exhaustion of the adaptive reserves.

A word of caution should be inscrted on the use of liver glycogen as an indicator or activity of the carbohydrate-active corticoids. Under carefully controlled conditions in the laboratory such measurements are very useful, but they do require rigid controls and precise, highly standardized procedures. Nar.torano (1957) has studied the variables having important effects on liver glycogen. The amount of time between killing and enzymatic immobilization, the manner of killing, and a variety of other factors can alter glycogen levels. Glycogen and glucose levels in the liver decline rapidly after death. There also is a daily cycle in the levels of liver glycogen which is associated with feeding and activity (Martorano, 1957). Therefore, reported liver glycogen levels must be critically examined in relation to the procedures used and their reliability.

$n$. Changes in the digestive organs and gastrointestinal tract. Alarming stimuli, ACTH, or cortisone produce a marked loss of the acidophilic zymogen granules and basophilic cytoplasmic pentose nucleic acids from the acinar cells of the pancreas and serous salivary gland cells (Ehrich and Seifter, 1948; Selye, 1950). The pituitary adrenocortical system also profoundly affects the function of the mueosa of the stomach and intestinal tract (Baker and Abrams, 1954; Baker and Bridgman, 1954; Gray and Ramsey, 1957). The adrenocortical hormone apparently stimulates the secretion of pepsinogen by gastric zymogenic cells (Cray and Ramsey, 1957; Mason, 1959), which is reflected morphologically by a loss of the intracellular pepsinogen granules and increased cytoplasmic basophilia (Baker and Bridgman, 1954). Additional changes may not be noted histologically in the mucosal cells of the intestine (Baker and Bridgman, 1954). 


\section{Generalized Effects of Physiologic Adaptation}

a. Growth. Associated with acute adaptation there is usually a suppression of growth (Selye, 1950). Selye (1950) has suggested that during actuation of the pituitary-adrenocortical system there is a decreased production of the other hormones of the adenohypophysis, including growth hormone. However, injected eorticoids or ACTH into intact animals also results in suppression of growth and ACTH will inhibit growth produeed by growth hormone in hypophysectomized rats (Jones, 1957). It was pointed out earlier in this chapter that the adrenal earbohydrate-aetive corticoids increase protein catabolism, prevent the growth of bone, lymphoid tissue, skin, connective tissue with the production of eollagen, and block mitoses in general. Therefore the suppression of growth by alarming stimuli may reflect decreased seeretion of growth hormone as well as the direct suppresive action of the carbohydrate-action corticoids on mitoses and therefore growth and development. Diminished thyroid activity during periods of actively increased adrenocortical activity may also play a role in this phenomenon. Selye (1950) has pointed out that most of the observed changes following an alarming stimulus serve to maintain life and that other, less immediately important, functions are suppressed.

b. Inflammation and granulation. The adrenocorticoids, cortisone, hydrocortisone, and to a lesser extent corticosterone, exert powerful antiinflammatory effeets which stem from the suppression of growth of eonneetive tissue, the depression of lymphocyte activity, and interference with the phagocytic process (Dougherty, 1953; Robinson and Smith, 1953; Thomas, 1953), but in addition they prevent the mobilization of all of the usual elements of an inflammatory response around the site of injury (Taubenhans and Amromin, 1950; Dougherty, 1953; Robinson and Smith, 1953; Gordon, 19.55; Dougherty and Schneebeli, 195.5). Androgens and estrogens potentiate these anti-inflammatory responses. They decrease the destruction of fibroblasts and the invasion of polymorphonuelear leucoeytes and macrophages. The appearance of epithelioid maerophages, giant cells, and formation of new fibroblasts and macrophages are suppressed by cortisone, either injected or implanted as pellets (Baker, 1954). Dougherty and Schneebeli (1955) explain the inhibition of the inflammatory response around the site of injury in the following way: When there is cellular injury, substances are released from the injured cells which trigger a series of responses which comprise inflammation (Menkin, 1955; Dougherty and Schneebeli, 1955). Cortisone or hydrocortisone inhibit the inflammatory response by protecting the surviving cells from the actions of the released produets of eellular injury. Growth hormone, deoxycorticosterone, and aldosterone appear to exert a stimulating effect on inflammation and the 
development of granulation tissue (Selye, 1955; Dougherty and Schnecbeli, 1955). These hormones, however, also inhibit the anti-inflammatory action of cortisone, hydrocortisone, and corticosterone and may enhance the local inflammatory response by increasing the susceptibility of the cells to the inflaming stimulus (Dougherty and Schneebeli, 1955). Therefore, if there is a reduction in the seeretion of growth hormone with a simultaneous inerease in the production of ACTH and the adrenocortical steroids, there will not only be a direct suppression of the inflammatory responses to infection or injury, but also a withdrawal of the factors which ordinarily would stimulate such activity. The effects of various alarming stimuli or hormones on inflammation and granulation in the intact animal have been studied and measured by using experimental granulomas (Meier et al., 1950; Selye and Bois, 1954; Robert and Nezamis, 1957; Christian and Williamson, 1958) or other means of inducing inflammation and granulation. It has been shown that in addition to suppressing inflammation the carbohydrateactive corticoids also suppress the formation of granulation tissue and healing, primarily by preventing conneetive tissue growth. There can be little doubt that the normal defenses against infection are severely depressed in stressed animals.

c. Antibody formation. Antibodies are formed mainly in the lymphatie tissues (Keuning and Van der Slikke, 1950; Kass et al., 1953a; Kelsall and Crabb, 1958). One school maintains that this function resides primarily in the plasma cells, while another group holds that lymphatic cells in general are eapable of manufacturing antibodies (Keuning and Van der Slikke, 1950; Dougherty, 1953; Kelsall and Crabb, 1958). We do not intend to enter into this controversy at the present time, but it seems relatively certain that the lymphoid tissues are primarily responsible for the production of antibodies. A variety of experiments have shown that injected corticoids or the increased secretion of endogenous corticoids, brought about either by injected ACTH or in response to alarming stimuli, markedly suppress the formation of antibodies (Kass et al., 19.33a). Protein manufacture, and therefore the formation of antibodies, requires the presence of nucleic aeids in the cells, especially in the cytoplasm, and antibody formation is normally assoeiated with an increase in nucleic acid content of the lymphoid organs (Kass et al., 1953a; Kelsall and Crabb, 1958). Therefore, when there is interference with nucleic acid metabolism or its formation, there is an accompanying reduction in the rate of formation of antibodies (Kass et al., 1953a; Kelsall and Crabb, 1958). We have seen that in addition to actually destroying lymphoid tissue, the glucocorticoids reduce the PNA content of the remaining lymphatic cells. The ability of the reticuloendothelial system, and possibly other cells, to dispose of phagocytized particulate material is also impaired (Thomas, 19.5) even though phago- 
cytosis may be stimulated (Gordon and Katsh, 1949; Thomas, 1953). By these several mechanisms the production of antibodies may be seriously impaired following activation of the adrenal cortex although there appears to be a dose-response relationship (Dougherty, 1953; Dougherty and Schneebeli, 1955). While most of these effects were demonstrated most clearly by injecting adrenocortical hormones, the same effects have been shown repeatedly following stimulation of adrenocortical secretion in the intact animal.

d. Resistance to infection. The three immediately preceding topics all deal with factors involved in the resistance to infection. It stands to reason that reducing the inflammatory response to, and depressing the formation of antibodies against, infectious agents will inevitably impair the ability of an animal to resist infection. Cortisone, hydrocortisone, and ACTH have been shown to decrease resistance to a variety of experimental infections caused by a variety of infectious agents including streptococcal, pneumococcal, tuberculosis infections in mice, rats, and guinea pigs, brucellosis, malaria in monkeys, and others (Kligman et al., 1951; Schmidt and Squires, 1951 ; Selye, 1951; Kass et al., 1953b; Le Maistre et al., 1953; Robinson and Smith, 1953). The pathogenicity of various agents has been increased by cortisone injection. For example, the virulence of Coxsackie infections in mice was greatly enhanced by cortisone (Boring et al., 1955), and poliomyelitis can be made a paralytic disease in the normally resistant hamster by cortisone or hydrocortisone (Shwartzman and Aronson, 1953). Viremias may likewise be prolonged appreciably by the adrenal glucocorticoids (Whitney and Anigstein, 1953; Pollard and Wilson, 1955). The list of experimental infections which have been made more virulent, prolonged, or otherwise increased in their pathogenicity by treatment with carbohydrate-active adrenocorticoids or ACTH (Selye, 1951) is long, and there is no point in listing them in detail here.

Whenever experiments with injected hormones are considered the question arises whether or not the same events may occur as a result of increased endogenous secretion of the same or similar hormones. A criticism frequently made of experiments with exogenous hormones, especially with large doses, is that the results are pharmacologic rather than physiologic. However, it is by using isolated hormones in highly controlled situations that an understanding of the basic mechanisms is gained. Nevertheless, before one can extrapolate from these data to natural events, comparable changes must be shown to occur in natural or seminatural conditions. Changes in host resistance may result from adverse environmental stimuli, possibly as a result of adrenocortical activity. It has long been common knowledge that excess fatigue, chilling, and a variety of comparable stimuli increase the susceptibility of humans to colds and other infections. It should 
be apparent now that most of these same stimuli also increase the secretion of adrenocortical steroids. However, commonly accepted truisms still do not constitute experimental evidence and proof of such conclusions, but several experiments have shown that host resistance is decreased by exposing the animals to stimuli which are known to increase adrenocortical activity. When mice are exposed to $4^{\circ} \mathrm{C}$. for a period of time, Coxsackie infections become much more pathogenic, spreading especially to the heart and liver in adult mice (Boring et al., 1956). Diminished resistance of mice to trichinosis and to tuberculosis has been demonstrated by procedures which also produce increased pituitary-adrenocortical activity and depress inflammation and granulation (Christian and Williamson, 1958; Tobach and Block, 1956; Davis and Read, 1958). Some aspects of these studies will be considered in more detail later.

e. Reproduction. Suppression of reproduction is a very important aspect of the endocrine adaptive responses (Selye, 1939, 1950). The decrease in reproductive function is in many ways a more sensitive measure of the existence of altered physiologic functions in response to adversity than the increase in adrenocortical activity and some of its sequelae (Christian, $1955 \mathrm{a}, \mathrm{b}, 1956,1959 \mathrm{~b}, \mathrm{c}$ ). The bulk of the experimental evidence indicates that, like growth hormone, the secretion of pituitary gonadotropins is suppressed in response to alarming stimuli which evoke an increased secretion of ACTH (Selye, 1939, 1950; Christian, 1956, 1959b, c). It is another indication that the immediate restoration of the normal internal environment takes precedence over functions which are less important to the immediate survival of the individual. Cold, heat, disease, trauma, severe emotional stress, and other stimuli will depress normal reproductive functions (Marrian and Parkes, 1929; Selye, 1939; Bohanan, 1939; Poindexter, 1949; Denison and Zarrow, 1955; Barnett and Manly, 1956; Christian and LeMunyan, 1958; Christian, 1959b). The suppression of gonadotropin secretion is evidently the primary cause of the inhibition of reproductive function (Mulinos et al., 1939, Selye, 1950; Srebnik et al., 1958), although there may be direct effects of increased adrenocortical activity and altered thyroid function, as mentioned earlier (Brynes and Shipley, 1950; Baker et al., 1950; Smith, 1951; Brimblecombe et al., 1954). The secretion of gonadotropins seems to be regulated principally by the hypothalamus (Anderson and Haymaker, 1948a, b; Markee et al., 1952; Everett and Sawyer, 1953; Hammond, 1954; Critchlow and Sawyer, 1955; Nalbandov et al., 1955; Laqueur et al., 1955; Fortier, 1957; Greer, 1957; D'Angelo and Traum, 1958 Everett, 1959), although there is evidence that the secretion of luteotropin by the pituitary may be independent of the hypothalamus (Everett, 1956). The hypothalamic centers involved in the regulation of the secretion of the gonadotropins apparently are distinct from those 
responsible for the regulation of ACTH and TSH seeretion (Laqueur et al., 1955; Greer, 1957; D'Angelo and Traum, 1958). In addition there are neurogenic factors involved in ovulation which consist of adrenergic and neurogenic components (Markee et al., 1952; Everett and Sawyer, 1953; Nalbandov et al., 1955) and probably also in the milk let-down reflex (Grosvenor and Turner, 1959a). Finally, as in other endocrine regulating mechanisms, the level of circulating sex steroids seems to exert a regulating effect on the release of the particular gonadotropin responsible for their release (Selye, 1947; Sturgis, 1950; Byrnes and Shipley, 1950; Bymes and Meyer, 1951). Whether these steroids exert their effects on the hypothalamus or on the anterior pituitary itself is not known. In any event, withdrawal of stimulation by the gonadotropins leads to atrophy of the gonads and decline in the production of their respective steroids (Burrows, 1949). The decline in the production of sex steroids is in turn followed by atrophy of those accessory organs and secondary sex eharacteristics which depend on the sex steroids for their activity and maintenance (Burrows, 1949).

If a male animal is subjected to adverse circumstances for any length of time, there is a marked decrease in spermatogenesis and a reduction in the secretion of androgens as indicated by atrophy of the seminal vesicles, prostate, and preputial glands (Christian, 1956; 1959b; Christian and LeMunyan, 1958). These changes may all be followed by the changes in their weights, but weight changes should be assessed by appropriately selected histologic examination of the organs. In young animals there may be either a delay in the onset of puberty or a total suppression of the development of puberty, as indicated by the development of spermatogenesis and the sex accessories or, in the mouse, by a failure of the $\mathrm{X}$-zone to involute at the usual time (Christian, 1956, 1959a, b). If mature animals are subjected to severe stimuli, there may be easily discernible degenerative changes in the tubules of the testes and cells of the spermatogenic series.

Changes in the female reproductive function are less easily scen than in the male, as organ weights do not provide as useful a eriterion of changes. Nevertheless, changes in estrus are usually evident and ean be detected by vaginal perforation or by vaginal smears. Depending on the severity of the inducing stimulus, estrus may be prolonged or totally suppressed. Uterine weight may reflect reproductive suppression, and would be especially valuable in demonstrating a delay in the onset of puberty in female animals. In some circumstances there may be a total suppression of reproductive activity. It is in actual reproductive performance that the effects of suppression of the reproductive endocrine system becomes most evident. Depending on the severity of the stimulus and on the species involved, there may be a complete failure to become pregnant, which may be due to a failure of ovulation or a failure of the shed ova to implant in the uterus or, 
if the females do become pregnant, there may be a marked increase in fetal mortality with increased resorption of the embryos (Christian, 1959a, b).

The causes of fetal mortality in these circumstances have not been explored in detail, but a number of factors may be involved. It is well known that cortisone and hydrocortisone have scrious effects on the fetus, apparently by inhibiting growth and development (Glaubach, 1952; Fraser et al., 1953; Davis and Plotz, 1954; Kalter, 1954). The resultant defect probably depends to a large extent on the developmental stage of the fetus when these hormones are active, as is the case with nutritional deficiencies (Lutwak-Mann, 1958). A variety of congenital defects have been produced experimentally by the injection of these hormones. However, high doses are required to produce these effects; furthermore, with chronic injection of cortisone some fetuses do not seem to be affected (Seifter et al., 1951). Hydrocortisone and cortisone increase the incidence of fetal mortality in rats during the second half of preguancy. The mechanism is unknown, but it has been suggested that it may be due in part to premature "aging" of the vasculature of the placenta (Seifter et al., 1951). Increased mortality may reflect fatal defects in the growth of the embryos due to the action of these hormones. In other words, there may be time-dosage relationships which determine whether the effects of these glucocorticoids will be fatal or will result in "congenital defects," such as cleft palate or cardiac anomalies. However, these explanations leave unanswered the question why many embryos subjected to the same influences are born viable and free of defects, even from the same pregnancies. However, there are many other factors to consider when discussing intrauterine mortality resulting from activation of physiological adaptive systems. One must consider the increased secretion of adrenal androgens by those species in which androgens or proandrogens constitute a major secretory product. These compounds possibly may directly inhibit the action of estrogens and progestins. For example, it has been shown that testosterone can completely inhibit the feminizing action of estrogens on developing rat embryos (Greene et al., 1941). More importantly, the adrenal androgens can inhibit the secretion of gonadotropins, especially of FSH (Byrnes and Shipley, 1950; Byrnes and Neyer, 1951; Dorfman and Shipley, 1956). Whether the decline in the secretion of gonadotropins with increased ACTH secretion is due to inhibition by increased circulatory levels of adrenal androgens or whether it is independent of the androgens, cannot be said. However, it seems probable that the androgens are not important or, if so, only in a limited number of species. The adrenal secretion of androgens or their precursors in many species does not appear to be sufficient to account for the effects seen. For example, in humans pituitary blockage requires much higher doses of testosterone than is required to produce overt androgenic responses. Very 
likely the reciprocal relationship between ACTH and gonadotropin secretion is independent of the adrenal androgens in most species, although the latter possibly may cnhance a pre-existing inhibition of the gonadotropins. However, they may be of considerable importance in house mice, rats, and other species which secrete appreciable amounts of adrenal androgens. There undoubtedly are many other factors contributing to intra-uterine mortality which have not been diseussed here. In general, however, it is evident that factors increasing pituitary-adrenocortical activity are associated with increased intra-uterine mortality, although the details of the mechanisms are largely unknown.

Finally, if the young are born, there may be a failure of lactation due to deficiency in the hormones normally required for the maintenance of adequate lactation (Christian and LeMunyan, 1958). The changes in reproductive function following inanition have been shown to be due primarily to a decrease in the secretion of gonadotropins, as the gonads remain responsive to injected gonadotrophin (Srebnik et al., 1958). Most of these alterations in reproductive function will be diseussed in more detail later.

$f$. Acute visceral degenerative changes. Acute degenerative changes of the liver, pancreas, and parotid glands have been described as occurring during an alarm reaction to a variety of stimuli (Selye, 1950; Ehrich and Seifter, 1948). Selye (1950) states that similar changes also occur during the "exhaustion" phase of adaptation.

The most constant hepatic changes are pyenosis, cloudy swelling, and stromal edema. Oceasionally there is fatty infiltration, severe atrophy, focal necrosis, and leucoeytic infiltration. However, many of these changes are duplicated by a variety of specific stimuli and cannot be considered as diagnostic in any sense unless all other possible factors, other than increased pituitary-adrenocortical activity, have been ruled out.

The salivary glands, especially the parotid, also exhibit degeneration and necrosis of the parenchyma, often with suppurative inflammation, during an alarm reaction (Ehrich and Seifter, 1948). In addition to the zymogen discharge already alluded to, the pancreatic parenchyma may exhibit focal necrosis and inflammation comparable to that seen in the salivary glands (Selye, 1950). The cytoplasmic nucleic acids are markedly decreased during acute adrenocortical stimulation or in response to injected adrenocorticoids, and may precede the degenerative changes. Loss of cytoplasmic basophilia in hepatic cells under the same cireumstances reflects the same kind of influence of adrenal steroids on eytoplasmic nucleic acids.

g. Other effects. In addition to those effects of physiologic adaptation which have been discussed, there are others which stem from a general shift in physiologic functions or as yet have not been related to the activity of any specific hormone. Among these is enlargement of the intervertebral 
dises (nucleus pulposus) which has been found in voles (Microtus orcadensis) subjected to emotional stress (Chitty et al., 1956). The enlargement of the intervertebral discs could result from altered fluid and electrolyte balanees brought about by increased aetivity of adrenal corticoids, or might follow alterations in the ground substance by eortical hormones; however, these explanations are conjectural as the mechanism is as yet unknown.

Physiologic adaptation may be accompanied by a variety of shifts in the composition of the blood with changes in hematocrit, electrolyte concentrations, and various metabolites. Blood lipids and sugar may also shift markedly, as we have mentioned earlier. However, these factors are discussed in considerable detail elsewhere (e.g., Selye, 1950; Hartman and Brownell, 1949; Jones, 1957) and will not be eonsidered further in this discussion.

\section{Part 2. Physiologic Adaptation and Mammalian Populations}

\section{Introduction}

The foregoing account dealt largely with the basic endocrine and other physiologie adaptive mechanisms that serve to maintain physiologic homeostasis in the face of a variety of stimuli tending to alter the internal environment and to equip the animal to meet the demands of emergeney situations. In general, any stimulus which imposes physiological demands on an organism beyond those ordinarily met in undisturbed idyllie daily life ealls into play a series of feedback meehanisms that regulate the secretion of hormones responsible for the maintenance of a relatively constant internal environment. These mechanisms act upon the distribution of the internal environment via the cireulatory channels, the composition of the internal environment with respect to fluids, clectrolytes, glucose, fats, and a variety of other metabolites and metabolie products, and on the supply of readily available nutrients and oxygen, especially for the skeletal muscle and nervous system. We have seen that these effects are not achieved without sacrificing funetions less immediately vital to the individual, such as reproduction, growth, and resistance to infectious disease and parasitism. We also have pointed out the error in thinking that the responses to all adverse stimuli are the same and that all necessarily are associated with increased secretion of adrenal carbohydrate-active corticoids. 
The mechanisms, hormonal effects, and general responses so far described have been confined largely to the results of experiments in the laboratory with the usual laboratory species. Very little work has been done on native mammals in the laboratory, and comparative studies are certainly needed. In addition, the work has been to a great extent limited to studies of the effects of injected hormones or of subjecting laboratory animals to extremely severe conditions.

The role that physiologieal adaptive meehanisms play under natural conditions was not investigated in most of these studies. However, it was postulated in 1950 that these same responses could be eroked by increased population density and that these same physiologieal reactions could serve as a feedback to regulate the growth of mammalian populations, their declines, and the mass mortality which occasionally terminates the build-up of a natural population to extremely high densities (Christian, 1950b). It was first suggested that the intraspecifie strife and soeial competition that force animals into adverse circumstances, together with all of the other adversities which become aggravated by high population densities, would elicit adaptive responses such as those which have been deseribed. Therefore one would anticipate a direct relationship between adrenocortical activity and a more or less reciprocal relationship between reproductive function and population density. The hypothesis that physiological mechanisms were active in all populations in response to changes in density and that most environmental deficiencies acted through this mechanism was inherent in the original postulate. This hypothesis implied that social competition or pressure was the sole factor, always present in all populations, which could logieally be expected to elicit the gamut of adaptive responses in every population. However, these relationships had to be demonstrated, and it was necessary to show that changes in population alone could induce a proportional increase in pituitary-adrenocortical activity, decrease in reproductive activity, decrease in resistance to disease, or even death from shock, and all the other reactions and responses which have been described in the preceding section.

Since that time considerable evidence has been accumulated from the laboratory and from natural populations which indicates that these responses to population density do occur and that they can regulate population growth. The balance of this chapter will be devoted to a presentation of the experimental evidence for the response of physiologieal adaptive mechanisms to social competition and therefore population density, and the evidence implicating these mechanisms, acting as a fecdback system, in the regulation of mammalian populations. Finally there will be a discussion of the pertinence of this evidence to the regulation and control of natural populations. 
The general plan of the following diseussion will be to explore first the ability of purely social factors to affect adrenocortical and reproductive function. Next the relation between population density and adrenocortical, reproductive, and other functions as well as alterations in resistance to disease, will be investigated. In general the plan will proceed from populations of fixed size in the laboratory to freely growing populations in the laboratory, and finally to natural populations. Under each of these experimental categories adrenocortical function, reproductive function, disease resistance, and mortality will be discussed with all appropriate experiments and species. The effect of population density on growth will be discussed where appropriate, and the effects of food and other environmental factors on social interactions and endocrine function will be discussed. It is not possible to stay strictly within this framework, as it is somewhat artificial, but it does seem to offer the most logical means of presenting the available information as it progresses from the most artificial but most highly controlled experiments to natural populations which are controlled with great difficulty, if at all.

\section{Endocrine Responses to Social Pressures and to Population Density}

\section{A. Experiments in the Laboratory with Populations of Fixed Size}

One of the basic tenets in the theory that physiological feedback mechanisms can regulate population growth is that a fundamental regulating factor must be present and active in all populations. Whether or not this particular factor is the proximate factor limiting population growth in a given instance is not important if it is universally present. The only known element common to all populations is social interaction, or intraspecific competition. Basically, competition depends on the behavioral characteristic of the species, but some sort of social organization or mutual intolerance is exhibited by all species of mammals.

\section{Social Factors, Adrexocortical and Reproductive Functions}

Social interactions may arise from two kinds of situations: one in which there is invasion of the private territory of one animal by another; and another in which there is conflict involved in the establishment and maintenance of a hierarchical situation. The first requirement of the hypothesis that physiologic mechanisms can and do control population growth is to show that purely behavioral or social interactions, acting through the central nervous system, can induce endocrine responses, especially of the pituitary-adrenocortical and reproductive systems, and to be able to rule 
out other factors which are known to produce the observed changes. That pure sociopsychologic factors can produce these effects is clearly demonstrated by the following experiments.

When a strange vole (Microtus agrestis) of either sex was placed daily for 27 days in a cage containing a resident pair of voles, and therefore in what was essentially their private territory, vicious fighting ensued (Clarke, 1953). The introduced voles lost weight and exhibited a significant hypertrophy of the adrenals and spleens and atrophy of the thymus compared to the residents. In similar experiments with Microtus orcadensis there were significant increases in the size of the livers, adrenals, intervertebral discs, and spleens, and decrease in the thymus in the "stressed" animals, those introduced as strangers to resident pairs (Chitty et al., 1956). Clarke (1953) attributed the changes in organ weight to the actual fighting, stating that fighting is a very effective form of stress since it involves vigorous muscular exercise in addition to the trauma of wounds (Selye, 1950). However, the fact that the resident voles had to fight as much as those which were introduced, and yet did not reflect this with increased adrenocortical activity, apparently was overlooked. This problem may be explored by data on albino mice (Christian, 1959d). An analysis of the relationship between scars from fighting and adrenal weight from 280 male albino house mice from 55 populations of 4,5 , or 6 each showed that, although the mean adrenal weight of every population increased appreciably with respect to isolated controls, there was no relationship between the amount of scarring, as an indication of the severity of fighting, and adrenal weight. Furthermore, the presence or absence of scarring in a population made no difference in adrenal weight. The results were similar irrespective of whether the analysis involved only the differences between populations or the difference between individuals. Adrenal weight increased the same amount in populations in which there was no fighting, or so little that none of the mice had injuries from biting, as it did in populations in which fighting was severe enough that most of the animals were badly scarred. It should be pointed out that these injuries were superficial and for the most part represented bites through the skin only. These results indicate quite clearly that fighting or injury per se are not the stimuli responsible for stimulating increased adrenocortical activity with thymic involution when animals are placed together. Conclusive evidence that sociopsychologic pressures alone are mainly responsible for evoking these physiologic adaptive responses lies in the following experimental results (Davis and Christian, 1957).

When house mice are placed together in groups of six, there is immediate fighting which soon ceases with the establishment of a social hierarchy with one mouse dominant over the others and another subordinate to all the 
others. The remaining mice arrange themselves in some sort of hicrarchy in between. It was found that adrenocortical hypertrophy was greatest in the most subordinate animals and was slight or absent in the dominant mice. The adrenals of those in between tended to fall in line in between in a reciprocal relationship to their dominance rank. Fighting cannot have been an important stimulus to increased pituitary-adrenocortical activity in these experiments, as the dominant animals fought as much as, or more than, any of the subordinate animals. The mice in these experiments also cxhibited changes in the weights of their reproductive organs consistent with suppressed secretion of gonadotropins coincident with increased pituitary-adrenocortical activity. There was no clear-cut decline in the woights of the reproductive organs with decreasing social rank, but the dominant mice had much heavier reproductive organs, especially the preputial glands, then the subordinate animals (Davis and Christian, 1957). These results were confirmed and intended in dogs by Eik-nes (1959), who found that the dominant dogs in groups secreted about half the amount of corticoids that the subordinate dogs secreted. Therefore there can be little question that there are significant differences in adrenocortical function associated with differences in social rank.

In another series of experiments Southwick (1959) demonstrated that moving mice into a new environment daily could induce a marked increase in adrenocortical activity as determined by eosinophil counts. That the eosinopenia was not a result of handling was shown by the fact that mice handled in the same way but not placed in a strange situation responded with only a slight fall in circulating eosinophils. The mice transferred to new cages for a period of time every day adapted to the situation, as the eosinophil count returned to normal levels by the end of the 8-day experimental period. A third series of animals were placed in groups of four each day and these animals exhibited an $80 \%$ mean decline in their eosinophil counts and these counts remained low as long as the animals were placed in groups. Presumably the mice responded to grouping with a marked increase in adrenocortical activity and did not adapt to the situation. It is clear from these results that merely placing mice in a strange situation is an emotional stimulus sufficient to result in a decline in circulating eosinophils and presumably in adrenocortical activity and that grouping constitutes a more profound stimulus to which animals fail to adapt. As we have pointed out previously, it camnot be concluded finally that the cosinopenia was due to increased adrenocortical activity, although it seems likely, until increased secretion of epinephrine is ruled out as a causative factor.

Similar results were obtained when male Norway rats were placed in groups (Barnett, 1955). The subordinate males, subjected to severe fighting for short periods, at first showed marked decreases in adrenocortical sud- 
anophilia, whereas prolonged exposure to less severe fighting resulted in normal sudanophilia with adrenal hypertrophy in the subordinate animals. None of the dominant rats showed adrenocortical hypertrophy or changes in sudanophilia in spite of the fact that they fought as much or more than the subordinate animals. Barnett (1958) has recently published additional evidence on a smaller number of rats from which he coneluded that the adrenal cortices of both subordinate and dominant animals hypertrophy. Nevertheless his data show that the mean adrenal weight, relative or absolute, of subordinate animals in a group of rats introduced into a colony was appreciably greater than that of dominant animals. Barnett used very small numbers of rats and based his conclusions on absolute rather than on relative adrenal weight, in spite of the fact that his animals varied from 250 to $400 \mathrm{gm}$. at the start and 170 to $420 \mathrm{gm}$. at the end of the experiment. He concluded, on the basis of thirty rats, that there was no relationship between adrenal weight and body weight for rats weighing more than $150 \mathrm{gm}$. This conclusion may be questioned for several reasons. In the first place the mean adrenal weight of his animals increased with increasing body weight, athough the differences were not signifieant. However, had larger numbers of animals been used it is more than likely that a significant increase in adrenal weight with increased body weight eould have been shown. Other data on over 1200 wild Norway rats shown conclusively that there is a definite increase in adrenal weight with increasing body weights varying from 50 through $600 \mathrm{gm}$., and that there was a significant linear relationship between the logarithm of the adrenal weight and body length or weight (Christian, 1954; Christian and Davis, 1955). Finally, it is well known that there is a definite tendeney for the larger animals to be dominant, therefore adrenal hypertrophy in smaller, subordinate rats would tend to make the adrenals of dominant and subordinate animals weigh the same. It is of considerable interest, however, that rats from colonies of mixed sex were appreciably larger than those from all-male colonies. In general the amount of sudanophilia in the zona fasciculata coincided with the weight data. Nevertheless, Barnett's results appear to agree with those established earlier as well as with the results of the experiments of other investigators.

The above experiments warrant the general conclusion that in house mice and Norway rats adrenal weight tends to be inversely related to social rank in that dominant animals exhibit little or no inerease in adrenal weight, while subordinate animals show a marked increase. A word of eaution should be directed with regard to the interpretation of results with adrenal weights. A hyperactive adrenal with a loss of cortical lipids may weigh less than a less active gland containing a large amount of lipids. This has been shown to be the case in mice (Christian, 1955a, 1959b) and evidently is also true in Barnett's (1958) experiments in which the adrenals of his "interloper" 
rats weighed somewhat less than those of subordinate rats, but the adrenals of the interlopers contained little or no lipid.

The relationship between social dominanee and adrenal activity is, however, not as elear eut as these experiments at first indicate and suggests that a great deal of work needs to be done on the factors which affect dominance-subordinance relationships and what constitutes "social stress" in mammals. The adrenals of the dominant animal in a group do not always weigh the least and occasionally may be the heaviest, even though the average weight of the adrenals of the dominant animals is appreeiably less than those of other ranks. Furthermore, the amount of fighting varies greatly from group to group. Finally, the amount of scarring on a mouse in a group is in a general way a measure of its rank. Observation has shown that it is generally safe to assume that the unscarred mouse in a group is the dominant animal, and yet, as we have shown, there is no relationship between the amount of searring and absolute adrenal weight in groups of albino male mice although there was clearly a tendeney for adrenal weight relative to body weight to increase with increased scarring (Christian, $1959 \mathrm{~d}$ ). These results apparently indicate that fighting is a poor measure of social rank and is related only indirectly to it. These results appear to contradict the results of other experiments, although these differences would probably be reconcilable if more detailed information were available on social behavior in these animals. Rather rigid and simple criteria are used to determine rank in animals, such as physical dominanee-subordinance relationships, and it is quite clear that social interactions are far more complex than this. However, the apparently contradictory results with relation to fighting and dominance in no way invalidate the conclusions that purely psychological social pressures are responsible for stimulating inereased adrenocortical and decreased reproductive activity in groups of mice and rats and that fighting per se has little or no effect on the adrenal hypertrophy observed in groups of animals. One can speculate with some reason that a massive stimulation of the nervous system and adrenal medulla occurs in subordinate animals when they are suddenly confronted with a dominant, aggressive male. Observation shows that these animals are aware of their rank and eower in front of the dominant animal. When there is no escape from constant contact, it seems inevitable that the subordinate animals must suffer from emotional anxiety resulting from a desire to escape from the situation and the inability to do so. Chronic stimulation of the pituitary-adrenocortical system presumably results from the chronic continuation of such a situation. Admittedly this somewhat anthropomorphic interpretation of the situation is largely speculative, but observations of mice in groups inevitably lead one to such a conclusion.

Finally, Mason (1959) has shown that when rhesus monkeys are kept in 
groups for prolonged periods of time there is a significant increase in their urinary tetrahydroxycorticoids, the metabolites of the carbohydrate-active corticoids, excreted by grouped monkeys is greater than their combined daily production of corticoids when individually caged. Furthermore, the production of corticoids remains high for the entire period of grouping. It has also been observed that when human bomber crews are housed as a group, their production of urinary corticoids is increased over the combined individual production of corticoids by the same men, paralleling the findings for monkeys (Mason, 1959). In the ease of monkeys and men there can be no question that the stimulus to increased corticosteroid production is psychological, resulting from social interactions.

There can be little doubt that social pressures can increase pituitaryadrenocortical activity. We have also suggested that there is a depression of reproductive function in male mice, as indicated by the weights of the sex accessories, coinciding with increased adrenocortical function in relation to social factors. These results are in agreement with the earlier work of Crew and Mirskaia (1931) and Retzlaff (1938), who showed that increased population density depresses reproduction in female mice. The reproductive performance of female mice was inversely related to population sizes in populations of $1,4,8$, or 12 pairs. Retzlaff (1938) also indicated that reproductive performance was best in the socially dominant females in each population. He made several additional observations of interest in these experiments. He noted that there were aggressively dominant females that attacked and viciously fought introduced females or females which had been removed and were later replaced. The subordinate females suffered death or injury, and, of particular interest, any infections that they had were greatly exaggerated. This is one of the early experimental indications of decreased resistance to disease following social stress, and is further evidence that resistance is decreased primarily in the subordinate animals. He also noted that in the largest populations, 12 pairs of mice, there was a sufficient confusion among the mice to offer the subordinate animals partial protection from attack. Similar effects were later noted in populations of 32 male mice (Christian, 1955a, b). Finally, it was found that a reduction in environmental temperature of approximately $16^{\circ} \mathrm{C}$. resulted in a significant decline in mean litter size for mice from populations of 1,2 , or 8 pairs, but not from populations of 4 or 12 . The protection against a reduction in litter size by decreased temperature probably reflects huddling as a means of maintaining body heat and therefore diminishing the need for increasing thyroid activity. Retzlaff could not explain the reduction in populations of 8 , but evidently it was due to severe social strife rather than the reduction in temperature. It is conceivable that severe strife prevented huddling due to mutual intolerance; so that both factors could play a causative role in 
reducing reproductive performance. The preceding experiments usually used populations consisting entirely of male or mixed male and female mice or rats (Crew and Mirskaia, 1931; Retzlaff, 1938; Barnett, 1958). However, a depression of reproductive function occurs when only female mice are placed in groups (Andervont, 1944). In these latter experiments estrous cycles began at an earlier age, were more frequent, and lasted until a greater age in segregated female mice than in their littermates kept in groups of 8 each. Bullough (1952) showed that "overerowding" mice (16 to a cage) for 3 weeks resulted in a 30\% increase in the cross-sectional area of the adrenal cortex and an $80 \%$ increase in medullary area. These changes were accompanied by a $60 \%$ reduction in epidermal mitoses, which was attributed to an increased secretion of adrenocortical steroids. Finally, Chitty (1955) showed that liver glycogen was appreciably lower in voles maintained in the laboratory in groups than in those maintained under segregated conditions.

2. Adrenocortical and Reproductive Responses to Population DeNsity

The preceding experiments indicate that sociopsychologic factors involved in social interactions between mammals can elicit physiologic adaptive responses with increased pituitary-adrenocortical and decreased reproductive function in voles, house mice, both wild and albino, Norway rats, rhesus monkeys, and humans. However, with the exception of the experiments of Crew and Mirskaia (1931) and Retzlaff (1938) there was no indication that these functions were altered in relation to population density. Although an inverse relation between population size and reproductive performance was shown in the experiments of Crew and Mirskaia (1931) and Retzlaff (1938), these authors did not investigate adrenocortical function. If social competition, as a stimulus to increased pituitaryadrenocortical and decreased reproductive activity, is responsible for regulating the growth and decline of mammalian populations, then there must be a relationship between population density and the magnitude of the endocrine responses. The existence of such a relationship has been demonstrated in a variety of experiments.

If male mice which have been segregated since weaning are placed together in groups of $1,4,8,16$, or 32 per cage for a week, there is a hypertrophy of the adrenal glands and atrophy of the gonads and sex accessories which progresses more or less linearly as the logarithm of the population increases (Christian, 1959b). There is a decline in thymus weight from that of the isolated controls, but the decrease is not related to the population size. The increase in adrenal weight was found to result primarily from 
cellular hyperplasia and hypertrophy of the zona fasciculata, although the glands were not critically examined for medullary hypertrophy. The decline in the weights of the testes was a reflection of the generalized reduction in body weight in one series of experiments (Christian, 1955a), but in another series the testes declined in relative testicular weight as well as in absolute weight with increasing population size (Christian, 1955b). Body weight was significantly less in populations of 4,8 , and 16 .

These experiments were repeated using wild house mice raised in the laboratory in populations of $1,3,4,6,8,9$, and 17 with similar though more pronounced results (Christian, 1955b). The thymus weight of these mice decreased markedly as population size increased. The adrenocortical response was also much more pronounced in these mice than in the albino mice, although the mean weights of the adrenals of segregated albino and brown male mice were identical. The increase in adrenal weight of albino mice reached a maximum of $8 \%$ above the control levels in populations of 16, whereas that of the wild stock attained a maximum increase of $21 \%$ above the control levels in populations of 9 . In populations of 32 albino mice and 17 wild mice the mean adrenal weight was less than in the next smaller population size in each case. This was found to be due to a loss of lipid and a marked decrease in the size of cells in the zona fasciculata although the degree of hyperplasia was greater than in the adrenals of mice from the preceding population size (Christian, 1959b). The wild mice are much more alert, reactive, and aggressive than albino mice, and the differences in adrenal reactivity probably reflect such behavioral differences.

The decline in the weights of the accessory reproductive organs, seminal vesicles, and preputial glands indieated a diminished secretion of androgens from the testes with increasing population size, based on the assumption that these organs accurately refleet androgen levels (Burrows, 1949; Rennels ct al., 1953). This conelusion is strengthened by the fact that relative testicular weight also declined in one group of experiments (Christian, 1955b) and absolute weight declined in all experiments with increased population size. Since all indications point to a decline in the secretion of androgens, the decline in thymus weight must therefore represent involution by increased amounts of circulating corticoids.

The progressive hypertrophy of the adrenals and atrophy of the thymus and reproductive organs do not reflect a diminished space per mouse per se, as the results were essentially identical where populations of $1,4,6,8$, and 17 each of male wild mice were placed in cages with 42 times the area of the cages used in the preceding experiments (Christian, 1959b). The similarity of the results from these two series of experiments with vastly different amounts of area per mouse also suggests that the amount of exercise or activity was not a factor in the observed endocrine responses. 
It has been established that close confinement with adequate feeding ean result in adrenocortical atrophy and lymphoid hypertrophy in a variety of speeies of mammals (Christian and Rateliffe, 1952), but these limits of confinement evidently were not approached in the above experiments, as indicated by the adrenal weights in the large and small cages.

These results apply to male mice. When female mice are grouped, there is evidence of increased adrenocortical activity, increased corticoids and androgens being secreted (Christian, 1960). The degree of hypertrophy is small, however being nowhere nearly the amount seen in grouped male mice or even in females from populations of mixed sex. The inference is that the cortical hypertrophy seen in females from populations of mixed sex must to a large degree reflect a situation created primarily by the males.

\section{Food and Social Competition; Splenic Hypertrophy}

In all the experiments with mice so far diseussed, food and water were provided ad libitum. Food was scattered over the cage and water was available from several sources in order to avoid competition for food which might result in inanition in the subordinate animals and might constitute stimuli to the endoerine adaptive responses. These preeautions were taken even though observation had indicated that feeding and drinking were more or less random and on an individual basis, and that there was no observable competition for these items. Also Uhrich (1938) and Strecker and Emlen (1953) had indicated that a limited supply of food did not increase competition among house mice. Nevertheless, the question whether or not the location and amount of food was a major factor in eliciting the observed changes in grouped mice in the pituitary-adrenal and pituitarygonadal systems was answered by specifieally designed experiments. In one series of experiments, male mice, some in groups of four each and some segregated, were provided food either ad libitum or limited to $4.0 \mathrm{gm}$. per mouse per day for a 7-day experimental period. This amount of food restricted weight gain but did not produce weight loss. The food given the grouped animals, whether limited or ad libitum, was scattered for half of the populations and supplied from a feeder for the other half. Therefore food was given the mice in one of the four following ways: scattered and limited in amounts, from a feeder and limited, scattered and ad libitum, or from a feeder ad libitum. The experiment was repeated using groups of five each and a food limitation of $3.5 \mathrm{gm}$. per mouse per day, an amount which produced an appreciable weight loss in all the mice during the week of the experiment. This amount of food per mouse per day can therefore be said to produce inanition. It was found that neither the location of food, whether seattered or from a feeder, nor the amount of food had any effect 
on adrenal weight in the segregated or grouped mice. (irouping produced signifieant adrenal and splenic hypertrophy along with atrophy of the thymus and reproductive organs, and these changes were not altered by the source or amount of food. The splenic hypertrophy produced in the albino house mice in these experiments by grouping paralleled the splenic hypertrophy in voles following social "stress" (Clarke, 1953; Chitty et al., 1956). However, restricting the amount of food resulted in an atrophy of the reproductive organs, thymus, and spleen which was related to the degree of food restriction. The source of food was without effect on the adrenals, thymus, spleen, or reproductive organs. Therefore the source or amount of food did not increase the level of social competition above that already present as a result of the establishment of a social order. The amount of food provided had no effect on the adrenal glands, therefore it was concluded from these and the experiments of others (Mulinos and Pomerantz, 1941; D'Angelo ct al., 1948; Baker, 1952) that inanition does not constitute a stimulus to the pituitary-adrenocortical system of house mice and rats.

On the other hand, Frank's (1953) experiments with confined populations of meadow voles (Microtus arvalis) suggest that a deficient supply of food may increase social competition in this species, as a marked increase in fighting oceurred following the development of a food shortage. Whether or not a food shortage will increase competition very likely depends on the time relationships of the feeding behavior of the species. One would not expect to find increased competition among animals which feed randomly with respect to time, otherwise one would have to attribute to these animals the ability to predict, ahead of time, the development of a shortage, as there would be no appreciable increase in competition for food at any given moment. This consideration also implies that dominant and subordinate animals would lose weight equally in the presence of a defieient food supply. Such was actually the ease in the experiments with house mice (Christian, $1959 \mathrm{c})$. On the other hand, an increase in competition would be expected to follow the development of a food shortage among mammals which habitually feed during the same period of time every day. In this situation one would not expeet the dominant animals to lose weight to the same degree as the subordinate animals, if at all. Frank's (19.53) results coincide with this latter situation. If these conjectures are correct, it is apparent that the temporal feeding relationship and behavior of any species will assume paramount importance with respect to the production of competition and the physiologic responses to it, and should therefore be investigated critically for a variety of mammals. The experiments with house mice should be repeated with voles and other species and any increases in competition determined by observation and the accompanying adrenocortical responses. 
Although inanition and starvation may not be stimuli to increased pituitary-adrenocortical activity in mice and rats, they may be in guinea pigs (D'Angelo et al., 1948) and white-footed mice (Peromyscus leucopus) (Sealander, 1950). However, a limited amount of food does depress reproductive activity in house mice as well as in other species which have been investigated (Lutwak-Mann, 1958), possibly as a result of a protein deficiency, as it has been shown that a dietary deficiency of protein diminishes the secretion of gonadotropins (Srebnik et al., 1958; Lutwak-Mamn, 1958). The striking declines in the weights of the preputial glands, and especially of the seminal vesicles, brought about by a limited amount of food (Christian, 1959c) indicate that the secretion of androgens by the testes was markedly depressed. Whatever the mechanism by which a food deficiency depresses the secretion of gonadotropins, it was not by eliciting a generalized adaptive response involving the pituitary-adrenocortical system. These results lead to several conchisions, at least as far as mice are concerned. One is that when food is supplied ad libitum, competition for food is not a factor in eliciting pituitary-adrenocortical responses in relation to population. A second is that inanition and starvation per se are not stimuli to increased pituitary adrenocortical activity in mice or rats. The third is that food restriction depresses reproduction by diminishing the secretion of gonadotropins without eliciting a more widespread response, and therefore a limited supply of food can limit population growth specifically by depressing reproduction without operating through the pituitary-adrenocortical system. However, we have seen that there is some indication that these relationships may not be universally true. Perhaps food supplies and social competition ean act independently to limit population growth. Food conceivably may not be important to natural populations as long as subordinate animals are frec to move elsewhere. Calhoun $(1949,1950)$ has indicated that social competition is a more important factor than food supply per se for Norway rats. One fact is abundantly clear as a result of these experiments, and that is that sociopsychologic factors stimulate increased pituitary-adrenocortical function and depress reproductive function in proportion to population density; this reaction system is therefore active at all levels of population in the control of population growth, whereas a limitation in the food supply will exert its effects on reproduction only when it results in inanition in the members of a population. These considerations are of obvious importance to the investigator interested in the control and regulation of mammalian populations.

The production of splenic lypertrophy in highly inbred albino mice by increased population density is especially interesting in view of Chitty's (1957) conclusion that the splenic hypertrophy in voles (Microtus agrestis and $M$. orcadensis) was due to inherited genetic factors. This conclusion 
was based on the fact that splenie hypertrophy, which had been observed previously only in natural populations of high density, had made its appearance recently in laboratory stocks of these voles. The only factor which seemed to account for this appearance was the introduction into the laboratory breeding population of voles from natural populations exhibiting splenic enlargement. The fact that a similar hypertrophy was observed in response to increased population density in a highly inbred strain of mice, maintained under constant conditions for a good many years with no exposure to natural conditions, makes a genetic explanation of the sort postulated by D. Chitty unlikely in such an inbred strain of presumptively genetically stable mice. Similarly, as was pointed out earlier, a comparable hypertrophy of the splcen was observed in a few inbred rats subjected to alarming stimuli. The hypertrophy, when examined critically, has been found to be due to increased hematopoiesis (Dawson, 1956), possibly in response to the increased stimulation of erythropoicsis by hormonal factors. It is also well known that splenic erythropoiesis can be stimulated by any stimulus that produces anemia. Therefore, conclusions regarding the causes of splenic hypertrophy, due to increased erythropoiesis, must be interpreted with caution; although it does not seem likely that it is genetic in origin, at least in inbred albino mice with splenic hypertrophy following increased population density.

\section{Reproductive Fuxction in Female Mice: Lactation, Reproduc-} TION

Female mammals have frequently been observed to respond to adverse stimuli with a reduction of reproductive function. When rats are suddenly moved from a temperature to which they have become accustomed to a different temperature, either higher or lower, there is retardation of growth and prolongation of the estrous cycle (Bohanin, 1939). Selye (1939) has listed a variety of agents which will inhibit ovarian function and estrus. Reproduction is also depressed in female mice by increased population density. We have noted that Crew and Mirskaia (1931) and Retzlaff (1938) found that reproductive performance of female albino mice declined with increasing population density. In another series of experiments no young were produced and no females became visibly pregnant when mice were crowded 20 males and 20 females to a cage for 6 wecks (Christian and LeMunyan, 1958). It is not known whether there was a marked suppression of ovarian function with diminished ovulation, a failure of the ova to implant, or intra-uterine loss early in pregnaney, but more than likely all these factors were involved. When the population size was reduced to 10 males and 10 females, all the females became pregnant, but the number of 
implanted ova was reduced significantly and only 7 of the 10 females delivered young. The remainder lost their progeny in utero during the early stages of pregnaney. The onset of pregnancy also was considerably delayed in these animals. Therefore there was decreased fertility, decreased implantation, and a marked increase in intra-uterine mortality. These results indicate that female mice respond to increased population density with a depression of reproductive function at all stages of the processes. These results correspond to those seen in males as indicated by the weights of their reproductive organs. Reproductive suppression was also observed when female mice were grouped without males (Andervont, 1944; Whitten, 1959; Christian, 1960).

Chitty (1952) noticed that young voles from natural populations of high density were reduced in size and were unusually susceptible to increased mortality. He hypothesized that the young were adversely affected in utero by the physiologic derangements in the mothers which resulted from high population densities (Chitty, 1952, 195t). Later it was shown in a limited series of experiments that social "stress" diminished lactation in voles, as measured by the weights of progeny nursed by the mothers subjected to social pressures which had previously been shown to result in increased pituitary-adrenocortical activity (Chitty, 195.5). These experiments were repeated on a larger scale using laboratory white mice (Christian and LeMunyan, 1958). It was found that progeny nursed by previously crowded mothers weighed appreciably less at weaning than those nursed by mothers which had always been segregated. The effect on the progeny was greater in the larger litter sizes, suggesting a quantitative rather than a qualitative deficiency in the supply of milk. When these young which had been nursed by crowded mothers were themselves bred, the progeny which they in turn nursed were significantly lighter at weaning than their controls. Again the defect was greatest in the larger litters, but the difference was not manifest until a larger litter size had been reached than in the preceding generation. These differences in the second filial generation cannot be attributed to grouping. Crowding depresses all the other reproductive functions, therefore it is not surprising that lactation is also suppressed, as it is to a large degree under the control of pituitary gonadotropins and sex steroids in addition to oxytocin, thyroxine, and growth hormone (Folley, 1956; Grosvenor and Turner, $1959 \mathrm{a}, \mathrm{b}, \mathrm{c}$, ). These results are consistent with others which show that lactation can be inhibited by a variety of stimuli which stimulate increased pituitary-adrenocortical activity and diminish increased reproduction and growth (Selye, 19.54); in fact, lactation can be limited by a deficiency of any one of a variety of hormones necessary for its fulfillment (Grosvenor and Turner, 1959a, b, c). Since the young mice in litters of small size were unaffected in the above experiments, it is unlikely that any 
substance, such as adrenal hormones, contained in the milk were responsible for the decrease in weights of the progeny. The mechanism by which these effects were carried over into the second generation of progeny is not known. Presumably the second generation of young may reflect the inanition suffered by the first generation of progeny as a result of deficient lactation. However, the fact that androgens can produce subsequent permanent sterility when injected into mice less than 10 days old suggests the possibility that increased amounts of adrenal androgens may reach the nursing young via the milk and exert similar partial effects if some escape metabolism in the liver. The weights of the young at birth were unaffected by the earlier crowding of their mothers. Although the precise mechanisms are not understood, these results support and extend D. Chitty's results with voles and help in providing an explanation for the prolonged effects of high density on surviving young observed in natural populations, such as Chitty's $(1952,1954)$ observations on young roles from natural populations.

\section{Growth}

The effects of increased population density on growth have not been studied to the same degree as its effects on other aspects of endocrine physiology, although suppression of the secretion of pituitary growth hormone presumably is a part of the response to stimuli which also result in the increased secretion of ACTH (Sclye, 1950). However, there are a few cxperiments which clcarly indicate that the growth of house mice is depressed in response to increased population density (Vetulani, 1931; Christian, 1955b). It has been shown also that there is suppression of growth in all but the dominant and second-ranking mice in a group of six (Christian, 1961). The degree of inhibition was related to rank.

6. Inflamation, Resistance to Infection, and Population DenSITY

In earlier sections of this account the inhibition of the inflammatory reresponse, granulation, and antibody formation, and therefore resistance to disease, by adrenal carbohydrate-active corticoids was discussed. Most of the experimental evidence cited was based on the results of injecting cortical hormones. A few examples of decreased resistance to infectious agents brought about by stimuli, such as cold, which stimulated increased adrenocortical secretion were given. However, even though these experiments indicated that such effects might occur under natural circumstances, they did not establish this possibility, and especially they did not show that increased population density could stimulate a sufficient increase in the 
secretion of adrenal carbohydrate-active corticoids to inhibit inflammation, granulation, and antibody formation suffieiently to decrease resistance to disease, although Retzlaff (1938) had indieated that there was decreased resistance to infection in the subordinate mice in his population studies. Experiments have been conducted which do establish these points to a limited degree, but more experiments with more species are needed.

An efficient method of inducing an inflammatory response and the formation of granulation tissue in rats or mice is to implant subcutaneously cotton pellets moistened with turpentine (Meier et al., 1950; Christian and Williamson, 1958). Later these pellets and the surrounding tissue can be removed and weighed. In this fashion the degree of the inflammatory response and formation of granulation tissue can be assayed under a variety of conditions. This technique has been used to show that ACTH and the adrenal earbohydrate-active corticoids suppress these responses (Meier et al., 1950). By this procedure it was found that when mice were placed in groups of 5 each there was approximately a $20 \%$ reduetion in the amount of granulation tissue formed in 1 week compared to the amount formed by the segregated control mice (Christian and Williamson, 1958). These experiments clearly indicated that increased population density is a sufficient stimulus to the pituitary-adrenocortical system to decrease significantly inflammation and granulation.

Davis and Read (1958) conducted a series of related experiments in which they demonstrated that placing wild-stock house mice in groups markedly increased the susceptibility of the mice to invasion by the larvae of Trichinella spiralis. Each mouse was infected parenterally with approximately 125 embryonated Trichinella larvae. Each mouse was maintained in a separate eage, but from day 3 through 11 after infection 11 of the mice were placed in two groups, one of 5 and the other of 6 mice, for 3 hours a day, while 11 others were left segregated. The mice were sacrificed the 15 th day after infection, the gastrointestinal tracts were digested, and the larval worms were recovered. Only 3 of the segregated mice were infected with an average of 9 worms apiece, whereas all the grouped mice were infected with an average of 32 worms each, a $250 \%$ inerease. The experiment was repeated with 6 segregated and 5 grouped mice, but they were not sacrificed until the 30th day post infection, and the encysted larvae were recovered. All the mice, segregated and grouped, were infected, but the grouped mice had $48 \%$ more encysted larvae than their segregated controls. In both experiments the differences in the number of worms between segregated and grouped mice were highly significant and in both experiments the mean adrenal weight was greater in the grouped than in the segregated mice, $8 \%$ $(P<0.20)$ in the first experiment and $20 \%(P<0.01)$ in the second experiment. 
In earlier experiments it had been shown that either cortisone or ACTH increased the invasiveness of Trichinella larvae by suppressing the defensive inflammatory response of the host's intestinal wall and possibly by prolonging the sojourn of the adult females in the gut by suppressing immune responses to the worms (Stoner and Godwin, 1953). Thus, these hormones decreased the resistance of mice to invasion by the larvae of Trichinella spiralis by inhibiting inflammation and possibly antibody formation. Grouping evidently stimulated a sufficient increase in the secretion of adrenal corticoids to produce similar effects. This conclusion is supported by the demonstration that grouping was sufficient stimulus to pituitary-adrenocortical activity to diminish granuloma formation appreeiably. These experiments with trichinosis in house mice have been repeated with albino mice with similar results, confirming the original results and demonstrating that albino and wild-strain house mice react similarly (Davis and Read, unpublished).?

The effects of crowding on trichinosis in mice is presumably primarily due to the effects of increased adrenocortical secretion on the inflammatory response to the worms. Therefore a similar series of experiments were performed which were more specifically designed to demonstrate the effects of grouping on antibody formation (Davis and Prudovsky, 1959). In this case mice were injected with tetanus toxoid and challenged 10 days later with 13 mouse MLD of tetanus toxin. The dosage of toxin was selected to cause death in $50 \%$ of segregated mice. Mice were placed in groups either 5 or 3 days prior to giving the toxoid and left in groups until 5 days after the toxoid. Control mice were left segregated in individual cages throughout the experimental period but otherwise were treated identically to the experimental animals. Grouping appreciably decreased resistance and increased mortality of mice to the challenge dose over the control levels. This effect was less marked when the mice were grouped 3 days before administering the toxoid. The effects of grouping resembled the effects of injected cortisone at the appropriate times and in appropriate doses. These results indi-

${ }^{2} \mathrm{~A}$ - report was published recently which implied that cortisone or ACTH are without effect on the course of trichinosis in mice, but which showed that cortisone markedly increased mortality (Lord, 1958). These experiments require some critical comment. The procedure used to infect the mice was similar to that used by Davis and Read (1958), but injections of cortisone and ACTH were not begun until 6 days after infection. One would not anticipate that these hormones would affect the course of trichinosis at that late date, as invasion by the larval worms, the inflammatory response to them in the intestinal wall, and immune responses to them are fairly well accomplished facts by that time. Therefore one would not anticipate an alteration in the resistance to invasion by the worms. The doses of cortisone used in these experiments were extremely high pharmacologic doses: $76 \mathrm{mg}$. in 39 days, beginning with $3 \mathrm{mg}$. a day per mouse. It is not surprising that cortisone increased mortality. 
cate that the adrenocortical response to grouping is sufficient to depress antibody formation as well as inflammation. From these results one can easily see that behavioral factors associated with increases in population density, or grouping, can sufficiently increase adrenocortical activity to profoundly affect host resistance to infectious disease and parasites.

The effects of hormones on murine tuberculosis are somewhat complex. Cortisone enhances tuberculosis infections in mice, especially by converting a smoldering chronic infection into a fulminating acute process (Hart and Rees, 1950). However, ACTH was without effect on the early development of tuberculosis in mice (LeMaistre et al., 1953). From these experiments it would appear that an increased secretion of endogenous adrenal corticoids was ineffective in enhancing tuberculosis in mice whereas injected carbohydrate-active corticoids markedly enhanced the infection. Therefore, it is especially interesting that Tobach and Block (1956) were able to show that crowding significantly altered the courses of acute and chronic tuberculosis in mice. Crowding after infection decreased the survival time of mice of both sexes suffering from an acute tuberculous infection. Crowding after infection had essentially no effect on the course of chronic tuberculosis in female mice, whereas it enhanced the chronic disease in male mice. These experiments also indicate that crowding prior to infection may enhance host resistance.

These experiments, although quite limited in number, all confirm the fact that crowding (increased population density) can reduce host resistance to disease. The evidence from experiments with increased population density is completely in accord with the results of experiments with injected corticoids. These results, considered in the light of the mass of this evidence on the effects of corticoids and ACTH on experimental infections, can only mean that increased population density sufficiently stimulates the pituitary-adrenocortical system to lower resistance to disease by inhibition of inflammation, granulation, and probably antibody formation by endogenous corticoids.

\section{Production of Mortality Directly}

In the preceding section the role of behavioral factors and increases in population density in decreasing resistance to disease was discussed. It therefore follows that grouping can increase mortality by this means and the more subordinate animals will be the ones most often affected. However, grouping is often followed in a very short time by sudden death preceded by alternate prostration and convulsions of many of the animals (Christian, 1955b), and the greater the number which are placed together, the greater the number which suceumb in this fashion. (J. J. Christian, umpublished), 
although there is considerable variation from population to population in the numbers which die in populations of the same size. The more reactive and aggressive wild-stock house mice are much more prone to succumb in this fashion than the usual albino mouse in the laboratory (Christian, $1955 \mathrm{~b})$. The proximate cause of sudden death in these animals has not been investigated, although its onset, behavior, and general symptomatology are highly suggestive of hypoglycemic shock and closely resembles the deaths from "shock disease" observed in natural populations at peak densities (Christian, 1950b). Frank (1953) observed similar deaths in voles (Microtus arvalis) after placing them in groups, as well as in natural populations, and was able to demonstrate that glucose could prevent the fatal termination of the syndrome, and also showed that the symptomatology of insulin hypoglycemia was identical to that observed in voles dying naturally following "crowding." Furthermore, he showed that the voles which were on the verge of dying had markedly decreased levels of blood sugar and liver glycogen. These results make it appear probable that the convulsions and death which follow shortly on the social strife produced by grouping mice are due to hypoglycemic shock. There is reason to suggest that a massive discharge of the adrenal medulla, with its subsequent exhaustion may lead to a failure to further mobilize glucose and the animals die in a hypoglycemic episode. However, this hypothesis requires further testing.

In any event, social strife and the physiologic responses to it may result in mortality directly as well as by affecting host resistance.

\section{Summary of Results from Populations of Limited Size}

Experiments with populations of fixed size have demonstrated that sociopsychologic interactions can and do stimulate increased pituitary-adrenocortical and decreased reproductive activity and growth in mice, voles, and Norway rats. Increased adrenocortical activity is related to social dominance-subordinance relationships, the more subordinate animals exhibiting a greater response than the dominant animals. These responses apparently are unrelated to fighting per se. Fighting is evidently a sign of social interaction, just as is the endocrine response, and not a causative factor. There is a progressive increase in the pituitary-adrenocortical response and decrease in reproductive function in male and female mice with increasing population size. All phases of reproductive activity are depressed, including fertility, implantation of blastulae, intra-uterine survival, estrus, lactation (with persistent effects for at least two generations), and the size and activity of the male gonads and sex accessories. Inhibition of reproductive function apparently stems from a depressed secretion of pituitary gonadotropins. Crowth is also inhibited by increased population density. 
In addition to these primary effects of physiologic adaptation to the social pressures associated with increased population density, there is a decrease in resistance to parasitism and infection. It was shown that increased density produced an inhibition of inflammation, granulation, and probably antibody formation. A deerease in host resistance to trichinosis and tuberculosis was observed which apparently was due to these factors.

It was also shown that food was not a factor in these effects, either indirectly by inereasing social competition or directly by inanition in the subordinate animals. A deficieney of food is not a stimulus to inereased adrenocortical activity in mice, or rats, although it may be in other species. A food deficieney, however, does result in a marked suppression of reproductive function, probably by decreasing the production of pituitary gonadotropins.

\section{B. Freely Growing Populations}

\section{Introduction}

Experiments with populations of limited size, although indicative, do not establish that these same responses occur in freely growing populations. In the first place, experiments with limited populations were conducted for limited periods of time, usually too brief to permit adaptation to the situation. Furthermore, in every ease the experimental approach involved suddenly placing strange animals together under rather artificial circumstances. Therefore, these experiments provided no evidence that mammals which have been more or less in contact with each other from birth would respond similarly. House mice (Mus musculus) and voles (Microtus pennsylvanicus, Microtus agrestis) from free growing populations show the same physiologic responses to increased population density as do mice from populations of fixed size but to an even greater degree.

\section{Population Density and Adrenocortical Function}

Increased pituitary-adrenocortical activity has been related to increased population density in a number of experiments with freely growing populations of house mice and voles (Christian, 1956: Louch, 1956; Christian, $1959 \mathrm{a}, \mathrm{b})$. A number of experimenters have shown that the growth of freely growing confined populations is self-limited in spite of the fact that food, water, nesting material, and nesting space were provided ad libitum and well scattered (Strecker and Emlen, 1953; Clarke, 1955; Southwick, 1955a; Christian, 1956; Louch, 1956; Croweroft and Rowe, 1957 ; Christian, $1959 \mathrm{~b})$. Competition for food or a lack of availability of food by particular individuals was not a factor (Christian, 1956; Crowcroft and Rowe, 1957). 
The growth form of these populations was approximately sigmoid, indicating that an intrinsic damping factor was operating to regulate and limit the growth of populations throughout their histories. These populations were started by introducing a small number of animals of both sexes into confined quarters and allowing the population to grow of its own accord. The populations were either sacrificed, at maximal and submaximal levels, to obtain organ weights and histologic material, or blood samples were taken for eosinophil counts to assess functional changes, especially of the adrenal cortex.

The zonae fasciculatae of the adrenal cortices were hyperplastic and hypertrophic in house mice of both sexes from populations of maximum (asymptotic) size. The number and size of the cells of the fasciculata were increased. Adrenal weight reflected the fascicular hypertrophy by increases of $25 \%$ in the males and $14 \%$ in the females. The adrenal cortical hypertrophy was approximately half as great in mice from populations of approximately one half the maximum size.

The presence in mice of an adrenocortical $\mathrm{X}$-zone which is involuted by androgens has been described. This zone complicates the interpretation of adrenal weight from immature or puberal male or nulliparous female house mice unless histologic studies accompany the data on weights. In the case of the freely growing populations of house mice there was a pronounced hump in the adrenal weights in relation to body weights for mice from the experimental populations in the $13-19.0$-gm. weight range, even though the fascicular hypertrophy was proportionately constant for all weights. The curve of adrenal weight on body weight for the segregated control mice was more or less regular with no pronounced irregularities, although there is a tendency for the slope to decrease with increasing body size. This apparently excessive adrenal hypertrophy in 13-19-gm. mice from intermediate and high populations resulted from a failure of the $\mathrm{X}$-zone to involute normally rather than from a true hyperplasia or hypertrophy. The width of the $\mathrm{X}$-zone in segregated male house mice begins to decline in mice in the 10-12.9-gm. weight group, and involution is essentially complete by the time a body weight of $16 \mathrm{gm}$. is reached. The decline did not begin in the experimental animals until a weight of $16 \mathrm{gm}$. or more had been reached and was not complete until a weight of $19 \mathrm{gm}$. Therefore, along with a marked hyperplasia of the zona fasciculata, suggesting an increased secretion of corticoids with increased density, there was a delay in the onset of puberty, presumably with an insufficient production of androgens to involute the $\mathrm{X}$-zone of male mice. A great variety of steroid hormones, including corticoids, have been tested for their ability to involute the $\mathrm{X}$-zone in male house mice (McPhail and Read, 1942b; Antopol, 1953; Allen, 1954; Christian, 1954), but only those with pronounced androgenic activity (e.g., 
testosterone) have been effective, although Delost (1954) has reported that cortisone involutes the $\mathrm{X}$-zone in voles. This delay in androgen production presumably indicated an inhibited secretion of gonadotropins from the anterior pituitary, although there apparently were sufficient gonadotropins to maintain the $\mathrm{X}$-zone, as luteinizing hormone presumably is responsible for maintaining this zone (Jones, 1949b, 1950, 1952).

Thymic involution is effected by carbohydrate-active corticoids, estrogens, and androgens, with variations in the mode of involution (cf. above). Therefore thymic involution, as a measure of increased adrenocortical secretion, must be interpreted with caution if there is reason to suspect differences in the levels of circulating sex steroids. Such was the case in the experiments under discussion, but additional information makes it possible to state with reasonable certainty that the weights of the thymus reflect increased corticoid secretion. The thymuses of $13-19-\mathrm{gm}$. male mice from high populations weighed more than their segregated controls, whereas the mean thymus weight of 19-23-gram mice from the high populations was less than that of the segregated mice. The greater thymus weight coincides with the greater width of the adrenal $\mathrm{X}$-zone in the experimental mice and probably, as in the case of the $\mathrm{X}$-zone, represents inhibition of androgenic activity which is not overridden by the increased amounts of circulating corticoids. It has been shown that the natural adrenal secretory products of mice will produce thymic involution and lymphopenia (Bahn et al., 1957; Wilson et al., 1958). However, depression of the thymus weight to below the control levels in the larger mice from high populations can only reflect increased adrenocortical activity. The mean thymus weights of mice from the intermediate populations were greater than those of the controls or experimental mice from high populations in the 16-19-gm. body weight range. Data from the $\mathrm{X}$-zone indicate that the secretion of androgen (or at least its activity) was inhibited to the same degree in the intermediate and high populations, whereas adrenal weights and width of the zona fasciculata were less in the intermediate than in the high populations. Therefore the greater thymus weights in mice weighing 16-19 grams from the intermediate populations may have resulted from a less marked increase in adrenocortical activity than occurred in the high-density populations, whereas androgen secretion was depressed equally in populations of both sizes. These results might be interpreted to mean that at increased population densities the younger, and presumably subordinate, animals are the ones predominantly affected by increased density. Since these results are obtained by sacrificing an entire population at one time, such a conclusion would be valid if it were not for the fact that the evidence indicates that the increase in pituitary-adrenocortical activity involved all weight groups and therefore all ages. Nevertheless, as we shall see below, a few of the heaviest 
animals, probably including the dominant animals, were less affected by increased density than those in any other weight group.

It was mentioned earlier than in response to stimuli which evoke increased adrenocortical activity there is often inhibition of growth resulting either from direct inhibition of growth by carbohydrate-active corticoids, by inhibition of the secretion of pituitary growth hormone, or both. Evidence has been presented indicating that there is inhibition of growth with increased population density. Therefore, one might question the comparison of organ weight data from mice from populations of high density with those from segregated mice, as mice from the high-density populations may be older than segregated controls for the same body weight. However, if it were possible to correct for age, the differences with respect to body weight between high-density populations and segregated controls would be even greater. Therefore, comparisons with respect to body weight will err on the conservative side.

The zonae fasciculatae of female mice of all sizes from high populations were appreciably wider than their controls as a result of cellular hyperplasia and hypertrophy, although the presumptive increase in adrenocortical secretion was not indicated by the thymus weights. If anything, the thymuses of females from the experimental populations were heavier than those of the segregated controls, possibly a reflection of a diminished secretion of the sex steroids.

On the other hand the preputial glands respond to stimulation by ACTH (Jacot and Sclye, 1951; Hess et al., 1952, 1953; Rennels et al., 1953), and the preputials of female mice from high-density populations were heavier than those from their segregated controls. Therefore, it is possible that the increase in the weights of the preputial glands of these mice may have resulted from an increased secretion of ACTH. This problem will be discussed in more detail subsequently.

The effect of increased population density on adrenocortical function of voles (Microtus pennsylvanicus) in freely growing confined populations has also been studied. The experimental procedure used for these populations was essentially the same as for the house mice except that cosinophil counts were used to measure adrenocortical function in the three populations of voles (Louch, 1956). The use of eosinophil counts has an obvious advantage over adrenal weight for assessing adrenocortical function in that the animals do not have to be sacrificed, especially when various factors contributing to variation in the counts are taken into consideration (Louch et al., 1953). In such long-term studies as this with repeated counts, there can be little doubt that the eosinophil counts reflect adrenocortical function rather than medullary. There was a significant negative correlation between 
mosinophil counts and population density, indicating a progressive decline decline in the number of circulating eosinophils with increasing population density, but the correlation between eosinophil counts and population density was not significant in the third population. The latter population, however, never reached 30 animals in size. The published figures indicate a striking parallel between the rate of population growth and eosinophil counts for all three populations, and in all three there were significantly fewer circulating eosinophil when the populations of these voles were greater than 30 than when they were less than 30 (Louch, 1956). These results indicate that adrenocortical function increased progressively with increasing population density. The variability in Louch's data reflect to some extent the difficulties in obtaining precise eosinophil counts, even though he was fully aware of these problems and took every step possible to aroid the usual pitfalls (Louch et al., 1953; Louch, 1956). The problems involved in using eosinophil counts as indices of adrenocortical function have been discussed fully elsewhere (Thorn et al., 1953; Louch et al., 19533; Rosemberg et al., 1954; Speirs, 1955; Visscher and Halberg, 1955; Louch, 1956; and earlier in this chapter). Handling will cause an adrenal medullary and cortical discharge due to fear, excitement, and possibly rage in wild mammals and therefore can effect eosinophil counts (Southwick, 1959). Consequently it is possible for variability to result from handling, as well as by individual and perhaps more importantly, by unknown factors, unless appropriate precantions are taken (Louch, 19.58). However, in spite of these problems. cosinophil counts offer a promising means of investigating adrenocorticalpopulation density relationships until simple, more direct tests become available. Probably the best procedure at the present time is to follow the population with eosinophil counts and to substantiate the changes with adrenal weights at the termination of the study. Louch's results provide evidence of increased adrenocortical function in voles with increased population density.

In a similar experiment, a marked increase in adrenal weight, as an index of cortical activity was found in both male and female voles (Microtus pennsylvanicus) from a frecly growing confined population which had reached its maximum size (Christian, 1959b). The adrenals of mature male voles were increased $39.6 \%$, and those of mature females $36.6 \%$ over their segregated controls. It was pointed out earlier that adrenal weight-body size relationships may vary with species and with sex; therefore, if animals covering a wide range of sizes are to be used, the correct relationship in these must be determined. It was found that in male voles over $115 \mathrm{~mm}$ long, adrenal weight did not vary with further increases in the size of the animal, therefore the absolute adrenal weights could be used for compara- 
tive purposes. However, in female voles adrenal weight increased with increase in body size; therefore for females it was necessary to use relative adrenal weights for purposes of comparison.

\section{Reproduction}

a. Male. Reproductive function was depressed in proportion to increases in population size in both male and female house mice from populations of fixed size. The results of experiments with freely growing confined populations of house mice and voles also show a progressive decrease in reproductive function with increasing density. We have seen that there was a delay in the onset of puberty in male house mice from frecly growing populations of high density, as demonstrated by the delayed involution of the adrenal $\mathrm{X}$-zone and the development of the testes, seminal vesicles, and preputial glands. The size of the gonads and sex accessories was less than their controls from segregated mice of all but the heaviest body weight. Rapid development of the seminal vesicles and preputial glands did not begin until a body weight of $16 \mathrm{gm}$. was reached, which coincides exactly with the beginning of $\mathrm{X}$-zone involution in animals. These results strengthen the conclusion that androgen secretion was delayed. In the segregated controls, the sex accessories had begun rapid growth by the time the animals had reached a weight of $13 \mathrm{gm}$. These results were confirmed by histologic examination of the testes. The size of the testes primarily reflected the advancement of spermatogenesis and the development of the seminiferous tubules. Spermatogenesis was inhibited to the same degree as the sex accessories (Christian, $1956)$.

Male mice from freely growing populations of intermediate size exhibited an inhibition of the reproductive organs which was approximately intermediate between that exhibited by the males from the high populations and the segregated controls (Christian, 1956).

It is of additional interest that testes and sex accessories of male mice weighing $28 \mathrm{gm}$. or more, the heaviest weight group in the experimental populations, were about the same size as those of the isolated controls. Furthermore, there was no inhibition of spermatogenesis in these animals. In general, this weight group contained the dominant animals in the four high-density populations examined and was represented by a total of 21 animals. These mice represented the initially introduced males and those males from the first litter or two that had established their dominance early in the histories of the populations. These observations are confirmed by Croweroft and Rowe (1957), who observed that the productive females in in confined populations of house mice were the introduced animals or those from their first few litters. Detailed observations relating the weights of 
reproductive organs to specific animals were not made, but it is likely that these results parallel those from populations of limited size in which it was found that the weights of the reproductive organs were greatest in the socially dominant animals. We have indicated that the weights of the accessory reproductive organs appear to be the most sensitive indications of changes in population density as well as social pressures and differences in rank. This also appears to be the case in these experiments with freely growing populations. Reproductive function of male mice has not been examined in detail, usually not at all, but other investigators studying freely growing populations, although Strecker and Emlen (1953) did find all the males in a self-limited population with epididymal sperm 6 months after population growth had ceased.

It might reasonably be asked why, during the 6 months or so that most of these populations lasted, there was not adaptation to the situation with a diminution of the effects of population density on the adaptive responses of the adrenals and reproductive organs. Brown (1953) and Southwick (1955b) have both pointed out that in a well-stabilized social hierarchy of mice there is a relatively low level of fighting, usually used as an indication of the amount of sociopsychologic pressure and interaction, but that when shifts or disruptions in the hierarchy occur, due to the maturation of new individuals or death of old ones, social pressures increase, as indicated by an increase in the amount of fighting. Southwick (1955b) has pointed out that these factors are constantly disrupting the social order in rapidly growing populations, therefore, as the population increases it is inevitable that social pressures increase apace. Comparable results have been observed in other freely growing populations, but there is not necessarily fighting (Christian, 1956, 1959a, b). Furthermore, female mice become particularly aggressive prior to parturition (Brown, 1953; Crowcroft, 1954), and so the total amount of aggressiveness contributed by females would tend to increase with the population. For these reasons it appears that the amount of social pressure in a population will increase with the size of population. It has been observed also that the self-limited maximum size varies greatly from population to population (Southwick, 1955a, b; Christian, 1956) apparently as a function of the amount of social pressure within the population, the variation being contributed by individual differences, the stability of the social structure, and similar factors. For example, Southwick (1955b) has described the individual differences between males in the amount of territory they will fight over. Southwick (1955b) has discussed in considerable detail some of the factors involved in the composition of social competition between mice. One population has been observed in which the social order of the population was disrupted at about half the estimated maximum size by the death of an old, tyrannical male (Christian, 1956, and unpub- 
lished). Severe fighting broke out in this population and the growth of the population eeased completely until social order was reestablished 6 weeks later. The cessation of growth resulted from a complete cessation of all aspeets of reproductive aetivity. These considerations, plus the fact that the physiologie responses are equivalent in populations of maximum selflimited size irrespective of the number of mice (Christian, 1956), make it likely that the growth of populations of house mice, and possibly voles, is limited by the total amount of social pressure rather than by the number of animals.

The reproductive competence of males has not been examined in detail for any species from freely growing populations other than house mice; and no studies have adequately explored the problem. In most studies on population density the criteria of male fertility are position of the testes, gross size of the testes, visibility of the epididymal tubules, and occasionally the presence or absence of sperm in the epididymes (Strecker and Emlen, 1953; Brown, 1953; Southwick, 1955a; Croweroft and Rowe, 1957). These criteria are aetually poor indicators of relative fertility and only suggest whether or not an animal is mature. Detailed morphologic studies provide more evidence of fertility (Christian, 1956), but they do not provide conclusive evidence. No information on subtle changes in fertility is provided by any of these criteria. The ability of males to fertilize females with respect to population density has not, to my knowledge, been investigated.

Many influences which depress reproductive activity cause striking degenerative ehanges in the germinal cells in the testes (Selye, 1947). The formation of giant cells from cells of the spermatogenic series is a common indicator of such changes (Selye, 1950; Steinberger and Dixon, 1959). Furthermore, influences which can produce severe degenerative changes or inhibit spermatogenesis completely can produce more subtle changes if the damaging stimulus is less severe (Steinberger and Dixon, 1959). It seems likely that the conspicuous changes that oceur in the testes of house mice as a result of increasing population density [a delay in the onset of spermatogenesis, reduction in the number of mature spermatoza, and formation of giant cells (Christian, 1951, 1956) ] ean also be extended to include less obvious abnormalities of the sperm, such as decreased motility and viability. There also may be a decrease in the number of sperm produced, and therefore in the number of sperm in the ejaculate. All these factors presumably affeet fertility (Chang and Pineus, 1951). To these must be added the likelihood of an altered medium for the sperm which would be suggested by the changes in the sex accessories associated with testicular ehanges, as indicated by the decline in their weights (Burrows, 1949; Lutwak-Mann et al., 1949; Leathem, 1950; Mann, 1954). Atrophy of sex aceessories carries the implieation that their secretory products may be 
abnormal, deficient, or decreased in amount, and that the eomposition of the ejaculate will in all probability not provide an optimal medium for sperm (Burrows, 1949; Lutwak-Mann et al., 1949; Cavazos and Melampy, 1954). Therefore it is possible that male fertility is depressed appreciably by increased population density, although the gross examination or relatively minor changes in weight would provide no indication of such a reduction in male fertility. Male fertility is assessed in most studies on small mammals by (1) the position of the testes, (2) whether or not the epididymal tubules are clearly visible, or (3) possibly by epididymal smears to determine the presence or absence of spermatozoa (Southwick, 1955a; Louch, 1956; Strecker and Fmlen, 1953; Croweroft and Rowe, 1957). Degenerative changes can be detected in testicular smears (Christian, 1950a, 1951), but with much less assurance than properly prepared, stained, and critically examined sections of the testes. Morphologic studies of the testes, if properly done, ean be extremely revealing, but the weights of the testes and aecessory organs, while providing a useful index of reproductive function in general, do not tell whether or not the male is actually fertile unless inhibition is severe. In the absence of good evidence to the contrary it is generally assumed that the males in a population are fertile--an assumption that may be misleading. Studies of male fertility in relation to population density are needed that make use of sperm counts, determination of the motility and viability of the sperm, and possibly direct determination of fertility by mating with proven females. Admittedly such a program would present problems, but they are by no means insurmountable. Furthermore, once the techniques are worked out for obtaining ejaculates from the males in populations of small mammals, it should be a valuable and useful procedure for routinely assessing male fertility and reproductive competence in freely growing populations of small mammals. But it is incorrect to assume that male fertility is not affected by increased population density, especially in view of the evidenee indicating that the secretion of testicular androgens is extremely sensitive to changes in population density or to social rank.

Southwick (1955a) observed a decrease in fertility in female mice from populations which were slowly declining from peak levels. He also noted, as have others (Southern, 1948; Calhoun, 1949), that in populations of high density or with poor social organization (Calhoun, 1949, 1950) that "copulation pressure" on females in estrus was high; that is, a number of males gathered around her and attempted to copulate although they were frequently pushed off by others, so that the mean copulation time per male was reduced. "Copulation pressure" was used as a possible explanation for the decline in fertility. This explanation overlooks several facts. The populations were progressively deelining from peak levels and did so for six 
months, and so did pregnancy rates and fecundity rates (correspondence in the declines in these latter two was used as an argument against the possibility of increased intra-uterine mortality). It is therefore unlikcly that a steadily declining fertility can be explained by copulation pressure in a constantly decreasing number of animals. Male fertility was assessed by uncritical procedures and female fertility by performance. Assessing fertility by female performance of course does not provide a way to assess the possible role of depressed male fertility. It scems much more likely that both male and female fertility were depressed. Crowcroft and Rowe (1957) noted a decline in fertility in female house mice with increasing density as well as during the period of asymptotic stability in populations of housc mice. These authors indicate the failure of population growth resulted entirely from inhibition of female reproductive function. Louch (1956) was unable to correlate diminished fertility with "copulatory pressure," although it was observed in his population of voles. We have seen that the females in extremely dense populations became pregnant but failed to bear litters and that recovery from the effects of chronically depressed fertility was extremely slow (Christian and LeMunyan, 1958). Increasing age may have been a factor in these freely growing populations, especially if fertility was already partially curtailed.

This discussion is meant neither to be a criticism of the observations made by these investigators nor to provide the correct explanation for them. Rather, these examples have been used to indicate pitfalls in experimental procedures presently in general use to assess male fertility and the effects of various factors on fertility in populations of small mammals, and to call attention to reasonable and perhaps more probable explanations other than those usually provided. The literature contains many examples which could have been used. Finally, it should be noted that the populations in Southwick's studies had reached peak densities and had been declining slowly for 6 months before the animals were examined, so it would be difficult to determine the effects of increasing density on fertility in mice of either sex. The results of those studies made during the period of increase of a population or at the time it reached maximum density are not comparable to studies made so much later, and it is not correct to extrapolate from one to the other, as was done in several instances. The results of other experiments in which male fecundity was assessed during the period of population increase indicate that male fertility, in terms of the whole population, has a striking negative correlation with density (Christian, 1956, 1959a, b; Croweroft and Rowe, 1957). The secretion of gonadotropins is apparently depressed with increasing pituitary-adrenocortical activity in the greater proportion of subordinate animals associated with increases in density. Evidence has been presented which shows that increased adreno- 
cortical activity in mice results in an increase in the secretion of weakly androgenie steroids, and that these can depress the secretion of gonadotropins.

b. Female. Reproductive competence of female mammals can be assessed grossly more easily than that of males during studies of growing populations simply because the number of young produced can be counted. Some comments have been made about the relationships of female fertility and reproduction to population density in animals from freely growing populations. Most of the pertinent evidence indicates that fertility and reproduction in female mammals is depressed by increased density. Two kinds of information has been gathered on female productivity in relation to population density. In one ease the number of young born at intervals throughout the history of the population has been determined, and in the other female fertility is assessed by external examination of the vagina, which is closed by a thin membrane during anestrus in many species, or by examination of the animals at autopsy. Information of both kinds is desirable, although neither is sufficient by itself.

It has been shown in studies with house mice and voles in freely growing confined populations from which adequate data are available that the birth rate for mature or potentially mature females in the population declines steadily with increasing population size (Clarke, 1955; Christian, 19.56, 1959b, 1961; Crowcroft, and Rowe, 1957). The decline in the number of young born per female appears to decline approximately linearly as the $\log _{10}$ of the population increases (Christian, 1959b).

The declines in productivity apparently resulted from suppression of all phases of reproductive functions. Crowcroft and Rowe (1957) showed there was complete suppression of estrus, as indicated by closure of the vaginal orifice, in an increasingly larger proportion of females as the size of a population increased. It was shown that closure of the vaginal orifice was a reliable index of fertility in female house mice in these experiments by the very high correlation between the condition of the vaginal orifice and the development of the reproductive organs. In these populations the majority of the young were produced by the introduced females initially or by females from the first few litters. These results eorrespond to those from populations of limited size in which it was indicated that the reproductive competence of female mice was related to their social status. Similar results were also obscrved in confined populations of Norway rats (Calhoun, 1949, 1950). Reproductive function was completely suppressed in the majority of female born into these populations.

These results are comparable to those from experiments with freely growing populations of house mice (Christian, 1956), thus indicating that reproductive function in female mice was depressed for all body-weight 
groups. In these experiments the weights of the preputial glands of mice from populations of high density were significantly greater than those from segregated controls. For their stimulation to full development and function, the preputial glands depend primarily on the more potent androgens (Burrows, 1949), but the weakly androgenic steroids (Huggins et al., 1955), ACTH (Jacot and Selye, 1951; Asling et al., 1951), and pituitary growth hormone (Huggins et al., 1955) all have a mild stimulatory action on the preputials. The evidence for the effects of estrogens on the preputials is conflicting. On the one hand, estrogens appear to have a mild stimulatory action (Burrows, 1949; Beyler and Szego, 1954) whereas other experiments indieate that they inhibit the preputials (Rennels et al., 1953). Since other evidence from the experiments with mouse populations indicates that the production of sex steroids by the gonads and growth hormone by the pituitary is inhibited at high densities, it seems probable that the increased size of the preputials in these intact females must have been due to increased $\mathrm{ACTH}$ and possibly increased adrenal $\mathrm{C}_{19}$ steroids. In any event the data on the preputials are strongly suggestive of increased pituitary-adrenocortical function. Attention should be directed to the fact that the preputials were appreciably larger than those from segregated mice with full reproductive competence. Therefore there is no reason whatsocver to implicate estrogens in these effects; so ACTH and adrenal androgens must be responsible for stimulating preputial development to well above the control levels. Presumably the levels of estrogens declined in these females as a result of a decreased secretion of pituitary gonadotropins in association with increased secretion of ACTH. These conelusions were supported by the fact that there was also (1) a marked increase in the proportion of females of adult size with infantile uteri and ovaries, and (2) a $20 \%$ decline in number of females with mature reproductive organs that were also pregnant, (3) a $13 \%$ decrease in the mean number of viable embryos per pregnaney, and (4) a $58 \%$ increase in the number of resorbing embryos per pregnancy. Thus there was diminished fertility and increased losses between orulation and implantation, and implantation and birth. Decreased fertility was shown in some animals by a total inhibition of reproduction and in others by a markedly diminished number of viable embryos per pregnancy. These results agree with those of Crowcroft and Rowe (1957). The same phenomena apparently occurred in populations of voles (Clarke, 1955; Louch, 1956; Christian, 1959b). Birth rates declined steadily as the populations increased for house mice and voles (Christian, 1959b, 1961). Therefore it seems that reproductive function is inhibited in female mice and voles in proportion to population density, either in terms of the proportion of reproductively competent adult females in a population or of the degree of reproductive function in individuals. Stated another way, reproductive 
function in female mice and voles is reciprocally related to population density. The degree of inhibition apparently may vary from none to complete in the females of a given population, within limits depending on social relationships of the particular individuals.

We have already criticized the concept of "copulation pressure" which has been used to explain the decline in female fertility seen in populations of high density. As an explanation it is inadequate because it does not coincide with the facts, even in those cases in which it has been used as an explanation (Southwick, 1955b). Furthermore, no correlation could be shown between "copulation pressure" and fertility in other studies (Louch, 1956). In the light of those studies in which female reproductive function was more precisely assessed, it appears that reproductive function was at least partially inhibited in those populations in which "copulation pressure" was used to explain the decline in fertility, and that the means used to assay female fertility were not sufficiently sensitive. "Copulation pressure" conceivably could have an effect on female reproductive performance, but until the problem is reexamined more critically it must remain an unaceptable concept, especially in view of the preponderance of evidence indicating that reproductive function is inhibited, either individually or on a population-wide basis, as part of the adaptive responses to increased density. A broad view of this evidence suggests considerable variability from population to population in the degree of reproductive inhibition with respect to the numbers of females involved as well as to the magnitude of inhibition in particular individuals. These variations are evidently related to social factors, and this aspect of the problem needs examination in greater detail than heretofore.

Prenatal mortality of the fetuses was an appreciable factor in reducing the number of births in dense populations, presumably largely owing to a suppression of gonadotropins, but also possibly to the effects of increased adrenal corticoids and androgens. There seems to be some kind of "dual response" to density in depressing female productivity. Judging from the results described in this and preceding sections, it appears that the least "dose-response" effect occurs when fertility is partially diminished, as by decreased numbers of ova and increased losses between ovulation and birth, without a total suppression on the ability to bear young. A greater effect apparently is total inhibition of estrus and reproduction in potentially mature animals. Delayed attainment of maturity probably reflects partial inhibition. The possible mechanisms involved in producing intra-uterine mortality have been discussed in an earlier section.

c. Litter survival. A marked decline in litter survival with increasing population density was observed in all but a few experiments with freely growing populations of house mice or voles (Strecker and Emlen, 1953; 
Brown, 1953; Clarke, 1955; Southwick, 1955a, b; Christian, 1956; Louch, 1956). Litter survival apparently declines approximately linearly as the logarithms of the population increases (Christian, 1959b). However, litter mortality is not always a factor in diminishing the rate of population growth. Crowcroft and Rowe (1957) found very little mortality of young mice in their populations and furthermore were able to attribute all the observed mortality to interference on their own part in making routine censuses. The decline in productivity in their populations was due almost entirely to prenatal loss or complete inhibition of all reproductive activity. It has been suggested (cf. above) that diminished fertility probably was more of a factor than was realized in several other investigations; nevertheless litter mortality has bcen an important factor in many populations. Decreased litter survival may be due to diminished lactation (Christian, 1956; Christian and LeMunyan, 1958) or to behavioral factors (Brown, 1953; Strecker and Emlen, 1953; Southwick, 1955b).

Partial inhibition of lactation with increased density was described earlier. The results suggested that lactation, controlled by complex endocrine mechanisms, was partially inhibited owing to inhibition of gonadotropin secretion and a resultant diminished production of estrogens and progestins. Lactation therefore reflected population density in a similar fashion to other reproductive functions. It was also pointed out that the progeny nursed by mothers subjected to increased density were profoundly affected by the inhibition of lactation. Experiments with freely growing populations of house mice suggest that deficient lactation may be an important factor in decreasing litter survival (Christian, 1956). These young were stunted and were weaned early. At autopsy their stomachs contained little or no milk. Calhoun $(1949,1950)$ suggested that the socially subordinate rats in freely growing confined populations were incapable of raising their young, even though they were protected from disturbance. He indicated that physiologic and psychologic disturbance in the subordinate females affected the young through either poor fetal nutrition or a breakdown of maternal instincts.

Diminished lactation may have been an appreciable factor in reducing the survival of young in the above experiments, and diminished lactation is a logical consequence of a generalized inhibition of reproductive function presumably due to diminished gonadotropin secretion.

Decreased litter survival was attributed entirely to social or behavioral factors in a number of experiments with freely growing populations of house mice or voles. Brown (1953), Southwick (1955b), and Louch (1956) related litter survival to the type of nest constructed and maintained. Brown and Louch each noted that litter survival was good so long as the female mice or voles were able to maintain covered or bowl types of nests, 
but that this was frequently impossible because of destruction of the nests and interference with the attempts of the females to maintain or reconstruct them. Only 49 of the 72 attempts to build and maintain either of these two types of nest were successful in these populations (Brown, 1953). Southwick (1955b) noted a similar relationship between the type of nest and litter survival, but he also found that litter survival was good in bowl or covered nests regardless of the number or sex of the adults which were also present. Litter survival was zero with no nests. These and Brown's (1953) results imply that not all the females were prevented from maintaining proper nests and nourishing their litters irrespective of the conditions and interference. These results are revealing, as they suggest strongly that more is involved in litter mortality in these experiments than simple interference or the type of nest maintained. Similar results were noted in other experiments (Christian, 1956) except that in this case even those with no nests sometimes survived. In these latter experiments it was also noted that if litter mortality occurred when the populations were less than one-half of maximum density, it occurred within the first four postnatal days and might be attributed to interference which resulted in too much movement of the litter by the mothers. Of additional importance is the fact that litter survival declined regularly with increasing density and that this began to occur well before interference could be significant. If the young survived the first 4 days at these lower densities, they almost always were successfully weaned. However, at higher densities the increased litter mortality usually occurred after the young were 10 days old. Until this age survival equaled that seen at lower densities. These facts suggest that the defect was primarily nutritional, even though there still were some deaths of young less than 4 days old attributable to interference. The age of death of the young is not known for most of the reported experiments, so that this distinction was not possible. However, the fact that crowding and interference was not a factor in litters with proper nests suggests that perhaps the behavioral changes which resulted in poor nest maintenance may be based on an underlying alteration in maternal behavior, possibly due to endocrine changes. Admittedly this is speculative, but the available evidence suggests some such explanation. The problem certainly must be investigated in greater detail. It is possible that the capacity to lactate properly and the ability to maintain proper nests are closely related by a common causal basis. However, until evidence for or against such a hypothesis is available, it must be assumed that postnatal litter mortality at high population densities may result from (1) inhibition of lactation, (2) interference by other animals, or (3) changes in maternal behavior, which apparently decreases with increasing population density.

We are inclined to the view that interference of various sorts probably 
plays a more or less constant, probably unimportant, role in causing litter mortality at all levels of population density, whereas inhibition of lactation and changes in maternal behavior are density dependent and probably reflect physiologic (endocrine) responses to changes in population density. Sonthwick (1955b) noted that aggressiveness, as indicated by fights per mouse per unit of time, was reciprocally related to litter survival at high densities, but that this relationship did not necessarily hold at lower densities. On the other hand, there was a correlation between fighting and density in only one of three populations of voles (Louch, 1956). The behavioral changes observed in relation to litter survival may be outward manifestations or "symptoms" of more profound physiologic changes at higher population densities.

A final consideration of importance is that fecundity and litter survival are sufficiently sensitive to changes in the population to be responsive to a variety of different circumstances affecting density. Litter survival was markedly increased in a population of house mice in which there was an appreciable mortality of adults (Christian, 1959b). The growth form and eventual size of the population were comparable to those of populations without appreciable adult mortality. The loss of adults in this population was compensated for by increased litter survival. Similar relationships were observed in three populations of voles (Louch, 1956). There was a significant correlation between fertility and population density in one population of voles in which there was no correlation between density and adult mortality. In a second population there was a good correlation between density and adult mortality, but none between density and fertility. The third population was intermediate between these two. Therefore, there appears to be sufficient flexibility in the physiologic adaptive responses to compensate for losses of adults from populations of voles or mice. This compensation apparently can occur at any stage of the reproductive process from ovulation to weaning.

d. Effects of food supply. It is often assumed without question that food shortage is responsible for limiting population growth. However, we have seen that the growth of a population may be entirely self-limited without a shortage of food. The role of a limited food supply in the regulation of population growths has been investigated (Strecker and Emlen, 1953; Strecker, 1954). Clarke (1955), Christian, (1955b, 1956, 1959b), Southwick (1955a, b), and Louch (1956) have emphasized the fact that in their experiments food was always abundant and usually scattered so that any animal could obtain food irrespective of other animals present. Furthermore, experiments with populations of fixed size indicated that inanition was not a stimulus to increased adrenocortical activity in house mice, nor did it constitute a factor for increasing competition when it (food) was 
limited either in source or amount (Christian, 1959c). 'These results are consistent with those from experiments with freely growing confined populations of house mice or voles in which competition for food in every case was rare or absent (Strecker and Emlen, 1953; Louch, 1956; Southwick, 1955a, b; Christian, 1956). Strecker and Emlen (1953) provided two confined populations with a limited amount of food but the growth of only one of these was limited by the limited food supply. Growth of this population was rapid and ceased abruptly when the limit set by the food supply was reached. Growth ceased because reproduction stopped abruptly with involution of the reproductive organs, especially of the females, all of which were reproductively quiescent at autopsy, although the animals otherwise appeared to be in excellent condition and usually excessively fat. Several points of interest emerge from these experiments. One is that population growth ceased very abruptly with a truneated growth curve, indicating that a limited food supply does not act as a damping factor, but exerts its effects at one point on the growth curve. Therefore, it is unlikely that a typically sigmoid growth curve would result from a food shortage. The second point of interest is that reproduction was so sharply limited by the limited food supply. These results agree with those from populations of fixed size and indicate that the food limitations completely inhibited reproduction, probably by suppressing the secretion of gonadotropins, without producing increased activity of the pituitary-adrenocortical adaptive system. The last point is that the population immediately adjusted to the supply and the general condition of the mice was unaltered by the limitation in food. The inhibition of reproductive activity in all the females contrasts sharply with the results of other experiments conducted on self-limited freely growing populations of mice or voles supplied with an excess of food. In the latter populations there were always some reproductively active females, although the actual proportion in the population varied with each population. Data on adrenal activity were not collected in these experiments on the effects of food limitation on freely growing populations. It is probable that there would have been density-dependent increases in adrenocortical activity, but the effects of food limitation were independent of any density-dependent effects. In another series of experiments, the food supply was limited similarly, but egress from the population was allowed (Strecker, 1954). In these populations there was a low constant rate of egress until the food limitation was reached and then a high rate of egress began and continued until the experiment was terminated three months later. The egress apparently involved all segments of the population except the youngest. Reproduction continued at a good rate in this population in sharp contrast to the confined population without egress. The implications of these experiments are clear: (1) food limitation can limit population 
growth by inhibiting reproduction, but the growth curve becomes sharply truncated; (2) if egress from a population is permitted, food limitation has no effect on reproduction; (3) feeding must be random and food cannot have been a factor increasing competition, otherwise all the mice would not have been affected cqually. Extrapolating from these results to natural populations, it is evident that a limited supply of food will not affect reproduction or otherwise affect the population so long as emigration is possible, but if emigration becomes impossible, owing to a complete saturation of the available habitat, reproduction will cease without any other apparent effect. Whether or not there is competition for food in specics other than house mice must be determined, as increased competition would increase pituitary-adrenocortical activity with all its sequelae. There may be increased competition for food in voles (Frank, 1953), although it seems unlikely that in general there will be an increase in competition over that already present due to social factors (cf. above). However, the usual result of emigration from a population is increased mortality: the animals which leave enter strange territories and very rapidly become mortality statistics (Errington, 1943, 1954b; Calhoun, 1948; Davis, 1953). Physiologic adaptive mechanisms apparently are always operative in relation to population density and therefore would reflect the relative density whether or not the growth of a population was limited by food, and a distinction betwcen foodlimited and self-limited populations should be possible by comparing the reproductive activity of the populations and the general conditions of the individual animals. Adrenal weights and other parameters of increased pituitary-adrenocortical activity should also differentiate between the two unless a population was food-limited at a point close to its maximum selflimited size.

These statements and conclusions are based on the results of experiments with house mice and seem fairly conclusive for this species, possibly even for voles, but it is difficult to believe that food is not a competitive factor for all species and for all populations. The results of experiments to date with populations of fixed size or freely growing indicate that food is not even a competitive factor within the existing social structure, as all animals are apparently equally affected irrespective of their social rank (Strecker and Emlen, 1953; Christian and LeMunyan, 1958; Christian, 1959b). It should be remembered that if ever and when ever food becomes an object of competition, shortages then necessarily will act through the generalized physiologic adaptive responses and limit population growth in a densitydependent fashion just as does purely social competition. The net conclusion from these results is that a shortage of food per se probably rarely limits population growth as the peculiar combination of effects which result from a food shortage is seldom seen. However, this statement is not 
to be interpreted to indicate that acute starvation camnot ensue in local populations or that a limited food supply is not at times important. The relationships between food, competition, reproduction, and the growth of populations require much more investigation, especially for different species. Until additional critical information is available, any conclusions regarding food and population growth must remain tentative.

\section{Growth}

In a recent experiment with a freely growing population of house mice, it was shown that there was appreciably inhibited growth in all but the mice originally introduced and those from the first litters (Christian, 1961). In this experiment all mice were toe-clipped so that their ages were known. In general the results from the reproductive organs and adrenal glands confirmed those of earlier experiments and were even more pronounced because the results could be equated with age rather than bodyweight categories.

\section{Summary of Results from Freely Growing Experimental Popu-} LATIONS

We have seen that, for the most part, freely growing populations of house mice and voles are self-limited, primarily as a result of a densitydependent activity of physiologic adaptive mechanisms. The activity of these mechanisms is reflected in both sexes by a progressive inhibition of reproduction and stimulation of the pituitary-adrenocortical activity with increasing population size. The results agree with those obtained from populations of fixed size. Diminished reproductive function in the female may be apparent at any one or all phases of reproductive activity: inhibition of estrus, increased intra-uterine mortality, reduced numbers of implanted ova, increased postparturient mortality due to suppression of lactation, and possibly by diminished maternal behavior. Inhibition of male reproductive ability is shown by decreases in the weights of the sex accessories and depressed spermatogenesis, although the problem of male fertility needs further investigation. The decreases in reproductive function in both sexes apparently stem from decreases in the secretion of pituitary gonadotropins with a secondary decline in the production of estrogens, progestins, and androgens. Evidence for and against the role of "increased copulation pressure" in diminishing the fertility of female mice and voles was discussed. Without much more conclusive eridence, it is likely that "copulation pressure" is an unimportant factor and that fertility is depressed by inereased density operating through sociopsychologic and physiologic pathways. Inereased adrenocortical activity was shown by increased adrenal 
weights, eosinophil counts, and histologie studies. Thymus weights refleeted these changes. Inereasing infant mortality with inereasing density may be due to diminished lactation, but may also result from social and behavioral factors. Food limitation may restrict population growth by completely inhibiting reproduction, but is without any other apparent effects.

Finally, it is evident that the growth of confined populations of mice and voles is regulated and limited by density-dependent physiologic responses which are activated by sociopsychologic pressures. Whether or not these relationships hold for all species remains to be determined.

\section{Natural Populations}

Evidence gathered in the laboratory relating physiologic adaptive mechanisms to changes in population density cannot prove that these responses oceur in natural populations. Even for freely growing confined populations in the laboratory, the environmental conditions are quite altered by the removal of the effects of elimatic and seasonal variability from those found in natural situations. Preventing egress is another artifieially imposed condition; although it seems more likely that confinement parallels the situation when populations reach densities where egress into neighboring areas becomes possible. Clearly, evidence from natural populations is necessary before any eonclusions are justified regarding the role of physiologie adaptive mechanisms in the regulation of mammalian populations under natural conditions. Conclusive results are much more difficult to obtain from natural populations than from confined populations, as might be expected, due to the eomplexity and variability of these populations and techineal difficulties in collecting the required data. Adequate controls and proper assessment of environmental factors are often severe obstacles, and obtaining samples of adequate size from populations at very low densities is intrinsically very diffieult. Nevertheless, evidence has been obtained to indieate that the density-dependent physiologic responses in natural populations are similar to those seen in experimental populations. Finally, social strife has been implicated (Kalela, 1957) as an important factor in producing the effects assoeiated with inereased density; territorial mutual intolerance increases greatly with sexual maturity in Clethrionomys rufocanus, which serves to increase tension even with decreased density (Kalela, 1957). Therefore behavioral factors again appear to be important.

\section{Adrenocortical Activity}

The available evidence relating adrenocortical activity to changes in population density has been obtained largely from studies on populations 
of Norway rats, both urban and rural, and voles (Microtus sp.), although suggestive results have been obtained for a few other species.

Adrenal weight in urban Norway rats of both sexes was shown to be related to population "density" in a study of 21 populations in Baltimore, Md. (Christian and Davis, 1956). Each population was confined to a city block, the latter acting as an island (Davis, 1953). The capacities of the blocks for rats varied considerably, as did the sizes of the populations themselves, which resembled confined freely growing populations of house mice in this respect. Therefore, the populations were categorized by their position on their own growth eurves at each sampling. A hypothetic growth curve was divided into low stationary, low increasing, high increasing, high stationary, and decreasing, the progression from low increasing through decreasing being a progressive increase in relative density, although the "density" status of low stationary is equivocal (Christian and Davis, 1956). Each time a sample was collected, the population from which it came was put in one of these categories. "Density" is obviously not strictly in terms of area, but in relation to the carrying capacity of the block and the social characteristics of the population. As populations increased in relative density from low increasing to decreasing, the mean adrenal weight was increased with each relative density; so that rats of both sexes from decreasing populations had $19 \%$ heavier adrenals than those from low, increasing populations. Thymus weight was reciprocally related to adrenal weight in female rats, but there was no apparent relationship between thymus weight and adrenal weight, with respect to population density, in male rats. The weights of the pituitary glands of male rats were positively related to the adrenal weights. That is, changes in adrenal weight were reflected by changes in pituitary weight in the male rats (Christian and Davis, 1956). However, the functional significance of these changes in the weight of the pituitary glands is not known. The weights of the pituitaries of females and thyroid glands of both sexes bore no apparent relationship to population density. The data from the low stationary populations are difficult to evaluate. The adrenals of female rats were almost as light as those from low increasing populations, and their thymus glands were the heaviest found in any category. On the other hand, the adrenals of male rats were heavier than in any other category. Low, stationary populations are extremely difficult to evaluate, as the actual numbers of rats are so small that even proportionately large changes are difficult to detect. However, these difficulties with their inherent errors in properly assigning populations to a category do not explain the divergence in the male and female adrenal weights, nor do they alter the fact that these populations were at very low levels of density. It should be pointed out that the decreasing populations were at maximum "density" because they were declining naturally and therefore were at or above the environmental capa- 
city. It should also be emphasized that food was available in excess of needs for all populations and from numerous sources; so that food cannot be considered to be an imprtant factor with respect to these populations.

Some of the variability between urban populations of rats may be explained by the fact that the rat population in each block is divided into several diserete eolonies when the total population is at a relatively low density (at high density these colonies coalesce and the population evidently becomes a single unit throughout the block). The colonies comprising several of the block-populations were evaluated individually with respect to relative density in the same way as the populations for the entire blocks (Christian and Davis, 1956). Relative density values were assigned and compared with the adrenal weights of the rats from these colonies. A suggestive correlation between adrenal weights and relative population densities of these colonies was found, although the differenees were not signifieant, probably owing to the small number of samples. The sample size was limited by the relatively few colonies from which a sufficient number of rats were collected for comparative purposes.

Although adrenal weight is greatest in naturally declining populations (Christian and Davis, 1956), it was shown in another study that artificially reducing populations of rats produces an immediate and proportional reduction in adrenal weight (Christian and Davis, 1955). Adrenal weight in both sexes of rats from three populations was reduced $38 \%$ by intensive trapping. The adrenal weights were maintained at this level for 5 months by maintaining the populations at the reduced level.

In another study a rural population of Norway rats was followed by monthly sampling for two years (Christian, 1959b). An index of the size of the population was obtained each time a sample was collected by using a standardized trapping procedure. The weights of the adrenals, pituitaries, and thyroids were determined for each sample. There was a highly significant correlation between adrenal weight and population index for both sexes, and an even more significant correlation between pituitary and adrenal weight for both sexes for the 24 monthly samples in the two-year period. The functional significance of the pituitary changes is not known; it can only be inferred from data on weight. Evidently changes in the rate of ACTH secretion, as indicated by adrenal weight, are accompanied by parallel changes in pituitary weight. Changes in thyroid weight and in thymus weight were not eorrelated with changes in the population size, adrenal weight, or pituitary weight.

The results of the foregoing experiments indicate that adrenocortical activity in Norway rats from natural urban or rural populations is related directly to changes in population density and that both sexes respond in the same way. Therefore it appears that physiologic adaptive mechanisms are 
operative in natural populations of Norway rats, just as they are in freely growing confined populations of house mice or voles.

Another series of experiments with populations of rats produced results which at first glance appeared to contradict the foregoing conclusions. Alien rats were introduced into stationary and increasing populations to verify earlier observations that such a procedure profoundly affected the population (Calhoun, 1948). When stationary populations of rats were increased $20 \%$ by adding alien rats of one sex or the other, the populations declined abruptly to about three-fifths their original values. When large numbers of rats were substituted (native rats were removed and replaced by an equal number of aliens) into increasing populations, the populations promptly ceased growing. These procedures presumably produced severe social strife with a marked increase in mortality (Calhoun, 1948; Davis, 1953). However, the adrenal weights of rats taken at regular sampling intervals failed to reflect the increased strife and closely followed the course of the population size. The explanation of this apparent paradox is elear. The introduced rats were, of course, aliens--hence subordinate rats-in a strange environment (see Clarke, 1953; Barnett, 1955, 1958) and therefore became mortality statisties. The native rats which succumbed were probably subordinate animals in the original population. The adrenal weights were only from survivors and therefore probably from dominant animals. It already has been pointed out that adrenal weight is least in dominant animals, and so the increase in adrenal weight which might have been anticipated from a superficial examination of the situation obviously and simply failed to appear. Had the adrenals of the rats that died been obtainable, they unquestionably would have exhibited profound changes.

Natural populations of voles (Microtus) have also been studied to determine the relation between adrenal function and population density. Adams, Bell, and Moore (Christian, 1959b) periodieally colleeted frequent samples from a natural population of Microtus montanus in Montana for four years to obtain indices of population density, adrenal weights, and data on reproductive activity. The number of males collected was inadequate for valid comparisons. However, population density and the adrenal weights of female voles were closely correlated. The population was at peak densities in late summer of 1952 after which there was a continued general overall decline in the population until the summer of 195.5. The adrenal weights of females were maximum in the late summer of 1952 and then underwent a gradual overall decline, generally following the population. However, within each year there were marked seasonal changes in the population which were reflected by equally marked changes in adrenal weights. The population reached its annual maximum density in late summer or early fall and then declined sharply to very low levels where it remained until late winter or 
early spring when breeding recommenced and the populations increased sharply. Changes in adrenal weight closely followed these animal eyeles in population density. Peak adrenal weights were found in early fall and coincided with a cessation of reproduction and the beginning of the autumnal decline in the populations. Adrenal weights were minimal by November and remained there until Mareh, ${ }^{3}$ when the population again began to increase. Adrenal weights were maximum in the last summer of 1952, averaging about $15 \%$ greater than in any other year for a comparable time of year. The mean weights in winter were roughly $60 \%$ less than the maximum. These results indieate that the supposedly severe winter conditions of the Rocky Mountain Region of Northern Montana do not impose physiologic hardships on voles. Climatic conditions, if they affected the animals at all, apparently were unimportant compared to changes in population density. The sharp declines in the population every fall evidently were due to a cessation of breeding and continuing random mortality, not to a declining enviromment, otherwise the adrenal weights would not have decreased so spectacularly (Christian, 1959b). It is possible that physiologic responses to high densities in late summer contributed to the annual declines in population density, but it seems unlikely except in 1952. In that year it is probable that physiologie adaptive responses were at least partly responsible for the decrease in the size of the population. It is noteworthy that the rapid decline from peak densities in the late summer and early fall of 1952 was not caused by increased mortality from disease (Adams et al.; cited in Christian, 1959b).

Adrenocortical-density relationships were studied by Louch (1958) in two natural populations of voles (Microtus pennsylvanicus). He used eosinophil counts, supplemented by adrenal weights, as indices of adrenocortical activity. Relative adrenal weights were high and cosinophil counts remarkably low in one population at peak density. Following a sharp deeline in this population, indicated by an $85 \%$ drop in the population index, the eosinophil counts rose sharply about $500 \%$ and continued to rise more gradually for the subsequent nine months of the study. Adrenal weight averaged $59 \%$ lower during the period of low density than during the preceding period of high density: $14.5 \mathrm{mg}$. $/ 100 \mathrm{gm}$. compared to $23.0 \mathrm{mg}$. $/ 100$ $\mathrm{gm}$. The size of the second population remained low throughout the experimental period. The eosinophil counts began high and gradually rose to higher levels. Adrenal weight declined about $27 \%$ (from a mean of 19.4 to a mean of $15.2 \mathrm{mg} . / 100 \mathrm{gm}$.) in the second population over the same period

${ }^{3}$ Subsequent work has suggested that the low winter adrenal weights are partly due to sexual inactivity and regression, although not entirely as a number of the voles were not sexually regressed. In other words the adrenal weights should be low due to density factors (cf. October), but these were too low [see Christian (1961, 1963b)]. 
as the $59 \%$ decline in the first population. However, the decline was not nearly so great and the initial weight was $19 \%$ less than in the first population. The lower adrenal weight was reflected by a higher mean eosinophil for the same period of time. Even though the reason for the lower adrenal weight in the second population during the second period is not known, adrenal weight and eosinophil counts in these studies generally reflected differences in population density (Louch, 1958).

Methods for estimating most natural populations of mammals are at best relatively crude, insensitive, and subject to many errors. At best they can detect only relatively large ehanges in population density. The catch per trap-night or any comparable index of population density obtained by some sort of uniform trapping effort is probably as good an index as any readily usable procedure. The eatch per unit of time was used in these studies of vole populations by Louch (1958) and Adams et al. (Christian, $1959 \mathrm{~b}$ ), as well as in the studies of rural populations of Norway rats (Christian, 1959b). Errors in estimating population density in this fashion may account for most of the observed discrepancies. If the existence of physiologic responses to population density is established for a variety of species, it is likely that the magnitude of these responses will provide a much more precise index of relative population density (the important figure for practical purposes) than any existing indirect method for determining population size. ${ }^{4}$

Limited studies with natural populations of other species have been made. A $68 \%$ decline in the index of the size of a population of white-footed mice (Peromyscus leucopus) from one July to the next was aceompanied by a $58 \%$ decline in their mean adrenal weight (Christian, 1959b). Similar, but less conclusive, results have been obtained with natural populations of short-tailed shrews (Blarina) (Christian, 1954, and unpublished). Beer and Meyer (1951) studied the seasonal changes in the endocrine and reproductive organs of muskrats and found a marked peak in adrenal weight in adults of both sexes in early fall and a second minor peak in Mareh and April, especially in adult females. These seasonal changes are similar to those we have noted in Microtus montanus and may be related to behavioral and social changes.

Preliminary work on the relationships of the weights of deer adrenals to

${ }^{4}$ The persistence of immature zonation in the adrenals of males and lack of hypertrophy due estrogenic stimulation in females may explain some of the discrepant results with Microtus, and possibly Lemmus, as these would seriously confound adrenal weight relationships if the population under study is composed of an appreciable percentage of sexually immature, regressed, or otherwise sexually inactive animals. Winter or high-density populations will consist lingely of such animals [see Christian (196il, $1963 \mathrm{~b})]$. 
various factors indicates that adrenocortical physiology in this species, as in others, reflects environmental changes, and the adrenal was therefore said to be an indicator of conditions as in any other spceies. (Hughes and Mall, 1958). However, sika deer (Cervus nippon) respond to changes in population density in a manner quite similar to that seen in rats (Christian et al., 1960). A $60 \%$ decline in the population of these deer was accompanied by a proportional decline in adrenal weight. The decline of this population was brought about by a mass mortality which apparently was due to metabolic disturbances resulting from the prolonged adrenocortical hyperactivity associated with a high density of the population. There is evidence that potassium deficiency resulting from cortical hyperactivity may have contributed directly to the mortality. Marked stunting of growth was also scen during the period of high density and especially during the year of die-off. Other possible causes of the die-off, such as malnutrition, could be ruled out.

More experimental work is needed to relate adrenal function with population density for a number of species in natural populations. The problems are numerous and not the least of these is being able to rule out extraneous factors or else to assess their role in producing the measured effects.

\section{Reproductive Function}

A large number of studies suggest that reproductive function is depressed with increasing density of natural populations, but studies sufficiently discriminative to attribute changes in reproductive function to changes in density per se without additional complications are few. Therefore a great deal of the evidence is circumstantial and tentative at best. The present discussion is limited to those studies which have been conducted with sufficient care and attention to a variety of details so that one is confident that the factors have been properly considered which possibly might affect reproduction. However, a brief discussion of various problems involved in evaluating reproductive function is in order before considering the evidence implicating physiologic responses to population density in the suppression of reproductive function.

First there is the problem of the food supply of the experimental population. As we have seen, there is ample evidence that food deficiencies can curtail reproductive function independently of other adaptive mechanisms. However, the effects of food and of increased density on the reproductive system and growth are so similar that unless careful assessments are made of the food supply (preferably these should be accompanied by an evaluation of adrenocortical and other adaptive functions, no separation can be made between the effects of food and of density, especially at relatively 
high population densities. Furthermore, it is very likely that the adaptive reactions to density and their effects may overlap food shortages, so that their effects are mutually augmentive at critical densities. However, the aim of the present discussion is to assemble the evidence that adaptive reactions to density occur irrespective of whether or not the effects of inadequate food or other environmental factors are superimposed. The growth of populations unquestionably can be limited by environmental factors which may either act through the physiologie adaptive mechanisms or independently of them, especially in localized populations. However, the basic eoncept of physiologic adaptation to population density is that these mechanisms are always operative, and will regulate and can limit population growth. Finally, limitations of environmental factors, even of food, may increase competition directly and therefore indirectly produce inereased activity of the physiologic adaptive mechanisms. Pitelka's (1957b) statement that "the interest in the stress mechanisms has led some students to overlook the point that such a mechanism does not evolve without linkages to critical variables extrinsic to the population" bear repeating and is emphasized and supported by an immense amount of work with rat populations (Davis, 1953). Nevertheless, it is extremely important to distinguish between the direet action of environmental variables on populations and their indirect action through sociopsychologic and physiologic mechanisms; and experimental evidence to date emphasizes the general importance of the latter mode of action. Evidence of the direct action of food shortages in depressing reproduction in natural populations of Peromyscus boylii and P. maniculatus is cited by Jameson $(1953,1955)$, and population declines evidently followed the shortages as a result of continuing normal mortality in the presence of a lull in reproductive activity with no recruitment into the populations. The evidence presented by Jameson, supported by the controlled experiments previously described, strongly favors the interpretation that inadequate food supplies were directly responsible for the inhibition of reproduction. However, the possibility that the shortages induced increased competition and increased activity of adaptive responses were not ruled out completely. Davis (1951c), in a study of Norway rats from natural populations, provided definitive evidence that a deficient food supply ean inhibit reproduction under natural circumstances. However, whether the effects were direet or produced by increasing competition with stimulation of generalized physiologic adaptive response is unknown. It was determined later that the adrenals of these rats were responsive to changes in population density (Christian, 1959b). Other examples could be cited, but the majority could serve only to emphasize the fact that discrimination between the effects of food and other factors is usually not attempted. 
Another, perhaps more serious problem in evaluating the effects of density on reproduction is the lack of adequate criteria to determine the age of most small mammals; so that weight and length measurements are usually used as eriteria of age. Since one of the major effects of increased activity of physiologic adaptive responses is diminished growth, as well as inhibition of reproductive function, there is no valid way to separate normal immature animals from older animals which should be mature but in which both growth and reproductive maturity are inhibited. Several serious misinterpretations can result from this situation: (1) a shift of age composition of the population toward greater age will be obscured; and (2) reproductive competence is usually assessed on the basis of the prevalence of pregnancy, prevalence of lactation, and similar criteria in those animals which are obviously mature, therefore total suppression of reproduetive activity with a delay in the onset of puberty will be missed altogether. Of course in such a situation, the reproductively active and obviously mature animals represent only the portion of the population which is least affected by density factors, a situation to which attention has been called earlier. The studies of Crowcroft and Rowe (1957) offer a clear illustration of this situation in controlled populations in which the ages of the animals were known. How would the nonreproductive mice in these populations have been classified in the usual studies of natural populations with trapped animals? Kalela's (1957) studies on the effects of population density on Clethrionomys rufocanus from a natural population are of singular value beeause (1) he had a means of determining the age of the animals by the rooting of their molars and was therefore able to state with certainty that growth and maturity were totally inhibited, and (2) he eliminated the possibility that food was an etiologic agent in producing these phenomena. Of course, all gradations of these effects may oceur to further confuse the situation. Most studies which evaluate changes in reproductive function of small mammals, for which there is no adequate way of determining age, in relation to density in populations must be viewed with considerable skepticism. It has already been pointed out that the usual means of determining reproductive competence do not detect changes short of almost total inhibition, especially in males.

A third problem, less frequent in its occurrence, is the failure to take into consideration changes in age eomposition when evaluating changes in litter size, although this frequently results from the inability to determine age. Hamilton (1937) reported that litter size in Microtus pennsylvanicus increased with increasing population density and yet there was increased intra-uterine mortality at the higher densities. It is well known that litter size inereases with parity for the first few litters and that there usually is a direct relationship between body weight and litter size for most litter- 
bearing mammals (Watt, 1934; Hoffman, 1957; Flick et al., 1959). Therefore it is entirely possible that the apparent increase in litter size noted by Hamilton was a result of a shift in age composition, and even if this is not the case, the point is generally valid and is well illustrated by Hamilton's data. These data bring up another point which often proves confusing; that is there is no word in use to designate an in utero group of embryos. "Litter" used for both postpartum and antepartum young with the result that "litter size" is frequently determined from embryo counts and conclusions drawn therefrom regarding litter size at birth. Although the authors are themselves usually quite clear on these differences, their descriptions in the literature often are not, and the reader will arrive at false conclusions or else be unable to make any at all. This situation can be corrected by the use of the collective noun gravidum to describe the in utero counterpart of "litter" (Snyder and Christian, 1960).

Finally, there is a wide divergence in the descriptions of the effects of population density on reproduction. It seems clear from experimental and field data that the reproductive processes can be inhibited at a number of different points, evidently depending on the severity of the stimulus, duration of the stimulus, age at which the stimulus was first effective, the position of any given animal or groups of animals in the social structure, probably individual physiologic and psychologic differences, as well as other unknown factors. The details of these various relationships are not known, but experimental results suggest that the smallest stimulus inducing an increased adaptive response with inhibition of reproduction in mature animals will affect lactation, increase litter mortality, and possibly depress fertility; a greater stimulus will result in increased intra-uterine mortality and an even greater stimulus will totally suppress reproductive function. It is suggested that as the alarming stimulus increases in severity there is a progressively greater suppression of the secretion of gonadotropins. It is equally evident that young mammals reaching maturity are far more susceptible to a given alarming stimulus than an animal which has already reached maturity. It is known that immature rats and mice are many times more sensitive to suppression of gonadotropins by steroids than the mature animals (Byrnes and Meyer, 1951). The results of experiments discussed in the preceding sections show that these statements apply to the effects of population density. The effects of vitamin and other nutritional deficiencies follow a similar pattern; furthermore, the earlier in the process of reproduction the deficiency begins, the more profound is its effect on the developing fetuses (Lutwak-Mann, 1958). Usually a deficiency beginning at the start of pregnancy or earlier will produce a very high percentage of resorptions. These decrease the later the deficieney begins. It is noteworthy that only "borderline" deficiencies appear to produce congenital anomalies. 
So it appears to be with population density. Only those female mice which were moderately affected by density produced young which were then permanently affected by reduction in lactation, whereas those mice more seriously affected failed to produce at all and exhibited $100 \%$ resorption of embryos (Christian and LeMunyan, 1958). Therefore, when one consider's the enormous number of variables affecting a population, it is not surprising that there is so much variation among individuals and among populations with respect to the effects on reproduction. Reproduction should include the entire process of producing young from maturation of the parents to weaning when discussing or measuring the effects of physiologic adaptive mechanisms and the effects on the endocrine organs.

In spite of these problems, a number of studies on natural populations of small mammals have been sufficiently detailed and eritical to permit examination of the relationships between population density and reproduction. The most conclusive of these have been carried out on Norway rats (Rattus norvegicus) and voles (Microtus sp. and Clethrionomys), but there have been others on muskrats (Ondatra), cotton rats (Sigmodon), woodchucks (Marmota), and hares (Lepus americamus). Most of these suffer to one extent or another from a lack of dependable criteria for determining age, as discussed above.

The reproductive performance of Norway rats from increasing, decreasing, and stationary populations was investigated (Davis, 1951a). These rats have a major reproductive season in late winter and early spring and a secondary minor peak in reproductive activity in the fall. Inereasing populations have a higher prevalence of pregnancy than either decreasing or stationary populations. These differences are especially marked in the spring breeding season when the prevalence of pregnancies was $41.6 \%$ in increasing, $25.3 \%$ in decreasing, and $14.4 \%$ in stationary populations. The incidences of pregnancy were, repsectively, 6.1, 6.3, and 3.8 per year. However, there were no apparent differences in litter size and the prevalence of lactation was essentially the same in all three categories although the data on lactation could not be analyzed separately for the two breeding seasons. However, an analysis of lactation by seasons could not account for the similarity of the overall figure and one must conclude paradoxically that, although the pregnancy rate was highest in increasing populations, there was also a greater parturitional mortality and at the same time a better survival of those young which were not lost soon after birth.

Similar results were obtained when reproductive performances of Norway rats from a rural population were compared at two different relative population densities. The pregnancy rate was $48.3 \%$ a month after artificially reducing the population a third, compared to $14.4 \%$ before the reduction. 
These results indicate that reproductive performance is inversely related to population density in Norway rats, at least for increasing and stationary populations. The status of decreasing populations is equivocal and may represent a partial reeovery of reproductive function in declining populations, although decreasing populations are presumably at maximum density. However, we already have noted that adrenocortical hypertrophy is greatest in populations spontaneously decreasing from asymptotic levels. These results represent a large number of populations and carry considerable weight; nevertheless in another experiment in which three urban populations of Norway rats were artificially reduced about $50 \%$ there was no corresponding increase in the rates of pregnancy (Davis and Christian, 1958). Two factors may have obscured such an occurrence. One was that the populations were increasing and therefore had a fairly high pregnaney rate at the time of the reduction $(30.2 \%)$, and the other was that the main breeding season was declining at the time the next samples were eollected (April, May, and June) from which a mean pregnancy rate of $32.2 \%$ was obtained.

Experiments designed to analyze reproductive function in relation to density of populations of rats suffer from the inability to determine the age of the animals, therefore attainment of maturity and other reproductive end points are based on the sizes of the animals. There is evidence to indicate that rats in increasing populations grow faster, and so are larger at a given age, than rats from stationary populations (Davis, 1951b). Therefore inhibition of growth in populations of high density may tend to obscure evidence of partial inhibition of reproductive function, such as delayed puberty, since it is likely that reproductive function and growth are equally inhibited. Thus, these results are evidence to support the earlier comments regarding the desirability of determining age in mammals when evaluating reproductive function.

One of the more conclusive studies to date on the relationship between density of population and reproduction was conducted by Kalela (1957) on red-backed voles (Clethrionomys rufocanus) in Finland. A major factor contributing to the value of this study was the ability to determine and approximate age of the animals by the root development of the first molars. These molars are rootless at the end of September of the year of birth, but the roots are visible by the following spring and continue to develop further (Zimmermann, 1937; Kalela, 1957). The question might be raised whether the growth and rooting of the molars would be suppressed along with suppression of growth, but molars were rooted in animals in which growth and maturity were definitely suppressed (Kalela, 1957); so that any suppression of molar growth and rooting would make the results even more dramatic. [This and other means of estimating age in roles has been more fully re- 
viewed by Bourlière (1951).] Kialela also determined that mature spermatozoa are present in the testes of these voles when the testes are seven or more millimeters long and defined maturity on this basis. Females which were pregnant or parous were defined as fecund.

During the first summer of Kalela's studies in 1954, moderate population densities were achieved after a spring characterized by a very small population which had overwintered from the preceding fall. Males and females from the litters born early in the breeding season matured without exception. Four-fifths of the mature females of late summer were young of the year. A large number of the young of the year which reached maturity had two litters and some even had three during their first summer. The old overwintering females had as many as four litters. The following spring, 1955, the population started with a much larger number of overwintering animals. The rate of growth of this population at first was as high or higher than the rate of growth of the population in the preceding year, but it rapidly declined as the density of the population increased. Peak densities were reached in this year. A good many of the males born in the first part of the breeding season reached maturity, but males with enlarged testes were rarely found in August, and there was an accumulation of immature males weighing between 20 and $24 \mathrm{gm}$. at this time (mature red-backed voles normally weigh more than $24 \mathrm{gm}$., usually between 30 and $40 \mathrm{gm}$.). This weight range represents the maximum size achieved by male or female red-backed voles which fail to mature, irrespective of their age. There was no accumulation of voles in this weight category during the preceding year of low population densities. Female young of the year exhibited the same cessation of growth and failure to mature, except for those born early in the breeding season. Less than half of the mature females in late summer were young of the year and there was a marked accumulation of immature females in the 20-24 gm. weight class. Furthermore, the pregnancy rate fell sharply and had fallen to well below the 1954 levels by mid-August. The overwintering females had up to three litters, but none had four litters. Those young of the year which reached maturity had no more than two litters and only a few had this many. One area (Malla) had twice the density of the others under study at this time, and none of the young of the year, male or female, attained sexual maturity or grew beyond the 20-24 gm. category.

The population had undergone a major decline in numbers by the spring of 1956 and was well below the 1954 levels for the same period, and yet the rate of growth of the population was extremely slow. The overwintering animals, as well as the young of the year, were distinctly smaller than normal. Reproductive performance was essentially identical to that of the preceding summer of 1955 when the density of the population was extreme. 
The rates of fecundity, pregnancy, and other measurements of reproductive competence were unchanged from the preceding year. There was no apparent change in litter size or intra-uterine mortality throughout the period of the study of this population. There was no shortage of food at any time during these studies, and especially not during periods of active reproduction. Therefore, the changes in reproductive function are not attributable to varying food supplies. Climatic factors, although variable, did not correlate with the functional changes and were probably noncontributory in producing functional changes.

This study shows, beyond reasonable doubt, that the shifts in reproductive function were reciprocally related to the density of the population in 1954 and 1955 and that increased density was the factor responsible for the decline in reproductive function (with a total suppression of reproduction in the young of the year in one area) and attainment of maturity in 1955. It also shows that the primary effect of increased density was to inhibit maturation so that large numbers of immature animals accumulated by late summer. At the same time, growth beyond a general level of 20-24 gm. was suppressed in both sexes. The data from the area of extreme density, in which no animals matured are especially convincing. No direct measures of endocrine function were made, but these occurrences in natural populations of Clethrionomys rufocanus conform with the results from the more highly controlled experiments with populations of mice or voles in the laboratory.

The apparently paradoxical failure in 1956 of reproduction to return to the 1954, or higher, levels, together with the apparent stunting, also fits the conclusions discussed earlier. The effects of increased density on lactation and subsequent growth of the young and in turn their inability to lactate adequately were discussed earlier, and attention was called to the profound long-range effects of increased density on the animals experiencing the increase as well as two generations of their progeny (Chitty, 1955; Christian and LeMunyan, 1958). The red-backed voles which overwintered until the spring of 1956 had been subjected to increased density and had experienced its effects. It was noted that these voles were unable to mature in the year of their birth and were stunted. The following year their reproductive performance and that of their offspring reflected a similar situation, closely resembling the effects of increased density on laboratory populations. The population decline was consequently due to the high mortality of those young born during the period of maximum density and probably reflects decreased resistance to a variety of stimuli coincident with increased activation of adaptive mechanisms. A very high rate of infant and juvenile mortality was a major factor in the cessation of growth in laboratory populations, but in these there was no appreciable mortality of the adults. 
There is a continuous high rate of mortality of all age groups in natural populations of these small mammals so that a failure of reproduction and a sharply increased mortality rate of jurenile animals make a collapse of the population easily understood. The prolonged recorery from this decline also can be attributed to the effects of increased density, apparently through defects in lactation as well as other endocrine reproductive functions, although the details of these mechanisms require much more investigation. It should be noted in these populations; that while all phases of reproduction were affected the particular functional aspect which was most severely affected appeared to depend on the level of density and on the age of the animals involved - the young being most severely affected, as was the case in laboratory populations. It appears that the responses of this natural population to density were identical in every respect to those seen in laboratory populations with the exception of the ever-present mortality which occurs in the wild.

Reproductive function evidently is inversely related to density in natural populations of red-backed voles (Clethrionomys), and the inhibition of reproduction and growth probably resulted from a suppression of the secretion by the anterior pituitary of gonadotropins and growth hormone. There is no other explanation evident which fits the known facts, even though there was no direct assessment of pituitary function. The apparent paradox of a low population exhibiting a marked inhibition of reproduction and growth following a decline appears to result from the prolonged effects of high population densitics on these animals. These conclusions derived from Kalela's studies carry particular weight, as food, and apparently other environmental factors, appear not to have been causative agents. Kalela (1957) discusses the social problems in these populations.

A quite similar study was conducted on Microtus montanus (Hoffmann, 1958) with comparable results. This population was followed for 3 years and there was a peak between the second and third breeding seasons which was followed by a marked decline in the size of the population. The population density was moderate in the spring of 1952, increased during the summer and early autumn, and was followed by the annual decline in density subsequent to the annual cessation of brecding. The population in the spring of 1953 began moderately and increased sharply until September. The spring population of 1954 was higher than in 1953 , but the population declined rather than increased during the breeding season and was at a very low level in the spring of 1955 . Thus the history of this population of Microtus montanus was in many respects similar to that of Kalela's population of Clethrionomys. The published data indicate that Microtus montanus may differ from most other small mammals in that litter size remains constant with respect to age, weight, and parity (Hoffmann, 1958). There- 
fore changes in mean litter size apparently do not need to be corrected for these variables. During each breeding season of the study there was an appreciable decline in litter size as measured by embryos and uterine implantation sears and this decrease was also paralleled by a decline in the number of corpora lutea. There was also an increase in the general level of the population for comparable months from 1952 to 1953. This general increase coincided with an overall decline in mean litter size which, however, was not significant. These changes were inversely related to the annual increase in population density for the first two years. However, the population declined sharply from spring to fall in the third year and yet the mean litter size also declined.

Prenatal mortality was directly related to population density in the first breeding season, inversely in the third and bore no conspicuous relationship in the second. Prenatal mortality was greatest after the population had declined in September 1954. The mean prevalence of pregnancy also declined with each successive year.

The peak of the population was evidently in September of 1953 , and the decline followed shortly thereafter. Hoffmann noted that at this time the proportion of fertile males began to decline and at the same time $24 \%$ of the mature females weighed less than $33 \mathrm{gm}$. This figure increased to approximately $45 \%$ in June and July of the following season. The proportion of mature males weighing less than $35 \mathrm{gm}$. also rose to reach a maximum of $33 \%$ in July of 1954 . These morphologic functional changes resemble those observed by Kalela and probably reflect suppression of growth at high densities. The fact that there was a decline in male fertility as well as in the incidence of pregnancy further supports such a conclusion. Hoffman called attention to the fact that prenatal mortality was lowest at peak densities and highest the following year. However, examination of his data reveals that the proportion of multiparous females rose steadily throughout the 1953 season to reach $100 \%$ in September, undoubtedly accounting for for the low proportion of prenatal losses for two reasons: (1) it is likely that the older parous females suffer less prenatal loss at any time; and (2) more important, they probably represent the dominant animals and therefore those least affected by high densities in accordance with the results of laboratory experiments (Retzlaff, 1938; Christian and IeNunyan, 19.58). The age of the animals could not be determined in these studies; immature animals were classed as such and were not further divided into those old enough to have matured and young animals. Had it been possible to determine age, it is likely that these results would have been more decisive. There was a low mortality of weanlings and juveniles during the period of build-up in this population, but apparently there was a marked increase in mortality in all age groups during the decline. 
These data, like those from the preceding study, indicate that the effects of high densities on reproduction and growth persist for some time after the peak has been reached and passed. It should be remembered that all animals beginning the breeding season after a peak are survivors from the peak densities. Although the highest numerical size of Hoffmann's population was in September of 1953 , the actual peak could just as easily have been the relatively high spring population of 1954 . The data suggest this to be the case, as there was a brief period at the beginning of the 1954 (June) breeding season in which prenatal and infant mortality were lower than usual and then climbed sharply. Hoffmann concluded that ovulation rate and litter size tend to vary inversely with population density and thus to run counter to the cyclic trend of the population, except during the crash decline period in Microtus montanus, when lowered litter size may contribute to the drop in density. He also concluded that speed of attainment of reproductive maturity remained rather constant despite changes in density, which is quite the reverse of Kalela's results and conclusions. This conclusion is, however, based on the assumption that there is 110 inhibition of growth or that it is totally independent of changes in reproductive function. Since the age of these voles could not be determined, age was assumed to be reflected in body weight.

In view of the likelihood that inhibition of growth and reproduction are not independent, but rather are two manifestations of one reaction, as we have seen for Clethrionomys as well as for Mus in experimental populations, the conclusion is not justified that the speed of attainment of maturity remains constant in the presence of changes in density. Furthermore, Hoffman's figures indicate that a high proportion of mature animals were much smaller in peak and declining populations than usually found at lower densities. Of course these data could be interpreted to indicate an early attainment of maturity with respect to size, but this interpretation is most unlikely in view of the other data on reproduction and the results of other experiments. This problem could be settled by determining the age of these animals; a problem already discussed at some length.

Chitty (1952) also studied a population of voles (Microtus agrestis) and obtained results similar to those of Kalela. During the peak breeding season of 1937 he found that young males did not increase in weight above 22.2 gm., whereas normally adult males seldom weigh less than $22.3 \mathrm{gm}$. after May. Young females weighed more than $22.2 \mathrm{gm}$. only while pregnant; otherwise the heavier females in this population were old adults with a few exceptions in September and October. The prevalence of pregnancy in the peak year was apparently less than in other years and appeared to be due mainly to a failure of maturation in the young of the year, as older animals exhibited a uniformly high pregnancy rate. In one area all age groups ceased 
breeding in August of the peak summer and the survivors did not breed the following spring after the population had declined. Other populations which were at peak density the following year continued to breed from May to October and survivors bred again the following year, after the decline, although no recovery in population occurred. D. Chitty concluded that lowered fertility was not invariably associated with a decline in numbers. The decreases in size in all these populations primarily was due to an excessive juvenile mortality before August which was attributed to some adverse effect of high population density on the ability of the young to survive.

Hoffmann (1958) also studied a population of Microtus californicus and noted a general inverse relationship between density and reproductive function which occurs on a seasonal basis. In other words, the annual increase in the population also has density effects on its members. Fitch (1957) noted a decline in litter size in Microtus ochrogaster with progression of the breeding season and increase in the population, as noted by other author's, but he attributed this to an increasing number of young of the year reaching maturity and having small first litters. However, this explanation does not account completely for the observed progressive decline in the population. A similar relationship has also been noted in muskrats (Ondatra zibethica) (Errington, 1948, 1951, 1954a). Errington (1954a, 1957) has also shown that reproduction may be depressed during the low years following high densities and increase during an increasing phase of the population. The depressed reproduction probably is another example of the prolonged effects of high population densities on succeeding generations.

The pregnancy rate was high early in breeding season during the peak year in a population of voles (Microtus montanus) studied by Adams, Bell, and Moore (Christian, 1959b), but dropped precipitously after June so that the percentage of pregnancy was below $30 \%$ for August and Septembermonths of peak breeding activity in subsequent years with lower population densities.

Hamilton (1937) reported an increase in litter size with increasing population density and a decrease in litter size during the decline. He also reported increased intra-uterine mortality. However, although the ages of these animals were not determined, it is probable that the age composition of the population shifted so that it is consisted of a higher proportion of older animals as the peak approached. The litter size of Microtus pennsylvanicus increases with parity (Hatfield, 1935; Martin, 1956; Fitch, 1957) differing from $M$. montanus in this respect; so that the increase in mean litter size could reflect such an occurrence. The decline in litter size during the decrease in population size is consistent with the preceding studies.

Odum (1955) also reports a greater mean litter size for Sigmodon in 
"high" density years than in "low" density years. Once again weight was used as an age criterion and so the possibility exists that Odum's figures for age composition in "low" versus "high" density populations may reflect complete suppression of reproduction and growth in young animals at high densities, rather than a shift in age composition toward younger animals as his figures suggest.

Tanaka (1956) reported complete inhibition of reproductive function in Anteliomys smithii (Clethrionomys smithii) irrespective of the age or sex of the voles during a season of peak densities. The reproductive organs were in a condition of complete quiescence. The possibility that a deficiency of food may have been instrumental in producing these effects cannot be ruled out. However, Tanaka $(1956,1957)$ points out that maximum densities, or vole "outbreaks," may occur either in concurrence with or independently of widespread flowering of bamboo grasses. The fact that the voles apparently were equally numerous and showed similar reproductive inhibition regardless of whether they were living in areas of living or areas of dead bamboo strongly suggests that the inhibition of reproduction was density dependent.

Lemmings, because of the conspicuousness of their population cycles, have been the subjects of study by a number of investigators. Lemmus trimucronatus has been reported to experience a complete suppression of reproduction during periods of peak densities of their populations (Rausch, 1950 ). In the spring of 1949 , prior to a precipitous decline in their population, there was an absence of young animals and no evidence of reproductive activity: none were pregnant, there were no uterine implantation scars, few females had perforate vaginas, and the testes of the males were small and incompletely descended. Similar results were obtained by Barkalow (1952) in a study of the tundra mouse (Microtus oeconomus) and lemmings (Lemmus trimucronatus and Dicrostonyx groenlandicus) at Barter Island, Alaska. Barkalow in summarizing the results of his and other studies on vole and lemming populations in the Aretic states that cessation of breeding: prior to and during a cyclic decline is in his opinion a population characteristic and that large litters are characteristic of the build-up phase of a population cycle, especially in its early stages. Rausch (1950) stated that although there was a reduction in the tundra vegetation there was no suggestion that the decimation of the population resulted from a deficiency of food. Thompson (1955) takes the view that exhaustion of the supply of food and cover is the important factor, and well it may be in a local situation. However, the bulk of the evidence suggests that a limited supply of food is not usually the factor limiting population growth. Lemmings have an annual decline in reproduction with the annual increase of density, especially in prepeak or peak years of density similar to that seen in the other 
microtines diseussed (Pitelka, 1957b). Pitelka (1957a, b) also makes the point that the cessation of reproduction at peak densities may oceur and produce a decline in the lemmings before their predators have a chance to exploit the lemming population. Lemming populations have not been studied for a long enough period of time or with sufficiently detailed evaluation of the various factors which might inhibit reproduction to warrant any conclusions at present. However, it is apparent that there can be inhibition of reproduction with increased density, but the extent to which food supplies may also contribute has not been sufficiently appraised. The evidence presently available suggests that failure of reproduction in response to extreme densities is the major cause of the eyclic declines in lemming populations.

Many studies indicating a decline in reproduction with high population densities in other species, such as hares (Preble, 1908; MacLulich, 1937), but in none of these is it possible to ascribe the alteration in reproduction solely to density factors, even though it may well be that such is the case. Many other studies could be listed which illustrate the same sort of thing, but no useful purpose would be served by doing so here. More recent studies with Microtus pennsyluanicus indicate that in this species the annual reproductive eycle is governed by population density, but this does not appear to be true for Peromyscus maniculatus (Christian, 1961).

The main point of interest is that reproduction does respond to changes in population density in natural populations of voles and rats and probably in other species as well. In some studies there was a reciprocal relationship between adrenocortical and reproductive function, in other's only one or the other of these general responses was studied, but the results conform to this general relationship. It seems undeniable, especially in the light of studies on laboratory populations, that physiologic adaptive responses are operative in natural populations and are refleeted by curtailment of reproduction and growth and stimulation of increased pituitary-adrenocortical activity in natural populations. However, detailed and well correlated studies on a variety of species still are required and for a variety of different populations in order to assess the relative importance of these mechanisms in eurtailing free-for-all population growth.

\section{Disease Resistance and Mortality}

One of the major points of the hypothesis that physiologic mechanisms could control population growth as a feedback system acting in response to changes in density was that the sudden mass mortality seen at the end of a build-up of populations to peak densities could be accounted by exhaustion of the adaptive responses (Christian, 19.50b). It was suggested that the 
symptoms so frequently observed (Hamilton, 1937; Green and Larson, 1938; Green, et al., 1939) were the result of hypoglycemic shock as a result of pituitary-adrenocortical exhaustion. The subject remains at that point today, except that Frank (1953) has succeeded in showing that hypoglycemic shock, which is uncorrected by epinephrine but is corrected by glucose injection, occurs in Microtus. Frank (1953) also succeeded in producing mass mortality from hypoglycemie shock by crowding voles in enclosures. However, he believes that in addition to increased density alone, which sets the conditions for a mass mortality, a trigger, such as eompetition for food must also be present. This seems a reasonable suggestion which perhaps can be modified to be included in density-dependent factors. On the basis of Frank's and other experimental data it appears that competition for any factor is also a matter of relative population density and inseparable from it. In this case it seems that the voles were reducing the environmental capacity at high densities and were therefore themselves setting up the conditions for increasing competition among themselves above the level that was already present.

It has been mentioned that mass mortality in sika deer was probably a result of electrolyte imbalances brought about by prolonged adrenocortical hyperactivity. There were indications of a prominent role of the zona glomerulosa, presumably with increased secretion of aldosterone, to explain the evidence of potassium defieiency (Christian ct al., 1960; Christian, 1963).

Frank (1953) was able to eliminate infectious disease and climatic factors as causative agents in precipitating the mortality in these animals, as were Adams, Bell, and Moore (Christian, 1959b) in Microtus montanus. Rausch (1950) also indicated that neither parasitism nor infectious disease could account for the sudden decline in the lemming population he was studying. In a study of mortality in an experimentally induced epidemic in Norway rats, Davis and Jensen (1952) found that mortality rate was not significantly changed and probably would be so only under special eircumstances. A precipitous population decline due to mass mortality from hypoglycemic shock is probably only one of several meehanisms which may operate to reduce population density drastically and it may be a rather special occurrence. Our knowledge of the physiologic mechanisms involved in invoking "shock disease" in natural or experimental populations is little better understood now than previously. It was originally postulated that adrenocortical exhaustion was the cause, but it is just as likely, if not more so, that readily mobilizable sources of glycogen and glucose become totally exhausted with continued overstimulation of the adrenal medulla and cortex. There may be exhaustion of other parts of the responsive system, such as the pituitary, hypothalamus, or of the adrenal medulla. None of these 
possibilities has been explored and in retrospect the idea of attributing hypoglycemic shock solely to adrenocortical exhaustion seems naive. That the hypoglycemic shock syndrome does occur cannot be denied, but its physiological causes remain to be determined. Such indirect evidence as is available indicates that it is a very complex affair. Mice and voles have been observed to die of hypoglycemic shock shortly after being placed placed together in groups (Frank, 1953; Christian, 1955a), but if moribund animals are removed from their cagemates, they recover (Frank, 1953; J. J. Christian, unpublished). The rapidity with which this occurs suggests something other than cortical exhaustion, perhaps medullary exhaustion or exhaustion of readily available glycogen reserves. In addition, there is evidence that adrenal cortical exhaustion may not occur per se, but may be an apparent result of exhaustion elsewhere in the adaptive system (Rosenfeld, 1958).

At the present time it appears that a failure in reproductive function may account for a decline in a population much more frequently than shock. It has been observed in several species that the young born during peaks of density appear to be much more susceptible to various mortality factors, and their deaths largely account for precipitous population declines (Green and Evans, 1940a, b, c; Chitty, 1952, 1954; Godfrey, 1955). This disproportionate mortality of young animals may result from three different factors that have already been discussed: (1) they are the subordinate animals in a population and therefore, as we have seen, are much more severely exposed to sociopsychologic pressures than older and more dominant animals; (2) they appear to be more sensitive to these pressures and respond accordingly; and (3) they are adversely affected by deficient lactation on the part of their mothers, and their progeny in turn are affected. The combination of these factors would be expected to have profound effects on the young, and we have already seen that their reproductive function is severely depressed. Excessive stimulation of the adaptive responses in these animals would also be expected to result in exhaustion and shock. Frank (1953) has shown that socially inferior voles are the ones primarily affected and that succumb to shock.

In addition to these effects of social pressure and increased activity of physiologic adaptive responses, one would anticipate a marked decline in resistance to infection, parasitism, or other harsh environment experiences. It is not surprising that epidemics are frequently observed following peak densities and a general decrease in host resistance. It is particularly significant that a variety of agents may be found to produce death in a given population, apparently as a result of decreased resistance (Chitty, 1954).

It is not the intent of this account to dwell on the causes of mortality in natural populations except insofar as a decreased resistance may result from 
increased physiologic adaptation to increased density. A causal relationship between population density and parasitism in hares is suggested by the studies of Erickson (1944). Parasites were scarce when population densities were low and maximum parasitism was found in populations of hares at peak densities. The hare populations declined but the parasites remained abundant in the surviving animals. There was no evident correlation between shock disease and the parasites in these hares other than that parasitism was at high levels when the population began to decline owing to shock. Similarly, a close correspondence between the level of parasitism of rabbits by Eimeria stiedae and population density was found in New Zealand (Whittle, 1955) and it was suggested that the differences in the incidence of severe infections were correlated with differences in host resistence, which in turn may have been a function of host density (Bull, $1955,1957,1958$ ). It is of interest that young rabbits were the ones primarily affected in these populations (Tyndale-Biscoe and Williams, 1955; Bull, 1958). In a study on parasitism by lice on populations of Microtus pennsylvanicus and Peromyscus maniculatus it was found that the level of parasitism varied with season in both the roles and the deer mice, but that the size of the louse population changed significantly with season only in the male voles (Cook and Beer, 1958). It was suggested that the spring maximum in the louse populations on the male voles eame at the period of greatest "stress" and therefore the increase in the louse population at this time resulted from decreased host resistance.

The evidence for a decrease in host resistance to specific diseases or parasitism in natural populations is not conclusive, but is certainly suggestive. However, there can be little question that there are severe physiological derangements associated with high population densities which result in high mortality rates in voles, lemmings, and hares (Green and Erans, 1940b; Elton, 1942; Rausch, 1950; Chitty, 1952, 1954; Frank, 1953; Godfrey, 1955), and that this mortality cannot be accounted for by infectious disease, even though the prevalence of infection and parasitism may be increased. It is equally evident that young animals are more severely affected than older animals and that the effects are prolonged and account for continued declines in populations for appreciable periods following a sharp reduetion in population density (Chitty, 1952, 1954, 1955; Christian and LeMunyan, 1958; Christian, 1959a, b).

In summary, there is much indireet evidence indicating that a decline of a population from peak densities may be caused by a density-dependent activation of physiologic adaptive mechanisms and exerting their effects in one or all of several ways:

1. There may be direct mass mortality due to physiologic exhaustion following prolonged, excessive stimulation of the adaptive mechanisms. 
2. There may be increased mortality due to parasitism or infectious disease due to decreased host resistance (presumably from suppression of inflammatory and immume defense mechanisms by increased adreoncortical activity).

3. There may be partial or complete inhibition of reproductive function, especially in the younger animals and with prolonged effects on the next generations of progeny, apparently initiated by deficient lactation on the part of mothers subjected to increased density, but also owing to the fact that the young probably are most severely affected by increased density.

It is assumed that in the absence of mass mortality normal or moderately increased mortality rates can produce striking declines in density when reproduction is partially or wholly suppressed. The bulk of evidence in the literature indicates that this is more usually the case than mass mortality. Furthermore, food shortages, epidemics, or predation may be the immediate cause of the mortality, although it is equally clear that the mortality still occurs when these factors are absent (Elton, 1942; Rausch, 1950; Chitty, 1954; Godfrey, 1955; Pitelka, 1957a, b). It is also possible that some precipitating factors may act through the physiologic adaptive mechanisms by increasing intraspecific strife, as was shown to be the case in experimental populations of voles (Frank, 1953).

\section{Summary: Natural Populations}

There is considerable evidence to indicate that there is a density-dependent increase in the activity of physiologic adaptive mechanisms in natural populations of Norway rats, voles (Microtus, Clethrionomys, Dicrostonyx, Lemmus, Ondatra), hares (Lepus), rabbits (Oryctolagus), whitefooted mice (Peromyscus), and probably other species. A relationship between population density and adrenocortical activity has been demonstrated for Norway rats, meadow voles (Microtus montanus and $M$. pennsylvanicus), white-footed mice (Peromyscus leucopus), and sika deer (Cervus nippon). An inverse relationship between density and reproductive function cxists for Norway rats, red-backed voles (Clethrionomys), meadow voles (Microtus agrestis, iI. montanus, M. californicus, M. pcnnsylvanicus), muskrats (Ondatra), cotton rats (Sigmodon), and hares (Lepus). However, in few of these studied were reproductive function and adrenocortical function correlated in the same population. Furthermore adequate assessment of other environmental factors has not been made. In many investigations there were no adequate means of determining age, so that a true indication of the degree of inhibition of reproductive function was not possible, espccially with a simultancous suppression of growth. Determinations of age should be possible in a variety of species if a concerted effort is made to find the proper criteria. There is evidence that, as in the laboratory, 
there is suppression of lactation, as well as of other reproductive functions, with increased density which may account for the prolonged effects of high density on the young. However, the effects of high density may also be exerted on the young animals directly. There is also evidence that resistance to disease and parasites is decreased, especially in the young, at high population densities. In extreme cases, there may be mass mortalities of populations due to hypoglycemic shock, presumably following exhaustion of some part of the entire physiologic mechanism or of the reserves of readily available necessary metabolites or both.

None of the data from natural populations is conclusive evidence that density-dependent responses of physiologic adaptive mechanisms are active in the regulation and control of the growth of mammalian populations. However, when the available evidence is viewed in the light of the results obtained from carefully controlled studies on laboratory populations, there is sufficient reason to conclude that these mechanisms are operative in natural mammalian populations, and more precisely in populations of rodents and lagomorphs. It is equally apparent that sociopsychologic pressures are the stimuli to the various physiologic responses involved. Therefore, there is a wide range in responses in the individuals of a population, the subordinate, and therefore younger, animals being the most severely involved. Finally, it appears that thesc sociopsychologic-physiologic mechanisms are basic controlling factors in populations of mammals and other factors are secondarily important in most cases.

There is a great need for a coordinated study on a small rodent for which there are criteria for determining age in which adrenocortical function, reproductive function, resistance to disease, mortality, social factors, and environmental factors, can be adequately studied for a complete cycle of the population. Until the results of such a study are arailable, conclusions regarding density-dependent physiologic mechanisms must lean heavily on extrapolations from data gathered from experimental populations in the laboratory. There are in addition to the general problem many specific problems that need investigation. Many of thesc have been mentioned in the course of the discussion, but a few have not. A major problem is what factors determine where the reproductive process will be affected in a particular population. For example, why is it intra-uterine mortality in one case, total inhibition of reproductive function in another, and depressed lactation in a third? The explanations are largely conjectural at present, but that these differences exist cannot be questioned, yet all represent a common mechanism and effect: inhibition of reproduction. The answer to another question may provide the answers to those already asked: how do social pressures affect the individual members of a population? 


\section{Conclusion}

The first part of this chapter reviewed briefly the endocrine adaptive mechanisms which are of unquestionable importance in enabling mammals to meet and adapt to their constantly changing environment and the visicsitudes of daily existence. The mechanisms discussed are by no means the only adaptive responses which are evoked by environmental exigencies, but they are the better known ones and at the present time those which are most likely to be of interest to mammalogists, ecologists, and students of mammalian population dynamics. The first part of the chapter was in itself a summary review of these responses; so no useful purpose would be served by further condensing and summarizing at this point. However, it should be emphasized that one is not justified in regarding any single response, for example increased adrenocortical activity, as an isolated phenomenon complete unto itself and independent of any other changes. Any adaptive response initiates and is a part of an extremely complex series of physiologic changes which probably involve every aspect of the host's physiology and metabolism. Furthermore, the adaptive responses are not static affairs, but a system of dynamie changes in the nature of feedback mechanisms which operate to maintain a constant internal environment and life. As stated earlier, these mechanisms are undoubtedly constantly active and responding to even minor changes encountered in daily life. Emphasis is lent to this statement by the hypoactive physiologic state observed in closely confined, inactive, and overfed mammals. Therefore one should regard the adaptive responses that are customarily studied and reported merely as quantitative deviations from "normal" daily experience. Finally, we should regard physiologic adaptations as flexible in that the available evidence indicates that qualitatively different stimuli probably do not elicit qualitatively similar responses even though cursory examination may seem to contradict this statement. The components of the responses probably differ proportionally from each other with differing stimuli. It is true that certain organ systems are primarily responsive to the demands of external change, but their responses appear not to be identical, either quantitatively or qualitatively, to all stimuli. For example, we have seen that heat and cold elicit quite different responses, while emotional stimuli evoke a third and different set of reactions, although all three may have certain features in common. Therefore a plea is again made to examine adaptive responses in the light of the specific stimuli by which they were elicited. Generalization serves an extremely useful purpose conceptually, but it may be misleading in the interpretation of results from critical studies designed to explore physiologic adjustments to specific stimuli. 
Finally, what has been presented here only represents a selected stopping point in a rery rapidly advancing field of research and must, perforee, be used as such and modified in the light of newer developments.

The second part of this chapter attempted to review critically the evidence from the laboratory and from the field relating physiologie adaptive responses to changes in population density, largely in response to poorly defined sociopsychologic factors. There seems to be little doubt that endocrine adaptive responses to sociopsychologic pressures are of basic importance in the regulation of population growth, at least for a limited number of species. It also is obvious that a great many gaps remain to be filled before the evidence for the physiologie regulation of natural populations can be considered conclusive one way or the other except for a few species of rodent. These gaps have been pointed out in the appropriate portions of the foregoing discussions. Nevertheless, considerable support has been derived in the last ten years for the original hypothesis that factors intimately related to population density are stimuli to physiologic feedback mechanisms and that population growth and decline are largely controlled by changes in density. It has also become evident that "density" in terms of manmalian populations is related only indirectly to numbers of mammals per unit of area, being more directly related to intraspecific competition, social strife, sociopsychologic pressures, or whatever other comparable designition one may choose to use for the interactions between mammals in the same population. It is obvious that these factors require a great deal more study in order to define them precisely.

Figure 1 is presented as a schematic summary of the physiologie feedback regulation of population growth as it is envisioned today in the light of the available experimental evidence. It should be re-emphasized that this is a dynamic and flexible system and that the importance of various components may vary with respect to each other. The broad ascending arrows on the left of the figure indicate that as population size increases, social pressures increase accordingly. The dotted lines above and below the arrow for population increase serve to indicate that the actual population size may vary considerably from population to population for a given degree of social pressure, which we see hypothetically as the fundamental growth-regulating and growth-limiting factor in all mammalian populations and the factor to which physiologic adaptive mechanisms respond. However, that this factor is always present and always operative does not necessarily imply that it is always the factor which limits population growth. Environmental factors probably operate through this mechanism by increasing or decreasing social pressures, although conclusive evidence for this statement presently is lacking. There is good evidence, however, that the social factors will limit population growth despite an abundance of all environmental requirements and that the degree of social density is related to the behavioral composition 


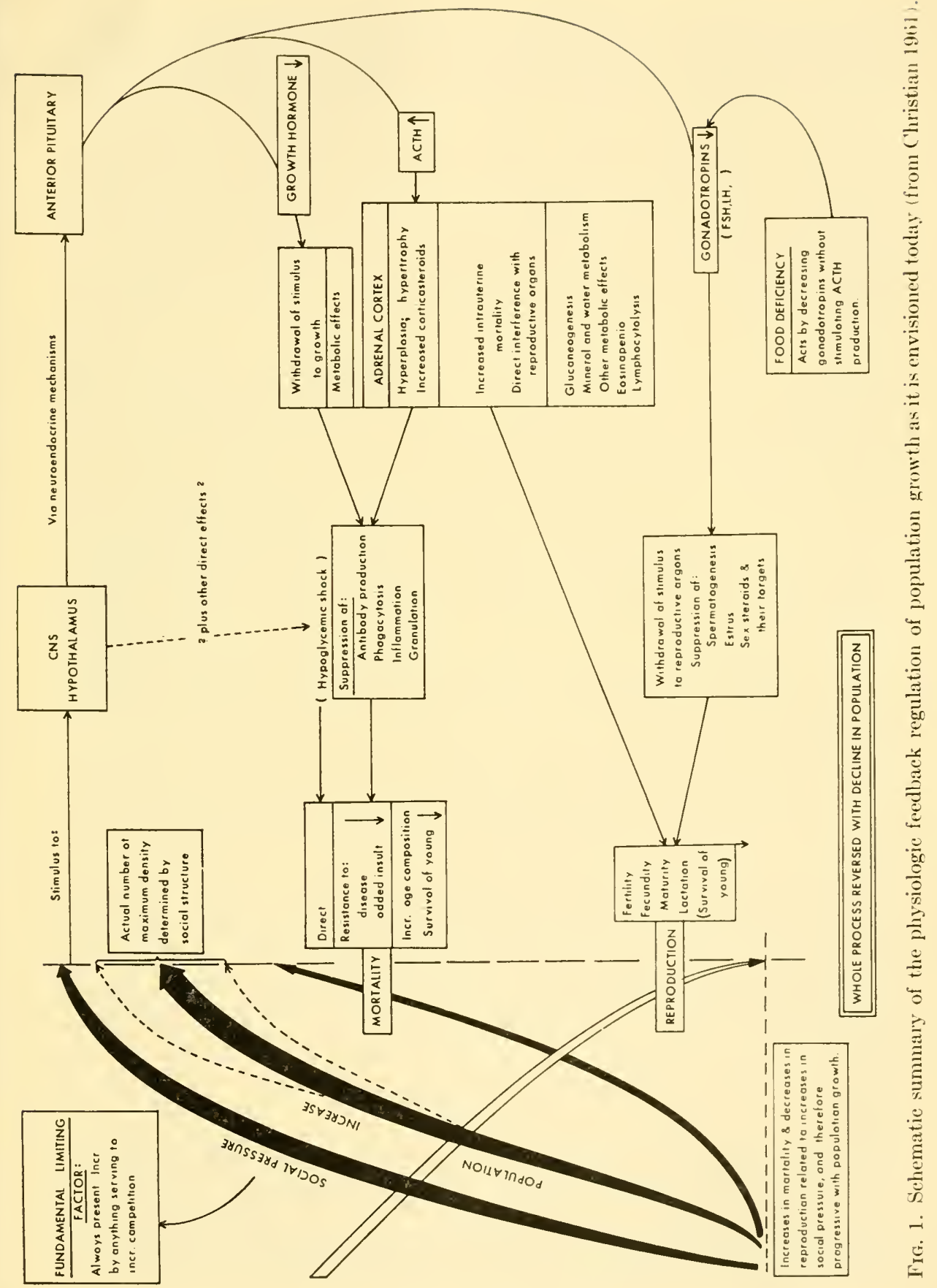


of the population. As social pressure increases, it acts as a progressively stronger stimulus to physiologic adaptation via the mechanisms on the right of the broken vertical line. These in turn act to decrease reproduction and increase mortality, and therefore the rate of population growth declines with increasing density and eventually ceases, as indicated by the "reproduction" and "mortality" arrows on the left. We have already seen that reproduction may cease altogether at maximum densities and that mortality may achieve precipitous proportions. An alteration in age composition of the population is assumed to be inherent in these effeets. The details of the various mechanisms have been discussed earlier, and will not be discussed further here. However, it is hoped that this diagram will help in visualizing the dynamic interrelationships between the endocrine responses in the members of the population to changes in the population as a whole. It will be observed that a food deficiency has been shown as deereasing directly the production of gonadotropins, but, as we have discussed earlier, it may serve to increase competition and therefore effective density under some circumstances. Theoretically it seems unlikely that a food deficiency would reach proportions in natural populations which would enable it to exert its effects directly on the pituitary without competitive factors first becoming operative. This diagrammatic hypothesis appears to fit the facts arailable for a limited number of studies on a few species of rodent, and undoubtedly it will be necessary to modify it as information from more critical studies become available. It is of primary importance to obtain results from careful studies on ungulates, similar to those already conducted on rodents, before regulation of population growth of ungulates by sociopsychologic-physiologic feedback mechanisms can be considered to be more than hypothetical. However, at present it is very probable that these mechanisms are basically important in regulating the growth of populations of rodents and lagomorphs.

An attempt has been made in this chapter to point out the areas where eritical data are lacking and some of the problems and pitfalls which may be encountered in investigating these physiologic phenomena in relation to population density. It is hoped that it will serve as a useful guide for further research by investigators who wish to explore the mechanics of population dynamics.

\section{ReFERENCES}

Ackerman, N. B., and Arons, W. L. (1958). The effect of epinephrine and norepinephrine on the acute thyroid release of thyroid hormones. Endocrinology 62, 723-737.

Agate, F. J., Jr. (1952). The possible role of the adrenal cortex in fluid shifts in the hamster following renal damage. Ann. N.Y. Acad. Sci. 55, 404-411.

Albert, A., Tenney, A., and Ford, E. (1952). The effect of cortisone and corticotropin on the biologic decay of thyroidal radioiodine. Endocrinology 50, 324-326. 
Allen, J. C. (1954). Effect of cortisone on the morphology of the mouse adrenal with special reference to changes in the X-zone. Bull. Johns Hopkins Hosp. 94, 349-350.

Allen, J. M. (1957). A chemical and histochemical study of aliesterase in mouse adrenal cortex under hormonal influences and altered sodium intake. Endocrinology 61, 368374.

Alpert, M. (1950). Observations on the histophysiology of the adrenal gland of the golden hamster. Endocrinology 46, 166-167.

Anderson, C. H., McCally, M., and Farrell, G. L. (1959). The effects of atrial stretch on aldosterone secretion. Endocrinology 64, 202-207.

Anderson, E., and Haymaker, W. (1948a). Influence of the hypothalamus on sexual functions, Part I. J. Am. Med. Women's Assoc. 3, 402-406.

Anderson, E., and Haymaker, W. (1948b). Influence of the hypothalamus on sexual functions, Part II. J. Am. Med. Women's Assoc. 3, 457-461.

Andervont, H. B. (1944). Influence of environment on mammary cancer in mice. $J$. Natl. Cancer Inst. 10, 579-5.51.

Antopol, W. (1953). Anatomical changes with excessive doses of cortisone in the normal mouse, the pregnant mouse, the fetus, and the offspring. Am. J. Pathol. 29, 612-613.

Asling, C. W., Reinhardt, W. O., and Li, C. H. (1951). Effects of adrenocorticotropic hormone on body growth, visceral proportions and white blood cell counts of normal and hypophysectomized male rate. Endocrinology 48, 534-547.

Aycock, W. L., and Ingalls, T. H. (1946). Maternal disease as a principle in the epidomiology of congenital anomalies. Am. J. Med. Sci. 212, 366-379.

Ayres, P. J., Gould, R. P., Simpson, S. A. S., and Tait, J. F. (1956). The in vitro demonstration of differential corticosteroid production within the ox adrenal gland. Biochem. J. 63, 19p.

Bachman, R. (1954). Die nebeuniere. In "Handbuch der mikroskopischen Anatomie des Menschen” (W. v. Möllendorff, ed.). Vol. 6. Springer, Berlin.

Badrick, F. E., Brimblecombe, R. W., Reiss, J. M., and Reiss, M. (1954). The influence of stress conditions on the uptake of $1^{131}$ by the rat thyroid. J. Endocrinol. 11, 305-313.

Badrick, F. E., Brimblecombe, R. W., and Reiss, M. (1955). Responses of hypophysectomized rats to stress. J. Endocrinol. 12, 205-208.

Bahn, R., Wilson, H., Kedda, L., Furth, J., and Anderson, E. (1957). Metabolic changes associated with transplantable ACTH-produeing pituitary tumors of the mouse. Can. J. Biochem. and Physiol. 35, 1057-1065.

Baker, B. L. (1949). Modification of body structure by adrenocortical secretions with special reference to the regulation of growth. Am. Assoc. Advance. Sci. Symposium on Pituitary-Adrenal Function, pp. 85-95.

Baker, B. L. (1952). A comparison of the histological changes induced by experimental hyperadrenacorticalism and inanition. Recent Progr. in Hormone Research 7, 331-373.

Baker, B. L. (1954). The connective tissue reaction around implanted pellets of steroicl hormones. Anat. Record 119, 529-539.

Baker, B. L., and Abrams, G. D. (1954). Effect of hypophyscetony on the eytology of the fundii glands of the stomach and on the secretion of pepsin. Am. J. Physiol. 177, 409-412.

Baker, B. L., and Bridgman, R. M. (1954). 'The histology of the gastro-intestinal mucosa (rat) after adrenalectomy or administration of adrenocortical hormones. $A \mathrm{~m} . J$. Anat. 94, 363-397.

Baker, B. L., Schairer, M. A., Ingle, D. J., and Li, C. H. (1950). The induction of involution in the male reproductive system by treatment with adrenocorticotropin. Anat. Record 106, 345-359. 
Baker, B. L., Ingle, D. J., and Li, C. H. (1951). The histology of the lymphoid organs of rats treated with adrenocorticotropin. Am. J. Anat. 88, 313-349.

Baker, J. R., and Ransom, R. M. (1932). Factors affecting breeding of the field mouse (Microtus agrestis). Part II. Temperature and food. Proc. Roy. Soc. B112, 39-46.

Barkalow, F. S., Jr. (1952). Life history and ecologic observations on the tundra mouse (Microtus oeconomus) and lemmings (Lemmus trimucronatus and Dicrostonyx groenlandicus) at Barter Island, Alaska. J. Elisha Mitchell Sci. Soc. 68, 199-205.

Barker, S. B., and Schwartz, H. S. (1953). Further studies on metabolism of tissues from thyroxine-injected rats. Proc. Soc. Exptl. Biol. Med. 83, 500-502.

Barrett, S. A. (1955). Competition among wild rats. Nature 175, 126-127.

Barnett, S. A. (1958). Physiological effects of "social stress" in wild rats. I. The adrenal cortex. J. Psychosomat. Research 3, 1-11.

Barnett, S. A., and Manly, B. M. (1956). Reproduction and growth of mice of three strains, after transfer to $-3^{\circ} \mathrm{C}$. J. Exptl. Biol. 33, 325-329.

Barraclough, C. A. (1961). Production of anovulatory, sterile rats by single injections of testosterone propionate. Endocrinology 68, 62-67.

Barraclough, C. A., and Gorski, R. A. (1961). Evidence that the hypothalamus is responsible for androgen-induced Sterility in the female rat. Endocrinology 68, 68-79.

Barry, M. C., Eidinoff, M. L., Dobriner, K., and Gallagher, T. F. (1952). The fate of $\mathrm{C}^{14}-$ progesterone in mice and rats. Endocrinology 50, 587-599.

Bartter, F. C. (1956). The role of aldosterone in normal homeostatsis and in certain disease states. Metabolism Clin. and Exptl. 5, 369-383.

Bartter, F. C. (1957). The role of aldosterone in the regulation of body fluid volume and composition. Scand. J. Clin. \& Lab. Invest. 10, 50-61.

Bartter, F. C., and Gann, P. S. (1960). On the hemodynamic regulation of the secretion of aldosterone. Circulation 21, 1016-1023.

Bartter, F. C., Liddle, G. W., Duncan, I. I)., Jr., Barber, J. K., and Delea, C. (1956). The regulation of aldosterone secretion in man: The role of fluid volume. J. Clin. Invest. 35, 1306-1315.

Bartter, F. C., Biglieri, E. G., Pronove, P., and Delea, C. S. (1958). Effect of changes in intravascular volume on aldosterone secretion in man. In "Aldosterone," Intern. Symposium, Geneva, 1957 (A. F. Muller and C. M. O'Connor, eds.), pp. 100-110. Little, Brown, Boston, Massachusetts.

Bartter, F. C., Mills, I. H., Biglieri, E. G., and Delea, C. S., (1959). Studies on the control and physiologic action of aldosterone secretion. Recent Progr. in Hormone Research, 15, 311-344.

Bastenie, P. A., and Ermans, A. M. (1958). Effect of cortisone on the action of thyroxine in hypophysectomized rats. Endocrinology 62, 245-250.

Beer, J. R., and Neyer, R. K. (1951). Seasonal changes in the endocrine organs and behavior patterus of the muskrat. J. Mammal. 32, 173-191.

Bell, G. H., Davidion, J. N., and Scarborough, H. (1950). "Textbook of Physiology and Biochemistry." Williams \& Wilkius, Baltimore, Maryland.

Benua, R. S., and Howard, E. (1950). A earbonyl reaction differentiating the fetal zona reticularis of the human adrenal cortex from the mouse X-zone. Bull. Johns Hopkins Hosp. 86, 200-213.

Berson, S. A., and Yalow, R.S. (1952). The effect of cortisone on the iodine accumulating function of the thyroid gland in euthyroid subjects. J. Clin. Endocrinol. and Metabolism 12, 407-422.

Beyler, A. L., and Szego, C. M. (1954). Steroid hormone interactions as evidenced by modification of $\beta$-glucuronidase activity of the preputial glands of the rat. Endocrinology 54, 334-337. 
Bing, R. J. (1952). The role of coronary circulation in shock. Ann. N. Y. Acad. Sci. $55,367-369$.

Birmingham, M. K., and Kurlents, E. (1955). Inaetivation of ACTH by isolated rat adrenals and inhibition of corticoid formation l,y adrenocortical hormones. Endocrinology 62, 47-60.

Bloch, E., and Cohen, A. I. (1960). In vitro steroid production by normal and adrenal tumor-bearing mice. J. Natl. Cancer Inst. 24, 97-107.

Boas, N. F., and Bates, R. IV. (1954). Role of the thyroid and anterior pituitary glands in the maintenance of the Harderian glands of the rat. Endocrinology 55, 601-612.

Bogdanove, E. M., and Halmi, N. S. (1953). Effect of hypothalamic lesions and subsequent propylthiouracil treatment on pituitary structure in the rat. Endocrinology $53,274-292$.

Bogoroch, R., and Timiras, P. (1951). The response of the thyroid gland of the rat to severe stress. Endocrinology 49, 548-556.

Bohanan, E. H. (1939). Effects of environmental factors on the length of estrus cycles in the rat. Am. J. Hyg. 29.4, 1-10.

Bois, P., and Selye, H. (1957). Enhancement by thryoxine of the adrenal-stimulating effect of somatotrophic hormone. Endocrinology 15, 171-179.

Boring, W. O., Angevine, D. M., and Walker, D. L. (1955). Factors influencing hostvirus interactions. I. A comparison of viral multiplication and histopathology in infant, adult, and cortisone-treated adult mice infected with the Conn.-5 strain of Coxsackie virus. J. Exptl. Med. 102, 753-766.

Boring, W. O., ZuRhein, G. MI., and Walker, 1). L. (1956). Factors influencing host-virus interactions. II. Alterations of Coxsackie virus infection in adult mice by cold. Proc. Soc. Exptl. Biol. Hed. 93, 273-277.

Botkin, A. L., and Tew, J. T. (1952). Thyroict and adrenal response to varying dose levels of epinephrine. Proc. Soc. Exptl. Biol. Mer. 79, 37x-379.

Bourlière, F. (1951). "Vie et moeurs des mimmifères," pp. 1-2.j). Payot, Paris.

Bourne, G. H. (1949). "The Mammalian Arrenal (iland," 1)p. 1-239. Clarendon, ()xford.

Bradlow, H. L., and Gallagher, T. F. (19.77). Metabolism of $11-\beta-1 y$ yroxy- $-^{4}$-androstene3,17-dione in man. J. Biol. Chem. 229, 50;-iss.

Bradlow, H. L., I)obriner, $K$., and Callagher, T. F. (1954). The fate of cortisone-T in micc. Endocrinology 54, 343-352.

Brimblecombe, R. W., Halkerston, I. D. K., and Reiss, II. (1954). The effect of various ACTH extracts on the gonads. $J$. Endocrinol. 10, 291-301.

Brown, R. Z. (1953). Social behavior, reproduction, and population changes in the house mouse. Ecol. Monographs 23, 217-240.

Brown-Grant, K. (1955). Inhibition of the release of thyroidal radioiodine in the rat by cortisone. Endocrinology 56, 607-609.

Brown-Grant, K., Von Euler, C., Harris, (A. IV., and Reichlin, S. (1954a). The measurement and experimental modification of thyroid activity in the rabbit. $J$. Physiol. (London) 126, 1-28.

Brown-Grant, K., Harris, G. W., and Reichlin, S. (1954b). The effect of emotional and physical stress on thyroid activity in the rabbit. J. Physiol. (London) 126, 29-40.

Brown-Grant, K., Harris, G. W., and Reichlin, S. (1954c). The influence of the adrenal cortex on thyroid activity in the ribbit. $J$. Physiol. (Landon) 126, 41-51.

Brown-Grant, K., Harris, (. W. , and Reichlin, S. (1957). The effect of pituitary-stalk section on thyroid function in the rabbit. J. Physiol. (London) 136, 364-379.

Bruce, H. M., and Sloviter, H. A. (1957). liffect of destruction of thyroid tissue by radioactive iodine on reproduction in mice. .J. Endocrinol. 15, 72-\$2.

Bull, P. C. (1955). Population regulation in rabhit nematodes. Nature 175, 215. 
Bull, P. C. (1957). Changing incidence of parasites in a declining population. Proc. New Zealand Ecol. Soc. 5, 11-12.

Bull, P. C. (1958). Incidence of Coccidia (Sporozoa) in wild rabbits Oryctolagus cuniculus (L) in Hawke's Bay, New Zealand. New Zealand J. Sci. 1, 289-328.

Bullough, W. S. (1952). Stress and epidermal mitotic activity. 1. The effects of the adrenal hormones. J. Endocrinol. 8, 265-274.

Bullough, W. S. (1955). Hormones and mitotic activity. Vitamins and Hormones 13, 261-292.

Burn, J. H., and Rand, M. J. (1957). Reserpine and adrenaline in artery walls. Lancet 273, 1097.

Burn, J. H., and Rand, M. J. (195Sa). Noradrenaline in artery walls and its dispersal. Brit. Med. J. I, 903-908.

Burn, J. H., and Rand, M. J. (1958b). The action of sympathomimetic amines in animals treated with reserpine. $J$. Physiol. (London) 144, 314-336.

Burrows, H. (1949). "Biological Actions of Sex Hormones," 2nd ed. Cambridge Univ. Press, London and New York.

Bush, I. E. (1953). Species differences in adrenocortical secretion. J. Endocrinol. 9, 95100.

Byrnes, W. W., and Meyer, R. K. (1951). The inhibition of gonadotropic hormone secretion by physiological doses of estrogen. Endocrinology 48, 133-136.

Byrnes, W. W., and Shipley, E. G. (1950). Estrogenic and gonadotrophic hormone inhibiting activity of some adrenal cortical substances. Proc. Soc. Exptl. Biol. Med. 74, 308-310.

Cain, A. J., and Harrison, R. G. (1950). Cytological and histochemical variations in the adrenal cortex of the albino rat. J. Anat. 84, Pt. 2, 196-226.

Calhoun, J. B. (1948). Mortality and movement of brown rats in artificially supersaturated populations. J. Wildlife Management 12, 167-171.

Calhoun, J. B. (1949). A method for self-control of population growth among mammals living in the wild. Science 109, 333-335.

Calhoun, J. B. (1950). The study of wild animals under controlled conditions. Ann. N.Y. Acad. Sci. 51, 1113-1122.

Cannon, W. B. (1915). "Bodily Changes in Pain, Hunger, Fear and Rage." Appleton, New York.

Cannon, W. B. (1932). "The Wisdom of the Body." Norton, New York.

Cater, O. B., and Stack-Dunne, M. P. (1953). The histological changes in the adrenal of the hypophysectomized rat after treatment with pituitary preparations. J. Pathol. Bacteriol. 66, 119-133.

Cater, O. B., and Stack-Dunne, M. P. (1955). The effects of growth hormone and corticotrophin upon the adrenal weight and adrenocortical mitotic activity in the hypophysectomized rat. $J$. Endocrinol. 12, 174-184.

Cavazos, L. F., and Melampy, R. M. (1954). Cytological effects of testosterone propionate on epithelium of rat seminal vesicles. Endocrinology 54, 640-648.

Chang, M. C., and Pincus, G. (1951). Physiology of fertilization in mammals. Physiol. Revs. 31, 1-26.

Chitty, D. (1952). Mortality among voles (Microtus agrestis) at Lake Vyrnwy, Montgomeryshire in 1936-9. Phil. Trans. Roy. Soc. London B236, 505-552.

Chitty, D. (1954). Tuberculosis among wild voles with a discussion of other pathological conditions among certain mammals and birds. Ecology 35, 227-237.

Chitty, D. (1955). Adverse effects of population density upon the viability of later generations. In "The Numbers of Man and Animals," pp. 57-67. Oliver \& Boyd, Edinburgh. 
Chitty, D. (1957). Self-regulation of numbers through changes in viability. Cold Spring Harbor Symposia Quant. Biol. 22, 277-280.

Chitty, H., and Austin, C. R. (1957). Environmental modification of oestrus in the vole. Nature 179, 592-593.

Chitty, D., Chitty, H., Leslie, P. H., and Scott, J. C. (1956). Changes in the relative size of the nucleus in the intervertebral disc of stressed orkney voles (Microtus orcadensis). J. Pathol. Bacteriol 72, 459-470.

Christian, J. J. (1950a). A field method of determining the reproduction status of small male mammals. J. Mammal. 31, 95-96.

Christian, J. J. (1950b). The adreno-pituitary system and population cycles in mammals. J. Mammal. 31, 247-259.

Christian, J. J. (1951). The mammals of the Mazinaw lake region of Ontario; their reproduction and population dynamies. Ann. Carnegie Museum 31, 339-386.

Christian, J. J. (1954). The Relation of the Adrenal Cortex to Population Size in Rodents. Doctoral dissertation, Johns Hopkins School of Hygiene and Public Health, Baltimore, Maryland.

Christian, J. J. (1955a). Effect of population size on the weights of the reproductive organs of white mice. Am. J. Physiol. 181, 477-480.

Christian, J. J. (1955b). Effect of population size on the adrenal glands and reproductive organs of male mice in populations of fixed size. Am. J. Physiol. 182, 292-300.

Christian, J. J. (1956). Adrenal and reproductive responses to popultaion size in mice from freely growing populations. Ecology 37, 25S-273.

Christian, J. J. (1959a). Control of population growth in rodents by interplay between population density and endocrine physiology. Wildlife Disease 1, 1-3S.

Christian, J. J. (1959b). The roles of endocrine and behavioral factors in the growth of mammalian populations. In "Comparative Endocrinology," Proc. Columbia University Symposium, 1958 (A. Gorbman, ed.), pp. 71-97. Wiley, New York.

Christian, J. J. (1959c). Adrenocortical, splenic and reproductive responses to inanition and to grouping. Endocrinology 65, 189-197.

Christian, J. J. (1959d). Lack of correlation between adrenal weight and injury from fighting in grouped male albino mice. Proc. Soc. Exptl. Biol. Med. 101, 166-16s.

Christian, J. J. (1960). Adrenocortical and gonadal responses of female mice to increased population density. Proc. Soc. Exptl. Biol. Med. 104, 330-332.

Christian, J. J. (1961). Phenomena associated with population density. Proc. Natl. Acad. Sci. U. S. 47, 42S-449.

Christian J. J. (1963a). The pathology of overpopulation. Military Med. 128, 571-603.

Christian, J. J. (1963b). Interrelated social and endocrine factors in populations of rodents. (Abstract.) Proc. 16th Intern. Congr. Zool., Washington, D. C., 3, S-13.

Christian, J. J., and Davis, D. E. (1955). Reduction of adrenal weight in rodents by reducing population size. Trans. N. Am. Wildlife Conf. 20, 177-189.

Christian, J. J., and Davis, D. E. (1956). The relationship between adrenal weight and population status in Norway rats. J. Mammal. 37, 475-486.

Christian, J. J., Flyger, V., and Davis, D. E. (1960). Factors in mass mortality of a herd of sika deer. Chesapeake Sci. 1, 79-95.

Christian, J. J., and LeMunyan, C. D. (1958). Adverse effects of crowding on lactation and reproduction of mice and two generations of their offspring. Endocrinology 63, $517-529$.

Christian, J. J., and Ratcliffe, H. L. (1952). Shock disease in captive wild mammals. Am. J. Pathol. 28, 725-737.

Christian, J. J., and Williamson, H. O. (1958). Effect of crowding on experimental granuloma formation in mice. Proc. Soc. Exptl. Biol. Med. 99, 385-387. 
Christianson, M., and Jones, I. C. (1957). The interrelationships of the adrenal glands of mother and fetus in the rat. $J$. Endocrinol. 15, 17-42.

Chu, J. P. (1944). The influence of the thyroid on pregnancy and parturition in the rabbit. J. Endocrinol. 4, 109-114.

Clarke, J. R. (1953). The effect of fighting on the adrenals, thymus, and spleen of the vole (Microtus agrestis). J. Endocrinol. 9, 114-126.

Clarke, J. R. (1955). Influence of numbers on reproduction and survival in two experimental vole populations. Proc. Roy. Soc. B144, 68-85.

Clayton, G. W., Bell, WT. R., and Guillemin, R. (1957). Stimulation of ACTH-release in humans by non-pressor fraction from commercial extracts of posterior pituitary. Proc. Soc. Exptl. Biol. Med. 96, 777-7ъ9.

Cole, D. F. (1957). Effect of aldosterone on renal excretion of intravenously administered saline, Endocrinology 60, 562-565.

Cook, E. F., and Beer, J. R. (195S). A study of louse populations on the meadow vole and deer mouse. Ecology 39, 645-659.

Crew, F. A., and Mirskaia, L. (1931). The effects of density on an adult mouse population. Biol. Generalis 7, 239-250.

Critchlow, B. V., and Sawyer, C. H. (1955). Electrical activity of the rat brain correlated with neurogenic stimulation of the adenohypophysis. Federation Proc. 14, $32-33$.

Croweroft, P. (1954). Aggressive behavior in wild house mice (Mus musculus L.) Ministry of Agr. and Fish Infestation Control Division, Research Rept. No. 35.

Croweroft, P. and Rowe, F. P. (195i). The growth of confined colonies of the wild house mouse (Mns musculus L.). Proc. Zool. Soc. (London) 129, 359-370.

Curley, F. J., and Ingalls, T. H. (1957). Hypoxia at normal atmospheric pressure as a cause of congenital malformations in mice. Proc. Soc. Exptl. Biol. Med. 94, 87-88.

I)'Angelo, S. A. (1955). Pituitary regulation of thyroid gland function. Brookhaven Symposia in Biol. 7, 9-29.

1)'Angelo, S. A., and Traum, R. E. (1958). An experimental analysis of the hypothalamic hypophyseal-thyroid relationship in the rat. Ann. N. Y. Acad. Sci. 72, 239-270.

I) Angelo, S. A., Gordon, A. S., and Charipper, H. A. (194S). A differential response of the rodent adrenal gland to acute starvation. Proc. Soc. Exptl. Biol. Med. 68, 527531 .

Davidson, C. s. (1937). Effect of adrenotropic extract upon the accessory reproductive organs of castrated rats. Proc. Soc. Exptl. Biol. Med. 36, 703-705.

1)avidson, C. S., and Mloon, H. D. (1936). Effect of adrenocorticotropic extracts on accesiory reproductive organs in castrate rats. Proc. Soc. Exptl. Biol. Med. 35, $281-2 \times 2.2$.

I)avis, D. E. (1951a). The relation between level of population and pregnancy of Norway rate. Ecology 32, 459-461.

Davis, D. E. (1951b). The relation between level of population and size and sex of Norway rats. Ecology 32, 462-164.

Davis, D. E. (1951c). The relation between the level of population and the prevalence of Leptospira, Salmonella, and capillaria in Noway rats. Ecology 32, 465-46S.

Davis, D. E. (1953). The characteristics of rat populations. Quart. Rev. Biol. 28, 373-401.

Davis, D. E., and Christian, J. J. (1957). Relations of adrenal weight to social rank of mice. Proc. Soc. Exptl. Biol. Med. 94, 728-731.

Davis, D. E., and Christian, J. J. (1958). Population consequences of a sustained yield program for Norway rats. Ecology 39, 217-222.

Divis, D. E., and Jensen, W. L. (1952). Mortality in an induced epidemic. Trans. N. Am. Willlife Conf. 17, 151-160. 
Davis, D. E., and Prudovsky, S. (1959). Effect of behavior on develepment of resistance to tetanus toxin. Unpublished.

Davis, D. E., and Read, C. P. (1958). Effect of behavior on development of resistance in trichinosis. Proc. Soc. Exptl. Biol. Med. 99, 269-272.2.

Davis, J. O. (1963) The role of the adrenal cortex and the kidney in the pathogenesis of eardiac edema. Yale J. Biol. Mert. 35, 402-42s.

Davis, J. O., Goodkind, M. J., Pechet, M. M., and Ball, W. C., J1. (1956). Inereased exeretion of aldosterone in urine from dogs with right-sided congestive heart failure and from dogs with thoracic inferior vena eava constriction. Am. J. Physiol. 187, $45-50$.

Davis, J. O., Pechet, M. M., Ball, WV. C., Jr., and Croodkind, M. J. (1957). Increased aldosterone secretion in dogs with rightsiderl eongestive heart failure and in dogs with thoracic inferior vena eava constriction. J. Clin. Invest. 36, 659.

Davis, J. O., Kliman, B., Yankopoulos, N. A., Peterson, R. E., and Casper, A. (1958). Increased aldosterone seeretion following acute constriction of the inferior vena cava. J. Clin. Invest. $37,17 \$ 3-1790$.

Davis, J. O., Bahn, R. C., Yankopoulos, N. A., Kliman, B., and Peterson, R. L. (1959a). Acute effects of hypophyseetomy and diencephalic lesions on aldosterone secretion. A m. J. Physiol. 197, 380-386.

Davis, J. O., Yankopoulos, N. A., and Holman, J. (19591)). Chronic effects of carotid sinus denervation cervical vagotomy and aortic depreson nerve section on aldosterone and sodium exeretion. Am. J. Physiol. 197, 207-210.

Davis, M. E., and Plotz, E. J. (1954). The effects of cortisone acetate on intact and adrenalectomized rats during pregnancy. Endocrinology 54, 384-395.

Dawson, J. (1956). Splenie hypertrophy in voles. Nature 178, 1183-1184.

Deane, H. W., and Morse, A. (1948). The eytological distribution of ascorbic acid in the adrenal cortex of the rat under normal and experimental conditions. Anat. Record $100,127-141$.

Deane, H. W., and Seligman, A. M. (1953). Evaluation of procedures for the cytological localization of ketosteroids. Titamins and Hormones 11, 173-204.

Deane, H. W., Shaw, J. H., and Greep, R. (). (1945). The effect of altered sodium of potassium intake on the width and eytochemistry of the zona glomerulosa of the rat's adrenal cortes. Endocrinology 43, 13:3-153.

Deanesly, R. (1928). A study of the adrenal cortex in the mouse and its relation to the gonads. Proc. Roy. Soc. B103, 523-546.

DeBodo, R. C., and Altzuler, N. (1958). Insulin hypersensitivity and physiological insulin antagonists. Physiol. Revs. 38, 389-145.

Delost, P. (1951). Structure histologique et étude l'activité phosphatasique alcaline du canal deférent du campagnol des chanps (Microtus arvalis, P.) état du cortex surrénal. Compt. rend. soc. biol. 145, 373-377.

Delost, P. (1952a). Le cortex surrénal du campagnol des chanps (Microtus arvalis, P.) et ses modifieations après castration. Compt. rend. soc. biol. 146, 27-31.

Delost, P. (1952b). Modifications histochimiques du cortex surrénal du eampagnol des champs (Microtus arvalis, P.) après castration. Compt. rend. soc. biol. 146, 1080-1083.

Delost, P. (1954). Action involutive de la cortisone sur la zone $\mathrm{X}$ surrénalienne du campagnol des champs (Microtus arvalis, P.). Compt. rend. soc. biol. 148, 1161-1163.

Delost, P. (1955). Role de l'ovaire et du cortex surrénal dans la stimulation de la prostate femelle chez les rongeurs sauvages. Compt. rend. soc. biol. 149, 1336-1339.

Delost, P. (1956a). Les corrélations génito-surrénaliennes chez le campagnol des champs (Microtus arvalis, P.). Ann. Sci. nat. Zool. 11. 18, 392-567. 
Delost, P. (1956b). Donnees expérimentales comparées sur la zone $\mathrm{X}$ surrénalienne. Action de la cortisone et de la testosterone. Compt. rend. soc. biol. 150, 1330-13332.

Delost, P. (1957). Recherches sur le cortex surrénal et la zone $\mathbf{X}$ des musaraignes (Sorex araneus L. et Crocidura russula H.). Compt. rend. soc. biol. 151, 484-187.

Delost, P., and Delost, H. (1954). Existence d'une zone X surrénalienne chez le campagnol roussâtre (Clethrionomys glareolus, S.). Compt. rend. soc. biol. 148, 1788-1793.

Delost, P., and Delost, H. (1955). Modifications ponderales et histologiques des glandes surrénales du campagnol agreste (Microtus agrestis, L.) en fonction de l'état sexual. Compt. rend. soc. biol. 149, 910-914.

Dempsey, E. W. (1948). The chemical cytology of endocrine glands. Recent Progr. in Hormone Research 3, 127-157.

Dempsey, E. W., and Uotila, U. U. (1940). The effect of pituitary stalk section upon reproductive phenomena in the female rat. Endocrinology 27, 573-579.

Denison, M. E., and Zarrow, M. X. (1955). Changes in estrous cycle of rat during prolonged exposure to cold. Proc. Soc. Exptl. Biol. Med. 89, 632-634.

Desclin, J. (1959). Masculinisation spontanée et expérimentale des glandes sous-maxillaires chez la souris femelle. Compt. rend. acad. sci. 248, 597-600.

Dorfman, A. (1953). The effects of adrenal hormones on connective tissues. Ann. N. Y. Acad. Sci. 56, 698-703.

Dorfman, R. I. (1949). The bioassay of adrenocortical steroids. Ann. N. Y. Acad. Sci. 50, 556-575.

Dorfman, R. I. (1959). Comparative biochemistry of the adrenocortical hormones. In "Comparative Endocrinology," Proc. Columbia University Symposium, 1958 (A. Gorbman, ed.), pp. 613-623. Wiley, New York.

Dorfman, R. I. (1960). Steroid metabolism in endocrine tumors. In "Biological Activities of Steroids in Relation to Cancer"' (G. Pincus and E. P. Vollmer, eds.), pp. 93110. Academic Press, New York.

Dorfman, R. I., and Shipley, R. A. (1956). "Androgens. Biochemistry Physiology and Clinical Significance." Wiley, New York.

Dougherty, T. F. (1952a). Studies of the antiphlogistic and antibody suppressing functions of the pituitary adrenocortical secretions. Recent Progr. in Hormone Research 7, 307-330.

Dougherty, T. F. (1952b). Effect of hormones on lymphatic tissue. Physiol. Revs. 32, 379-401.

Dougherty, T. F. (1953). Some observations on mechanisms of corticosteroid action on inflamnation and immunologic processes. Ann. N. Y. Acad. Sci. 56, 748-756.

Dougherty, T. F., and Schneebeli, G. L. (1955). The use of steroids as anti-inflammatory agents. Ann. N.Y. Acad. Sci. 61, 32S-348.

Drager, G. A. (1950). Neurosecretion following hypophysectomy. Proc. Soc. Exptl. Biol. Med. 75, 712-713.

Ehrich, W. E., and Seifter, J. (1948). Role played by salivary glands in the alarm rereaction. A. M. A. Arch. Pathol. 45, 239-245.

Eik-nes, K. (1959). Comments on a paper by J. W. Mason. Recent Progr. in Hormone Research 15, 380-381.

Eisenstein, A. B. (1957). Pantothenic acid and adrenocortical hormone secretion. Endocrinology 60, 298-302.

Eisenstein, A. B. (1959). Relationship of vitamin $B_{6}$ to adrenocortical function in the rat. Proc. Soc. Exptl. Biol. Med. 100, 111-114.

Eisenstein, A. B., and Hartroft, P. M. (1957). Alterations in the rat adrenal cortex induced by sodium deficiency: Steroid hormone secretion. Endocrinology 60, 634-640. 
Elias, H. (1948). Growth of the adrenal cortex in domesticated Ungulata. Am. J. Vet. Research 9, 173-1S9.

Elias, H., and Pauly, J. E. (1956). The structure of the human adrenal cortex. Endocrinology $\mathbf{5 8}, \mathbf{7 1 4 - 7 3 8 .}$

Elmadjian, F., Hope, J. M., and Lamson, E. T. (1955). Excretion of epinephrine and norepinephrine under stress. Recent Progr. in Hormone Rescarch 14, 513-553.

Elton, C. (1942). "Voles, Mice and Lemmings." Clarendon, Oxford.

Engel, F. L. (1952). The significance of the metabolic changes during shock. Ann. N. Y. Acad. Sci. 55, 381-393.

Eränkö, O., and Hopsu, V. (1958). Effect of reserpine on the histochemistry and content of adrenaline and noradrenaline in the adrenal medulla of the rat and the mouse. Endocrinology 62, 15-23.

Eränkö, O., and Räisänen, L. (1957). Adrenaline and noradrenaline in the adrenal medulla during postnatal development of the rat. Endocrinology 60, 753-760.

Eränkö, O., Hopsu, V., and Räisänen, L. (1959). The effect of prolonged administrations of nicotine on the medullary volume and the distribution of noradrenaline in the adrenals of the rat, the mouse, and the guinea pig. Endocrinology 65, 293-297.

Erickson, A. B. (1944). Helminth infections in relation to population fluctuations in snowshoe hares. J. Wildlife Management 8, 134-153.

Errington, P. L. (1943). An analysis of mink predation upon muskrats in North-central United States. Iowa State College Agr. Expt. Sta. Bull. No. 320.

Errington, P. L. (1918). Ecology of the muskrat. Report on Agr. Research for year-ending June 30, 1948. Iowa State College Agr. Expt. Sta. pp. 230-232.

Errington, P. L. (1951). Concerning fluctuations in populations of the prolific and widely distributed muskrat. Am. Naturalist 85, 273-292.

Errington, P. L. (1954a). On the hazards of overemphasizing numerical fluctuations in studies of "cyclic" phenomena in muskrat populations. J. Wildlife Management 18, 66-90.

Errington, P. L. (1951b). The special responsiveness of minks to epizootics in muskrat populations. Ecol. Monographs 24, 377-393.

Errington, P. L. (1957). Of populations, cycles and unknowns. Cold Spring Harbor Symposia Quant. Biol. 22, 287-300.

Ershoff, B. H. (1948). Effects of thiouracil feeding on resistance to low environmental temperature. Endocrinology 43, 36-39.

Evans, E. S., Simpson, M. E., and Evans, H. M. (1955). The role of growth hormone in calorigenesis and thyroid function. Endocrinology $63,836-852$.

Everett, J. W. (1956). Functional corpora lutea maintained for months by autografts of rat hypophyses. Endocrinology 58, 786-796.

Everett, J. W. (1959). Neuroendocrine mechanisms in control of the mammalian ovary. In "Comparative Endocrinology Proc. Columbia University Symposium," $195 \mathrm{~S}$ (A. Gorbman, ed.), pp. 74-1S6. Wiley, New York.

Everett, J. W., and Sawyer, C. H. (1953). Estimated duration of the spontaneous activation which causes release of ovulation hormone from the rat hypophysis. Endocrinology 52, 83-92.

Farrell, G. L. (1958). Regulation of aldosterone secretion. Physiol. Revs. 38, 709-728.

Farrell, G. L. (1959a). The physiological factors which influence the secretion of aldosterone. Recent Progr. in Hormone Research 15, 275-310.

Farrell, G. L. (1959b). Steroidogenic properties of extracts of beef diencephalon. Endocrinology 65, 29-33. 
Farrell, G. L., and Laqueur, G. (1955). Reduction of pituitary content of ACTH by cortisone. Endocrinology 56, 471-473.

Farrell, G. L., Rauschkolb, E. W., and Royce, P. C. (1955). Secretion of aldosterone by the adrenal of the dog. Effects of hypophysectomy and ACTH. Am.J. Physiol. 182, 269-27:.

Farrell, G. L., Fleming, R. B., Rauschkoll, E. W., Yatsu, F. M., MeCally, M., and Anderson, C. H. (1958). Steroidogenic properties of purified corticotropins. Endocrinology 62, 506-512.

Feldman, J. D. (1950). Histochemical reactions of adrenal cortical cells. Anat. Record $107,3+7-358$.

Feldman, J. D. (1951). Endocrine control of the adrenal gland. Anat. Record 109, 41-69.

Fitch, H.S. (1957). Aspects of reproduction and development in the prairie vole (Microtus ochrogaster). Univ. Kansas Museum Nat. Hist. Publ. 10, 129-161.

Flexner, L. B., and Grollman, A. (1939). The reduction of osmic acid as an indicator of adrenal cortical activity in the rat. Anat. Record 75, 207-221.

Flick, S., Davis, D. E., and Christian, J. J. (1959). Effect of constant length of day on reproduction in albino mice. Brit. J. Hyg. 57, 193-197.

Flückiger, E., and Verzár, F. (195̃)). Thyroid function after adrenalectomy. J. Endocrinol. 13, 39-45.

Folley, S. J. (1956). "The Physiology and Biochemistry of Lactation." C. C. Thomas, Springfield, lllinois.

Fortier, C. (1956). Hypothalamic control of the adrencorticotrophic function 20th Intern. Congr. Physiol., Brussels, Abstr. of Revs. 1-1S.

Fortier, C. (1957). Neuroendocrine relationships. Progr. in Neurol. and Psychiat. 12, $252-261$.

Fortier, C. (1959a). Pituitary ACTH and plasma free corticosteroids following bilateral adrenalectomy in the rat. Proc. Soc. Exptl. Biol. Med. 100, 13-16.

Fortier, C. (1959b). Effect of hydrocortisone on pituitary ACTH and adrenal weight in the rat. Proc. Soc. Exptl. Biol. Med. 100, 16-19.

Frank, F. (1953). Untersuchungen über den Zusammenbruch von Feldmausplagen (Microtus arvalis Pallas). Zool. Jahrb. Jena. 82, 95-136.

Fraser, F. C., Fainstat, T. D., and Kalter, H. (1953). The experimental production of congenital defects with particular reference to cleft palate. Nco-Natal Studies 2, $43-58$.

Fredrickson, 1). s., and (iordon, 1R. S., Jr. (195s). Transport of fatty acids. Physiol. Revs. $38,555-630$.

Freedman, H. H., and (Fordon, A.S. (195i)). Endocrine factors in response of the hypothyroid rat to cold. Endocrinology 56, 135-142.

Gaddum, J. H., and Holzbaucr, M. (1957). Adrenaline and noradrenaline. V'itamins and Hormones 15, 152-205.

Gallagher, T. F. (1958). Steroid hormone metabolism and the control of adrenal secretion. Harvey Lectures Ser. 52, 1-22.

Gallagher, T. F., Kappas, A., Hellman, L., Lipsett, M. B., Pearson, O. H., and West, C. I). (19:5). Adrenocortical hyperfunction in "idiopathic" hirsutism and the Stein-Leventhal syndrome. Abstr. Endocrine Soc. Program, p. 15.

Ganong, W. F. (1959). Adrenal-hypophyseal interrelations. In "Comparative Endocrinology," Proc. Columbia University Symposium, 1955 (A. Corbman, ed.), pp. 157-201.

(iannt, R. Renzi, A. A., and Chart, J. J. (1955). Aldosterone: a review. J. C'lin. Endocrinol. and Metabolism 15, 621-646. 
Gersh, I., and Grollman, A. (1939). The nature of the X-zone of the adrenal gland of the mouse. Anat. Record 75, 131-154.

Gersh, I., and Grollman, A. (1941). The vaseular pattern of the adrenal gland of the mouse and rat and its physiological response to changes in glandular activity. Carnegie Inst. Wash. Publ. No. 525, 111-126.

Gerwing, J. (1958). The effect of continued toxic stress on the activity of the thyroid gland in the rat and the guinea-pig. J. Physiol. (London) 144, 243-249.

Gerwing, J., Long, D. A., and Pitt-Rivers, R. (1958). The influence of bacterial exotoxins on the activity of the thyroid gland in different speeies. J. Physiol. (London) 144, $229-242$.

Giroud, C. J. P., Stachenko, J., and Piletta, P. (1958). In vitro studies of the functional zonation of the adrenal cortex and of the production of aldosterone. In "Aldrosterone," Intern. Symposium, Geneva, 1957 (A. F. Muller and C. M. O'Connor, eds.), pp. 56-72, Little, Brown, Boston, Massachusetts.

Giroud, C. J. P., Stachenko, J., and Venning, E. H. (1956). Secretion of aldosterone by the zona glomerulosa of rat adrenal glands incubated in vitro. Proc. Soc. Exptl. Biol. Mcd. 92, 154-158.

Glaubach, S. (1952). Effect of exeessive doses of cortisone, ACTH and prolactin in pregnant and nursing mice. J. Mt. Sinai Hosp. N. Y. 19, st-90.

Glick, D., and Ochs, M. J. (1955). Studies in histochemistry: Quantitative histologieal distribution of cholesterol in adrenal glands of the cow, rat and monkey, and effeets of stress conditions, ACTH, cortisone and desoxyeorticosterone. Endocrinology 56, 285-298.

Godfrey, G. K. (1955). Observations on the nature of the decline in numbers of two Microtus populations. J. Mammal. 36, 209-214.

Goldberg, R. C., Wolff, J., and Greep, R. O. (1957). Studies on the nature of the thyroidpituitary interrelationship. Endocrinology 60, 38-52.

Goldfien, A., Zileli, M. S., Despointes, R. H., and Bethune, J. E. (1958). The effeet of hypoglycemia on the adrenal seeretion of epinephrine and norepinephrine in the dog. Endocrinology 62, 749-757.

Goodman, H. M., and Knolil, E. (1959). Mobilization of fatty aeids by epinephrine in normal and hypophysectomized rhesus monkeys. Proc. Soc. Exptl. Biol. Med. 100, 195-197.

Gordon, A. S. (1955). Some aspects of hormonal influenees upon the leukocytes. $1 \mathrm{nn}$. N. Y. Acad. Sci. 59, 907-927.

Gordon, A. S., and Katsh, (. F. (1949). The relation of the adrenal cortex to the strueture and phagocytic aetivity of the maerophagic system. Ann. N. Y. Acarl. Sci. $52,1-30$.

Grad, B., and Lchlond, C. P. (1949). The necessity of testis and thyroid hormones for the maintenance of the serous tubules of the submaxillary glands in the male rat. Endocrinology 45, 250-266.

Gray, I., and Beetham, W. P., Jr. (1957). Changes in plasma concentration of epinephrine and norepinephrine with museular work. P'roc. Soc. Exptl. Biol. Med. 96, 636-638.

Gray, S. J., and Ramey, C. G. (1957). Adrenal influence upon the stomach and the gastric responses to stress. Recent Progr. in Hormone Research 13, 583-617.

Green, J. D. (1951). The comparative anatomy of the hypophysis, with speeial reference to its blood supply and innervation. Am. J. Anat. 88, 225-312.

Green, R. G., and Evans, C. A. (1940a). Studies on a population of snowshoe hares on the Lake Alexander area. I. Gross annual censuses 1932-1939. J. Wildlife Management $4,220-238$. 
Green, R. G., and Evans, C. A. (1940b). Studies on a population of snowshoe hares on the Lake Alexander area. II. Mortality according to age groups and seasons. $J$. Wildlife Management 4, 267-278.

Green, R. G., and Evans, C. A. (1940c). Studies on a population of snowshoe hares on the Lake Alexander area. III. Effect of reproduction and mortality of young hares on the cycle. $J$. Wildlife Management 4, 347-35s.

Green, R. G., and Larson, C. L. (1938). A description of shock disease in the snowshoe hare. Am. J. Hyg. 28, 190-212.

Green, R. G., Larson, C. L., and Bell, J. F. (1939). Shock disease as the cause of the periodic decimination of the snowshoe hare. Am. J. Hyg. 30B, 83-102.

Greene, R. R., Burrill, M. IV., and Ivy, A. C. (1941). Experimental intersexuality: The effects of combined estrogens and androgens on the embryonic sexual development of the rat. J. Exptl. Zool. 87, 211-232.

Greep, R. O., and Deane, H. W. (1947). Cytochemical evidence fo the cessation of hormone production in the zona glomerulosa of the rat's adrenal cortex after prolonged treatment with desoxycorticosterone acetate. Endocrinology 40, 417-425.

Greep, R. O., and Deane, H. W. (1949a). Histological, cytochemical and physiological observations on the regeneration of the rat's adrenal gland following enucleation. Endocrinology 45, 42-56.

Greep, R. O., and Deane, H. W. (1949b). The cytology and cytochemistry of the adrenal cortex. Ann. N. Y. Acad. Sci. 50, 596-615.

Greer, M. A. (1951). Evidence of hypothalamic control of the pituitary release of thryotrophin. Proc. Soc. Exptl. Biol. Med. 77, 603-60s.

Greer, M. A. (1952). The role of the hypothalamus in the control of thyroid function. J. Clin. Endocrinol. and Mctabolism 12, 1259-1268.

Greer, M. A. (1957). Studies on the influence of the central nervous system on anterior pituitary function. Recent Progr. in Hormone Research 13, 67-104.

Gross, F., and Lichtlen, F. (1958). Further evidence for a qualitative difference between aldosterone and cortexone. In "Aldosterone," Intern. Symposium, Geneva, 1957 (A. F. Muller and C. M. O'Connor, eds.), pp. 39-55. Little, Brown, Boston, Massachusetts.

Gross, J. (1955). The distribution of radioactive thyroid hormone in tissues. Brookhaven Symposia in Biol. No. 7, 102-110.

Grosvenor, C. E., and Turner, C. W. (1959a). Effect of growth hormone and oxytocin upon milk yield in the lactating rat. Proc. Soc. Exptl. Biol. Ned. 100, 15S-161.

Grosvenor, C. E., and Turner, C. W. (1959b). Effect of growth hormone upon thyroid secretion rate in the rat. Proc. Soc. Exptl. Biol. Med. 100, 70-72.

Grosvenor, C. E., and Turner, C. W. (1959c). Thyroid hormone and lactation in the rat. Proc. Soc. Exptl. Biol. Med. 100, 162-165.

Guillemin, R. (1955). A re-evaluation of acetylcholine, adrenaline, noradrenaline and histamine as possible mediators of the pituitary adrenocorticotorphic activation by stress. Endoerinology 56, 24S-255.

Guillemin, R., Hearn, W. R., Cheek, W. R., and Housholder, D. E. (1957). Control of corticotrophin release: Further studies with in vitro methods. Endocrinology 60, 488-506.

Guillemin, R., Clayton, G. W., Smith, J. D., and Lipscomb, H. S. (1958). Measurement of free corticosteroids in rat plasma: Physiological validation of a method. Endocrinology 63, 349-35s.

Hagen, P., and Welch, A. D. (1956). The adrenal medulla and the biosynthesis of pressor amines. Recent Progr. in Hormone Research 12, 27-44. 
Halmi, N. S., Spirtos, B. N., Bogdanove, E. M. and Lipner, H. J. (1953). A study of various influences on the iodide concentrating mechanism of the rat thryoid. Endocrinology 52, 19-32.

Hamilton, W. J., Jr. (1937). The biology of microtine cycles. J. Agr. Research 54, 779790.

Hammond, J., Jr. (1954). Light regulation of hormone secretion. Vitamins and Hormones 12, 157-206.

Hamolsky, M. W., Gierlach, Z. S., and Jensen, H. (1951). Uptake and conversion of radioactive iodine ( $\left.\mathrm{I}^{131}\right)$ by the thyroid gland in vivo and in vitro in tourniquet shock in rats. Am. J. Physiol. 164, 35-53.

Harris, G. W. (1955a). The reciprocal relationship between the thyroid and adrenocortical responses to stress. CIBA Foundation Colloq. on Endocrinol. 8, 531-550.

Harris, G. W. (1955b). "Neural Control of the Pituitary Gland." Arnold, London.

Harris, G. W. (1959). Neuroendocrine control of TSH regulation. In "Comparative Endocrinology." Proc. Columbia University Symposium, 1958 (A. Gorbman, ed.), pp. 202-222. Wiley, New York.

Harris, G. W., and Fortier, C. (1954). The regulation of anterior function, with special reference to the secretion of adrenocorticotrophic hormone. In "4th Anmual Review of Stress" (H. Selye and G. Heuser, eds.), pp. 106-127. Acta, Montreal.

Harris, G. W., and Woods, J. W. (1958). The effect of electrical stimulation of the hypothalamus or pituitary gland on thyroid activity. J. Physiol. (London) 143, $246-274$.

Harrison, R. G. (1957). The adrenal circulation in the rabbit. J. Endocrinol. 15, 64-71.

Harrison, R. G. (1951). A comparative study of the vascularization of the adrenal gland in the rabbit, rat, and cat. J. Anat. 85, 12-23.

Harrison, R. G., and Asling, C. W. (1955). The anatomy and functional significance of the vascularization of the adrenal gland in the rhesus monkey (Macaca mulatta). J. Anat. 89, 106-113.

Hart, P., and Rees, R. J. W. (1950). Enhancing effect of cortisone on tuberculosis in the mouse. Lancet ii, 391-395.

Hartman, F. A., and Brownell, K. A. (1949). "The Adrenal Glands." Lea \& Febiger, Philadelphia, Pennsylvania.

Hartroft, P. M., and Eisenstein, A. B. (1957). Alterations in the adrenal cortex of the rat induced by sodium deficiency: Correlation of histologic changes with steroid hormone secretion. Endocrinology 60, 641-651.

Hatfield, D. M. (1935). A natural history study of Microtus californicus. J. Mammal. 16, 261-270.

Hellman, K., and Collins, J. (1957). Thyroid, salivary and Harderian glands in mice exposed to heat. J. Endocrinol. 15, 145-150.

Hess, M. (1953). Effect of thyroidectomy in adrenal weight in adult male rats. Proc. Soc. Exptl. Biol. Med. 84, 127-130.

Hess, M., and Finerty, J. C. (1952). The effect of hypo- and hyper-thyroidism on the pituitary-adrenal response to stress. Texas Repts. Biol. and Med. 10, 554-570.

Hess, M., Hall, O., Hall, C. E., and Finerty, J. C. (1952). Endocrine factors effecting the weight and ascorbic acid content of rat preputial glands. Proc. Soc. Exptl. Biol. Med. 79, 290-292.

Hess, M., Rennels, E. G., and Finerty, J. C. (1953). Response of preputial and adrenal glands of hypophysectomized rats to ACTH. Endocrinology 52, 223-227.

Hill, R. T. (1937). Ovaries secrete male hormone. I. Restoration of the castrate type of seminal vesicle and prostate glands to normal by grafts of ovaries on mice. Endocrinology 21, 495-502. 
Hill, R. 'T. (1948). Grafted mouse ovaries and their adrenal cortical function. Endocrinology 42, 339-351.

Hoffmann, R.S. (19.58). The role of reproduction and mortality in population fluctuations of voles (Microtus). Ecol. Monographs 28, 79-109.

Hofmann, F. (i. (1956). Observations on in vitro adrenal steroid synthesis in the albino mouse. Endocrinology 59, 712-715.

Hofmann, F. G. (1957). The in vitro bydroxylation of 21-earbon steroids by rat adrenal glands. Endocrinology 60, 3\$2-389.

Holnes, W. N. (1955). Histological variations in the adrenal cortex of the golden hamster with special reference to the X-zone. Anat. Record 122, 271-294.

Horn, E. H. (1955). Nutritional and hormonal influenees upon reproductive maturation, organ weights and histochenistry of immature male rat. Endocrinology 57, 399-40s.

Howard, F. (19:2). A transitory zone in the adrenal cortex which shows age and sex relationships. Am. J. A nat. 40, 251-293.

Howard, E. (1938). The representation of the adrenal $\mathrm{X}$-zone in rats, in the light of observations on $\mathrm{X}$-zone variability in mice. A m. J. Anat. 62, 351-373.

Howard, E. (1939). A note on the reactions of the mouse uterus to ovariectomy in the presence of the X-zone. Butl. Johns Hopkins Hosp. 65, 341-347.

Howard, E. (1946). The effect of adrenalectomy on the accessory reproductive glands of mice eastrated for short periods. Endocrinology 38, 156-164.

Howard, E. (1959). A complementary action of corticosterone and dehydroepinandrosterone on the mouse adrenal with observations on the reactivity of reproductive tract structures to dehydroepiandrosterone and 11-hydroxy-androstenedione. Endocrinology 65, 7.5. 801 .

Howard, E., and Bemua, R. S. (1950). The effeet of protein defieiency and other dietary factor's on the X-zone of the mouse adrenal. J. Nutrition 42, 157-174.

Hsieh, A. C. L., and Carlson, I. D. (1957a). Role of adrenaline and noradrenaline in ehemical regulation of heat production. Am. J. Physiol. 190, 243-246.

Hsieh, A. C. L., Carlson, L. D., and Gray, G. (1957b). Role of sympathetic nervous system in the control of chemical regulation of heat production. Am. J. Physiol. 190, $247-251$.

Huggins, C., Parsons, F. M., and Jensen, E. V. (1955). Promotion of growth of preputial glands by steroids and the pituitary growth hormone. Endocrinology 57, 25-32.

Hughes, E., and Mall, R. (1958). Relation of the adrenal cortex to condition of deer. Calif. Fish Game 44, 191-195.

Hume, D. M. (1953). The neuro-endocrine response to injury, present status of the problem. Ann. Surg. 138, 548-557.

Hurst, V., and Turner, C. W. (1947). The thyroik secretion rate of growing and mature mice. Am. J. Physiol. 150, 686-692.

Ingalls, T. H. (1956). Causes and prevention of developmental defeets. J. Am. Med. Assoc. 161, 104s-1051.

Ingalls, T. H., and Philbrook, F. R. (1958). Monstrosities induced by hypoxia. New Engl. J. Med. 259, 558-564.

Ingle, D. J. (1949). Some studies on the role of the adrenal cortex in organic metabolism. Ann. N. Y. Acad. Sci. 50, 576-595.

Ingle, D. J. (1950). The biologie properties of cortisone: A review J. Clin. Endocrinol. $10,1312-1354$.

Irving, J. T. (1957). A eompar son of the influence of hormones, vitamins, and other dietary factors upon the formation of bone, dentine, and enamel. Vitamins and Hormones 15, 291-232. 
Isler, H., Leblond, C. P., and Axelrod, A. A. (1958). Mechanism of the thyro d stimulation produced by sodium chloride in the mouse. Endocrinology 62, 159-172.

Jacot, B., and Selye, H. (1951). A non-adrenal mediated action of AC'TH on the preputial glands. Proc. Soc. Exptl. Biol. Med. 78, 46-48.

Jameson, E. W., Jr. (1953). Reproduction of deer mice (Peromyscus maniculatus and $P$. boylii) in the Sierra Nevada, California. J. Mammal. 34, 44-5S.

Jameson, E. W., Jr. (1955). Some factors affecting fluctuations of Microtus and Peromycus. J. Mammal. 36, 206-209.

Jones, G. E. S., Delfa, E., and Foot, E. C. (1946). The effect of thiouracil hypothyroidism on reproduction in the rat. Endocrinology 38, 337-344.

Jones, I. C. (1948). Relationship of the $\mathrm{X}$ zone of the mouse adrenal. Proc. Soc. Exptl. Biol. Med. 69, 120-121.

Jones, I. C. (1949a). The action of testosterone on the adrenal cortex of hypophysectomized prepuberally castrated mouse. Endocrinology 44, 427-438.

Jones, I. C. (1949b). The relationship of the mouse adrenal cortex to the pituitary. Endocrinology 45, 514-536.

Jones, I. C. (1950). The effect of hypophysectomy on the adrenal cortex of the immature mouse. Am. J. Anat. 86, 371-403.

Jones, I. C. (1952). The disappearance of the $\mathrm{X}$ zone of the mouse adrenal cortex during first pregnancy. Proc. Roy. Soc. B139, 398-410.

Jones, I. C. (1957). "The Adrenal Cortex." Cambridge Univ. Press, London and New York.

Jones, I. C., and Roby, C. C. (1954). Some aspects of zonation and function of the adrenal cortex. I. The effects of hypophysectomy on the adrenal cortex of the adult male monse. J. Endocrinol. 10, 245-250.

Jones, I. C., and spalding, M. H. (1954). Some aspects of zonation and function of the adrenal cortex. II. The rat adrenal after enucleation. $J$. Endocrinol. 10, 251-261.

Jones, I. C., and Wright, A. (1954a). Some aspects of zonation and function of the adrenal cortex. III. Self-selection after adrenal enucleation in rats. J. Fndocrinol. 10, 262-26.5.

Jones, I. C., and Wright, A. (1954h). Some aspects of zonation and function of the adrenal cortex. IV. The histology of the adrenal in rats with diabetes insipidus. J. Endocrinol. 10, 266-272.

Josimovich, J. B., Ladman, A. J., and Deane, H. W. (1954). A histophysiological study. of the developing adrenal cortex of the rat during fetal and early post natal stages. Endocrinology 54, 627-639.

Kalela, O. (1957). Regulation of reproduction rate in subaretic populations of the vole Clethrionomys rufocunus (Sund.). Ann. Sci. Fennicae, Ser. A. Iv. Biol. 34, 1-60.

Kalter, H. (19.54). The inheritance of susceptibility to the teratogenic action of cortisone in mice. Genetirs 39, 185-196.

Kalter, H. (1956). Modification of teratogenic action of cortisone in mice by maternal age, maternal weight and litter size. Am. J. Physiol. 185, 6.)-68.

Kass, E. H., Kendrick, M. I., and Finland, M. (19.53a). Effect of certain corticosteroids and of growth hormone on mucleoproteins of lymph nodes. Ann. Y. Y. Acad. Sci. 56, $737-741$.

Kass, E. H., Lundgren, M. M., and Finland, M. (1953b). Observations on the effect of corticosteroids and growth hormone on resistance to experimental pneumococeal and influenza virus infections. Ann. N. Y. Acad. Sci. 56, 765-771.

Kass, E. H., Kendrick, M. I., and Finland, Ml. (1955). Effects of corticosterone, hydrocortisone and corticotropin on production of antibodies in rablits. J. Exptl. Merl. $102,767-774$. 
Kelsall, M. A., and Crabb, E. D. (1958). Lymphocytes and plasmacytes in nucleoprotein metabolism. Ann. N. Y. Acad. Sci. 72, 293-338.

Keuning, F. J., and Van der Slikke, L. B. (1950). The role of immature plasma cells, lymphoblasts, and lymphocytes in the formation of antibodies, as established in tissue culture experiments. J. Lab. Clin. Med. 36, 167-182.

Kligman, A. M., Baldridge, G. D., Rebell, G., and Pillsbury, D. M. (1951). The effect of cortisone on pathologic responses of guinea pigs infected cutaneously with fungi, viruses, and bacteria. J. Lab. Clin. Med. 37, 615-620.

Knigge, K. M. (1954a). The effect of hypophysectomy on the adrenal gland of the hamster (Mesocricctus auratus). Am. J. Anat. 94, 225-271.

Knigge, K. M. (1954b). The effect of acute starvation of the adrenal cortex of the hamster. Anat. Record 120, 555-582.

Knisely, M. H., Bloch, E. H., Eliot, T. S., and Warner, L. (1947). Sludged blood. Science 106, 431-440.

Kraatz, C. P. (1939). Effect of brief experimental hyperthyroidism on reproduction in the rat. Proc. Soc. Exptl. Biol. Med. 40, 499-502.

Kracht, J. (1954). Fright-thyrotoxicosis in the wild rabbit, a model of thyrotrophic alarm-reaction. Acta Endocrinol. 15, 355-367.

Kramer, J., Meyers, V. M., McCarthy, H. H., and Simay Kramer, M. (1957). Further study on the endocrine relations of capillary resistance. Endocrinology 60, 589-596.

Kuhl, W. J., Jr., and Ziff, M. (1952). Alteration of thyroid function by ACTH and cortisone. J. Clin. Endocrinol. and Metabolism 12, 555-559.

Lanman, J. T. (1957). The adrenal fetal zone: Its occurrence in primates and a possible relationship to chorionic gonadotropin. Endocrinology 61, 6\$4-691.

Laqueur, G. L., MeCann, S. M., Schreiner, L. H., Rosemberg, E., Rioch, D., and Anderson, E. (1955). Alterations of adrenal cortical and ovarian activity following hypothalamic lesions. Endocrinology 57, 44-54.

Leathem, J. H. (1950). Seminal vesicle response as an assessment of androgenic activity. Trans. N. Y. Acad. Sci. [2], 12, 234-235.

LeMaistre, C. A., Tompsett, R., and MeDermott, W. (1953). The effects of corticosteroids upon tuberculosis and pseudotuberculosis. Ann. N. Y. Acad. Sci. 56, $772-778$.

Levy, A. C., and Ramey, E. R. (1958). Effect of autonomic blocking agents on depot fat mobilization in normal adrenalectomized animals. Proc. Sor. Exptl. Biol. Mcd. 99, 637-639.

Li, C. H., Fønss-Bech, P., Geschwind, I. I., Hayashida, T., Hungerford, G. F., Lostroh, A. J., Lyons, W. R., Moon, H. D., Reinhardt, W. O., and Sideman, M. B. (1957). Corticotropins (AC'TH) X. Biological investigations of $\alpha$-corticotropin. J. Exptl. Med. 105, 335-360.

Liddle, G. W., Duncan, L. E., and Bartter, F. C. (1956). Dual mechanism regulating adrenocortical function in man. Metabolism 5, 369-353.

Lillie, R. D. (1954). Histopathologic Technic and Practical Histochemistry. MeGrawHill (Blakiston), New York.

Lord, R. A. (1958). Studies on the use of cortisone and ACTH in trichinosis. $4 \mathrm{~m} . J$. Trop. Med. Hyg. 7, 611-617.

Lostroh, A. J., and Li, C. H. (1957). Stimulation of the sex accessories of hypophysectomized male rats by non-gonadotrophic hormones of the pituitary gland. Acta Endocrinol. 25, 1-16.

Lostroh, A. J., and Li, C. H. (1958). Effect of growth hormone and thyroxine on body weight of hypophysectomized C3H mice. Endocrinology 62, 484-492. 
Lostroh, A. J., and Woodward, P. (1955). Changes in the adrenal of hypophysectomizerl C3H mouse with $\alpha$-corticotropin and grow th hormone. Endocrinology 62, 495-505.

Louch, C., Meyer, R. K., and Emlen, J. T. (1953). Effect of stress on diurual fluctuations in eosinophils of the laboratory mouse Proc. Soc. Exptl. Biol. Med. 82, 66S-671.

Louch, C. D. (1956). Adrenocortical activity in relation to the density and dynamies of three confined populations of Microtus pennsylvanicus. Ecology 37, 701-713.

Louch, C. D. (1958). Adrenocortical activity in two meadow vole populations. $J$. Mammal. 39, 109-116.

Lutwak-Mann, C. (195S). The dependence of gonadal function upon vitamins and other nutritional factors. Vitamins and Hormones 16, 35-75.

Lutwak-Mann, C., Mann, T., and Price, D. (1949). Metabolic activity in tissue transplants. Hormone induced formation of fructose and citric acid in transplants from accessory glands of reproduction. Proc. Roy. Soc. B136, 461-471.

McCann, S. M. (1953). Effect of hypothalamic lesions on the adrenal cortical response to stress in the rat. Am. J. Physiol. 175, 13-20.

MeCann, S. M. (1957). The ACTH-releasing activity of extracts of the posterior lobe of the pituitary in vivo. Endocrinology 60, 664-676.

MeCann, S. M., and Brobeck, J. R. (1954). Evidence for a role of the supraopticohypophyseal system in regulation of adrenocorticotrophin secretion. Proc. Soc. Exptl. Biol. Med. 87, 318-324.

McCann, S. M., and Fruit, A. (1957). Effect of synthetic vasopressin on release of adrenocorticotrophin in rats with hypothalamic lesions. Proc. Soc. Exptl. Biol. Med. 96, 566-567.

McDonald, R. K., Wagner, H. N., Jr., and Wiese, V. K. (1957). Relationship between endogenous antidiuretic hormone activity and $\mathrm{ACTH}$ release in man. Proc. Soc. Exptl. Biol. Med. 96, 652-655.

MacLulich, D. A. (1937). Fluctuations in the numbers of the varying hare. Univ. Toronto Studies Biol. Ser. 43, 1-136.

McPhail, M. K. (1944). Androgenic activity of the adrenal gland with special reference to the X-zone. Rev. can. biol. 3, 312-327.

McPhail, M. K., and Read, H. C. (1942a). The mouse adrenal. I. Development, degeneration, and regeneration of the X-zone. Anat. Record 84, 51-73.

McPhail, M. K., and Read, H. C. (1942b). The mouse adrenal. II. The action of certain hormonal substances on the adrenal gland of the mouse with particular reference to their action on the X-zone. Anat. Record 84, 75-89.

Mann, T. (1954). On the presence and role of inositol and certain other substances in the seminal vesicle secretion of the boar. Proc. Roy. Soc. B142, 21-32.

Maqsood, M., and Reineke, E. P. (1950). Conference of environmental temperatures and thyroid status on sexual development in male mouse. Am. J. Physiol. 162, 24-30.

Marine, D., and Bauman, E. J. (1945). Hypertrophy of adrenal medulla of white rats in chronic thiouracil poisoning. Am. J. Physiol. 144, 69-73.

Markee, J. E., Everett, J. W., and Sawyer, C. H. (1952). The relationship of the nervous system to the release of gonadotrophin and the regulation of the sex cycle. Recent Progr. in Hormone Research 7, 139-163.

Marrian, G. F., and Parkes, A. S. (1929). The effect of anterior pituitary preparations administered during dietary anestrus. Proc. Roy. Soc. B105, 24S-25S.

Martin, E. P. (1956). A population study of the prairie vole (Microtus ochrogaster) in Northeastern Kansas. Univ. Kansas Museum Nat. Hist. Publ. 8, 361-416.

Martorano, J. J. (1957). Variation in liver glycogen levels of intact and adrenalectomized mice. Naval Med. Res. Inst. Res Rept. 15, 191-198. 
Mason, J. W. (1959). Central nervous system factors in the regulation of endocrine secretion. Recent P'rogr. in Hormone Research, 15, 345-3\$9.

Meier, R., Schuler, W., and Desaulles, P. (1950). Zur Frage des Mechanismus der Hemmung des Bindegewebswachstume durch Cortisone. Experientia 6, 469-471.

Menkin, V. (1955). Factors concerned in the mobilization of leukocytes in inflammation. Ann. N. Y. Acad. Sci. 59, 956-958.

Miller, R. A. (1949). Cytological criteria of activity and inactivity in the glomerular fascicular zones of the adrenal cortex in mice. Anat Record 103, 489.

Mills, I. H., Casper, A., and Bartter, F. C. (1958). On the role of the vagus in the control of aldosterone secretion. Science 128, 1140-1141.

Money, W. L. (1955). The interrelation of the thyroid and the adrenals. Brookhaven Symposia in Biol. No. $\gamma, 13 \overline{7}-168$.

Money, W. L., Kirschner, L., Kraintz, L., Merrill, P., and Rawson, R. W. (1950). Effects of adrenal and gonadal products on the weight and radioiodine uptake of the thyroid gland in the rat. J. Clin. Endocrinol. 10 1282-1295.

Moon, H. D., Simpson, M. E., Li, C. H., and Evans, H. M. (1950). Neoplasms in rats treated with pituitary growth hormone. II. Adrenal gland. Cancer Research 10, 364-370.

Moon, H. D., Simpson, M. E., Li, C. H., and Evans, H. M. (1951). Neoplasms in rats treated with pituitary growth hormone. V. Absence of neoplasms in hypophysectomized rats. Cancer Research 11, 535-539.

Morin, G. (1946a). Médullo-surrénale et régulation thermique. I. Action calorigène de l'adrénaline. Démonstration, signification. Rev. can. biol. 5, 121-134.

Morin, G. (1946b). Médullo-surrénale et régulation thermique. II. Action adrénalinosecrétrice du froid. Rev. can. biol. 5, 385-400.

Mosier, H. D. (1957). Comparative histological study of the adrenal cortex of the wild and domesticated Norway rat. Endocrinology 60, 460-469.

Moss, M. L. (1955). Morphological changes in the growing rat skull following administr:1tion of cortisone acetate. Proc. Soc. Exptl. Biol. Med. 89, 645-650.

Mulinos, M. G., and Pomerantz, L. (1941). Hormonal influences on the weight of the adrenal in inanition. Am. J. Physiol. 132, 368-374.

Mulinos, M. G., Pomerantz, L., Smelser, J., and Kurzrok, R. (1939). Estrus-inhibiting effects of inanition. Proc. Soc. Exptl. Biol. Med. 40, 79-83.

Nalbandov, A. V., Moore, W. W., and Norton, H. W. (1955). Further studies on the neurogenic control of the estrus cycle by uterine distension. Endocrinology 56, 225-231.

Nelson, 1). H. (1955). The signifieance of plasma corticosteroids as a measure of adrenal cortical function. In "Symposium on Adrenal Functions in Infants and Children," N. Y. Univ. Med. Ctr., Syracuse, 1954. NMRI (Naval Med. Res. Inst.) L and R $55-1$.

Nichols, J. (1950). Effects of captivity on adrenal gland of wild Norway rat. Am. J. Physiol. 162, 5-9.

Noble, R. I. (1955). Physiology of the adrenal cortex. In "The Hormones" (G. Pincus and K. V. Thimann, eds.), Vol. III, pp. 68.5-\$19. Academic Press, New York.

Nowell, N. W. (1959). Studies in the activation and inhibition of adrenocorticotrophin secretion. Endocrinology 64, 191-201.

Odum, E. P. (1955). An eleven year history of a Sigmodon population. J. Mammal. 36, $368-378$.

Parmer, L. G., Katonah, F., and Angrist, A. A. (1951). Comparative effects of ACTH, cortisone, corticosterone, desoxycorticosterone, pregnenolone on growth and development of infant rats. Proc. Soc. Exptl. Biol. Med. 77, 215-218. 
Paschkis, K. E., Cantarow, A., Eberhard, T., and Doyle, D. (1950). Thyroid function in the alarm reaction. Proc. Soc. Exptl. Biol. Med. 73, 116-118.

Pauly, J. E. (1957). Morphological observations on the adrenal cortex of the laboratory rat. Endocrinology 60, 247-264.

Peron, F. G., and Dorfman, R. I. (195S). Quantitative analysis of steroids isolated from the urine of normal and ACTH treated guinea pigs. Endocrinology 62, 1-8.

Perry, W. F. (1951). The action of cortisone and ACTH on thryoid function. Endocrinology 49, 284-285.

Pitelka, F. A. (1957a). Some aspects of population structure in the short-term cycle of the brown lemming in Northern Alaska. Cold Spring Harbor Symposia Quant. Biol. $22,237-251$.

Pitelka, F. A. (1957b). Some characteristics of microtine cycles in the arctic. Biol. Colloq. Proc. 15th Colloq. 73-8s.

Poindexter, H. A. (1949). Experimental animal colony in tropical West Africa. Public Health Repts. (U. S.) 64, 57-62.

Pollard. M., and Wilson, B. R. (1955). The prolongation of murine typus rickettsemia by cortisone. Texas Repts. Biol. and Med. 13, 126-128.

Poore, W., and Hollander, V. P. (1957). Plasma ketosteriod studies in normal mice. Endocrinology 61, 651-656.

Porter, J. C., and Jones, I. C. (1956). Effect of plasma from hypophyseal-portal vessel blood on adrenal ascorbic adid. Endocrinology 58, 62-67.

Porter, J. C., and Rumsfeld, H. W., Jr. (1956). Effects of lyophilized plasma and plasma fractions from hypophyseal-portal vessel blood on adrenal ascorbic acid. Endocrinology 58, 359-364.

Preble, E. A. (1908). A biological investigation of the Athabaska-Mackenzie region. U.S. Dept. Agr. N. Am. Fauna No. 27.

Puntriano, G., and Mcites, J. (1951). The effects of coutinuous light or darkness on thyroid function in mice. Endocrinology 48, 217-224.

Ramey, E. R., and (ioldstein, M. S. (1957). The adrenal cortex and the sympathetic nervous system. Physiol. Revs. 37, 155-195.

Randall, H. T. (1952). The shifts of fluid and electrolytes in shork. Ann. N. Y. Acad. Sci. 55, 412-428.

Ratcliffe, H. L., and Cronin, M. T. I. (1958). Changing frequency of arteriosclerosis in mammals and birds at the Philadelphia Zoological Gardens. Circulation 18, 11-52.

Rausch, R. (1950). Observations on a cyclic decline of lemmings (Lemmus) on the aretic coast of Alaska during the spring of 1949. Arctic 3, 166-17 7 .

Rawson, R. W., Rall, J. E., and Sonenberg, M. (1955). The chemistry and physiology of the thyroid. In "The Hormones" (G. Pincus and K. V. Thimann, eds), Vol. III, pp. 433-519. Academic Press, New York.

Reichlin, S. (1957a). The effect of dehydration, starvation, and pitressin injections on thyroid activity in the rat. Endocrinology 60, 470-4S7.

Reichlin, S. (1957b). The effect of hypothalamic lesions upon the thyroid response to partial thyroidectomy. Endocrinology 60, 567-569.

Reif, A. E., and Longwell, B. B. (1958). Extraction of corticosteroids from adienal vein blood of rats and rabbits. Endocrinology 62, 573-586.

Rennels, E. G., and Drager, G. A. (1955). The relationship of pituicy tes to neurosecretion. Anat. Record 122, 193-203.

Rennels, E. G., Hess, M., and Finerty, J. C. (1953). Response of preputial and adrenal glands of the rat to sex hormones. Proc. Soc. Exptl. Biol. Med. 82, 301-309.

Retzlaff, E. G. (1938). Studies in population physiology with the albino mouse. Biol. Generalis 14, 238-265. 
Richards, J. B., and Pruitt, R. L. (1957). Hydrocortisone suppression of stress-induced adrenal 17-hydroxycorticosteroid secretion in dogs. Endocrinology 60, 99-104.

Richardson, J. A., and Woods, E. F. (1959). Release of norepinephrine from the isolated heart. Proc. Soc. Expll. Biol. 1Ied. 100, 149-151.

Richter, C. P., Rogers, P. V., and Hall, C. E. (1950). Failure of salt replacement therapy in adrenalectomized recently captured wild Norway rats. Endocrinology 46, 233-242.

Ring, G. C. (1942). The importance of the thyroid in maintaining an adequate production of heat during exposure to cold. A m. J. Physiol. 137, 582-5S8.

Robert, A., and Nezamis, J. E. (1957). The granuloma pouch as a routine assay for antiphlogistic compounds. Acta Endocrinol. 25, 105-112.

Robinson, H. J., and Smith, A. L. (1953). The effect of adrenal cortical hormones on experimental infection. Ann. N. Y. Acad. Sci. 56, 757-764.

Rogers, P. V., and Richter, C. P. (1948). Anatomical comparison between the adrenal glands of wild Norway, wild alexandrine and domestic Norway rats. Endocrinology 42, 46-55.

Rosemberg, E., Cornfield, J., Bates, R. W., Anderson, E. (1954). Bioassay of adrenal steroids in blood and urine based on cosinophil response: a statistical analysis. Endocronilogy 54, 363-383.

Rosenfeld, G. (1958). The function and capacity of the adrenal cortex immediately before radiation sickness death. J. Lab. Clin. Med. 51, 198-210.

Rosenfeld, G., and Bascom, IW. D. (1956). In vitro studies of the influence of corticotropin, somatotropin, thyrotropin and gonadotropin on adrenal steroidogenesis. Endocrinology 59, 497-499.

Rothballer, A. B. (1953). Changes in the rat neurohypophysis of painful stimuli with particular reference to neurosecretory material. Anat. Record 115, 21-11.

Saffran, M., Schally, A. V., and Benfey, B. G. (1955). Stimulation of the release of corticotrophin from the adenohypophysis by a neurohypophyseal factor. Endocrinology 57, 439-444.

Sakiz, E. (1956). Le cortex surrénal excrce-t-il une action hormonale sexuelle? Compt. rend. soc. biol. 150, 2172-217t.

Salter, W. T. (1949). The metabolic circuit of thyroid hormone. Ann. N. Y. Acad. Sci.50, 358-376.

Sandberg, A. A., Nelson, D. M., Palmer, J. G., Samuel, L. T., and Tyler, F. H. (1953). The effects of epinephrine on the metabolism of 17 -hydroxycorticosteroids in the human. J. Clin. Endocrinol. and Metabolism 13, 629-647.

Santisteban, G. A., and Dougherty, T. F. (1951). Comparison of the influence of adrenocortical hormones on the growth and involution of lymphatic organs. Endocrinology $54,130-146$.

Sayers, G., and Sayers, M. (1919). The pituitary-adrenal system. Ann. N. Y. Acad. Sci. $\mathbf{5 0}, 522-539$.

Schally, A. V., and Guillemin, R. (1959). Concentration of corticotropin releasing factor by chromatography on carboxymethylcellulose. Proc. Soc. Exptl. Biol. Med. 100, 138-139.

Schapiro, S., Marmorston, J., and Sobel, H. (1958). Mobilization of the antidiuretic hormone and the secretion of ACTH following cold stress. Endocrinology 62, 27S-2S2.

Scharrer, E., and Scharrer, B. (1954). Hormones produced by neurosecretory cells. Recent Progr. in Hormone Research 10, 183-240.

Schindler, W. J., and Knigge, K. M. (1959a). Adrenal cortical secretion by the golden hamster. Endocrinology 65, 739-747.

Schindler: W. J., and Knigge, K. M. (1959b). In vitro studies and adrenal steroidogenesis by the golden hamster. Endocrinology 65, 74S-765. 
Schmidt, L. H., and Squires, W. L. (1951). The influence of eortisone on primate malaria. J. Exptl. Med. 94, 501-,520.

Schmiterlöw, C. G. (194S). The nature and occurrence of pressor and depressor substances in extracts from blood vessels. Acta Physiol. Scand. 16, Suppl. 56, 1-113.

Scudder, J. (1952). Introduction to shock syudrome. Ann. N. Y. Acad. Sci. 55, 349-350.

Sealander, J. A., Jr. (1950). Effect of environmental temperature and starvation on adrenal glands of the white-footed mouse. Am. J. Physiol. 163, 92-95.

Seifter, J., Ehrich, W. E., and Hudyma, G. M. (1949). Effect of prolonged administration of antithyroid compounds on the thyroid and other endocrine organs of the rat. A. M. A. Arch. Pathol. 48, 536-547.

Seifter, J., Christian, J. J., and Ehrich, W. E. (1951). The effect of cortisone and other steroids on the hibernating gland of the pregnant white rat. Federation Proc. 10, 334.

Seifter, J., Ehrich, W. E., Baeder, D. H., Butt, A. J., and Hauser, E. A. (1953). Evidence for the direct effect of steroids on the ground substance. Ann. N. Y. Acad. Sci.55, 696-697.

Seifter, J., Baeder, D. H., Zarafonetis, C. J. D., and Kalas, J. (1959). Effect of adrenals, pituitary, liver, and mucopolysaccharides on blood lipids. In "Hormones and Atherosclerosis" (G. Pincus, ed.), pp. 265-2\$1. Academic Press, New York.

Selye, H. (1939). The effect of adaptation to various damaging agents on the female sex organs in the rat. Endocrinology 25, 615-624.

Selye, H. (1947). "Textbook of Endocrinology." Acta, Montreal.

Selye, H. (1950). "Stress." Acta, Montreal.

Selye, H. (1951). The influence of STH, ACTH and cortisone upon resistance to infection. Can. Med. Assoc. J. 64, 489-494.

Selye, H. (1954). Stress and lactation. Rev. can. biol. 13, (4), 377-3S4.

Selye, H. (1955). Interactions between corticoid and folliculoid hormones in the regulation of anabolism, lymphatic tissue and inflammation. Acta Endocrinol. 20, 1-1S.

Selye, H., and Bois, P. (1954). On the design of experiments to appraise the role of the adrenals in biologic reactions. In "4th Annual Report on Stress," (H. Selye and G. Heuser, eds.), pp. 533-552. Acta, Montreal.

Share, L., and Stadler, J. B. (195S). Alterations in sodium and potassium metabolism following hind-leg fracture in the rat: Role of the adrenal cortex. Endocrinology 62, 119-128.

Shwartzman, G., and Aronson, S. M. (1953). Poliomyelitis infection by parenteral routes made possible by cortisone. Ann. N. Y. Acad. Sci. 56, 793-798.

Simpson, M. E., Evans, E. S., and Posenberg, L. I. (1959). Re-evaluation of the evidence for a pituitary erythropoietic hormone. Endocrinology 64, 592-599.

Slusher, M. A. (1958). Dissociation of adrenal ascorbic acid and corticosterone response to stress in rats with hypothalamic lesions. Endocrinology $\mathbf{6 3}, 412-419$.

Smelser, G. K. (1943). Changes induced in the Harderian gland of the guinea pig by the injection of hypophyseal extracts. Anat. Record 86, 41-56.

Smith, E. K. (1955). Interrelationships of anterior pituitary and adrenal cortex in the rat ovarian hyperemic reaction. Endocrinology 56, 567-574.

Smith, R. W., Jr. (1951). Augmented excretion of urine gonadotrophius during ACTH administration. Proc. Soc. Exptl. Biol. Med. 78, S6S-\$72.

Snyder, R. L., and Christian, J. J. (1960). The reproduction cycle and the litter size of the southern woodehuck. Ecology 41, 64i-656.

Sollman, T. (1957). "A Manual of Pharmacology," Sth ed. Saunders, Philadelphia.

Southern, H. N. (1948). Sexual and aggressive behaviour in the wild rabbit. Behaviour 1, 173-194. 
Southwick, C. H. (1955a). The population dynamics of ronfined house mice supplied with unlimited food. Ecology 36, 212-225.

Southwick, C. H. (1955b). The regulatory mechanisms of house mouse population; social behavior affecting litter survival. Ecology 36, 627-634.

Southwick, C. H. (1959). Eosinophil response of C57BR mice to behavioral disturbance. Ecology 40, 156-157.

Speirs, R. S. (1955). Physiological approaches to an understanding of the function of eosinophits and basophils. Ann. N. Y. Acad. Sci. 59, 706-731.

Speirs, R. S., and Meyer, R. K. (1949). The effects of stress, adrenal and adrenocorticotrophic hormones on the cirulating eosinophils of mice. Endocrinology 45, 403-429.

Speirs, R. S., and Meyer, R. K. (1951). A method of assaying adrenal cortical hormones based on a decrease in the circulating eosinophil cells of adrenalectomized mice. Endocrinology 48, 316-326.

Srebnik, H. H., Nelson, M. M., and Simpson, M. E. (1958). Response to exogenous gonadotrophins in absence of dietary protein. I'roc. Soc. Exptl. Biol. Med. 99, 57-61.

Stachenko, J., and Giroud, C. J. P. (1959a). Funetional zonation of the adrenal cortex: Pathways of corticosteroid hiogenesis. Endocrinology 64, 730-742.

Stachenko, J., and Giroud, C. J. P. (1959b). Functional zonation of the adrenal cortex site of ACTH aetion. Endocrinology 64, 743-752.

Stanbury, S. W., Gowenlock, A. H., and Mahler, R. F. (195S). Interrelationships of potassium deficiency and renal disease. In "Aldosterone," Intern. Symposium, Geneva, 1957 (A. F. Muller and C. M. O'Connor, eds.), pp. 155-166. Little, Brown, Boston, Massachusetts.

Steinberger, E., and Dixon, W. J. (1959). Some observations on the effeet of heat on the testicular germinal epithelium. Fertility and Sterility 10, 578-595.

Stevens, C. E., I' Angelo, S. A., Paschkis, K. E., Cantarow, A., and Sunderman, F. W. (1955). 'The response of the pituitary thyroid system of the guinea pig to low environmental temperature. Endocrinology 56, 143-156.

Stoner, R. D., and Godwin, J. T. (1953). 'The effects of ACTH and cortisone upon susceptibility to trichinosis in mice. Am. J. Pathol. 29, 943-950.

Story, J. L., Melby, J. C., Egdahl, R. H., and French, L. A. (1959). Adrenal cortical function following stepwise removal of the brain of the dog. Am. J. Physiol. 196. $583-588$.

Strecker, R. L. (19.54). Regulatory mechanies in house mouse populations: The effect of limited food supply on an uneonfined population. Ecology 35, 249-253.

Strecker, R. L., and Emlen, J. T. (1953). Regulatory mechanisms in house mouse populations-the effect of limited food supply on a confined population. Ecology 34, 375-38.5.

Sturgis, S. H. (1950). The mechanism and control of primate ovulation. Fertility and Sterility 1, 40-52.

Sundberg, R. 1). (1955). Lymphocytes and plasma cells. Ann. N. Y. Acad. Sci. 59(5), 671-6s9.

Swanson, H. E. (1956). Interrelationships between thyroxin and adrenalin in the regulation of oxygen consumption on the albino rat. Endocrinology 59, $217-225$.

Swanson, H. E. (1957). The effect of temperature in the potentiation of adrenalin by thyroxine in the albino rat. Endocrinology 60, 205-213.

Sweat, M. L., and Farrell, G. L. (1952). Resolution and quantitative anatysis of steroids in adrent.l-vein blood. J. Clin. Endocrinol. and Metabolism 12, 165.

Sweat, M. L., and Farrell, G. L. (1954). Deeline of corticosteroid secretion following hypophysectomy. Proc. Soc. Exptl. Biol. Med. 87, 615-61S. 
Sydnor, K. L., and Sayers, G. (1954). Blood and pituitary ACTH in intact and adrenalectomized rats after stress. Endocrinology 55, 621-636.

Sydnor, K. L., Burks, R., and Brown, D. (1955). Blood ACTH in the stressed adrenalectomized rat after intravenous injection of hydrocortisone. Endocrinology 56, 204-208

Symington, T., Currie, A. R., O'Donnell, U. J., Grant, J. K., Oastler, E. G., and Whyte, W. H. (1958). Hyperplasia and tumours of the human adrenal cortex: histology, coenzymic changes and corticoid production. CIBA Foundation Colloq. on Endocrinology 12, 102-121.

Tamura, Y. (1926). Structural changes in the suprarenal gland of the mouse during pregnaney. Brit. J. Exptl. Biol. 4, 81-92.

Tanaka, R. (1956). Fluctuations of vole populations following the widespread synchronous flowering of bamboo-grasses on MIt. Turugi. Bull. Kochi I1'omen's College $\mathbf{4}$, 61-68.

Tanaka, R. (1957). An ecological review of small-mammal outbreaks with special reference to their association with the flowering of bamboo grasses. Bull. Kochi Women's Univ. Nat. Sci. Ser. 5, 20-30.

Tata, J. R., Rall, J. E., and Rawson, R. W. (1957). Metabolism of L-tlyroxine and L-3:5:3'-triodothyronine by brain tissue preparations Endocrinology 60, 83-98.

Taubenhaus, M. (1953). The influence of cortisone upon granulation tissue and its synergism and antagonism to other hormones. Ann. N. Y. Acad. Sci. 55, 666-673.

Taubenhaus, M. and Amromin, D. (1950). The effects of the hypophysis, thyroid, sex steroids, and the adrenal cortex upon granulation tissue. J. Lab. Clin. Med. 36, $7-18$.

Thomas, L. (1953). Cortisone and infection. Aun. N. Y. Acad. Sci. 56, 799-814.

Thompson, D. Q. (1955). The role of food and rover in population fluctuations of the brown lemming at Point Barrow, Alaska. Trans. N. Am. Hildlife Conf. 20, 166-176.

Thorn, G. W., Jenkins, D.. and Laidlaw, J. C. (1953). The adrenal response to stress in man. Recent Progr. in Hormone Research 8, 171-215.

Thorn, N. A. (1958). Mammalian antidiuretic hormone. Physiol. Revs. 38, 169-195.

Tobach, E., and Bloek, H. (1956). Effect of stress by crowding prior to and following tubereulous infection. Am. J. Physiol. 187, 399-402.

Tyndale-Biscoe, C. H., and Williams, R. M. (1955). A study of natural mortality in a wild population of the rahbit, Oryctolagus cuniculus (L.). New Zealand J. Sci. Technol. B36 $(6), 561-5 \$ 0$.

Uhrich, J. (1938). The social hierarchy in albino mice. J. Comp. Psychol. 25, 373-413.

Vander Laan, W. P. (1955). The biological significance of the iodide concentrating mechanism of the thyroid gland. Brookharen Symposia in Biol. No. 7, 30-39.

Vander Laan, W. P., and Caplan, R. (1954). Observations on a relationship betwees total thyroid iodine content and the iodide concentrating mechanism of the thyroid gland of the rat. Endocrinology 54, 437-447.

Vander Laan, W. P., and Greer, M. (1950). Some effeets of the hypophysis on iodine metabolism by the thyroid gland of the rat. Endocrinology 47, 36-47.

VandeWiele, R., and Lieberman, S. (1960). The metabolism of dehydroisoandrosterone. In "Biologieal Activities of Steroids in Relation to Cancer" (Ci. Pineus and E. P. Vollmer, eds.), pp. 93-110. Academie Press, New York.

Van Middlesworth, L., and Berry, M. M. (1951). Iodide metabolism during anoxia, nephrectomy, trauma, avitaminoses and starvation in the rat. Am. J. Physiol. 167, 576-550.

Verzár, F., and Vidovic, V. (1952). Action of T.S.H. and cortisone on uptake of ${ }^{131} 1$ by thyroid gland of hypophysectomized rats. J. Endocrinol. 8, 321-32s. 
Vetulani, T. (1931). Untersuchungen über das Wachstum der Säugetiere in Abhangigkeit von der Anzahl zusammengehaltener Tiere. Biol. Generalis 7, 71-98.

Visscher, M. B., and Halberg, F. (1955). Daily rhythms in numbers of eirculating eosinophils and some related phenomena Ann. N. Y. Acad. Sci. 59, 834-849.

von Euler, U. S. (1951). The nature of adrenergic nerve mediators. Pharmacol. Revs. 3, 247-277.

Wallach, D. P., and Reineke, E. P. (1949). The effect of varying levels of thyroidal stimulation on the ascorbic acid content of the adrenal cortex. Endocrinology 45 , $75-81$.

Waring, H. (1935). The development of the adrenal gland of the mouse. Quart. J. Microscop. Sci. 78, 329-366.

Waring, H. (1942). Effect of hormones on degeneration of the x-zone in the mouse adrenal. J. Endocrinol 3, 123-131.

Watt, L. J. (1934). Frequency distribution of litter size in mice. J. Mammal. 15, 1S5-1S9.

Weaver, J. A. (1955). Changes induced in the thymus and lymph nodes of the rat by the administration of cortisone and sex hormones and by other procedures. J. Pathol. Bacteriol. 69, 133-139.

Weber, A. F., McNutt, S. H., and Morgan, B. B. (1950). Structure and arrangement of zona glomerulosa cells in the bovine adrenal. J. Morphol. 87, 393-416.

Wexler, B. C., Runfret, A. P., Griffin, A. C., and Richardson, H. C. (1955). Evidence of pituitary control of the lipid content of the zona glomerulosa of the rat adrenal cortex. Endocrinology 56, 120-123.

Whitney, O. M., and Anigstein, L. (1953). Effect of cortisone on experimental rickettsioses and on antibody formation. Texas Repts. Biol. and Med. 11, 561-569.

Whitten, W. K. (1959). Occurrence of anoestrus in mice caged in groups. $J$. Endocrinol. $18,102-107$.

Whittle, P. (1955). An investigation of periodic fluctuation in the New Zealand ralsbit population. New Zcaland J. Sci. Technol. B37(2), 179-200.

Williams, R. H., Jaffe, H., and Kemp, C. (1949). Effect of severe stress upon thyroid function. Am. J. Physiol. 159, 291-297.

Wilson, H., Borris, J. J., and Bahn, R. C. (1958). Steroids in the blood and urine of female mice bearing on ACTH-producing pituitary tumor. Endocrinology 62, 135-149.

Woodbury, D. M. (1958). Relation between the adrenal cortex and the central nervous system. Pharmacol. Revs. 10, 275-357.

Woods, R., and Carlson, L. D. (1956). Thyroxine secretion in rats exposed to cold. Endocrinology 59, 323-330.

Wright, A., Jones, I. C., and Phillips, J. G. (1957). The histology of the adrenal gland of the prototheria. J. Endocrinol. 15, 100-107.

Yamada, T. (1959). Studies on the mechanism of hypothalamic control of thyrotropin secretion: Effect of intrahypothalamic thyroxine injection on thyroid hypertrophy induced by propylthiouracil in the rat. Endocrinology 65, 216-224.

Yamada, T., and Greer, M. A. (1959). Studies on the mechanism of hypothalamic control of thyrotropin secretion: Effect of thyroxine injection into the hypothalamus or the pituitary on thyroid hormone release. Endocrinology $\mathbf{6 4}, 559-566$.

Yankopoulos, N. A., Davis, J. O., Kliman, B., and Peterson, R. E. (1959). Evidence that a humoral agent stimulates the adrenal cortex to secrete aldosterone in experimental secondary hyperaldosteronism. J. Clin. Invest. 38, 1278-1289.

Zalesky, M. (1934). A study of the seasonal changes in the adrenal gland of Citellus with particular reference to its sexual cycle. Anat. Record 60, 291-321. 
Zimmermann, K. (1937). Die markische Rotenmaus. Analyse einer population. Markische Tierewelt 3, 24-40.

Zweifach, B. W. (1952). Functional deterioration of terminal vascular bed in irreversible hemorrhagic shock. Ann. N.Y. Acad. Sci. 55, 370-380.

Zweifach, B. W., Shorr, E., and Black, M. M. (1953). The influence of the adrenal cortex on behavior of terminal vascular bed. Ann. N. Y. Acad. Sci. 55, 626-633.

Zwemer, R. L. (1936). A study of adrenal cortex morphology. Am. J. Pathol. 12, 107-114.

Zwemer, R. L., Wooton, R. M., and Norkus, M. G. (1938). A study of corticoadrenal cells. Anat. Record 72, 249-264. 



\section{Author Index}

$\mathrm{N}$ umbers in italics indicate the pages on which the complete references are listed.

Abrams, G. D., 242, 253, 329

Ackerman, N. B., 233, 328

Agate, F. J., Jr., 244, 328

Albert, A., 232, 328

Allen, J. C., 196, 282, 329

Allen, J. M., 196, 201, 213, 329

Alpert, M., 194, 196, 329

Altzuler, N., 227, 395

Amiot, L. W., 97, 187

Amromin, D., 209, 254, 851

Auderson, C. H., 202, 203, 204, 209, 213, 329,338

Anderson, E., 206, 212, 218, 257, 258, $283,285,329,344,348$

Andervont, H. B., 269, 275, 329

Angevine, D. M., 256, 331

Angrist, A. A., 208, 210, 346

Anigstein, L., 210, 256, 352

Antopol, W., 282, 329

Arons, W. L., 233, 328

Aronson, S. M., 210, 256, 349

Asling, C. W., 196, 292, 329, 341

Austin, C. R., 222, 333

Axelrod, A. A., 238, 343

Aycock, W. L., 221, 328

Ayres, P. J., 200, 329

Bachman, R., 193, 329

Badrick, F. E., 233, 235, 32!

Baeder, D. H., 209, 241, 349

Bahn, R., 212, 218, 293, 329

Bahn, R. C., 203, 206, 207, 208, 209, 212, $218,219,283,329,335,352$

Baker, B. L., 209, 211, 222, 242, 253, 254, $257,272,329,330$

Baker, J. R., 221, 330

Baldridge, G. D., 256, 344

Ball, W. C., Jr., 204, 335

Barber, J. K., 204, 330

Barkalow, F. S., Jr., 318, 390

Barker, S. B., 229, 330

Barnett, S. A., 131, 185, 257, 265, 266, 269, 303,330
Barraclough, C. A., 221, 390

Barry, M. C., 249, 330

Bartter, F. C., 201, 202, 203, 204, 330, 344, 346

Bascom, W. D., 213, 348

Bastenie, P. A., 232, 330

Bates, R. W., 240, 28.5, 331, 348

Bauman, E. J., 227, 345

Beer, J. R., 305, 322, 330, 334

Beetham, W. P., Jr., 223, 226, 339

Bell, G. H., 223, 224, 225, 330

Bell, J. F., 225, 253, 320, 340

Bell, W. R., 215, 384

Benfey, B. G., 214, 215, 348

Benua, R. S., 196, 197, 380, 342

Berry, M. M., 235, 351

Berson, S. A., 232, 330

Bethune, J. E., 223, 2:26, 339

Beyler, A. L., 292, 330

Biglieri, E. G., 202, 203, 204, 330

Bing, R. J., 244, 331

Birdsell, J. B., 97, 99, 185

Birmingham, M. K., 216, 331

Black, M. M., 209, 353

Bloch, E. H., 206, 244, 331, 344

Block, H., 257, 279, 351

Boas, N. F., 240, 331

Bogdanove, E. M., 230, 232, 331, 341

Bogoroch, R., 235, 331

Bohanan, E. H., 257, 274, 331

Bois, P., 238, 255, 381, 349

Boring, W. O., 256, 257, 331

Borris, J. J., 206, 207, 208, 209, 212, 21s, $219,283,852$

Botkin, A. L., 233, 831

Bourliere, F., 312, 331

Bourne, G. H., 193, 194, 331

Bradlow, H. L., 206, 207, 219, 249, 331

Braidwood, R. J., 97, 99, 185

Bridgman, R. M., 242, 253, 329

Brimblecombe, R. W., 233, 235, 257, 329, 331 
Broatbent, D. E., 70, 185

Brobeck, J. R., 213, 345

Broderick, C. B., 100, 187

Brown, D., 215, 351

Brown, R. Z., 257, 2SS, 294, 295, 381

Brownell, K. A., 193, 194, 195, 223, 224, $227,228,261,341$

Brown-Grant, K., 231, 232, 233, 234, 235, 236,331

Bruce, H. MI, 239, 331

Bull, P. C., 322, 331, 332

Bullough, W. S., 198, 210, 269, 332

Burks, R., 215, 351

Burn, J. H., 225, 332

Burrill, M. W., 259, 340

Burrows, H., 211, 21s, 219, 25s, 270, 2ss, $289,292,332$

Bush, I. E., 206, 207, 208, 212, 219, 332

Butt, A. J., 209, 349

Byrnes, W. W., 211, 212, 220, 257, 258, $259,309,332$

Cain, A. J., 195, 332

Caldwell, 'T. MI., 82, 184

Calhoun, J. B., 4, 6, 26, 32, 39, 43, 44, s1, $52,57,92,93,97,101,132,138,140$, $148,150,161,165,168,185,273,259$, $291,294,298,303,382$

Cannon, W. B., 224, 332

Cantarow, A., 234, 235, 350

Caplan, R., 230, 351

Carlson, L. 1)., 228, 234, 342, 352

Carpenter, C. R., 90, 91, 185

(asby, J. U., 4, 6, 26, 39, 4:3, 44, \$1, 165, 185

('isper, A., 204, 3.35, 3.46

('ater, O. B., 195, 2-13,3,395

Cavazos, 2N9, 333

Cervantes, L. F., 100, 187

Chang, M. (.., 248, 332

Charipper, H. A., 2272, 273, 334

Chart, J. J., 199, 201, 388

Chrek, W. R., 215, 216, 340

Chitty, 1)., 89, 179, 185, 242, 2.51, 261, 264, $27 \cdot 2,273,275,276,313,316,321,322$, $323,332,383$

Chitty, H., 222, 242, 261, 234, 272, 333

Christian, J. J., 159, 197, 202, 210, 2200, $221,222,225,226,242,244,246,247$, $250,251,25.5,257,254,259,260,262$, $264,265,266,267,265,269,27), 271$,
$272,273,274,275,276,277,279,280$, 281, 282, 285, 286, 287, 288, 289, 290, 291, 292, 294, 295, 296, 297, 29s, 299, $301,302,303,304,305,306,309,310$, $311,313,315,317,319,320,321,322$, $327,333,334,349$

Christianson, MI., 195, 334

Chu, J. P., 239, 334

Clarke, J. R., 242, 251, 263, 272, 2\$1, 291, $292,294,296,303,334$

Clayton, G. W., 213, 215, 216, 334, 340

Colien, A. I., 206, 331

Cole, D. F., 204, 334

Collias, X. E., 96,186

Collins, J., 2:34, 235, 240, 341

Cook, E. F., 322, 334

Cornfield, J., 28.5, 348

Crabb, E. D., 2.5., 344

Crew, F. A., 268, 269, 274, 334

Critchlow, B. T., 257, 394

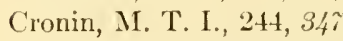

Croweroft, P., 221, 281, 2\$6, 287, 2\$8, 289, $290,291,292,294,308,334$

Curley, F. J., 221, 394

Currie, A. R., 19s, 205, 217, 249, 351

Dahlberg, B. I., 95, 96, 185

D'Angelo, S. A., 231, 232, 233, 234, 257, $258,272,273,334,350$

Davidson, C. S., 212, 213, 219, 38/4

Davidson, J. N., 223, 224, 225, 330

Davis, D. E., 247, 257, 264, 265, 266, 277, $278,295,301,302,303,306,307,309$, $310,311,320,333,334,395$

1)itvis, J. O., 202, 203, 204, 335, 352

D:tvis, М. E., 210, 259, 385

1):wson, J., 242, 274, 335

1)(2unc, H. W., 194, 195, 198, 201, 217, 24s, $335,340,343$

1) tuanesly, R., 196, 197, 33!'

DeBodo, R. C., 227, 335

Deevey, E. S., Jr., 97, 185

Delea, C. S., 202, 203, 204, 330

Delea, C., 204, 330

Delfa, E., 239, 343

Delost, H., 196, 336

Delost, P., 196, 197, 218, 219, 220, 283, 335,396

Dempsey, E. W., 195, 198, 217, 238, 248, 253,836

Deeney, R., 163, 186 
Denison, M. E., 238, 239, 257, 336

Desaulles, P., 255, 277, 346

Deselin, J., 219, 836

Despointes, R. H., 22:3, 224, 399

Dixon, W. J., 28s, 350

Dobriner, K., 207, 249, 330, 331

Dorfman, A., 209, 336

Dorfman, R. I., 206, 207, 208, 209, 212, $218,219,250,253,259,336,347$

Dougherty, T. F., 208, 209, 210, 211, 249, $250,254,255,256,336,348$

Doyle, D., 235, $\$ 47$

Drager, G. A., 214, $396,39 \tilde{4}$

Duff, W., 99, 186

Duncan, L. D., Jr., 204, 330

Duncan, L. E., 202, 203, 204, 34 '

Eberhard, T., 235, $34 \%$

Egdahl, R. H., 213, 350

Ehrich, W. L., 209, 210, 235, 242, 253, 259, $260,336,349$

Eidinoff, M. L., 249, 330

Eik-nes, Ki., 265, 336

Eisenstein, A. B., 200, 222, 336, 3.1

Elias, H., 194, 195, 196, 337

Eliot, T. S., 244, 344

Elmadjian, F, 223, 226, 227, 244, 387

Elton, C., 82, 186, 322, 323, $38 \%$

Emlen, J. T., 271, 281, 2S4, 255, 2s7, 288, $289,293,296,297,295,345,350$

Engel, F. L., 209, 244, 337

Erachkoe, O., 198, 225, 227, 387

Erickson, A. B., 322, 337

Ermans, A. M., 232, 330

Errington, P. L., 298, 317, 397

Ershoff, B. H., 234, 397

Evans, C. A., 321, 322, 339, 340

Evans, E. S., 228, 337,349

Evans, H. M., 199, 227, 229, 346

Everett, J. W., 257, 258, 337, 345

Fainstat, T. 1)., 210, 221, 259, 838

Farrell, (․ 1., 199, 202, 203, 204, 206, 204, $209,213,215,329,337,338,350$

Feldman, J. I)., 195, 338

Finerty, J. C., 237, 270, 254, 292, 341, 347

Finland, M., 20S, 209, 210, 211, 255, 256, 343

Fitch, H. S., 317, 338

Fleming, R. B., 202, 203, 209, 213, 398

Flexner, L. B., 195, 398
Flick, S., 309, 338

Flueckiger, E., 232, 388

Flyger, V., 306, 320, 332

Foenss-Bech, P., 209, 213, 344

Folley, S. J., 275, 328

Foot, E. C., 289, 343

Ford, E., 232, 328

Fortier, C., 213, 215, 216, 257, 388, 341

Frank, F., 225, 253, 272, 280, 298, 320, 321, $322,323,338$

Fraser, F. C., 210, 221, 259, 338

Fredericson, E., 47, 186

Fredrickson, D. S., 227, 338

Freedman, H. H., 234, 238, 338

French, L. A., 213, 350

Fruit, A., 214, 345

Furth, J., 206, 212, 218, 283, 329

Gaddum, J. H., 225, 224, 225, 226, 227, 338

(rallagher, T. F., 206, 207, 218, 219, 249, $330,331,338$

Gamn, P. S., 203, 204, 330

Ganong, W. F., 338

Gaunt, R., 199, 201, 338

Gersh, I., 194, 196, 339

(ierwing, J., 236, 237, 399

Gesehwind, I. I., 209, 213, 344

Gierlach, Z. S., 235, 341

Giroud, C. J. P., 199, 200, 202, 339, 350

Glaubach, S., 210, 259, 389

Glazer, N., 163, 186

(iliek, D., 196, 339

(iodfrey, (i. K., 321, 322, 323, 339

Godwin, J. T., 278, 350

Goldberg, R. C., 232, 339

Goldfien, A., 223, 226, 339

Goldstein, M. S., 209, 223, 347

Goodkind, M. J., 204, 335

Goodman, H. NI, 227, 339

Gordon, A. S., 211, 234, 238, 249, 251, 252, $254,256,272,273,334,338,339$

(iordon, R. S., Jr., 227, $33 s$

Gorski, R. A., 221, 330

Gould, R. P., 200, 329

Gowenlock, A. H., 201, 350

Grad, B., 240, 339

Grant, J. K., 195, 205, 217, 248, 351

Gray, G., 22S, 342

Gray, I., 223, 226, 339

Gray, s. J., 242, 25:3, 339 
Green, J. D., 213, 214, 339

Gren, R. G., 225, 253, 320, 321, 322, 399, 3.40

(ireene, R. R., 259, 340

Greep, R. ()., 195, 198, 201, 217, 232, 248, $335,339,340$

Greer, M., 230, 351

(rreer, M. A., 232, 234, 257, 258, 340, 352

(iriffin, A. C., 213, 352

Grollman, A., 194, 195, 196, 338, 339

Gross, F., 199, 201, 340

Gross, J., 231, 341

Grosvenor, C. E., 258, 275, 340

(iuettinger, R. C., 95, 96, 185, 186

Guillemin, R., 213, 215, 216, 33. $, 340,3: 4$

Hagen, P., 223, 340

Halberg, F., 285, 352

Halkerston, I. D. K., 257, 331

Hall, C. E., 202, 284, 341, 348

Hall, C. S., 89,186

Hall, O., 284, 341

Hallowell, A. I., 98, 186

Halmi, N. S., 230, 232, 331, 341

Hamilton, W. J., J'., 30s, 317, 320, 341

Hammond, J., Jr., 257, 341

Hamolsky, M. W., 235, 341

Hardin, G., 77, 186

Harris, ( . W. . 213, 231, 232, 233, 234, 235, $236,331,341$

Harrison, R. G., 194, 195), 196, 332, 341

Hart, P., 279, 341

Hartcroft, P. M., 200, 386, 341

Hartman, F. A., 193, 194, 198, 223, 224, $227,228,261,341$

Hatfield, D. MI., 317, 341

Hauser, E. A., 209, 349

Hayashida, 'T., 209, 213, 344

Haymaker, W., 257, 329

Hayne, D. W., 81, 186

Hearn, W. R., 215, 216, 840

Hellman, K., 234, 235, 240, 341

Hellman, L., 218, 219, 388

Hess, M., 237, 239, 270, 284, 292, 341, 3/4

Hill, R. T., 220, 341, 342

Hoffmann, R. S., 314, 317, 342

Hofmann, F. G., 206, 309, 342

Hollander, V. P., 206, 209, 3.47

Holman, J., 204, 395

Holmes, W. M., 196, 197, 342
Holzhauer, .11., 223, 224, 225, 226, 227, 339

Hope, J. M., 223, 226, 2.27, 24., 937

Hopsu, V., 225, 227, 337

Horn, E. H., 238, 342

Housholder, D. E., 215, 216, 340

Howard, E., 195, 196, 197, 212, 217, 330, 342

Hsieh, A. C. L., 228, 342

Hudyma, G. M., 238, 349

Huggins, C., 292, 342

Hughes, E., 306, 342

Hume, D. M., 216, 342

Hungerford, G. F., 209, 213, 344

Hurst, V., 235, 240, 342

Hutchinson, C. E., N., 77, 186

Ingalls, 'T. H., 221, 329, 334, 342

Ingle, D. J., 208, 209, 211, 253, 257, 329, 380,342

Irving, J. T., 210, 342

Isler, H., 238, 343

Ivy, A. C., 259, 340

Jacot, B., 213, 284, 292, 343

Jaffe, H., 233, 235, 352

Jameson, E. W., Jr., 307, 343

Jenkins, D., 285, 351

Jennings, H. H., 154, 186

Jensen, E. V., 292, 342

Jensen, H., 235, 341

Jensen, W. L., 320, 335

Jones, G. E. S., 239, 343

Jones, I. C., 193, 195, 196, 197, 199, 200, 201, 206, 207, 208, 209, 213, 215, 217. $218,219,254,261,283,394,343,347$, 352

Josimovich, J. B., 195, 343

Kabat, C., 96, 186

Kalas, J., 241, 349

Kalela, O., 221, 300, 308, 311, 314, 843

Kalter, H., 210, 221, 259, 338, 343

Kiappas, A., 218, 219, 338

Kass, E. H., 208, 209, 210, 211, 255, 256, 343

Katonah, F., 208, 210, 3/6

Katsh, G. F., 256, 339

Kedda, L., 206, 212, 218, 283, 329

Kelsall, M. A., 255, 344

Kemp, C., 233, 235, 352 
Kendrick, M. I., 208, 210, 211, 255, 343

Keuning, F. J., 211, 255, 344

Kew, MI., 99, 186

Kirschner, L., 232, 233, 250, 346

Ǩligman, A. M., 256, 344

Kiliman, B., 203, 204, 335, 352

Knigge, K. M., 194, 196, 206, 344, 348

Linisely, M. H., 244, 34t

Knohil, E., 22- 2839

Iiraatz, C. P., 239, 344

Kracht, J., 235, 344

Iiraintz, L., 232, 233, 250, 346

Iiramer, J., 209, 344

Kuuhl, W. J., Jr., 232, 344

Kurlents, E., 216, 331

Kurzok, R., 346

Ladman, A. J., 195, 3.43

Laidlaw, J. C., 285, 851

Lamson, E. T., 223, 226, 227, 244, 337

Lanman, J. T., 195, 344

Laqueur, G. L., 215, 257, 258, 338, 344

Larson, C. L., 225, 253, 320, 340

Leathem, J. H., 2SS, 344

Leblond, C. P., 238, 240, 399, 343

LeMaistre, C. A., 210, 279, 344

Le Munyan, C. D., 257, 258, 260, 274, 275, $290,294,295,313,315,322,333$

Leopold, A. S., 21, 186

Leslie, P. H., 242, 261, 264, 272, 333

Levy, A. C., 227, 344

Li, C. H., 199, 209, 211, 212, 213, 219, 227, $257,292,329,330,344,346$

Lichtlen, F., 199, 201, 340

Liddle, G. W., 202, 203, 204, 330, 344

Lieberman, S., 206, 212, 219, 351

Lillie, R. D., 253, 344

Lipner, H. J., 230, 341

Lipscomb, H. S., 213, 215, 216, 340

Lipsett, M. B., 21S, 219, $38 s$

Long, D. A., 236, 237, 399

Longwell, B. B., 206, 347

Lord, R. A., 278, 344

Lostroh, A. J., 199, 209, 212, 213, 219, 289, 344,345

Louch, C., 2\$4, 2\$5, 345

Louch, C. D., 252, 281, 2\$4, 285, 290, 292, $293,294,296,297,304,305,345$

Lundgren, M. M., 209, 210, 256, 343
Lutwak-Mann, C., 222, 259, 273, 288, 289, $309,310,345$

Lyons, W. R., 209, 213, 344

McCain, R., 24, 186

NicCally, N., 202, 203, 204, 209, 213, 329, 338

McCann, S. M., 213, 214, 257, 258, 344, 345

MeCarthy, H. H., 209, 344

MeDermott, W., 210, 279, 344

McDonald, R. K., 215, 345

Mac Lulich, D. A., 319, 345

Ic Nutt, S. H., 196, 352

McPhail, M. II., 196, 197, 217, 282, 345

Mahler, R. F., 201, 350

Manly, B. M., 257, 330

Mann, T., 2ss, 2s9, 345

Mall, R., 306, 342

Magsood, M., 238, 345

Marine, D., 227,345

Markee, J. E., 257, 258, 345

Marmorston, J., 215, 348

Marrian, G. F., 222, 257, 345

Nartin, E. P., 317, 345

Nartorano, J. J., 253, 345

Mason, J. W., 244, 253, 267, 268, 346

Neier, R., 255, 277, 346

Meites, J., 235, 347

Nelampy, R. M., 289, 382

Melby, J. C., 213, 350

Menkin, Y., 254, 346

Merrill, P., 232, 233, 250, 346

Meyer, R. K., 211, 212, 220, 252, 258, 259, $281,285,305,930,332,345,350$

Neyers, I. MI., 209, 344

Miller, R. A., 196,846

Mills, I. H., 202, 203, 204, 330, 346

Mirskaia, L., 268, 269, 274, 384

Money, W. L., 232, 233, 234, 237, 250, 346

Moon, H. D., 199, 209, 212, 213, 219, 22.' $394,344,346$

Moore, W. W., 257, 258, 346

Mora, P. MI., 97, 187

Moreno, J. Is., 154, 186

Morgan, B. B., 196, 352

Morin, G., 22S, 346

Morse, A., 195, 335

Mosier, H. D., 195, 346 
Moss, M. L., 210, 211, 346

Mulinos, M. G., 257, 272, 346

Nalbanov, A. V., 257, 258, 346

Nelson, D. H., 206, 207, 208, 346

Nelson, D. M., 216, 348

Nelson, M. MI., 222, 257, 260, 273, 350

Nezamis, J. E., 255, 348

Nichols, J., 202, 346

Noble, R. L., 208, 346

Norkus, M. G., 247, 353

Norton, H. W., 257, 258, 346

Nowell, N. W., 215, 216, 346

Oastler, F. G., 198, 205, 217, 248, 351

Ochs, M. J., 196, 339

O'Donnell, U. J., 198, 205, 217, 248, 351

Odum, E. P., 317, 346

Palmer, J. G., 216, 348

Parkes, A. S., 222, 257, 34.5

Parmer, L. G., 20S, 210, 346

Parsons, F. M., 292, 342

Paschkis, K. E., 234, 235, 347, 350

Patric, E. F., 67, 85, 186

Pauly, J. E., 194, 19;, 33\%, 34\%

Pearson, O. H., 218, 219, 398

Pearson, O. P., 175, 186

Pechet, M. M., 204, 3.35

Peron, F. G., 206, 34i

Perry, W. F., 232, 347

Peterson, R. E., 203, 204, 335, 352

Philbrook, F. R., 221, 342

Phillips, J. G., 195, 352

Piletta, P., 199, 200, 339

Pillsbury, D. M., 256, 344

Pincus, G., 288, 332

Pitelka, F. A., 307, 319, 32:3, 347

Pitt-Rivers, R., 236, 237, 399

Plotz, E. J., 210, 259, 835

Poindexter, H. A., 257, $34 \%$

Pollard, M., 210, 256, 347

Pomerantz, L., 257, 272, 346

Poore, W., 206, 209, 347

Porter, J. C., 214, 215, $34 \%$

Preble, E. A., 319, 347

Price, D., 288, 289, 345

Pronove, P., 204, 330

Prudovsky, S., 278, 385

Pruitt, R. 1., 215, 348

Puntriano, G., 235, 347
Raeisaenen, L., 198, 227, 397

Rall, J. E., 229, 230, 231, 238, 347, 351

Ramey, E. R., 209, 223, 225, 227, 344, 347

Ramey, C. G., 242, 253, 399

Rand, M. J., 225, 332

Randall, H. T., 244, 347

Ransom, R. MI., 221, 330

Ranson, S. W., 82, 185, 186

Ratcliffe, H. L., 202, 225, 244, 250, 271, 333,347

Rausch, R., 318, 320, 322, 323, 347

Rauschkolb, E. W., 199, 202, 203, 208, 209, 213,398

Rawson, R. W., 229, 230, 231, 232, 233, $238,250,346,347,351$

Read, C. P., 257, 277, 278, 335

Read, H. C., 196, 197, 217, 282, 34.5

Rebell, G., 256, 344

Reed, C. A., 97, 99, 185

Rees, R. J. WV., 279, 341

Reichlin, S., 231, 232, 233, 234, 235, 236, $237,331,347$

Reif, A. E., 206, 347

Reinhardt, W. O., 209, 213, 344

Reineke, E. P., 237, 238, 345, 352

Reinhardt, W. O., 292, 329

Reiss, J. M., 233, 235, 329

Reiss, M., 233, 235, 257, 329, 331

Rennels, E. G., 214, 270, 284, 292, 341,347

Renzi, A. A., 199, 201, 398

Retzlaff, E. G., 268, 269, 274, 277, 315, 347

Richards, J. B., 215, 848

Richardson, H. C., 213, 352

Richardson, J. A., 223, 348

Richter, C. P., 195, 198, 202, 348

Riesman, D., 163, 186

Riney, T., 24, 186

Ring, G. C., 234, 348

Rioch, D., 257, 258, 344

Robert, A., 255, 348

Robinson, H. J., 210, 254, 256, 348

Roby, C. C., 196, 343

Rogers, P. V., 195, 198, 202, 348

Rosasco, E. M., 80, 85, 187

Posemberg, E., 257, 258, 285, 344, 348

Rosenberg, L. L., 349

Rosenfeld, G., 213, 253, 321, 348

Rothballer, A. B., 214, 348

Rowe, F. P., 221, 281, 286, 288, 289, 290, $291,292,294,308,334$ 
Royee, P. C., 199, 202, 203, 208, 213, 338 Rumsfeld, H. W., Jr., 214, 215, 347

Runfret, A. P., 213, 352

Sacks, J. G., 82, 185

Saffran, M., 214, 215, 348

Sakiz, E., 218, 348

Salter, W. T., 230, 348

Samuel, L. T., 216, 348

Sandberg, A. A., 216, 348

Sanders, R. D., 96, 186

Santisteban, G. A., 208, 211, 250, 348

Sawyer, C. H., 257, 258, 334, 337, 3.45

Sayers, G., 195, 197, 195, 215, 243, 246, $248,348,351$

Sayers, M., 195, 197, 198, 243, 246, 248, 348

Scarborough, H., 223, 224, 225, 330

Schaefer, E. S., 143, 155, 162, 186

Schairer, M. A., 257, 329

Schally, A. V., 214, 215, 348

Schapiro, S., 215, 348

Scharrer, B., 214, 348

Scharrer, E., 214, 348

Schindler, W. J., 194, 206, 348

Schmidt, L. H., 256, 349

Schmitenloew, C. G., 224, 349

Stchneebeli, Cr. I., 20s, 209, 210, 254, 255, 256,336

Schneirla, T. C., 89, 186

Schorger, A. W., 96, 186

Schreiner, L. H., 257, 258, 344

Schuler, W., 255, 277, 346

Schwartz, H. S., 229, 330

Scott, J. C., 242, 261, 264, 272, 333

Scudder, J., 244, 349

Sealander, J. A., Jr., 273, 349

Seifter, J., 209, 210, 238, 241, 242, 253, 259, $260,336,349$

Seligman, A. M., 194, 385

Selye, H., 192, 208, 209, 211, 212, 213, 217, $220,236,238,242,243,244,250,253$, $254,255,256,257,258,260,261,264$, $274,275,276,284,288,292,391,343$, 349

Share, L., 216, 349

Shaw, J. H., 195, 201, 335

Shipley, E. G., 211, 212, 220, 257, 258, 259, 309, 332
Shipley, R. A., 206, 208, 212, 218, 219, 250, 259,336

Shiras, G., 96,186

Shorr, E., 209, 353

Shwartzman, G., 210, 256, 349

Sideman, M. B., 209, 213, 344

Simay, Kramer, M., 209, 344

Simpson, M. E., 199, 222, 227, 228, 257, $260,273,346,349,350$

Simpson, S. A. S., 200, 329

Sloviter, 239, 331

Slusher, M. A., 215, 216, 24s, 34.4

simelser, G. K., 240, 349

Smelser, J., 3.46

Smith, A. L., 210, 254, 255, 348

Smith, E. K., 220, 349

Smith, J. D., 213, 215, 216, 340

smith, R. W., Jr., 257, 348

Snyder, R. L., 309, 349

Sobel, H., 215, 348

Sollman, T., 229, 230, 231, 238, 3.49

Sonenberg, M., 230, 231, 23S, $34 \mathrm{\gamma}$

Southern, H. N., S9, 179, 185, 2\$9, 34.9

Southwick, C. H., 252, 265, 281, 285, 287, $288,289,293,294,295,296,297,350$

spalding, M. H., 195, 343

Speirs, R. S., 211, 252, 285, 350

Spirtos, B. N., 230, 341

squires, IV. L., 256, 349

Srebnik, H. H., 222, 257, 260, 273, 350

stachenko, J., 199, 200, 202, 339, 350

Stack-Dunne, M. P., 19.5, 213, 332

Stadler, J. B., 216, 349

Stanbary, S. W., 201, 350

Steinberger, E., 2\$\$, 350

Stevens, C. E., 234, 350

Strecker, R. L., 271, 281, 287, 288, 289, $293,296,297,298,350$

Stewart, J. O., 97, 186

Stoner, R. D., 278, 350

Story, J. L., 213, 350

Sturgis, S. H., 258, 350

Sundberg, R. D., 211, 350

Sunderman, F. W., 234, 350

Swanson, H. E., 228, 229, 234, 350

Sweat, M. L., 206, 350

Swift, E., 96, 186, 187

Srdnor, K. L., 215, 351

Symington, T., 19S, 20., 217, 24s, 351

Szego, C. M., 292, 330 
Tait, J. F., 200, 329

Tamura, Y., 196, 198, 351

Tanaka, R., 31S, 351

Tata, J. R., 229, 351

Taubenhaus, M., 208, 209, 210, 254, 351

Tenney, A., 232, 328

Tevis, L., Jr., 24, 186

Tew, J. T., 233, 891

Thomas, L., 210, 254, 255, 256, 351

Thompson, D. Q., 31S, 351

Thorn, G. W., 285, 351

Thorn, N. A., 241, 851

Timiras, P., 235, 331

Tobach, E., 89, 186, 257, 279, 351

Tompsett, R., 210, 279, 344

Traum, R. E., 231, 232, 233, 234, 257, 25s, 334

Turner, C. W., 235, 240, 258, 275, 340, 342

Tyler, F. H., 216, 3.48

Tyndale-Biscoe, C. H., 322, 351

Uhrich, J., 271, 351

Uotila, U. U., 23s, 336

Vander Laan, W. P., 230, 351

Van der Slikke, L. B., 211, 255, 344

Vande Wiele, R., 206, 212, 219, 8.51

Van Middlesworth, L., 235, 351

Venning, E. H., 200, 339

Verzár, F., 232, 338, 352

Vetulani, T., 276, 352

Vidovic, V., 232, 352

Visscher, M. B., 285, 352

Ton Euler, C., 231, 331

Von Euler, U. S., 223, 234, 236, 352

Von Foerster, H., 97, 187

Wagner, H. N., Jr., 215, $\$ 45$

Walker, D. L., 256, 257, 331

Wallach, D. P., 237, 352

Waring, H., 196, 197, 852

Waruer, L., 244, 344

Watt, L. J., 309, 352
Weaver, J. A., 211, 249, 250, 352

Webb, W. L., 32, 80, 82, 85, 185, 187

Weber, A. F., 196, 352

Welch, A. D., 223, 340

West, C. D., 218, 219, 338

Wexler, B. C., 213, 352

Whitney, D. MI., 210, 256, 852

Whitten, W. K., 275, 352

Whittle, P., 322, 352

Whyte, W. H., 163, 187, 198, 205, 217, 248,351

Wiese, V. K., 215, 345

Williams, R. H., 233, 235, 352

Williams, R. M., 322, 351

Williamson, H. O., 255, 257, 277, 38't

Wilson, B. R., 210, 256, 347

Wilson, H., 206, 207, 208, 209, 212, 218, $219,283,329,352$

Wolff, F., 232, 339

Woodbury, D. M., 213, 216, 352

Woods, E. F., 223, 348

Woods, J. W., 231, 232, 233, 234, 236, 341

Woodward, P., 213, 345

Wooton, R. MI., 247, 353

Wright, A., 195, 349, 352

Yalow, R. S., 232, 390

Yamada, T., 234, 352

Yankopoulos, N. A., 203, 204, 335, 352

Yatsu, F. M., 202, 203, 209, 213, 398

Zalesky, M., 196, 353

Zarafonetis, C. J. D., 241, 349

Zarrow, M. X., 23S, 239, 257, 336

Ziff, M., 232, 344

Zileli, MI. S., 223, 226, 339

Zimmerman, C. C., 100, 187

Zimmermann, K., 311, 353

Zippin, C., 26, 187

ZuRhein, G. MI., 25i, 391

Zweifach, B. W., 209, 244, 353

Zwemer, R. L., 247, 353 


\section{Subject Index}

AAF, 216

Aborigines

Australian, 99

ACTH, 197, 200, 209, 214, 220, 232, 243

assaying, 248

basal release rate, 215

\lrcorner-1 fraction, 203

endogenous, 249

exogenous, 249

inhibiting the release, 215

mice, 237

rabbits, 237

rats, 237

regulation of the release, 213

release, 216,217

release fraction, 215

secretion, 237,258

secretion of $\mathrm{ADH}, 241$

secretion of aldosterone, 202

synthesis, 216

Activity

hyperactivity, 9

minimal, 9

normal level, 16

normal nocturnal period, 16

2.t-hour rhythm, 9

endocrine glands of, 192

evolutionary, 123

Adaptive mechanisms, 190

atrophy, 244

Adaptive responses

measurements of the endocrine, 24:2

Adenohypophysis, 214, 233

arterial supply, 213

ADH, 201, 214, 241

Adirondacks, 55

Adjustment

learned, s9

Adrenal

androgen, 206

effect on nursing young, 276

atrophy, 238

ascorbic acid, 248

depletion, 192

Callothrix argentata, 196

cat, 195

Cercopithecus, 195, 196 chimpanzee, 195

cholesterol, 192, 193, 245

circulation, 195

Citellus tridecemlineatus, 196

Clethrionomys glareolus, 196

colobus monkey; 196

Colobus polykomos, 196

cortex, 191, 192, 193, 217, 245, 246

hormones, 199

human beings, 212

lipid vacuoles, 194

measurements of increased function, 245

Microsorex hoyi, 246

parenchyma, $19 j$

Sorex cinereus, 246

Sorex dispar, 246

Sorex fumeus, $2+16$

Sorex palustris, 246

secretory activity, 248

sex steroids, 219

sex steroidal activity, 218

sudanophilia, 197

$\mathrm{X}$-zone, 217

$\mathrm{X}$-zone of mice, $2 \$ 2$

zona fasciculata, 194

zona glomerulosa, 194, 199

zona reticularis, 194

zonation of, 194

demedullation, 22s

cow, 196

Crocidura, 196

gland, $19: 2$

effects of hypophysectomy on, 196

fetal zones of the, 195

general morphology of, 193

ground squirrels. 196

hamsters, 196

histologic measurements, 247

histology, 195

liumans, 195,196

hypertrophy, 234, 24ti

lipid content, 193

Loris, 196

Macaca mulatta, 195

macaque, 195, 196

marmoset, 196 
Adrenal-continued medulla, 193, 198, 223, 224, 245, 246 hypertrophy, 199

Microsorex hoyi, 246

Sorex dispar, 246

Sorex fumerus, 246

Sorex cinereus, 246

Sorex palustris, tumors, 199

Mesocricetus auratus, 196

mice, 196

Microtus agrestis, 196

Microtus arvalis, 196

monkeys, 195, 196

Ornithorhynchus, 195

Oryctolagus, 195

Pan, 195

Perodicus potto, 195

pituitary, 197

Pitymys, 196

potto, 195

primates, 195

rabbits, 195

laboratory rabbit, 195

rats, $195,196,198$

Norway rat, 195

wild rats, 195,

Rattus alexandrinus, 195

Rattus norvegicus, 195

regulation of hormones, 213

reticularis, 195

samples, 247

slow loris, 196

Sorex araneus, 196

starvation, 196

steroids, 219

steroid secretion, 19:2

androgenic metabolites, 219

synthesis, 219

Tachyglossus, 19.;

transplantation, 218

ungulates, 196

vascularization, 19.;

weight, 192,245

factor, 216

fighting, 264

variation, 246

X-zone, 196

Adrenalectomized animals, 201

Adrenocortieal activity

indicess, 211 in natural populations, 300

sudanophilia, 248

Adrenocortical-gonadal interrelationships, 218

Adrenocorticotropin, 196, 197, 205, 213, 220,222

release, 216

secretion, 215

secretion of aldosterone, 202,205

inaccuracy of determining, 308

Age

determination by teeth, 311

Alarm stimulus, 192

Alberta, 54

Albino mice (see also mice)

trichinosis, 278

Aldosterone, 199

release, 217,243

secretion

decrease in blood volume stimulates, 204

and pulse pressure, 204

stimulation by decrease in blood volume, 204

regulation, 202, 203

renal hemodynamics, 204

stimulation of, 203

Aliesterase, 200

Alley

emotional activity, 175

Alountta palliata, 90

Alpha individuals, 59

central, 59

Amines

pressor, 225

Amine oxidases, 223

Androgen, 217

adrenocortical, 219

cortical, 220

precursors, 206

Androgenic steroids

house mice, 220

man, 220

rats, 220

Androstenedione gonadotropic activity, 220

Animal (see also specific animal names), 239

laboratory, 262

Anteliomys smithii

inhilition of reproductive function, 318 
Antibody formation, 255,278

inhibition, 210

Antibodies stored, 211

Antidiuretie hormone, 201

Antigonadotrophic activity, 211

Area determination, 84

Ascendency dominant, 55 psychological, 55

Ascorbic acid, 217, 237 adrenal, 216, 248 factor, 216

Associations intraspecific, 64

Audition, 38

Australian aborigines, 97

Avoidance of a strange field, 17

$\Lambda$ wareness of self, 150

$\mathrm{AIFF}, 216$

Bainbridge cardiac reflex, 230

Balance electrolyte, 192 fluid, 192

Barter Island, Alaska, 318

Basic group size, 113, 125

Basic number, 3

Bats (see also generic names such as Myotis), 193

Behavior, 15, 158

alterations, 94

cultural origin, 124

exploratory, 175

genetic origin, 124

Behavioral sink, 93 development by Norway Rat, 92

Beta individuals, 59

Bivariate normal distribution, 20 function, 4, 39

Blarina, 29, 30, 31, 32, 36, 38, 52, 61, 64, $66,68,69,72,75,76,78,80$ population, 305

Brain subcortical portion, 15
British Columbia

indians of, 99

Broadbent's theory, 70, 72

Brown fat, 194

Cages

"life-space," 179

Callothrix argentata, 196

Capture cumulative probability, 21

Carbohydrate-active corticoids suppressive effects, 209

$\mathrm{C}_{19}$

steroids, 206, 217, 219

ketosteroids, 205

Caribou, 122

Catch comparative, $29,30,31,32$ decline, 56

Huntington Wildlife Forest, 29, 30 Maine (1950) and Maryland (1953), 31

Peromyscus and Clethrionomys, 32

Cattle, 194

Cells

inflammatory, 210

Census

30-day, 53

Central nervous system, 191

integrative role, 242 memory store, 134

Cercopithecus, 195, 196

Cervus nippon, 323 population density, 306

Chadwick Woods, 76

Changes environmental, 190 liver, 260 pancreas, 260 parotid, 260 visceral degenerative, 260

Chimpanzee, 195

Chromaffin cells, 198 tissue, 195, 223, 225

Circulatory collapse, 244

Citellus tridecemlineatus, 196

Clethrionomys, 27, 30, 31, 32, 33, 34, 35, 36, $52,53,54,55,61,67,68,69,70,75$, $78,80,83,85,88,314,323$

inhibition of growth and reproduction, 316 
Clethrionomys - continued relationship between population density and reproduction, 310

reproductive function, 314

Clethrionomys glareolus, 196

Clethrionomys rufocanus, 311 population density, 308,313 territory, 300 inhibition of reproductive function, 318

Close confinement effects of, 271

Codominants, 67

Cold

adaptation, 228

exposure, 234

Colobus polykomos, 196

Colony

compact, $\$ 6$ evolution of, 88

Howler monkeys, 90, 92

Norway rat, 92

formation, 87

stability, 89

Communication, 38, 125

low frequency, 59

Communication

reduction, 67

vocal, 70

Communication constant, 133

Communication function $(\mu), 126$

Community, 2, $8,32,35,49,55,74$, is

Blarina: Peromyscus, 73

one-species, 73

psychological apex, 79

small-manmal, 75

Sorex: Clethrionomys, 73

subordinate member, is

Competition, 78

food and social, 271

social, 262

"Competitive exclusion," 77

Configuration active rejection of new, 179

physical nonsocial, 183

seeking of new, 178

Conformity, 162

Constellation, 85

acljoining, 60

derivation of compact colonies from, 86

expected viability in, 61

formation, 57 individuals forming, 63

loosely knit, 88

number of individuals forming, $6 \mathrm{i}$

periphery, 60

theoretical, 62

Contact

between individuals, 105

decrease of responsive-responsive, 107

encounters, 106

frequency, 38, 106, 139

frequency of responsive-responsive, 106

individual, 106

refractory, frequency of, 105

responsive-refractory, 103

responsive-responsive, 103

social perception, 136

variables determining, 101

Copulation pressure

criticized, 293

Cortex (see also adrenal cortex), 72

adrenocorticotropin, 197

cellular hyperplasia, 213

fasciculata, 198

hypertrophy, 213

Cortical glomerulosa, 216

Cortical hormones relationships, 212

Cortical hyperplasia cortical, 218

Cortical mass

increase, 135

Cortical stroma

(arnivores, 194

rodents, 194

Corticoids carbohydrate-active, 220,$24 ;$

secretion, 222

Corticosteroids

release, 213

Corticosterone, 205, 206 mice, 208

rats, 208

Corticotropin peptides, 200

Corticotropin-releasing factor, 214

Cortisone, 196, 197, 205, 232

tetratozenic effects in mice, 210

Cotton rat, (see also Sigmodon), 323

Cow, 196

Creativity, 162

Crocidura, 196 
Crocidura russula, 197

Cultural disturbance, 99

Cynomys ludovicianus, 122

Cytochrome, 230

Cytochrome oxidase, 223, 230

d-gene, 134

differentiation in a similarity rank hierarchy, 142

dominant, 128, 141

recessive, 128,141

transformation of recessive, 159

DCA, 205

DOC, 205

DOCA, 205

Deer

adrenals, 305

Northern Wisconsin, 95

white-tailed, 95

yarding, 95

Deer mice (see also Peromyscus), 322

Dehydroepiandrosterone, 206, 212

Density

actual, 53

high, 53

increase, 138

low, 53

low spring, 54

Density function, $4,6,44,45,4 \overline{7}$

Cartesian coordinates, 4

Deoxycorticosterone, 196, 199

Diabetes, 241

Dicrostonyx (see also lemming) 323

Dicrostonyx groenlandicus

population cycles, 318

Diestrus, 239

Differences morphologic, 190

Diffuse motor activity (DMA), 175

Digestive organs effect of stimuli, 253

Diiodotyrosine, 230

Disease resistance in dense populations, 319

Distance between neighbors, 44 methods of calculating data, 44

Distribution spatial, 58,59

uniform, 81
Dog

adrenal medulla, 225

dominant, 265

hypophysectomized, 233

release of radioiodine, 233

subordinate, 265

secretion of aldosterone, 203

Dominance

psychological, 74, 75, 77, 79

Effector, 134

Eimeria stiedae, 322

Electrolyte balance, 201

11-Deoxycorticosterone, 205

11-Deoxy-17-hydrocorticosterone, 205

11B-Hydroxytestosterone, 211

11-OH4AD, 206, 211

Elk, 122

Embryo resorption of, 310

Emotional activity alley, 181

Encounters, 106

Endocrine

adaptive mechanisms, 191

adaptive responses, 189

function

inhibition, 221

organs

relationships, 227

Endogenous corticosteroid secretion, 211

Environment

abnormal, 108

external, 190

impact on, 43

of all individuals, 44

initial hyperactivity in a strange, 15

internal, 190, 261

optimum uniform utilization, 60

shifts, 190

structured, 11

two-dimensional, 8

Environmental factors, 190

Eosinopenia, 252

Eosinophil

as index of adrewocortical activity, 211

counts, 252, 285

as index of population density, 284

Epinephrine, 209, 216

action, 215

effects, 223,224

secretion, 226,227 
Estradiol, 208

Estrus, 239

changes, 258

Evolution, 51

eultural, 98,122

of a new species or genus, 87

of compact colony, 88

of a filtcring device, 134

proluable cause, 74

man's social, 184

social, 3,137

\section{Facilitation, 151}

Factor

communication-inhilsiting, 112, 139

contact-blinding, 112, 139

Families

human, 100

$\mathrm{F}: \mathrm{B}$ ratio

monkeys, 206

mice, 206

rabbits, 206

rats, 206

Feedback mechanisms

physiologic, 190

Female

asexual, is

estrus, 89

Ferguson activity alley, s, is

Fertility

density, 290

female, 239

male, 239

mouse, male, 290

Fetus

congenital defects, 210

growth and development, 2.59

prenatal mortality, 293

resorption, 95

Fighting

as a measure of soeial rank, 267 as form of stress, 264

\section{Filter}

Broadbent, 71

neural, 71,74

system, 77

Food

limiting population growth, 298
Food

seareity, 98

Food supply

effects on population growth, 296

Frequency

of meeting of responsive individuals, 112

of meeting of responsive-nonresponsive individuals, 112

Frustrations

optimum, 119

FSH, 259

Funetions

interaction, 113

Gene

$d-, 128$

frequency, 125

mutant, 125

Gene pool, 125

Gamma individuals, 59

Gastrointestinal traet

effect of stimuli, 253

integrative role, 242

Glomerular filtration rate, 201

Glomerulotropin, 203, 205

depression, 204

secretion, 204

(ilucagon, 240

Glucocorticoids, 24j

Glucose utilization inhibition, 209

Glycogen

exhaustion, 253

showshoe hares, 253

voles, 253

levels

factors altering, 253

Glycosuria, 209

Cionadal endocrine cells

steroids, 219

Gontdectomy, 219

Gonadotropin, 217, 219

inhibition, 211, 220

production, 211

secretion

suppression, 257, 309

Granulation

effect of corticoidson, 254 
Gravidun, 309

Ground squirrels thirteen-lined, 196

Group dynamics formulation, 101

Group size

basic, 3

change, 125

effect on reproduction, 91

optimum, 2

saltatorial changes in the basic, 122

satisfaction and frustration as a function 116

Growth, 254

effects of increased population density, 276

house mice, 299

population, 189

Guinea pig

harderian glands, 240

hydrocoltisone, 236

medullary hypertrophy, 227

pituitary-adrenocortical activity, 2,:3

Guinea pig

pneumococcal infection, 256

streptococcal infection, 256

thyroid activity, 236

tuberculosis infections, 256

Habitat, 42

marginal, 45

one-dimensional, 8

unstructured one-dimensional, 9

unsuitability, 80

Hamster, 196

golden, 194

poliomyelitis, 256

sudanophilia, 248

Hare (see also Lepus), 319, 32:3

mortality, 322

population density and parasitism, 32.2 relationships between population density and reproduction, 310

Harvest mice, 6

Hematopoiesis, 251

Hibernating gland, 194

Hierarchical situation, 263
Hierarchy formation

reductions in velocity of, 153

Histamine, 216

Homeostasis

circulatory, 192

electrolyte, 202, 205

fluid, 202, 205

Home range, 20, 25, 36, 38, 42, 164

behavioral origins of the bivariate normal, 8

bivarate normal, 4

center, $4,23,39,42,43,47,57,55,72$

actual, 64

"ideal" interval between, 45

optimum interval, 51

uniform distribution, 59

clumping, 67

contracted, 54

enlargement, 76

expansion, 68,79

fixed, 77

inhibition, $53,78,80$

intraspecific differences in size of, is

mutual inhibition of, 79

overlapping, 67

periphery, 51

reduction, 70

relative, 165

relative, of constellation nembers, 166

schematic

for Blarina, 65

for Peromycus, 6.5

for Pitymys, 66

sigma, 5, 6

size, 53

social inhibition, 70

travel-path, 24

IIomo sapiens, 57

Hormone

antidiuretice, 241

anti-inflammatory, $20 \mathrm{~S}$

fascicular action, 204

regulation of secrotion of the fascicular, 213

Host resistance

decrease, 210

effect of crowding, 279

infectious disease, 279

parasites, 279 
House mice (sce also mice, mouse, Mus), $140,14 \mathrm{~S}, 1 \mathrm{~S} 0,197,251$

ACTH and gonadotropin relationship, 260

adrenals, 206

adrenal cortex, 217, 282

adrenal weight, 266

albino, 269

birth rates, 292

competition, 271

competition for food, 297

crowding mortality, 280

decrease in reproduction, 286

food, 296

growth, 276

inanition, 272

inhibited growth, 299

intra-uterine mortality, 221

litter survival, 294

nests, 294

pituitary-adrenocortical activity, 273

resorption of the embryos, 221

responses to increased population density, 281

responses to sociopsychologic factors, 269

self-limited populations, 299

social pressure, 285

spermatogenesis, $28 \mathrm{~S}$

splenic hypertrophy, 272

trichinosis, 278

wild, 269

wild-stock, 280

Howler Monkeys, 90, 121

Humans, 196

Humans

dominant, 132

hypothyroidism, 238

responses to sociopsychologic factors, 269

thyroid, 232, 236

Hum field, 41, 50

Humoral substance, 16

Huntington Forest, 27, 61, 82

Hyaluronidase, 209

Hydrocortisone, 205, 236

Hydrocortisone

cats, 206

ferrets, 206

guinea pigs, 206 humans, 206

mice, 208,

monkeys, 206

rats, 208

sheep, 206

Hyperactivity, 175

in a strange environment, 16

Hyperglycemia, 209, 224, 226

Hyperthyroidism, 239

Hypertrophy cortical, 216

Hypoglycemic shock, 320, 321

Hypophysectomy secretion of aldosterone, 202, 203

Hypothalamic centers, 216

Indians

Kúunghit Haida, 99

Individuals

alpha, 59, 61, 165

beta, 59, 165

gamma, 59, 165

Infection

resistance to, 256

Inflammation

effect of corticoids on, 254

Inflection point

arithmetic, 121

Inhibitory influenee, 35

Input

rates, 56

Insulin, 240

Intensity of action, 112

Interaction

constant, 115

frequency, $10 \mathrm{~s}$

frustrating, 119

intensity, 115

maximum frequency, 114

positively affective, 112

refractory, 109

responsive, 109

variables determining, 101

Interconstellation matrix, 60, 65

Intraspecific competition, 263

Intrauterine mortality, 259

Invasion

induced, 80

Iodine, 230

Iraq, 99

Islets of Langerhans, 240 


\section{Isolation spatial, 69 \\ Isolation cage, 17}

Jarmo site, 99

Kendall's compound A, 205
B, 205
E, 205
F, 205

\section{Lactation}

failure, 260

inhibition, 294

Lemming (see also Dicrostonyx and Lemmus), 320

populations

cyclic decline, 319

mortality, 322

population cycles, 318

Lemmus, 305, 323

Lemmus trimucronatus population cycles, $31 \mathrm{~S}$

Lepus (see also hare), 323

Lepus americanus relationships between population density and reproduction, 310

Life-space quadrants shifts into the second and fourth by rats, 159

Lipid mobilizing factor, 241

Lipogenesis, 209

Litter mortality

factors causing, 295

mice, 294

size, 239

survival

effect of increasing density on, 295

effect of nests on, 295

factors, 294

mice, 293,294

voles, 293,296

Liver, 260

glycogen, 252

L.MF, 241

Loris, 196

Lymph nodes weights, 249, 250

Lymphocytes

as indices of adrenocortical activity, 211 criteria of stress, 251

counts, 251

Lymphocytolysis, 211

Lymphocytopoiesis

depression, 211

Lymphocytopoiesis

depression, 211

Lymphoid organs nucleic acid content of, 255

Lymphoid tissue growth, 250

involution, 250

Macaca mulatta, 195

Macaque, 196

Maine, 32, 54

Iisle

dominant, 89

rats, pansexual, 162

reduction of, 90

territorial, 161

Mammals (see also specific names)

adrenalectomized, 208

European, 196

Man (see also human) 121, 173, 194

anestrus, 220

basic numbers for, 97

corticosteroid production, $26 \mathrm{~s}$

ovarian atrophy, 220

Marking

with red dye, 76

Marmoset, 196

Marmota, 193

relationships between population density and reproduction, 310

Marmota monax, 122

Maryland, 32

Montgomery County, 69

Meadow mouse (see Microtus)

Medulla (see also adrenal)

adrenal, 198

basophilic granules, 198

chromaffin cells, 198

Medullary hyperplasia

mice, 198

ungulates, 198

Mesocricetus auratus (see also hamster), 194,196

Metestrus, 239 
Mice (see also house mouse, Mus, whitefooted mice, mouse), 26, 148, 170, 193, $196,198,212,218,222,257,264,277$, 293,309

adrenals, 200

adrenal weights, 246

adrenocortical responses, 270

albino, 264

androgen, 221

anestrus, 220

antibody formation, 278

blood level of TSH, 2:37

corticosterone, 236

Coxsackie infections, $256,2.57$

deeline in birthrate, 291

during pregnancy, 196

effect of new environment, 26i.

effect of overerowding, 269

effect of population density, 274

effect of social pressures, $26 \mathrm{~s}$

eosinophil counts, 252

fighting social competition, 2אA

gonadotropins, 309

harderian glands, 240

hierarchy, 265

hypoglyc'emic shock, 321

hypophysectomized, 212

inanition, 222

inbred, 274

inbred albino, 273

laboratory, 196

lactation, 274

medullary hypertrophy, 227

method of indueing an inflammatory response, 277

nursing, 210

ovarian atrophy, 220

ovaries, 220

pituitary-adrenocortical activity, 273

preumococcal infection, 256

preguant, 210

preputial glands, 292

radiothyroideetomized, 239

release of iodine, 238

reproduction, 274

reproductive suppression, 275

resistance to infection, 277

shock death, 225

social hierarey, 264

sociopsychologic interactions, 280 splenic hypertrophy, $242,273,274$

streptoeorcal infection, 256

susceptibility to trichinella, 277

testes, 238

thymus, $28: 3$

thyroid aetivity, 23.5

trichinosis, $25 \overline{7}$

tuberculosis, 257,279

tubereulosis infections, 256

Microsorex hiyi, 193, 246

Ificrotus (see also voles), 77, 301, 305, 323

hypoglycemic shock, 320

natural populations, 303

relationships between population density and reproduction, 310

"shock disease", 22.5

Microtus agrestis, 196, 2.51, 273, 323

behavior, 222

estrous pattern, 222

inhibition of growth and reproduction, 316

number of births, 22:2

responses to increased population density, 281

sociopsychologic factors, 264

splenic hypertrophy, 273

Microtus arvalis, 196, 218

ronfined populations, 272

crowding mortality, 280

sex accessories, 219

Microtus californicus, 323

reproduction, 317

Microtus montanus, 305, 316, 317, 320, 323 population density, 303, 314

population fluctuation, 303

Microtus ochrogaster

reproduction, 317

Microtus oeconomus

population eycles, 318

Microtus orcadensis

nueleus pulposus enlargement, 261

sociopsyehologic factors, 264

splenic hypertrophy, 273

Microtus pennsylvanicus, 323

adrenocortieal-density relationships, 304

adrenal weight, 285

body-adrenal relationships, 247

effeet of inereased population density, 284 
lice, 322

litter-size, 308, 317

population fluctuations, 304

productive cycle, 319

responses to increased population density, 281

Migrations

lemming, 86

Milk let-down reflect, 25 s

Monkey (see also Rhesus moukey), 194

brucellosis, 256

colobus, 196

corticoids, $26 \mathrm{~s}$

malaria, 256

Moose, 122

Mortality

alterations, 94

effect of crowding on, 279

immediate cause, 323

increase, 323

in dense populations, 319

mass, 322

of young

factors, 321

prenatal, 315

Mlotor activity, 16

Mt. Descrt Island, 33, 53

Mouse (see also mice), 196, 170, 175

adrenalectomized laboratory, 201

adrenal gland, 196

adrenal weight, 197

albino, 280

copulation pressure, 289

crowding mortality, $2 \$ 0$

development of, 196

dominaut, 264

fertility, $28 \mathrm{~s}$

fights, 296

male fertility, 289

Noninovolution of $\mathrm{X}$-zone, $25 \mathrm{~s}$

puberty, 197

Mouse (see also Clethrionomys)

red-backed, $27,52,54$

subordinate, 264

tundra, 318

velocity in a hierarchy, 149

Mule deer, 122

does, 24

fawns, 24

males, 24
Mus (see also mice and mouse)

inhibition of growth and reproduction, 316

Muskrat (see also Ondatra), 323

endocrines, 305

relationships between population density and reproduction, 310

reproduction, 317

reproductive organs, $30 \tilde{5}$

Mus musculus (see also mice and mouse), 196,217

medullary hypertrophy, 227

responses to increased population density, 281

Myotis, 193

Iyotis grisecens, 122

Myotis lucifugus, 122

IIyotis yumanensis, 1'2

Hyotis velifer, 122

Natural populations, 323

Natural selection, 122

Neighbors

contacting, 45 , 46

distance between, 50

number of, 51

perception of, 51

signal field, 47

sign field, 47

Nerves

postganglionic sympathetic, '22:3

splanchnic sympathetic, $22: 3$

Nervous system

sympathetic, 223

Neurohypophysis, 214, 241

Neutrophil

count, 252

New York, 33, 54

New Zealand, 322

Niche

characterization, 7

primary component of, $7 \bar{\tau}$

specializations, 74

NIH Emotional Activity Aller, 17

Norepinephrine, 209

effeets, 223,224

secretion, 226,227

source, 225

North American Census of Small Mimmals, 26 
Norway Rat (see also rat, Rattus), 87, 180,

$195,320,323$

adrenalectomized, 202

adrenal weight, 266,301

behavioral sink development by, 92

body-adrenal relationships, 247

domesticated, 163, 175

inhibition of reproduction, 307

intra-uterine mortality, 221

laboratory, 195

relationships between population density and reproduction, 310

reproductive depression, 291

resorption of the embryos, 221

responses to sociopsychologic factors, 269

rural population, 302

social competition, 273

sociopsychologic interactions, 280

subordinate males, 265

wild, 179, 183, 195, 265

Number

total of individuals, 110

Oak, 64

Ondatra (see also muskrat), 323 relationships between population density and reproduction, 310

Ondatra zibethica reproduction, 317

Open-field emotional behavior, 89

Orders, 122

Organ isolated, 191

Ornithorhynchus, 195

Oryctolagus, 193, 323

Os penis

stimulation, 212

Ovary, 194

Ovaries

androgens, 220

Pan, 195

Pancreas, 260

Pancreatic islets, 240

Pantothenic acid

deficient, 222

Parasitism, 322

Parotid, 260
Particle

basic, 2 general elasses, 3

Partner

choosing of, 154

data regarding the ehoosing of, 156

Perception, 89, 139

Perodicus potto, 195

Peromyscus, 29, 30, 31, 32, 33, 34, 35, 36, $52,53,54,55,61,64,66,67,68,69$, $70,75,76,77,78,79,80,88,323$

Peromyscus boylii inhibition of reproduction, 307

Peromyscus leucopus pituitary-adrenocortical, 273 population, 305

Peromyscus maniculatus inhibition of reproduction, 307 lice, 322

productive cycle, 319

Personality, 158

Phagocytosis

inhibition, 210

Physiologic adaptation generalized effects, 254

Physiology

elassical, 184

evolution, 60

reproductive, 95

Pigs, 194

Pine, 64

Pineal, 204

Pipistrellus (see also bat), 193

Pitressin, 214, 232

Pituitary

anterior, 197, 220

antidiuretic, 241

gonadotropins, 257

hormones, 218

lipid mobilizing factor, 241

posterior, 241

secretion of aldosterone, 203

Pituitary-adrenocortical exhaustion, 226 tumors, 218

Pitymys, 32, 52, 64, 66, 69, 70, 76, 196

Pitymys subterraneus, 196, 197

Population, 189

collapse, 314

densities, 190, 238

fertility, 290 
fixed size, 263

food-limited, 295

freely growing, 281

individuals of, 103

limited size, 280

methods for estimating natural, 305

natural, 190, 262, 300, 306

physiologic adaptation, 261

reproductive function, 306

resident, 56

self-limited, 298

size, 240

uniformly distributed, $5 \mathrm{~S}$

Population density, 226, 262, 287, 305

adrenocortical and reproductive responses, 269

effect on adrenocortical function, $2 \$ 1$

endocrine responses, 263

index of relative, 305

inflammation, 276

resistance to infection, 276

schematic summary, 326,327

Potassium, 204

excretion, 201

levels, 204

level of body, 202

secretion of aldosterone, 204

Potto, 195

Preconstellation phase, 58

Pregneninolone, 210

Preputial development

adrenal androgens, 292

stimulating, 292

stimulation, 212

Probability

formulas, 144

$P_{i}{ }^{(A)}, 144$

$\mathrm{P}_{\mathrm{i}}^{(\mathrm{a})}, 144$

terminating trips, 12

vacillating at the termination of trips, 18

Proestrus, 239

Psychology

classical, 184, 185

Rabbit, 193, 323

adrenalectomized, 236

corticosterone, 236

domestic, 236

embryo

resorption, 239 parasitism, 322

release of radioiodine, 233

thyroid, 232

thyroid activity, 235

thyroidectomy, 239

thyrotoxicosis, 235

wild, 235,236

Radial distance, 623

Radioiodine

release in domestic rabbits, 236

release in wild rabbits, 236

uptake, 232

Rat (see also Norway rat, Rattus), 13s, $159,182,194,196,212,218,309$

$\mathrm{ACTH}$ and gonadotropin relationship, 260

adrenals, 201, 206

adrenal atrophy, 237

adrenal cortex, 200

adrenalectomized, 253

adrenalectomized laboratory, 201

adrenal hypertrophy, 237

adrenal weight, 302

adrenocortical secretion, 222

androgen, 221

anestrus, 220

albino Osborne-Mendel strain, 181

blood level of TSH, 237

chorionic gonadotropins, 220

corticosterone, 236

domesticated albino strain, 171

domesticated Norway, 8

effect of temperature changes on, 274

gonadectomized, 219

gonadotropins, 212, 309

growth, 210

harderian glands, 240

house, 212

hypertrophy of medulla, 227

hypophysectomized, 216, 233, 254

hypothalamic-hypophyseal-thyroidal relationships, 233

hypothyroid, 234, 239

inanition, 272

in a strong environment, 303

inbred, 274

infant, 210

length of estrous cycle, 238

method of inducing an inflammatory response, 277 
Rat-continued

newborn, 210

Norway (see Norway rat)

Osborne-Mendel, 17

ovarian atrophy, 220

pituitary, 236

pituitary-adrenocortical activity, 273

pneumococcal infection, 256

postparturitional loss, 221

pregnant, 210

release of iodine, 238

splenic hypertrophy, 274

streptococcal infection, 256

submaxillary glands, 240

subordinate, 303

testes, 239

of immature, 238

thiouracil treatment, 239

thyroid, 232

thyroid activity, 235, 237

thyroidectomized, 228

tuberculosis infection, 256

uptake of radioiodine, 233

white, 237

wild, 195

Rat embryos

action of estrogens, 259

Rat society, 161

velocity in high-density, 168

velocity-rank relationships in, 168, 169

Rattus (see rat, Norway rat)

Rattus alexandrinus, 195

Rattus norvegicus, 195

adrenalectomized, 202

medullary hypertrophy, 227

relationships between population density and reproduction, 310

Recapture radii, 5, 6

Receptor, 50

Refractory period, 49, 141, 153, 176

duration, 103, 112

frustrating, 110

frustrating type, 131

frustrating producion, 112

increasing, 130

satisfaction producing, 112

time spent in satisfying, 112

Refractory state

maximal, 109

optimal, 109
Regression line, 81

Reichstein's compound S, 199, 20j

Reithrodontomys, 6

Rejection psychological, 183

Relationships spatial, 64

summary of presumed, 137

Relative density, 78

Relative probability, 45

Removal study

Chadwick Woods, Montgomery County, Maryland, 29

Rich Lake Island, New York, 27

Removal trapping observed data, 52

Renal nephron

tubular cells, 201

Reproduction, 190, 256-291

evaluating the effects of density on, 308

inhibition, 309

mice, female, 291

mice, male, 286

sexual, 133

suppression, 222, 257

Reproductive

inhibition, 220

function, 292

evaluation, 306

inhibited by population density, 292

inhibition, 323

organs

accessory weights, 287

Reserpine, 225

Response

avoidance, 93

degree, 192

endocrine, 191

inflammatory, 210

intensity, 151

neural, 191

neuroendocrine, 191

nonspecific, 192

vascular, 191

Response-evoking capacities, 143, 146

Response-evoking capacity

$S$

behavioral origin, 140

circumplex depiction of behavior and personality superimposed upon, 157 
circumplex, 155

formulae, 128,143

$\mathrm{S}^{(\mathrm{A})}, \mathbf{1 4 3}$

$\mathrm{S}^{(\mathrm{\Omega})}, 143$

$\mathrm{S}_{\mathrm{i}}{ }^{\mathrm{A}}, 12 \mathrm{~S}$

$\mathrm{S}_{\mathrm{i}}{ }^{(\mathrm{a})}, 128$

$\mathrm{S}^{(\mathrm{V})}, 143$

$\mathrm{S}^{(\mathrm{v})}, 143$

Responsive state, 140

Rhesus monkey (see also monkey), 267 hydrocortisone, 236

responses to sociopsychologic faetors, 269

thyroid activity, 236

lodents (see also specific categories), 212

Runaways

underground, 76

Salivary glands, 260

Sasctions, 151, 163

sicent, 35

Schaefer's autonomy, 143

Schaefer's control, 143

Schaefer's hostility-rejection, 128,143

Schaefer's love-acceptance, 12S, 143

Serotonin, 216

Sex accessories, 2:34

involution, 218

Sex steroids, 206,217

Sheep, 194

Shock, 192, 243, 244

"Shock disease," 226, 280, 320

Shrews (see also Sorex and Blarina), 26, 27, $75,193,197$

long-tailed, 246

population, 30;

short-tailed, 305

Sight, 38

Sigmodon (see also Cotton rat), $32: 3$

litter size, 317

relationships botween population density and reproduction, 310

Sign, 47

Sign field, 49

Signal, 53, 57, 167

detection, 41

inhibitory, 68

intensity, 48,49

learning of, 39,50

neural, 78 noxious, $7 \mathrm{~S}$

simultaneous, 48

sound, 41

Signal field, 47, 49

Sika deer, 323

mass mortality, 320

population density, 306

Size

basie group, $\$ 6$

effect on response-evoking eapacity, 130

Slow loris, 196

Snowshoe hare (see also hare), 253

Social behavior, 128

more effective, 136

Social dominanee, 74

adrenal aetivity, 267

changing, 89

Social factors

adrenocortical functions, 236

interspecific, 77

reproductive functions, 263

Social groups

budding off, 121

Social hierarchy evolution, 70

Social interaction, 133

basic processes, 116

eonsequences and examples, 148

frequency of satisfactory, 117

frustration from, 110

as a function of density, 117

heightened frequency, 9.5

intensity, 152

model, 101

optimum satisfaction, 118

and refractory period, 117

satisfaction from, 110

withdrawal from, 173

Social phenomena, 3

Soeial pressure

endocrine responses, 263

Soeial rank, 64

Soeial relations

instability, 67

Social response

blocking, 104

mechanism, 104

Social subordination

interspeeifie, 67 
Social system, 3,4 semiclosed, 100

Social withdrawal, 145

Society human, 184 rat, 161

Sodium: potassium ratio serum, 204

Sodium retention, 201

Sorex, (see also shrew), 27, 29, 31, 32, 36, $38,52,61,68,69,70,72,75,77,78$

Sorex araneus, 196,197

Sorex cinereus, 193, 246

Sorex dispar, 193, 246

Sorex fumeus, 193, 246

Sorex palustris, 193, 246

Soricidae (sce also shrew, Sorex, and Blarina)

adienal, 246

Space

general theory of use, 34

interspecific and intraspecific use, 34

social use, 2

theoretical conceptualization of use, 70

Spatial equilibriun, 57

Species, 3

alpha, 36, 37

beta, 36,37

distance between neighbors, 42

dominant, $36,52,67,68,78$

incipient, 74

relationship between two dominant, 52

removal captures, 55

socially dominant, 55,57

subordinate, $36,38,52,61,67,68$, $7 \mathrm{~S}$

survival, 122

spermatogenesis,

decrease, 258

spleen

house mice, 251

hypertrophy, 251

Microtus agrestis, 251

volves, 251

weights, 249, 251

Splenic hypertrophy, 271

Standard area, 5

State

nouresponsive $P, 140$

Stations

feeding, $9 \bar{j}$
Status

subordinate, 68

Steady state

evolutionary, 108

Steroid hormones, 218

Steroids, 248

sex, 258

site of action, 216

urinary, 249

Stimuli

alarming, 212, 215

Stimuli

auditory, 70

emotional, 243

conditioned, 75

Strange object reaction, 89

Strange-object response, 179

Stress, 8S, 193, 196, 213, 235

generalized state, 182

resulting from group size change, 118

Stressful state

amount of time spent, 112

Stressors

nonspecific, 119

Stretch receptors

secretion of aldosterone, 204

Strife

intraspecific, 262

Sudanophilia, 248

Supraopticohypophyseal tract, 214

Survival

threshold of, 132

Sylvilagus (see also rabbit), 193

Sympathetic nervous system ganglia, 198

System

hypothesized communication, is

two-species, 36

Tachyglossus, 195

Target diameter

determined by variability of hehavioral traits, 140

genotype, 140

hypothesized divergenee, 132

of individual, 110

variability, 127

Territory, 263

of male mice, 287 
Testis

interstitial cells, 194

Testosterone, $20 \mathrm{~S}$

Thetas satisfaction and frustration, 131

Thiouracil, 237

Thymicolymphatic system, 249

Thymus, 283

cortex, 249

involution, 208, 211, 249, 250

medulla, 249

weights, 249

Thyrocarotid artery, 204

Thyroglobulin, 230

Thyroid

activity

factors regulating, 231

index, 231

mice, 235, 236

$\mathrm{NaCl}$ intake, 238

rabbit, 236

rat, 235,236

gland, 191, 228

hormone

actions, 229

formation, 230

inhibition, 236

responses to external stimuli, 234

Thyroidal-gonadal interrelationships, 238

Thyroidectomy, 228, 234

Thyrotropin, 231

inhibition, 236

Thyroxine, 228, 229, 231

effects, 230

Tonus

vascular, 224

Trap, 20, 37

"Havahart," 184

probability of encountering, 52

Trapline, 53, 82

standard, 26

Trapped-out area invasion, 82

Trapping, 76

comparison of results between shortterm and long-term removal, 28

continuous removal, 26,37

removal, 76

short-term removal, 26
Tribe

as a social entity, 98

Trichinella increased invasiveness, 278

Trichinella spiralis, 277,278

Trichinosis

effects of crowding, 278

Triiodothyronine, 228, 229, 231

TSH

mice, 237

rabbits, 237

rats, 237

rate of release, 231

release, 232,236

secretion, $237,25 \mathrm{~S}$

Tuberculosis

crowding, effect on, 279

murine, 279

Two-dimensional field

use, 19

Tyrosine, 230

Ungulates

captive, 198

domesticated, 196

Urine

metabolites, 207

Vaginal orifice

closure, 291

Vagus nerve, 204

Vasopressin, 214

Velocity, 164

biological, 111

effect of interval between $E$ 's on, 181

effect of tranquilizer on, 172

effect on fat, 174

effect on weights of adrenals, kidneys, and heart, 174

formulae, 111, 112, 113

$\alpha, 112$

aaa, 112

$\alpha$ ap, 112

$\alpha \mathrm{faa}, 112$

$\alpha \mathrm{f}^{\prime}$ ap, 112

$\alpha_{\mathrm{m}} \mathrm{f}_{\mathrm{ag}}(\mathrm{m}), 113$

d, 110

faa, 112

f'ap, 112

i, 112 
$\mathrm{i}^{2}, 112$

$\mu^{\prime}, 112$

N, 110

$N_{b}, 113$

$\mathrm{N}^{(\mathrm{m})}, 113$

$\mathrm{N}_{0}, 113$

$\theta \mathrm{a}, 112$

$\theta_{\mathrm{a}}{ }^{(\mathrm{m})}, 113$

$\theta_{\mathbf{a}}{ }^{(0)}, 113$

$\theta^{\text {(a) }}, 112$

$\theta$ f, 112

$\theta^{(\mathrm{f})}, 112$

$\theta_{\mathrm{a}}{ }^{(\mathbf{m})}, 113$

$\mathrm{V}, 111$

minimal, 173

rank, $17 \mathrm{I}$

reduction in bierarchy of mice, 148

Visitation frequency, 165

Vitamin A

effects on behavior, 170

Vitamin B

insufficiency, 222

Vocalization, 38, 48, 75

loss of, 77

Voles, (see also Microtus, Clethrionomys, and Pitymys), 193, 196, 253, 261, 293, $317,318,322,323$

adrenalectomized, 218

adrenal weight, $285,286,301$

adrenocortical-density relationships, 304

anestrus, 220

birth rates, 292

competition for food, 297

confined populations, 272

copulatory pressure, 290

crowding mortality, 280

decline in birthrate, 291

decrease in reproduction, 286

depression of fertility, 221

effects of high density, 276

effect of increased population density, 284

effect of overcrowding, 269

effect of population densities, 27.5

eosinophil counts, 252

female, 286

fights, 296

hypoglycemic shock, 320,321

inhibition of growth and reproduction, 316 intraspecific strife, 323

laboratory, 274

litter survival, 294

meadow, 196, 218, 272, 323

northern montana, 304

mortality, 322

natural populations, 303

nests, 294

ovarian atrophy, 220

pine, 196

population fluctuation, 312

red-backed, 196, 311, 312, 314, 318, 323

relationships between population density and reproduction, 310

reproductive function, 314

responses to inereased population density, 281

responses to sociopsychologie factors, 269

self-limited populations, 299

social pressure, 2SS

sociopsychologic factors, 264

sociopsychologic interactions, 280

splenic hypertrophy, 242, 272, 273, 274

$\mathrm{X}$-zone, 283

White-footed nice (see also Peromyscus), 273,323

White-footed mice

population, 305

Withdrawal, 162

Withdrawal state, 180

Woodchuck (see also Marmota), 193

relationships betwcen population density and reproduction, 310

Wound healing

delay, 210

X-zone

acidophilic cells, 197

androgens, 197

basophilic nuclei, 197

Crocidura russula, 197

experimental treatments, 196

function, 217

hormones, 196

pituitary luteinizing hormone, 197

Pitymys subterraneus, 196, 197

reactions to, 196

shrews, 197 
Sorex araneus, 197

voles, 196

Zona fasciculata, 217

carbohydrate-active corticoids, $2 \mathrm{CO}$

hormones, 205

hyperplasia, 246

hypertrophy, 246

regulation of hormones, 213
Zona glomerulosa

aldosterone, 213

beef cattle, 200

hypertrophy, 246

rats, 200

Zona reticularis

carbohydrate-active corticoids, 200

function, 217

sex steroids, 217 






